


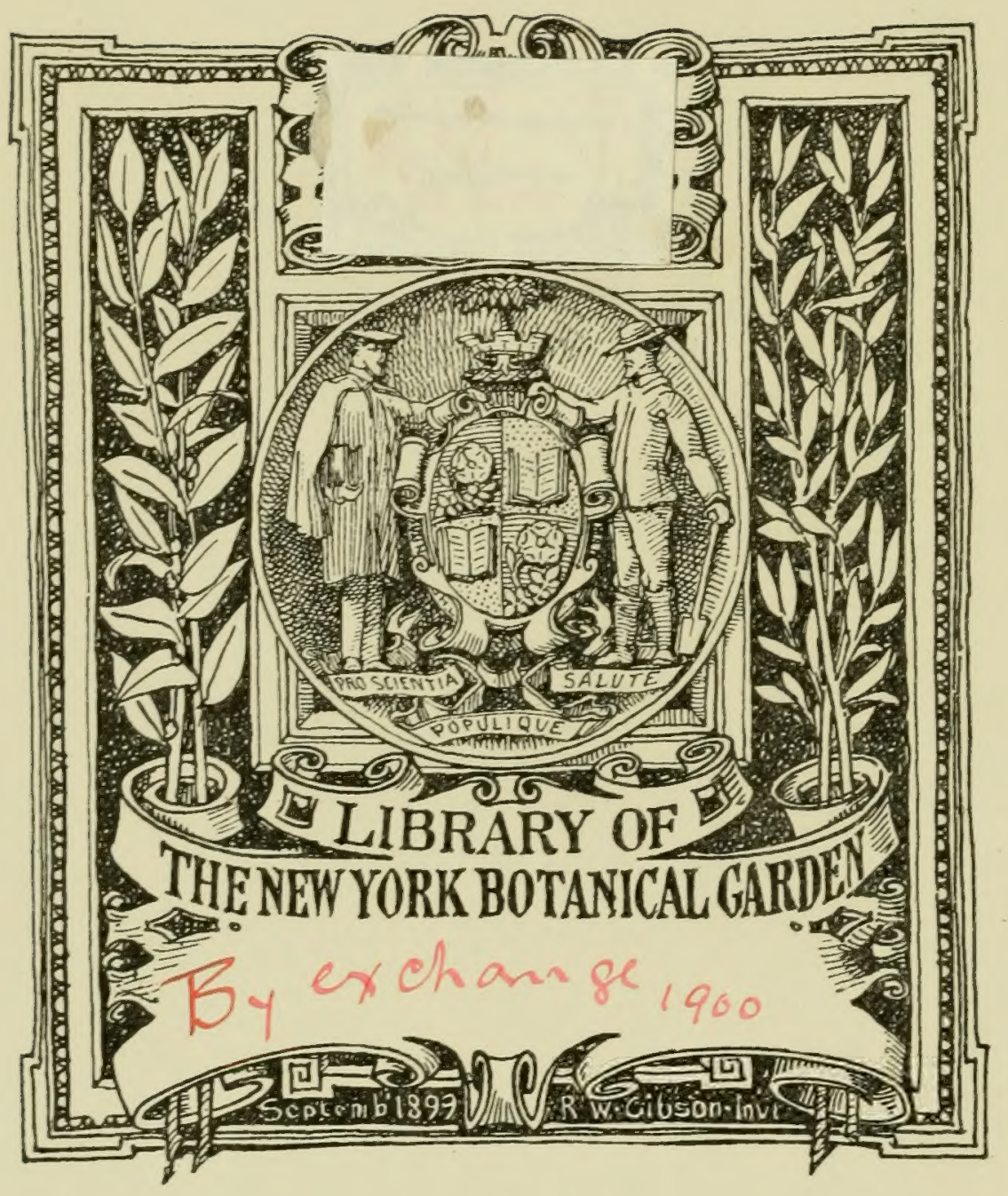






Moonographia

Rosarum Europae. Orientis. terrarumque ajacentium

auctore

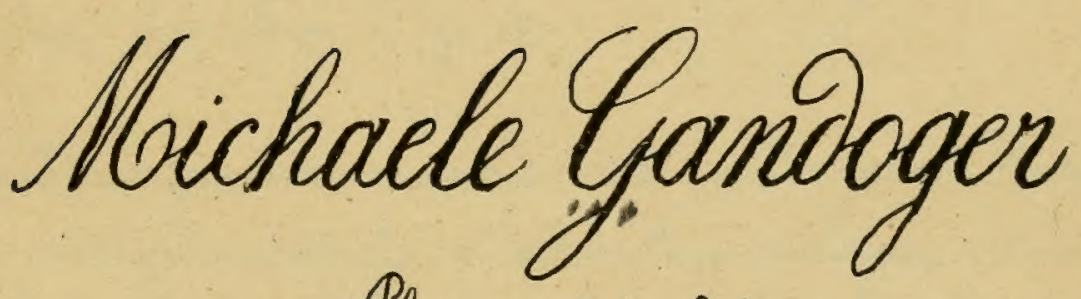

Slurim. societ sodali.

\section{$T_{\text {ome }}$ III}

complectens Canineas Hispiass, Tubescentes, Collinas et Gomentellas (scilicat subgeneris Crepiniace tribus Stictopososs, Brichophyllas et torenophoras).

Sarisuis, 7.93. Praillière et fill, bitliopolace, 19, via Hautepenille. 


$$
\begin{aligned}
& \text { Q1.145 } \\
& . R 6 \\
& 629 \\
& +3
\end{aligned}
$$


Monographia Rosarum Europae as Orientis terrarumque abjacentium.

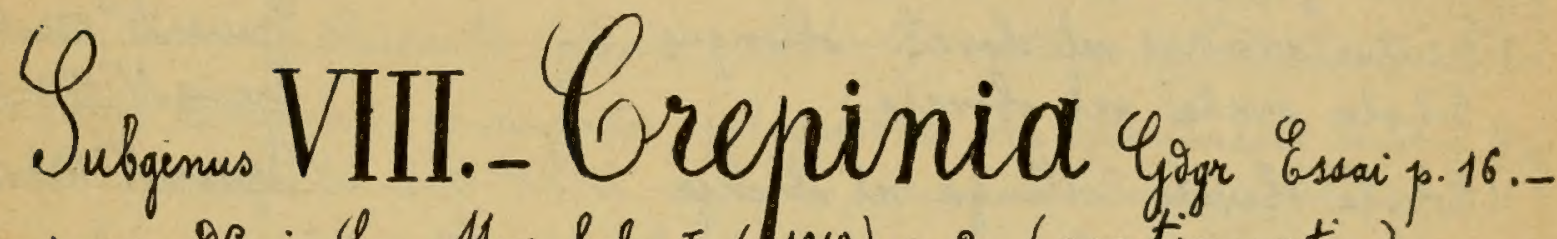
Camineare DC. in Gor. Mus. helr. I (1818),p.3. (continnicatio).

B.- Stictopodae Giogr Essai p. 28, Gorgr Eab. p. 148. Comineare hispisae Giép. Guim. I, p. 18.

Foliola unitique ylaberima, simpliciter pal argute bicereato, 1irentia, subtus vero glaucesentia et as costam mesiam plorumque glan-

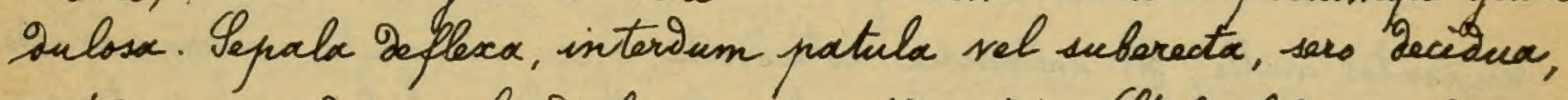
eatus saepins gorso glanioulosa, pimatipartita. Styli liberi, glaber; out villosi, wetrax descum capitati. Cosolla rosex, mekiscris rel ampla. Fructus pulpossus. Tedunculi semper glandulosi vel hropis: Accules phes minus falcato - Dilatati, nonnum quam ininophic ac in setis Glaniouliferis (of ramos proesertion flariforos) abeuntibus. Madia quantopque reptans.

Foliola ommino simpliciter serrata

a. Euandegavensed.

1 Folola sat persistentia, alia simplicitor, alia biserrata. B. Maciioe. Foliola aminia argute biserrata c. Omaphyllac.

a.- Euandegarrenses Goge Essai p.28; Gogr bab.p.149.

Tolislis simpliciter sereatis, sepalis post an thesin gefleacis moxque seciduis; aculeis conformibus; radice non reptante. 
$-2-$

Gtylu glabi aut vix hirtelli

1 S Styli hirsuti vel lanati

Rami Ploriferi inermes

2 Mrami florales aculeati

Foliola orbiculata, ovata vel oborata

3 Toliola sblonga

Seprala garso eglanioulosa

4 Sepala oorso glaniulosa

2

5

3

4

Sheries $\mathcal{N}^{2}+1106-1113$.

$\varphi_{\text {pecies }} N^{\prime}: 1114-1120$.

Srecies $N: 1121-1123$.

Ipecies $\mathcal{N}^{-1124-112 \mathrm{~g}}$.

Fructus sblongus

5 Fructus globosus, rotundatus vel ovactus

6 Fructus ovoidens vel obovato - oblongus Foliola ovata rel obavata

6 Folisla obarato - oblanga vel oblonga

$\varphi_{\text {pacies }} \mathcal{N} \cdot 1144-1150$.

Inecies $\mathcal{N}^{0}=130-1132$.

Species $N^{-1133-1137 . ~}$

Opecies $\mathcal{N}=1138-1143$

1. Styti glabri ant rix hirtell:

a. Rann floriferi inermes:

+ Foliola arbiculata, svata rel obovata.

1106. Crepinia semifoliosa Gorgor mss.

teculei tenmes, inclinati, parum oilatati, as ramos flor-basi semper ienuiatos nulli; fol mediscria, rotuniata, apice vix acuta, inferne coroata, intense vïidia, as costam eglanaulosa, Dentes lanceolato - convargentes, simplies; pet.culeati, glanaulosi, vic sparse pilosuli; stip. latae, virises, auriculis rectis; pest 1, vix hispiai, $7-8 \mathrm{~m}$. longi; tubus tratus, glaber; sep. extus ommins eglanioulosa, finmulis foliaces-incisis, instructa, icumine lato, integro; styli sublaberrimi, disco plano; cor, parro, albo-subcarnea, eciliata. pruct. notungatus, utrinque vix depressus, laevis.

Hab. Belgrim, Hainaut: is Harrengt (M. Ganooger).

1107. Crepinia hirtella (Rip. in Chist Rosen ger Gchw. p. 161). - C.camina f. hartella (Christ l.c.). Gigg Gab. N.1924;

Bculei pariciores, faleato-gilatati, is ramos flor-milli, fol ovatolaneolata, rcuminata, utrinque virisia, Dentes simphices, convergentes; fut. aculeati, sparse glanillosi, hine inde pilosi, stip. anguster, auricutis Sivergentibus, pes. hispisi; tubus oblongus, hanis; sep. extus eglaniarLosa, brevia, pinnata; styli glabri, grises cornis, cor. lacte rosea, fuct oblongus.

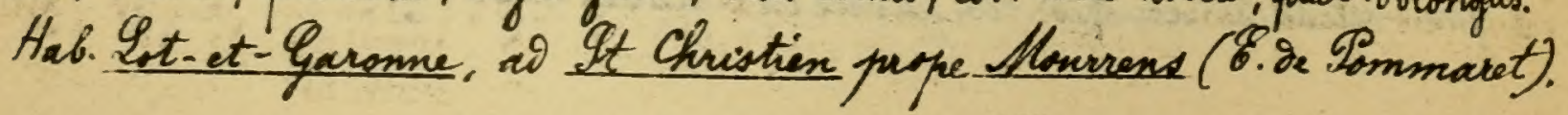


$-3-$

1108. Gepinia litigioss (Grip.) in Gogre Eab. N-1925. (Grip. Trim. I, p.18). toculei Debiles, dos ramos flor, brevissimos plerumque nulli; fol minuta, srata, vel orato-elliptica, ettringue attenuata, subacuminata, is costam saepe eglanioulosex; Dentes simplices; pet. glabri, aculeati, parce glanioulosi; stip. longuisculae, sat angustac, aurieulis lanceslatis, Divergentibus; pers. breves, parce ofaniulosi; tabus ellipsoiéens, loevis; sep. pinnata, gorso eglandulosa; - tygli glabri; cor....... fruct. oblangus.

Hab. Hérault, Montpelher: at viam La Gaillarde (H. Loret).

110g. Crepinia Salvatoris Googr Eab. N-1926.

toculei faleats-Dilatati, as ramos flor. nulli; fol: oborato-oblonga, acuta, basi subtruncata, glauca, crassa, ai istam eglanaulosa; dentes simplices, lanceolato-convergentes; pet. inermes, vix glaniulosi, inferne unifariam pubescentes, stip. gilute rubentes, auriculis givergentibus; ped. 1-3, hasi laves, caeterum tenuiter glanialosi, 8-10 m. longi; tubus oblongus, usope in $1 / 2$ inf. plerumque hispiom; sep. gorso glandulosa, pinnulis angustis, integris instructa, scumine angusto, styli globbi, gises comico; cor. eciliata; fruct. oblongus, utringue attenuatus.

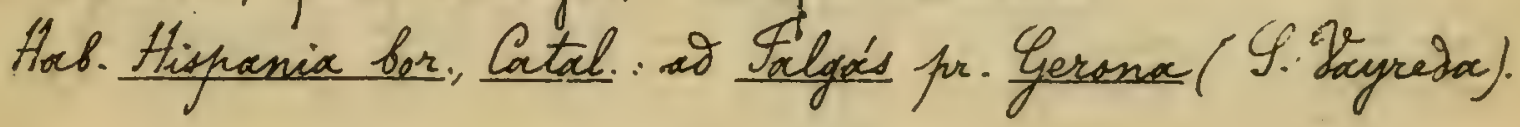

1110. Crepinix arenosa CGogr mss.

tcculei breves, defiles, parum bilatato-subadunei, at ramos flor-laxe toliosos mulli; fol parva, oborata, nix auta, basi subrotuniacta, supra glauca, as costam eglandulosa; dentes lanceolato-subconvergentes, simphies; pet glandulase; glabri, plerumique inermes; stip. latoe, breves, rebrae, auriculis rectis; pet. 1, sat glanioulosi, $12 \mathrm{~m}$. longi; tubus anguste clongatus, toto glanioulosus, sep rubra, Dorso glaniaulosa, fuinmulis omnitus pariesm latis sat gentato-glanioulosis instructa; stegli sparse pilosuli, griscs parum conico; cor. parva, sat amoene rosea, poulo ciliata; fruct. anguste oblongus, utringue langius attenuatus.

Hab. Iame-et-Lave, in arenosis as It Laurent-g'tandenay (H. Gagr).

1111. Crepinia cauresans Gogr Bab. F-1928.

Acculi rari, gebiles, harum faleato- Dilatati, ao ramos flor. mulli; fol. obovato-oblonga, rcuta, basi abrupte etternuata, at costam glanisulosa; Dentes profunde lanceolati, simplices, pet sparse glandulosi, ceuleati, in- 
$-4-$

ferne putosuli; stip. mediseres; ped. 1-2, glandulasi, $12-15 \mathrm{~m}$. longi; tubus oblangus, toto sporse aculeolatus; sep. Dorso glanioulosa, pirnulis angustis, longatis instructa; styli fore coaleti, glabri, gisses subeonics; cor......; fruct. Silangus, utringue attenuatus, apice valise strangulatus.

Hab. Rhone, inter Trancheville et Chaponost (I. Chabert).

1112. Crepina myrophylla Gogr Gab.1927.

to pracecesente giffert Coliolis minoribus, utrinque aequaliter attenuates, frevius dentatis, petislis inermibus, pesunculis brevisuibus $\left(6^{1} / 2-g-m\right.$.), tubo glabro, stylis sessilibus, fructer ovisises, apia subapresso, basi abrupte et valde attenuato- recurrenti.

Hab. Phône, at Irancheville et Charbonières (I. Chabert).

1113. Greninia Menchinati (Ggar et Debeause in Debx Mater. Ros. Dyren. $1878, p .20)$, C Gogr \&ab. N=1929.

Fculei valist, falcato. Silatati, as ramos flor. folissos nulli; fol oboratoelliptica, scutiuscula, basi subtruncata, glaucescentia, crassa, at costam glaniulosa; gentes simphices, lanceolato - subconvergentes; pet. glaniaulosi, sculeati, sat pubssentes; step. magnae, auriculis rectis; per. 1-3, acculeslati, 9-11 m. longi; tubus sbovato-oblongus, laevis; sep. asso temiter glaniaulosa, pinnulis integris, longis, latis instructa, acumine lato; styli glabri, Disco conico, cor-nosea, eciliata, fuct.magnus, obovats-sblongus, basi saepius rotuniatus, apioe beeviter attenuatus.

Hab. Iyrenaci Orient., circa Perpignan (O. Debeacux).

t+ Foliola oblonga.

1114. Crepinia pictariensis Gogr Gab. N-1930.

Aculei Defibs, rari, as ramss flor. melli; fol oblongo- acuta, basi roturiata, costam eglaniulosa; Sentes simplices, lanceolato-recti; pet. Ylabui, inermes, vix glanianlosi; stip. angustoe, Dilute rubentes; pes. 1-2, parce hispiivi, 10-13 m. longi; tubus oblongus, temuiter glandulasus; sep. iorso minute glaniulosa, pinnulis angustis instructa; styli glabri, lisco rat camio; cot.pallive rosea, pauls ciliata; fruct. obovato-sblongus. Hab. Lux - Serres, ad 2a Hothe-It-Heray (Naillaro). 
$-5-$

1115. Gepinia glabristyla Gogr Eab. N-1931.

Acculei rarissimi, falcato- Silatate, as ramos flor mulli; fol. oblongolanceolata, acuminata, basi breviter attenuata, ad costam eglanioulosa sed foliolo terminali inferne 1-3-aculeolato; Dentes simplices, magni, lanceolato-recti; pet. parce aculeate, eglanioulosi, inferne unifariam pubescentes; stip. angustae, auriculis ivergentibus; peo.1-3, glanoulosi, 16-20 m. longi; tubus oblongus, glaber; sep. Dorso eglanianlosa, pinnulis latiusculis, eglanoulosis instructo; styli glabri, Tiseo conico; con. intense rosea; fruct. obovato-oblangus, fasi rotuniatus, apice strangulatus.

How. Lot-et-Garonne, ad It Christien, Nowrrens (G.De Ponmaret).

1116. Grepinix Bavouxii Gogr Gab. N-1932. - Billot exs. N-3722.

Bculei panciores, purvi, parum falcato-Dilatati, ad ramos flor nulk; fol. oblonga, utringue attenuata, ao costam eglaniulosa; dentes recti, simplices, pet. soepius inermes, glanibulosi, fere toto uniforisim laxe pilosi; stip. Congre, auriculis aivergentikus; ped. 1-3, glaniaulosi, g-14 m. longi; tubus elongatus, hispiasus; sep. Dorso temuiter glanoulosa, pinnulis angustis, eglanDulosis instructa; styli nune glabri, nune subrilosuli, Dises paulo conico; cor. pallidius rosea; fruet. oblongo-claviformis, apice attenuatus, fasi gecurrens. Hab. Doub, Besaneon ad Rosemont (Mavoux et Poillot).

1117. Crepina latens Goggr mss.

Acculei parvi, breves, temuiter falcati, os ramos flor. mulli; fol. ample oblango-acuminata, basi acuta, superne lucide ateovirentix, ad costam eglaniulosa; Jentes simplices, late lanceslati, recti; pet. inermes, eglaniaulosi, basi subpulosuli; stip. virioes, angustissinae, longae, auriculis sat givergentibus; pes-1, vix glanoulosi, $18 \mathrm{~m}$. longi; tubus sblongus, glaber; sep. 90 rso eglanioulosa, pinmulis onnibus longis, filiformibus, integris et eglanoulosis

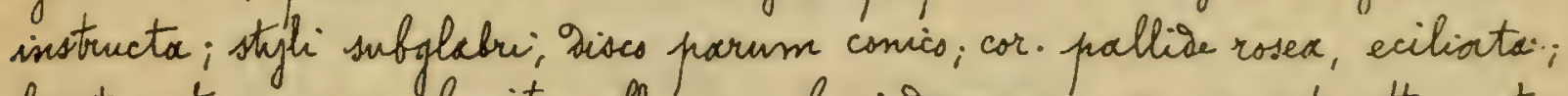
fruct. sat magnus, breviter oblongus, basi gepressus, apice sat attenuatus. Hab. Saane-et-Loire, as It Laurent-2'Aondenay (M. Ganooger).

118. Grepinia biturigensis Gogr mss.

toculei breves, parvi, parum Silatati, valse falcati, as ramos flor. mulli; fol mediscria, oblongo-acuta, basi subrotuniata, pallive riridia, as csstam eglanioulosa; dentes simphies, lanceslato-recti; pet-eglanioulosi, 
$-6-$

inermes, basi unifariam pilosuli, stip. rubentes, angustae, auriculis suboivergentibus; ped. 1-1, parce glanioulosi, 14-16 m. longi; tubus oblongus. loevis; sep. prorsus eglandulosx, pinnulis angustis, 2-4-2entatis instructo, acumine integro; styli glabri, Tisco valie comico; car. parva, subalba, eci; liatar; fruct. oblongus, utrinque attenuatus. Hab. Cher, ad Arcay (Mb. Canooger).

1119. Gepinia tubicalyx Gogr \&ab. N-1g33.

Foculei rarissimi, falcato-gilatati, à ramos flor mulli; fol oblonga, utrinque attenuarta, ad costam eglanialosa; Dentes simplices, salto profunisi; pet inermes, parce glaniaulosi, inforne uniforiam pilosuli; stip. mesiscres, pes. 1-2, hiogisuli, 10-11 m.longi; tubus elongatus, toto glanimlosus; sep. brevia,, gorso glandu-

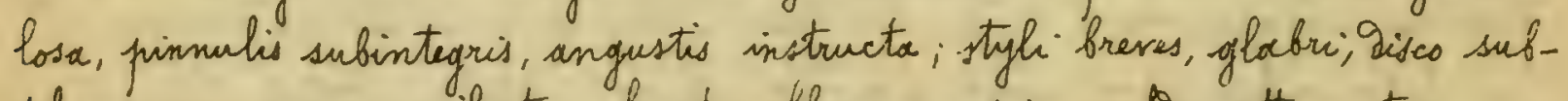
plano; cor. rosea, eciliata; fruct. sblongus, apuice valie attenuatus.

Hab. Mhone, at It Germain-au-Mont-g' Cr et Mobymieux (9. Chabert).

1120. Gepimia orthoosa Ceiogr miss.

Acculei longi, parum falcato - Yilatati, ad ramos flor. plerumque nulli; fol-mesiocria, oblongo-lanceolata, brest longe attemusta, supra glacica, as costam eglandulosa: Dentes simplices, lanceolato-aperti; pet-inermes, basi hinc inive piloso-glanioulosi; stip.longae, pareim latae, virieses, auriculis rectis; pes-1-3, sparse glandulosi, $8 \mathrm{~m}$. longi; tubus sblangus, glaber; sep. Dorso glavouloso, pinmulis omsibus integris, angustis instructa, styki glabri, 2iscs plans; cor-méaiserio, lacte rosea, eciliata; fuct. oblongus, basi attemuatus, ad apicem valse contractus.

Hab. Saone-et-Laire, ad St Micaud (16. Gandoger).

b. Rami florales aculeati.

+ Sepala gorso eglanioulosa.

1121. Crepinia eita (Désegl. Cat. p.178 non Gojge, Gogre Gab. N-1934.

teculei inchinato-gilatati, as ramos flor. pauciores; fol. elliptico-acuminata, basi coriata; Dentes simphices; pat- aculeati, sporse piloso-glanioulosi; stip. laeves; pes. 4-10, alii heres, alie piarce glansulosi; tubus ovoideus, glaber; sep. Forss aglanisulosa, pinmelis integris, latis instructa, styghi glabri, sisco plans; cor. alba; fruct. parrus, subglobosus, apice attennatis. Hab. Cher, ad Celle-Irviore (to. Disighise). 
$-7-$

1122. Crepinia castellana Googr \&ab. N- 1935.

toulei breves, valise falcati, wo ramos flor geminati, fol. late ovato. clliptica, abrupte subacuta, basi truncata, coriacea, al costam glanoulosa; dentes lanoeolato-convergentes; pet aculeate, glanoulosi, sparse pilosuli; stip. latissinae, virises, surviculis rectis; per. 2-3, sublaeves, $12-15 \mathrm{~m}$. longi; tiubus oblongus, glaber; sep. Sorss eglanioulosa, primulis et acumine integris, angustis instructa; styli glabri, Gisco comico; cor. pallide rosea, eciliata; frect. ollongus, utrinque attemuatus.

Hab. Hespamia centr., as El Escarial (No. Laguna).

1123. Grepinia ianensis Gogor mss.

toculei longi, cylinarici, inchinato - subilatati, as ramos flor. geminati; fol.médiacria, obovato-oblonga, acuta, basi subrotuniata, lacte virioix, ai costam eglañoulosa; Dentes simplies, lanceolato-aperti, pet. glabwi eglanioulosi, inermes; stip. latoe, breves, virides, auriculis rectis; pas.1-2, tantum apicem subalandulosi, $10 \mathrm{~m}$. longi; tubus oblongus, basi pauls glaniulssus; sep. Dorso prarce (aut non) glandulosa, prinmulis brevibus, onnonibus integris, angustis, eglanaulosis instrueta; styh subhirtelli, aises poulo conic; cor....; fruct. oblongus, basi attenuatus, apice valve contractus.

Hab. foin, in esitis ad Montanges (M. Ganioger).

T† Yepala dorso glaniulosa.

124. Gepinia Characias (Gogr Ros.nor. I. p. 16); Gogr bab. N- 1936.

Acculei valixi, at ramos flor late silatato-subfalcati; fol ample obosato-oblonga, attenuata, basi coridata, laete virisia, xi costam eglanioulosa'; Tentes simphices, magni, subconvergentes, pet parce glanoulosi, sacpies inermes, basi pilssuli, stip. virentes; pes. 2-3, hispisi; tubus obivato-obtongus, toto aculeolatus; sep. Dorso glanimulose, pinnulis angustis, gentatis instructa, rcumine longo, suiforlatato; styli subglaberrimi, asses fere plano; cor sosen, basi paulo ciliata; fruct. ovoideus, hispidus.

1.ab. Rhóne, at tornas (N6. Ganooger).

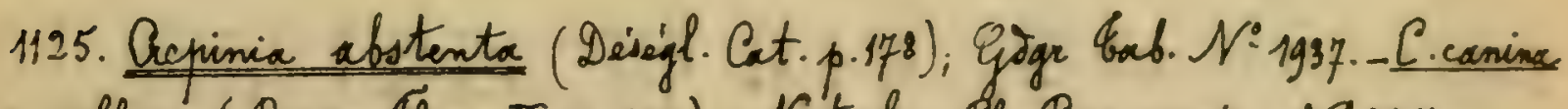
var collina ( Mroiss. Fl. or. II, p.685). - Kotschy 9l. Ters, auste. N-547. Foculei parri, falcato-gilatati, foll orato-elliptica, firma, nervosa, 
$-8-$

viriti-glaucescentix, as costam glandulosa; Dentes simplices, acuti; pet. paulo pilosulo-glanaulosi, aculeati; stip. auriculis acutis, rectis; pes.1-2, glanoulosi, tubus rotundatus, hispiaus; sep. Oorso glandulosa, pinnulis filiformibus instructa; styli glabri, gisco comico; cot......; fruct. globosus. Hab. Persia merid, in monte Kuh-Delu ju. Schiras (8h. Kotschy).

1126. Crepinia magna Gogr Gab. N-1938.

toculei falcato-Silatati, is ramos copiosisimi; fol. late oforato-acuta, fasi sensin attenuata, subglaucescentix, id costam glaniulosa; Dentes simplices, aperto - triangulares; pet. aculeati, glanioulosi, usque ad apicem inaíque pubescentes; stip. virides, maximae, auriculis rectis; pes. 2-3, sparse glañoulosi, 10-13 m. langi; tubus ovoirsens, glaber; sep. Dorso glanaulosa, pinmulis angustis, eglaniulosis instructa, acumine lato; styli subhirtelli, Dises conics; cor.....; fruct. maximus, voideus, basi rotungatus, apice paus lo attenuatus.

Hab. Rhone, at Sommeirs (No. Gangoger).

1127. Crevinia Rousselii (Rip. in Disegl. Cat. p. 184), Gogr Eab. N : 1939. - C. Dubia (Mast. ined. 1815, non gYib.).

toculei subilatate, inclinati vel rectiusculi, as ramos flor.geminati; fol. oborato-acuta, petiolulato; Dentes simplices; pet. aculeati, sparse pilosulo-glanoulosi ; stip. auriculis acutis, rectis; per. saepe 1, hispidi; tubus orvideus, glandulosus; sep. Torso glangulosa, breviter prinnato; styli exserti, sparse pilosuli, giscs consics; cor.magna, pallise roser; fruct. oroivens.

Hab. Cher, of Eurly, ete. (Mipart).

1128. Genimia glaucocarpa Gogr \&ab. N'1940.

Aculei gebiles, folcato-gilatati, di ramos flor.panciores; fol. obovatoscuminata, basi attemuata, parum glauca, ad costam parce of lanoulose; Dentes simplices, lancealato-convergentes; pet. aculeati, glanaulosi, inaeque et parce pilosi; stip. virides, auriculis Sivergentibus; ped. 2-3, in $1 / 2$ inf. laves, cacterum parce gtaniulosi; tubus oblongus, in $1 / 3$ inf. hipious, sep. gorso minute et parum glandulosa, pionuclis ommitus integris, angustis instructa; styli giabui, disco conico; cor. eciliata; fruct glacucus, elangatus, aprice praesertion attenuatus.

Hab. Garit, at taslas prope Le gigan (D. Euezkinvicy). 
$-9-$

1129. Crepinia trichella Gogr \&ab. N-1941.

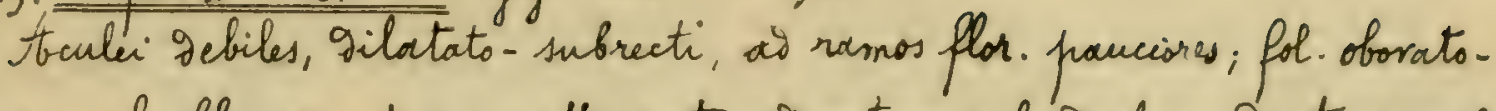
sblonga vel oblonga, utrinque attenuata, ad costam eglanioulosa; Pentes simplices, recti; pet. aculeati, eglaniulosi, inferne uniforiam pilosi; stip. Tilute unbentes, latiuscular, auriculis subrectis; peo. 1-2, hispiti; 7-11 m. longi; tubus obovato-oblongus, basi setulasus; sep. Forso sparse glavioulaso, pinnulis sat latis instructa; styli late capitati, subhirtelli, Giseo paulo comico; con..... i fruct. oborato - oblongus.

Hab. Haute-Savoie, ad Habeie-Luthin (Suget).

2. Styli hirsuti vel lanati.

a. Fructus oroideus vel obovato-oblongus.

1330. Crepinia extranea Gogr Gob. N-1942.

treculei ad ramos flor. falcato-gilatati; fol. oblongo-attemuata, basi via contracta, ad basin costare pausho glandoulosa, Oentes simplices, recti, put. aculeati, hine inse pilosulo-glaniuloss; stip. longae, auriculis aivergentibus. peos-1-2, sparse glanaulosi, 7-13 m. longi; tubus obovatus, jlaber; sep. gorso eglanoulosa, pinnulis angustioribus instructo; styli villosi, Dircs subplano; cor.....; fruct. ovoidens, basi rotuniatus, apuce attenuatres.

Hab. Basses- Pyrénées, at Biaurity (H. Iosiòre).

1131. Crepinia explanata Gogr mos.

toculei tenves, rectiuseuli, cylinirici, temuter acuti, ad namos flor copiostis fol. obovato-acuta, basi subrotuniata, glawea, as costam glansulosa, ientes simplices, triangulares, aparti; pot aculeati, inferne sibhirtelli.ac vix glanIulosi; stip. virides, angustae, auriculis girergentebus; pes. 2-3, sparse aculerlat, 13- $15 \mathrm{~m}$. longi; tubus ovoideus, glaber; sep. Torso eglanioclosa, puinnulis $l_{\alpha}$ tis, 1-3-Pentatis instructa, acumine porum latt, integro; styli villosi, Sisco plans; cor..... ; fruct-ovoiveus, basi gepressus, aprice abrupte strangulatus.

Hab. Cancasus, Carthahimia: OS Gore prope fhur. Anra (Mrotherus).

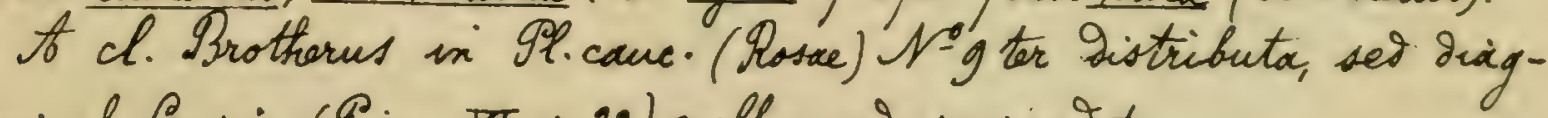
nosi cl. Crepin (Trim. DT, p.22) nullo modo responiset.

1132. Crepinia Carthalimiae Gogr mos. - Rosa....... Crép. Prim. VI, p.11.Grotherus MP. cauc. (Mos.) N:18. 
$-10-$

tbentei panciores, inchinati, sat temues, suboilatati, id ramos flor. saepe mulli; fol! ample obovato- blonga, abrupte cuspidata, basi subrotunioata, glauca, at costam eglandulasa; gentes simplices, lanceolato-subconvergentes; pet. aculeati, glabri, eglanioulosi; stip. virides, angustissimac, auriculis vivergentibus; pes.1. pparse setulosi; 8-9 m. longi; tubus obovato- oblongus, glaber; sep. Dorso eglandulosa, pinnulis onnibus integerrimis, subfoliaccis instructa; styli villosi, giseo conico; cor. magna, roser, eciliata; fruct. probabilius sbovato-oblongus, utrinque attenuatus.

Hab. Caucaous, Carthalinia: ad Borshom prope fl. Kira (Brotherus). Corolla 42-44 mill. Siam. lata ideoque magna nee sat paiva ut falso griet $\mathrm{Cl}$. Crepin cu' exdem ac meinss specimina sifpeditavere.

6. Fructus sblongus.

f Foliola ssata rel obovate.

1133. Crepinia anisgavensis (Mast. F9. Maine-et-Loire p. 189; Gren-et. Goor. Fl. De Fr. I. p.558; Koeh Gyn. of. 22 p. 251; Ledeb. Fl. ross. II. p. Fy; Goiss. Fl. or. II. p. 685 a.; For. Fl. Ju Centre ed. 3- II. p. 225; Carist Etride

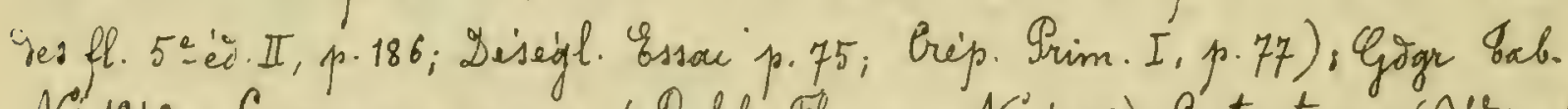

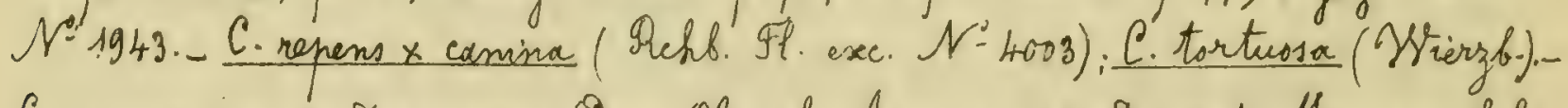
C.camina var andegavensis (Desv. Obs. pl. Ang. p. 157; Dumort. Mon. ros. belg. p. 60; Christ Rosen ger Gchw. p. 160).- Rei. at thory Ros. 5g. - Billot exs. N.1476; Déségl. Herb. ros. Nos 17 et 18.

Accules foleato-silatati i fol amphiscule orato-acuta, glanca, ic costan poulo glaniulosa; dentes simplices; net. glabri, parce glanioulosi, aceleati; stip. glabra, gilatator; pes. hispivi; tubus oblongus, setrilosus; -dep. pinnat, carso glanialasa; stghli villasi; cor. rosea; fruct oblangus.

Hab. Europa plux

1134. Greninia gysphoroiea Gogr mos. $\mathcal{Y}-1945$.

taculei falcato-dilatati, so ramos flor. mox denubatos milli; fol. ofoxata, utrinque paulation attenuata, as costam eglanisulosa; gentes simplices; pet. inermes, eglaniulosi, plerumque glabri; stip. virides; pes. 1-2, parce glaniulosi, 10-15 m. longi; tubus oblongus, glaber; sep. Sorss vix glaniaulara, pinmulis angustis, brevibus instructa; styli hirsuti, gisco conics; cor...... fruct. oflongus, basi athematus, aprice strangalatus. Hab. Sthine, as Charnpogre et Dardilly (M. Chabert). 
$-11-$

1135. Crepinia gracilispina. Gogr bab. N 1946 .

toculei graciles, subvilatato-curvuli, as ramos flor. sat copiosi; fol. late obovato-oblonga, basi breviter truncata, apice subrotuniata, as costam

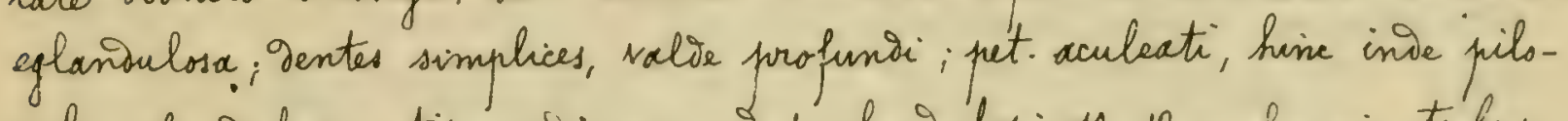

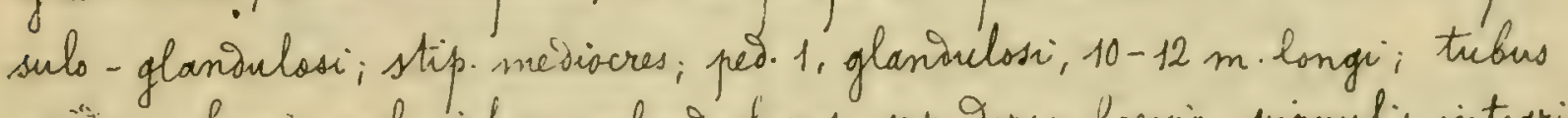
voitens, laevis, vel inferne glanionlosus; sep. Forso Laevia, pinnulis integris, anqustis instructa; styli hirsuti, gisco subplano; cor.......; fruct.ovoideus, intrinque abrupte sed parum attenuatus.

Hab. Rhone, ad Montonelas (M. Ganooger).

1136. Brepinia longa Gogr Eab. No1947.

toculec gebiles, genninati, falcato-gilatati, ar ramos flor paneiores; fol. obovata, utringue breviter attenuata, os costam saenins eglanioulosa; Contes simplices, lanceolato - xperti; pet. inermes, eglanoulosi, inferne vix pitsschi; stip. breviusculoe, auriculis givergentibus: pes. 1-2, glanoulosi, 8-11 im. Songi; tubus elongatus, hispious; sep. Dorso glanioulosi, pirnulis angustiribus instructa; styli villosi, sises subplano; cor.......; fruct. anguste elangatus, utrinque val'se attemuato-strangulatus.

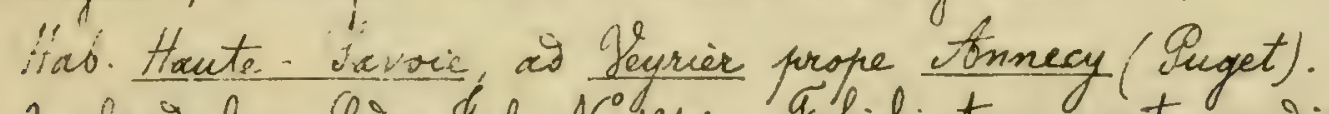

P. cinanidulosa Gogr bab. No 1949 . Foliali term costa media plus mimis glaniulosa; pet. aculeati, glanioulasissini; sepala late pinnata. Hab. cam type.

$\gamma$ affinis Cgrogr Gab. N 1948. - Petioli sup paulo aculeati, hinc inde ondulosi; pes. longiores (1/1/2-2 cent.) it et tubus, subglabici.

Hab. curn typo.

1137. Crepinia Morelii Gogr toab. N-1950.

Aoculei Dilatato-subfaleati nel inchinati, ad ramos flor. copiosi; fol. oforato-attenuata, basi rotundacta, glauca, coriacea, ad basin costac paulo- jlandulasa; Dentes simplios, minuti, patulo- triangulares; pet. glanoulosi, aculeati, sparse pilosi; stip. angustoe, auriculis parum givergentibus; pio. 1-5, hispinissinn:, 2-2\% cent. longi; tubus oblongus, inferne glandoulosus; sep. Dorso sparse glandulosa, pinmulis mediseribus instructa; styli villosi, Sises subplano, car. pallice rosea; fruct.magrnus, oblongo-conices, basi rotuniatus, apice selie attemuatus.

Hab. Irkône, ad Ecully (Norel; M. Ganooger). 
$-12-$

tT Foliola obovato-oblonga vel oblonga.

1138. Grepinia povita (Désegl. in Journ. of Mot. 1874; extr.p.2); Gigr Eab. N-1951. - C.acuta (Crép. Prim.I, p. $4 \mathrm{~g}$ non Fisch.); C. cariacex (Crép. in Yan Heurck Ofs. fot. I, 1870, p. 3o; Boiss. Fl. or. II, p. 685 mon Opiz nee Hallb), C. laristanica (Gjogr Essai p.28). - Kotschy Pl. Pers. for. N-263 et 653 .

toculei falcato-Dilatati, fol. ample oblongo-acuminata, coriacea, nervosa, sentes arguti; pet. pubruli, glansulosi, aculeati; stip. glabrac, serrulatac, pes. corymbosi, elongati, setulosi; tubus oblongus, hispidiclus; sep. pinnata, granoulosa; styli lanati; cor. rosea; fruct-oblongus.

Hab. Tervia bor. in monte Elbrus ad Iman Lader Tbrachim frope

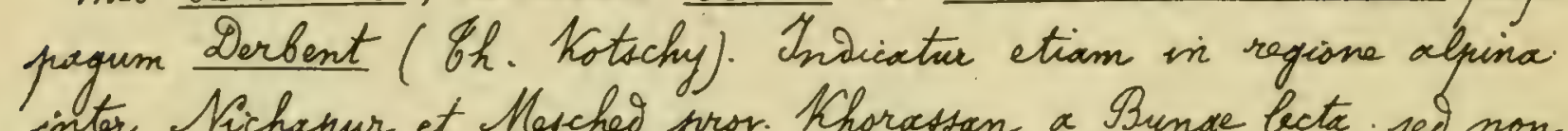
inter Nichapur et Nesched prov. Khorassan a Bunge becta, sed non risi.

Rosa acuta Fisch.ex Trvect (e. Caucass) ut et Rosa coriacer Halls. Hist ros. p. 223 mihi ignotie sunt. Rosa corioucea Ofin in Presl Fl. cech. pertiret ad yregem Crep. tomentellace (Lem.).

113g. Grepinia arelatensis Gogr tof. N-1952.

toculei longi, recti, cylingrici, at ramos flor. nulli; fol. oblong-acuimirata, basi rotunisata, ab costam eglaniulosa; gentes simplices, patulosublanceolati; pet. aculeati, glanoulosi, glabri, stip. longae, virides, auriculis suboivergentibus; pet. 1-2, tenuiter et parum glandulosi, 10-12 m. longi, tubus oblongus, basi hispious; sep. Dorso parce glaniaulosa, pinnulis latis, gentatis instructa; styli villosi, gisco paulo comico; cor.......; fruct. oblongus, basi rotuniatus, apuce attemeatus.

Hab. Gallopror. ao Raphile prope torles (A. toutheman).

1140. Crepinia quercetorum Gige mss.

Feculei breves, parum folcato-gilatati, ad ramos flor. rarissimi, fol. mediocria, oblong-lanceolata, basilonge cuneata, lacte viridia, ad costam eglaniaulosa; sentes simplices, lanceolato-convergentes; pet. subinermes, basi sparse pilosulo-glanioulosi; stip. longac, angustace, virides, auriculis subqivergentibus; ped.1-2, basi laeves, apice tantum vix glanioulosi, $12 \mathrm{~m}$. ingi; tubus oblongus, laevis; sep. gorso glaniulosa, pinmulis parum latis, 2-3-Dentatis instructo, acumine lato, integro, styli villosi, gisco paulo 
$-13-$

comics; cor......, fruct. oblongus, utrinque longuis attenuatus.

Hab. Sarne-et-Laire, as Yt Laurent-g'tondenay (M. Ganioger).

1141. Crepinia foetose Cogre mss.

Acculei obsiles, falcato-subsilatati, as ramos flor. stipulares; fol. late oblanga, breviter acuminata, basi attenuata, atrovirentia, as basin costace paulo glaniarlosa; dentes simplices, triangulari-subconvergentes; pet-eglansulosi, parce aculeati, inferne pauls pubercentes; stip. virides, magnae, auriculis sat givergentibus; pes. 1-2, mediocriter glanisulosi, $12 \mathrm{~m}$. longi; trebus oblongus, laevis, sep. Dorss sparse glaniaulosa, pinmelis onnibus subfoliaceis, integris, eglaniculosis instructa; ityli villosi, gisco parum comis; cor. magna, rosea, ciliata; fruct magnus, oblongus, utrinque attenuatus, turgiivus. Hab. Saone-et-Loire, at St Laurent-g'.tandenay (H. Ganooger).

1142. Beninia Pugeti Gogr Eab. N:1953.

Acculei falcato-gilatati, as ramos flor. copiosi; fol-ample oblongo-acute, basi subroturioata, is costam eglaniulosa, Dentes simplices, lanceolato-convexjentes; pet. aculeati, eglaniulosi, toto temiter pubescentes; stip. maximac, auriculis subsivergentibus; peo. 1-3, vix glanoulosi, $12-13 \mathrm{~m}$. Longi; tubus sllongus, laevis; sep. prorsus eglaniulosa, pinnulis latis, copiose instructa, xumine foliaces; styli lanati, disco conico; cor......; fruct. oblongus, utrioque attenuatus.

Hab. Houte- Savoie, ad Habie- Sulbin (Suget).

114.3. Grepinia oblangifera Gogr mss. - Rosa ....... Crep. Prim. VI, p.16.Protherus IP. cauc. (Ros.) $N=19$ bis.

toculei graciles, inclinato-subsilatati, ot ramos flor plerumque mulli; fol. ample sborato-oblonga, utrinque attemuata, mesis dilatata, parum glavea, at costam eglansulasa; gentes simplices, late lanceolatoconvergentes; pet. aculeati, inferne vix pilosuli, eglanibulosi; stip. virides, longre, sat latae, auriculis givergentibus; pes. 2-3, vix glandulosi, 15-17 $m$ - longi; tubus oflongus, loevis; sep. Torso eglanisulosa, pirmulis 3-4gentato-glandulosis instructa, acumine integro, parum lato; styli inferne hirsuti, gises conico; cor-magno, alba, eciliata; fruct. probabihius oblongus, base gecurrens, apice sttemuatus.

Hab. Courcous, Carthalinia: oo Strasneakop frope fluvium Kura (Mrotharus). 
$-14-$

c. Fructus globosies, rotuniatus vel ovatus.

1144. Greninix Rauni (Gratt. Ros. mon. II, p. 35; Rehb. Fl exc. N-4003;

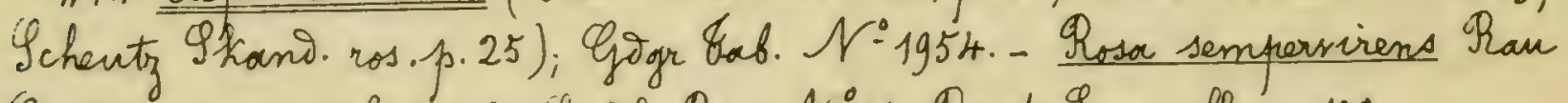
Erum. p. 120 (excl syn.); Linit. Tros. N-g8; Bast. Essai fl. p. 188.

Aculei falcato-Dilatati; fol-orata, pallice viridia, crassa; Dentes simplicer; pet. aculeati, pilosulo-glanduelosi; stip. Denticulatae, lacres; poo. breves, hisfisi; tubus ovatus, setrulosus; sep. Dorss glanbulosa, late pinnata; styli lanati; cor. rosea; fruct. suboglobosus, turgigus.

Hab. Bavarix, ete.

1145. Crepinia hebegyna Gogr Gab. N-1955.

Acculei falcato-Silatati, as ramos flor copiosi; fol. obovata, utriname vix attenuato, as costam folioli mesic inferne 1-2-aculestato vel pauilo glaniulosa; Dentes convergentes; pet aculeati, egtanioulosi, inferne unifariam pubescentes; stip. magnac, auriculis Divergentibus; pe⿻-1-4, atii haeves, alic parcissine glanaulosi, 15-22 m. longi; tubus orotus, bxeris;

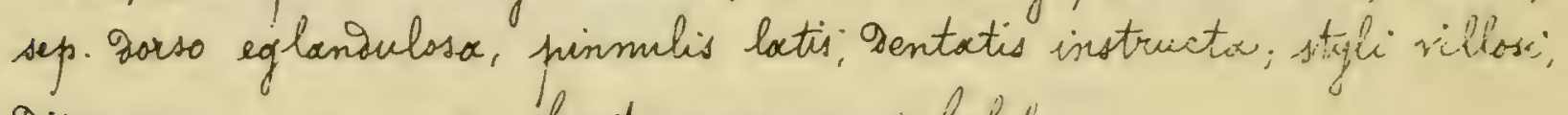
giseo camic; cor rosea; fruct. magrus, sufoglotosus.

Hab. Haute- Savori, Habere-Sullin (Puget).

1146. Grepinia cyclomapha Gogor bab. N-1956. - Gogor Herb. ros. N. 322.

Fcculei recurrentes, inchinato - suboilatati, ad ramos flor. sat copvisn; fol. obovato-oblonga, utrinque haud aut vix atternata, firma, as costam glanioulosa, Dentes simplices, recti; pet. xculeati, hine inoe glanituloso-pilosuli, stip. mediacres, auriculis Divergentións; pes. 1-2, crassi, parce setulosi, 8-10 m. longi; tubus orxtus, glaber; sep. Porss eglanoulosa, pinmulis sat angustis instructa; styki villosi, Disis subplane; cor.........; fruct. globosus, laeris

Hob. Fyrenace hisp. ai Moucharo (H. Bordere).

1147. Crepinia abupta yogr 8ab. N=1957.

Afb antecedente recedit foliolis accutis, minus aeque dentatis, pectislis mayis glandulosis at acubis copiosionibus.

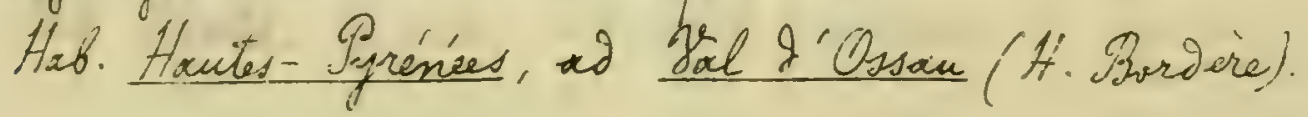


$-15-$

1148. Grepinia transmota (Gép. Trim. I, p. 18); Gogr 8ab. N. 1958. C. psilophylla (Gor. Fl. Iu Centre 32's. p.226 non Rau).

Fculei debiles, ad ramos flor. longi, falcati; fol. orato-rotuniata, sacpe subbitusa, basi subattenuata, ad costam parce glaniulosa; Dentes simphices. pat glabui, glanoulosi, aculeati, stip. sat latac, auriculis lanccolatis, givergentibus; ped. 1-3, setulosi; tubus ovatus, laevis; sep. dorso paulo glandulosi, pinmulis parce dentatis instructa; styli villosi; con. rosea, majus cula; fruct. ovatus.

Hob. Gallia ocil., prope Aingers (Borean).

1149. Crepinia megalostyla Gogr bab. $\mathcal{N}-1959$.

Aculei falcato-dilatati, as ramos Har. rubros sparsi; fol. ample of. langa, utrinque breviter attemuata, crassa, is costam eglaniulosa; isentes simplices, aperto-triangulares; pet. aculeati, eglanisulosi, inferne uni proriam pubescentes stip. maximace, gilatatas, auriculis rectis; ped.1-2, sparse glanoulosi, g-11 m.longi; tubus svatus, glaber; sep. Dorso sparse glaniulosa, pinnulis integris, angustis instructa, acumine magno; styli magni, lanati,

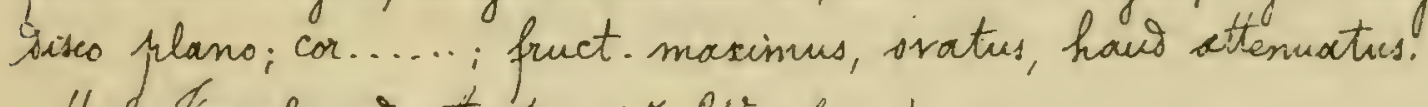

Hab. Inrol, it Ititon (Y. Yistaur).

to ilontanas (Ozanonia) forsen pertinet.

1150. Crepunia surculasa ( Hoods in brans. Linn. soc. XII, p.228, Gratt. Thos.mon. II, p.262; Maker in Limn. Journ. XI, p. 226, Dumort. Mon. ros. filg. p. 10; Déségl. Cat. p.177); Gogr Goub. N'1960. - C. Martivis (Gren. Fl jur. p. 242).

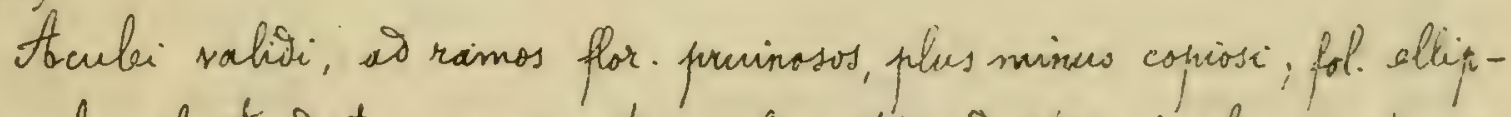

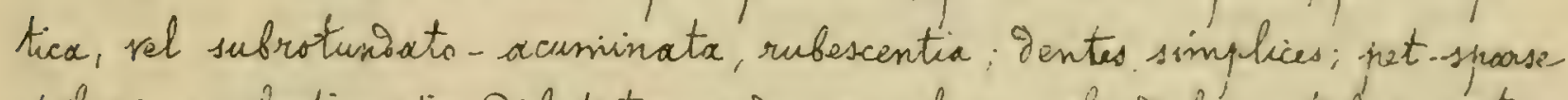

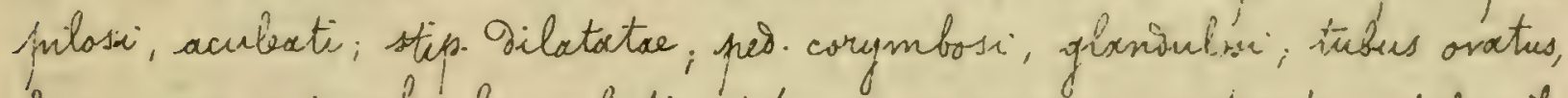
lauris, seps. pinmelis lancealatis, intagris copiasissinne sinstisceta, styli villoni, giscs conico; cor lacte rosea; fruct. maynues, ovatius.

Hab. Angliac.

B. minar Goser Gab. N-1961. - fo typo siffert ramis rubentibus nec pruinosis minusque floriferis, Sisco subplano. Hab. Inglia. 
f. - Moxrcidale Gogr Essai p.29; Giger Gab. p. 151.

Foliolis mune simpliciter mune crebre biserratis, ylancescentibus, quam in spreciebus sectionis praceidentis serius Decidvis; sepalis post anthesim mox decidvis; aculeis plerumque conformibus; radice haud reptonte.

1 Yepala garso eglaniulasa

Styli glabri vel subhirtelli

2 Gtyli firsuto-lanati

Eructus ovatus, oroidens vel obovato-oblongus

$3\{$ Fructus oblongus

Inecries N-1151-1156.

Folicla fasi cordata vel rotuniata

4 Fohila basi phus minus attenuata

Speries $\mathcal{N}=1157-1164$.

Species $\mathcal{N}: 1165-1168$.

Getioli sparse hirtelli; fol. basi attenuata, oi costam vix

$\left\{\begin{array}{l}\text { Slanidioli minute hirsuti, foliola fasi rotuniata, ai }\end{array}\right.$

Ipecies $\mathcal{N}-1169-1176$

costam semper glaniculos $\alpha$

Styli glabri val glabrescentes

Sparies $\mathcal{N}: 1177-1181$

6 Styli villosi vel lanati

Setioli glabri vel sparse pilasi

7 Setioli plus minus laxe et unidique pubescentes

Gubus saltem usque ai $1 / 2$ inf. partem glanivulosus

8 Gubus laevis vel tantum basi infirna glaniulosis Fructus rotunatus vel ovatus

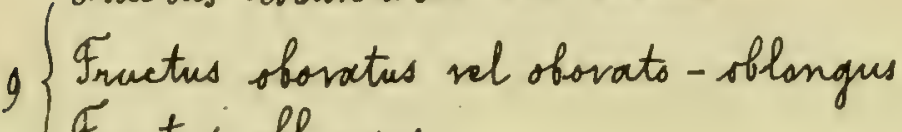

Fructus oblongus

Fructus rotuniatus vel ovatus

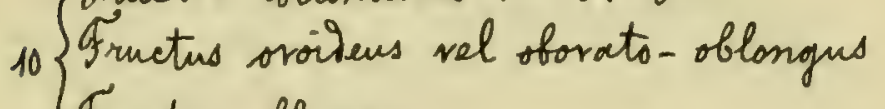

Fructus oblongus

9pecies $\mathcal{N}^{\circ} 1206-1213$.

Qpecies $N^{\prime}: 1200-1205$. Spaies $N=1182-1186$

Inecies $N: 1187-1193$.

$\varphi_{\text {precies } N-1194-1199 .}$

Inecies $N-1214=1221$.

Species N:1222-122g.

Ipecies $N=1230-1236$.

1. Sepala garso eglaniulosa.

a. Tyli glabri vel subhitelti.

+ Fructus oflongus.

1151. Gepinia leptodes Gogr Gab. $N^{\circ} 1962$. 
$-17-$

Aculei rarissimi, Debiles, teniter folcati, as ramos flor minutos mulli, fol. ovato-elliptica, breviter attenuata, basi rotuniata, viridia, as costam glaniulose; Dentes fere ormes biserrati, profunoc, lacimiati, aperti; pet. aculeati, glansulosi, sparse pilasuli; stip. virides, latoe, aurieulis rectis: ped. 1. gracillimi, hispibi, $15-19 \mathrm{~m}$. longi; tubus oblangues, glaber, sep. Dorso eglanDulosa, primulis latis, 3-4-Dentato-ylaniulasis instructa, acumine integro, lato, styli glabri, gises conics; cor. cciliata; fruct-anguste oblongus, utrinque longe ottenuatus.

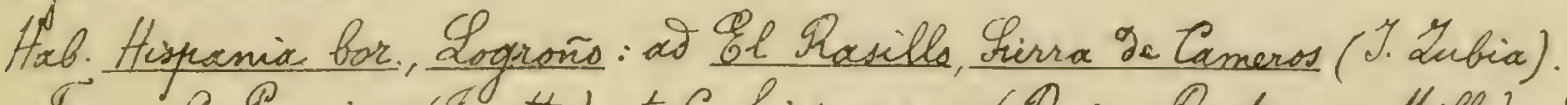
Facies C. Pouzini (Gratt.) et C. hisfanicace (Boiss. Peut. nec Hill.), sed folisla nec ommins biserrata.

1152. Crepinia gymnophlaca Gagr Eab. N-1963. Gogr Herb. nos. N-30. Inermes, vel caules inf. aculeis gebilibus, faleato-gilatatis soli instructi, fol. obovato-oblonga, magna, attenuata, basi subrotuniacta, id basin costace paulo oflandulosa; Dentes inaequales, biserrati; pet. aculeati, glandulosi, sparse pilasi; stip. angustiones, auriculis givergentibus; pros. 1-4, hispidi, 13(19) m. longi, tubus oblongus, laevis; sap. Tantum marginibus paulo glanoulosa, pirnulis longis, dentatis, angustis instructa; sityli glabri, Disco conico; cor-rosea; fruct. oblongus, utringue depressus.

Hab. Hispania for-orient, ad Barcelone (Machis).

1153. Crepinia apricana Goor mss.

Acculei elosyati, parum foleato-gilatati, as ramos flor-copiosi; fol. meaiocria, obovato-oblonga, basi attenuata, pise subrotundata, viridia, ad costam paulo glanioulosa; Dentes valde profunioi, convergentes, fre omnes biserrati; pot-aculeati, glanioulosi, glabri; stip. brevissinne, latae, auriculis rectis; ped. 1, basi laves, apice glanaulosi, $15 \mathrm{~m}$. longi; tubus oblongus, laevis; sep. Dorso eglanisulosa, pinnulis anmibus parum latis, 2-4-Dentatis instructor; styli sparse pilosi, gise plans; cor...... fruct. sat anguste oblongus, utringue longius attenuatus.

Hob. Algeria arrent, ad Abotna (Hinon).

Habitus onmino C. Souzini (bratt.) sed foliola inaeque serracta.

1154. Crepinix castaneasfolix Gogr 8ab. N-1964.

Acculei debiles, inclinato-subsilatati, ad ramos flor. nulli; fol amiplis- 
$-18-$

sime oflong-acuminata, basi attenuate, à costam eglanaulosa; dentes profunoi:, Divergentes, fere onnes simplices; pet inermes, sparse glanduloso-pitosuli; stip. mediscres; pet. nune laeves, munc parce glandulssi; tubus oflonjus, plerumque laenis; sep. Dorso eglaniulosa, pinnulis angustis, 1-4-Dentatis instructa; styli glabri, exserti, Dises conves; cor porrva, rosea, eciliata; fruct. oblangus, basi attenuatus, apice contracto-strangulatus.

Hab. If hine, as Chaponost versus aquareinct. roman. (T. Chabert).

1155. Crepinia ascensionis Gogr Gab. No 1965.

Abculei debiles, inchinato-subsilatati, as ramos flor.geminati; fol. obovato-oblonga, utrinque acuta, ad costam eglanoulosa; dentes lanceslati, fere omnes simphices; pot. aculeati, vix glanoulosi, hine inde pilosuli; stip. virides; pes. 2-3, parce setulosi; tubus oblangus, nunc laenis, nune basi glaniulosus; sep. Oorso eglanaulosx, pinnulis angustis, 1-4-gentatis instructa; styli breves, glabri; giscs pauls conics; cor....... fruct. oblongus, basi depressus, apice valde attemuato-strangulatus.

Hab. Rhone, ad Limonest (9. Chabert).

1156. Crepinia bactica Goor Gab. N:1966.

Fculer gebiles, inclinato - gilatati, dio ramos flor-sparsi; fol.minutissima, obovato-acuta, basi rotundacta, ad costam glaniulasa; gentes aperto-triangulares, fere osnnes simplices; pet: aculeati, glanisulosi, inferne vix pilosuli; stip. rubentes, parvae, auriculis rectis; pod. 1, hispidi; 19-20 m. longi; tubus oblongus, in $1 / 3$ inf. hispitus; sep. Dorso eglanoulosa, pinmulis omnibus an. gustis, frevibus, subsentatis instructa; styhi glabri, disco comic; cor rosea; fruct oblongus, utringue attenuatus.

Hab. Hispania merid, Murcix: in Sierra Calar del Mundi, Jegura (M. Carradx).

Facies C. Pouzini (Gratt.), sed Dentes fere omned simplices.

t Fructus ovatus, svoideus val oforato-oblongus.

\$. Foliola basi cordata vel rotundata.

1157. Crepinia intervemens (Desigh Cat.p. 185), Gogr Gab. N:1967. Acculei gimorphi, alii falcato-gilatati, alii setacei, fol ovato-subobtusa, basi: rotundata, pallide viribia, Dentes inaequales; pat. glandulosi, 
$-19-$

aculeati, glabri; stip. angustore, auriculis Divergentibus; pod.1, hispidi; tiebus ovoiveus, laevis; sep. Oorss eglaniulosa, acumine elongato, lato; styli subglabarrimi, diseo fere plano; cor-rosex; fruct. ovoideus, basi notunioatus, apice attenuatus.

Hab. Cher, as Erouy (At. Déséglise).

1158. Crevinia cordifflia Gouge \&ob. N-1968.

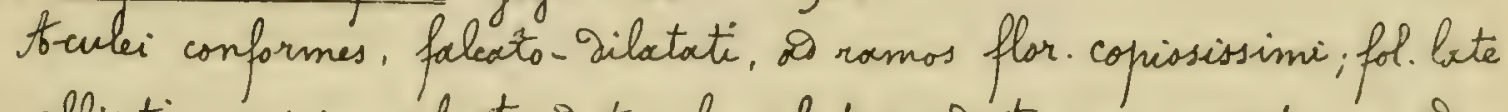
ovato-elliptica, apice subroturioata, basi late coriato-emorginata, viridiglaucescentia, à costam eglaniulosa, Dentes triangulares, aperti, fere - smmes simplices; pet aculeati, glanioulosi, inferne paulo pubercontes: stip. latac; virises, auriculis rectis; peo. 1-2, hisfidissimi, 9-12 m. longi; tubus oroiteus, laevis; sep. Torso eglanioulosa, pirnulis latis, pauls gentatis instructa, styli aisperse pilosali, Dises plano; cor.......; fruct. magnus, breviter oviodeo-conicus, basi rotuniactus, aprice attenuatus. Hab. Anghix, prope Gilmaton ( H. Ley).

1159. Prepinia almicola

teculei breves, inclinato-subbilatati, ad ramos flor pauciores; fol. mediscria, ovato-elliptica, parum acuto, basi cordata, id costam glanZulosic; Dentes lanceolato-rectr, fere ornnes simphices; pet acculeati, hinic inive vix glaniulosi, inferne uniforiom pubescentes; stip. virites, angustac, auriculis givergentibus, peo. 1-2, parcissine glanioulosi, 10-12 m. longi; tubus woideus, laevis; sep. Dorso eglarioulosa, pinnulis sat latis, 1-2-ineisis instructa, acumine lato, integro; styli sparse pilosuli, Dises plano, cor. albosubrosea, eciliata; fruct. magnus, svato-ellipticus, utringue vix gepressus. Hab. Belgium, Hainaut: ad Harvengt (M. Ganooger).

1160. Crepinia incisa Gogor bab. N-1969.

teciulei gilatati, volise falcati; fol. late ovato-attemuata, basi coridata, lacte viridia, à costam paulo glanoulosx. fohiolo terminali inferne 1-3aculeolato, Dentes valis profundi, sublaciniati, fere amnes fiserrati; pet. aculeati, glanionlosi, unifariam pubescentes; stip. angustae, aurriculis givergentibus, perd 1-4, minute et parce glanoulosi, 10-15 m.longi; tubus oborato-oblongus, laevis; sep. Tarso eglaniulosa, finmulis latiusculis, copriosis, paulo Dentatis instructa; styli glabri, Disco subarmico; cor. rosea; 
$-20-$

fruct, ovoioens, utrinope attemeatus.

Hab. Tifrenari cente, ad Gavarmie (H. Bordère).

1161. Crepinia oligoblephara Gogr \&ab. N-1970. - Gogre Herb. ras. No go. Aculei longi, fachato-Dilatati, is ramos flor-copiosi; fol. obovato-oblonga; abrupte et breviter acuta, basi cordata, is costan ylansulasea; Sentes inaequales, profunon; pet. eglanbulosi, aculeati, vic pilosuli; stip. meiveres; poo. 2-4, pubescentes et simul paulo glandulosi, 10-12 m. longi; tubus ovoideus, laevis; sep. Dorss glanoulasa, pinnulis subintegris instructa; styli fere glabri, Disco subplano; con......; fruct. ovoidens, strinque gepressess. Hab. Gañne, ad Aornas (M. Ganiager).

1162. Crepinia editorm Gogr mss.

Aculei freves, inchinato-Dilatati, à remos flor. panciores; fol.parva, oborato-obionga, utrinque sensin rotuniatx, virides, ab basin costae paulo glaniulosx; gentes profundi, lanceolato - recti, fere onnes biserrati; pat. accebati, glanoulosi, inforne umifariam pubescentes; stip. breves, latae, auriculis ovvergentibus; frio. i, hispidi, 10-12 m.longi; tubus ellipticus, lavis; sep. sorso sed marginibus glanianlosa, pinnulis cmnibus latuisculis, sat sentato. glarioulosis instructä, ocumine integro; sty'i glabri; giseo subplano; car. pallide rosea; fruct ovato-ellipticus, utrinopes gepressus.

Hob. Hismama for. Learoño: Ol Rasills, Sierra de Cameros (M Mubia).

1163. Creninia aborzana (Debeave in litt. 1879).

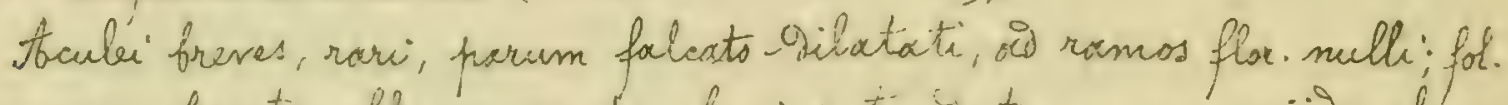
sat parra, obovats-oblanga, acuta, basi rotirnacta, coriacea, viridi-glawea, ad costam glandulosa; dentes profunis: hanceslats-recti, acopue simplices et biserrati; pet. xcula ati, glanvulosi; inferne sporse pilosi; stis magnace, aurniulis subsivergentibus; peo. 1-3, sparse et vise alaninlosi, 1 cent.longi; tubus obovato-sitongus, leveris; sep. Darss eglanisulasa, latius prinnata; styli pauciores, glabri, Gisco valte conics; cor...... ; fruct. magnus, ovoidens, utrinque sensim attenuatus, turgious.

Hab. Syrenaci orient, ad Collioure Consolation (O.Debeux).

1164. Creminia grwiens Gjogr Gob. No 1971.

toculei breves, tenuiter at valie falcato-gilatati, as ramss flot. mulli; 
$-21-$

fol. oborato-subacuta, bas rotundata, as costarm eglandulosa; dentes inacequales, lanceolato - subconvergentes; pat vix glasinulosi, inferne proulo pubescentes, sup. inermes; stip parrar, virides, auriculis divergentibus; pod. 1, hispioi, 10-12 m.longi; tibus oforato - oblongus, lacris; sep. Tantum marginibus paculo glaniulosa, pinmulis folioces-Dentatis instructa, acumine integro; stagli -glabri, Disco valde conico; cor....... fruct. sbovato - oblongus, fasi gepreasies, apice strangulatus.

Hab. Houtes-Pyrénées, ad Benqué (H. Bardère).

§§. Tolis la basi plus minus attenuata.

1165. Brepinia erythrella ( Rip. in Disengl. Cot.p. 161), Gogr Gab. N: 1972.

Acculer falcato-gilatati, ad ramos flor plerumque nulli; fol ovato-acuta, Pasi attemata, viridia, ad costam paculo glaniaclosa; Dentes inaeguales; pet aculeati, hinc inis pilosulo-glandulosi; stip. orso glanduloso-sculeslatar, auriculis sup. rectis; ped. 1-9, parce setulosi; trebus ovoidens, laevis; Ar. pinnulis meiveribus, gentato - glaniulosis instructa; styli sacepius glabri, jisco conics; cor.magna, rosea; fruct. ovoidens.

Hab. Cher, wo Les toix g'tongillon (Mipart).

1166. Ripartia calamares. Gogr bab. N: 1973.

toculei subilatati, purmm falcati, ad ramos flor bebiles; fol obovatooblanga, acuta, basi attemuata, as costam eglangulosa, gentes iscaequales. recti; pet inermes, fivic inde pilosulo-glaniulosi; stip. breves, aciriculis - sivergentibus; pet. 1-2, hispici, g-12 m. longi; tulus ovoideri, hippiars; sep. - arso eglaniulase, pinviclis parum latis instructa; styu oflabri, gisco subplans; car....... fruct. svoidens, basi valde attenuatus.

Hab. Gallix ovient, ad Hant-Cindre frope Lyon (Wib. Eiandoger).

1167. Crepinia ambigens Govar toab. No 1974 .

Therlei validi, late falcato-gilatati, à ramos flor. minores; for obovatsacuta, basi breviter attemuata, magna, lacte virioix, wio costam parce gleonSulosa, foliolo terminali basi 1-3-aculeolato; Tentes valse profundi, fere omnes biserrati; pet-glandulosi; aculeati, unifariam pubescentes; tip.angustas, auriculis Sivergentibus; ped.1-4, parce ghandulosi, $10-15 \mathrm{~m}$. longi; tubus obovato - oblongus, laevis; sep. Dorso eglangulosa, pirmulis latuesculis, 
$-22-$

gentato-glaniulosis copiose instructa, acumine foliaces-Dentato; styli glabri, gisco pauls comis; con. rosea; fruct-avoideus, utringue attemuatus. Hab. Syrensei cente, as Gavarmie (H. Barière).

1168. Crepinia umbraticola Gogr \&ab. $\mathcal{N}^{-19} 75$.

foculei gilatato-subinchinati, od ramos flor. copiosi; fol oblongo-lancolata, basi attemuata, intense viridia, ad costam glandulosa; dentes lanceolato-aperti, aeque simplices et biserrati; pet. aculeati, hine inie glanguloso-pilosuli; stip. Dilute rubentes, auriculis subrectis; ped. 1, temuissime et vix glaniulosi, 10-12 m.longi; tubus obovato-oblongus, laevis; sep. gorso eglandulosa, pinnulis angustis, sat dentato-glanivilosis instructa, jeumine subfoliaces-Oentato; styli sparse pilosuli, Disco camio; cor. eciliata; fruct. obovato - oblongus, utrinque paulo Depressus. Hab. Hispania bor., prope Logroño (J. Lubia).

6. Ytyli hirsuti vel lanati

8. Setioli sparse hirtelli, fol. basi attemata, as costam vix glanioulosa.

1169. Crepinia phalacrostema (Gogr Ros. nov. II, p.17); Gogr Eac. N-1976.Goor Herb. ros. N'207.

to eulei longi, faleato-gilatati, as ramos flar. sat copiasi; fol.oblonga, utrinopue acuta, ar costam glanaulosa; Dentes inaequales, lanceolatorecti; pat glandulosi, hine inde vix pilosuli, inf-inermes; stip. mediseres; ped. 2-4, nunc laeves, nunc glandulosi, 12-17 m. longi; tribus ovoideus, Laevis; sep. Iarso eglanoulosa, pinnulis angustis valie pectinato-glaniulosis instructa, styli hirsuti, gisco plano; cor. rosea, eciliacta; fruct-magnus, scicieus, utrinque depressus.

Hab. Rhóne, in monte It Bonnet supra Montmelas (Nh. Ganioger).

1170. Crepsinia lavissima Giagr mss.

A-ulei a ramos flor parum gilatat, temuiter falcati, fol. ample oblong-rhomboidalia, utrinque paulation attersuata, medis gilatata, tenuia, nervosa, as costam eglanivelosa; dentis inaequales, profuniri, aperti, net. subinermes, eglanaulosi, inferne paulo pubescentes; stip. sat latoe, aurriculis aivergentibus, pes. 1-2, parce glondulosi, 3-6 m. longi; tubus obovato-oblongus, laevis; sep. gorso eglanaulosa, pinsulis angustis, longis 
$-23-$

instructa; styli villosissimi, gises plans; car. rosea; frect. parves, obovato-oflangus, apice attenuatus.

Hob. Hante- Lavoie, ad Bellevaux (Muget).

1171. Grepinia brachystema (Gogr Ros. nov. I, p. 8); Goog \&at. N-1977.Gogr Herb. ros. N-537.

Acculei falcato-dilatati, ad ramos flor-copiasi; fol.elliptico-attemucta, basi cariata, glaucescentia, ad costam glandulosa; orentes indequales, subconvergentes; pet. glanoulosi, parce aculeate, hine inde pilosuli; stip. virives; ped. 2-4, nunc glabri, nunc glandulosi, 9-14 m. longi; tubus oboratooblangus, laevis; sep. Darss eglandulosa, pinmulis latis, pectinato-gentatis instructa; styli lanati, disco sat conics; cor. pallide iosea; fruct. ovoidens, utrinque attemuatus, medis turgious.

Hab. Rhone, at Gleize' (M. Gundoger).

1172. Crenimia rinealis (Thip. in Billotia 1866, p.36). Cariot Etude des fl. 6:id. II, p.240); Gogr Eab. N-1978. - Désigh. Herb ros. $N$-19.

A-cilei gilatato - foleati sel subincurvi, ad ramos flor. sparsi; fol. ovata, utringue attenuata, vividia; Dentes inaequales; pet. aculeati, glabri, parce glandulosi; stip. laves, auriculis givergentibus; ped. glanaulosi; tubus of longus, lavis; sep. Darso eglanoulosa, pinnato-gentata; styli lanati, gises sat conico; cor.magna, intense rosea, fruct. magnus, oblongus

Hou. Cher, ad Grouy, La Grange - It Hean (Ripart).

1173. Crepinia phlebophora Gogr Eab. N-1979.

Acculei falcato-dilatati, as ramos flor. foliosos mulli; fol obovata, $u$ trinque attemuata, ad costam eqlonioulosa, Dentes inaequales, subconvergentes: pet. inermes, eglanoulosi, hine inse pilosuli; stip. longac, angustiores, auriculis parum givergentibus; ped-1-3, alii glabri, alii paulo glanioulosi; trebus oblongus, lacvis; sep. Forso eglaniolosa, pinnulis angustis instructa, acumine subsilatato; styli villosi, gisco convico; cor...... fruct. blongus, utringue
attenuatus.

Hab. Haute-Grvai, ad St Gervais-les-Sains (Suget).

1174. Crepinia Dehiciosa Gogr tab. N:1980.

Aculei longi, dilatato - subfalcati, as ramos flor. pauciores ifol. oblonga. 
$-24-$

utringue attemuata, lacte virisia, as costam sacpe eglanioulosa, dentes tanceslato-aperti, fere onnes simplices; pet. rubentes, aculeati, sparse glanioulosi, unifariam hirtell; stip. Hlaves-rubentes, auriculis parum givergentibus;

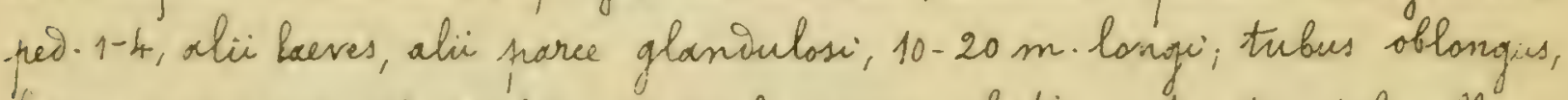
laevis; sep. Dorss eglanioulosa, prnnulis parum latis instructa; styli villosi, aiscs paulo conico; cor. rosea; fruct. oblongus, utrinque attenuatus.

Hab. Firenai centr., ad Gère (H. Sactiere).

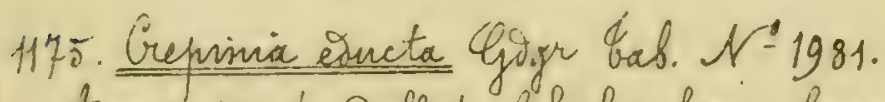

if praciesente giffert fohiolis basi breniter attenuatis vel subrotuniatis, temuius Dentatio, costam meriam semper minute glanioulosis, petalis intense roseis, is unguem lutescentibus.

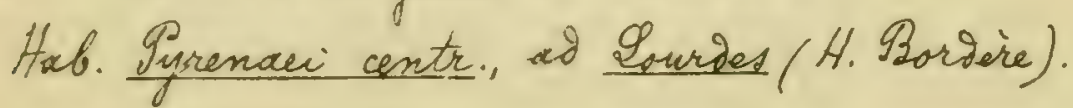

1176. Crepinia vergens Gagr Herbor. Fyren. (1884) p. 43.

foculei recti unt subinchirati, parum gilatati, temues, longi, ad ramos Plor-copiosissimi; fol oborato-oblonga, acuta, basi rotuniata, atrovirentia, ai costam valle glanoulosa: Intes inaequales, lances lato-recti, pet. glanDulosissimi, aculeati, hinc inie pilasulc; stip. pirides, latae, gorso glandulosac, auriculis subrectis; ped. 1-3, in. $1 / 3$ inf laeves, cacterum temiter hispiduli; 13-15. m. longi; twhus oblongus, laevis; sep. 2oros eglanialosa, pinnulis latiusculis, dentato-glaniulesis instructo; styli villosi; Disco sulplano; cor.

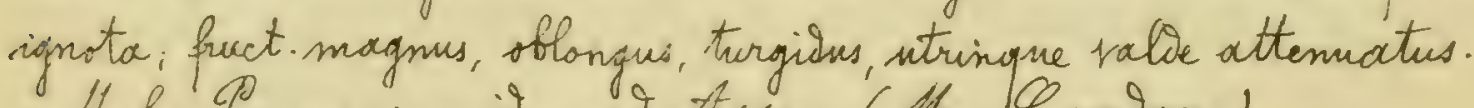
Hab. Prenaci occio, at Itson (Ab. Gandoger).

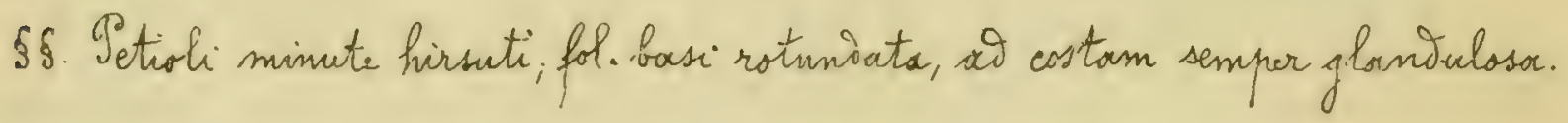

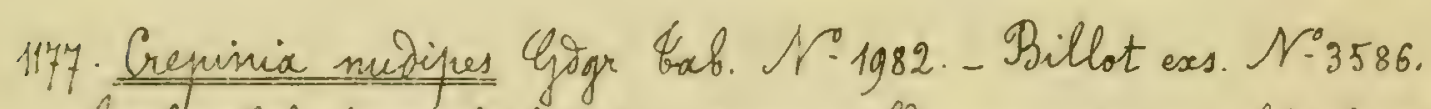

Frauli forlcato-subiclatati, à namos flor copiosissimi; fol covorato-attenurta, fasi rotuniata, ad costam glanialosa; dentes inf biserracti, subecti: pat glanialosissini, temuitex villosi, aculeati, stip. latoc, auriculis oivergentibus; per. 1-4, fere onnes laeves, $15-20 \mathrm{~m}$. longi; tukus oblongus, lacvis; sep. Oorso eglanioulosa, pinmulis paucioribus, parum latis, gentatis instructs; styli villosi, Discs plano; cor rosea; fruct. oborato-oblongus, apice attermiatus.

Hab. Rhone, as Charbonnières (Moullu). 
$-25-$

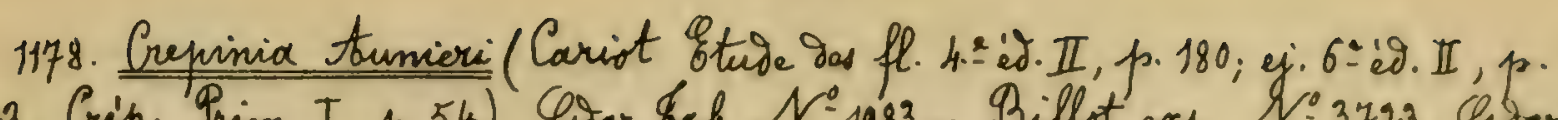
243 ; Crip. Prim. I, p.54), Gogr bab. N-1983. - Billot exs. N:3723, Giogr Herb. ros. $\mathcal{N}=381$.

Acculei falcato-gilatati, as ramos flot-minoras; fol-ovato-rotuniacta, utrinque subattemuata, glauca, nervosa, a costam glaniulasa; Dentes indequales, convergentes, pet. villoso-glanibulosi, subinermes; stip. oorso glanioulosac; ped. 2-5, hispidi, 1 1/2 cent. Congi; tubus ovoivens, laevis; sep. Dorss eglanDulose, pinnulis angustis, gentatis instructa; styli lanati, gises subplans; cor. magna, rosea: fruct. magnus, ovatus, apice attenuatus. Hab. Thone, id Francheville (9. Chabert, 1851).

1179. Crevinia opacifolia Gogr 6ab. $N^{-1984 . ~}$

Acculei Debiles, inclinato-Dilatati, ramos flor. pacuciores; fol obovatosubacuta, basi rotuniata, coriocea, crassa, id costam glaniaulosa; Dontes lancolats-operti, inaequales; pot. glandulosi, aculeati, tenuiter pubescentes; stip. latar, inf. Dorso glandulosae, auriculis rectis, por. 3-5, hispisissimi, 11-13 m. longi; tubus ovoideus, basi infima hispidus; sep. Dorso eglanaulosa, pinnulis longes, angustioribus, glaniulosis instructa, acumine longs, sat lato; styli lanati, gisco paulo conico; car-magna, rosea, cciliata; fruct. ovoideus, apice atten. Hab. Hungaria, ad Tresburg (Mchneller).

1180. Creninia myriocarpa Gogr Eab. N-1985. - Gogr Herb. ros. N:134.

Acculei faleato-gilatati, wo ramos flor. copiosissimi, verticillati; fol. ovatoelliptica, utrinque rotundata, al costam parce glandulosa, Dentes falcati, fere ornnes simplices; pat aculeati, glansulosi, inferne tenuiter pubescentes; stip. latissimac, auriculis subrectis; pas. carymbosi; 8-10, hispibi, 8-16 m. longi; tubues obovatus lavis; sep. Dorss Laevia, pinnulis angustis, parce gentato - glassulosis instructa; styli lonati, disco plano; car. magna lacte rosea; fruct. magnus, oflongo-pyriformis, apice attenuatris.

Hab. Mhone, ad 2oordilly (Mb. Gantoger; Hort. bot. Lyon, an. 1867-73).

1181. Grepinia potens Gojor Gab. N-1986. - Gogr Herb. ros. N- 323.

Aculei Quri, falcats - gilatati, as ramos flor.copiosi; fol. oborata, utrinque rotuniata, coniacea, a' costam glanisulosa; gentes ciallosi, subconvergentes, fere omnes simplices; pet. glaniulosi, aculeati, parce pilosuli; stip. magnac; pes. gense ramsso-corymbosi, hispivi, 11-20 m. longi; tubus laevis, 
oborato - oblongus; sep. Forso lavia, pinnulis paucioribus, sat angustis, dentatis instructa; styli lanati, gises subplano; cor. rosea; fruet. magnus, ob. longus, apice attenuatus.

Hab. Irhone, at Chorbonmieres (H. Ganooger, Hort bot. Lyon, an. 1867-73).

f Petioli glabri sel sparse pilosi.
§. Eubus saltem usque as $1 / 2$ inferiorem partem glaniulosus.
〜. Fructus rotuniatus vel ovatus.

2. Seprala do'ss glanioulosa.

a. Styli glabi vel glabrescentes.

1182. Crepinia brachycacca Gogr Sab. $\mathcal{N}$ : 1987.

Acculei panciores, parvi, falcato - subsilatati, as ramos flor mulli; fol. obovato-oblonga, acuta, basi rotundata, al costam glaniulosa; rentes apertic, fore annes simplices; pet. lasiulose, inferne unifariasm pubescentes, inf.inermes; stip. mediscres; peo. 1-9, ylaniulose; 16-18 m. longi; tubus oractus, hispioulus; sep. Darso glanoulosa, pinnulis brevibus, angustis instructa; styli glabrescentes, Dises sufplans; cor.....; fruct. parves (scent. latur), rotuniatus.

Hab. Rhane, Berument: at aquaeduct. roman. (9. Chabert).

1183. Creninia Irpex Gogr Eab. $N=1988$. Ggogr Herb. nos. $N=32$.

teculei falcato - subsilatati, al ramos flor-geminati; fol. obovato-oblonga, dcumionata, basi attemuata, glaucescentia, as costam parce glandulosa, Dentes lanceolati, fere onnes simplices; pat aculeati, sacpe glabic et eglanDulosi, auriculis Divergentibus; pes. 1-3, hispisi; 10-114 m. longji; tubus breviter orrideus, toto aculeatus; sap. gorso valde glandulosa, pinnulis integris, angustis instructa; styli hine inive pilosuli, gises subplans; cor.magna, rosea, ecilista; fruct. sot magnus ( $131 / 2-14 \mathrm{~m}$. latus), subrotundatus.

Hab. Mhone, Montmelas: in monte It Monnet (M. Gandoger).

1184. Crepinia mesostema (Gjogr Ros. nor. II, p. 17)i Gogr \&ab. N-1989. Gogr Herb. ros. $\mathcal{N}^{\circ} 163$.

Aculei as ramos flor. faleato. Dilatati, fol. oborato- acuta, basi subro-

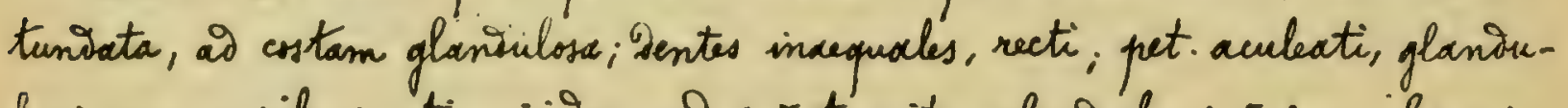
Losi, sparse pilasi; stip. virides; pee. 2-5, tenuiter glansulosi, 5-8 m. longi, tubis oratus, usoque ad $2 / 3$ aup. saltem hispiaulus; sep. gorso glasioulosa, 
$-27-$

pirnulis integris, mediseribus, longriuseulis instructo, styli sparse pilosuli, Dises subplano; cor ...... ; funct. oratus, basi rotundactus, apuice breviter attemuatus, $12-13$ me giam. latus.

Hab. Rhône, al gille-sut-Jormioux (M. Ganooger).

1185. Crepinix Jimpiphocycla Gogr \&ab. N-19g1.

Acculei termes, inclinato-subsilatati, as ramos flor. sagpins mulli; fol. late sbovato-elliptica, abrupte attemuata, basi coriata, as costam plus minues glanidulosa; Dentes fere smes biserrati; pet, inormes, sparse glanoulosi, hasi unifariam pubescentes; sthp. latoe; pes. 2-4, hispidi; 11-17 m. longi; tubus ovoideus, to to glaniulosus; sep. Dorso glandulosx, pinnulis integris, angustis instructa; styli glabri; disco subplass, cor.......; fruct. mence ovatus, mene breviter ovoideus, apice semper attemuats-strangulatus.

Hab. Thone, Grézieux-la- Haremne (P. Chabert).

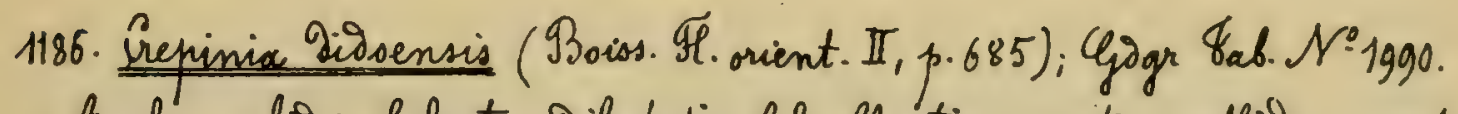
Acculei valioi, falcato- Oilatati; fol. elliptico-acuta, pallise virentix; Dentes inaequales, orguti; pet glabri, aculeati; stip. Dilatatae, serrulator; peos. 1, aculeati; tubus oratus, dense hispidus; sep. pinnata, gorso glanioulosa; styli glabrescentes; cor pallice cariea; fruct probabilius ovatus.

Hab. Cancesus ot, Daghestan: Divo, Boris Neidan (Muprecht).

$\sim \sim$. Fructus obovatus vel borato-oblongus.

1187. Greninia generensis Gojg 8ab. N-19g2.

Acculei breves, parum falcati, so ramos flot mulli; fol ample allipticooborata, abrupte acuminata, basi truncata, io costam glanioulosa; Pentes maximi, lanceslato-conrergentes, fere onnes simplices; pet. sculeati, glanoulosic inferme praulo pubescentes; stip. longee, virides, auriculis rectis, pes. 1, hispiai; 14-16 m. langi; tubus sbovato-oblangus, toto glanaulosus; sep. Porso tenuissine olsendulosa, pirnmlis omnibus in tegris, longis, latiusculis instructa, acumine foliaces; styli exserti, glabri, gisco conico; cor. eciliata; fenct. oborato-oblongus, utrinque sensin attemuatus.

Hab. Helvatix, Geneve: ad La Croix-2e-Mozon (Mandog). 
$-28-$

1188. Genimia fastitita Ggar Eab. N-19g3.

foculei gilatati, valde falcati, as ramos flor. mulli; fol oborato-oblonga, acuta, basi paulation attemuata, as costam vix glanisulasa, gentes triangularisubconvergentes, fere onnes simplices; pet. aculeati, glanialosi, sparse pilasi, stip. freves, virides, auriculis Divergentibus; peo. 1, hisfinis; 10-11 m.longi; tubus sbovato - oblongus, glandulosus; sep. Porss pauls glandulosa, pismulis angus. tioribus, 3-4-gentatis instructa, acumine integro; styli glabii, gisco conics; cor. meariocris, eciliata; fruct - oborato-oblongus, basi subrotunisatus, apice attenuatus. Hab. Gyrenaei centr., ad Gière (H. Sordère).

1189. Orepinia asperulosa Gogr mss.

feculei rectrisculi, cylinivici, tenues, to ramos flor geminati; fol parwa, obovato-sblonga, breviter acuta, basi rotuniata, glauca, so costam paulo glaniaulosa; Dentes fere onner biserrati, triangulari-convergentes; pet. aculeati, sat glanoulosi, inferne pilosuli; stip. virides, breves, sat angustac, acuriculis givergentibus i. pes. 1-2, sat hispiai, $8 \mathrm{~m}$.longi; tubus oborato-oblongus, hinc inde glangulosus, sep. Oorss glandulosa, pinnulis onnibus latiusculis, dentatis instructa; styli subglaberrimi, gises pauls conico; cor......; fruct-meidiocris, obovato- oblangus, basi rotuniactur, apice abrupte attenuatus.

Hab. Eranssilvania, Eorba tranyos: as Gorda (L. Michter).

11g0. Crepinia cinis Gogr toab. N-1994.

Acculei sparsi, gebiles, faleato-gilatati, ar ramos flor sacpins milli; fol. obovato-oblonga, breviter attenuata, basi rotundata, os costam plerumque eglanoulosa; dentes profunis:, inaequales; pet. inermes, sparse glanoulosi, inferne paulo prebescentes; stip-mesiocres; per. 2-3, glandulosi; 7-18 m. lorigi; tubus oblongus, toto glanidulosus; sep. Dorso sparse glandulosa, pinnielis anigustis, 1-g-gentatis instructo, styh hine inde pilssuli, gises subplans;cor........; fruct. oborsto- blongus, aprice semper strangulatus, centralis basi attematus. Hab. Rhone, Le Iont-g'tlai of Francheville (9. Chabert).

1191. Crepinia perturbata Gjgr Eab. N-19g5.

Atculei Debiles, vix inchimati, subsilatati, ad ramos flor pauciores; fol. oblong-lanceslata, basi acuta, ad costam eglaniulosa; Dentes givergentes, lanceolati; pet. inermes, paulo glasiaulosi, basi uniforiam pilasuli; stip. majuscular; pet.1-3, glanivulosi, 14-16 m. longi; tibus obovato-oblongus, 
$-29-$

glancus, toto hispiisus; sep. 9orss glanidulosa, pinnulis angustis, 1-3-Dentatis instructa; styli glabre;, subcolumnares, Disco proulo comis ; cor..........; fruet. shorato-oblongus, basi subrotunidatus, apice attimuato - strangulatus. Hab. Thâne, as Grézicux-lo- Sorenne (P. Chabert).

1192. Crepinia nematostipula Gigr \&ab. N-19g6.

Rubens; aculei gilatato-subfalcati, à ramos flor interdam nulli; fol. obovats-elliptica, breviter attenuata, basi subtruncato, as costam parce glangulosa; gentes inaequales, aperti; pet. glanoulosi, basi paulo pubescentes, inf. inermes; stip. mubro-sanguineace; pres. 1-2, hispisuli, 8-10 m. longj; tubus oblongus, in 1/2 inf. temuiter glanioulosus; sep. gorso parce glandulosa, pirnulis integris, angustis, langis parce instructa, styli subcohumnares, glabri; oises plans; cor. rosed; fruct. oborato-oblangus.

Hab. Ahoone, in montibus as Haugneray (9. Chabert).

1193. Crepinia cinnamomicolor Gogr Eab. N:1997. Gogre Herb, ros. N-337.

Acculai salisi, gilatato-subfalcati, as ramos flor. lucise bruness sat copiosti fol. obovata, utrinque parum attemuata, firma, is costam eglangulosa; Dentes convergentes, fere onnes simplices; pett parce glanoulosi, hinc inde pilosuli, inf inermes; stip. magnae, virides; pes. 2-4, glanaulosi; 10-13 m. longi; tubus obovato - oblongus, toto glanioulosus; sep. Dorso glanioulosa, pirnmlis latiusculis, Dentactis, elangatis, copiose instructo; sty li breves, sparse pilosuli, gises plano; cor-rosea, subciliator; fruct. blovato-oblongus. Hab. Iuy-De-Deme: ad Montbaubier (No. Dubosclart).

$\sim \sim \sim$ Fructus oblangue.

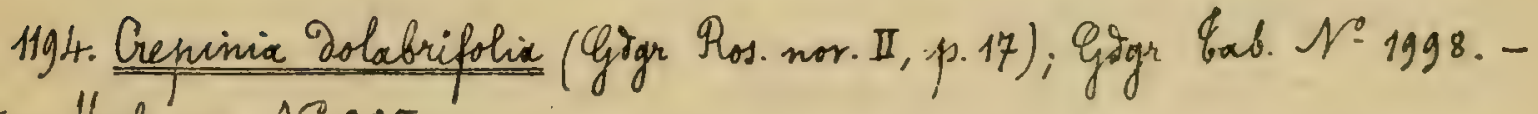
Gogr Herb. ros. N-285.

Acculei rarissimi, validi, falcats-Vilatati, as ramos flor-mulli; fol. obovato-oblonga, attemuata, basi truncata, ad costam eglanialosxi Dentes incequales, aperti; pet. inermes, hinc inse pilosulo-glansuloss; stip. longace; pes.1-3, glondulosi; $14-19 \mathrm{~m}$. longr; trebus anguste oblongus, toto glandulosus;

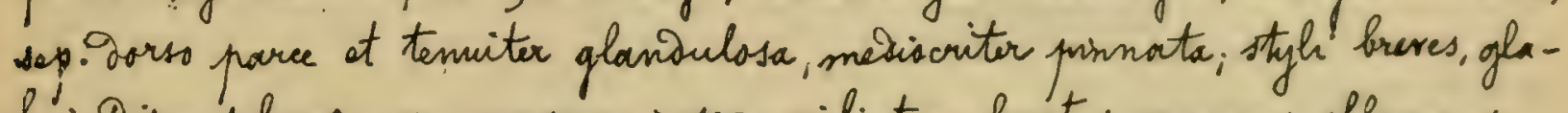
bri: Diseo plano; cor.magna, rosea, eciliata, fructus madmus, oblongus, mesio turgious, utrinque depressus, centralis vel solitorius claviformis. Hof. Thóne, à tormas (Nh. Ganooget). 
$-30-$

1195. Crepinia actinophlase (Gogr Ros. nor. II, p. 18), Ggogr Gab. N: 199g.Gogr Herb. ros. N'q7.

Glauca; aculei gabiles, faleato - Dilatati, al ramos flot patulos garninate vel verticillati; fol. orata, utrinque breviter attenuata, as costam eglaniseloss: Tentes recti, incequales; pet. plerumque glebri et eglanduloss; saepins inermes, stip. longae, auriculis subirengentibus; pes. 1-4, glandulosi, $15-20$ m. longi; tubus oblongus, fere to to sculeolates; sep. Forss glanoulosa, pinmulis angustis instructs; styli basi sparse pilosuli; Dises plans; cor. Oi. late rosea; fruet. oblongus, aprice attenuatus.

Hab. Ihone, at Mantmelas : in monte It Bonnet (Nh. Gandoger).

1196. Grepinia marcescens (Giogr Thos. nov. I, p. g); Gojgr Gab. N-2000. C. hirsuta (Deisigl. et Ozanon in Tsull. soc. Tauph. 1882, p. 328). - Giogr Herf.ros. N=113; Goc. Druph. exs. $N=2855$.

Fculei Debiles, inclinati, ad ramos flor pauciores; fol.oblanga, utringue paulation attenuata, gir persistentia, at costam eglanioulosa, gentes inacequales; pet. inermes, eglanioulosi, parse pilssuli; stip. sat parrace, auriculis farum givergentibus; pes. 1-5, hispiovi, 12-20 m. longi; tubus oblangus, Hoto setulosies; sep. Oorso glanivulosa, pinmulis angustis instructa; styli. glabri, sics plans, cor majuscula, rosea; fruct. oblongus, utrinque atten. Hab. Thone, ad Montmelas (No. Gandoger).

1197. Crepinia Carrac Gogr Gab. N.2001. - Gojgr Herb. ros. N=520.

Yalise glavea; aculei folcato-Dilatati, as ramos floc. sacpe mulk; fol. lates obovato-oblonga, acuta, basi breviter attenuata, at costam glansulosa, foliolo mesio basi aculeslato; Dentes valse profunsi, faleati, fere smnes bisernatis; pet aculeati, glanoulosi, saepius glabri; stip. Catore, auricuhis rectis; ped-1-5, hippiai, 8-12 m. longi; tubus elongatus, fere toto setulosus; sep. Dorso glandulasa, pinmulis linearibus instructa; styli glabri; gisco conico; cr. rosex; fruct. oblengus, apuice attemuatus.

Hab. Rhone, in montosis od Milb-sur-Jamisuxe (M. Ganooger).

1198. Creninia Lisibuñax (Gremier et Taillot in Gren. Inppl. fl. jur.), CGogr bab. N'2s02.- C. Kosinsciana (Taillat in Tiellotha 1865, p. 121 non Tress.). Biliot exs. N-3722, Flora Yequan. exs. N-36g.

Feculei falcato-gilatati, ad namos flor mulli; fol late oborats-acute, 
$-31-$

basi parum attemuata, ai costam eglarioulosa, Dentes aperti, fere onnes simplices; pet. inermes, glaniouloss, hine inive pilosuli; stip. longace, aurieulis subrectes; ped-1-3, parce glasialosi, 10-12 m. longi; tubus oblongus, to to sparse glanioulosus, sep. Torso parcius glanioulosa, pinnulis integris, longis, angustionibus instructa; styli glabre; gises sat comis; cor.rosea, eciliata; fruct. oblongus, utrinque attemuatus.

Hab. Gallix orient., Mresaneon: at Movemont (Y. Taillot).

1199. Grepinix expallens Giogr tob. N:2003.

Aculei pauciones, Pilatati, gebiles, so ramos flor. mulli; fol obovata, abupte utringue attermata, pallide virisia, as costam eglarioulosa; gentes inneequales, recti; pet.inermes, vix glanioulosi, pilosuki; stip auriculis subrectis; pes. 1-3, glanisulosi, $10-15 \mathrm{~m}$. longi; tubus anguste sblongus, toto glanisulosus; sep. Dorso olangulosa, pinnulis longis instructa, acumine foliaces-laciniato; styli glabri, gesce sat comis, cor. rosea ; fruct. anguste oblongus, aprice attenHob. Rhöne, is Irancherille (to. Carist).

88. Gubus laevis vel tantum basi infina glanoulasus.

1200. Crepinia agraria (Rip. in Disegl. Cat. p. 181); Ggogr Eab. N-2004.C. agrestina (Crip. Trim I 18). - Deségl. Herb. ros. N-18?

Acarlei parni, graciles, cylinorici, rectiusculi; fol-minuta, orato-elliptica, brenter acuta, basi subrotuniacta, as costam parce glanisulosa; Pentes inaequales; pet glabri, aculeati, sparse glasioulose; stip. breves, angustar, auri-

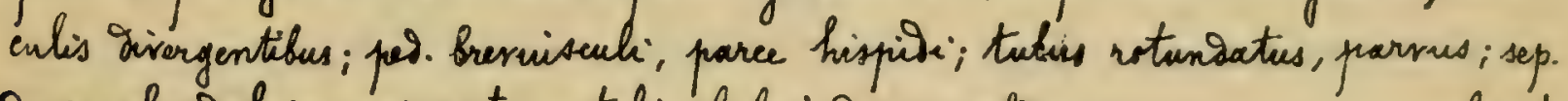
Darsso glamiulosa, pinnato; styli glabri, giscs valse conics, cor........; fruct. minor, rotuniatus, locevis.

Hab. Heirult, at Ganges, unie Sescripsi (H. Laret); Gallix meria, ete.

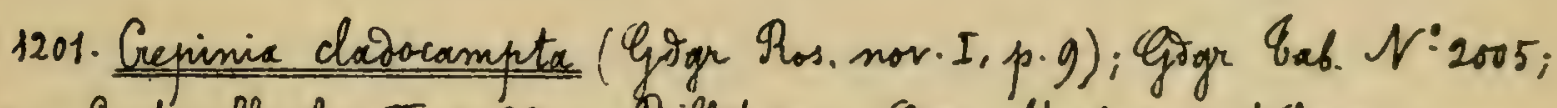
Ejogr Contr. fl slav. II, p.33. - Gillot exs., Giogr Herb ros. N-30\%.

Acculei valios, falcato-gilatati, as ramos flor. panciores; fol. elliptica, glancescentia, as costam parce glanioulosa, pentes couvergentes, fere omnes simplices; pet. aculeati, hine inie pilaso-glasioulossi; stip. hreves, latiusculoc, laeres, auriculis rectis; peo brevissimni, paree glanovelosi; tebus obovatooblangus, saepuis laevis; sep. gorso glasaulosa, pinnulis angustionibus istrue- 
$-32-$

ta; styli breves, glabri, Jisco subplano; cor magna, gilute nosea, inferne ciliata, fruct. ovoidens.

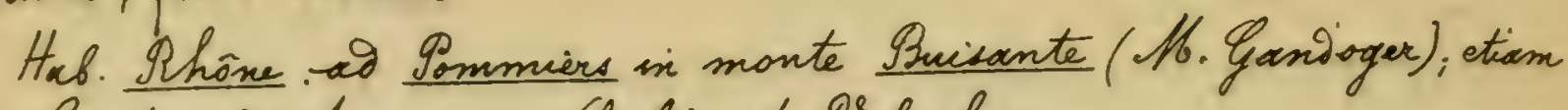
in Croatia ad Toram, in Serbia et Malachia.

1202. Grepinia Lemaitrei (Rip. in Gerlot Cat. Dauph. p. 9g4; Déségl. Cat. p. 182); Gigr Gab. N'2006; Gogr Contr. Fl. slar. II, p.33.-Gige Horb. ros. N-530. Acculei longr; as ramos flor. falcato-subililatati; fol ovato-acuta, glancescentix, à basin folish meaï culeslato; Dentes inaequales, pet. aculeati, glanoulosi, sparse pilosi; stip. Angustac, auriculis rectis; pes. 1-3, breves, hispiovi; tubus oroideus, parce glanoulosus; sep. gorso glanoulosa, pinnulis brevibus instructa; styli glabri, gises plans; cor. alba aut silute rosella; fruct. oroigens, basi paulo glaninulsus.

Hab. Gallia, Chex, Haute- Savoie, ete.

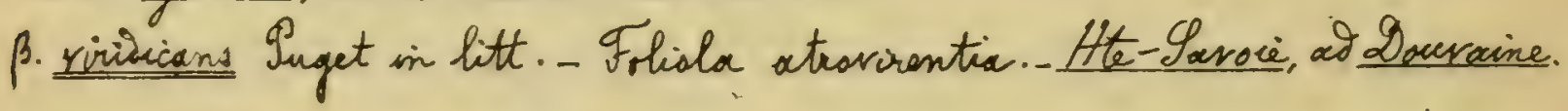

1203. Grepinia Suberti (Rip. in Disegl. Cat. p. 183; Carist \& twire Jes fl.

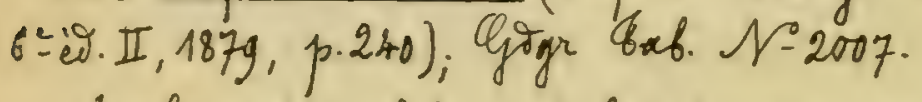

tculei valivic, falcato - Silatoti, as ramos flor geminati; fol late ovato-

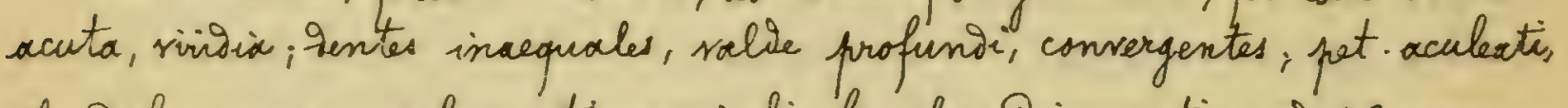
glanoulosi, sparse pilass; stip. auriculis brevibus, divaricatis; pes. 1-3, parce setulosi; 11- 15 m. longi; tubus elongatus, basi hispious; sep. Porss glaxSulosa, pinnulis longis instructo, acumine lato; stgli glabri, gisco comico; cor-magna, albo-rosea; fruct. elongatus, utrinque valde attenuatus.

Hab. Gallia centr. Cher (Ihipart), Hante- Savoci, Ghorens! (Ingeit).

1204. Creninia stenoges Goger mos.

trulei validuli, parum falsats-Silatati, ad ramos flor. stipulares; fol-parra, oborato-oblonga, acuta, basi rotuniacta, lacte viridia, ad costam glanoulosissima; Dentes profunisi, lanceolato-aperti, fere onnes biserrati; pet. aculeati, eglaniulosi, -inferme vix pilasuli, stip. parwace breves, rubentes, anriculis rectis; ped-1-2, hispisissimi, $18 \mathrm{~m}$. longi; tubus oblongus, Pasi in-

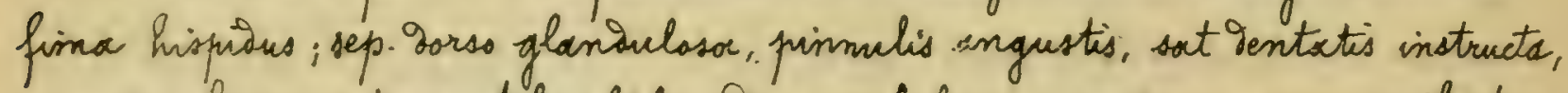
acumine longo, integro; styli glabri, disco subplano; cor rosea, parva, eciliate; fruct. anguste oblangus, basi apressus, apice Langissime attemuatus. Hab. Hispansia erient., Yelencia: as Segabe, Cabrera (C. Dau). Facies C. Douzini (Gratt.), sei folisla inasque nec hiserrata. 
$-33-$

1205. Gepinia macroton Ggo \&ab. N-2008.

tourler falcato-ivilatati, as ramos flor.copiosi; fol-obovats-asuts, basi attic-

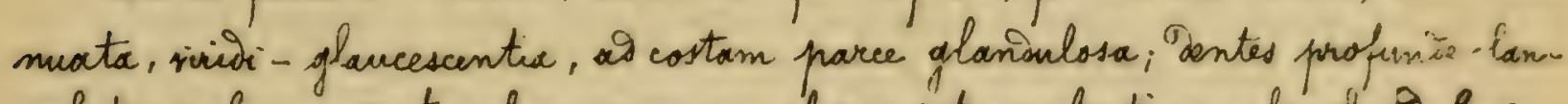
colato-subconvergentes, fere omnes simplices; pet. aculeati, poulo glasbereln-si; inferme uniforian pilosuli; stip. magnae, longoe, aurriculis subrectis; ped. 1-9, parce glanoulosi, $7 \mathrm{gm}$. longi; tubus oslongus, lavis; sep. Dorso paice glan. gulosa, pinnulis onnibus linearibus instructa; styli glafri, gisco csmic; cor. ignota; fruct. oblongus, basi notuni tuss: xpice longe attermatus.

Hab. Hehretix, Yalaic: as St Nawrice, Sex (L. Favrat).

If Petioli plus minus laxe et imique pubescontes.

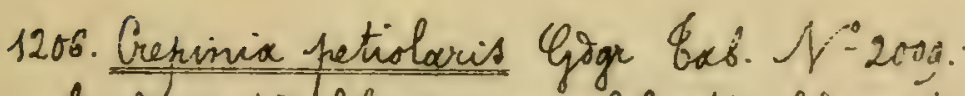

Aculir sat defiles, porum falcati; fol-ovato-elliptur, acuta, bass attemeate, petiolulata, os costam eglanouls-so, santes inacquales, pet. villos-plan-

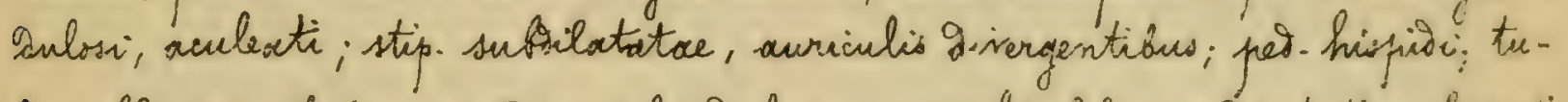

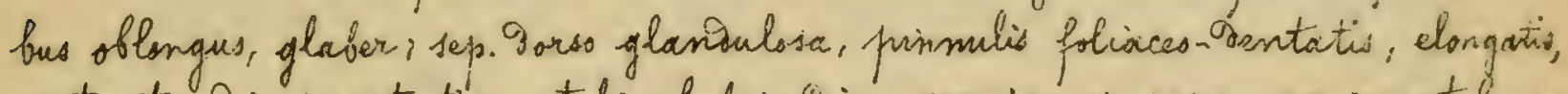

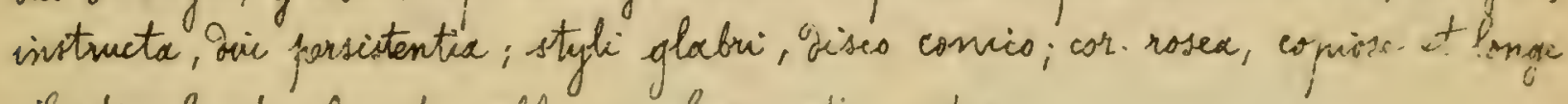
ciliata; fruct. sborato - sblongus, basi atienuatus.

Hab. Dhane, as Limonest: La Bérollière (P. Chabert).

1207. Gepinia ptylodes Gigr \&ab. N-2010.

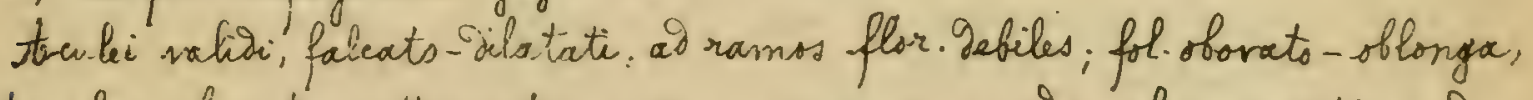
acuta, basi franter iteruata, coriacea, nervosa, siribi-glaucescentia, as costam glanoulosx.; Dentes profunivi, lanceolate; fere omnes simphies; pet. acu -

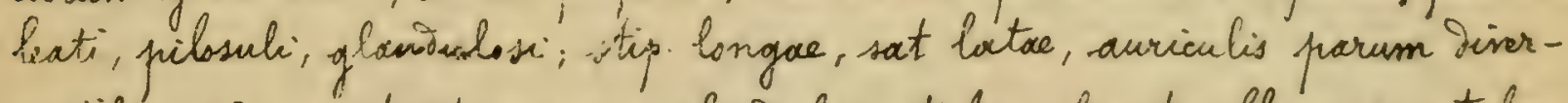
gentibus; pes. 1-2, taritim apise glandulose; tubus oborato-oblongus, setulosus; sep. Isrso glaniulssice, pinmelis integris, latiusculis, longis instructa; styh: glabri, subecalite, disco parvo, connico; cor........; fruct. ovoidew, basi rotunratus, apice truncato - strangulatus.

Hab. Hacucluse, at Lallen de la Baume (fo. Tutheman).

1207. Crepinia ixcounojosentron (Gögr Ros. nor. II, p. 18); Goger \&ab. N:2011. Gogr Herb. ros. N:196.

Gracilis, flexuoss; aculer Debiles, faleato-gilatati, breves, ramos flor. rari; fol.minuta, oborato-iuboblonga, utrinque acuta, pallide et lucide vi- 
$-34-$

rivia, ai costam temiter glanialosa; gentes lanceolato-recti, fere omnes biser. rati, pet-lase et parce pubascentes, glanoulasis, aculeati; stip. latiusculae, ouriculis rectis; peg. 1-2, sparse hispituli, 10-12 m. longi; tubus oblongus, in 1/3 inf-glaniulosus; sep. Darso glaniulosa, pinnulis longis, integris, angustioribus instructa; styh glabri, 2isco valoe comics; cat. pallide rosea; fruct-pervus, anguste sblongus, utrinque, sed aprice praesertion, attenuatus. Hab. Phone, at tomas (M. Gavidoger).

120g. Crepinia nemausensis Gogr $q_{\text {ab. }} \mathcal{N}: 2012$.

tbulei inclinats-Bilatati, do ramos flor. copiasi; fol. paura, obovatoacuta, basi attenuata, ad costam glanisulosa; ientes lanceslato-convergentes, fere onnes biserrati; pot-glansulosi; aculeati, in $1 / 2$ inf laxe villosi; stip. rubentes, latae, auriculis rectis; ped. 1-2, hispidi, 4-6 me Longi; tubus oblongus, toto sparse glanimlosus, sep. Sorso minute glanioulasa, pinnulis latis, 2-3-rentatis instructa, reumine in tegro; styghi glabri, Disco canico; cor.eciliata; fruct. oblongus, utrinque attenuatus.

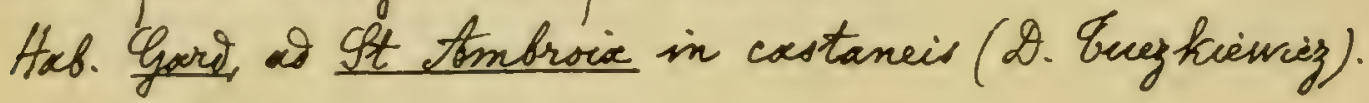

1210. Grepinia eviora Gggr Eab. N-2013.

Acculei pauciores, subfaleato-Silatati, oid ramos flot. saepe mulli; fol. paira, ovata, vix acuta, basi rotunsata, ad costam parce gllanisulosa; Tientes recti, inarquales; pet-glanialssi, unifariam laxe longeque villosi, inf-inermes; tip. meviscres, auriculis sisergentilus; pad saepe 1, hispidali, 12-15 m. longi; tubus elongatus, gracilis, laevis; sep. vivivia, gorso glavioulosa, anguste pinnata; styli glabri; discs conico, cor. rosea, fruct. elongatus, utringue valie attenuatus.

Hab. Houte-Savoie, ad Singy (Suget).

1211. Bepinia callichroa Gogr Eab. N-2014.

Aculei copiosi, falcato-gilatati, ad ramos flor-geminati; fil ovatoacuita, basi cororta, as costasm glangulosa; antes inarquales, profunis; convergentes; pet parce aculeati, glandulosi, toto laxe pilosi; stip. mesioeres, auriculis ciliatis, givergentibus; ped. 1-3, hispisali; 8-15 m. longi; tubus oblosegus, plerumque basi setosus; sep. rubra, gorss glansulosa, pirnulis angustis, elongatis instructa; styli glabri, Sisco valde comico; cor-nosea; fruct. blongus, apuie attenuatus.

Hab. Haute- Lavore as Annecy et Siningy (Suget). 
$-35-$

1212. Gepinia nematochroa Gögr mss.

Intense propures-violacen; vculei faleato-dilatati, al ramos flor. geminati; fol. elliptico-obovata, acuta, basi rotunaseta, virioia, as costam glanioulosa, dentes inaequales, profunisc, lanceslacto-subconvergentes; aeque biserrati et simplices; pat. aculeati, glanidulosi, inferne pubescentes; stip. latare, rubentes, auriculis Divergentibus; peo . 2-3, hispidi, 8-9 m. Longi; tubus sblongus, in $1 / 2$ inf glaniulosus; sep. 9orso sparse glanoulosa, pinnulis onsibus parum latis, 5-6-6 sentatis instructo, acumine integrs; styli glabri; Dises paulo conics; cor-magna, rosea, eciliata; fruct. oblongus, basi subrotuniatus, apice attenuatus.

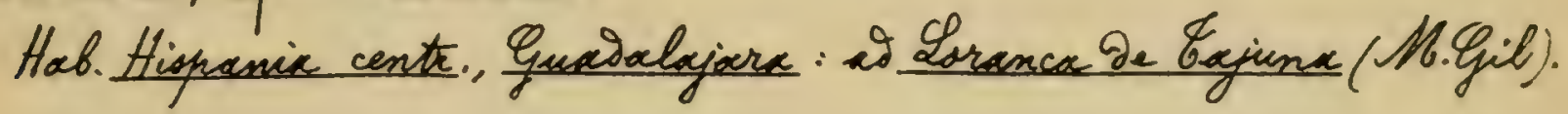

1213. Crepinia mirarhyncha Goge 8nb. N-2015.

toculei falcato-gilatati, as ramos flor-panciores; fol. late svata, subattemuata, basi rotuniacta, ai costern glanoulosa, Dentes inaequales, necti; pot-glandulosi, fore toto laxe pilose; inf. inermes; stip. magnoe, auriculis rectis; pes. 1-3, glanioulosi, $10-16 \mathrm{~m}$. longi; thehes oblongus, laevis vel basi paulo glanoulosus; sep. virisia, gorso glaniulosa, pinnato-subsentata, accumine lato; styli glabri, Disco comics; cor.rosea, fruct. oblongus, ad apicem freviter attenuatus.

Hab. Houte-Savoie, is tonnecy-le-griux (Muget).

b. Ityli villosi vel lanati.

f Fructus rotuniatus val ovatus.

1214. Crenimix cyclacalyx Gogr Gab. N-2016.

Acculei rarissimi, gebiles, as ramos flox. mulli; fol oblongo-acuminata, basi attenuata, ai costam eglañoulosa; Dentes inaequales, aperti; pet inermes, parce glanaulosi, unifariam tenuiter pubescentes; stip. Congae, auriculis $g_{i-}$ vergentibus; ped. 1-4, glandulosi, $14-21 \mathrm{~m}$. longi; tiebus orato-rotundatus, toto sparse glaniaulosis; sep. Forso glanamloss, pinmulis angustis instructor, styli villosi, Misco paulo comico; cor rosen; fruct. subolobosus.

Hob. Haute-Savori, as Habire-Zullin ('Puget).

1215. Grepinia streptomoupha Gagr Gab. N-2017. Gogr Herb. ros. N $36 g$. Acurei varissimi, falcato-Silatati, as ramos flor articulato-tortuosos 
$-36-$

mulli; ist. lats obovata, etringue parum attenuata, os costam glangulosa, Sm.t. vividia, "sentes inasquales, longi, profunisi, aperti; pet aculeati, glan-

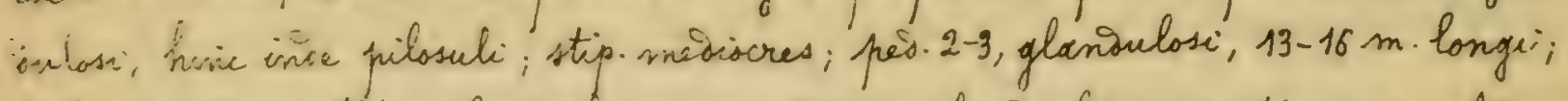
tiubus vorieus, toto glanioulsis, sep. Darso glaniulosa, erecta, pinnulis angustis, longis, copiose instructa, scermine lato; styhi exserti, hirsuti, diseo subrlans; cor.palis rosea; fruct. arato-rotunistus.

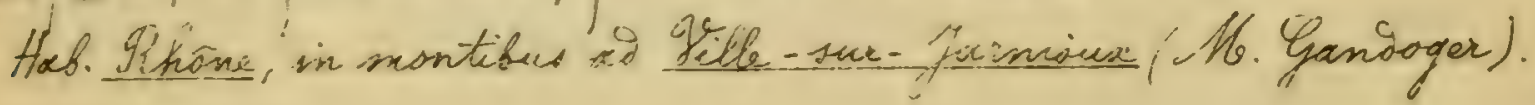

1216. Crejpiria rethervica yiger miss.

Heculei sefives, temuter facicati, as rams fur milli; fol late oborato-

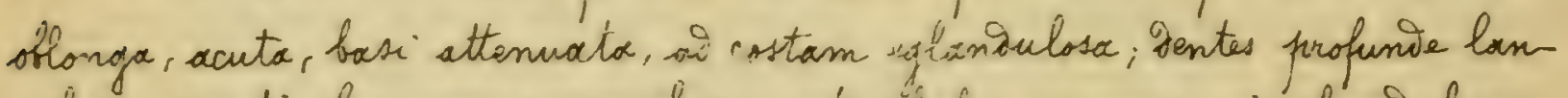
colati, aperti, Lere smnes simplices; pet-glefori, inarmes, vix glandulosi; stip. brerss, virices, auriculis rectis: heo. 1, givnsulosi, 6-8 m.longi; trebus svato-ellipticus, lasvis; sep. Sorso temuiter glanisulosa, pinnulis hinearibus, Dentatis inotructo, acumine latione, intedis; stegli hirsuti, discs plans; cor. ecihata; frinct-orsto-ellipticus, besi rotunatus, apice vix attenuatus.

Hab. Tossia centr. as Coula ('inger).

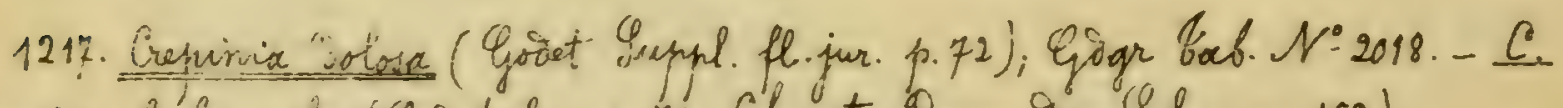
camina f. firmula (Goot l.c.p. Fi; Christ Trosen ger Ychw. p-102).

Greciem sohue male cognitam sic exposit $C$. Christ l.c. : Ferschieden Guren schwächern Hinchs, (1 Meter). Sinne, stark verzweigte Foste, leicht gebogene bis gerave kurze uno dinne Stacheln, ire haufig in ren obern Sheilen ges Ftrauchs ganz fellen, fast totalen Mangel an Dirisen, Kleine, oval-lanzettliche bis lanzettliche, lang gespitzte oor kiufig breit abgestumplte Mlattchen mit etwas keiliger Masis, spiegehoer Oberfläche, Zähne lanzettlich tief, gross einfach bis appelt. Bliethen inzzeln, Klein, Kalchzipfel mit linealex, arïsenlosen twhingseln. Griffel Kahl orar schwach behavit. Tetalen rosa bis weisshich. Frucht lang gestielt, oval bis Krgelig. Klein. tonorert ab mit sticlorisigen Bhithenstielen uni einzelnen Driusen auf Dem Riveken der Kelchzipfel. Auf den Gempenflien steht in Strauch mit auffallenis Kartigen, festen un gerben Gglittihen, arisigem Mlattsial, untern Glattzïhnen uns II lithenstiel.

Hab. Halvetix, in Jurasso, ete.

to procedentibus ramis floriferis plerumque sculeis statim Sistinguitur. 


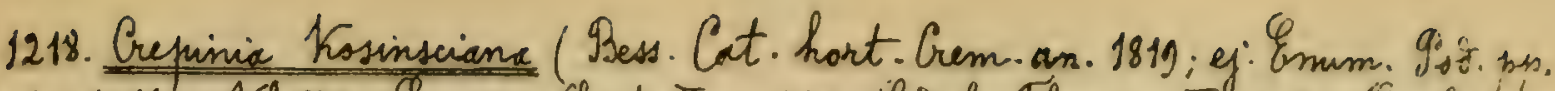

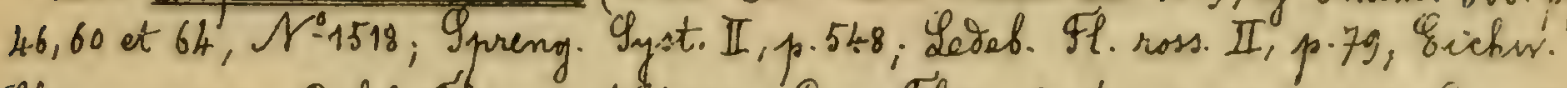

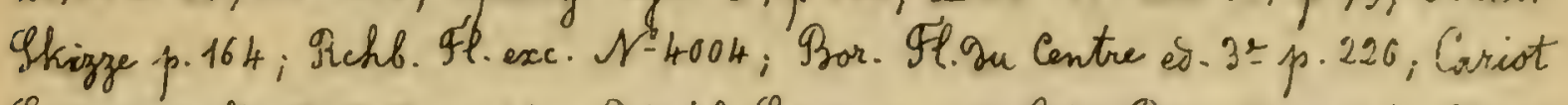

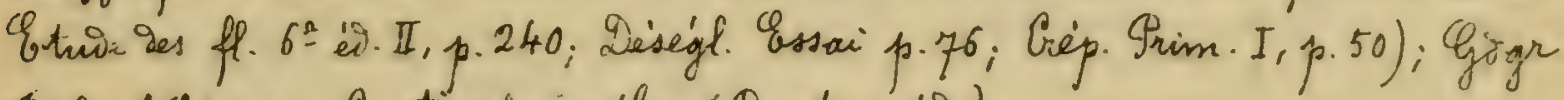
Tab. N-201g. - i stipulaciantha (Mrest. inéd).

Aculei treves, falcats-Silatati, as ramos flor. saepe stipulares; foll am-

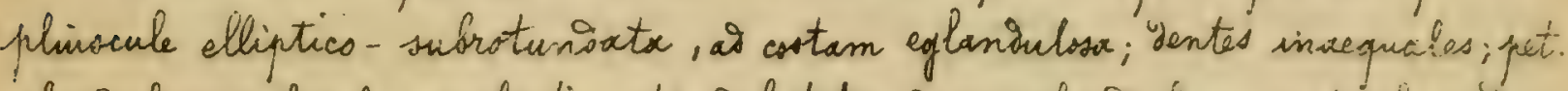

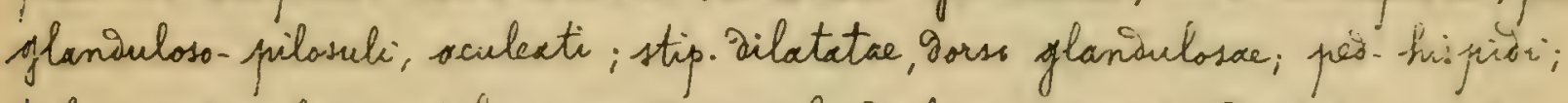

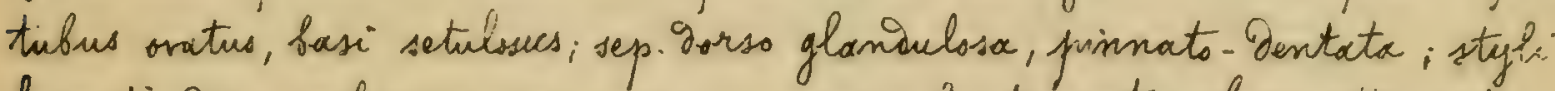
hirsuti, Tisco subconico; cort magna, rosea; fruct. ovatus, basi attemuatus.

Hab. Europa mesia.

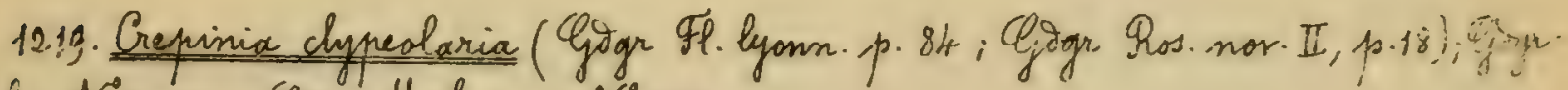
tab. M:2020. - Gogr Herb. ros. N'227.

Acculei valiosi, falcate-jilatati, as ramos flor. copiosi, foll paros, allipticsacuta, basi abreipte triencata, glauca, ai costam glanivilosa; ientes arguti, fere onnes biserrati; pet. aculeati, glanoulosi, inferme pubescentes; stip. latar; per. 2-5, glaniulosi;. 7-10 m. longi; tubur ovoideus, basi lacris aut glaniouisiss; sep Darto parce glandulosa, pinnulis angustis instructa; styli parce villosi, gisco subplano; car parva (vix 3 cent. diam. lata), albo-rosella; fruct. parwus, ovatus, basi returiactus, apice attenuatus.

Hab. Rhone, at tornas (Mb. Ganooger).

1220. Grepinia pseno-Chaberti Gigr tab. N:2021.

Acculer valivi, ad ramos flor. falcato- Dilatati; fol-ovato vel elliptica, crasse, virivia; Dentes breves, convergentes, fere omnes simplices; pot aculeati, glansulosi, sparse pubescentes; stip. rabentes, auriculis rectis; pes-1-3, breves, vix setulosi; tubus ovatus, laevis, glaneus; sep. Oorss glanoulosa, oir persistentia, pinnulis brevibus, rentatis instructs, styli hirsuti, Disco plano; cor-magna, rosea; fruct. maximus, oratus, utrinque attenuatus.

Hob. Thone, at Charbonsieres (At. Carist).

1221. Crepinia psendo-Ozanomia Gogr mss.

Aculei breves, sat parvi, inchinato-subsilatati vel rectiviculi, ad ramos flor-copiosi; fol-parva, sbovato-sblonga, abrupte acuta, besi attemuata, conincer, glaucescentia, ad costam glasiaulosx; gentes profunive lanceslato- 
convergentes, fere onnes biserrati; pat. aculeati, glanisulosi, inferne toto pubescentes; stip. sinises, latae, breves, glabrae, auriculis subvivergentibus; pes.1-2, parcissime glanisulosi, $12 \mathrm{~m}$. longi; tubus ovoideus, sparse glanaulosus; sep. Sorso glensulssa, pirnulis onnibus subfoliaceis, sentatis instructo, acumine integro; styli villosissimi, Sisco subplano; con.........; fruct -magnus; oratoellipticus, fasi rotusidatus, appice abrupte strangulatus.

Hab. Iranssilvamia, Garda Aramyes: at Eoria (2. Richter).

Habites cujusiam Montance (Ozanonia).

\section{t† Fructus ssiditens vel oborato-sblongus.}

1222. Crenimia Hriosomana Gogr tab. N-2022.

teculei panciares, inclinato - Dilatati, as ramos flor. mulli; fol. oblongo-lancoolate, basi attenuata, viriti-glauca, ad costam glasioulosa; gontes apertotriangulares, fere snnes biserrati; pet sculeati, glaniulosi, inferne paulo pubescentes; stip. sivioses, latissimoce, auriculis rectis; per. 2-3, temiter glanouLosi, 16-18 m. longri; tubus ovoisens, lasis, sep. Dorss glasimlosa, pinmulis latis, melse dentato-glandalosis instrueta, acumine lats, dentato; styli hirsuti, asses plans; cor........ ; fuct-parvus, oroidens, utrinque valse attermatus. Hab. Anglia, Stafforshire: io Renn (J. Fraser).

\section{Crepinia puttide Gogr mss.}

Acculei panciores, falcato-2ilatati, breves, is ramos flor. mulli; fol mediocria, oblong-lanceolata, acuminata, basi subrotundata, glaucescentia, do costam glantulosa; dentes triangulares, subpatuli, fere onnes biserrati; pet. subinermes, sat glaniulosi, usque as 2 a foliola toto pubescentes; stip. viriDes, angustae, sat breves, glabrace, auriculis girergentibus; pos. 2-4, parce glan-

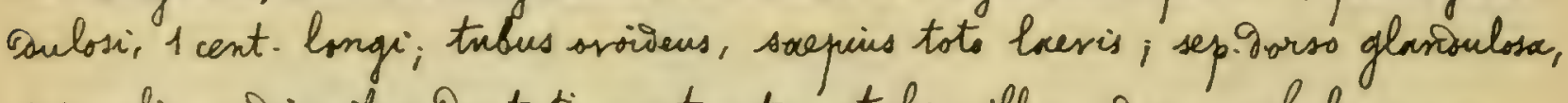
pinnulis midiocribus, Dentatis instructa, styli villosi, gises subplan, cor. ignota; cor....... fruct mesiocris, aroines-comicus, basi rotuniatus, apice longe attemuato-strangulatus, centralis vero inferne contractus.

"Hab. Eransihescia, Eordx toranyos: ad Eorayki' (2. Richter).

1224. Crepinia puinogenita Gögr mss.

toculei breves, parum dilatati, valse et temiter folcati, ad ramos fler. copiosi, fol.contigua, mesiseria, sborato-acuta, base subcoidata, glauea, as 
$-39-$

basin costoce glanoulosa; gentes triangulares, recti, fere annes biserrati; pet: aculeati, glanioulosissimi, in $1 / 2$ inf prorsus pubescentes; stip. Bilatatoe, longae. glanes - virides, inf. Dorso glanisubsae, arriculis subrectis; pes.1-2, glangulosi, 1cent. longi; tubus oroiteus, toto glaniulosus; sep. Darso glanioulosa, pinmulis brevibus, latis, subsentatis, acumine integrs; styli villosi, aises sub. plano; cor........; pruct. majusculus, oroideus, miais turgious, utrinque gepressus. Hab. Eranssihamia, Kolozs: ad Ieloi"- Iebes! L. Thichter)

1225. Creminia parex Gogr mss.

Aculei brevissimi, defiles, suboilatato-falcati, ad ramos flor. geminati; fol-midiseria, oborato-subacuta, basi paulation rotuniata, glduea, at costam glasiaulosa; Dentes arats-convergentes, fere omnes biserrati; pet-aculeati, sat glaniulosi, nic hinc inis pilosuli; stip. sirites, latar, longiusculae, glabrac, auriculis rectis; peo. 1-2, tantum apuse glanioulosi, 1 cent. longi, tubur ovoirens, toto glaniulosus, sep. Dorss glanoulosa, pinmulis mediocribus, dentatis instructs; styli villosi, gisco subplano; cor.........; fruct. mediscris, ar videns, basi rotuniatus, apuice attemuatus.

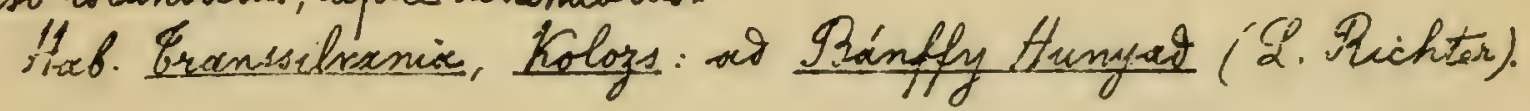

1226. Grepinia enemacharis (Gigr Ros. nov. II, p.19); Gggr Gab. N-2023. C. Sisparilis (Lucani et Ozanon in Mall. soc. 2auph. 1881, p.328). - Gogre Herb. ros. N:17, Soc. dauph. exs. $N \cdot 2849$; Magmier Fl. sel. $N=533$.

Aculei debiles, falcato-subdilatati, ad ramos flor. saepe nulli; fol. oblonga, utringue sension attenuato, io costam pauls glandulosa; dentes acuti, fere mones biserrati; pet. glabri, parce olaniulosi, aculeati; stip.latoe; per.1-2, hispiouli, $11-14 \mathrm{~m}$. Congi; tubus oblongus, sep. Sorss glaniulosa, pinnulis bresibus, angustis instructa; styli villosi, Discs conics, cor....... fruct-magnus, oforato-oblongres, utrinque attemuatus.

Hob. Thone, ad It-Cyr-2e-Châtoux: in monte Sevelette (H. Gandoger):

1227. Grepinia cultellata Gogrmss.

Aculei tenues, parri, minute falcato-dilatati, \&s ramos flor. stipulares, fol-parsa, anguste oblong- lancealata, fasi sensim rotunsata,

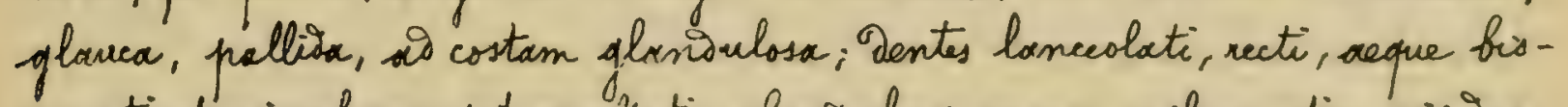
serrati et sienphlices; pat acuiexti, glanisulosi, sparse pilosi; stip. virides, grabrace, treves, angustare, auriculls rectis; pas. 1, toto glanisulosi, 1 cent. 
$-40-$

Longe; tubus ovoidseu, toto plandulosus; sep. Dorso glanisulosa, pinnulis axgustis instructo, stygli villosi, giscs paulo conico, car......., fruct. sat parvus, oroideus, basi subrotunsatus, apice breviter attenuatus.

Hab. Eranssihrmix, Kolozs: ad Mainfly Humyad (2. Richter).

1228. Crevinia ruralis Gìgr mss.

taculei breves, gilatati, valde falcati, is ramos flor-geminati; fol. sat parva, obovata, utrinque subattemuata, lacte viridix, al costam glanioulose; sentes tirangulares, recti, pere omnes biserrati; pet. vix glanoulosi, aculeati, hine inde pilosuli; stip. sirides, angustae, breves, superne medis puberulore, auriculis Sivergentisus; ped. 1, parce glanidulosi; $8 \mathrm{~m}$.longi; tubus obovato. oblongus, toto gense setulosus; sep. Dorso glanoulosa, puinnulis sat latis, dentatis instructa; styli villosi', Lisco subplano; cor........ fruct - obovato- oblongus, basi abrupte subsecurrens, mevio turgious, apuic gepressus.

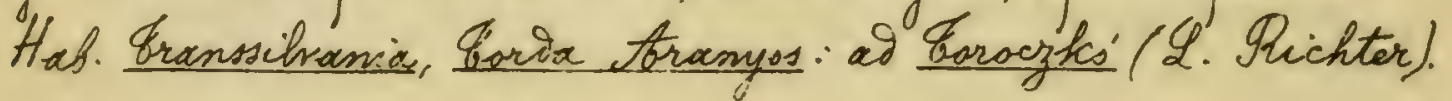

1229. Crepinia sunposito Goar mss.

Acculer minute, rix ailatati, tenuissime subfaleate, at ramse flor. sparsi; fol. lativisurle oblang-acuta, basi notuniata, intense sirictia, ad costarm saepe eglaniulosa; dentes late triangulares, recti, fore onnes simplices; pet. aculeati, parce glanaulosi, basi unifariom pubescentes; stip. virides, glabrace, longinscular, sat latae, auriculis subvivergentibus; ped-1-2, dense hispidi, $8 \mathrm{~m}$. Longi; tebus suboblongus, toto setosus; sep. Oorso glandulosa, pinnulis latuisculis, Dentatis instructa; styli villosi, disco subplans; car........ fruct. sat magnus, oborato - oblongus, basi abrupte decurrens, apice substrangulatus. Hab. Iransilvania, Kolozs: ad Kalota Iz. Kiraly (2. Sichter).

T+T Fructus oblongus.

1230. Greninia platycalyx Gogr \&ab. N-2024. $^{\circ}$

Eota purpurea; aculei falcato-gilatati, abior, as ramos flor nulli; fol. oblong-acuta, basi paulatim attenuata, as basin costar prara glanioulosa; Dentes inacquales, recti; pet. acubati, glongulose; sparse pilosuli; stip.latar aurienlis rectis; per. 1-4, hispidri, $11-15 \mathrm{~m}$. longr; tubus oblongus, laevis; sep. Lorso glanivilose, pinnulis angustioribus instructa; styli hirsuti, dises subplann; cor....... fruct. magnus, oblongus, basi rotundatus, ad 
apicem valde attenuato-strangulatus.

Hab. Heire, in mant. Le Lappey (Moullu).

1231. Crepinix oculus solis Ggogr mos.

fruler Debiles, falcato-Dilatati, ar ramos flor. sparsi; fol. obovatsoflonga, acumixata, basi attemuata, glauca, as costam glandulosissima; Dentes lanceslato-canvergentes, fere omnes biserrati; pet. aculeati, valve glandulosi, sparse pulasi; stip. rubentes, batac, auviculis sat givergentibus; pes. 2-3, hispidi, 8-11 m. langi; tubus oblangus, toto et parce hispidus; sep. Darso glandulosa, pinnulis angustis, 5-6-Dentatis instructes, scumine integro; styli hirsuti, elongato - subcoaliti, gises plans; cor. eciSiarta; fruct - ovideus, base gepressus, apuice attemuatur.

Hab. Hispania for, Catal. : M Marai fer. Gerona (I. Yayreta).

1232. Crepinia angustituba Gogr mss.

Aculei brevs, parvi, parum faleato- Dilatati, as ramos flor. genvinati; fol-meviocria, obovata, utrinque subrotundata, atronirentia, as costam. parce glengulosa, Dentes ovato-subconvergentes, fere ornnes simplices; pet. acule ati, paulo glanivulosi, sparse pelosuli; stip. viriedes, angusta, freves, glaibrace, arriculis rectis; ped. 1-2, tantum apice jlandulosi, 1 cont. longi; tubus oblongus, toto glanaulosus; sep. Iorso glanduclose, pinnulis sat angusAis, is entatis instructa; styli villosi, gises sat conics; cor........, fruct anquste oflongus, utrinque longe attenuatus, apice valise strangulatus.

Hab. Eranssihania, Exida toranyos: ad Goria Hasedek (2. Rechter).

1233. Crexinix conflictata Gigr mss.

Acculei valisi, falcato-Dilatati, as ramos flor-copiosi, fol.ampluiscule -blong-acutor, basi subattenuata, glaucesontix, ai costam glansulosisimai ientes late triangulares, aperti, aeque simplices et biserrati, pet. aculeati, copiose glanoulosi, basi unifariam pubercentes, stip.viridies, angustae, Freviusculae, apice gorsi paulo glansulosac, auriculis rectis; pet. 2-3, hispiauli, 12-13 m. longi; tibus suboblongus, toto sparse glaniaulosus; sep. Dorss glaniulosa, pinnulis latis, Pentatis instructa, styli sublanxti, Dises paulo conics; cor........; fruct. magnus, suboblongus, basi rotunitatus, aprie conicus t attenuato-strangulatus.

Hab. Eransilvamix, Goriox tranyos: as simfoha (L. Phichter). 
$-42-$

1234. Crepinia slancensis (Mancic) in Gogr bab. N-2025. - Gogr Contr. ad fl. slar. II, p. 34.

Atculei pauciores, parni, faleato-Dilatati, as ramos flor-mulli; fol. oflongo-acuta, basi attenuata, glavea, al costam eglanioulosa; gentes lancoolato-sperti, acope simplices et biserrati; pat. aculeati, hinc inoe glanguloso-pilosuli; stip. virises, angustac, surriculis divergentibus; pod-1-2, hispidi, 7-9 m. longi; tubus oblongus, toto glandulossus; sap. Oorss tenuiter glanoulosa, pinmulis latiusculis, 2-3-Dentatis instructa, acumine hineari, integrs; stygh villosi, 2ises valse comico; cor lacte rosea, eciliata; fruct. ollongus, utrinque xttenuatis.

Hab Serbia bou. ad Slanci (\%. Tancic).

1235. calyciera Gogr \&ab. N:2026.

beulei folcato-gilatati, at ramos flor. mulli; fol oblongo-acuta, basi attenuata, glacescentix, as costam eglanoulosa, sentes lanceslats-convergentes, inseguales; pet-inermes, eglaniulosi, inferne unifariam pubescentes; stip. latae, longae, auriculis bivergentibus; ped. 2-3, parecis sime glangulasi, 19-22 m-longi; tubus sblongus, laenis; sep.gorso sparse glanouloss, pinnulis latis, longissimis coprisse instructa, acumine gilatato; styli pubesantes, gises plans; cor. rosea, eciliata; fruct. oblongus, wtringue attemuntus. Hab. Saxania, as Nebra (C. Hoursknecht).

1236. Geninix formifoha Ggogr mss.

to-culei parri, breves, parum gilatati, temicter falcati, ad namos flor. copriasi; fol ample oborato-suboblonga, paulo acuta, basi rotundata, firrno. coriacea, intense rivitia, ad costam rix glanisulosa, dentes late ovatoconvergentes, fere omnes biserratic; pet. aculeati, copiose glaniulosa, plerumque glabri; stip. Sarso rubentes, amplac, longae, auriculis givergentibus; pes. 1-2, tantum in $1 / 2$ sup. glaniulosi, cacterum laeves, $1 / 2$ cent. longi i tubus oblangus, toto glanaulasus; sep. Dorso glanionlosissima, pinmulis ventatis, brevibus instructa, acumine integro; styh lanati, disco subcomico; $\operatorname{cor} . . . . . .$. ; fruct. oblongus, basi gepressus, ai aprice longissime attenuato-strangulatus:

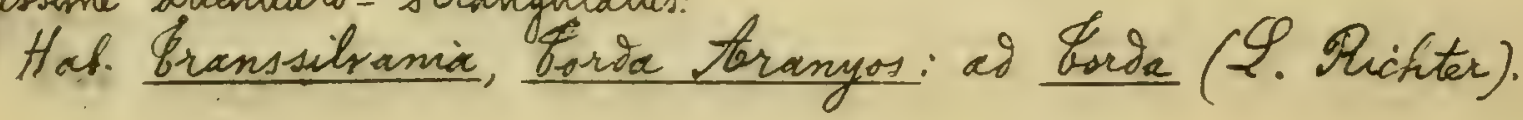




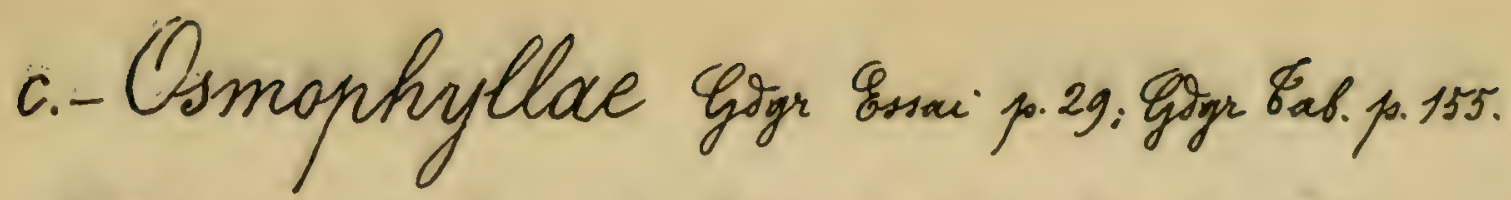

Foliolis ommins et argute biserratis, odoratis, at costam megiam glandulosis, subcorciccis, nervosis; sepalis post anthesine intersum erectis, usque as colorationem frectus persistentibus, dein caducis, sculeis saepe conformibus, intergum in setos glansuliferas frodeuntibus. - Ravic nownumquam reptans, tune prutices nel suffertices humiles, graciles ramisopic tenciibus frocesiti.

Acculei conformes

1 foculei Simorphi, alii setaces-ylaninlifere; ahii asunci

Sepala dorsoeglaniulosa

2 Sepala orso jlanoulosa

Styli glabri

3 Styli sillosi vel lanati

Fructus svoideus val obsvato-oblongus

4 \{ructus oblongus

Fructus svoidens val sbovato - oblingus

$5\left\{\begin{array}{l}\text { Fructus oblongus } \\ \text { Fresctus svatus }\end{array}\right.$

Gubies laevis

$6\{$ Eubus phus minus hispidus

7 Discus planus; foliola basi subattenuata

7 Lis-cus plus minus conicus, folis la basi coriacta

Styli glabre val subhirsuti

8 Gtyle villosi rel lanati

Tarbus lavissimus

9 Gubus plus suines hispions

Fructus ellipticus, ovoidens val obovato-oblangus

$10\{$ Fructus oblongus

Foliola basi rotuniata

$11\{$ Folisla basi plus minus attenuata

Foliola basi rotuniacta vel coriata

8

2

Irecies $\mathcal{N}^{2}-1237-1241$.

Speves $N-1242-1248$.

6

Inecies $N-1266-1271$.

Priecies N=124g-1250.

Qrecies $\mathcal{N}^{\circ}+262-1265$

Qhecies N-1251-1253

precies N:1254-1261

$12\{$ Foliola bosi plus minus attenuata

Qpeais $\mathcal{N}-1272-1278$.

Irecies $N-127 g-1283$.

qrecies $N=1284-1288$.

Species $N=1289-1201$. 
$13\left\{\begin{array}{l}\text { Frliola parra, oborata } \\ \text { Foliola ample slonga }\end{array}\right.$

Speries $N: 1292-1298$.

\{ Folisla ample oblonga

Fructus ovoidens

fhecies $N$ :12gg-1300.

15

$14\{$ Fructus obs rato - oblongus, vel oblongus

Fructus rotuniatus vel ovatus

\&ubus glaber rel tantum basi infina glandulosus

Ipecies N-1301-1307.

16

$15\{$ Eubus saltem usque ad $1 / 3$ inf. hispiass

Foliola parma, ovato - obtusa

16 Foliola ample obovato - oblonga vel oblonga Eubus glaber

17 Gubus plus misms hispidus

Tearenculi onnes plus minus glangulosi

18 Geamneuli ali laeves, alii glanoulosi

Foliola basi attenuata

$19\{$ Foliola basi rotuniata

Tetioli glabri vel sparse pilasi

20 Setioli toto hirsuti

Inecies $N=1308-1314$

yperies $N-1315-1317$.

Species $N: 1318-1324$.

Lpecies $N \cdot 133 g-1348$

Inecies $N-1325-1328$ Ipecies $N-132 g-1331$. Ipecies $\mathcal{N}-1332-1338$. Irecies N $1349-1355$. Inecies $N=1356-1360$

1. Aculei conformes.

a. Sepala Dorso eglanialosa.

+ Ityli glabri.

\$. Fructus ovoideus val obovato - oblongus.

1237. Crepinia macramedas Gogr tab. M-2027.

tealei panciores, parum gilatati, valde falcati; ar ramos flor. mulli; fol. ample oborato-elliptica, subrotunoata, basi rotundato-subcordata, as costam eglaniulosa; dentes magni; biserrati, aperti, pet. virises, aculeati, sparse glangulosi, usque as 2a foliola unifarian pubescentes; stip angustre, scurriculis givergentibus; ped. 1-3, termes, setosi, 11-17 m. longi; tubus oblongus, lacris; sep. Dorso eglanouloso, pismulis parum latis, paucioribus instructa, acumine subfoliaces; styli glabre; subcohumnares, gisco paulo comic; cor. rosea; fruct. parvus, sborato - oblongus, utringue depressus.

Hab. Hautes- Tyrénés, as Camprieil (H. Bordere).

1238. Crepinia abhorrens Goge mss.

Acculer breves, falcat-gilatati, as ramos flor. mulli; fol. parra, ofo- 
$-45-$

vato-oblonga, ncuta, basi rotuniata, conincea, intense virivia, as costam glaniaulosa; Dentes valde profundi; lanceslato-subconvergentes; pet-glarioulosi, aculeati, hinc inde vix pilasuli; stip. virides, breves, dorso eglandulosac, auriculis sat 9 ivergentibus; pes. 1-3, aculeati, 16-20 m. longi; tubus orideus, laeris; sep. Darss eglaniulosa, pinmulis parum latis, sat gentatis instructa; styli glabri, gisco subplano, minimo, fruct.minimus, ovideus, basi rotungato-umbili:catus, apice longe attenuato-comicus; cor. ignota.

Hab. Hispania centr. Mairid: at Escorial, Herreria (M. Compaño).

1239. Crepinia lepiada Goor Herbor. Gyren. (1884), p. 44.

Acculer elangati, inclinato - suboilatati, as ramos flot geminati; fol. sat parva, oborato-blonga, acuta, basi rotuniata, vividi-glaucescentia, as costam tenuter glansulosa; gentes biserrati, triangulares, recti; pet. glabri, aculeati, glanoulosissimi; stip. virides, breves, Dorss glandulosac, suriculis subrectis; ped. 1, temuiter hispiduli, 14-16 m. longi; tubus oroidens, laevis; sep. Dorss eglanDulosa, pinnulis latis, Dentatis instructa; styli breves, glabri, Dises paulo comic, $\operatorname{cor} . . . . .$. ; fruct. svoidens, basi rotunistus, apice sbrupte strangulatus. Hab. Basses_- Prénées, at Nay (M. Gañoger).

1240. Crepinia apposita Gigr tab. N-2028.

Acculer alongati, temiter faleati, as ramos flor. panciores; fol parra, elliptico-oftusciescula, basi subrotunoata, suprac paulo glauceseentic, as costam parce glanidulosa; dentes biserrati, lanceslato-aporti, pat-glaniulosi, aculacti, inferne paulo pubescentes; stip. virides, auriculis rectis; pod. 1 , inferne sublaeves, 12 -14 m. longi; tubus obovato-oblongus, laevis; sep. gorso eqlaniulosa, pinnulis anguotis, 3-5-Dentatis instructa, acumine angusto, subsentato; styli glabri, gises paulo comics; cor-eciliata; fruct. svoideus, wringue sensim atternatus.

Hab. Hispania bor, Logroño: ad Rasillo, Lierra he Cameros (J. Lubic).

1241. Crepinix pyenocantha Gogr mss.

Aculei longi, parum falcati, rubentes, ad ramos flor copiosissimi; fol. parra, elliptic-acuta, fasi rotunstata, as costam glansulosa, lacte viridia, corrincer; ontes biserrati, triangulares, aperti, pet. unbentes, glasioulosi, aculeati, inferne sparse pilosi; stip. rubentes, subtus evlanoulose, frevs, latac, auriculis subsivergentibus, pas. 1-2, parce setulosi, $10 \mathrm{~m}$. longi, tubus 
$-46-$

oftongus, laevis; sep. Oarso eglanimulosa, pirmulis onnibus dentatis, latiusculis, copiose instructa, styli glabri, Disco comis, cor parra, eciliata, fruct obovato-oblongus, utinque atternuatus.

Hab. Flgeria, Constantine: 2o 2jebel Orack (Y. Mebour).

$\S \S$. Fruetus oblongus.

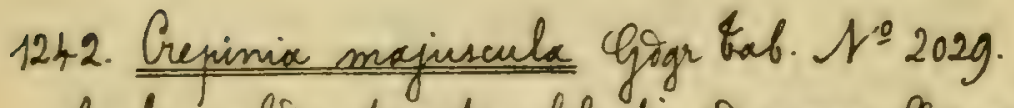

Aculei salisi, temuiter falcati, as ramos flor. mulli; fol late obovatooblonga, reuta, basi coriacta, intense virioric, subglauca, at costam eglanioulosa; gentes biserrati, triangulares, aperti, pet glabri, glanioulosi, subiner mes; stip. virios, latissimae, auriculis Sivergentibus; pos. 4-10, hispitissimi, $15-17 \mathrm{~m}$. longi; tubus oblongus, laevis; sep. Porss eglanialosa, pinnulis latis, 4-6-gentatis instructa, acumine subfoliaces, in tegro; styli glabri; Siscs plans; cor-magna, rosea, eciliata, fruct ample oblongus, basi depressus, aprice longe attenuatus.

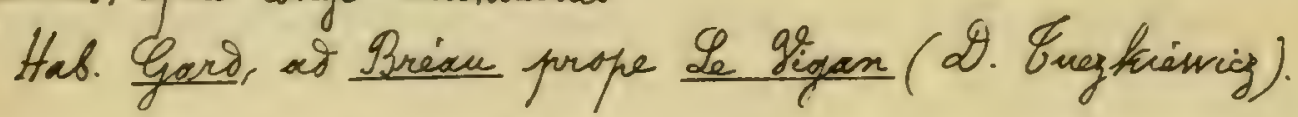

1243. Crepinia Drepanoven Giogr mss.

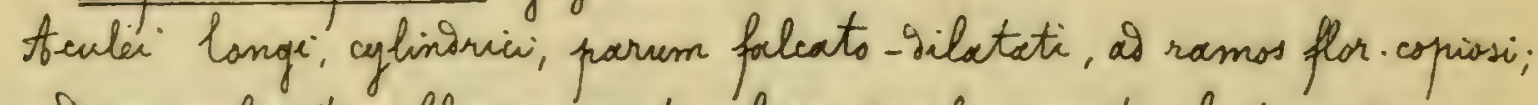
fol mediacric, obovats-oblanga, scuta, hasi paulo cuneata, bacte vrivica, parum coriacex, ai costasi glanialssissima identes biserrati, profunai, lanceolato-recti; pet. glansulosa, aculeati, glabru; stip. breves, viribes, gorso acubeslatae, auriculis rectis; peg.1-3, to to hispioi; 11/2 cent. longi; tubus oblongus, laeris; sep. Torss eglarioulosa, pinmulis omnibus parum latis, 2-5-Dentatis, acumine integro; styli glabri, Visco plans; cor majuscula, eciliata; pruct-parrus, oblongus, utringue longe attenuatus.

Hab. Hisnamia orient, gelencia: at foltura (C. Sau).

1244. Crepinia umblyotis Gogr Gab. N-2030.

Acculei falcato - rilatati, av ramos flot. geminati; fol. latiuscule obovato-elliptica, paulo acutor, hasi sensim rotunioato, subylouca, ad costam glanoulssa; sentes biserrati, triangulares, ape:ti; pet aculeati, grancioulosi,

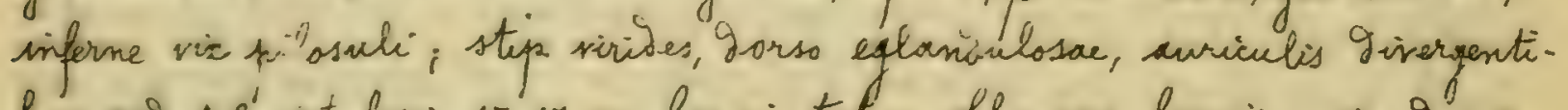
bus; pes. 1-2, st. losi, 15-17 m. longi; thebus sblongus, laevis; sep. 90 orso eglanioulosa: pinnulis latis, valse sentato-glanidulosis instructo, acusnire 
$-47--$

Pliaces-laciniats; styli glabri, gisco plano; cor. eciliata; funct. oblongus, uitrinque attermatus.

Hab. Hispania bot., Catal.: al Olt (M. Gayreda).

1245. Crepinie orbicularifolia Gogr \&ab. N-2031.

toculei elongati, temuiter folcati, as tamos flor. mulli; fol orbiculatooftusa, basi coriata, uninque viritio, at basin costae glasioulosa, bentes biserrati, fuofunive lanceslats - aperti, pet glanoulosi, aculeati, glabri, stip. rubentes, latiosimne, auriculis aivergentibus; per - 2-5, sparse hiopios; 10-12 m. longi; tubus elongortus, gracilis, laenis, sep. Dorso aglanioulosa, pirmulis latis, valse laciniato-glanioulasis copiose instructa, acumine lato, laciniats, styli glabri, Gises comics, cor eciliata, fruct parrus, anguste oblonopus, utimque attemiativ.

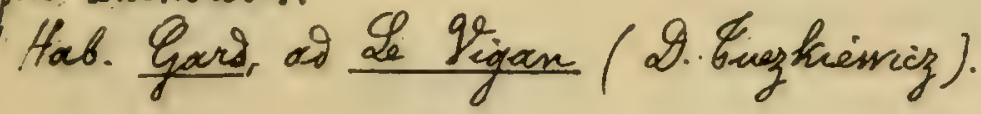

1246. Crepinia unicolor Googr bab. Ni2032.

Areulei rarissimi, falcato-jilatati, al ramos flar. mulli; fol. laste oborata, breviter acuta, basi rotuniacto, unisigue intense virivia, ad costem praniulosa, dentes biserrati, late aperto-triangulares, pet subinermes, glabici, - Ranoulosi, stip. latoe, gorso glanaulosse, auriculis givergentibus; pas. 1-3, thispituli, g-11 m. longi; tubus oblongus, loevis; sep. Dorss eglanidulosa, pinmilis sat latis, 3-4-2entatis instructa, acumine parum lato, integro, styli glabri, gises paulo comis; cor ecchiacta; fruct. oblongus, basi roturiactus, aproe attemuatus.

Hab. Gart, at Bréan prope Le gizwen (D. Enezhiewizy).

1247. Gepinia stephanophora Gojgr Gab. N-2033. Gotyr Harb. ros. N-214. Acculei elongati, temieter falcati, parum rilatati, as ramos flor. copiosissimi fol. late sborato-acutor, basi rotunoatoc, gilatator, as 'costam glansulos ; Ventes latissimi, fiserrati, aperti; pet rubentes, aculeati, spave pilosulo-granaulosi; stip. angustae, Torso eylanisulosac, aurriculis girergenHbus; per. 1-3, graciles, hispituli, 11-18 m. longe; tubus oblongus, haeris; sep. Donso eglanoulosa, pinnulis fohiaces-dentatis, coprose instructa, acumine loliaces; styli glabri, gises comics; cor pallise rosea; fruct. obovatus, apuice attemuats-strangulatus.

Hab. Syrenaci cente, ad Gieire (H. Bordère). 
$-48-$

1248. Crepinia sulalauce Goge bab. Ni2034.

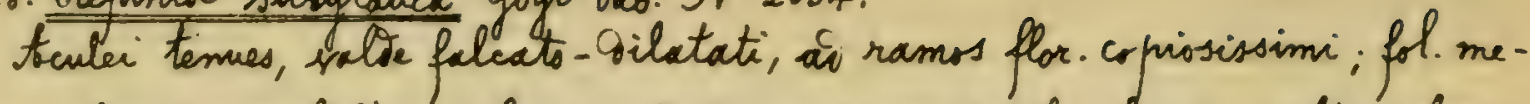
Discria, obovato-subbotusa, basi coriata, suparne pauls glanca, subtus glaucsOiscolorix, ad costam glanisulsais sima; Dentes biserrati, triangulares, aperti; pet-glanoulosi, ceuleati, glabri; stip. virites, Dorso eglanisulosar, auriculis rectes; pes. 1-2, glanioulosi; inforne laves, $13-15 \mathrm{~m}$.longi; tubus oblongus, laevis; sep. Forso eglanioulosa; pismulis parum latis, 3-5-Dentatis instructe, acumine lato, integro, styli glabri, Disco prauls conico; cor eciliata; fruct.minimus, oblangus, utimque atternatus.

Hab. Hispamia bot, Catal.! al Ialgás pu. Gerona (9. Gayreida).

T+. Styli villosi vel lanati.

§. Fructus ovatus

1249. Cepinia tenmispina Gogre Gab. N-2035.

Rubens, acubi tenves, elongati, rectiusculs vel fere subulati, cylinarici, ai ramos flor.copississimi; fol-ovato-elliptica, ralis coriaced, nervosa, is costam parce glaniulasa; Dentes biserrati, aperti; pet. sparse glanioulosi; aculeati, glabri; stip. angustac, laeves, auriculis parum givergentibus; pes.1-3, hispituli, g-13m. longi; tubus ovoideus, laevis; sep. Forso eglandulasa. erecto-connirentia, gir persistentia, parce pinnata, acumine lato; styli lanati, breves, giseo plano; con....... funct. ovatus.

Hab. Hautes - Athes: ao Chaniun prope Gap (G. Burle).

Forsan of Montanas (Ozanomiac) fertinet of aculeorm formam spalarumque evolutionem.

1250. Crepinia Chabortiaides Gogr Gat. N-2036.

Atculei as ramos flor falcato-gilatati; fol late ovato-elliptica, lacte virivia, subtus glaucescentia, Dentes profungri, acuti; pet. aculeate, sparse pi-

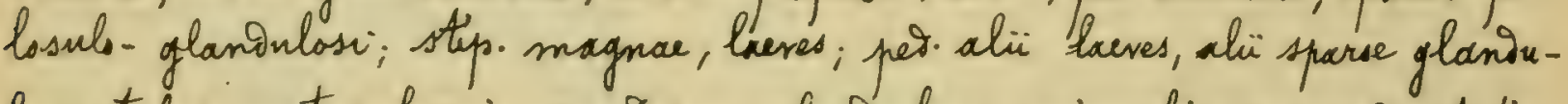
losi; tubus oratus, laevis; sep. Torso eglanionlosa, pinnulis parum gentoctis, pancioribus instructis; Styli villosi; Qisco pauls comico; cor magna, rosea; fruct. ovatus, glaber.

Hab. Thone, as Charbornieres (. Bouller). 
$-49-$

\$. Fructus ovivieus vel oborato-oblongus.

〜. Gubus beeris.

$x$ Diseur planus; foliola basi subattenuata.

1251. Gepinia àdenodontos Gjogr \&ab. N-2037. - Goger Herb. ros. N-192.

Acculei falcato-gilatati, at ramos flor. sat copiose; fol ample oblongorhomboialia, utringue paulation attenuata, at costam saepe aciculatam glansulosa; sentes valis biserrato-glartoulosi, profunide lanceolatic; pet. glanioulosi, reculeati, sparse pilosi; pet.1-2, hispianhi, 15-23 m. longi; tubus oblongus, lavis; sep. erecta, gorso eglanoulosa, pirmulis angustionibus, valde dentatoglanimlasis instructa, styli hirsuti, Sisco subplano; cor......., fruct. obovato-blongus, utrinque attemuatus.

Hab. Ysere, Charancieu (M. Ganorger).

1252. Gepinia oligadena Gigor mss.

Fcculei Dilatato-subfalcati, ad ramos flor mulli; fol. sat late oboratsacuta, basi rotuniata, intense virisia, ad costam colanioulosa; gentes biserrati, triangulores, subpatuk; pet. glaniaulosi, glabri, sup. inermes; stip.. virives, longae, auriculis sat sivergentibus; pes. 1-2, glaniulis 3-4 of siti, 7-9 $m$.longi; theus obovato - oblongus, laevis; sep. garso eglaniulosa, pinnulis snmibis latis valdeque gentato-glanionlosis instructa, styki lanati, gisco plans; cor....... fruct. obovato - sblongus, utringue Gepressus.

Hab. Luecia, at Megotige Hallaniaie (7. At. Gabrielsson).

1253. Grepinia Fernanderü Gigr mss.

Acculev gilatati, valde falcati, al ramos flar panciores, fol sat pawa, svato-elliptica, acuta, basi coroata, lacte virisia, as costam glandulesa, sentes biserrati, profunise lanceolati, recti; pet glanialosi, aculeati, hinc inde pilosuli; stip. virides, latae, apia garsi glainoulosas, auriculis rectis: pes. 1-2, hispidi, 15-17 m. longi; tubus oborato-oblongus, laevis; sep. dorso eglaniublosa, pirmulis ommibus angistis, genticulato-glanoulosis instructa; styli lanati, Disco subplano; cor.rosea, eciliata; fruct. oboratoslongus, basi gepressus, aprice oittemuatus.

Hab. Hispania centr, olguxialajara (Fernanies).

Notis invicatis a C. hispania (Moiss. et Rent. non Mill.) cui prosima, bene gistinguitar. 
$-50-$

XX Disens plus minus conicus; foliola basi coroata.

1954. Gepinia shinifolia Gogr Gab. N:2038.

foculei temiter it valie faleati, as ramos flor. panciores; fol-méviveria, oborato-acuta, basi coriata, paulo glauceseentia, ad costasn basi aculextam parce oflaniulosa; Dentes biserrati, triangulares, pperti; pet. glanisulosi, eculeati, glaberrisni; stip. paulo rubentes, auriculis rectis; peg. 1-2,

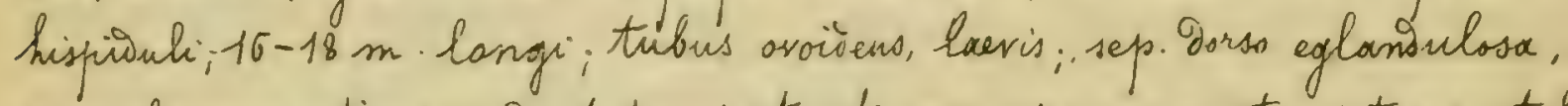
pinmulis angustis, 3-5-gentatis instructa, acumine angusto, integro; styli villosi, gisco conics; cor. eciliata; fruct. ovoideres, medro turgidus, utrinque atter. Hab. Gars, as bulas prope \& gigan (D). Buezkieswing).

1255. Crepinia Doonacana Gotgr mss.

toculei parri, inclinato - suboilatate, ad ramos flor. stipulares, geminati; fol. obovato-oblanga, apice subrotuniota, basi rotundata, firma, nersosa, as costrom glanisulosa; inentes biserrati, triangulares, recte; pat. aculeati, glaniulosissini, prarse filssuli; stip. virites, longae, dorso valde glanguIosae, auriculis rectis; pes. 1-2, in 1/2 inf. laeves, cueterem parce hispioi; tubus ovoideus, laevis; sap. gorss eglaraulosa, pirnulis omnibus latis ac valde Oentato-glariulosis instructa; styli:hirsuti, gisco plano; con. rosea, eciliata: fruct. ovoidens, utrinque subrotuniatus.

Hab. Selgium, Hainaut: as Hownengt (M. Gonvoger).

1256. Crefinia biner Gogr mss.

Aculei valioi, Dilatato - subfalcate, ad ramos flor saepe nulli; fol. parva, spato-acuta, mesis ailatata, basi subrotuniata, crassa, pallice virisia, is costam glandulosa; Dentes biserrati, triangulares, lanceolato. recti, pet. aculeati, copiose glaniulosi; basi unifariam pubescentes; stip. rubentes, lativisulac, auriculis Sivergentibus; ped. 1, parce glanoulosi, 7-8 m.longi; tubus oborats - oblangus, laevis; sep. Forso eglanoulosa, pinnulis parum latis, omnibus dentati instructa; styli parce villosi, Sisce ralde conico; cor....... fruct parvus, oborato - oplongus, basi subDepressus, apice attemuatus.

Hab. 'Sicilia Mont. Nebroteis: in monte Matonie ad Cesteburono (No. Lajocons). 
$-51-$

1257. Crepinia seltima Gogr mss.

Fculei validuli, parum Silatati, temiter at valde faleati, ad ramos flor.geminati; fol. ample oblongo-lanceolata, basi rotuniata, intense viridia, as costam glanidulosa; Dentes biserrati, late aperto-triangulares, pat. aculeati, glaniulosissimi, hinc inde pilssuli; stip. angustiores, parum longae, virides, gorso eglandulosace, auriculis rectis; po-1-3, tots gense his piouli, $18 \mathrm{~m}$. longi; tubud oblongus, laevis, sep. asorso eglandulosa, pinnulis annibus dentato-glandulosis, parum latis instructa; styli pubescentes, Sisce valde comic; cor........; fruct. sat magnus, obonato-oblongus, comicus, basi rotuniates, apice longissime attenuato-strangulatus. Hab. gor, og Armpues (folbert).

1258. Crepinix pronata Giver mss.

Aculei maximi, falcato-gilatati, as ramos flor geminati; fol sat late obovato-oblonga, in acumine torto prosucta, basi rotundsta, lacte virisia. at costam glanioulosa; gentes biserrati, triangulares, aperti, pet aculeati, glaniulosissimi, inferne pauls pubescentes; stip. rubentes, lativiculae, dorso eglanialosae, auriculis rectis; pos. carymbosi, basi laeves, caeterum sat hispiivi, $12 \mathrm{~m}$. longi; tubus oroileus, laevis; sep. Iarso eglanoulass, pinnulis - Annibus latis Dentatinque copiose instructa; styli pubescentes, gisico sat convies; cor......., fruct, frarwis, ovoidens, basi gepressus, apice strangulatus. Hab. Hispamia orient., gelencia: at Legorbe (C. Sau).

1259. Crepinia lentula Gogr toab. $N^{\circ} 203 \mathrm{~g}$.

toculei longi, temiter falcati, ad ramos flor.copiosi; fol. sat late disvata, apice rotuniacta, basi cordata, glauca, as costam glandulosa; gentes fiserrati, triangulares, aparti; pet-glanoulosissimi, aculeati, inferme paculo pubescentes; stip. sivides, auriculis rectis; pet. 2-3, hispidruli, 10-12 m. longi; tufus ovoigeus, laevis; sep. Inso eglaniulosx, pinmilis hinearibus, valde Dentato-glanaulosis instructa, acumine filiformi, integro, styli villosi, Dises praulo conico; cor. eciliata; fruct. pxoilens, utrinque paul attemuatus. Hab. Hisponia bor. Catal. ad Falgá pr. Gerona (9. gayreta).

1260. Greminia segobriensis Gogr mss.

Acculei abiles, parum faleato-Dilatati, is ramos flor. stipuclares; for. ample oborat-acuta, fasi rotuniato, pallide viriaix, ad costam flaniulosex, 
$-52-$

Ientes biserrate, latissine triangulares, perti; pot. rubentes, subinermes; glandulosi, inferne vix pilosuli, stip.magnae, latoce, virides, Dorso eglanioulosac, anriculis rectis, ped. corymbosi, prorsus dense hispioi;, 11/2 cent. longi; tubus oblongus, laevis; sep. Gorso eglarisulosa, pinnulis omnibus foliaceis, 2-4-Dentatis instructa; styli parce pubescentes, gisco comico; cor. parra, basi paulo ciliata; fruct. Fovato- obongus, basi opepressus, apuce strangulatus.

Hab. Hispania orient., galencia: at Segorbe (C. Sau).

1261. Crepinia albistigma Gogr mss.

Acculei parwi, valie breves, inclinato-subsilatati, as ramos flor.copiosi; fol oborato-sblonga, acuta, basi subrotunsata, coriacea, lacte viridia, as basin costar glanisulosa, dentes biserrati, triangulares, aperti, pet. glandulosi; sparse pilosi, sup. inermes; stip. breves, parum latae, sirides, auriculis rectis; pel.1-2, toto sat glanoulosi, $8 \mathrm{~m}$.longi; tubus abovats-oblangus, laevis; sep. Dorso eglansulosa, pinnulis parum latis, gentatis instructa, acumine integro; styli albissimi, lanati, dises poulo conico; cor.......; fruct. mediseris, obovato-oblongus, midio turgious, utrinque attenuatus.

Hab. Hispania meaix, Ad Alarracin (9. Zapater).

$\sim \sim$ Gubus plus minus glanoulosus.

1262. Crepinia inconspicia (Deségl. Cat. p. 188); Gogr Gab. N.2040.

Abculer copioss: falcato-dilatati, ad ramos flor verticillati; fol-mediocria, ovato-acuta, siriedia, as costam glanibulosa; Dentes biserrati; pet-aculeati, glanoulosi, sparse pilssi; stip. glabrae, auriculis rectis; ped.1-3, hispidi ; tubus ovoireus, plus minus hispious; sep. gorso eglanoulosa, purnulis latis, dentato-glanoulosis instructa; styli hirsuti, Disco parum consico; cor. rosea; fruct. ovioiens.

Hab. Gallic centr; Angl oxeid.; turstr. sup.

1263. Crenimix mayoson Gogr \&ab. N:2041.

taculer folcato-Vilatati, ad ramos flor. stipulares; fol mediveria, obovato-oblongr, acuta, basi vix attenuata, as costam paulo glansulosa, Denties biserrati, triangulares, aperti, pet. glanoulosi, aculeati, sparse pilasuli; stip. majusculae, aurriculis rectis; pos. 1-2, parce glanisulosi, 8-10 m. longi; tulies oborato-slonsus, sparse glanioulosus; sep. gorso Laevia, pinnulis brevibus, 
$-53-$

angustis, 1-3-Dentatis instructa, acumine angusto; styli pubescentes, gises prouls comic; cor....... fruct. ovoriens, apuce proesertion atternuatus.

Hab. Saxonia, at gralleniorf prope greimar (C. Haussknecht).

1264. Gepinia ambigua (Lej. Fl. O. Ipa p.gs; Ipreng. Syst. II, p. 548; Trehs. Tl. exc. N-4003, Bluff et Fing. Comp. Hl germ. I, pars 2a p.224; Dumort. Mon.

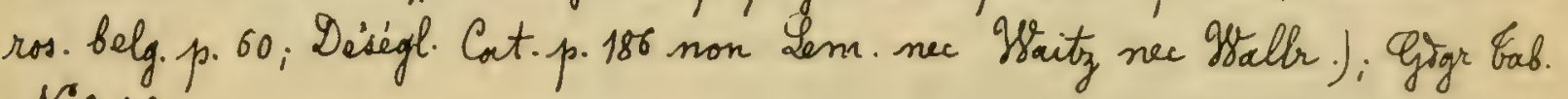
N-20t+2.

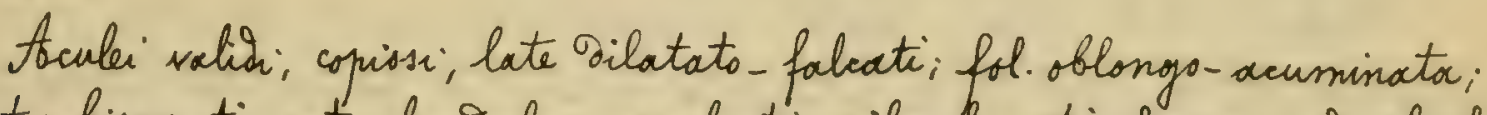
gentes biserrati; pet. glanoulosi, rculeati, pilosuli; stip.laeves; pes alii lacess, alii parce glasioulosi; tubus oroideus, hine inde setulosus, sep. Porso laeria, pinnato-subsentata; styli hirsuti; cor. pallive rosea; fruct oroidens.

Hab. Belgium, inter gerriers et Ginsival (Eejeune).

Leguentes mihi ignotare sunt sprecies: Rosoc ambigua Leman in Gull. phil. (1818) p. 11, N:15, forsan prope Saris crescens;

Rosa ambigux gsaitz ex Spreng. Syst. II, p. 548; DC. Prodr. II. Rosac N-38, ex Oriente sita:

Rose ambigua gralb. Hist. ros. p.234.

1265. Crepinia simixta Gogr Eab. N:2043.

Aculei falcento-2ilatati, as ramos flar copiosi; fol oblongo-lancealata, basi attemuata, glaucescentix, as costam tenuiter glandoulosa; gentes fiserrati, lancoolato-recti; pot. tenuiter glanimlosi, aculeati, inferne sparse pilosuli; stip. $v_{i}$ rises, auriculis valse givergentibus; pes. 2-6, setulosi; 14-16 m. longi; tubus obovato-oblongus, basi infima glanaulosus; sep. Dorso lavia, finmulis angustis, valie dentato-glanivulosis instructa, acumine lato, copiose dentato; sty i lanati, tises plans; cor....... fruct obovato - oblongus, claviformis, apice inflatus, $2 / 3$ sup. usque at basin longe attenuatus.

Hab. Anglix. Monmonths hire: at Llanthomy (to. Ley).

$\S \S$. Fructus oblongus.

1266. Cuepinia sispronites Cojor \&ab. Ni2044.

Abculei prenciores, valiar, inclinato- Silatati, as ramos flor mulli; fol. late oboratix, utringue abrupte attemuatai, glavea, as costam parce glanoulsa, iontes biserrati, lanceolati; pet. glanoulosi, subinermes, unifariam pubescentes; 
$-54-$

stip. latae, longas, auriculis girergentibus; pet. 1-2, parce et temuiter glanisulosi, 7-g m. longi; tubus oblongus, heris; sep. Torso glanioulosa, finnulis latis, integris, copiose instructa; styli villosi, gises subplano; cor magna, pallide rosea, eciliator; fruct. oblongus, apice attenuatus.

Hab. Gironie, ai It Nèoars (L. Notalay).

1267. Crepinia gransidentata Gogr mos.

Aculer validuli, falcato Silatati, romos flor. copiosi; fol. late ovata, fere onnes obtusa, basi ample sotunitata, firma, at costam basi acenleolatam glaniulasa; Dentes biserrati, lanceolato-aperti; pet. aculeati, hine inde glansuloso pilosuli; stip. virides, latae, gorso laeves, ourricalis sivergentibus; pes. 1-2, glanoulosis 2-5 obsiti, g-14 m. longis; tubus oblongus, laevis; sep. Dorso lacevia, pirmulis latis, 2-3-gentatis instructa, acumine subfoliaces, integro; sty $k_{i}$ parce villosi; Gisco planiusculo; cor. albo-rosen, eciliata; fruct. oblongus, $\mu$ trinque attemeatus.

Hab. Belgium, Hainaut: ad Harvengt (N6. Ganooger).

1268. Grepinia glancoxivens Giogr \&ab. N-2045.

Laete viridi-glauca; acculei valide; faleato - Silatati, as ramos flot. pauciores, fol-ovato-elliptica, utrinque paulation attemuata, as costam glansulosa; gentes biserrate, convergentes; pet. glanoulssi, aliquost inermes, basi pubescentes, cacterum sparse pilosi; stip. sat pauvae, inf. subtus glanioulosse, auriculis rectis; pes-1-9, temuiter glensiolosi, 7-14 m. longi; tubus oblongus, laevis,

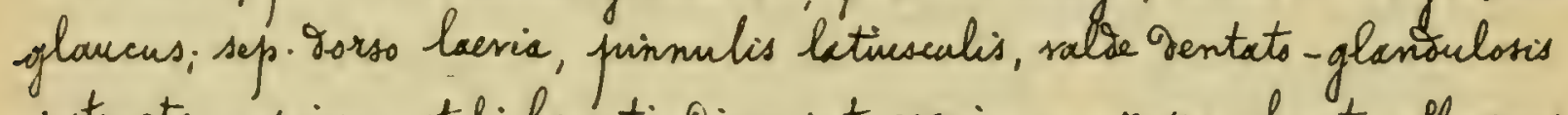
instructa, copiosa; styli lanati, lisco sat comico; cor. rosea, fruct oblangus. Hab. Haute- Savoie, at Pringy (Puget).

1269. Gepinia bavines Gögr Eab. N-2046.

Acculei raliar, ao ramos flor. falcato-gilatati, fol oborata, breviter attemuata, basi subrotuniata, at basin costace parce glanosulosa; sentes biserrati, lanceolato-recti; pet. glanisulosi, aculeati, saepins glabri; stip. longaci, Porso paulo glandulosae sed valie aculeolatre, auriculis subsirergentibus; pet-1-7, alï glabri, alii vix hispisuli; tubus oblongus, laevis; sep. saepe rubra. gorso eglanioulosa, pinmulis parum latis. dentatis instructa; styli lanati, excerti, Qiscs sat conces, cor........, fruct. ample obovato-oblongus vel oblongus, utrinque salis attermatus.

Hab. Heute-Savoie, as Draillant (Muget). 
$-55-$

1270. Fepinia germana Gigor mss.

Aculei longi, parum foleato-Dilatati, subteretes, ad ramos fior. stipulares; fol mediceria, obovato-oblonga, breviter acuta, basi rotundata, intionse sirivia, at costam glanioulosa et aculeolato; ientes breves, biserrati, perto.

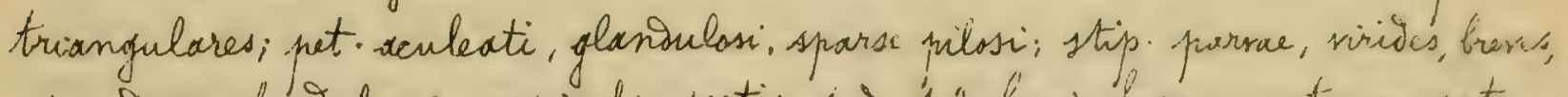
ahice To'si glandulosal, auriculis rectis; pas. 1-3, basi haves, cacterum sat

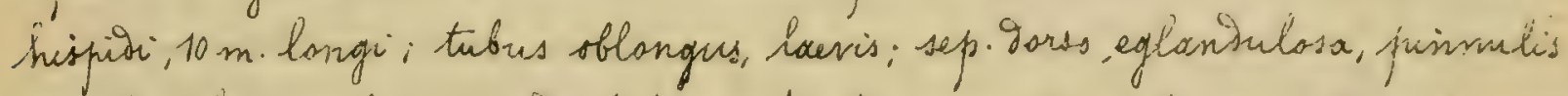
comsibus larius-eulis, 2-5-Dentatis instructa, vcumine integro; stryli pubescentes,

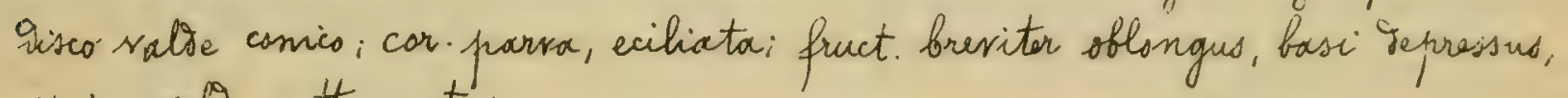
aprice valle attenuactus.

Hab. Hispanix orient, Halencix: at Segorbe (C. Tau).

1271. Gepinia Proterianx Gogr mss.

Acculei breres, faleato-dilatati, at ramos flor.copiosi; fol amphinscule

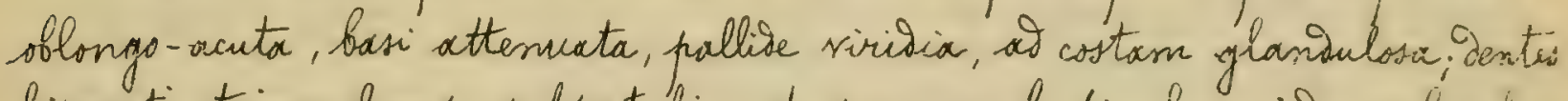
biserrati, triangulares, subpatuli; pet. parce acculeati, hric inde pillosuloglanouloss; stip. virides, angustae, gorso eglanioulsoa, anriculis semipatulis; pes. 1-2, in 1/2 inl. Laeves, caeterum parce hispinouh; tubus oblononis, laen's; sep. garso hereia, pinnulis omnibus latis, dentato-glanoulosis instructa: sight pubescentes, giscs conico; cor........ fruct ample suboblangus, bisiDepressus, appice strangulatus.

Hab. Lusitamia, at Cascaes (M. Coutinho).

b. Lepala iorso glaniulosa.

t Ityli glabri vel subhirsuti.

\$. Gubus plus misus hispions.

1272. Crepinia Renteriana Googr \&ab. N'2017.

toculai panciores, valie folcato-Dilatati, as ramos flor.mulk; fol mediocrior, obovato-obturinscula, basi paulation attemuata, virisia, coriacea, as basin costar paulo glaniulosa; Dentes biserrati, lanceolato-aperti; pet. subinermes, glansulosi, glabri, stip. rubentes, breves, auriculis rectis; pes. 1, hispidi, 9-10 m.longi; tubus oblangus, basi glanioulosus; sep. Dorso parce glanisulssa, pirmulis parum latis, fere onmibus integris instructa, acumine latiusculo, in. tegro; styli glabri, Sisco plano; cor eciliata, fruct. oblongus, utrinque sensim
attermatus.

Hab. Hispramia bors, 2ogroñs: Masillo in Sierra a Cameros (J. Lubia). 
$-56-$

1273. Crepinix gnara Gogr mss.

foculei longi, tenues, cylinivici, vix falcato- dilatati, at ramor flor-intersum ternati; fol parra, oblongo-acuta, basi rotuniata, cariacea, intense viridia, as costam glaniulosa; Dentes biserrati, parvi, triangulares, pactuli; pet glanoulosi, aculeati, rubentes, inferne vix pilosuli; stip minutar, rubentes, Sorss glansulosae, auriculis rectis; pes 1-2, toto hispiai, 11/2 cent. longi; tubus oblongus, basi infima hispidus, sep. Dores glantulosa, pinnulis ommibus hinearibus, gentatis instructa, scumine integro; styli glabri, disco subplano; car meniseris, eciliata; fruct. oblongus, angustus, basi sttenuatis, ad apuiem longe strangulatus.

Hab. Hispania orient, galencia: ad foltura (C. Sau).

1274. Creninia Chaubartians Ggor mss.

Acculei dilatati, temiter falcati, as ramos flor glanco-farinosos saepe vecticillati; fol-ampliuscule oborato-oblonga, acuta, basi sensin attemuata, as costam glanivulosa; Bentes biserrati, late aperto- triangulares; pet. copiose glanoulosi, sparse pilosuli, inf inermes, sup. vix aculeati; stip. rubentes, longae, latissimae, Torso glandulosae, auriculis divergentibus; peo.1-3, hisfisi; 7-8 m. longi; tubus ovodeus, toto glandulosus; sep. Darso glanoulosx, pinmulis ammibus parum latis, gentato-glaniulosis instructa, styli adsperse pilsuli, aisco plano; cor........ fruct. sat parrus, svoidens, basi rotundactus, apice parum attennatus.

Hab. Lot-et-Garonne, at Montban prope togen (O. Debeaux).

1275. Grepinia Dencalion Gogr \&ab. N:2048.

Acculei falcato-gilatate, as ramos flor copiosissimi; fol minutissima, oborato-oblanga, subattenuata, basi cordata, supra sirentia, subtus rubra, ai costam glanculosa; Dentes biserrati, triangulares, pperti; pet. glanoulosi, aculeate, hine inde pilosuli, stip. nubentes, prarvare, auriculis givergentibus; pes. 1-2, parce glanaulosi, 7-8 -m. longi; tubus oroisens, in 1/3 inf. hispiaus; sep. Forso glanivelsa, pinnulis onnibus angustis, integris instructa; styli subgheberrimi, Dises conico; cor.minuta, eciliata; fruct. ovoideus, basi rotuniatus, apice attinustus.

Hab. Hifnania for, circx Logroño (1. Hubix). 
$-57-$

1276. Crepinia houmatoges Goar \$ab. N-20kg.

Eota rubro-sanguinea; aculei dilatato-subfalcati, at ramos flor. subnulli; fol oblongo-acuta, basi breviter attenuata, nervosa, ad costam non. glaniulosa, Dentes biserrati, recti, pet. glanoulosi, inf.glabri et inermes, sup: vero parce aculeati et hiric inide pilosuli; stip. laceses, latace, longac, aurriculis Sivergentibus; ped. 1-3, hispidi; 7-13 m. longi; tubus suboblongus, setosus; sep. Forss glaninlosissima, pinnulis parum latis, subventatis instructa; styli glaberrimi, Sisco comico; cor. rosea; fruct. obovato-oblongus, basi Dopressus, aprice strangulatus. Hab. Houte- Savoie, as Pringy (Tuget).

1277. Crepinia Cotornui Gogr \&ab. N-2050.

Acculei foleato-Silatoti, at ramos flor. pauciores; fol parra obovato-oblorga, obtusiuscula, fasi paulation attenuata, crassix, intense virioica, jumicra autem ubentio, is costasn eglandulosa, sentes biserrati, triangulares, aparti,; pet- glanoulosi, aculeati, sparse pilosuli; stip. parvac, anguestac, auriculis ovivergentibus; pes. 1-4, hispitissimi, 8-10 m. longi; thibus obsvato-oblorgus. inferne setosus; sep. Garso texicater glarsulosa, pirnulis filiformibus, filiformibus, copiose instructa, acumine longissimo, angusto; styli glaberrimi, gisco parro, conico; cor. parra, pallide roses, eciliata, fruct minor, ovoileus, utrinque attenuatus.

Hab. Hispania marid.-or., Murcia: of Ihige (M. Coornic).

De grege C. Soupini (Eratt.) quacum conjuingi nequit of calycis tevburn base hiofitum.

1278. Grepinia aspratelis (Grép. Grim. I, p. 52); Grge Gab. N-2051. - C. espernata (Disegl. in Journ. of Bot. 1874, estr. p. 5; Desiegl. Cat. p. 188).

Acculer copiosi, breves, gilatati, recti vel inchinati, as ramos flor. sacpe geminati, fol parwa, sbovato-sblonga, utrinque attenuata, ad costam glansulosa; gentes biserrati, aperti; pet glanidulosi, hine inde pilosuli, parce aculeati; stip. breves, latac, apice garsi paulo glaniaulosae, acuriculis givergentibus; pes. 1-2, hispiouli, 7-g m. longi; tubus obovato-oblongus, toto ace pparse flansulosus; sep. Torso copiose glansuloss, pirnulis angustis, valde gentatis instructo; styli glabri, gises sat conico; cor.rosea; fruct. mrideus, apice atternuatus.

Hab. Haute-Garonne, I Coulouse unde Geserifsi) Gimbel-Lagrane? etiam in. Gaillia centr. et in tonglix sec. Désiglise. 
$-58-$

§§. Enbus lacrissimus (C. Pouzini).

$\sim$ Fructus ellipticus, voideus rel obovato-oblongus.

$X$ Folisla basi rotuniata.

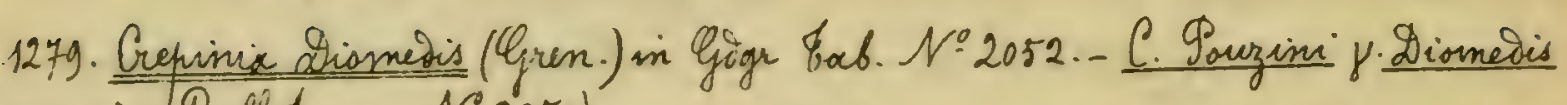
(Gren. in Fillot exs. $N=3850$ ).

Stculer faleato-jilatati, as ramos flor. panciones; fol parva, bovato-acutx, basi rotunioata, lacte viridia, as costam glandulosissima; Ientes biserrati, profunic lanceolato-aperti; pet. paree aculeati, glabri, valdo glanoulose; stip. virives, auriculis rectis; peo. 1, hispioi, 12-14 m. longi; tubus ovideus, lacvis; sep. Forso parce glandulosa, finnulis onnibus angustis, valis dentatoglaniulosis instructa; styli glabri, gises sat comico; cor.parva, eciliata; fruct ovoideus, basi rotunivatus, aquice attenuatus.

Hab. Gart, al Le Higan it Manoxgent (Dionide Enazkiewies).

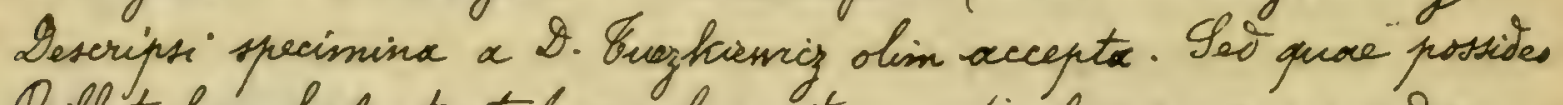
- Billot l-c., habent tubum elongatum, stipulas purfureas Discumque subplanum ideogue cum descriptione supra data non omnins congraunt exemplaria. Gidebitur si non elterx ac nova species exstet ulterius inrestigando.

Species 21 sequentes, nempe $\mathcal{N}$ 1280-1300 ad ipsamet $C$. Fouzini (oratt.) pertiment.

1280. Crepinia farbara Giog mss.

Acculir vatioi, longri, inchinati, valde gilatati, at ramos flor copiosissimi; fol-parra, elliptico-obovatus, vix acuta, basi subrotundata, intense viribia, parum criacea, ad costam glaninulosa; gentes biserrati, triangulares, aperti; pet. rubentes, aculeati, glanaulosi, basi vix pilosuli, stip. breves, rubrae, parvae, Dorss eglaniaulosse, auriculis rectis; ped. 1-2, hispisi; 1 cent longi: tubus oblongus, laenis; sep.extus paulo glanioulosa, pirnulis smonibus latis, gentatas instructa, styli glabri; Risco valie conico; cor minuta, eciliatar; fruct. sboratosblangus, utrinque attemuatus.

Hab. Flgeria arient. at Constantine (ge. Reboud).

1281. Gepinia xunthoucantha (Gogr Tas. nor. I, p. 10), Gogr Bab. N: 2053. - Gojor Herb. ros. $\mathcal{N} 561$.

toculei flarescentes, falcuto-gilatat, at ramos flor. plus minus copiosi; 
$-59-$

fol. parra, ovata, breviter acuta, basi rotuniata, as costam glaniulosa; sentes biserrati, triangulares: pet. glanimulosi, aculeati, vix pilosuli; stis. intense rubentes; per. 1-3, hisfiai; g-11 m. longi; tubus oblongus, laeris; sep. Larso glaniulose, anguste pinnata; styli subglaberrimis, sisco fere plans; cor. rosea, funct. suboblongus, utringue seo apice praesertion ottemuatus.

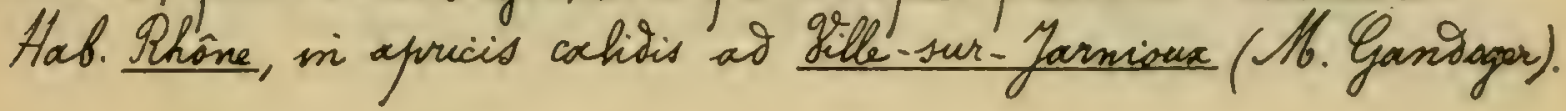

1282. Crepinia staurinies Gogr mss.

Aculei tenues, inchinati, vis gilatati, teretes, copiosi, as ramos flor saepe 2-3-tei fol-parva, obovato-oblanga, breviter acuta, basi rotungata, ad costam glaviouloso; gentes profunsi; biserrati, triangulares, recti; put. acenleati, glabri, glanioulosissimi; stip. minute, rubentes, Dorso glandulosar, awriculis rectis; per. 1, toto glaniulosi, 1 cent. longi; tubus svoiseus, lacvis; sep. Oorso glaniaulosa, pinmulis omnibus parum latis, Dentatis instrueta; styli glabru; Gises plans; cor.......; fruct. parrus, elliptico-conicus, basi rotiniarto- Silatatus, apice attemuatus.

Hab. gar, ad tompus (Albert).

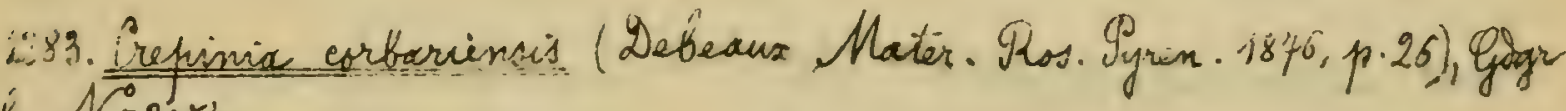
B..6. N-2054.

touki gilatati, tenuiter falcati, as ramos flor.copiosi; fol. parrac ablongo-acuticscula, basi rotusioata, saene rubrox, firma, as costam glanaulosa; Dentes parri, biserrati, triangulares, aparti; pet glaniulosi, aculentiv, unifariam sparse piloss; stip. parrace, rubentes, curriculis rectis; ped. 1- 3, hispiti; tubus obovato-oblongus, laevis; sep. gorso in $1 / 4$ inf haviar carteram glasiouloso-muricata, pinmulis latis, gentato-glanivulosis instructa, acumine latiusculo, integes: styli glabri, 2isco subplano; cor. parro, pallide rosea, eciliata; fruct sbovato - blongis, apice attenuatus.

Hab. Iyrénées Orientales, as It Taul-De-Fenouillet (O. Debeaua).

XX Folisla basi plus minus attenuata.

1284. Crepinia caibata Gogr Gab. N-2055.

Aculei valisi; falcato-gilatati, at ramos flor. saepe nulli; fol obo ato-oblonga, attenuata, fasi abupte truncata, ad costam glanoulosa, ientes biserrati, convergentes; pet. glanim losi, parce aculeati, fere glaberrini, 
$-60-$

stip. latue, virides; pres. 1-4, Mlii setulasi, alii sublaevissimi, 10-13 m.longi; tubus oblongus, laevis; sep. Oorso glanivelosa, anguste pinnata; styli glabri, parric, Gisco connis, magns; car......; fruct mediseris, rbovato- oflongus, utringue aequaliter attemeatus.

Hab. Syrénées Orient, in Mont-It-Martin prope Amélie-lesBains (O. Deberux).

1285. Crepinia Debeauxii (Gogr in Debeaux Mater. Ros. Pyren.p.19), Gogr Bab. $\mathcal{N}^{-} 2056$.

Aculei tenues, elongati (1 cent:), inclinati val subfalcati, parum $x_{i}$ latati, at ramos flor copississimi; fol elliptico-acuta, basi breviter acuta, as costam glandulosa; Dentes biserrati, profunai, aperti; pet. glanioulosi: aculeati, plerumque glabri; -stip. vïidss, auriculis acutisimis, rectis; ped.1-3, omnes soit hespiduli, $14-17 \mathrm{~m}$. longi; traus oblongus, laenis; sep. Iorso glanioulosa, sat anguste pinnata; styli brevissimi; glabri, gisco plano; cor..... i fruct parsus, oborato - sblongus, basi rotuniactus, apice conicus. Hab. Oyrénées Orintales, as Castelnau prope Ehuir (O. Debexua).

1286. Qrepinia ceratophylla Gogr tab. N-2057.

Aculei griser, falcato-gilatati, as ramos flor. tortuosos copiose; fol. parver, elliptica, utrinque breviter attenuato, ad costam glansulosa; Odentes biserrati, profunsi, folcati; pat-glaniulosi, aculeati, subglaberrimi; stip. angustae; pas. 1-2, hispiaissimi, 8-g m. longi; tubus ovoideus, laenis; sep. Dorss ghanaulosa, minara; styli glabri, sat exserti, Dises subplans; $\operatorname{cor} . . . . .$. , fruct minor ( $6 \mathrm{~m}$. giam. latus), oroideus, basi rotundatus, apice farum geporessus.

Hab. Iyrénés Qrient. at Camélas in Mt. Mt-Martin (O. Debeaux).

1287. Crepinia Pourimi (Gratt. 20s. mon.II, p.112; Carist Gtude des fl. 6:2'. II, p. 241; Grép. Prim. I. p.52), Gogr Gab. N.2058; Gogr Contr. fl. slar. II. p. 34 .

Acculei griser, Dilatato-subfalcati, subgeminati, copiosi; fol. mesiocria, anguste oblongo-acuta, glancescentia, as costam glanivulosa; Dentes bierrati, pet. glavoulosi, aculeati, hine inide pilosuli, stip. angustar; pet-gradeiles, setulosi; tubus oborato-oblongus, laevis; sp. oorso glaniulosx, minute pin-nata; styli glabri; cor carnea; fruct. parrus, obovato-oblongus.

Hab. Gallix merid; Hispania; Oslmatia; Allania. 
$-61-$

1288. Grepinix provincialis Gogr 8 ab. N-205g.

Rubens; aculei ralivi, falcato-gilatati, ro ramos flor.copiasi; fol. Katiuscule oblongo-acuta, price torta, basi attenneata, viridia, nervosa, cariacea, at basin costae paulo glavaulosa, Dentes biserrati, lanceolato-recti, pet. rubentes, parce rculeati, glanimlosissimi, hisic inde pilosuli; stip. ruberrisnace, latae, auriculis divergentibus; pò. 2-5, glanoulosi, 12-14 m. longi; tubus ovoideus, laevis; sep. Dorso parce glanioulosa, pinnulis hinearibus, 1-3-jentatis instructa, acumine latiusculs, integro, stygh hinic inoe subpilosuli, Sises paulo conics; cor........ f fuct. breviter svoideus, basi rotuniatus, at apicem abrupte strangulatus.

Hob. gar, in ralle St Clair os Rovesarques (H. Soux).

$\sim \sim$ Fructus oblongus.

$X$ Folisla basi plus minus attemuata.

128g. Creminia castaneicola Gogr bab. N-2060.

Acculei falcato - subsilatati, as ramos flor. saepe nulli; fol. obovata, utrinque attemuata, at costam parce glanoulosa, gentes biserrati, profunde lanceolati; pet. melentes, aculeati, glaniculosi; subglaberrimi, stip. angustae, rubentes; fer. 1-2, setulosi, 10-11 m.longi, tubus oblongus, laevis; sep. Dorso glanioulosa, anguste pinnata; stgli glabri; disco subplans; car.....; fruct. oblongus, fasi Depressus, apice attemuatus.

Hab. Pyrénées Orient: si castoneis al tomélie-les. Sains (O.Debrause).

1290. Brepinia Nixchylon (Gogr Ros. nor. I.p.g), Gogr 8ab. N-2061. - Gogr Herb. ros. No18g.

toculei cylinorici, rectivisouli, parum falcati, at ramos copiosi; fol. oborato-oblonga, acuta, fasi sensim attemuata, at costam glandulosa, foliols med is basi saepe 1-3-aculeolats; dentes biserrati, patuli: pet. glaniulosi; aculeati, unifariam sparse pilosuli; stip. breves, gorss glandulosace, auriculis Divergentibus; pes. saepe corymbosi, hispitic; 12-20 m. Congi; tubus elongatus, laevis; sep. Dorso glanoulosa, sat anguste pinnate; stght hine inve pibsuli, sises sufplano; con rosex; fruct. anguste oblongus, utringue attemuatrus.

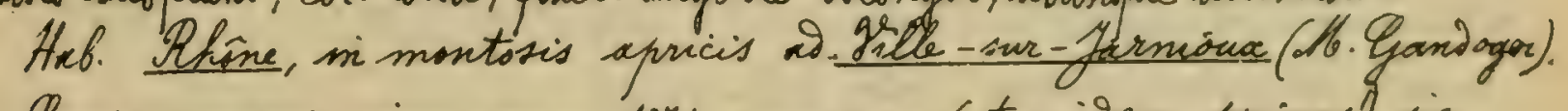
Ifecies insignis jim anno 1871 a meipso (et quidem frimses.) in hre regine isventa. Igrege C. Pouzini (Eract.), cujius limites septentrionales ejus 2 istributionis gergraphicae at localitetem supra memaratame
jidentur esse. 
$-62-$

1291. Grepinia Guirana Ggogr tab. N-2062.

Acculei sparsi, valisi, falcato-Dilatati, as ramos flor. 1-2; fol-ampliuscule oborata, brenter acuta, basi subattemuata, as costam parce aciculata; Dentes biserrati, sublanceolato-aperti, pot. glanoulosi, aculeati, inferne paulo pubescentes; stip. misiscres, virises, auriculis divergentibus; pes. 3, hispisi;, 15-16 m. longi; tubus oblongus, laeris; sorso paulo glandulosa, finmulis smmibus angustis, Pentato-glandulas is instructa, styli glabii, giscs comico; cor....... fruct. parrus, blongus, basi attemuatus, apice longe et valie sttemuato - strangulatus.

Hab. Hiphania merid.-or. Murcix: in regione superisci Treirra de Espuña (N. Cañata).

XX Foliola basi rotuniata vel corsata.

- Folisla parra, sbovata.

1292. Crepinia orocharis Goge tab. N-2063.

toculai longissimi, inclinato - subvilatati, ai ramos flor copioss; fol. parva, oborato-acuta, basi rotundata, at costam glantulosa; Dentes biserrati, valse profunivi, lanceolato-aperti; pet. aculeati, glabri, parce glandulosi; stip. virioes, parrac, auriculis subrectis, pes.1, hispiasi, $13-15 \mathrm{~m}$.longi; tubus elongatus, laevis; sep. Dorso paree glandulosa, pinnulis brevibus, angustis, subcontatis instructa; styli glabri, Sisco sat conics; cor. parva, eciliata; fruct. parvus, oblongus, basi subattemuatus, apice longe constrictus.

Hab. Hispania merid-arient, Murcia: as Campo de San Juan in Sierre ge Segura (Nh. Cañaia).

1293. Gepinia Harm Gogr \&ab. 1:-2064. - C. Ponzini (Cariot \&tuide des fl. t=id. II. p. 177 mon Gratt., e loco!). Millot exs. N-3721 bis.

toculai gebiles, falcato-gilatati, ad ramos flor. panciores; fol ovato-elliptica, rix attenuata, basi corvata, al costam parce glanoulosa, Dentes biserrati, aperti, pet. glanisulosi, parce aculeati, subglaberrimi; stip. brevas, oorso glaniulosac, auriculis parum givergentisus; pes. 1-4, hispiti; 11-14m. longi; trebus oblongus, laevis; sep. Oorso pare glaniulosa, pinmulis angustis, proucioribus instructa, stygli glabui, panciores, gisco paulo conico; cor. rosea; fruct. anguste sbongus, utrinque valde attenuatus.

Hab. Isère, as Gerna prope Ciemiux (Moullu). 
$-63-$

1294. Gepinia escurialensis Gogr 8ab. N-2065.

Acculei falcato-2ilatati, ao ramos flor. sparsi; fol. late elliptica, brenter acuta, basi rotuniata, subters valse glauca, valde coriacex, ab costasn glanDulosa; Dentes fiserrati, triangulares, aperti; pot glaniulasssimi, aculeati, hinc inde pilosuli; stip. rubentes, eglaniulosae, duriculis sivergentibus; pes. 2-3, Dense scriculati, 14-16 m. longi; thbus oblongus, laevis; sep. tantion apice vorsi glanoulosa, pinmulis onnibus linearibus, paulo dentatis instructa, reumine integro; styli glaberrimi, Disco conico; cor.magna, rosea, ecilisata; fruct. oblongus, basi sublepressus, aprice attenuatus.

Hab. Hippania centr., as \&l Escorial prope Madrit (No. Laguna).

1295. Gepinia muscaria Goggr Fob. N-2056. - Gogr Herb ros. N-88.

Accilei gilatato-inchinati unt subfalicati, ad ramos flor. plenumgue melli: fol late obrato attenuata, basi coraata, as costam glanoulosa; dentes biserrati, profungi, aperti; pet. glanaulosi, subglabri, parce aculeati; stip. parrac; pes. 1-3, hispiari, 7-11 m. longi; tubus elongatus, baevis; sep. 90roo Temiter glaniuloss, anguste pinnacta, styli glabri, asses plano; cor rosea; finct. anguste oblongus, basi attenuatus, in $1 / 3$ inf. inflatus, gevin usque ad apriesn longe contracts-strangulatus.

Hab. Rhone, in saxosis at gelle-sur-yarmioux (No.Gandoger).

1296. Grepinia toletana Gogr mss.

toculei temus, falcato-gilatati, as ramos flor. mulli; fol. parva, sbovatosubscutor, fasi roturiacto, coriacea, pallise viridia, at costam eglarialoss, gentes biserrati, profunde lancealato-recti, pet. glanoulosi, aculeati, inferne parlo pubescentes; stip. latae, Silute rubentes, auriculis rectis; pes-1. Tense setulosi, 9-10 m. longi, tubus oflongus, laeris; sep. Dorso copiose glanioulssa, pimmulis onnibus rentato-glandulosis, angustis instructa, xcumine lats, styli glabri, gisco plano; cor........; fruct. oflongus, utringpe attematus, torgines. Hab. Hispania centr., Enledo: id Harjalizax (E. Pomata).

1297. Grepinia Durifolia Gogr Gab. N-2067.

Acculei breves, falcato - Silatati, at ramos flor. praneiores; fol. latiuscule obovato-obtusa, fasi rotuniata, vinivia, Iura, coriacea, at hasin costae paulo glandulosa; gentes biserrati, valive profunor; lanceolato-subconvegentes; pst. sculeati, glabri, parce glanioulssi i stip. rubentes, auriculis rectis; 
$-64-$

pet. 1, glangulosi, $7-8 \mathrm{~m}$. longi; twbus oblongus, laeris; sep . Parso glanoulosa, pimmulis annibus latiussulis, integerrimis instructa; styli glabri; Oises subplano; cor eciliata; fruct oblongus, basi rotuniatus, apice attenuates.

Hab. Hispania bor., Logroño: a Rasillo in Sierex 2e Cameros (M. Tubia).

1298. Gepinia temuiscapa Googr 8ab. N-2068. - Billst exs. N-3721 ex parte.

Acculei gebiles, falcati, ai ramos flor minutos pauciores; fol oborato, utringue subrotuniata, à costam glaniulosissiona; Destes bisorrati, operti; pot. glaniulosi, via sparse pilosuli; saepins inermes, stip. angustac, auriculis givergentibus; ped.1-1, setulosi; $10-12 \mathrm{~m}$. longi; tubus oblongus, laevis; sep. Iorso glarioulosa, anguste pinnuta; styli glabici, magni, gisco subplans; cor rosea; fruct. anguste oblangus, atrinque depressus.

Hab. Gard, at Le gigan (D. Eughkianicy).

Iub eadem schioula ahia prostabent specimina e C. temvisicapa ivicrsissima, at C.confertifbram Gige (Mon.ros. II, p. 298) fartinentia.

o 0 Foliola majora, oblonga.

1299. Drepinia Durioeana Cojgr 8ab. N-206g.

foculei panciores, tenmiter falcato-gilatati, as ramos flor mulli; fol. latiuscule oblongo-acuta, basi paulation rotundata, viribia, as costam glanioulosa, basique aculeata; dentes biserrati, lanceolato- aporti; pet. glansulosi, aculeati, glabri; stip. pirides, auriculis rectis; ped-1, hispici, $19-22 \mathrm{~m}$. longi; tubus oblangus, laveris; sep. Dorso glanioulos a, pinnulis omvibus latis, it-6-2entatis instructa, acumine 2entato-subfoliaces; styli glabri, giseo plano; cor eciliata; fruct. oblongus, basi longins aprice autem praulo attemuat. Hab. Hispania bor., Logroño: at Rasills in Sierra de Camevos (1. Zubia).

1300. Crepinia valentina Gogr \&ab. N-2070.

A-culei panciores, temiter falcato-Dilatati, ad ramos flor. melli; fol. late oblongo-acutiuscula, basi rotuniato, supra virisia, subtus rubentia, ai costam copiose aculeolatam glanioulosa, Dentes biserrati, ovato - patuli; pet. glansulosi, aculeati, subglaberrimi; stip. rubentes, longace, auriculis $\partial i-$ vergentitus; peo, 1-2, hispioissimi, 18-21 m. longi; tubus oblongus, laevis; sep. Oorso parcissime glansulosa, pinmulis latis, 4-6. Dentatis instructa, eccumine integro, angusto; styli glabri; gises paulo conico; con-ecilinta; 
$-65-\ldots$

fruct oblongus, basi gepressus, apice longissime attenuatus.

Heb. Hisponix orient, Valencia: as 9. Felipe de Dátiva (F. Hegelmaier).

jt Ytyli villosi vel lanati.

6. Fructus rotuniatus vel ovatus.

1301. Crepinix verticillacanthx (Nerat FP. Paris. 1812, p. 190, DC. Proor. II, po.

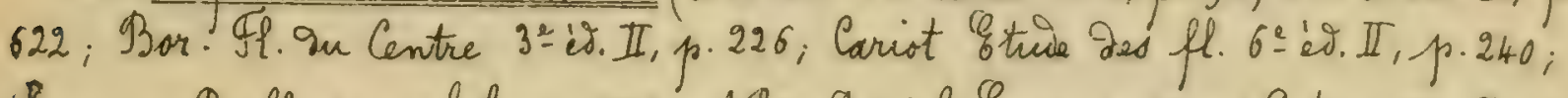
Zem. in T5ull.sse. philom. 1818, N-g; Déségl. Essai p. 67; ej. Cat. p. 188; Dumort.

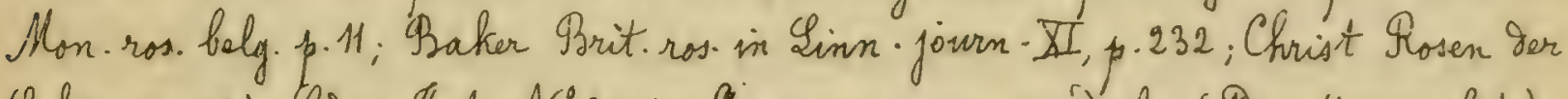

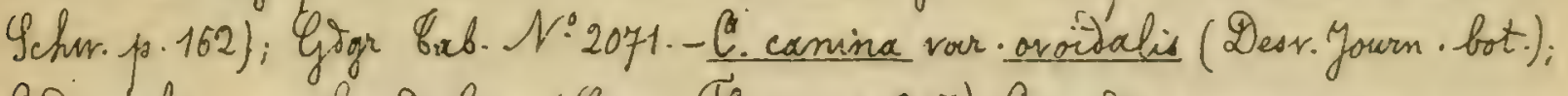
C. Aumalis glanaulosx (Gren. Fe. jur. p.215), C andegavensis var. innernsteinensis (Keller et Mrissbour in Hiesf. At Hoselbarger Rosenfl. Obersesterr.p. 12).

Ataculsi gebiles, copiosi, verticillati, fol ovats - acuta, glancescentie; Dentes biserrati; pet. villosuli, oflanoulosi, -aculeati; stip. Dilatatae, laeres, auriculis Osivergentibus; pet. hispini; tubus sratus, setulosus; sep. Lorso glandoulose, lativescule pinnata, tyli hirsuti; car meviseris, rssea; fruct. ovats-rotuniatus. Hob. Gallia, circa Saris; tongl.; Belg.; Helv; Bohem.

1302. Crepinix objurgeta Gjogr bab. N:2072.

Acculei termes, inchinati, havis aut vix dilatati, ag ramos flor mulhi fol. oblongo-xcuminata, basi paulatim rotundato, is costam glandulosa; rentes biserrati, lanceslato-recti; pot. glandulosi, parce aculacti, inferne pilosuli; stip. latae, virives; pet. 1-2, alii laeves, alii temuter et parum glanioulosi, 12-15 m.longi; trubus ovoideus, laevis; sep. Dorso glantulosa, pinmblis hinearibus, vix dentato-glanoulosis instructa; styli hirsuti, gises plano; cor........ fruct.oratus, utrinque rotundactus.

Hab. Prrenaici antr, et Giore in viam Héas (H. Brroire).

1303. Crenimia nitioula (Mess. Inppl. IV at Cat. Crem.p.20; j: Omum. Pol. ps.20,61 at 67; M. Bief. Fl. toun-canc. III, p. 347; Ledeb. Fl. ross. II, p. 79; Gichw. Ikizze p.164, Rchb. Fl. exc. N-39g1, Desighl. Cat.p.261); Gogr Eab. N-2073.

Aculer valivi, copiosi, falcato-dilatati; fol-ovato-elliptica, anta, ritioula; ventes biserrati; pet aculeati, glaniulosi; stip. Oenticulatar; pet-1, setosi; tubus subglobssis, laevis; sep. glandulosa, pinnata; styli villosi; cor. sat parre, carnea, fruct magmes, subrotuniatus.

Hab. Soghia, al Kazemenie (Bewer). 
$-66-$

Diseghise l.e. foliola subtus glanvulosa huic spesiei tribuit; sed Messer in Descriptione sux characterem hume gravem tacet. Notandum st opos quot schesula speciminis authentici herbarii Canobleani verosimiliter casu fortinter transmutata fint; unde error.

130\%. Creminix histricose (Grép.) in Gogr Gab N-2074.

toculei robusti, valie falcati, as ramos flor. copiosi; fol sat parva, 8vato-elliptica, inf.subobtusa, sup.acuta, basi attenuato-subrotunioata, as costam prarce glandulasa; dentes biserrati; pet. glabri; glasioulos; copiose aculeati; stip. ciliatae, auriculis subrectis; pet.4-5, hispisi, elongati, tubus ovatus, laevis; sep. Garso lanoulosa, finnata; styki hirsuti; cor......; fr.ovatus. Hab. Hérault, at Le Larzac, It Manice (H. Loret).

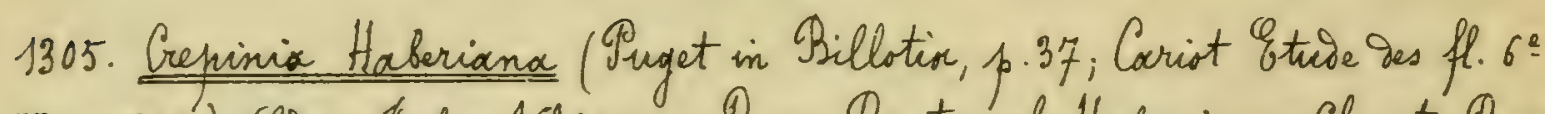
i. II p. 241); Goor \&ab. N-2075. - Rosa Renteri f. Haberiana Christ Rosen der Gihr. p. 168. - Pillet exs. N-3585; Deisegl. Herb. ros. N-20 et 20 bis.

foculei valioi, falcato-gilatati; fol ovato-elliptica, nervos, es costam - glanoulosa; Pentes biserrati; pet. aculeati, sparse pilosulo-glanoulosi; stip. silatetac, auriculis givergentibus; pes. hispidi; tubus svatus, lacris; sep. Forso glanoulosa, latiuscule pinnata; styli lanati, isce salie conico; cor maynin, intense rosea; fruct. magnus, svatus.

Hab. Haute- Savore, at Hobère-Lullix et Habeire-Soche (Tiget). Frnctus, a Dereglise elfipssifens vel obovoiden falso dictus, sec-exemplaria sucthentiea pherima quace suppeditaverunt, certe ovatus est.

1306. Crepinia cuneata Gogr \&ab. $\mathcal{N}=2076$.

trenlei as ramos flar. falcato-dilatati; fol obovata, utringue attemata, cuneata, is costam -glandulos-a; Dentes biserrati, recti; pet. glanisulosi; sparse pilosi, inacque armati; stip. latac, aurriculis divergentibus; pes. 1-4, sat hisfidi, 7-14 m. longi; tubus soratus, laevis; sep. Gorsos glaniulose, late pionnata; styli villosi, dises camcs; cor rosea, fruct. ovato-rotundatus. Hobl Hante- Saroie, at Habire = whin (Suget).

1307. Prepinia firma (Puget in Sillotia p. 118; (rep. Prim. I. p. 52), Civgr Gab. $\mathcal{N} 207.7$ - gillot exs. No3847.

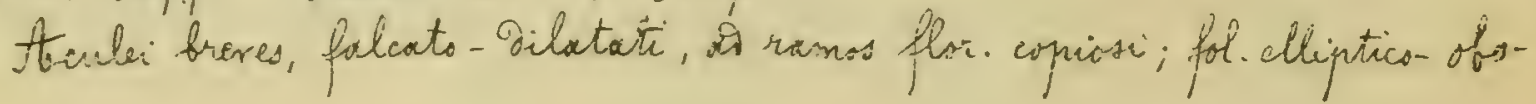


$-67-$

vata, subattemuata, basi abrupte contracta, so costam glanoulosa; gentes biserrati, subfalcati; pet aculeati, sparse glansulosi, pubescentes; stip. sape rubentes, anriculis rectis; pes. 1-4, parce hispiouli; 7-12 m. longi; tubus ovato-rotuniatus, laevis; sep. Forso glaniulosa, late pinnata; styli villosi, Disco conico; cor.magna, rosed; funct mognus, subglobosus.

Hab. Haute-Saroi, as Belbraux (Tuget).

$\S \S . F_{\text {ructus oxoisens. }}$

i. Eubus toto val saltem usque at $1 / 3$ infer. glanoulosus.

1308. Grepinia stictocarpa Gogr \&ab. N-2078.

Abculei gilatati, rectivsculi, ad ramos flor saepe geminati; fol-ovata, coroata, apice rotundata, as costam eglanovlosa; Dentes fiserrati, aperti; pet. aculeate, hine inde glaniuloso-pilasuli; stip. laeres, auriculis subrectis; hot.1-3, setulosi, 10-19 m. longi; tubus oridens, tots hispitus; sep. Dorso glanSulose, angustius pinnata; styli villosi, 2ises plano; con...... fruct orsiDeus, utrinque saepuis attermatus.

Hab. Yoère, of Jillard-De-Ians (Boullu).

130g. Orepinia subsisteglis (Morbás) mos.?

Atculer minuti, subulati, recti, ad ramss flor geminati; fol. ampluiscule oblong-acuta, basi paulation rotuniata, is costam parce glanoulosa, lacte virisia; Dentes biserrati, triangulares, recti; pot. glaniulosi, aculeviti, sparse pilosi; stip. lativimare sat breves, rubentes, Dorso glanoulosac, auriculis yirergentifus; ped. 1-2. Aparse glanionloss; 1 cent. longi; tubus oborato-oblongis, sparse setasus; sep. Dorso parum glaninlosa, pinnulis ommibus gentatis, sat angrestis instructa, styli villosi, Dises conico; cor. parva, eciliata; fruct. oroilens, utringue attenuatus.

Hab. Hungaria, Schemnity at Grencos Cabrod (Fmet).

1310. Creminia vornssica Gogr \&ab. N=2079.

Aculei panciones, parvi, recti, ad ramos flor. mulli; fol late oborrito - Plonga, ccuta, basi rotuniata, glanca, a costam glandulosa, isentes fiserrati, triangulares, aperti, pet. glaniulosi, aculeatr, glabri; stip. glaveac, jilatatar; pes.1, glanoulosi, 16-18 m.longi; tubus svideus, in 1/3 inf. ilaniulosus; sep. Orors glansulose, pinnulis parum latis, valse gentats- 
$-68-$

giansulosis instructa, coumine latisre, dentato; styil sublanati, disco fere plano; cor-maima, rosea, eciliata; fruct. oroidens, basi sepressus, aprice atternatus. Hab. Borussia, at Irremberg (Miess).

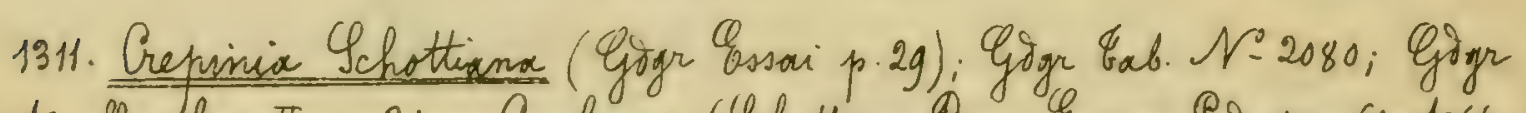
Conte. fl. Slar. II. p. 34. - C glauex (Ychott ex Gess. Envem. Pog. pp. 60 et $64, N^{\circ}$ 151; Ledeb. IP.ross. II, p.78; Bichw. Tchizze p. 164; Prep. Trim. I. p. 52 non Desf, nec Dierb. nee Vill.); C.virata (Gremli mss.). C. camina var. Gehottiana ( Per. in DC. Proor. II, p. 613; Dejegl. Cat. p. 18g).

Atculer rarissimi, at ramos sup misntos nulli; fol elliptica, glanca; Dentes biserrati; pat. glonoulosi, aculesti; stip. laeves; pes. hispiti; tubus oroidens, gracilis, in $1 / 2$ inf. glaninlosus; sep. Dorss glaniulosa, pinnata; styli hirsuti; car. rosea; fruct-aroitens, itimque attenuatus.

Hab. Porolix (Besser), Gilesix (Goimm. ot Grab. Fl siles. II, p. 83); Regis Tamub; Romania (Giger Contr. l.c.). (1)

1312. Crepinia nciculifera Gogr toab. N-2081.

foculei rarissimi, falcati, late gilatato-becurrentes, ad ramos flor.mulli; fol. parra, obovato-oblonga, utrinque attenuata, firma, nervosa, as costam egloniulosx; sentes biserrati, recti, callosi; pot. vis pilosulo-glansulosi, subinermes, stip. sngustare, laeres, auriculis givergentifus; per. 1, gense aculeolati, g-13 m. longi; trisus obovatus, in $1 / 3$ inf. vel $1 / 2$ aciculatus; sep. gorso glaniuloso, sat anguste pinnata, styli lanati, oisco subplano; cor. rosea; fruct. oboratus, aprice attemuatus.

Hab. Tsere, at Le Sappey (Moullu).

Tarsan ad Montanses (Ozanmiea) pertinet, set sepala decitux.

1313. Prepinia Dumantieri (Gogr Gssi p.29), Ggar Gab. N:2082. P.campestris

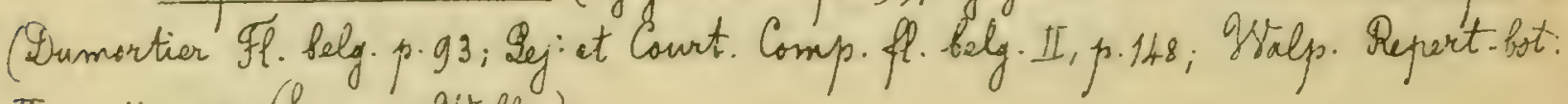
Iip.11, non Ser. nee Mralle.).

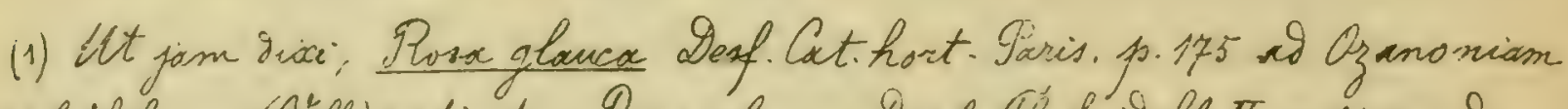
rubrifoliam (Gill.)pertinet; Rosa glauca Dierb. IP. heidelb. II, p.140 al

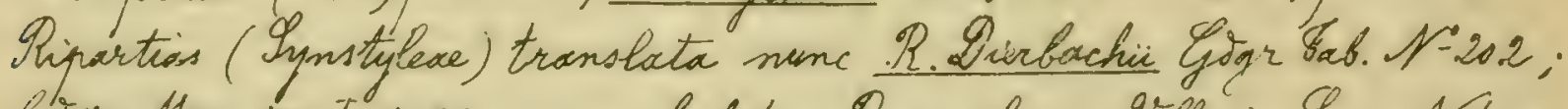

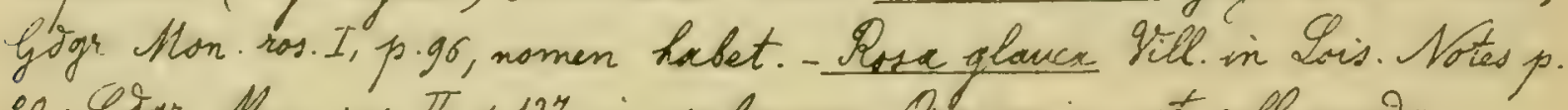
80; Cigar Mon. ras. II, p.127 in subgenere Ozanomix est collacanisa. 
$-69-$

Tocului rectiuseuli, subsilatati; fol. orata, firma, as costam glanisulosa; dentes biserrati; pot aculeati, glabri, glandulosi; stip. serruclatas; pie. hispiivi;

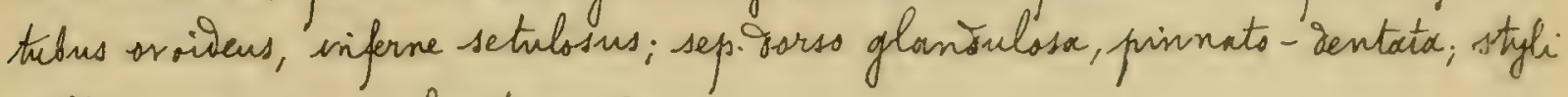
villosi; cor. rosec; fruct. ovoidens.

Hab. Melgium, ad Inxembourg (Dumortier).

C.campestris (Ir. in Fries Pl. hall p.86) at Prepinias Collineas pertinat, Ium C. campestris (Malk. Hist. ros. p. 110) at Cottetias (Iimpinellifoliae) spectare visetur.

1314. Creminia Legalliu Giar Gab. N-2083.-C.canina var. (2e Gall. Fl-morb.).

focula falcato-dilatati, at ramos flor-copiosi; fol. late sbovato-acuta, basi subattenuata, id costasm parce glaniulssa; gentes biserrati, breviter triangulares; pst. glaniulosi, aculeati, inferne sparse pilosuli; stip. longae, auriculis rectis, pet. 2-4, hispiti, 6-8 m. longi; tubus svoideus, toto hispiqus; sep. Dorso glanaulosa, pinmilis parum latis, dentactis instructa; styli lanati, gisco conic; cor. rosea, eciliata; fruct. ovoidens, gepressus. Hab. Finiotere, ad Daculas (C. Ghiebaut).

ن Gubus laeris, vel tantum basi infima glantulosus. $X$ Foliola parra, ovato-obtusa.

1315. Crepinia obtusa (Mip. in Diségl. Cat.p. 183); Grogr Gab. N-2084. toculei debiles, recti, parum Silatati, at ramos flor.breves; fol mesia svato-obtusx, sup. acutivisula, ao costam parce glandulosa, Dentes biserrati; pet-aculeati, sparse pilosulo-glanoulosi; stip. lneves, auriculis rectis; peos.1, parce hispisi.; tubus ovoidens, laevis; sep. Sorso glandulasx, frevix, pinnata; styli rillosi; cor. subalba; fruct - parrus, ovoidens.

Hob. Cher, wo burly (Ripart).

1316. Crepinia sardox Goor mss.- Reverchor Pl. Gars. (1882), A:301. foculailongi, gilatati, valte folcati, ad ramos flor-mulli; fol.obovatoobtusa, basi rotuniata, pallite virioia, nt costam eglanoulos $x$; Tentes biserrati, triangular - subconvergentes; pet. inermes, sparse glanimlosspilosuli; stip. rubentes, parrar, eflanoulosa, auriculis rectis; pes.1, hiopiai, 11-13 m.longi; tubus oroteus, laevis; sep. Sorso parce glaniulos:x, pinmulis annibus angustis, 1-3-Dentatis instructa, acumine integ-o; styli 
$-70-$

lanati, Disco plans; cor rosea, eciliata; fruct, magnus, ovoideus, basi atternuatus. Hab. Sorroinia bor., at rempio (E. Reverchon).

1317. Crepinia pleiacantha Gige mss.

Aoculei longissini, teretes, parum et temiter falcati, at ramos flor. coprosissimi, fol parva, obovato-oblonga, ecuta, basi subattenuata, pallise viridi-glavcescentia, ad costam glanoulosissima; Dentes biserrati, profunde lanceolato-aperti, stip. parve, breves, sirides, Porso eglanoulosae, auriculis rects; per. 1-2, valise glantulosi, 11/2 cent. longi; tubus sborato-sblangus, basi infima setosus; sep. Dorso glaniulosa, primulis onnibus parum latis, gentatis instructo; styli parce pubescentes, Lises comics; car....... fruct -ovoiDeo-comius, basi rotuniatus, apice longius attenuatus.

Hab. Far, at Asmpus (tolbert).

XX Folisla ample sorato-oblonga vel oblonga.

1318. Crepinia gicupnopoota Gogr Gab. N-2085.

Acculei breves, temuiter falcati, parum Dilatati, as ramos flor, saepins mulli; fol oborato-oblonga, acutiuscula, basi attemeato-subcuneata, intense viridio, ad costam glanoulssa; bentes bisersati, lanceslato-aperti; pat. parce wculeati, glaniulosi, inferne unifariam minutissine pubescentes; stip. viricies, longae, auriculis sivergentibus; pet.2, flexuosi; temiter glaniulasi, 16-18 m.longi; tubus avoideus, laeris; sep. Tarss glandulosa, prinnulis angustis, valts gentato-glanioulosis instructa, acumine parum lato; styli hirsuti, gisco plass; $\operatorname{cor} \ldots . . .$. ; fuct-oroinens, utrinque depressus.

Hab. Anglia, ad Gork (G. Hebster).

1319. Crepinia laxistyla Gogr bab. N-2086.

toculie valiai, parum falcati, as ramos flor sparsi; fol oborato-oblonga, subacuminata, basi rotuniata, corixcea, is costam glanoulosa; dentes biserrati, ovato-convergentes; pet-glansulosi, aculeati, vix sparse pilosuli, stip.

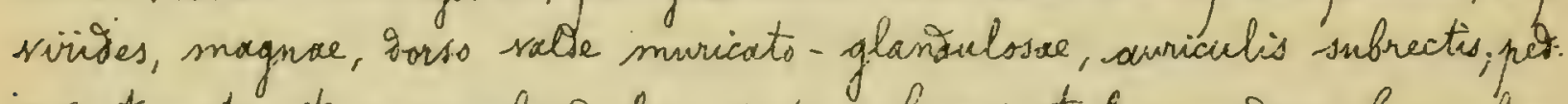
2-3, temiter et pare glanisulosi; g-11 m. longi; tubus ovoisens, basi glan-

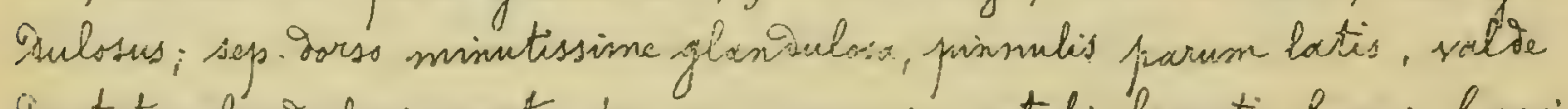
sintato-glangulosis instructi, cum acurnisin; styli lanati, longi, laxi,

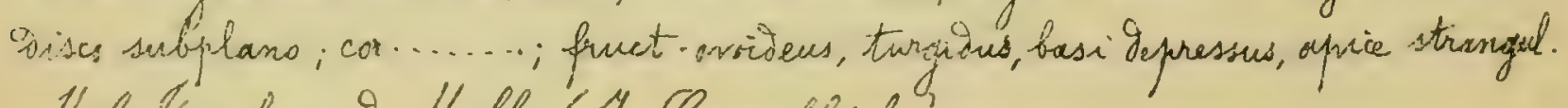
Hab. Eurot, at Hall (H. Greonbich). 
- 71 -

1320. Cepinia insolata Gogr toab. N-2087.

Rubens; aculei subteretes, ois ramos flor. Silatati, parum et tersitor falcati; fol oborato-oblonga, utringue paulation atternuata, at costam glanioulosa; Dentes biserrati, aperti; pet. subinermes, glaniulosi, hine inve piloseli; stip. longar, rulentes, aprice Silatatae, auriculis Sirergentilus; ped.1-2, hispiaic,

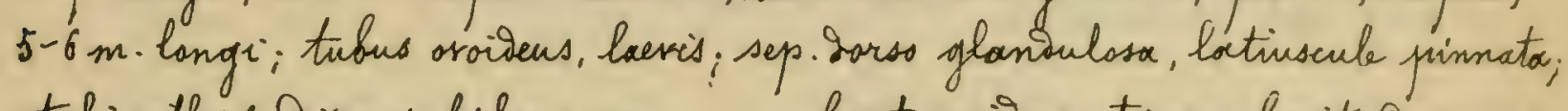
styli villosi, Gises subplans; cor......... funct. ovoidens, utrinque breviter depressus. Hab. Houte- Savoie, at Pringy (Muget).

1321. Grepinix ulmoides Gigr Gab. N-2088.

Acculei breves, valis falcati, of ramos flor. panciores; fol ample suboblongo-acuta, basi fere rotuniata, crassa, ad costam glandulosa; Fentes late triangulares, aperti, biserrati; pet. glanoulosi, parce aculeati, inferne paulo pubescentes; stip. longre, siviess, eglaniulosae, ped. 2-3, Dense setulosi, 19-21 m. tongi; tubus oroiteus, basi infina hispitus; sep. Dorso minute glandulosa, pinnulis paucioribus, amnibus angustis valieque dentato - glanioulosis instructo; styli sublanati, gisco conico; con....... fruct sroitens, basi depressus, is apicem abrupte strangulatus.

Hab. Anglia, Cheshire: as Dorkgate (y. Hoorford Lewis).

1322. Crepinia erassipes Gogr mss. - Tosa ....... Crép. Prim. VI.p.18. Brotherus 9l. cauc. (Mosa) N:2.

Aculei parri, breves, parum gilatati, temiter folcati, as ramss flor.copiosi: fol obovato oblonga, brenter acuta, basi subrotundata, laete viribia, ai costam glanimlosx; tentes biserrati, late lanceolato-aperti; pot. glaniulosi, aculeati, inferne subhirtilk; stip. latae, inf. Porss glandulosae, auricilis rectis; pes.1-3, parce aculealati, crassi, apice Demum carnosi, 21-23 m. Congi; tubus ovoiseus, basi aculeslatus; sep. crassa, tantuin apice gorsi glanoulosa, pirnulis omnibus latis, set copiose sentats-glasinulsis instructor; stegli sublanati; Sises sat conico; car. alba, eciliata; fruct. magnus, ovoìens, hasi Dapressus, aprice attemuatus, inflorescentia (pros. et tubus) violacea.

Hab. Coucasus, Carthalinia: ad Gori for Gi Kura (Mrotherus).

1323. Crepinia sommians Giagr toof. N'2089.

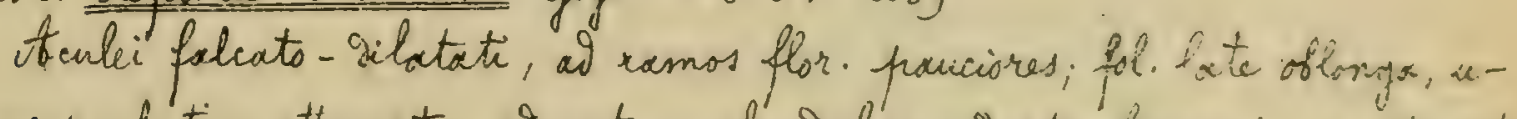

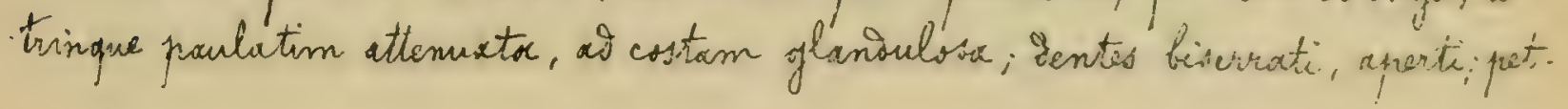


-72-

parce aculeati, hire inie glaniouloso-pilosuhi; stip. elongatore, gilatatre, auriculis ivirergentibus; pes. 1-2, parce setulosi, 7-12 m. longr; tubus oroideus, haevis; sep. Porso glanisulosa, late pinnata, acumine dentato-subfolixces; Styli sillosi, Oive rubrs, paulo conico; esr rosea; fruct maximus, ovoidens.

Hab. Haute-Savori, ad Habire-Poche (Theget).

1324. Crenimia prionoserala Gogr mss. - Rosa ........ Crep. Trim. VI, p. 18. - Protherus Ml.caue. (Rosa) N'G.

toculei maximi, felcato- Silatate, ad ramos flor. sparsi; fol. oblongolarculata, hasi attemuata, intersse virisia, ad costam eglanisulosa, Dentes biserrati, lanceolato-aperti; pet.hinc inse glaniuloss-pilssuli, parce aculecti; stip. latar, longor, Dorso oflansulosoc, auriculis sirergentrbus; pes. 6-8, rigigici, vis glasimlosi, $20-22 \mathrm{~m}$. longi; tubus voitens, lacris; sep. Tanturn apice iorsi parce glinaulosa, cactorum Laevia, pinmulis latis, valoe gentato-glanDulosis instructa, acumine lats, subfoliaces; styli pubescentes, Disco canics; cor........, fruct orotivens, basi rotunsatus, apice paulo strangulatus.

Hab. Concasus, Carthalinia: as Gori prope flur. Kura ( Brotherus). Pesunculi tubusque violacei, ut in N. $1322 \mathrm{~cm}$ sat accesit.

$\S \S$ - Fructus sborato-oblangus vel sblangus.

$\sim$ Gubus laeris.

$X$ Pesureuli alii laves, alii glandulosi.

1325. Brenimia psilonhylloides (Crip). in Giggr Gab. No 2090.

toculei raliai, falcato-gilatoti, io caules soepe verticillati; fol oboratoobionga vel oblengo-scuta, basi roturniata, glancessentia, ao costarn glanaulosa; Dentes fiserrati, recti; pet. glanoulosi, aculeati, tenuiter et toto pubeiuli; stip. longae, corso glansulosae, auriculis divergentibus, peo-1-3, ahii laives, alii $1-5$ glaniulis instructi, $10-19 \mathrm{~m}$. longj;, tubus oblongus, laeris; sep. Sorss qíanisulsa, pinnulis angustis, Dentatis instructa; styli villosi, Siscs subplano, cor. nosea? Fruct. oborato-oblongus, utrinque gepressus. Hab., Belgium, Samur: at Rochefort (F. Crépin).

1326. Creninia porlyphlaes Gyogr Bob. 1/2001.

Glavea; culei sebiles, late silatati, rectivisuli, as ramos flor. sat copiosi; fol-orata vel orats-elliptica, sacpins utrinque rotuniata, ad costom 
$-73-$

glanoulosa; gentes biserrati, breves, recti; pet glanoulosi, aculeati, hinc inde pilosuli; stip. majusculae, Darso glaniulosae, auriculis Sivergentebus; pas. 1-9,

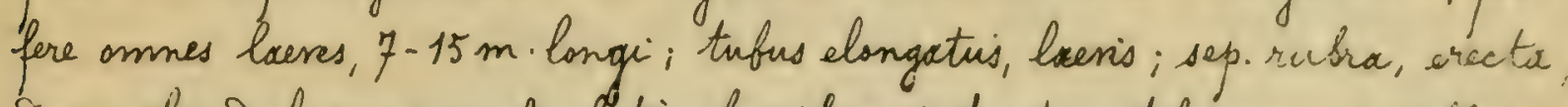
sorso glansulosa, pinmulis latis, brescibus inotructa; styli magni, villosi. isses plans; cor. rosea; fruct. magnus, oblongus, utringue attenuatius. Hab. Haute-Saroie, at Iringy (Muget).

1327. Crepinia lancaeformis Gagr bab. N-20g2.

Acculei rarissimi, gilatato - subfalcati, ai ramos flor mulli; fol. oblongsacuminata, fasi attemuata, as costam glanoulisa; Dentes bierrati, patuki; pet. glanoulosi, subinarmes, hine inde pilosuli; stip.longace, subtus glandulosse; pot. 1-3, nunc glabri, nune glingulosi; $17-23 \mathrm{~m}$. Congi; tubus oblongus, lacvis; sep. Iorss glaniulosa, fimmulis parum latis, valde gentato - glanzulosis instructa, stylivillosi, subcoaliti, Dises fere plans; cor.......; fruct. oborats-oblongus, apice attermatus.

Hab. Houte- Savoie, at Annecy-b-Sienx (Suget).

1328. Crepimia pretiosa Gagre Gab. N'20g3.

Abculei-parwi, breves, parum falcati, a ramos flor. submulli; fol. Eate sbovato-oblonga, acuta, basi attermata, glaucescentia, xo costam vix glarDulasa; grentes biserrati, triangulare-subconvergentes; pet glanisulosi, subinermes, paulo pubescentes; stip. latissimae, Silute rubentes, auriculis girergentibus; net. 2-4, tenuiter alii et parce glanoulosi, alii laeves, $13-21 \mathrm{~m}$. longi; tribus oborats-oblongus, laevis; sep. Dorso glanidulosa, pinnulis parum latis, valde gentato-glaninulosis instructa, acumine lato, copiose dentato; styli hirsuti, gises plans; cor. eciliats; fruct. ofovato - oblangus, basi rotuniatus, apice longe attenuatus.

Hab. Hippana bor, Iogroñs: at Rasillo in Sierra de Cameros (O. Tubia).

$X X$ Vesumanli omnes phes minus hisficio:

o Foliola basi attenuacta.

1329. Crepinia sinace (Maker Revies p. 32; y. Mrut. nos. in Linn. sac.

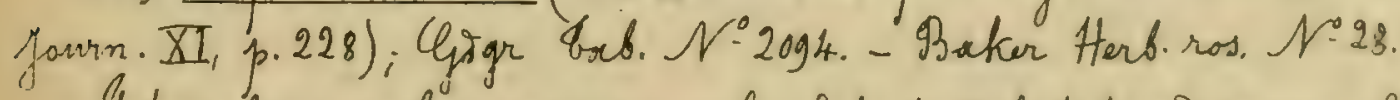

Sista plenco-rubra, rinosa, aculei faleato-gilatati, a ramos flor. sat copiasi; fil.oblongs-lanceslatia, basi attenuata, as costam g'anouloser, ider- 
$-74-$

tes biserrati; pat. aculeati, glandulosi, vix pilosuli; stip-mediscres, rubras; pes. smnes phes minus setulosi; tubus sblongus, laevis; sep. Sorso glandulose, pinnata, styli villosi, gises plans; car.rosea; fruct. oblongus. Hab. Anghia, Yorkshire: a Sowerby (J.G. Baker).

1330. Grepinia erectisepala Gogr Gab. N-20g5.

toculei rarissini, rebiles, falcati, aid ramos flor. mulli; fol. late obovatooblonga, obtusa, bisi paulation attenuato, as costam glanivulosa; gentes biserrati, late triangulari - convergentes; pet subinermes, hisic inde glanoulosopilosuli; stip. virides, latae, eglaniulosae, auriculis rectis; ped. 2-3, hispidi, 11-12 m. langi; tubus oblangus, laevis; sep. Dorso sparse muricata, erectosubcomniventia, pinmulis angustis, onnibus integris, paccioribues instructa, acumine subfoliaces; styli lanati, gises plano; car.magna, eciliata; fruct. magmus, oblongus, utrinque attenuatus, apice strangulatis.

Hab. Hautes - Pyrénées, at Nousea (H. Bordère).

1331. Crepinia aarcobasis Gogr tab. N'20g6.

Rubens; aculer breves, parri; falcato-gilatati, as ramos flor.mulli; fol. late oborato-acuta, basi paulation attemuata, subglauca, at costam parce glaniulasa; dentes biserrati, lancealato-aperti; pot praslo glaniulosi, glabri, aculeati; stip. rubentes, auriculis Divergentibus; pes.2-4, hispisi; 12-15 m. longi; tubus elongatus, laevis, sep. Oorso glaniulosa, pinnulis hinearibus, integris val 1-2-2entatis instructa, acumine parum lato, integerrimo; styli exserti, sublanati, Jises conico, cor........ fruct. magmus, elongatus, apice paulo iapressus, a $2 / 3$ sup. usque at basin longissime attenuato- recurrens. Hab. Anglia, gestmorelant: à Grasmere (H. T. Fisher).

00 . Folisla basi notuniata.

1332. Crepinia pringyersis Giver Gab. N-2097.

toculei temes, recti, parum Dilatati, at ramos flor. milli; fol parva, ova ta, subattenuata, basi subrotuniate-truncata, ad costam parce glandulosa; sentes biserrati, aperti, pet. subinermes, glanimlosi, saepius globui; stip. Dorso olansulosse, auriculis suboviergentibus, pet saepe 1, parea et tesviniter glanqulosi, 14-19m. longi; tubus oblongus, laevis; sep. gorso glansulosa, pinmuli: latis copiose instructa, styli villosi, aisco paulo comico, cor.lacte rosea, as 
$-75-$

unguem lutescens, fruct. anguste oblangus, utrinque atterneatus.

Hab. Haute-Savoi, at Pringy (Muget).

1393. Gepinia bractessa (Crép.) in Gogr \&ab. N-2ogs.

Acculei falcato-dilatati, at ramos flor panciores; fol ovato-elliptica, acuta, basi rotuniacta, at costam vix glanoulosa; ientes biserrati; pet. parce glanioulosi, glabri, aculeati; stip. masimace, aurieulis latis; pes. 1-3, copiose bracteati, parce glaniulosi; tubus sblongus, laevis; sep. Sorso Dense olaniulosa, pinnulis copiose dentatis instructa; styli villosi, gisco paulo comico; cor......., fruct. oblongs - pyriformis.

Hab. Balgium, Mamur: as Rochefort (F. Céfin).

1334. Crepinia focharii (Millb. in Irensk bot. IX, tab. 577; Rchb. Fl. esc.

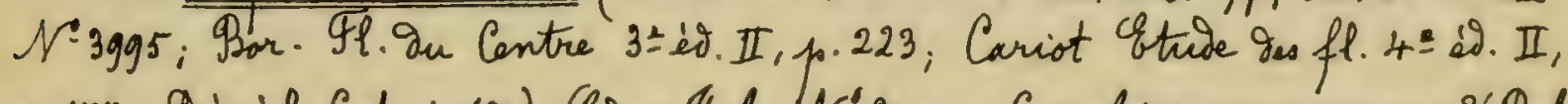
p.177; Diseigl. Cat.p.190); Gogr 8ab. Y'20g9. - C. nubiginasa x camina? (Rchb.

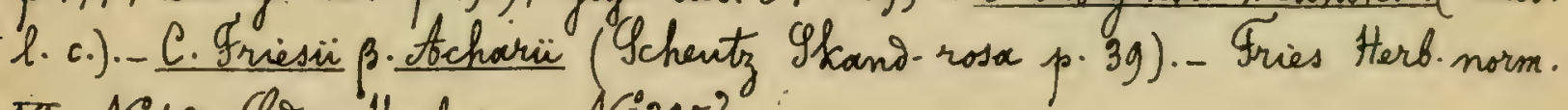
VI. N.42, Gojgr Herb. ros. N'385?

Acculei Silatati, temuiter falcati, as ramos flor. sat copiosic; fol oborata, subacuta, basi rotunisato, intense virivia, subtus pallisa, dentes lati, biserrati; pet. hinc inise glanioulosi, sculeati, plerumque glabri, stip. Dilatatae, Gorso glanoulosar; pes setosi; tubus oboratus, basi attematus, laev-is; sep. gorso hispita, erecto-conniventix, pinnata, acumine subillatato; styli magni, lanati, baises plano; cor sat mogna, lacte rosea, ad ungrem nitise albicans; fruct. magnus, sblongus; rectus, sepalis coronatus.

Hab. Suecia.

Descripsi ex ione citata. - Confer Crepin Sirm. I.p.55.

Li recte geprita sit ista species, propter calycis lacinias erectsconmirentes et forsa.r pervistentes, a caeteris Crepinis Giversissima est atoue in Montanis (Ozanonia) collocare Debnerat.

1335. Crepinia inconsideratax (De'segl in Mim aca9. or Maine -et-Love, XXVIII, 1873, p.117; extr. p.21; ej. Cat.p.175); Googr Eab. N-2100. C. his-

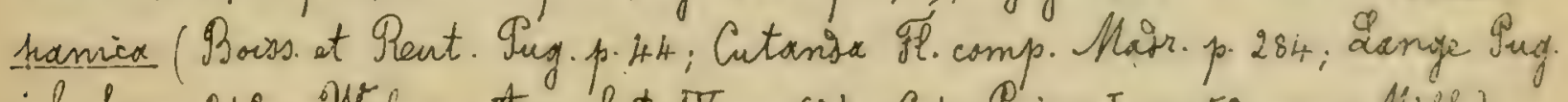
ph. hisp. 342; Halpers Jorm. fot. IV, p.654; Grep. Prim. I, p. 52 non Mill.);

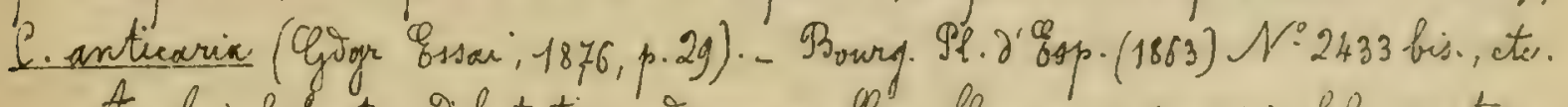
Atcenlei falcato-Dilatati, ad ramos flor. flexuosos spoorsi; fol ovata, 
mesiceria, acuta, basi rotuniate, oi costam glansulosa; Pentes biserrati, triangulares; pot aculeati, glaniulosi, hinc inis pilosuli; stip. latac, auriculis scutis, ped glandulosi, graciles; tubus oblongrs, utrinopue attenuatus, laevis; sep. Dorso glanioulosa, erecta, pinnata; styli villosi; cor- nosea; fruct. oblingus, apuice attematus.

Hab. Hispamix tota; primum in Sierra ge Anteonera getecta.

Fructus calycisque tibus a Boiss. At Reut. orati Licti rel suboblongi. evisenter sunt ollongi; sepalo abiisfem auctoribus persistentia orict, demum cabuca eveniunt; corolla variat a pollive as intensius rosea.

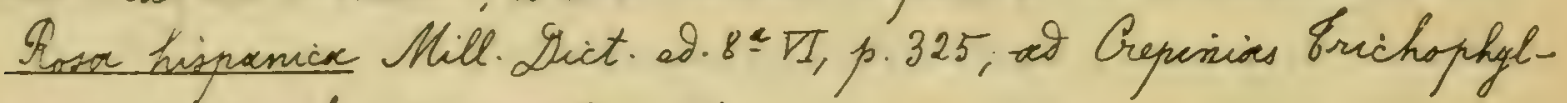
las pertinat at infra Descripta erit.

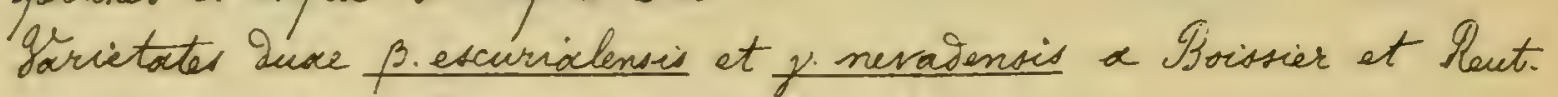
l.c. propositace species iviersas constituunt.

1336. Creprinia micradens (Gogr Ros.nor. II,p.19); Gogr Gab. N'2101. Gogr Herb. ros. N-177.

toculei gebiles, falcats-Dilatati, as ramos flor-geminati; fol oborata. breviter attemuata, fasi rotuniacta, nemota, as costam parcissime glanioulose; Sentes biserrati, aperti; pet. glanvalosi, parce aculeati, hinc inde pilssuli; stip. angustissimac, subtus paulo glaniulosa, auriculis givergentibus; fes. 1-3. temiter glaniulosi, 13-17 m. langi; tubus oblengus, laevis, sep. Sorso parce glanisulosa, latiuscule pinnata; styli hirsuti, tenves, Bis-s subplans, cor-roser; fruct-obovato-oblongus, apice attenustus.

Hab. Thone, ad Gleize' (M. Ganooger).

1337. Crepinia leporina Gogr mss. - Magnier Fe. sel. N-540.

Aculei robusti, inchinati, valie bilatati, al ramos flor. stipulares; fol. aspple sbovato-oblongas, acuta, basi rotuniata, glancescentia, ad costam glaniulisa; Dentes biserrati, late lanceolate-aperti; pet-glansulosi, aculeate, glabri; stip. magnae, latae, gorso laeres, auriculis rectis; pet.1-3, toto setosi, 1 cent. longr; tabus oblongus, laeris; ses Baras parce glanoulosa, pinnulis onnisus engustis, valie dentatis instructa; styfi pubescentes, Fisco plano; con parra, pallice rosea, esiliata; fruct. sat magnus, oforats-oblongus, Sasi sepressus, apice sat longe attermatus.

Hab. Fire, ad Serna (Boullu). 
- $77-$

1338. Crepinia opportuna Goor Gob. N-2102.

Abeulei longi, inclinati, as ros flor pauciores; fol late obovato - subacuta, hasi rotunsata, cariacea, cos costam eglaniulosa; Pentes biserrati, sublanceolato-recti; pot. eglandolssi, aculeati, sparse pilosuli; stip. latissimas, rubentes, eglanivilos ar, auriculis rectis; pes. 1-2, hispisis, g-10 m.longi; tubus oblongus, laeris, sep. Tarss glaniaulosa, pinnulis ommibus angustis, 1-2- tontatis instructa, acurnine integro; styli sublanati, Discs plano; cor......; fruct. magnus, suboblongus, basi pauls gepressus, apice attennatus.

Hab. Basses-Alpes, Bo Bouzolieres (Mroal).

$\sim \sim$ Eubus plus minus hispious.

1339. Creximia ruberrima Gigagr tab. N-2103.

Eota rubra; rculei cylingrici, longr; rectinsculi; as ramos flot. sparsi; foet.

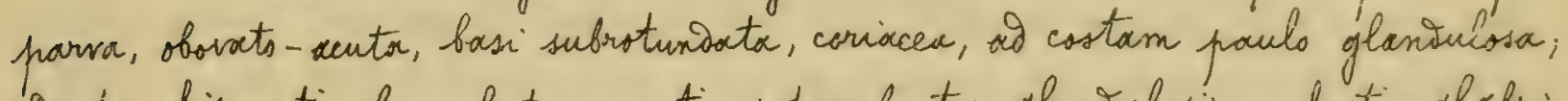
Dentes biserrati, lanceslats-aperti; pet rubbentes, ylanorulosi, aculeati, glabri; stip. rubentes, parrae, eglantulosor, avriculis rectis; pet. 1-2, hispiti, 8-g m. longi; inbus tblongus, Basi infima setosus; sep. Torso parce glaniulosor, pinnulis annibus linevrious, 1-2-gentatis instructa, acumine integro; staghi pulescentes, Fisco plans, cor......., fruct oflongus, basi depressus, aprice. tonige attemuat. Hab. Gaucluse, ad Mont- Sentoux (to. Toutheman).

1340. Crepinia popmlaris Goor mos.

Acculei falcato-Dilatati, valisi, à ramos flor.geminati; fol amplissime oborato-oblonga, acnta, basi roturnouta, intense virisia, ao costam glan2ulosa; ientes biserrati, triangulares, aperti; pet. copiose glanioulosi, aculeati, in $1 / 2$ inf. prorsus laxe pubescentes, stip. sirides, latae, elangator, Dorso glanioulosere, auriculis subrectis; pes. 2-6, cense setulosi, 5-7 m langi; tubus obvato-solongus, toto glainioulosus; sep. Dorss glanoulosa, pinmulis latis, Jentatis instructa, acumine Sentats; styfi villosi, gisco plano; cor...... fruct-mag. mus, obovats-sblongus, targitus, utrinque parum attenuatus.

Hab. Iranssilvamia, Kolozs: at Magyar í Kereke (2. Mrichter).

1341. Crefunia nevaiensis CGagr tab. N'2104 - C. hispamia y' nevaDensis (Mociss at Trent. Mug. p. 4t, e loses).

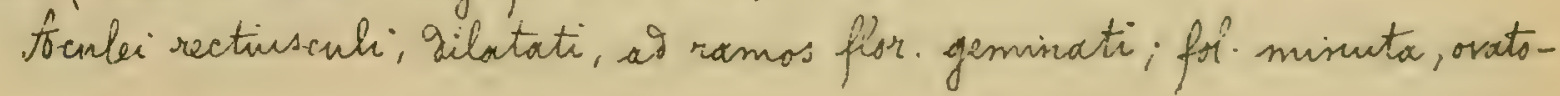


$-78-$

retuss, basi rotuniata, Dura, coriaces, superne intense viridia, subtus glaucas, it costam glanimloss; gentes breves, biserrati; pet glanioulosi, aculesti, glabri; stip.intense rubentes, eglangulasac, breves, auriculis rectis; ped.1, Densissime glanaulosi, 8-9 m. longi; tubus elongatus, basi infima glasioulosus; sep. gorso glanioulosa, vix pimnata, pinmulis raris, 2-3-sentatis instructa, acmmine filiforme; styli villosi; Qises plans, corminuta, rosea, eciliata: fruct anguste elangatus, utrinque attemuatus.

Hab. Hispania merid. Sierra Nevada: as S. Geromino (F. Hegelmacier). Hue pertinere viletur C.hispanica y. nevadensis (Boiss. Reut.l.c.) sic ab anctoribus Descripta! "Pracelenti" (Mosa hispanica) similis, sei foliola os serraturas glaniulosa, sepala vix appendiculata."

1342. Crepinia moranica Gogr Gab. N-2105.

Acenlei minutisimi, inclinati, vix Silatati, à ramos flor. plus mimus copiosi; fol. late orato-elliptica, obtusa, basi rotuniata, cressa, coriacex, unsigue glaucescentia, at costam glanioulosa, Dentes biserrati, lanceslatoconvergentes, pat. glandulosissini, aculeate, toto pubescentes; stip. basi angustiores, superne Dilatatae; pes. 2-4, hispini, incrassati, 14-16 m. longi; tubus oborato-sblangus, in 1/3 inf-glanioulosus, sep. gorso hispiza, purnulis latis, 5-7-Ventato-glasioulosis instructa, acumine lato, integros; stagli mag. mi, lanati, dises plans; cor magna, eciliato; fruct magmes, oborato - oblongus, utringue attemuatus.

Hob. Austrix, Morana: It Inaion (Oborny).

1343. Gresinia Gerloti (Grip. Grim. I, p. 53 nomen sol; Deisegl. Cat. p. 183; Cariot \&ture ges fl. 6: 's. II, p.240), Gogr bab. N-2106.

toculei folcato-Decurrentes, at ramos flor copiosi; fol ample oblangres, utringue attenuata, at costarn paree glaniulssa; Dentes biserrati, late apati; pet aculeati, hic inse glantuloso-pilosuli; stip. longose, laeves, auriculis subrectis; pes. 1-3, tense hispisis, 7-11 m. longi; tubus elongatus, sparse setulosus; sep. Dorso graninlosa, pirsmulis pancioribus, angus tis instructa, acu-

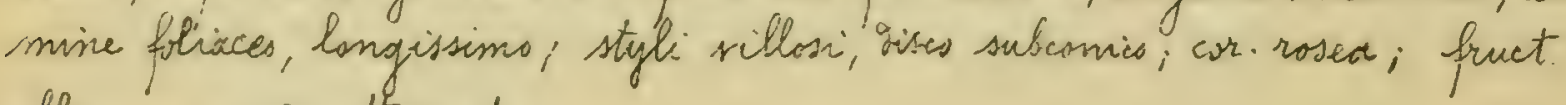
oblongus, aprice attemuatus.

Hab. Fiere, at Le Sappey (\%. 9. Yorlot).

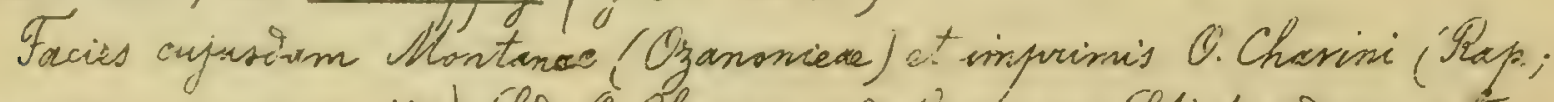

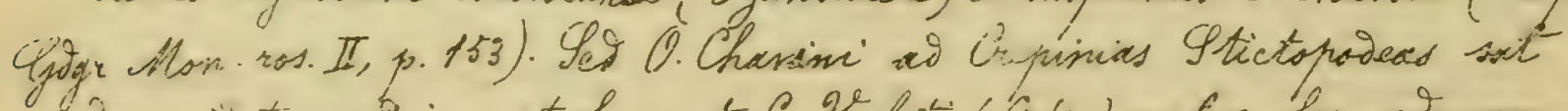

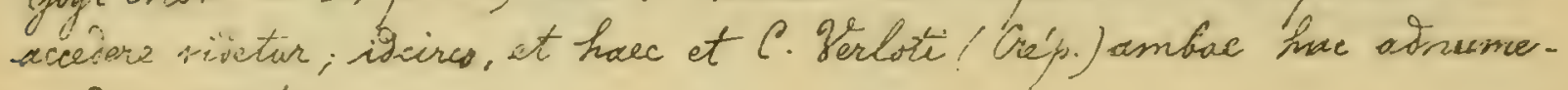
rarive sunt. 
$-79-$

1314. Grepinia Lubonici Gogr mss.

teculei temus, brevissimi, subsilatati, sulde falcati, as ramos flor paucisres; fol mesiocria, sblongo-acuta, basi attemuata, pallive glavea, ad costam parce glanioulosa, gentes biserrati, triangulares, aperti, pet. coppose glaniaulosi, aculeati, in $1 / 2$ inf prubescentes, stip. breves, sat angustae, virites, inf. apice gorsi pubescentes, auriculis givergentibus; pol. 1-2, Dense glanioulosi, $8 \mathrm{~m}$. longi; tubus oblongus, toto glanioulssus; sep. Dorso glanioulosa, pinnulis brevibus, sat angustis, Dentatis instructo, scumine integro, angusto; styli villosi; gusco subcomici cor........ fruct. parrus, oblongus, strinque longe atten. Hab. Granssilvania, Gsolnok Doboka: is Cerrefalu et Sinfalra. (Luovicus Richtor).

1345. Crepinia vicana Goor mss.

trenkei longissini, Silatati, parum falcati, as ramos flor copiass; fol latinscule oblongo-acuta, basi rotunisata, viritia, at costam glanidulose, irentes biserrate, trianqulares, subpatuli; pet rubentes, glanioulosissimi, aculeati, glabri; stis.magnae, longace, laeves, suriculis rectis ; pes.corymbosi, toto hispioi, 1/1/2 cent longi; tubus subblongus, basi infina setosus; sep. Toros gense glanDulosa, pinnulis onnibus foliaceis, valde gentatis instructor; t tygh pulascentes, isisc comico, cor......., fruct oborato - oblongus, basi gepressus, ad apicern longe strangulatus.

Hab. 些, ad Tompus (folbert).

1346. Grepinia recallata Googer mss.

Aculei breves, gilatati, valio falcati, ais ramos flor.copiosi; fol. oblongoacuminata, basi sensim attenuata, coriacea, nervosa, intense viridia, ad costasn nix Glanioulsax; - Dentes biserrati, profunise lanceslato-subeonvergentes, pet aculesti, hinc inde pilosulo-glandulosic; stip. longessimae, parum latac, virides, loeves, aviriculis subsivergentibus; pet-1, tantum apice glantoulosi, 1 cent longi: tubus oblongus, ive sparse glandulosus; sep. Dorso glansouloso, angustius pinnata; styfli villasi, Dises conics; cor......... fruct. oblongus, utrinque attematus, apuie stravigulatus.

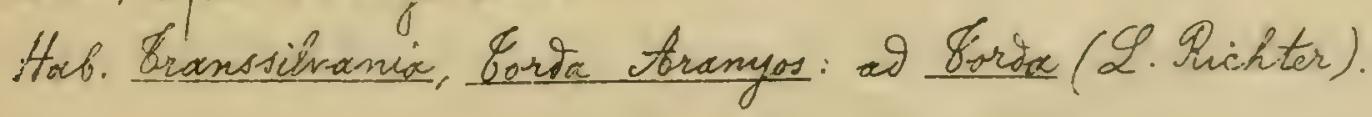

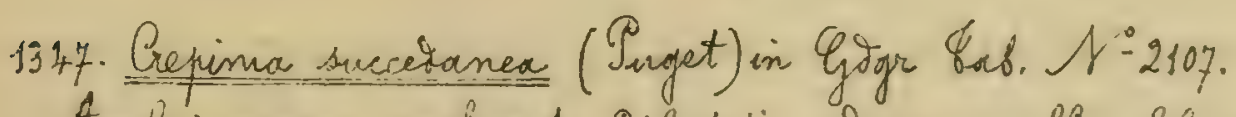

Acculei sparsi, inchirato-dilateti, so ramos flor. folisoss ac purpurios 
$-80-$

plerumgue mulli; fol. ample oblongo-lanceslata, acuminata, fasi breviter attenuata, lacte viridia, as costam eglaniulosa; dentes biserrati, aperte lanceslati; pat inermes, hinc inde piloselo-glanisulosi; stip.longae, laeves, suriculis 2ivergentibus; pet. 1-3, hispiai, 6-11 m. longi; thebus sblongus, toto et Dense aculeolatus, sep. Dorss glanmulosa, pinmulis ongustioribus, subintegris, crpise instructa, acumine spattmlats; styli sillosi, gises sat comics; cor....... i fruct magnus, oblongus, utrinque attenuatus.

Hab. Houte-Savoie, as Singy (Suget).

1348. Crepinia polyneura Gigr mos.

Aculei breves, volide falcato-gecurrentes, as ramos flor. nulli; fol ampliuscule oblonga, abrupte acuminata, basi attenuata, coriacea, prominule nervosa, atrovirentia, subtur albiox, as costam glandulosa; ventes biserrati, late lanceolati, recti; pet. glanaulosi, aculeati, basi prorsus pubescentes; stip. Nirises, latae, sat breves, apice dorsi glanbulosae, auriculis rectis; ped. 2-3, parce hispisuli, $8 \mathrm{~m}$. longi; tubus oblangus, toto setosus; sep. Dorso glaniulosx, pinnulis lativaculis, Dentatis instructa, acumine integro; styli villasi, discó comico; cor........; fruct. sat magnus, oblangus, utrinope attematus, medio turgidus.

Hab. Eransilvamia, Irohnok Doboka: ot Cernefala (2. Richter).

Of foliola at Montanas (Ozanoniea) accedit, sed sepala initis taqusti jorse Vecidux.

2. Aculai gimorphi, alii setaces-glanduliferi, alii setacei.

a. Petioli glabri, vel sparse pilosi.

1349. Crewinia mutata. (Rip.) in Ggogn Gab. N:2108. - C. techarii (Carist

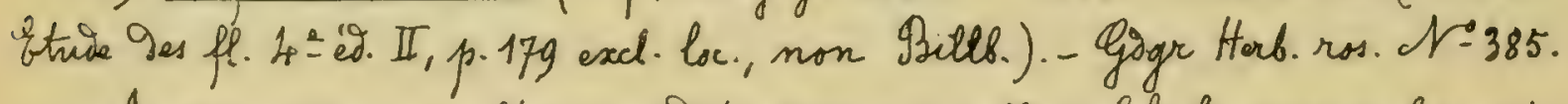
Ab sequente cui multum accedit, imprimis differt folislis majoribus, et stulis dense lanatis.

Hab. Rhane, ad Dardilly (9. Chabert).

1350. Greninio Hailstami (Maker Mep. Lond. 1867. p.7, g: Brit. ros. in Limn sor. Journ. XI, p.234); Cgogr Gab. N-210g.

Aculei co ramos flor. Oimorphi, munc setace-glanouliferi, nunc validi,

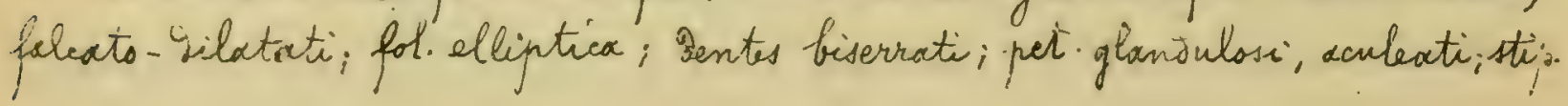


mesiacres; ped. setulosi; tubus onatur; sap. laceria, pinnata, deciouas; styli parce villosi; cor. rosea; fruct. ovatus.

Hab. Anglix, Yorkshire: is Sutton, Hhitstanchiff (Y. G. Baker).

1351. Crepinia limitanea (Crep.) in Cgoogr Bab. N-2110.

toculei gimorphi, ar ramos flor simul falcato-gilatati et glanduliperi; fol - late ovato-elliptica, apice subrotundata, potiolulata et basi breviter truncata, costam glandulosa, gevites biserrati; pet. glandulosi, sparse pilosuli, aculeati, stip. latar, virides; pet. 1-3, hisfisis; 6-12 m. longi; tubus obovatio, lavis; sep. Sorso glandulasa, rubentia, sat anguste purnata; styli villosi; cor-intense rosea; fruct. sborato-oblongus, utringue depressus.

Hab. Haute - Savie, at St Martin pa. trunecy; torgonnex (Suget).

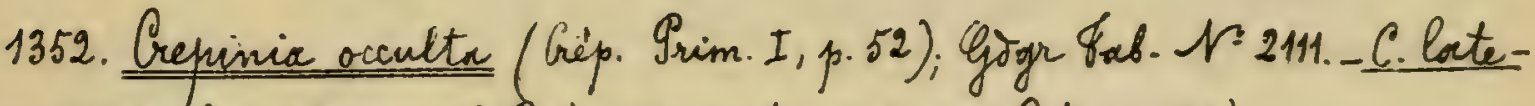

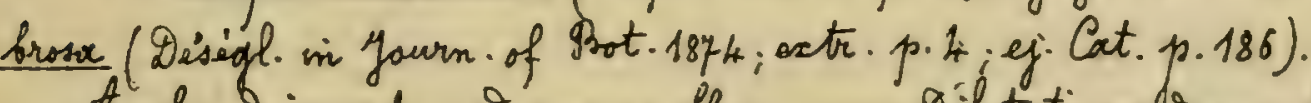

Aculei Simorphi, of ramos flor. nume gilatati valieque falcati, nune satacei; fol. parva, oforato-acuta, fasi attenuata, as costam glaninlosa; rentes biserrati, convergentes; pet-glanaulosi, aculeati, sparse pilosuli; stip.magnae, sup. Dorso glanaulosa, acuriculis subrectis; pes-1-2, hisptiuli, 12-14 m. longi; tubus sborato-oblongus, lavis; sep. Iorso glaniulosix, pinmulis sat lati, valle gentatis copriose instructa, styli lanati, gises subplans; cor....... ; fuct. ovoiseus, utringue attenuatud.

Hreb. Belgium, Simur: at Han-sur-Lesse et Belvawa (F. Créfin) unde descripsi; Gallix contr., Cher et tonglix (sec. De'séglise).

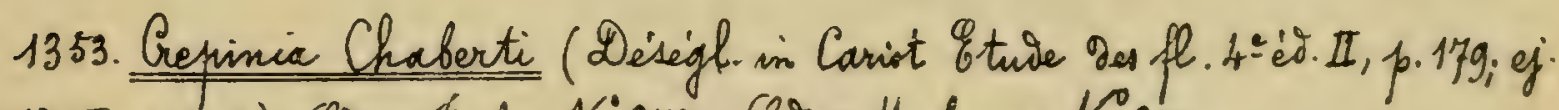
6-:2. II, p.242). Gogr \&ab. $N=2112$. Goyr Herb. ros. $N-391$.

A-culei gimorphi, faleato- Filatati et simul as ramoo flos glaniuliferosetacei; fol. late orata, intens-arentix, costrem glanoulosa; \&intes biserrati, magni; pet. glansulosi, aculeati, glabri; stip. gilatatae, glawas, gorso glandulosar; ped. 2-4, hiopiduli; tubus oblongus, havis, sep. gorso glansulosa, erecta, pisnato-Denticulata; styli magni, lonati; cor magna, roser; fruct ample oblongus

Hab. Rhone, is Dardilly ot Charbonnières (P. Chabert).

Ragix reptass, ut atian occurrit in pluribus speciebus hijus gregili. 
$-82-$

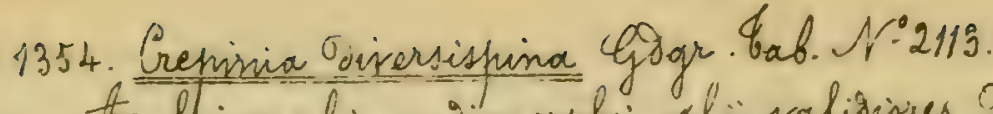

Acculic coulium simsrphi, alii valisiones, Bilatati, recti, alii autem setaces-filiformes, eglangulosi, copiosissirni; fol minutissima, obovata, breviter acuta, asi ro unioata, costam glanoulose; antes biserrati, pro-

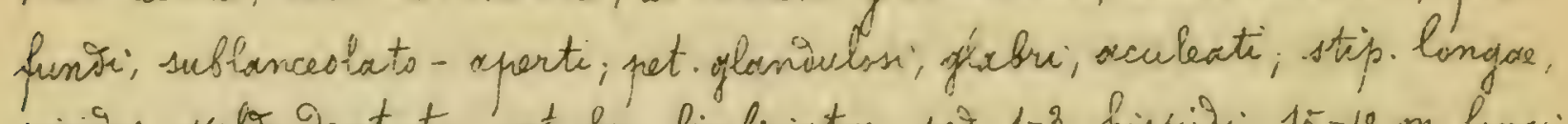

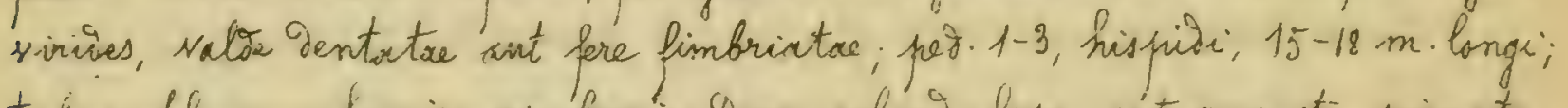
tubus oblonguis, taevis; sap. brevia, odorso eglaminulosa, sat anguste pinnato.

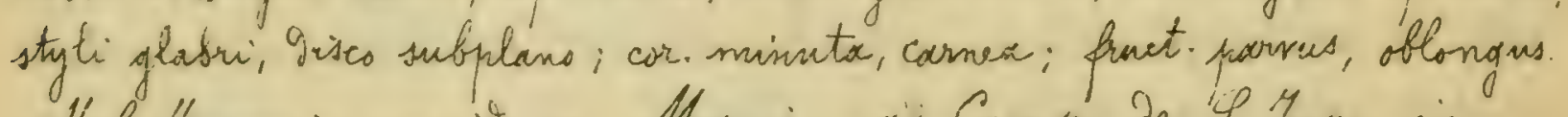

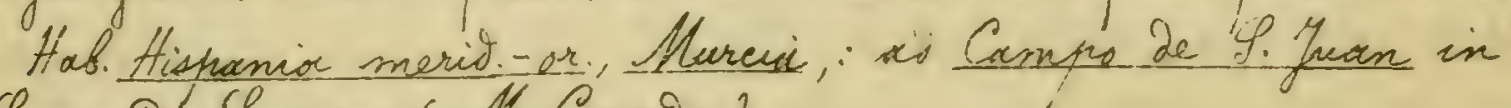
Sierra dé Yegura (N. Cañoia).

Sacies (Monini (Gratt.) cui valie affinis, sed aculei Gimarphi.

1355. Crepinia Dimorphacantha Giog cab. N'211\%.

Aculei valisi ot simul as ramos flor setacei, Somorphi; fol late iso-

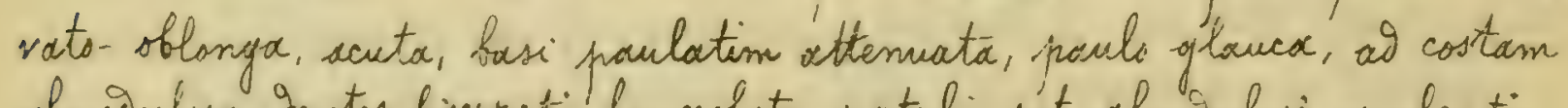
ylanivulosa; gentes biserrati, lanceolato-patuli; pét glavoulosio, aculeati, Thine inve pilosule; stip. Oorso glanoulosse, breves, auriculis subrectis; ped. 2-4, setulasi, 11-14 m. longi; ; tubus oblongus, laevis; sep. 9orso eglaniselosa, finmulis latis, valis gentato-glanialosis instructa ut et accumine; styli villosi, exserti, jisco crnico, cor......., fruct. ample oblongus, apice inflatus, Dein bresiter constructus, a $2 / 3$ sup. usque ad baxsin tonge atiemurto-claractus, subiecurrens.

Hab. Itonglia, as gyolverkamptore (J. Fraser).

b. Petioli toto hirsuti:

1356. Crepinia Gimeroyi (Chabert in Cariot Qtudo ger fl. 4. id. II, p. 180;

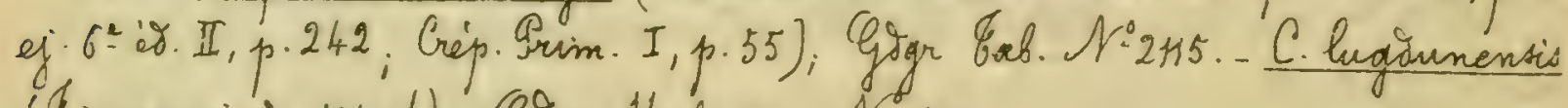
(Tirreroy iner. 1849!).- Gogr Herb. ros. N-179.

taculei imorphi, menc falcato sictatate, salio iores, nunc glanouliferosetaciei; foll late ovata, virioia, at costam glaniuloss; gentes lati, argute biserrati, pet. glanouloss-villosi, aculeatic; stip. gilatatae, Gorso glanoulosae; peos. hispiidi; tubus ovatis, laevis; sep. gorso glandulasa, copiose finnato-gentata, erecta; styli magni, lanati, arisco subconics, cor.magna, lacte rosea; fruct. ample ovatus, turgious.

Hab. Thöne, ad Francheville, Goleymieux, cte. (Oimerry; P.Chebert). 
$-83-$

In herbaris Bimeroy viti have tpeciem sub nomine Masace Luginnensis (Vim.non Désegl.), quamobrem 9. Chabert eam in R2. Gimeroyi mutarit.

$\beta$. Langiana (Keller at Haselberger in Griasb. et Haselb. IRosenfl. Oberoest. p.13). - At typo recesit folishis minoribus et fructu plerumque globosa. Hob. Fohemia, ai tonoorf (Lang er Mrisbour).

1357. Crepinia odontaceras (Gogr Gssai p.2g namen solum; Googr Ros. nov. II. p. 20); Goger 8ab. N:2116.

toculei simonphi, copiosi, alii gilatato-falcati, alii temuires, setacei vel glansuliferi; fol. obovata, utrinque attenuata, fasi ceneata, as costans glangulosa, tentes magni, argute biserrati, falcato-convergentes; pet xculeati, pibescentes, glanaulosi; stip. longae, garso glanaulosac, auriculis rectiusculis, pos.1-4, hispidi; 12-20 m. longi; tubus oroideus, basi infima setulosus; sep. sorso glanoulosa, erecta, sat anguste pirnnato; styli magni, lanati, Diseo subrlano, car. lacte rosea; fruct. oroiteus, apice attenuatus.

Hab. Cher, prope Bowiges in nemore Des Dames (to. Diseglise) et in silvelis circa Lyon.

1358. Crepinia Decumbens Googr \&ab. N'2117. C. insisioss (Mipart in litt. non Gren.).

Humilis, Secumbens, flexuosa; aculer Iimorphi, si ramos flor falcati simulque setaces-glaniouliferi; fol. subrotunisata, firma, subtus glauca, ad costam glanioulosa; rentes argutissime biserrati, pet. glanouloso-pucbescentes, aculeati; stip. glebrae, auriculis inf Divergentitus; pes. 1-3, hispisi; tubus oroidens, laevis, sep. Torso sparse glanoulose, erecta, pinnulis brevioribus, ientatis instructa; styli subglabri; Disco conico; cor-magna, laete roser; fruct. parrus, ovoidens, smepe abortivns.

Hab. Chir, as La Grange - It - Kean (Mipart).

1359. Grepinia gallica x canina (Renter in Gootet Fl. Jur. p.218; ejus in Taull soc. Haller. I, p.182; ej. Cat gener. 2zir. p. 73; Rapin Guite Jaui p. 195; Christ Rosen ger Gchr.p.202); Giogr Bab. No2118. C. opallica x lutsAiana (Christ l.e.).- Billot exs. N-3377.

Humilis, gracilior; culei Simorphi, mune validiores, falcato-2ilatati, nune setaces-glanisuliferi; fol ample orato-rotuniata val suborbiculata, 
$-84-$

at costam glanbuloso: "dentes lati, argute biserrati; pot glanivulose; pubescentes, aculeati; stip.glabrac, angustac, auriculis rectiusculis; pos. hispidi; tubus ovoideus, lacvis; sep. Dorss glanoulasa, copiose gentatopactinata, pinnata, gecioua; stjpli hissuti; cor.magna, lacte rosea; fruct. magmus, ovoidens.

Hab. Helvetia, Genere: as Compsières, Onex, etc.

1360. Gepinia psilophylla (Rau Enum. p.101; Rchb. Fl.exc. N-39g2; Koch Gyn. 2d. 2a p.251; G8imm. Grab. Tl. Files. II, p. 83; Gren.et Godr. Fl.

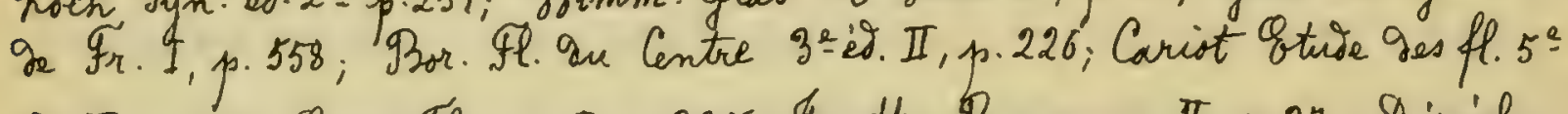
ig. II.p. 188; Gren. Fe jur.I,p.225; Gratt. Tos.mon.II, p.27; Déseigl. Gsai p.79; Grep. Frim. I.p.47; Lange Tug. pl. hisp.p. 342); Goar \&ab. N: 2119; Gogr Contr. fl. slar.II,p.35. - C.camina h. prilophylla (Dumort. Mon. nos. belg. p. 61). - C. gallica $\times$ biserrate (Christ Rosen ger Tehur p. 202). Goggr Herb.nos. N=384 et 405.

Aculei Dimorphi, validiores, folcato-Dilatati simulope ad ramos setaces-glansuliferi; fol orato-rotuniseta, glanca, ampliescula, as costam glaniulosa; gentes argute biserrati; pet.glaniuloso-pubescentes, aculeati; stip. glancescentes, denticulatre; pes. hispivi; tubus ovioiens, setulosus; sep. glanoulosx, pinnato, Decidua; styth lanati, Dises subconico; cor magna, lacte rosea; fruct. magnus, ovoirens.

Hab. Europa plur.

C. psilophylla (Deseg'l. Herb.ros. N:65), ut jam monuit cl. Crepin Trim. I, p-56 habet nervos folislornm subtus minute glandulosos, sigitur nec of Crepinias set ad Chavinias Icabrateas (Glanoulssexe) pertinet.

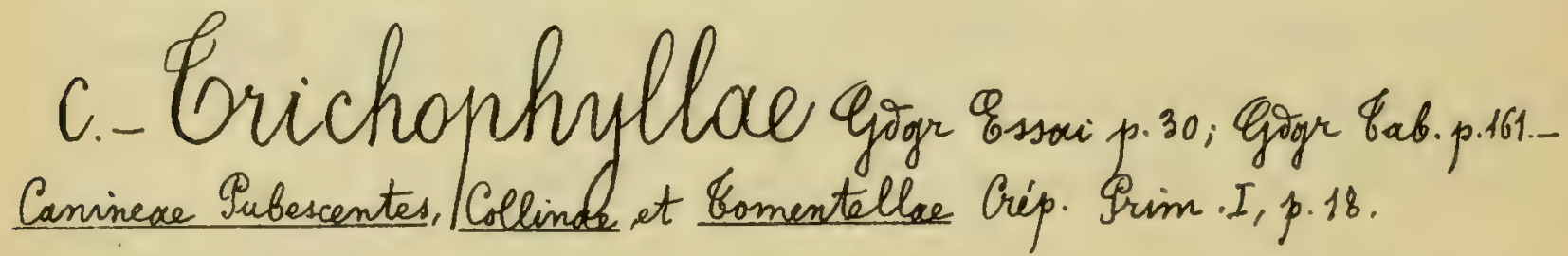

Folisla phus minus (subtus saltem at costam meriam) hirsuta, pillosa sel tomentosa, saepius simpliciter serrata. Sepals post anthesin reflexa, gein caducx (exceptis in quibustam sect. Solstitiales), extus farevis sel glantulosa, fimmatifuartita. Styli libere; ultra Sixcum phes minus capitati. Corolla albo vel recries intense rosex, meiriocris, interdum parva, tunc albo-achrolenca. Fructus pulfosus. Pesunculi gla- 
$-85-$

bri, villose, glanivulosi sel villoso-pubescentes simulopue setulosi, Aculei conformes, e basi late gilatata abunci, valide. Redix hou's reptans.

Pesunculi et sepala eglandulasa, aculer conformes I. Eupubescentes.

a Pesunculi et sepala plus minus glansulose; aculei nonnunquam II. A venophorac.

I. Orupubescentes Ggor Essai p. 30; Gogr Eab.p. 161. Caninear Gubescentes Gép. Gim. I,p.18.

Foliola plus minus villosa, at costam meriam aroinarie aglax-

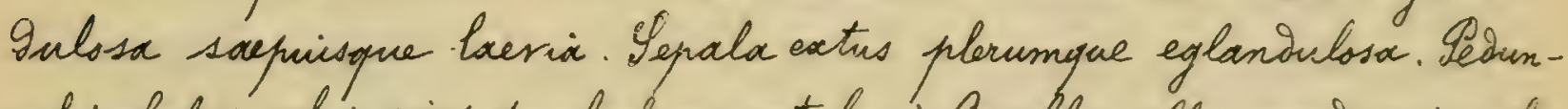
culi glabri val rarius pauluhm setulose. Corolla alba, mediocris, $a l$ pallide rosea. Fructus glaber. Ateulei valise; conformes.

Foliola subtus a costam mediam tantum paulo villosa 1. Decalvatae. Foliola subtus ai costam normaliter villosx, cum pilis raris at nervos secundarios obsucto

2. Semi-glabras.

Foliola subtus à nervos tantum villosa

3. Pilosirsculac.

A Foliola subtus at nerros villosa cume pilis raris hine inse

abluc obsita

4. Unbride.

Foliola subtus toto villosa, supra paulo hirsuta, gin glabra. 5. Pilosae.

Foliola aivulta unioique molliter pubescentix, fere tomentosa G. Solstitiales.

1.- Decalvatae Gigag Essai s. 3o; Gigar bas. p. 162.

Foliolis medies ramorum flariferorum subtus at costixn metiam tantum phusminus sillasis, serraturis esum simplicibus vel compositis.

$1\left\{\begin{array}{l}\text { Foliola ammin biserrata } \\ \text { Foliola elia simpliciter, alia biserrata } \\ \text { Foliola omnino simplicitex serrata }\end{array}\right.$

2

3

5 
$-86-$

Fructus globoses, ovatus vel oflongus

2 Iructus svoidens rel oborato. oblongus Setunauli glabri

3 Sesunculi villosi

$4\left\{\begin{array}{l}\text { Fructur globosus } \\ \text { Fructus svideses } \\ \text { Fructur sflongus }\end{array}\right.$

Pedunculi glabri

$5\{$ Sesunculi villosi

Iructus sroideus val oboreto-sblongus

$6\left\{\begin{array}{l}\text { Fructus oblongus } \\ \text { a }\end{array}\right.$

Fructus globosus, ovatus vel ovato-ellipticus Ityli hirsuti vel lanati

i\{ Glyli glabri

Discus planus vel subplanus

$8\{$ Discus comicus

Ityli glabri vel sparse pilosi

9 Styli hirsuti

Gructus ovatus sel oblongus

$10\{$ Fructus svoideus vel sovato-sblongus
Ypacies $N^{3}-1351-1364$

Qpecies $\mathcal{N}-1365-136 \mathrm{~g}$

4

Species $N-1370-1374$.

Ipacies $N=1375-1379$.

Species $N: 380-1384$.

Speries $N^{0} 1385-1380$

6

10

$\dddot{H}$

)

Inecies $\mathcal{H}^{\prime 1390-1397 .}$

8

Ypecies N-13ag-1401.

Grecies $N: 1402-1405$.

Species N-1406-1411.

Shecies $N^{\circ} 1412-1415$.

Species $N$-1416-1422.

Ppecies $\mathcal{N}=1423-1425$.

Irecies $N-1+20-1433$

1. Foliola mnins biserrata:

a. Fructur globosus, ovatus val oflongus.

1361. Gepinia cosmophylla (Gogr Ros. nor. I, p. 10), Gogr Bab. N:2120.Gogr Herb.ros. N-300.

Aculei rarissime; tenmes, ad ramos flor nulli; fol. amplissime sblongolancelata, scuminato, lucive atrovirentia, is costam paulo glaniuloso-hilose; jentes biserrati, valde profunor; fere incisi; pet aculeati, sparse pilosoglenoulosi; stip. latae, auriculis givergentibus; pes. 3-6, laeves, elongati; tubus tenmis, elongatus; sep. anguste pinnata; sty hi parce hirsuti, giseo salie conico; cot. pallise rosea; fruct. elongatus, utrinque attenuatus Hab. Rhone, ad tornas (Nh. Ganooger).

Ipecies, ramis prossus inermibus, foliolis lucidis, atrovientibus profunsissime serratis, laciniatis sel incisis, ib ommibus Vistinctisisia atque curias-a. 
$-87-$

1362. Crepinia mirioion Gogr mss.

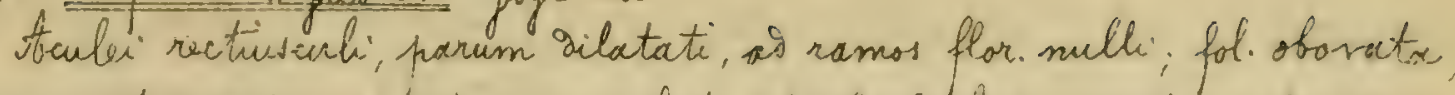

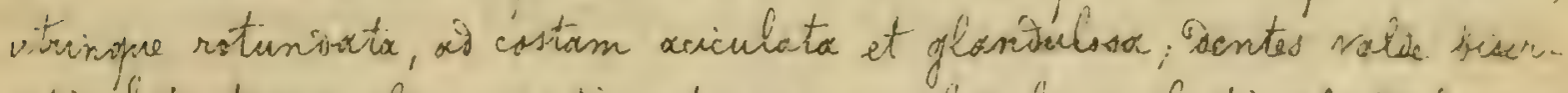

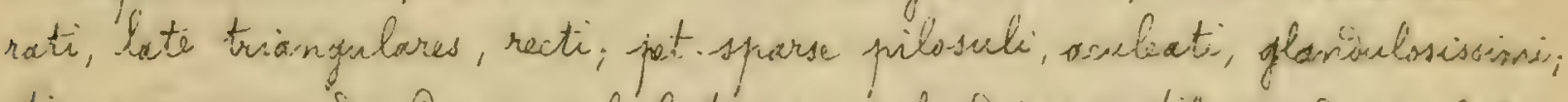

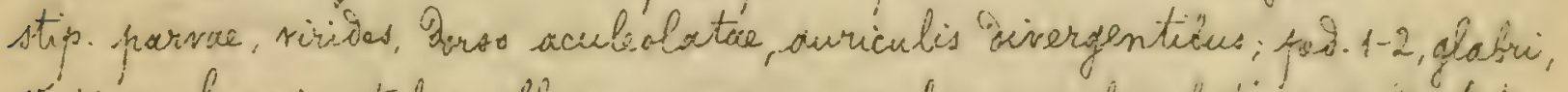
15-15 m. langi; tubus oblongus; sep. pinmulis onnibus latis, 4-6-deptateframoulesis instructa, acumine foliaces, subintegro, stepli puebescentes, is to plano; cor.......; fuit. oilonges, basi gepressus, aprice atternuatus.

Hab. Sicilia orient, at Stumianic (QR. Nicotra).

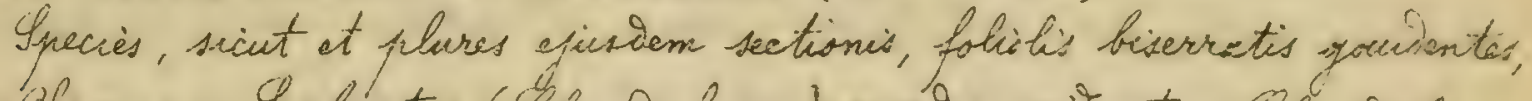

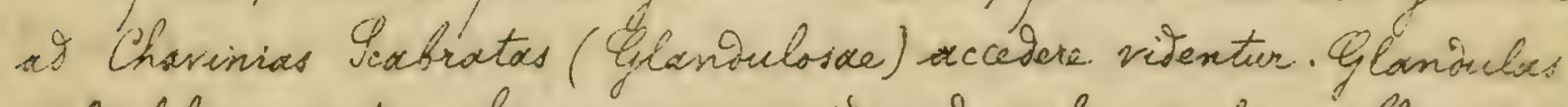

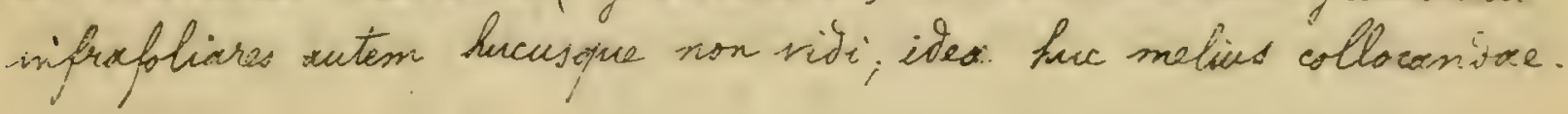

1303. Gepinix oontosticte Gogr Bab. N-2121. - Billot exs. N:3840.

foculei stipulares, falcato-dilatati, as namos flar. pancisres; fol obovato. silongia, utrinque breviter attenuate, is costam rix glansulosam toto villosa; oentes biserrati, sperti, cuspiracti; stip. glabroe, laeves, auriculis rectis; pes.1-2, glabri, $12-14$ m. longi; tubus ovatis, sep......... styli hirsuti. Gises fere plano; cor.........; fruct. parves, ovatus.

Hab. Thone, at dassin; Charbonnières (Boullu).

1364. Crepiania Gennarie (thet ger Pavillon in Genn. Pant. ligust. centr.

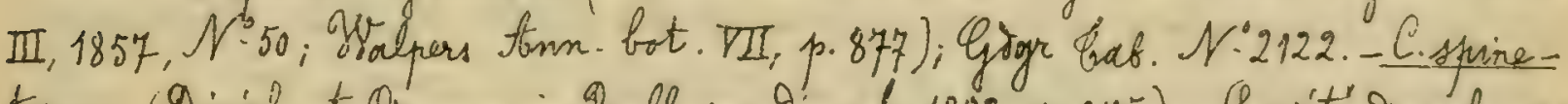

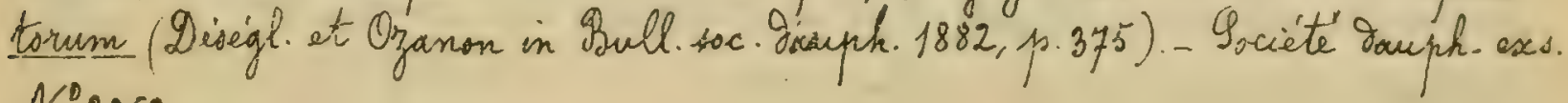
$N^{\circ}-2868$.

Aculei falcato. gilatati, fol ampliusarle oflonga, is costam pilosa; - Ventes biserrati, pet. villosi, parce aculeati; stip. late gilatatae, auriculis allinticis: per giabri; tubus subglobosus; sep. erector, eglaniclosa, pinnulis villosulis instructa; styli villose, exserti; cor sepalis frenior; fruct. globosus. Hab. Liguria arient, in monte torionx drato Nolle (Gennari).

b. Fructus vroideus vel obovato-oflongus.

1365. Crepinia glandulosa Giogr Gab. N-2123.

Aculei falcato-Dilatati, as ramos flor. nulli; fol. oborato-oblonga, attemuata, besi freviter contracta, id costam glanoulose; dentes valde fiserrati, 
$-88-$

triangulares, aperti, pet. glandulosissimi, parce aculeati, temuiter villosi; stis. Catar, ciliatar, auriculis rectis; per. 1-2, ylabri, 10-11 m. longi; tubus oborato- oblongus; sep. pinnulis longis, angustis, valie gentats-glanioulosis instructa, acumine folixes, styli pubescentes, gises paulo conics; cor........; fruct. obovato-oblongus, utrinque valse set basi praceration atten.

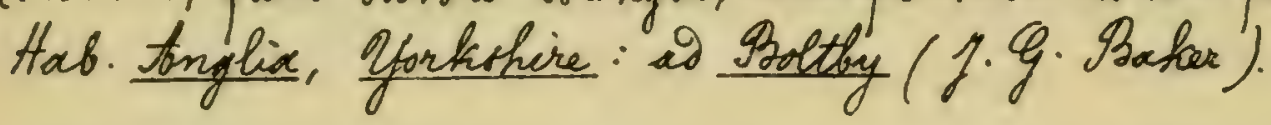

1366. Crepinia Oliveri (Debeaua) in Gogr \&ab. N:2124.

Acculai gebiles, inclinato-dilatati, as ramos flor. melli; fol. parra, sbovato-acuta, basi rotuniata, corcices, as costam pauls glandulosx; Dentes biserrati, triangulares, aperti, pat parce glavidulosi, aculeati, molliter pubescentes; stip. rivicses, wuriculis Givergentibus; pes. 1, glabrc; 8-gm. longi, tubus ovoiseus; sep. pinnulis latiusculis, 1-2-gentatis instructor, acumine lato, integro; styli parce villosi, disco salte conics; cor sub. alba, exiliata; funct. ovoident, utrinque vix Defressus.

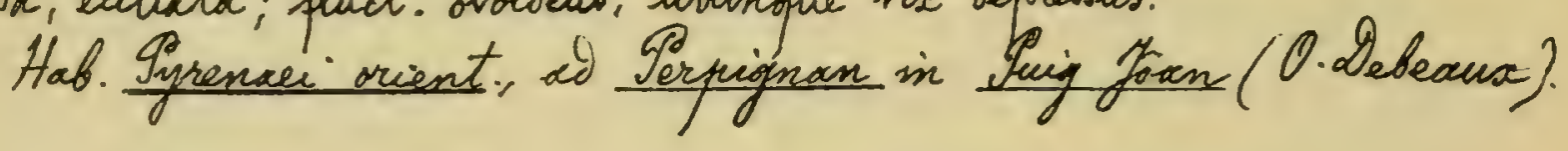

1367. Crefinix kullabargensis Giger mss.

Atculei gilatati, valle faleati, as ramos flor. copiosi; fol. anguste blong-lanceolata, base scuta, at costam glandulosa; dentes salde biservecti, patrilo- triangulares; pet. glandulosi, to to pubescentes, oculeati; stip. magnae, gilute rubentes, auriculis rectis; pe9.1-2, glabri, $14-16 \mathrm{~m}$. longi; tubus svoideus, sep-pinmulis angustis, valde dentats-glandeclosis instructa, scumine integro; styli pubbesentes, sises conico; car......; fruct. svoideus, utringue breviter attenuatus.

Hab. Suecia, Icania : in monte Kullaberg (\%. At. Gabrielsson).

1368. Crepinia stylosa Gogr Gab. $N=2125$.

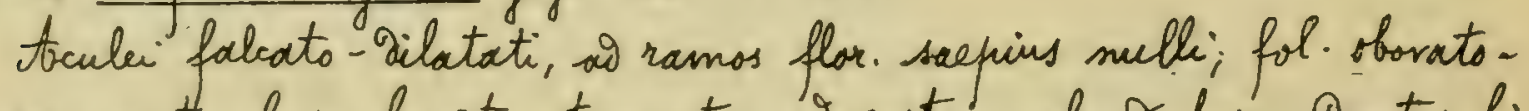
oblonga, acuta, basi breviter truncata, os costam glanoulasa; Dentes biserrati, lanceslato-aporti; pet. glandulosi, aculeate, parce pubescentes; stip. latoe, auriculis sublivergentibus; ped. 1-2, glabri, 4-6 m bngi; tutus ovoideus, sep. pinnulis longis, parum latis, eglanaulosis instructa; zueumine longs, latiusculo; styli glabri, exserti, discs conico; cor......... fruet. parrus, avoisens, basi subiepressus, apice valte attenuato-strangulatus. Hab. tonglia, Yorkshire: ad Shirsk ( $\% . G$. Gaker). 
$-89-$

1369. Creninia conifera Gogr bab. N:2126.

foculei debiles, subrecti, as rarnos flor. fere nulli; fol amphiesicule ob. longo-lanceolata, hase subtruncata, as costam glaniuclosa; dentes valie biserrati, lancelato-aperti; pet-glanidulose, sculeati, sparse pilosuli; stip. rubentes, auricilis Aivergentibus; pet. 1-2, ghabri; 7-8m. lmngi; tubus ovoireus; sep. pinnulis latiusculis, 1-Dentatis instructa; acumine integres; styli glabri, asises longe conics; cor........; fruct. svoides-conicus, basi rotunsatus, aprice longe attenuatus.

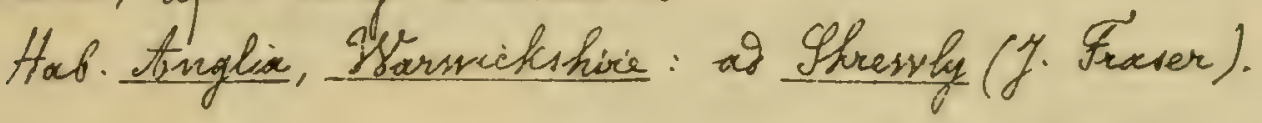

2. Foliola alia simpliciter, alia biserrata. *. Tesunculi villosi.

1370. Crehimia sclerovonta Gije bab. N-2127.

toculi valisi, faleato-dilatati, wo ramos flor. minores; for. sat parra, - ovato-subattenusta, basi rstundata, coriacea, as costarm interdum acuFeslata; Dentes inaequales, breves, Iuri, magni; pet eglaniulosi, parce acuiexti, toto laxe pubescentes; stip mediscres; ped. sacpe corymbosi, pubescentes, 4-g m. longi; tubus obsrato-oblongus; sep. pirnulis pacrum lati, rentatis instructa, acumine foliaces; styli vix as sperse pilosuli; gisco comics; $\operatorname{cor} . . . . . .$. ; fruct sforato - sflangus, aprie valde attenuatus.

Hab. Lavoie, as Salins (Suget).

1371. Benimia gecalvata (Geip) in Gogr Eab. Y'2128.

foculer ralivi, gilatato-rectiusculi; od ramos flor. pauciores; fol. ample oblongo-acuminata, basi attenuata, as costam eglanaulosa; Sentes incequales, aperti, cuspitati, pet. sglandulosi, aculeati, villosi; stip. Irsso sparse pubesentes, auriculis givergentibus; pot.1-3, pilosuli, p-11 m.longi; tubus oblangus; sep. pinmulis parvis, brevbus, filiformitus instructor; styli oparse pilose; cor. pallive rosea; fruct. oblongus, apuce attenuatus. Hab. Balguim, Namur: a Rochefort, Eprare (D. Ciefuin).

1372. Crepinia novella Gogr mss.

tbculei gebiles, inchinato-subsilatate, brevis, as ramos flor. geminati; fol. ample sborato - subacuta, basi rotuntacta, ad costam eglaniulosa; gontes fere onnes simplices, profunoi, ovato-subeonvergentes; pet. nubentes, villosi, 
- 90-

eglanaulosi, inf.inermes; stip.mognace, gilute rubentes, latac, auriculis sat givergentibus; ped. 2-3, pubescentes, $8 \mathrm{~m}$. longi; tubus sioiens; sep. primulis copiosis, smmitur foliaceis, 2-4-Dentatis instructa; styli hirsuti, gises conico; cor-eceliata; fruct. orsicieus, utrinque attenuatus.

Hab. Gar, ao Lollies-Eoncas (Albert).

1373. Crepinia trichopus (Gogr Essai p. 30 nomen solum; Gogr Ros. nor.II, p.21); Gjogr Eab. N-212g.

Aculei Debiles, rectiusculi, geminati, ris ramos flor. copiosi; fol. late oborato - attemuata, fasi pauls contracta, as basin costoe eglanivelosace paulo hirtella; Dentes fere onnes simplices, recti, pos villosissimi, eglanSulosi, aculeati; stip.longac, Dorso pubescentes, ouviculis ivivergentibus; pey. 1-2, villosissimi, 7-10 m. longi; tubus oblongus, basi pubessens; sep. Tue persistentic, pinnatoc, oglanioulosa, acumine lato; styli villosi; Sise plano; $\operatorname{cor} . . . . . .$. , fruct -maximus, oblanqus, apice valde strangulatus. Hab. Savoie, at Salins prope Noütiers ( Tuget).

137\%. Geninia fuccina Gogr mss.

Aculei breves, tenuiter falcati, Decurrentes, al ramos flor. Stipulares; fol amplissime obovato-subacuta, basi attenuata, mecis illatata, atrovirentia, as basin tanturn costac pauls pilssae eglanioulosa; Pentes profunia lanceolato-convergentes, fere onnes biserratic; pot. vix glanioulosi, acculeati, supra tantum usque ad $1 / 2$ inf puchescentes; stip. virisiss, longace, angustar, avriculis sat givergentibus; pes. 1-2, villosi, crassi, 1/2 cent. longi; tubus oblongus; sep. pinnulis armibus folixceis, brevibus, 2-4-gentato-glanoulosis instructa, styli lanati, Diseo comics, cor....... fruct-magmus, breviter oblanqus, a $1 / 3$ sup. inflato usque ad basin attemuatus.

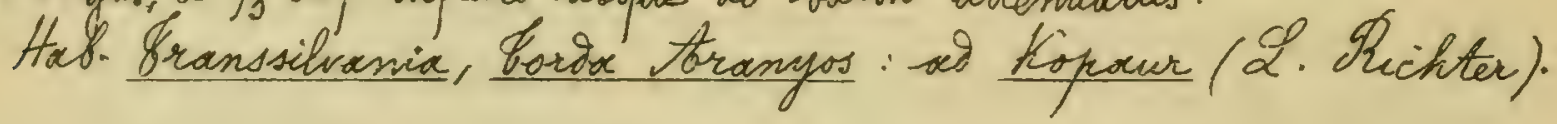

b. Peannculi glabri.

f Fructus glatosus, noturivatus vel ovatur.

1375. Creninia coroeta Gogr Fab. N:2130.

toculie rarissini, gebiles, inclinato-Dilatati, ai ramos flor. mulli; fol. Alliptico-attenuata, basi late coriata, as costam glanoulssam et basi subhirtella, folislo medis 1-3-aculeolato; pat. eglandulosi, aculeati, fasi 
$-91-$

unifariam pilosuli; stip. parvae, glabrae, auriculis givergentibus; ped.1-3, glabri; 7-14 m. langi; tubus ovatus; sep. pinnulis latiusculis, eglanaulosis sat copisse instructo; styli parvi, plarumque glabri, Disco plano; cor.pallise rosed; fruct minimus, subglobosus.

Hab. Hautes-Syrénées, at Sroumouse (H. Isordère).

1376. Crepinia sparse Gogr bab. N-2131.

toculei pouciores, subsilatati, minute et longe falcati, Dacurrentes, ad ramos flor mulli; fol ovets-elliptica, vix attemuato, basi corvata, ad costion glanitulosa; sentes late subconvergentes, fere omnes simphices; pet. lase pilosic, inermes, saepe onnes eglaniulosi; costa mesia basi pilasella, coeterum glanisuloso-aculeolata; stip. mesiscres; peo-2-3, glabri; 8-12 m longis tuhus ovatus; sep. primulis lativisculis, subsentatis instructa, acumine subfoliaceo-genticulato; styhi oflabri; Gises sat comis; cor-alba; fr.subglobssus. Hab. Hautes- Mejrénées: ad Monné prope Gidre (H. Bordère).

1377. Crepinia trichooonta Giogr Gab. N2132.

Focculei panciores, parri, inchinati, a ramos flor mulli; fll oblongoacuta, basi atternata, al costam eglanioulosa; ventes inaeguacles, lanceolatoaperti, ciliati; pet paulo glanoulosi, sculeati, pubescentes; stip. sivises, ouriculis sivergentibus; pet. 1-3, glabri, 8-g m. longj; tubus ovorto-roturiatus; sep. pinmulis angustis, integris vel 1-Dentatis instructa, acumine integro,

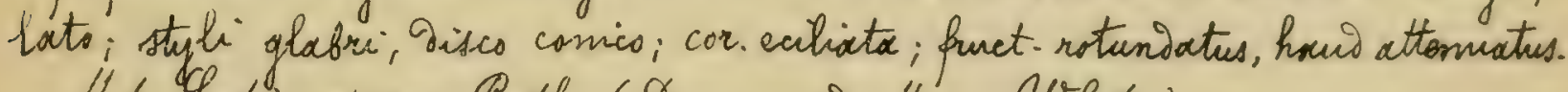
Hab. Cootia, prope Perth (Drummont-Hay; HShite).

1378. Crepinia latillora Gogr Gob. N-2133.

toculi: gebiles, falcato subsilatacti, al ramos flor. Seflexos plus minus copiosi; fol-parva, sbovata, utrinque sensim attenuata, at costam pilosula, Dentes pere onnes biserrati, pet. villosi, inermes, inferne paulo glanuulosi; costa media eglanisulosa; stip. rubentes; pes glabri, 3-5 m. longi; tubus ovotus; sep. rubescentio, late pinnacto-dentata, acumine subfoliaces; styli basi pubescentes, superne glabri, dises sat comico; cor magna, rosea, inferne subiliata; fruct. rotuniatus, basi attemuatus.

Hab. Rhane: ad Trancheville in Iont-2'Atlaiu (P. Chabert). 
$-92-$

1379. Crepinia agmina Oogr mos.

Fcculei breves, parvi, inclinato-Silatati, as ramos flor. pauciores; fol. amphinscule oblongo-subacuta, basi truncata, is basin costac proulo aculeslata; Dentes fore annes biserrati, aperto- triangulares; pet. sat glanoulosi; aculeati, tots pubescentes; stip. sirises, freves, angustor, auriculis sat givergentifus; ped. 1-2, glabri, 12 m.longi; tulus svatus; sep. pinnulis latiusculis, subintegris instructa, styli villosi, Disco comiso; cor........; fruct. me-siocris, rotun'sato-conicus, basi gilatatus, apie abrupte strangulatus.

Hob. Iransilvamia, Kolozs: ad Nogyar í Kerake (2. Michter).

†† Fructus orsidens.

1380. Crepinia villinaera Giagr Gab. $\mathcal{N}^{\circ} 2134$.

Acculei falcato-dilatati, so ramos Har. sat copiosi; fol. ample obovatoshlonge, atternata, basi truncata, lucise atrovichentia; costa mesia in $1 / 2$ inf. hivitella, inferne 1-2-aculeolatta, Dentes valio profunos; lanceslato-aperte, inceopuales; pet. aculeati, toto villosi, inferne glanoulosi; stip. virises, latoce, sorso pubesientes, auriculis givergentilus; pes- 1-3, glabri; $9-11 \mathrm{~m}$. Longi; tubus oborato-oblongus; sep-elorgats, pinmulis villosis, latiusculis, 1-3-Dentatsflavioulssis instructa; styli parce villosi, Disco comico, cor mediveris, alba, eciliata; fruct ovisens.

Hab. Tyrenaci centr. Gèìre in vism Héas (H. Morière).

1381. Crepinia uneata Gigr mss.

Acculei robusti, Securrentes, valto faleati, as ramos flor copississimi, fol. ampluiscule suboblongs-acute, basi rotunisato, lacte virisia, tantum as busin costare eglanioulosece pilsula; Dentes ovato-recti, fere omnes biserrati; pet. toto pubescentes, paulo glanoulosi, sup. inermes, stip. rubentes, longissimase, latoe, suriculis giviergentitus; pes.1-2, glabri, 1/2 cent. longi; tubus soritus, sep. pinmulis latiusculs, subintegris instructa; styli sublanati, isisco comico; cor........, fruct. meiviocris, oroivens, basi gepressus, afuice attenuatus, laterales autem saere comici; basi rotuniati.

Hab. Heongaria, Gzepes: as Locse (2. Michter).

1382. Bapinia pleioion Gopr mss.

Feculei panciores, inchinato-Qilatati, eid ramos flot. nulli; fol. sat 
$-93-$

parra, oborato-acuta, basi truncata, as costam eglanaulosa, ientes aperto triangulares, fere onnes biserrati; pet paulo glansulosi, inermes toto temiter pilosi; stip. virieses, auriculis rectis; pes.1, glabri, 6-8 m. longi; tubus ovoidens; sep. pinmulis onnibus integris, ingustis instructa; styli lanati, gisco plano; $\operatorname{cor} . . . . . . ;$ fruct. ovoidens, basi truncatus, apice longe strangulatus. Hab. Surcix, Imalond: a Dref (Hyltin-Caralliue).

1383. Creminix vindicans Gjgr mss.

Tarler meviocres, Dilatati, temiter falcati, à ramos flor. pasciores; fol. misioeria, oblongo-acuta, basi attenuata, subglaucescentia, costa meiva eglanoulosa, tantum inferne pilosuli; gentes fere smnes biserrati, patulo-trisngulares; pet. hinc inde glandulosi, aculeati, Dense pubescentes; stip. virides, angustar, longiuscular, auriculis subsivergentibus; pes. 1-2, glabri; $8 \mathrm{~m}$. longi; tubus ovisiteus; sep. pinnulis subintegris, angustis instructix; styli villosi;

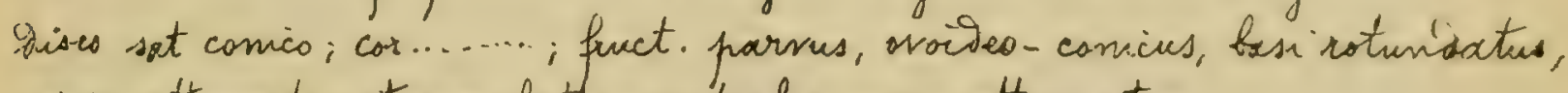
spice attenuato-strangulatus, centralis vero attenuatus.

Hob. Hungearia for" Lipti: ad Likanka (L. Richter).

1384. Grepinia sublaeris Gogr Gab. N-2135.

Atculei minutissini, falcati, vix Silatati, Decurrentes, gè ramos flor. sais pe mulli; fol-oborato-oblonga, acuta, basi subrotindata; costa medix toto villosa, glanoulosa; sentes inaequales, profuna lanceolnti; pet glansulosi, villosi, aculeati; stip. breves, sat angustoc, gorso pubescentes, auriculis -suhrectis; per. 1-2, glabri, 11-13 m.longi; tubus svoideus; sep. purnulis sat angustis instructa; stygli mune glabri, nume asperse pilosuli, disco conica, cor-rosea; fruct. parves, obovato-oblengus, 2-10 m. Siane. latis.

Hab. Rirenaci centr, ed Gidre in nam Lus (H. Marbère).

B. macrocarpa Gogr Gab. N-2136. - At typo aiffert costa mesia foliolor. tantum basi villosa, fructu majore, $14-15 \mathrm{~m}$. giam lats.

Hab. cum type.

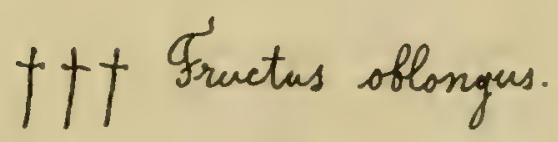

1385. Grepinia albogilva Goge toab. N-2137.

Acula masimi, falcato-Dilatati, decurrentes, longi, at ramos flor. copiosi, fol-oborota, attemuata, basi rotuniata, viriti-cinerea nó costam 
$-94-$

hirtellam glasioulosa; Dentes subconvergentes, fere omnes fiserrati; jet. glanioulosissimi, aculeate, nunc glabri, nunc lase vel pparse nubescentes; stip.elongatae, longae, inf. Dorso glansulosace, auriculis givergentibus; peovitt, oflabri, $11-16$ m longi; tubus oblangus; sep. pinmulis brevibus, latiusculis sentato - glanoulosis copisse instructa; styli hirsuti, giscs set comico; cor. rosec; pruct. oblongus, utrinque depressus.

Hab. Haute- Lavoie, ad Tringy (Muget).

1386. Geninia hoganata Googr bab. N.2138.

A-culei panciores, parum vilatati, Decurrentes, temiter valsesnce folcati, as ramos flor.mulli; fol ovato-elliptica, subattemuato aut fere ad apicems rotuniata, basi coriouta; costa mesia basi subhirtella et insuper glanduloso-aculeolato; Tentes lati, subconvergentes, fere onnes simphies; pet. laxe villosi, vix glanoulosi, parum aculectic; stip. anqustice, auricilis patulis: peo graciles, glabri, $17-28 \mathrm{~m}$. longi; tubus solongus; sep. latiuscule pinnator, acumine subfoliaces, gentato; styli glabri; gisco sat comico; crr. alba; fruct. oblongus, aproe valio attenuatics.

Has. Fyrenaci, as forran (H. Tardère).

1387. Crepini inacoueserrata, Giogr Fab. N'2139.

trenlei folcato_Oilatati, aid ramos flor sxepins geminatic foll oblongolanceolata, basi attenuats, as costam eglaniulosa; dentes lanceolati, profungi, inaequales; pet. aculeati, eglanivilosi, qubescentes; stip. langare, an gustare, ouriculis rectis; poo-1-2, glabri; 7-8-m. longi; tubus elongatus;

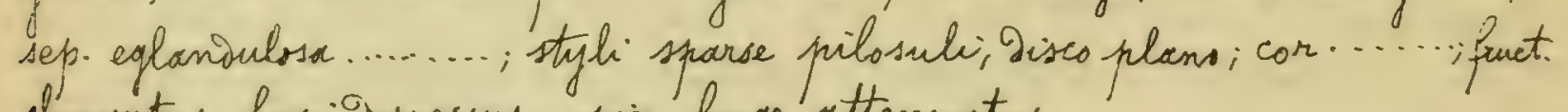
elongatus, basi repressus, aprice longe attemuatus.

Heb. Saxania, as Ettersberg prope Gseimar (C. Hausstnecht).

1388. Crepinia efferta Gigor Gab. N-2140.

focilei breves, temuiter et volive falcati, ad ramos flor-copiosi; fol. late obovato-subacuta, basi sensin attemuata, ad costam eglanidulosa identes late triangulares, aperti, inaequales; pet. laxe et toto pubescentes, glangulosi, aculeati; stip. virides, auriculis give tentibus; pod. 2-3, glabri, $12 \mathrm{~m}$. longi; tubus subblongus; sep. pinmulis latiusculis, 1-2-Dentatis instructa, aumine integrs, styli lanati, Disco plans; cor........ i fruct. Suboblonqus, Easi gepressus, apure abrupte strangulatus.

Hab. Angtia, Cheshire: at Horetan (F. Horbori-Lewis). 
$-95-$

1389. Creprina navarrensis Gögr Gah. $\mathcal{N}^{\circ} 2141$.

Feculei à ramos flor. forcato-dilatati ifol. obovato-acceta, basi attemua-

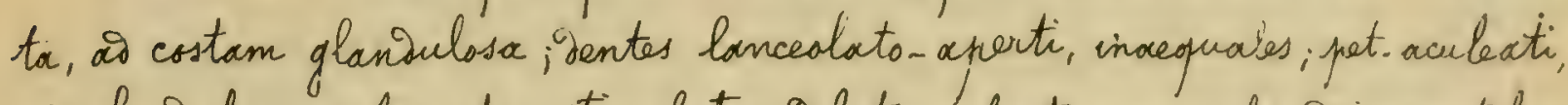
siz glanoulosi, pubescentes; stip. latre, gilute rubentes, -avriculis sivergentibus; pit-1-2, glabri, $14-15 \mathrm{~m}$. longi; tubus oflangus; sep. pinnulis angu-stis, 1-2-ventatis instructa, acumine angusto, integro; styli glabri, Yisco sat conics; cor........ fuct. oblongus, basi vepressus, apuse constrictus.

Hab. Hispamia bor. Navarra: at Larrion (F. Muiz-Casavieilla).

3. Folisla ommins simplicitex serrata.

a. Pesunculi glabri.

f Fructus globosus, rentundatus, ovarto-rot, ovatus vel ellipticus.

13g0. Crepinia falcilera Goger 8ab. N-2142.

Acculei valie falcati, parum silatati, ad ramos flor. saepuis mulli; fol.

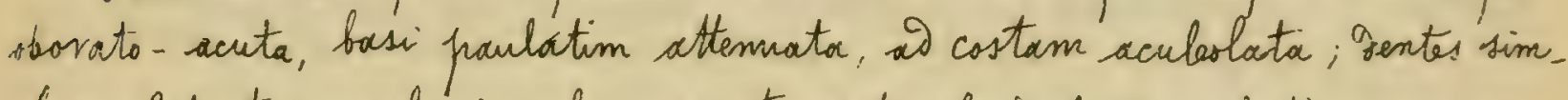
plices, late triangulari-subconvergentes, pet eglanisulosi; aculeati, parce pubesecentes; stip. virides, auriculis givergentisus; ped. 2-4, glabri; $11-13 \mathrm{~m}$. longic; tubus ovoidens; sep. puinmulis latiusculis, 2-3-Dentatis instruecta, accumine integro; styli glabiu; gisco poulo comiso; cor eciliata; fruct ovato-ellipticus, basi subrotungatus, apice attennatus.

Hab. Tyrenaci centr, ad Gèdre (H. Borière).

1391. Crefunia acora Gigor Gob. N:2143.

Foculei sparsi, Debiles, falcato-gilatati, as ramos flor interaum mulli; fol. parva, elliptica, acuta, basi coriata, lacte viridic, as costam eglanderlosam basi subhirtella et 1-3-aculeolata; Dentes simphies, rigidi, aperti; pet glanidulosi, aculeati, basi unifariam hirtelli; stip. breves, glabrac, awriculis givergentibus; pes. 1-3, glabri, g-13 m. longi; tufus ovatus; sap. pinmulis langis, latiusculis, eglinsulosis instructa, styli parri, glabri, Dises subplano; cor........ ; fuct. parruy, ovato-notundatus.

Hab. Pyrenari centr. a Lourdes (H. Bordère).

1392. Crapinia vascula Gogr mss.

Acculei rari, Debiles, parum falcato - Dilatati, oi ramos flor. mulli; fol amplissine oblongo-acuminata, fasi rotuniata, coriacea, lacte viriatia, 
$-96-$

as costam eglanoulosa; dentes simplices, breves, ovato-patreli; pot. ciglaninelosi, tomentelli, inf. inermes; stip. rivives, latac, lonainsculse, revisculis sat givergentibus; fas. 1-3, glabri, $12 \mathrm{~m}$. longi; tubus ovatus; ses. pinnulis latis, 1-2-2entatis instructa; styil subghaberrimi, Disco comics; cor........ fruct-maximus, subrotunarto-comins, basi gilatatis, apice attenuatus.

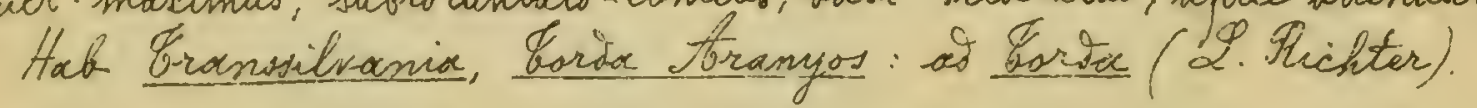

1393. Crepinia magnolivefolia Gogr tab. $N^{-2} 144$.

A-culei falcato-Dilatat, od ramos flor mulli; fol starilia maxima (4 cent. lata, 7-71/2 cent. longa), floralia autem late obovata, utimque subrotungata, ad costam oglarialosa, dentes simplices, frofunisi, convergentes; pet-eglanoulosi; pubescentes, aculeati; stip. sirises; pes. 1, glabri; $7-g$ m. langi, tubus svatus; sep. pinnulis latis instructa, acumine foliacer, stygli pubescentes, dises plans; cor........; fruct-globosus.

Hab Isere, is Recoin, in riam Saladru (No. Ganogyer).

1394. Cresinix affebilis Gogr mss.

Acculi minuti, cylindrici, suboilatati, tenuissime subincurvi; to ramos flor.copiosi; fol. ample oflongs-sublanceslata, basi attenuata, coviacea, glaucescentia, tantum as basin costace paulo glandulssoce pilosula; dentes simplices, triangulares, aperti, pet eglanaulosi, aculeati, toto breviter tomentelli; stip. rubescentes, maximac, auriculis divergentibus; per. 1-2, glabri, 3-4 m.longi, tubus ovato-rotundatus, sep pinnulis angustis, brevibus, 2-3-Dextates instructa; styli lanati, Disco plans; cor......... frect. minimus, subglobosus, basi rotuniatus, aprice sulattenuatus.

Hab. Hungaria for. Izepes: at Löse (2. Richter).

1395. Qresinia lasula Gigr Eab. N'2145.

Acculei valibi, folcato-gilatati, as ramos flor copiosi; fol ample oborato-oflonga, rhamboea, acuta, bosi attenuata, as costam to to villosam eglanaulosa; Tentes simplices, acuti, pot-eglanisulosi, basi pubescentes, aculeati; stip.magnie, auriculis aivergentibus; ped.1-3, glabri, 9-13m.longi; tubus oratus; sap. prinnulis paucioribus, sat angustis instrueto; stygli villosi, gisco comico; con........ fruct. rotundatus.

Hab. Houte- Savoie, at Habire-Lullion (Muget). 
1396" Crepinia Dolenida Gogr mos.

Aculei valivi, inchinato-Silatati, as ramss flor. glaucos, geninati; fol-mediocria, obsvato-oblonga, utrinque prabition atternata, glauca, nervosa; costa media cylanioulosa, tantum basi pilosula, sentes simplices, ovato-convergentes pet. aculeati, vix glanaulosi; toto pubescentes; stip. maximae, sirides, aurriculis sat sivergentibus; pet. 1, glabre; 2-4 m. longi; tubus subglsbosus; sep.pinmulis subintegris, latis instructa; styli lanati, Disco subcomico; cor....... fruct. mediocris, sulgloboso-comicus, basi dilatato-umbilicatus, anice via jepressus.

Hab. Hungaria bor, Gyapes: ad 2icse (2. Thichtor).

1397. Crepinia lociensis Googr mss.

Abeulei valiai, gilatato - subrecti, as ramos flor. saepe geminati; fol. oblonga, apice subroturiata, basi sension attenuata, lacte virisia, aid costam eglanoulosa; Ventes simplices, henceolato-convergentes; pat rubri, inormes, oglanisulosi, breviter pucesentes; stip. latar, longre, rubentes, auriculis subrectis; pos. sape 2, glabri, 2-4 m. longi; tubus arato-rotundactus, sep. innulis subintegris, latis instructa; styli lanati, Discs subplano; cor........, frect. meniocris, ovato-subglofosus, comins, basi umbilicatus, apice subsepressus.

Hach. Hungaria bor., Sapes: ad Licse (2. Richter).

\section{T† Fructus orovieus val obovato-oblongus.}

\$. Styli glabri.

1398. Grepinia Gibellii Gogr 8ab. N:2146.

trculei ralidi, falcato - gilatato, id namos flor nulli; fol ample oborato-acuta, basi rotuniata, a costam eglanaulosa; Dentes triangulares, a prerti, simplices; pet. colanimlasi, inermes, villosic; stip. rubestes, latae, auriculis givergentibus; por. 2-4, glabri, 13-15 m. longi; tubus svodeus; 1ep. pinnuhis foliaceis, 2-3-laciniatis instructe, et et acumine; styli glabri, iseo sat conico, cor-major, rosea, eciliata; fruct: maximue, ovrideus, basi esturioates, apice sension attenuatus.

Has. Mabia cente, ad Molene (G: Gibelli?

1399. Geninix pallitifolia Gigr mss.

Eota rusens, aculei minuti, inchinato-dilatati, as ramos flor. sipue- 
$-98-$

lares: fol parri, anguste pblongs-acuta, basi attenuata, corracea, pallida, is costam eglanoulosa, Tentes simplices, rubentes, lanceolato-recti; pet. eglaxoulssi, toto longe pilosi, parce aculeati, stip. rubentes, sat angustae, me.Diocres, auriculis suboivergentibus; pot. 1. of abri, $7 \mathrm{~m}$.longi; tubus suboblongus, sep. pirnulis mesiocribus, subintegris instructo; styhi glabui, givo subcomico; car........; fruct. oforato-obiongus, basi gepressus, aprice attenuatus.

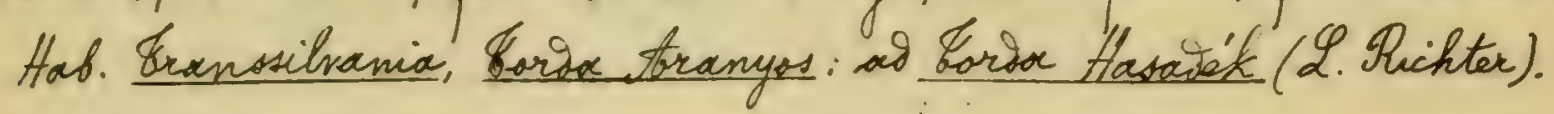

1400. Cresinia musescens Gogr Eat. N-2147.

tculer gabiles, falcato-gilatati, à ramos flor. sparsi; fol. oblongoattemuata, basi freviter contracta, pallide viridi-glaucescentia, estam glaniulose; Jentes magni; simplices, lanceolats - recti; pet. laxe villosi, aculeati, eglanimlosi; stip. sirises; pes. 1-2, globri, 10-12 m. longi; tubus oborato-sotongus; sep. punnulis anqustis, sat copiosis instructo; styli glabre; sises subplano; cor.........; fruct. parrus, ovoileus, attenuatus, as apriem pauls strangulatus.

Has. Hsire, a Charancieu (M. Ganooger).

1401. Genimia tequareatios Gogr 8ab. $N-2148$.

Freulei paucisres, mimuti, falcati, id ramos flor. tortussos nulli, fol. fol. parra, oborrato-acuta, basi attemuata, ad costam eglangulosa; Dentes simplices, lanceolato-aperti; pet. paulo glanisulosi, acculeati, longe pubescentes; stip. rubentes, auriculis givergentibus; pes. 1-2, glabri, 5-6 m.longi; tubus obovato - oblongus; sep. pinnulis onnibus integerrimis, parum latis instructa; styli glabri, give pauls conics; cor eciliata; fruct. suboblongus, basi fere rotunatus, apice attenuatus.

Hab. Galloprovincia, in monte Ste gevteire sufra teia (to. Thuthoman).

§§. Gtyli hirsuti val lanate.

$\sim$ Discus planus vel subplanus.

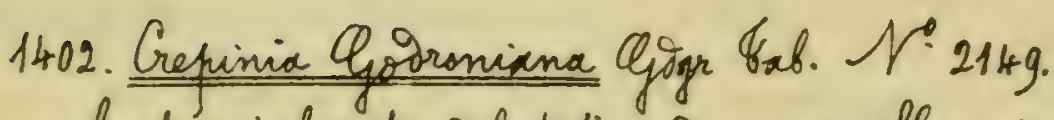

Acculei inclinato-gilatat, is ramos flor. pranciones; fol parva, obovats - ccuta, basi paulation attenuata, glauca, 2 costam eglanbubsa; ientes simplices, lanceolato-convergentes; pet. glaniulosi, wculeati, gense viltosi; step. virives, auriculis subrectis; pes. 1.glabri; 8-g m. longi; trbus oblongus; 
- 99--

sep. pinnulis sinnibus integerrimis; latiusculis instructa; stagli hirsutic, Misco plano; cor.......; fruct magnus, subollangus, basi fere rotunioatus, aprice breviter straingulatus, mesis turgious.

Hab. Qise, at Noyon (Ch. Magnier).

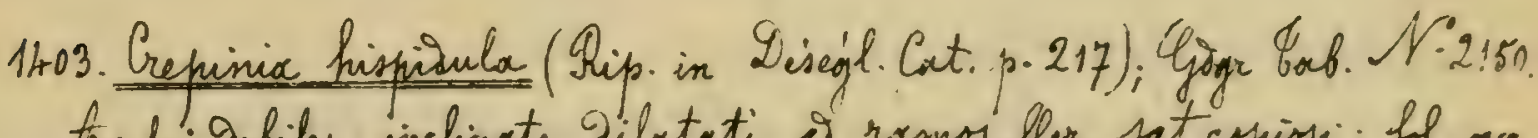
foculei gebiles, inchinato-dilatati, is rxmos flor. sat coniosi; fol ovato-acuta, atrovirentia, aid costam vix pilosula; Dentes simplices; pet. hinc inde glanisulosi, inermes, villosi, stip. Torso paulo pubescentes, vuriculis rectis; pes. 2-4, breves, glabri; tubus ovoideus; sep. breviter finnato, eglan"sulosa; styli hisuti, subeaaliti, gisco plano; cor rosea; fruct. ov ridens.

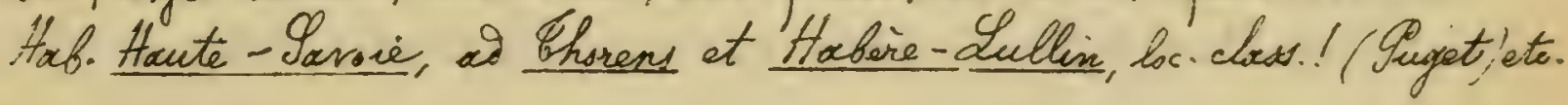

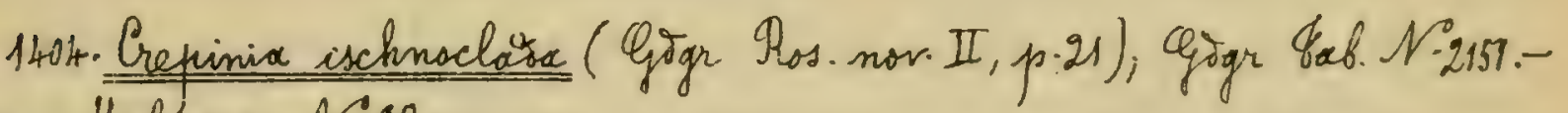
Gigor Herb. ros. Nig8.

Acculei longuiceuli, temues, parum silatati, ai ramos flor. Apansi, fol. parva, elliptico-oblonga, acuta, al costam vix inirtella; sentes profunioi,

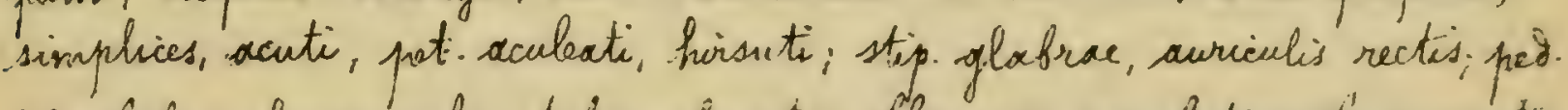

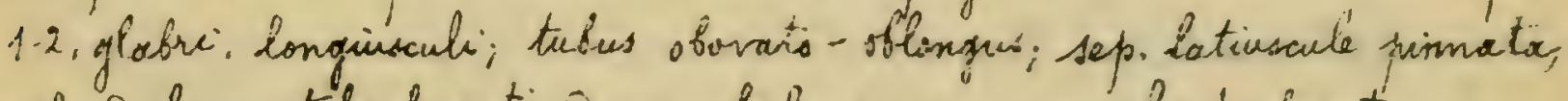
eglaniulass; stigli hirsuti, Dises subplann; cor. rosen; funct ofovatus.

Hat. Thane; at Aornad (No. Gandoger).

1405. Cepeinia perthinensis Goor \&a6. N-2152.

Acculei cylinarici, parum silatati, teniciter at parce folcati, as ramos flor-geminati, fol-anguste slongo-lanceolatio, basi acuta, od costam eglanoulosa; Dentes simplies: lanceslato-recti; pot-eglansulosi, aculeati,

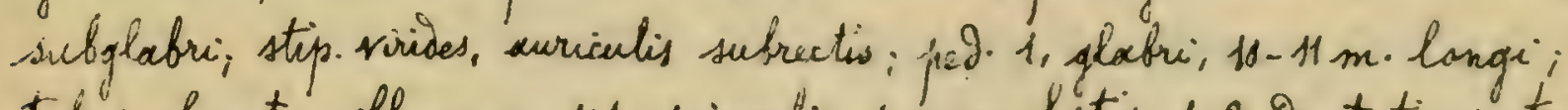
tuchs oborato-oblongus; sep. pinmulis prarum latis, 1-2-gentatis instereta, acumine integro; stijli muckescentes, Visis plano; cor........; fruct - ofovato: oblorgus, basi subisejressus, apios attemuatus.

Hab. Hungaria, frope Sesth (2. Shichter).

$\sim \sim$ Disens conicus.

1406. Gupinia bombax Googr \&ab. N=2153.

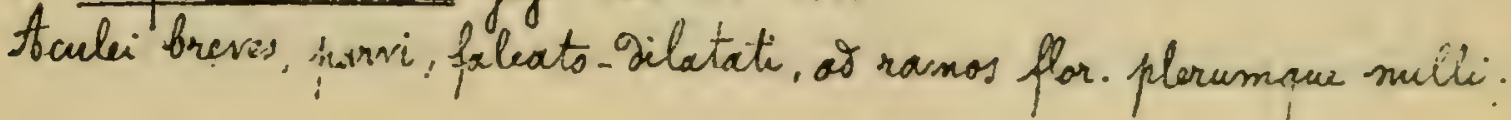


$-100-$

fol. oblong-lanceslata, basi attenuata, at costam paulo glandulosa; dentes simphices, lanceslato-recti; pet. vic glansulosi, villosi; aculeati; stip. virides, auriculis rectis; per-1-3, glabri, 10-12 m. longi; tubus sbovato-oblangus; sers pinnulis latis, 1-2-laciniatis instructa, acumine foliaces-dentato; styli hirsuti, gises valde comis, cr........ i fruct. maximus, oborato-oblongus, basi rotunaatus, apice attemuatus.

Hab. Irouches - Su - Mhone, at Martigues (At. toutheman).

1407. Crepinix moerens Gogr mas.

foculei parvi, breves, parum faleato-Dilatati, od ramos flor-geminati; fol ampluiscule oblongo-subacuminata, basi longe attenuato-cunexta, as costarr eglangulasa; dentes simplies, profunde lanceolato-recti; pet. toto pubescentes, aculeati, eglandulosi, stip. virides, magnac, auriculis rectis; ped. 1-?, glibri, $8 \mathrm{~m}$-longi; tubus ovoideus; sep. pinnulis onmibus integris et latis instructa; styli villosi, Gises comico; con frallide rosea, eciliata, fruct. orrieus, fai frocesertion attenuatus.

Hab. Lasne-et - Laire, as It Micaut (M. Ganooger).

1408. Creminia incisifolia Ggogr mss.

Trablei longi, necti, parum bilatate, as ramos flor-mulli; fol. oblangoacuta, basi subtrumeata; costa mesix glandulosa, at basix aculeolata; gentes simplices, triangulares, lancolato-recti, valse profuntr; pet-eglanimlosi, laxe rubescentes, aculeati; stip. sirises, anqustor, auriculis rectis; pes. 1. glabri, '11-13 m. longi; tubus oforato-sblongus; sep. pinnulis annibus integris, latis instructe, styli sublanati, deses ralie conics; cor........ fruct. obor atosolongus, sat gecurrens, apuie attenuatus, mesio turgious. Hab. Italia sicint, as Rimimi (2. Coldedi).

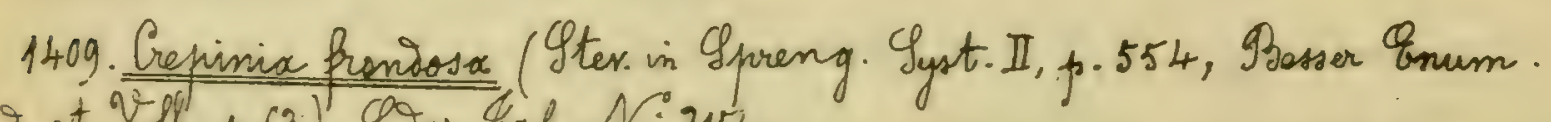
Gov. et Volh.p.63); Gogr \&ab. M:2154.

toculei pancires, falcato - Silatati, as rames flor. mulli; fol. ovata, as costam villosula; Oenter simplices; pot villosi; stip. subtus pulescentes; peos. lacves, solitarie; tubus risovato-oblongus; sep. longe pinnata, eglenaulosa; stepli villose; gises valde comic; car roses - alba; funct-obovato-oblongus, apice ratie constrictus.

Hab. Mossia merid, in Eauria (Mteren) et ix Moolia (Masser 1.c.p 63). 
$-101-$

1410. Crepinia platustigma Gogr \&ab. N-2155.

Fculci gebiles, falcati, ac ramos flor. geminati; fol. anguste of. orato-oblanga, utrinque breviter attenuata; gentes simplices, convergentes; pet. inermes, eglanialosi, to to longe pilosi; stip. longace, Dorso pubescestes, auriculis parum divergentibus; ped. 1-5, glabri', 5-11 m. longi; tubus oflongus; sep. pinmulis parallelis, parum latis instructa; styli magnsi, subcolumnares, lanati, Disco conico; cor. rosex; fruct. obovato-oblongus. Hab. Houte- Savoie, ad Ehorens (Suget).

1411. Gepinia ostrea Googr mss.

tbculei breves, parri, falcato- idilatati, somos flor. stipulares; fol. ampliuscule obovata, abrupte subacuta, basi cuncato, atrorirentia, tantum à basin costace eglasiaulosace pilosula; bentes simplicas, lanceolato-subconvergentes; pet. eglanoulosi, inf. inermes, basi paulo pubescentes; stip. inaximae, rubentes, curiculis rectis, ped. 1-2, glabri, 5-7 m. langi; tubus ovoidens; sep. pinnulis sublinearitus anguste instructa; styli lanati, gisco subcomico; cor...... fruct magnus, ovoideus, utringue subaltenuatus, medis valae turgianes.

Hab. Hungaria bor; Lipto. Liptó Hivair (L. Sichter).

tot Fructus oblongus.

\$. Ityli glabri vel sparse pilosi.

1412. Crepinia acutifolia Gogr Eab. N'2157.

Atulei gabiles, falcato-Jilatate, ad ramos flor. inormes; fol anguste of lango-lancolata, utionque scutissima, ad costam hinc inide glanialosx, Pentes simphies, lanceolato-aperti; pot. sillosi, eglandulosi, aculeati; stip. longae, auriculis rectis; fied. 1-2, glabri, g-10 m. longi; trubus oblongus; sep. pixnulis eglandulosis, ommibus sat latis instructa; styli olabri; disco plano; car. rosea, eciliata; fruct. oblongus.

Hab. Hanorre, at Gertenbach, Hitrenkausen (2abl).

1413. Crenimia felix Gogr mss.

Abculi breves, parum dilatati, tonuiter faleati, ad ramos flor geminati, fol. parva, rovato-subacuta, basi sensim notuniata, ad costam glanarelosa; Dentes simplices, parvi, aperto-triangulares; pet. aculeati, lase 
$-102-$

jubescentes, sat oflandulosi; stip. parrae, longuisculace, viresses, auriculis subsivergentibus; per. 1-2, glabri, $8 \mathrm{~m}$. longi; tubus oblongus; sep. pwivmulis brevibus, smnibus integris late instructa; styli glabri, disco comico; cor. magna, sciliata; fruct. parrus, oblongus, utrinque sed apicem praesertion attenuatus.

Hab. Yar, ad tompue (tblbert).

1414. Crepinia babensis Gogr mss.

fbculi pauciores, validi, falcato-gilatati, ar ramos flor mulli; fol. sat ample oblongo-acuta, basi sensim rotuniata, lacte virisia, as costam sglangulasa; dentes simplices, aperto-triangulares; pet. virides, tomentosi, eglanoulosi, sup.inermes; stip. sirides, meisiocres, ouviculis rectis; ped. 1-2, glabri, 1 cent. longi; tubus oblongus; sep. pinnulis subfoliaceis, 2-3-dentatis instructa; styli glabri, Disco sat conico; car......; fruct. magnus, oblongus, basi subsepressus, apice longe attenuatus. Hab. Eransilvania, Solnok Doboka, ad Mába (2. Richter).

1415. Crexinia Segurae Gogr Eab. $\mathcal{Y}-2156$.

toculei valisi, breves, salos falcato-gilatate, as ramos flor. sparsi; fol. crassa, oborato - subacuta, basi subrotuniata; costa media hinc inide pilosula, bosi paulo aculeolate, Dentes simphies, aporto-triangulares; pet. xculeati, eglanoulosi, unifariam toto pubescentes; stip. latae, subrubentes, auriculis rectis; pes. 3-7, glabri, 11-13 m. longi; tubus oblongus; sep. hirta, pinmulis annibus foliaceis, integris copiose instructa; styli sparse pilosuli, Sises conics; cor. alba, ecilista; fruct. oblongus, basi depressus, is apicem longe attenuatus.

Hab. Hisframia merid. Sierra de Segura: in regione supar. montis Calar del Mundo (No. Cañada).

§§. Styk hirsuti.

1416. Grepinia patentivamea Gogr Eab. N:2158.

foculi inclinati, paum silatati, ai ramos flor copiosi; fol. oblongs acuminata, basi subrotuniate, as costam eglantulosa; dentes simplices, aperto-lancealati; pet. villosi, aculeati, turie inde glanioulosi; stip. virides, suriculis Dirergentibus; pet. 1-2, glabui, 9-11 m. longi; tubus oblangus; 
$-103--$

sop-pinmulis integris, linearibus instructa, acumine angusto, styli hirsuti, Diseo subplans; cor......; fruct. oblongus, utrinque attenuatus, sed basi fresertion contractus.

Hab. Halix arient., Fonza (2. Calsesi).

1477. Gepinia lacertosa Giag mss. - Magnier Fl. Gall. sept. at Belg. N: 181. Acenlei breves, Dilatati, parum falcati, id ramos flor. saepius nulli; fol. oblongs-sublanceolata, basi rotuniata, à costam eglaniulosa, Pentes simplices, lanceolate, triangulares, recti, pet. toto pubescentes , hine inde glanidulosi, prarce aculeati; stip. ubentes, magnae, auriculis divergentibus; pes. 1-2, glabri, 8-10 m. longi; tubus suboblongns; sep: pinnulis smnibus integris, porum latis instructa; styli lanati, disc plans; cor......; fruct. suboblongus, utrinque attemuatus.

Hab. Qise in monte St Simén prope Moyon (Ch. Magmir).

1418. Geprimia mustentata Gagr mos.

Acculei parwi, gebiles, parum silatati, tenviter falcati, as ramos flor. stipulares; fol. sat late oborato-acuta, fasi sensim atternata, mesis silatata, atrovirentic, tantum a basin costae mediace eglaniulosace pilosule; Dentes simplices, profunde lanceolato - convergentes; pet. prorsus laxe villosi, subinernes, vix glanioulosi, stip. sivises, latissimac, longuisculace, auriculis Divergentibus; pè. 1-3, glabri, $8 \mathrm{~m}$. longi; tubus oblongus; sep. pinnulis subintegris, latis instructa; styli lanati, giscs parelo comic, cor....... fruct. magnus, oblongo-pyriformis, gilatatus, utringue longins attemuatus.

Hab. Hangria bor., Liptó: as Stankorán (L. Richter).

1419. Crepimia crassinervia Gogr Eab. N-2159. Gogr Herb. ros. N-371. Fculei ocbiles, falcato - subsilatati, ad ramos flor. Dense foliosos munc mulli, munc pauciores; fol. sat ample oblong-acuminita, basi valde attenuata, atrorirentia, frominule nervosa, at costam sglaniulosa, foliolo Aerminali saepe basi 1-2-aciculato; Tentes simphices, profunse convergentes; pet. eglaniulosi, aculeati, lase villosi; stip. longse; pes. 2-3, jlabri, 7-10 m. longi; tubus coxsuis, elongatus, sep. pinnulis hirtellis, anqustis copriose instructa; styli magni, sublanati, gises flano; cor.nosea, sciliata; fruct. oflongus, utrinque valde attenuatus, basi gecurrens.

Hab. Suy-2e-Dome, ad Dhim (M. Duboscloud). 
$-104-$

1420. Prepinia oligotricha Ggor mss. - Rose......... Grep. Prim.. VI. p.21; Brotherus Pl. cauc. (Mosce) N-10 bis.

Atculei mesiacres, tenmiter falcati, as ramos flor. sat copiosi, fol. Late oblongo-acuta, basi attemuata, virioia; costa media toto pubescens, eglanDulosa; Dentes simplices, magmi, lanoeolato-recti; pet. aculeati, to to hirsuti, eglanioulosi; stip.longae, virises, auriculis parum divergentibus; pè̀. 2, glabri, $17 \mathrm{~m}$. longi; trubus oblongus; sep. pinmelis glabris, smibus integerrimis, latis instructa, styli villosi, Disco comio, cor....... fruct. oblongus, hasi attemuatus, apice longe strangulatus.

Hab. Caucasus, Carthalinia : ad Gori prope fhur. hura (Mrotherus).

1421. Crepinia oranensis Ggor mss.

toculei Debiles, parum falcato- Dilatati, ad ramos flor. mulli; fl. ampliuscule oblong-subacuta, basi sensim attenuata, ab costam paulo glansulosa; Dentes simplices, profunise lanceolats-aperti, pat. villosi, hrice inse glangulosi, inf. inermes, stip. longae, latae, virides, auriculis givergentibus; pes. 2-4, glabri, $10 \mathrm{~m}$. longi; tubus oblongus; sep. pinmulis ormibus foliaceis, lateralibus 1-9-2antatis instructa, acumine inciso; styli sillosi, Oisso comics; cor....... fruct. magnus, oblongus, utringue sat attenuatus. Hab. Blgeria occit, Oran: at St-2enis-9u-Iig (O. Debeawa).

1422. Repinia amplior Gogr mos.

Acculei valioi, inclinato - Dilatati, a ramos flor. Debiles; fol amplissime oblongo-acuta, fasi attenuata, crassa, lacte viridia, as costam eglanioulosx, Dentes late ovatt-aferti, simpliees, pot inermes, tomentosi, inferne paulo glanioulosi; stip. sirides, magnae, auriculis rectis; ped. 1-2, glabri, $1 / 2$ cent. langi; techus sblongus; rep. pinnulis latis, subientatis instructa, styli lanati, disco plans; car......., fruct. magnus, oblongus, basi plus minusse attenuatus, apvice longius contractus. Hab. Hungaria bar. Irepes: ad Loese (2. Michter).

b. Sesunculi villosi.

+ Sructus ovatur sal oblangus.

1423. Crepinix Corriozio Gogr Eab. $N-2160$ - Gogr Herb. ros. $N-54$. toculai inclinati, gilatate, is ramos flar. glaucos langi; fol ovata rel 
$-105-$

elliptica, acuta, intense viridia; costa medir hine inve piloso-glanioulosa, 'antés simphices, profunsi, convergentes; pet aculeati, saepuis eglaninulosi, unifariam et toto pubescentes, stip. virides, auriculis rectis; ped.1-4, breves, sporse pielos: tubus oblangus; sep. pirnulis elongatis, eglanoulosis instructa; style hirsute, sisco plano; cor......; fruct. ample oblongus, utrinque attemuatus.

Hab. Phone: ad tornas (M. Gandoger) st as Tommers (So. Carties).

1424. Grepinia subglabrata (Gogr Ossai p. 20 , nomen sol.; Gggr Ros. II. p. 21) Gigr Conte. IL. Slar. II, p. 35; Gogr Sab. N-2161. - C semiglabra (Cariot Gtide des Al. $5 \div$ id. II. p. 190, non Désegl.).

Abculei falcato - compressi, subgeminati; fol amplriscule obovato-elliptica, freviter attenuata, basi rotundata, gentes simplices, pet. pubescentes, eglaniulosi, inf. inermes, stip. sillosulae, pes. 1-3, pilosi, 5-6 m. longi; tubus ovatus, sep. pinnulis eglanaulosis, angrestis longe insAructa, styli glabri, gisco subplans; car cornea, pruct. parsus, ovatus, apice abrupte attemuatus.

Hab. Qhone, ad bassin (Jo. Carist).

1125. Crepinia syntrichopoda Giogr Gaf. M:21E2.

toculi parum gilatite, tenuiter falcati, as ramos flor. valie tortoarticulatos sat copiosi; fol oborato-oblonga, acuta, basi subrotundata, folis medio basi saepe 1-aculeolato; costa mesia slandalose, parcissime pilosula; Dentes simphies, lanceolito-subpatuli; pet. parce aculeati, pubescentes, eglandulosi, stip. mexiocres; pod. 2-3, sillosi, 7-10 m. Longi; tubus svoideus; sep. pinnulis angustis instructo; styli albo-lanati, gisco subplano; car....... fruct. oratus, basi rotunoactus, apice parum attenuatus.

Hab. Mhone, id Dommiers: in ruprestibus m. Suivante (H. Gooroogar).

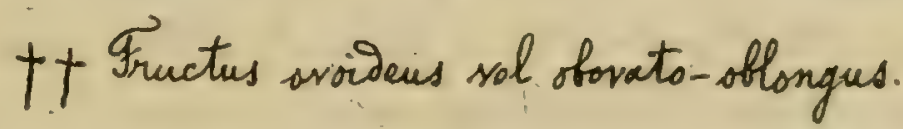

1426. Crepimia nummularioides (Gogr in Debeaux Mator. ros. Syren. 1878, p.20), Gogr Gab. N-2163.

toculei breves, Dilatati, salde falcati, ad ramss flor. sat copiosi: fol. harva, ovata, frevitor attenuata, fasi rotuniata, costa mesia glaniaulosa it insuper saepe saltem a basin sciculata; Dentes simplices, aperti, acuti, pet. aculeati, vix sparse glanidulosi, sapuis unifariom ct laxe prubes- 
$-106-$

centes; stip. brevissimac ; ped. 1-2, pubescentes, 11-12 m. longi; tubus ovsideus, sep.brevia, angueste pinnata, styli pauciores, glabii, gisco conico; cor......; fuct parvus, breviter ovoideus, basi subrotunidatus, price brexiter attemuatus. Hab. Iyrenaei orient, is tomélie-bs-Bains (O.Debexux).

1427. Crepinia galloecica Goge bat. $\mathcal{N}-2164$.

Abculei rarissimi, faleato- Silatati, ad ramos flor. mulli; fol. sat late -oblongo- scuta, basi sensin attenuata, a costam eglanaulosa; sentes simplices, lancealato-aperti; pet. hirsute, inermes, parcissime glaniulosi; stip. Latar, rubentes, auriculis parum givergentibus; per. 2-5, valie albotamentosi, 5-6 m. longi; tubus oborato-oblongus, basi pubescens, sep. pinmulis integris, parum latis instructa, acumine angusto; styli glabri, axserti, Diseo valde conico; cor. eciliata; fruct. svideus, basi rotuniatus, apice attenuat.

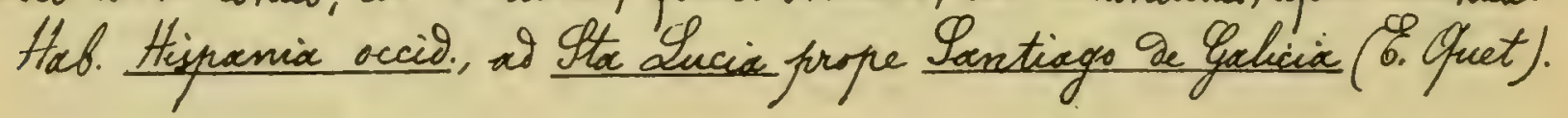

1628. Crefinia painsis Gogr Gab. N-2165.

toculei rarissimi, faleato. gilatati, a ramos flor. nulli; fol sat late sbovata, utrinque sed basi praesertion rotuniacta; costa medic paulo glanOulosa, inferne aculeolata; dentes simplices, triangulari-convergentes; pot. tomentosi, aculeati, eglandulosi; stip. gilute rubentes, auriculis subrectis; per. 2-5, villosi, g-10 m. longi; tufus oborato-oblongus; sep. finsuclis latis, 1-2- Ientatis instructa, acumine lato, integrs; styli glabri, Qises paulo conics; car. eciliata; fruct. obovato-oblongus, aprice attenuactus.

Hab. Halix mesia, Parme: is Taffora (G. Gibelli).

1429. Crenimia truthemami Goge \&as. N-2166.

Acculei falcato- Dilatati, somos flor. mulli; fol. coriacea, orato-elliptica; costa media glandulosa, in $1 / 2$ inf. hirtella ; dentas simplices, magmi, convergentes, pot. aculeati, eglaniaulosi, sup. sparse pubescentes; stip. Dorso rilCosae, auriculis subrectis, pres. 3-8, sparse pilosi, 6-10 m.longi; tribus or oideus, sep. pinmulis eglaniulosis, foliaceis copiosissime instructa, styli majusachi, hirsuti, Dises conics; cor. pallide rosea, eciliata; fruct. maximus, ovoideus, basi attenuatus.

Hat. Gouches-gu-Mhone, at Martiones (Tt. toutheman). 
$-107-$

1430. Crepinia fivides Gogr \&ab. N.2167.

taculei vix gilatati, temuiter falcati, as ramos flot. plerumgue nulli fol. obovato-oblonga, hhomboiadia, utrinque paulation rotuniata; costa gense villosa, basi paulo glaniulosa; dentes simplices, sat aperti; pat. glanSulosi, aculeati, tomentosi, stip. Torso pubescentes; per. 1-3, rillosi, 2-7 m. longi; tubus oblongus; sep......., styli villosissimi, Discs paulo conico; cor....... fruct. maximus, sbovato-oblongus, utringue gepressus. Hab. Rhone, a Chaponost (M. Chabect).

1431. Crepinia globata (Déségl. Catal.p. 205), Gggr Gab. N-2168.

Aculei folcatio-dilatati, os ramos flor. copiosi, geminati; fol oratoweuta, at basin costa villose; Dentes simplices; pet. villosi, parce aculeati; stip. Torss pubescentes, auriculis rectis, acutis; ped. 2-3, breves, basi sparse pilosi; tubus ovidens; sep. pinnata, suberecta, deciour, styli hirsuti, gises conico; cor. pallide rosea; fruct. magnus, oroitens, sorbide ruber.

Hab. Sabandia, at fomecy, Moutien (Maget) et alib:

1432. Brepinia nccedens (Rip.) in Gogr \&ab. N-216g.

teculei faleato-Dilatati, as ramos flar. articulatos copiosi; fol. ellipticooborata, costa mesia in $1 / 2$ inf. hirtella; dentes simplices, subconvergentes; pet. villosi, inermes, pleumque eglanioulosi; stip. Dorso villosce, ciliatoe; ped. 1-4, villosi, 3-10 m. longi; tubus avoideus, sep. sat anguste pinnata; styli oxserti, subcolunnares, magni, lanati, gises parvo, conico; car. subalba; fruct. parrus, ovoisens, apice attemuatus.

Hab. Iavoie, ad Salins propue Moûteirs (Miget).

1433. Creninia Gerardiana Gogr \&ab. $\mathcal{N}=2170$.

tcculei falcato-gilatati, 28 ramos flor. copiosi; fol parra, coriacea, ofsrato-sblonga, reute, basi sensim rotundata; costa mesia parce hirtella, glanioulosa, basi aculeolata; Dentes simplies, triangulares, aperti; pet. rculeati, eglanianlosic, usque as 2 um jugum fol. pubescentes, cactorum gla fri; stip. sirides, nuriculis rectis; ped. 1-3, paulo hirtelli, $7.8 \mathrm{~m}$. longi; trbus sbovats-oblongus; sep. pinsmelis latiusculis, omnibus 1-2-ientatis instructa, styli villosi, gises valse conico; cor eciliata; fruct mediscris, obavats-oflongus, basi subrotunaatus, aprice paub attenuatus. Hab. Boucher-2u-Phone, a Martigues (J. Toutheman). 
$-108-$

2. Semi-glabrac Gigr \&ssai p. 30; Giggr Lab. p. 164.

Foliolis medies ramorum flarifuorum supra glabris, subtur vero -at costam mesiam villosis, cum filis raris as nervos primarios; sevaturis intergum compositis.

$1\left\{\begin{array}{l}\text { Foliola ammino simpliciter serrata } \\ \text { Foliola alia simpliciter alia biserrata } \\ \text { Foliola omnino biserrata }\end{array}\right.$ Pesunculi glabri

2 Seaunculi villosi vel glanaulosi Fructus ovoideus vel obovato - oblongus Fructus oblongus vel elongatus

$3\{$ Fructus globosus vel rotunisatus Fructus ovatus vel breviter ovoidens Styli glabri vol subglaberrim:

4 Y Ytyli hirsuti val lanate Ityli glabri

$5\{$ Ityli paulo villosi Stuli Dense hirsuti

Gtigli hirsuti val lanati

$\checkmark\left\{\begin{array}{l}\text { Syli paulo villosi } \\ \text { Styli gabi }\end{array}\right.$ Ityli glabri

7 Discus planus
2

6

Yrecies $N=1478-1481$.

3

Ineries N:1470-1477.

4

5

Srevis N:1434-1437. Ipeais $N \cdot 1438-1445$ Ipecies $N-1446-1452$ Ypenes $N-1453-1457$ Inecied $N=1458-14.61$. \$pecies $\mathcal{N}: 1462-1464$ Precies N:1465-146g.

7

Yrecies $N^{\circ} 1482-1484$. ipreies Ni485-14ge Species N-1491-1495. 9hecies $N^{-1496-1498 .}$

1. Folish omnino simpliciter serrata.

a. Sesunculi glabri.

+ Fructus glabosus vel rotunaatus.

1434. Crepimia sphaerocarpa (Puget in Désegl. Catal p. 208), Ggogr Bab. N: 2172. - Billst exs. N-3587 bis; Déségl. Herb. ros. N-68.

toculei ad ramos densos validi, falcato-dilatati; fol elliptica, etrovirentix, prominule nervosa, gentes simplices, recti; pot. villosi, parce aculeati, stip. latae, glabrae; ped. 1-3, glabri, 7-13 m. longi; tubus rotundatus; 
$-109-$

sep. pimmulis latis copriose instructa; styli pubescentes, gisco subplano; cor. alba; fruct maximus, subglobosus.

Hab. Haute-Savoin, ad Habie - Lullin et Habire-Toche (Tuget).

1435. Grepinia basaltica Gigr Lab. N-2173.

Acculei Debiles, parum falcato-Dilatati, ad ramos flor pauciores; fol. blonga, utrinque acuta, a costam eglanioulos as ontes simphices, opertolanceolati; pot aculeati, pubescentes, eglanoulosi, stip. sirides, auriculis Divergentibus; peo. 1, glabri, 9-10 m. Congi; tubus roternoaties isep. pimnulis annifus integris, angustis instructa; styli sparse pilosi, Disco pla-no; cor......., fruct parsus, rotieniatus, basi rotuniatus as apricem Depressus. vel pauls attenuatus.

Hab. Hibernix, in basalticis as Noira, Lawn (S. To. Stewart).

1436. Greninia bueostyla Gogr \&ab. N-2174.- C. acanthina (Désigh at Ozanon in Isull. soc. Tauph 1882, p. 370). . Ooc. Tauph exs. N: 3264 . Magmir 531.

toculei gebiles, faleato - subsilatati, ad ramos flor. glaucos copiosi, fol. firma, umple oblango-lanceolata, basi notundata, atrovirentia, prominule newosx, ad costam eglandulosa; gentes simplices, aparti pet. tomentosi, hine inse glandulosi, inf. inermes; stip. Dorso pulescentes; peo. 1-9, glabri, 8-gm. longi; trebus rotundatres: sep........ styli magne; lanati, gises plano; cor...... fruct. globosus, bai depressus, apice prouls attenuatus. Hab. Fyrénés arient., of toméke-les-Mains (O. Debewx).

1437. Crepinix microsphoera Gagr bab. No2175.

Acculei falcato - delatati, ad ramos flor. geminati; fol late oblongoscuturseula, basi rotunsata, a costam eglanoulosa; Dentes simplices, aparto- triangulares, pet. villosi, aculeati, eglandulosi; stip. sirides, auriculis rectis; pes. 2-3, glabri, 12-15 m. longi; tubus rotuniatus; sep. pimnulis angustis, 1-3-Dentatis, acumine latuisculs, integro, instructa; styli lanati, sisco subplano; car...... fruct. parrus, globosus, utrinque roturiatus. Hab. Aonglix, as Ofork (G. Goebster).

†† Fructers onatus vel breviter ovoidens.

1438. Crepinix contorta Goar \&ab. N-2176.

tacubi rarissimi, Debiles, faleati, ad ramos flar patulo-tortuosos mulli; 
$-110-$

fol. oflonga, utrinque parum attemuata, ad costam eglanialosa; dentes simplices, profunivi, paulo convergentes; pet. tomentosi, eglanialosi, inf. inermes; stip. mesiocres; pes. 1-2, glabri, $11-12 \mathrm{~m}$.longi; tubus oratus; sep. pinmulis longiusculis instructs, acumine gilatato; styli villosi; Disco plano; cor...... fruct satus, basi rotumiatus, apice pauls attemeatus. Hab. Asere, a Recoin (M. Gandoger).

1439. Greninia haemantha Gogr Eab. N-2177. - Gogr Herb. ros. N-136. toculei vebiles, folcato-subsilatati, as ramos flor. mulli; fol. sat anguste oblango-acuta, basi attenuata, ad costam glaninclose, 2 entes simplices, convergentes; pet. villosi, eglanialosi, inf inermes; stip. virides; ped. 1-3, glabri, 7-10 m. longi; tubus svatus; sep. brevia, sat anguste puinnata; styli sillosi, Disco subplan, cor. lacte rosea, ad unguem sparse ciliacta; fruct. ovatus, gepressus.

Hab. Duy-2e-Dame, as Sary (Nb. Dubosclart).

1440. Prepinia tomostigma Gogr Eab. N-2178.

Acculi langi, id ramos faleato-Dilatati; fol. cariacea, obrato-oblonga, freviter sttenucta, basi subrotundata; Dentes simphies, corvergentes; pet. aculeati, tomentosi, sparse glansulasi, stip. lattar, aurieulis givergentibus; ped. 1-4,glabri, 3-g m. longi; tubus ovato-ellipticus; saps rubentia, pinnulis paucisoribus angustius instructa; styli magni; albo-lanati, aises plano; cor.......; fruct. magnus, syatus.

Hab. Houtes - thers, ad La Garte prope Gape (E. Surb).

14t1. Crefinia annuens Ggogr inss.

tocula bress, faleato-gecurrentes, so ramos flor. rari; fol ample obovato-acuta, basi rotuniata, intense viridia, a costam eglaniulosa; Dentes simplices, lanceolato-convergentes, pet laxe pilosi, apice fere glatri, aculeate, plerumque eglanoulosi, stip. medio subrubentes, latae, longae, auriculis sat avvergentibus, pe8. 1-3, glabri, 1/2 cent. longi; tubus ovat-ellipticus; sep. longa, primulis subintegris, sat angustis instructa: stagli lanati, Didso comico; cor....... fruct. oratus, basi rotuniatus, apice paulo attemuatus. Hat. Hungario bor, Lifté. Sotankora (2. Richter). 
1442. Crepinia Fempletomian Gogr bab. $\mathcal{Y}-2179$.

Aculei debiles, subrecti, iilatati, as ramos flor. pauciores; fol. oboratsoblonga, acuta, basi rotundata, as costam paulo glanaulosa, dentes simplices; pet. pubescentes, aculeati, eglandulosi; stip. virides, auriculis girergen. tibus; per. 1, glabri, 10-11 m. longi; tubus srato-ellipticus; sep. pirnuclis amnibus integris, angustis instructa; styli issporse pilosuli, Disco paulo camis; cor...... fruct. parsees, ovato-ellipticus, basi rotundatus, as apicem Nixe defressus.

Hab. Hibernix, ad New Forge prope Melfart) (I. A. Itewart).

1443. Crepinia paprostila Gogr bab. N-2180. - Gogr Herb ros. N-10g. toculei folcato-gilatati, as ramos flor. remotos plerumque mulli; fol ample obovato - slonga, medis dilatata, acuminata, basi attemuata, glanescentia, as costam eglanioulosa; Dentes simplices, profunisi, convergentes; pet. villosi, temuiter glanaulasi, inf. inermes; stip. 2ilatatac; pes. 2-4, glabori, 7-10 m. longi; tubus ovoidens; sep. pinnulis parum latis, longis instructa; style exserti, sublanati, Oisco subeonics, sulcato; cor....... fruet. breviter ovoideus, saltem basi attemuatus.

Hab. Hire, ad Charancieu (M. Gandoger).

1444. Rrepinia hallioula Gigr bab. N-2181.

Aculei inclinato-Dilatati, as ramos flor melli aut rari; fol. parve, slongp-acutiuscula, basi subrotuniata, od costam eglandulasa, gentes lanceolato - aporti, pet. pubescentes, aculeati, eglandulosi, stip.parvae, virides, auriculis givergentibus; poo. 1, glabui, 8-9 m. longi; tubbs ovoideus; sep. pinmulis latiusculis, integris, cum acumine instructa; styli poulo pubescentes, gises comics; cor....... f fruct. perrus, breviter oroisens, utrinque gepressus.

Hab., Anglia bor. as York (G. Hebster).

1445. Grepinix Stuartiana Gogr Gab. N-2182

Aculai parri, inclinato- Dilatati, as ramos flor. paviciores; fol. Late oborato-acutriescula, basi sbrupte cuneata, of costam eglanaulosa; gentes simplices, lancealato-convergentes; pet. villosi, aculeati, hinc inde-glanisulosi; stip.latre, auriculis rectis; pes. 2-3, glabri, 6-8 m. Longi; trebus ovoideus; sep.pinmelis parum latis, 1-2-gentatis instructa, acumine integro, angustiore; stylli glabri, isco sat comico; cor magna, cciliata; fruct. breviter orrideus, 
utringue vix gepressus.

Hab. Icotia, Gerthshire: at Dunkelt (Drummond-Hay).

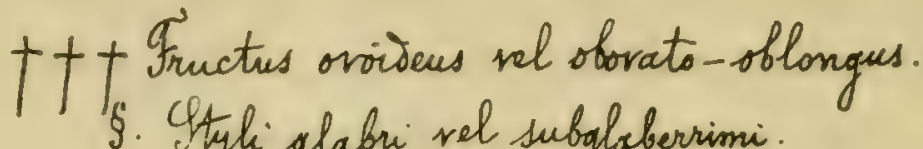

\$. Atyli glabri vel subglaberrimi

1446. Crepinia officiosa Gogr mss.

toculei rari, gebiles, parum falcato-dilatati, oo ramos flor. mulli; fol. oblongo-acuta, basi rotunixta, pallide virisia; costa inprne paulo glangulosa, Dentes simplices, triangulares, recti; pat. tomentosi, subinermes, hinc inse glanivulosi; stip. Latac, longac, Dilute rubentes, auriculis subrectis; poo. 1-2, glabri, $1 / 2$ cent. longi; tubus obovato-oblongus; sep. pinnulis subintegris, meviacribus instructa; styli sparse pilasuli, aises sat conics; cor....... fruct. sat parrus, svoideus, basi rotunioatus, aprice conico-attemuatus. Hab. Hungaric bor, Syeped: al Lucse (2. Richter).

1447. Grepinia lifertina Gògr ms.

toculei pauciores, validuch, faleato-Dilatati, as ramos flor. glaucos mulli; fol. amplissime obovato - oblonga, breviter acuita, basi rotundata, sat pallide viridia, as costam eglaniulosa; dentes simplices, profunde lanceolato-recti; pat. rubentes, tomentosi, inermes, eglaniulosi; stip. ruberrimae, maximas, auriculis subrectis; pes.1-9, glabri, 5-6 m. longi; tubus ovoideus; sep. pinnulis latis, subventatis instructa; styli glabri, giseo plano; cor....... fruct. sat magnus, oroideus, basi subjepressus, apice valie strangulatus, mesio turgious. Hab. Hungaria bor. Izepes: of Leise (2. Michter).

1448. Crepinia forinaum Gogr 8ab. N:2183.

Aculei breves, temiter faleati, subilatati, of ramos flor. sat copiosi; fol. obovato-elliptica, base subtruncate, apice brevitor acuta, of costam eglaniaclosa; dentes simplices, aperto-lanceslati; pat. hirsuti, eglanoulosi, parce aculeati; stip. apice prebescentes, auriculis subivivergentibus; ped. 1-3, glabri, 8-10 m. longi; tubus svioens; sep. pinmulis latis, longis, 1-2Oentats-glanisulosis instructa; styli glabri, disco paulo conico; cor.rosea, eciliata; fruct: ovoidens.

Hab. Givande, at Montferrand (L'. Motelay). 
$-113-$

1449. Crepinia infatuata Ggor mss.

Aculei dilatati, valbe falcati, as ramos flor. stipuclares; fol. Late oborato-acuta, cuspidata, basi rotuniata, as costam eglanioulosa; gentes simplices, triangulari-subconvergentes; pet.villosi; aculeati, eglanialosi; stip. sivides, longue, auriculis valte givergentibus; pes. 1, glabri, 5-7 m. longi; tubus sbovato-oblongus; sep. pinnulis amnibus latis, incisis instructa, styli glabri, Diseo plans; car....... fruct. obovato-sblongus, base abrupte securrens, apice strangulatus.

Hob. Inecia, Icania : It Drild (\%. t. Gabrielsson).

1450. Prepinia petulans Gogr mos.

Aculei robusti, dilatati, valie falcati, io ramos flor. sparsi; fol. amplissime oblongo-acuminata, basi rotuniata, crassa, intense viridia, as costam eglansulosa; Oentes simplices, profunivi, lanieslato-convergentes; pet. tomentosi, hinc inde vix glandulosi, sup. inermes; stip. longre, latissimae, rubentes, auriculis rectis; pot. 1-2, glabri; $8 \mathrm{~m}$. longi; tubus obovato-oblongus, sep. pinmulis subfoliacsis, Dentatis instructa; styli subglaberrimi, Disco comic, cor........ fruct. sat magnus, obovato-sblongus, utrinque sed basi prosertion abrupte attenuatus.

Hab. Hungaria, of Mar (2. Michter).

1451. Crepinia nuivicaulis (Gogr Ros. nor. II, p. 22); Gogr Eab. N:2184. Goge Horb. ros. $N-20$.

Demidata pareque foliosa; aculai Debiles, parum faleato-Dilatati, 8 ramos flax.copriosi, fol. amphiescule oblongo-acuta, basi sensim rotundata, xo costam eglangulosa; Dentes simplices; pat. pubescentes, aculeati, infone 1ix. langulasi; stip. latae; pas. 1-2, glabri, 14-16 m.longi; tulus oblongus; sep. pinmulis longis, eglinoulosis, latiuscule instructa; styli glabri, exserti, disco subconico; cor....... fruct. oborato - oblongus, utrinque attenuatu. Hab. Ysere, as Charancieu (Mb. Gandoger).

1452. Crepinia telonensis Gogr mss.

Acculei panciores, validi, falcats-2ilatati, io ramos flor mulli; fol. me. Discria, suboblong-acuta, basi rotuniata, costam inferne aculeolata; dentes simphices, profunide lanceolato-wherti, pat. villosi, aculeati, hine inde glanoulosi, stip. virides, parvas, awriculis aportis; pet. 1-3, glabir, 13-14 m. 
$-114-$

longi, tubus obovat -oblongus; sep. pinmulis annibus integris, latis instructa; styli sparse pilosuli; sisco paulo comico; cor......; fruct. ovoideus, conicus, basi rotuniato-vilatatus, apuice longe attenuatus.

Hab. gar, is La Garde prope Coulon (Jblbert).

\$§. Ityli hirsuti vel lanati.

1453. Crenimia antriace Gogr Eab. N-2185.

Acculei gilatati, parum falcati, as ramos flor melli; fol firma, late obovato-acuta, basi rotuniata, as costam eglanoulosa, Dentes simplices, orato-aperti; pet. aculeati, eglanaulosi, pulescentes; stip. breves, auriculis divergentibus; ped. 1-2, glabri, 5-7 m. longi; tubus avoideus, sep. pinmulis sat latis, eglaniulosis instructa, scumine lato; styli villosi, Sises pauls conics; car........; fruct. svoiveus, utrinque set apice procesertion attemiatus.

Hab. tustria inf, as Miinchenorf prope Mren (J. Hiesocur).

1454. Brenimia macrophylla Gigr Eab. N-2186.

tacula valivi, inchinato- Dilatate, ai ramos flor. nulli; fol amplissime oblangs-acuta, fasi attemuata, ad costam eclanisulose; dentes simplices, subpatulo-triangulares; pot. eglaniulosi, aculeati, lase et sparse pilosuli, stips sivides, auriculis rectis; ped. 2-9, glabri, 12-14, m. longi; tubus obovats - oblangus; sep......... styli hirsuti, gises conics; cor......., fruct. glaucus, ob. ovato-oblongus, utrinque atténuatus.

Hab. Italia orient, as Fanza (L. Calvesi).

1455. Crepinia Montswiana (Cnabert) in Gigr Gab. N-2187. Gigr Herb.ros. 398. trculei minute, temes, rectiv vel inclinati, ad romos flor. mubros at flexuosos sparsi; fol sat parva, elliptiex, as costam eglansulssa; gentes simplices, ciliati, pat pubescentes, eglanoulosi, aculeati, stip. auriculis paulo givergentibus, pes. 1. brevissinni, tennes, glabri; tubus sbovato-oblongus; sep. pinnulis alongatis, cspiose instructa, stigh hirsuti, exserti vel subcolusmores, gisco paulo comic, cor.pallise rosea; fruct. obovato-oblongus, apice attenuatus. Hab. Mhone, at Nontsuit prope Iyon (O. Chabert).

1456. Crepinia crispatula Gogr Bab. Ne2188.

taculei falcoto-iilatati, io namos flor. sape gemincti ; fol. parra, obo- 
$-115-$

rato- oblonga, acuta, basi attemuata, ad costam furpuream eglasiolosio; dentes simplices, crispo-unisulati, profunde lanceolato - convergentes; pot-aculeati, laxe pubescentes, sparse glanidulosi; stip. latoe, longatae, auriculis subrectis; pes. 2-4, glabri, 11-13 m. longi; tubres oborato-oblongus; sep. pinamilis latiusculis, 2-4-2entato-glaviaulosis, acumine longs, foliaces; styli sublanati, isiso fere plano; car. alba, eciliata; fruct. oborato-oblongus, utringue atten, Hab. Iyrénées arient, as Olette-les-Mains (O. Debeara).

1457. Crepinix elisabethopolensis Gogr 8ab. N-2189. - C. lencantha (Unis itiner. 1835, non NO3.).

tculei Debiles, purpurei, ad ramos flor. Dilatato- subfolcati, fol. firma, nerrosa, obovato-elliptica, utrinque paulation attenuata, foliolo terminali temiter aculeolato; Pentes simplices, breves, pactulo-asscententes; pat-villox; aculeati; stip. latae, inf.subtus toto pubescentes, auriculis givergentibus; peo.1-3, glabri, 7-11 m. longi, tubus oblongres; sep. brevia, pinmulis filiformibus parcissime instructa, styli lanati, gisco plano; cor. pallide rosea? cciliata; funct. probabilins obovato-oblongus, utingue depressus. Hab. tormenia orient., ad Elisabethopl (M. F. Hohenackar).

T†十 $\vec{F}_{\text {ructus }}$ oblongus vel elongatus.

\$. Styliglabri.

1458. Crepinia clavigera Gogr Gab. N:2190.

tculei gebules, Dilatati, parum falcati, ais ramos flor. copiosi; fol. oboirato-oblonga, attenuata, basi rotunisata, as costam eglanioulosa; dentes simplices, recti; pet. pubescentes, aculeati, basi 2-4-glandulosi; stip. mesiscres; ped. 1-3, glabui, 10-15 m. longi; tubus longissimus; sep. brevia, late pinnata; styli pauciores, glaberrimi, sessiles, bisco comics; cor......; funct. elongatus, strinque set apice procestion valte ettemuatus,

Hab. IRhore, inter Ecully et Chabonnièred (Fo Chabert).

1459. Greninia fusiformis Goor bab. N-2191. (Chabert in litt.).

Aaculei elongati, at ramos flor parum dilatati, rectiusculi vel inclinati; fol. ovata vel oborato-acuta, ai costam eqlanoulosa; dentes simplies, cilialati, convergentes; pat eglandulosi, longi pilosi, aculeati, stip. as apriem gilatatae, auriculis divergentibu:; pei. 13 , glabri, mesioeres; tubus anguste 
$-116-$

Clongatus; sap. pinmulis foliaceis, elongatis copiose instructa; styli glabri; breves, Gises connic, violaceo; cor. magna, anguste unguieulata, eciliata, fruct. enguste elongato-fusiformis.

Hab. Rhone, \& Charbonnieres (P. Chabert).

1460. Geninia Dumetorsides (Giép.) in Gogr \&ab. N-21g2.

trculei Debiles, parum faleati, as ramos flor. geminati; fol. obovato-oblonga, utrinope sensin attemuata, gentes simplices, aperti, pet-villosi, eglanoulosi, inf. inermes; stip. apice dilatatoe, auriculis givergentibus; pes.1-3, glabi:, 10-15 m. longi; tubus sblongus; sep. anguste et copiose pinnata; styli gla bri, oisco subplans; cor-rosea; fruct sblongues, utrinque depressus.

Heb. Inghix, Yorkshire: as Icanton (7. G. Baker).

1461. Crepinia livenice Gogr Eab. N-21g3.

tocula temiter falcati, gilatati, ai ramos flor copiosi; fol. obovato-oblonga, acuminata, basi truncata, ad costam eglanhulosa, rentes simplices, ovat-subconsergenter; pet-tamentosi, aglanoulosi, aculeati; stip. rubentes, suriculis givergentibus; peo. 2-4, glabri, g-11 m. longi; tubus oblangus; sep. pinmulis foliaceis, profunie 4-5-Dentats-glantulosis instructa, rcumine hineari, integro; styli glabri, Disco comico; cor. exiliata; fruct. oblongres, utrinque depressus, Hab. Rossix bor-acid., of Gbelshof prope Miga Miromiae (Eh. Miencit).

§. Ityli paul villosi.

1462. Crepina lenconoma Gigr \&ab. $N=2194$.

Aculai validi, is ramos flor. falcato-decurrentes; fol. oborato-acuta, basi subattermata; Dentes simplices, convergentes; pet vellosi, parce aculeati, eglan Dulosi; stip. hano Dilatata, gorso pubescentes, aviculis givergontibus; pes. 1-3, glabri, 5-10 m. longs; tubus oblongus; sep. pinsulis brevibus, sat latis, paucioribus instructa; styhi paulo villosi, ases subplano; cor. pallide rosea rut subalbs; fruct. oblongus, utrinque oppressus. Hab. Haute-Savoie, at Habire-Lullin (Iuget).

1463. Creninia amphoricarnas Goyr Eab. $\mathcal{N}-2195$.

toculi debiles, recti, ou ramos flor. mulli; fol. oborato-oblonga, subacute, basi rotuniata, as costam eglanivuloss, Dentes simplices, lanceolato- 
$-117-$

convergentes; pet. villosi, inermes, eglangulosi; stip. sirides, auriculis vatie givergentibus; pes. 1, glabri, 12-19 m. longi; tubus elongatus, sep. pinnulis parusm latis, 1-2-Ventatis instructa, acumine integrs; styli sparse pilosi, gisco paulo comico; cor....... fruct. oblongus, basi attemuatur, médio paculo turgious, apice longissime strangulatus.

Hab. Dise, at Noyon (Ch. Magnier).

1461. Crepinia 2arisiana Gogr bab. N-2196.

Aculei vefiles, inchinato-dilatati, is ramos flor. prancisres; fol. Late oborata, apice subrotundata, basi longe cuneata, ad costam eglanioulosa; ventes simplices, lanceslato-convergentes pet. pubescentes, aculeati, eglaniulosi; stip. rubentes, latissimae, auriculis rectis; pes. 2-5, glabri, 12-13 m. longi; tubus oblongus, sep. pinnulis angustis, 1-2-gentatis instructa, acumine lato, integro; styli pauls villosi, bises plano; cor, eciliata; fruct. oblongus, utringue attenuatus.

Hab. Ionglia bor., a York (G. Hebster).

$\S \S$ Stylidanse hirsuti.

1465. Crepinia rhepnchophora Gigr Eab. N:2197.

Abculei gebiles, temiter falcato-gilatati, is ramas flor-pauciores; fol. crassa, strovirentia, oborata, sensin acuta, basi truncata, costam paulo glaniaulosa; Dentes simplias, lati, aperto-triangulares; pet. subinermes, ntlosi, vix glanderlosi; stip. latae, auriculis rectis; ped.1-3, glabri, 17-10 m. longi, tibus oblongus; sep. pinnnlis angustis, 1-2-gentatis instructa; styli hirsuti, gisco plano; car. pallise rosea, ecihiata; fruct. slongres, basi gefressus, apuice anguste et longissime attemuato-strangulatus.

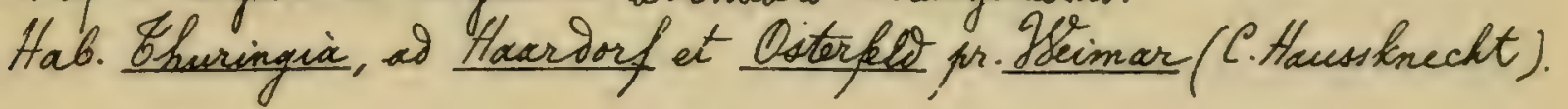

1466. Crepinia sharsipila Gogr Eab. N-2198.

toculei Debiles, ad ramos flor. temiter falcati; fol obovats-oblongr, utrinque attemuata, gentes simplices, pet. villosi, eglaniulosi, sup. parce -aculcati, stip. angustiores, garso villosac, auriculis Givergentibus; pet. 1-3, glabri, 5-11 m. longi; tubus elongatus; sep. late pinnata; stigli hirsuti, Sisco suoplano; cor.......; fuct. elongato - fusiformis, utringue valie attematus.

Hab. Haute- Marmis, as Mort- Sion (Muget). 
$-118-$

146\%. Orepinia amygarlina Gogr mos.

foculei longi, falcato-rilatati, ad ramos flor. stipulares; fol. anguste blongo-lanceolata, fasi sensim atternuata, lacte virisia, aid costam eglarioulos a i dentes simplices, triangulares, aperti, pet. tomentosi, inermes, eglanoxlosi; stip. virides, breves, latae, auriculis subrectis; pes. 1, glatri, 3-5 m. longi; tubus oblongus; sep. pinmelis subintegris, foliaceis instructa; stygh sublanati, gisco plano; cor....... fruct. solongus, besi Decurrens, apice longe attex. Hab. Hungaria bor. Lipte" at Mónsahegy-Marithegy (L. Michter).

1468. Crepinia pilinaeva Gogr 6 ab. N'219g.

Acculei gebiles, inclinato-rilatati, ad ramos flor panciores ac minutissimi; fol glauca, sat late obovato-acuta, basi rotuniata, is costam inferne pauls glanioulosa; bentes simplices, profunse lanceslato - subconvergentes, pet. villosi, inferne parcissime glandulosi, acceleati, stip. virides, angustar, auriculis parum givergentibus; pes. 1, glabri, 5-6 m. longi; tubus oblongus; sep. pinmulis amnibus integerrimis, angustioribus instructa; styli hirsuti, disco plans; cor......., fruct. oblongus, basi roturiates, apice abrupte ac longe strangulatics.

Hab. Oise, ad Moyon (Ch. Magnier).

1469. Orepinia veromensourum Gogr \&ab. N-2200.

Acculei minutissimi, falcats-gilatati, ad ramos flor. nulli; fol. oblongo-subacuta, basi truncata, as costam eglanoulosa, Dentes simphices, orato-aperti; pet. pubescentes, eglaniulosi, sup.inermes; stip. Latae, Silute rubentes, auriculis rectis; pes. 1-2, glabri, 5-7 m. longi; tribus elongatus, sep. pinnulis ommibus integris, foliaceis instructa, styti hirsuti, dises plano; cor......, fruct. elongatus, turgious, basi attemuatus, ad apicem longissime strangulatus.

Hab. trisme, as It Orention (Ch. Magnier).

b. Pesunculi villosi vel glanoulosi.

1470. Crepinix umbina (Tuget) in Cgigr trab. N-2201.

Acculei id ramos breves, parri; fol obovata, utrinque sensim attemuata, a costam eglanoulosa, Dentes simplices, recti; pet subinermes, villosi, inferne sparse glandulasi; stip. angustise, auriculis givergentibus; 
po. 1-2, basi prasertim villosi, 4-7 m. longi; tubus avoideus; sep. pismulis integris, angustis instructa; styli sillosi, gisco plano; cor.......; fruet. parvus, ovatus.

Hab. Haute-Lavoie, ad Tirenthon (Puget).

1471. Crepinix aequalis Gogr Gab. N-2202.

teulei debiles, Dilatato-inclinati, ad ramos flor.copiosi sapeque geminati; fol. elliptica, sape obtusa, basi rotuniata, a costam eglaniulosa; genter simplices, rotundati; pat. villosi, subinermes, parcissime glangulosi; stip. glabrae, suriculis givergentibus; peo. 1-3, breves, basi paulo pilsti; tubus borato-oblongus; sep. mediscriter pisnata, eglandulosa; stjli sparse pilosuli, gisis subplans, cor. rosea; fruct. ovoisens.

Hab. Rhone, ad tornas (H. Ganioger).

1472. Brepinix 1cleroirea Gogr mss.

Aculei inchinato-gilatati, ad ramos flor. Dense fohissos copiosissimi; fol. parva, oborats-acuta, basi attemuata, coriacea, is costam eglasisulosa, dentes simplices, profunio, triangulares, recti; pet. aculeati, laxe pubascentes, basi hinc inse glanoulosi, stip. virises, breves, sat angustare, auriculis subdivergentibus; per. 1-3, villosi; 7-10 m. longi; tubus orvidens; sep. pinnulis latirsculis, 1-2-Dentatis instructa, styli subglaberrimi, Disco pauls conico; cor...., fuct sat magnus, ovoives-conicus, fasi rotuniatus, apice strangulatus.

Hab. Eranssilvania, Eorda toranyos: as Eorocyla' (L. Tichter).

1473. Creninia semialabra (Mip. in Gép. Trim. I, p-1g et 59; Déseigl. Catal. p.204; Carist Etride aes fl. 6-è., 1879, II, p. 244), Gogr Gab. N.2203.

Acculer as ramos flor. inclinato subsilatati; fol. elliptica, viridia, as costam eglanoulosa; ontes simplices, convergentes; pet. subinermes, villosi, eglangulosi; stip glabrar, zuriculis girergentibus, ped. 1-3, pubescentes, g-13 m. longi; tubus oflsngus; sp. pinnulis longis, parum latis instructa; stygli hisuti, isco sat conico; cor.pallise rosea; fruct. ovoidens.

Hob. Savoie, ad toune; Haute-Pavoie, at Insecy-b-Gienx, Habre - Lullin (Duget), unte descripsi, et alifi in Gallix, ete.

1474. Brepinia Hanryana Gigr Tab. $N=2204$. 
$-120-$

toculei breves, jilatati, salive Oelatato-falcati, ad ramos flat geminati; fol. ample oborate, breviter acuta, basi rotuniata, coriacea, ad costam eglanoulosa; irentes simplices, triangulares, recti; pet laxissime villosi, aut sparse pilasuli, eglansalosi, parce aculeati, stip. virides, auriculis subotivergentifus; pes. 1-4, paulo pubescentes, 10-12 in. langi; tubus sbovato - oblongus; sep. pinnulis integis, angustis, acumine lato, instructs; styli hissuti, Uises valie conico; cor........ fruct. magnus, oborato- oblongus, utringue gepressus.

Hab. Bouches_du- Mhone, al Martigues (t. Tuthaman).

1475. Crepinia illigata Gige toab. N-2205.

toculei dilatato-subfaleati, id ramos flor. sparsi, fol-obovato-ellipticx, utrivase breviter attenuato, a costam eglañulosa, gentes simplices, cruvisuntes; het. nubentes, toto villosi, aculeati, eglaresulosi; stip. rebrae; pei. 1-2, prusciscentes, $14-16 \mathrm{~m}$. longi; tubus sblongus; sep......... styli villosi, Dises plans; cor....... fruet. oblongrs, basi depressus, apice attenuatus. Hab. Thine, Dardilly et Eculb (P. Chabert).

1476. Crepinia rossica Gogr bab. N:2206.

toculei parvi, inclinato- Dilatati, samos flor. geminati, fol. late sblong-lanceolata, basi subrotundata, inferne ai costam glandulosa i Dentes simplices, ovato-convergentes; pet. temiter villosi, eglanaulosi, inf inermes; stip. rubentes, auriculis rectis; pes. 1-3, pubescentes, 10-11 m. longi; tubus oblangus; sep......... styli villosi, Disco plano, cor........, fruct: magnus, oblongus, utrinque attemeatus.

Hab. Mossia accis., ad Mahilew (Laupmann).

1477. Crepinia Ponopica Gogr tab. N:2171.

Acculi breves, parri, falcati, omos flor sat copiosi, fol ample oblongo-rhomboidalia, utrinque sensime attennata, lacte virisia, ad costam eglanidulosa, inf. suporne pilis caducis ct raris obucta; dentes simphices, magm; pet. probscentes, eglanaulosi; parce aculeati, stip. longar, auriculis rectis; pes. 1-3, nume laeves, nume aspperse glanialosi, 10-14 m. longi; tubus obovato-oblongus, laevis; sep. Oarss sparse glandulose pinmelis latis, eglanidulosis instructa, acumine foliaces; styki lanuti, isco conics; cor pallide nosea; fruct.

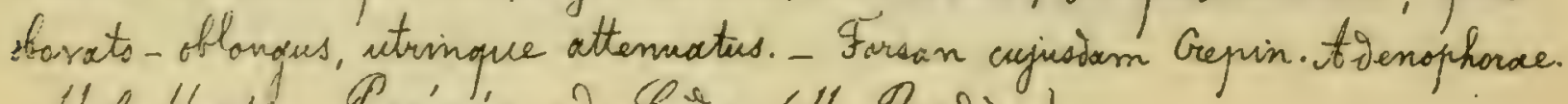
Hab. Hastas- Prénees, as Gedre (H. Fardère). 
$-121-$

2. Foliola annix biderrata.

1478. Crepinia anthophora Goge \&ab. N:2207.

treuler valide faleato-gilatati, al namos flot.copiosissimi; fol. late orata, basi rotundata, apice acuta, ad costam sparse aculeolato; gentes bisa. rati, lati, profunisi, pot. nune sparse pilasi, nunc pubasoentes, aculeati, paulo glanaulosi; stip. latre, glabrae, duriculis rectis; ped. 5-15, glabri; 13-17 m. longi; tufus freviter svatus; sep. pinmulis foliaceis, longis. 1-3Tentatis copiose instructa; styli magni, lanati, dises prauls conico; cor-alba, eciliata; fruct.magnus, subglobosus, basi rotuniatus, ofuce truncats-attenuat. Hab. Syrénées orient, at Serpignan (O. Debeaux).

1479. Crenimia gidymovonta (Gigr Ros: nor. II, p. 22), Gogr Eab. N-2208.Gillot exs. N-384g ax parte.

Aculei validi, falcato-gilatati, ad ramos flor. stipulares; foh anquste oblorgo-acuta, basi attemeata, ab costam intersum paulo glandulosa; ientes fiserrati, tennes, apati; pat. parce aculeati, villosi, sparse glandulosi; stip. breves, latae; ped. 1-3, glabri, 9-12 m. longi; tubus onatus; sep. brevia, pinmelis lacimiats-Dontatis copiose instructs, styli hirsuti, Disco sat conico; cor........ fruct. parrus, rotundatus vel subglobasus.

Hab. Rhone, at Hassin (Boullu).

1480. Crepinia affinita (Muget) in Gogr Eab. F-220g.

Aculei valisi, inclinato-gilatati, ar ramos flor. minores; fol. srato-rcuta, atrovirentia, ot costam intersum paulo glanaulosa; gentes biserrati, ciliati, pet. glaniallasi, aculeati, nune pubescentes, nunc glabrescentes vel sparse pilsoi, stip. glabrac, auriculis rectis; pet. 1-4, laeves, 11- $18 \mathrm{~m}$. longi; tubus op oidens; sep pionmilis latis, dentato-glaniulosis copisse instructa, acumine foliaces; stajli breviusculi, lanati, jisc plans, car. pallide rosex, fruct. ovoideus, utrinque depreossu Hab. Mhone, of Cullins et Charbonnieres (Boullu).

1481. Grepinia Euerkieswigü Gogr \&ab. N: 2210.

toculei parri, falcato-gilatati, as ramss flor.milli; fol parna, obovato polanga, acuta, basi subattemuata, id costam eglaniulosa; Oentes biserrati, aperto-triangulares; pet-villosi, parce glaniulosi, aculeati, stip. ruventes, auriculis parum givergentibus; per. 1-3, glabri, 11-13 m. longi; tutins ov sidens: sep. 
$-122-$

pirmulis paum latis, 1-2-Dentatis instructa, acumine lato, integro, styfei inllosi, Dites conics; cor. alb, eciliato; fruct. ovowiens, parum Depressus. Hab., Gous, at Le gigan (D. Eurzhiswion).

3. Folish alia simpliciter alia biserrata.

a. Ityli pauls villosi.

1482. Crepinia mesocarpa Gagr \$ab. N=2215.

tbculei parri, inchinato - Dilatati, as ramos flor. mulli; fol parva, oboratosubacuta, fasi sensim attenueta, at costam eglanialosa, Dentes inaequales, lanceslato-operti; pet. eglanoulosi, pubescentes, aculeati, stip. longae, angustac, auriculis rectis; pet. 1.2, glabri, g-11 m. longi; tubus ovoisens; sep.........; styli minores, paulo villosi, jisco plans, cor....... fruct. parrus, svoideus, basi notuniatus, apice attenuatus.

Hab. Halia arient. at Iaenzo (2. Calsesi).

1483. Gepinia glabrescens Gogr Gab. $N^{-} 2216$.

toculai recti, subilatati, cylinarici, it ramos flor. fere geminati; fol. oborato-oblonga, acuta, basi breviter attenuata, iocostam poulo glansulosa; Dentes fore onnes biserrati, lanceolato-aperti; pet. aculeati, hine inde glansulosi, Marse pilosi, stip. angustas, auriculis divergentibus; pes. 1-3, glabri, 13-15 m. longi; tubus oblongus; sep......... styli pauls villosi, gisco subplano; car....... fruct. oblongus, basi subrotunaxtres, apice attenuatus. Hob. Italix orient, at Faenza (2. Caliesi).

1484. Preninia Derbesiana Gogr tab. N-2217.

Arculei brevisencili, falcato-Dilatati, as ramos flor.copiosi; fol. late inato-elliptica, acuta, basi rotuniata, is costam sparse glaniulosa; dentes fore onnes simplices, ovato-convergentes; pet. eglanoulosi, villosi, aculeati; stips-siriges, auriculis subrectis; per.1-3, purbescentes, $7-g$ m. longi; tubus offengres; sap. pinmulis latis, 2-3-gentatis instructa, acumine subfoliaces-Dentato, styli exserti, paulo villosi, Disco valie comico; cor-eciliata; fruct. sblongus, utringue attenuatus.

Hab. Pouches - Du - Phone, at Le Réaltor (At. Abutheman). 
b. Ytyh glabri:

1485. Crepinia inaequalis Goger \&ab. N-2218.

toculei falcato-dilatati, as ramos flor-mulki fol obovato-oblonga, breviter acuta, basi attemuata, ad costam sparse glanoulasa; Dentes fere annes simplias, sublanceolato-aperti, pat. pubescentes, copiose glansulosi; inf. inermes; stip. virises, magnae, auriculis paulo givergentibus; pes. 1, glabri, 10-11 m. longi; tubus obovato - sblongus; sep. pinnulis amnibus integris, latis instucta; styli glabri, aises camici; cor........; fruct. obovato-oblongus, basi rotuniactos, as apram valte et abrupte strangulatus.

Hab. Eurol, in monte gasone prope Erente (\&. Gelmi).

\section{Grepimia pellita Gogr Eab. N-2219.}

Acculei breves, parri, falcato- Silatati, à ramos flor. mulli; fl. obovato-oblanga, scuta, basi abrupte truncata, id costam eglanialosa; gentes fere anmes simphices, lanceslato-aperti, pet. eglanioulosi, pulescentes, aculeati, stip. sirides, auriculis Sivergentibus; ped. 1-2, glabri, 12-14 m. longi; tubus ovatus; sep. pinmulis integris, linearibus instructa, acumine lato, integro; styli glabri, gises plano; cor....... fruct parvus, rotunsatus, as apicem brevissime strangulatus.

Hab. Anglia, ad York (G. Hebster).

1487. Crepinia tarraconensis Grogr bab. N-2220.

Abculei parri, breves; folcato- Dilatati, at ramos flor pauciores, fol. obovata, breviter acuta, basi truncata, or costam ealansulosa; Dentes fere comnes simplices, triangulari-subeonvergentes; pet. villosi, seulecti, inferne paulo glanoulosi; stip. sirises, auriculis givergentibus, pes. 1-2, glabri, graciles, 17-20 m. longi, tubus sblangus; sep. pinnelis parum latis, 1-2-Ventatis instructa, acumine integro; styli glabri, Disco conics; car........, fuct. oblongus, itrinque attemuatus.

Hab. Hispania orient. Earragone: ad Font Den Dorca (M. Bolois).

1488. Crepinia subcompositax Gogr \&ab. N.2221.

Abculei falcato-sublilatati, as ramos flor-copioss; fol ovato-elliptica,

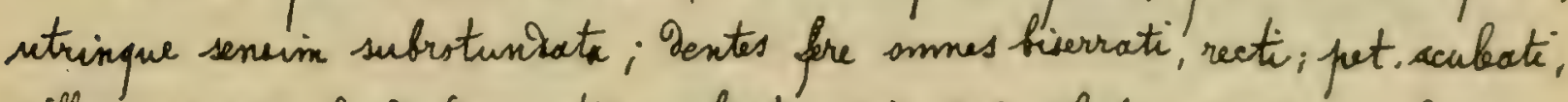
villosi, sparse glanoulasi; stip. rubentes; per. 1-2, glabri, 10-11 m. longi; 
tichus obovato-oblangus; seps. purmulis longis, parum latis, 2-4-2entato-glandulosis instructa; styli glabri, gisco plano; cor. prallive rosea, eciliata; fruct. obovats-oblongus, aprice attenuatus.

Hab. Inhone, at tornas (N. Gandoger).

1489. Crepinia transitoria (Gogr Iros.nor. II, p.22)i Gogr Eab.ros. N-2222. Giger Herb. ros. $N=165$.

ticulei asciles, subilatato-inclinatis, no ramos flor. Densos copiosi; fol. pbovata, utringue breviter attemuata, foliolo medio inferne aculeolato; Den- tes convergentes, inacque subbiserrati; stip. meniocres; ped. 1-3, glabri, 14-17 m. longi; tubus oblongus; sep. pinmulis latis, 2-4-gentato-glandoulosis copiose instructa; styli plerumque glabri, iiseo plano; cor. rosea, eciliata; fruct. orsidens vel obovato - blongus, basi suboepressus, aprice attenuatus. Hab. Ihone, ad tinas (M. Ganoger).

1490. Crepinia Cupaniana Gogr mss. - Rosa sp. Lojacono Pl. sicul rar. N-345. Accubi duri, parum faleatr, Dilatato-cylinarici, ad ramos plor. Danse foliosos copiosissinn; fol. minutissima, coriacea, orato-elliptica, subobtusa, basi. rotunisata; costa media glanoulosissima basique aculeolata; dentes profunisi; ovato - convergentes, fere onines simphies; pet rubentes, glanisulosi, valda aculiati, parce pubsecertes; stip. purpureae, breves, latae, auriculis parum vivergentibus: pet. 1. glabri, 5-7 m. tongiv; tulus magnus, oroisens; sep. pinmelis latis, 1-3 Dentatis copiosissime instructa, acumine foliaces, integro, styli glabri;, Disco plano; cor sat magna, eciliata; fruct. parrus, ovoides-conicus, basi depressus, aprie attemuatur.

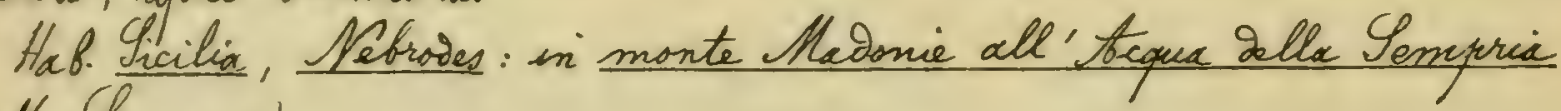
(No. Lojacono).

Spreies folislis minutissimis, cariaceis, potiolis acubatissimis, tebo magno, sepalis raloe pinnatis, etc. curiosissima.

c. Styli firsuti vel lanati.

† Discus planus.

1491. Crepinia unainella (Mass. Cat. hort. Cran. 1811, suppl. IV, p.2s et Cat. 1316,

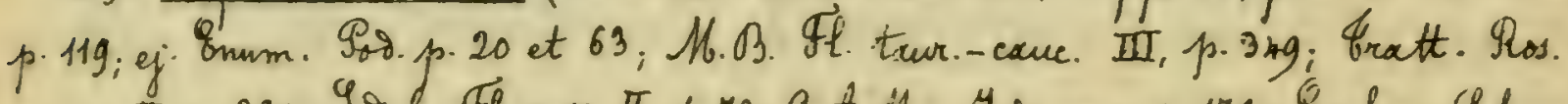

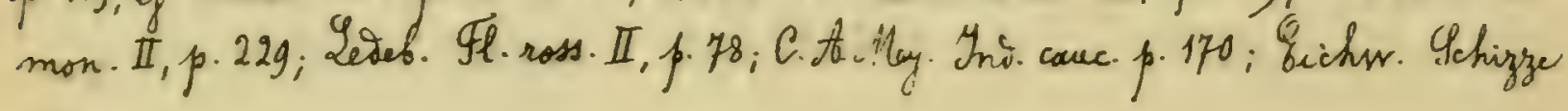




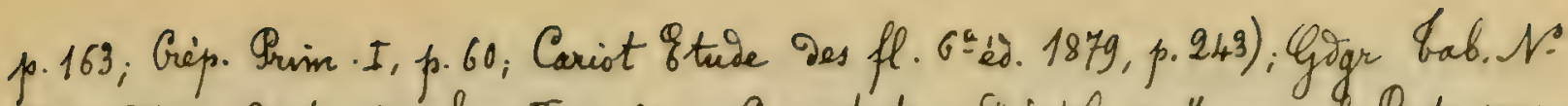
2211 ; Gogr Contr. fl. slar. II, p. 36 . - C. jactata (Disigl. in Joum. of Mot. 1874, ext. p.3, typo Bess. non Differt).

Acculei valiai, falcato-bilatati, ad ramos flor. breves vel nulli; fol. frarva, oncto-elliftica, breviter acuta, basi subattenvata; Dentes inceque subbiserrati; pet. villosi, inermes, eglandulosi; stip. glabrae, Denticulatae, auricutis acutes, givergentibus; pet. 1, pilosuli, $10 \mathrm{~m}$. longi; tubus ellipsidens; sep. pinmelis pectinato-subglanisulosis instructa, styli hirsuti, disco plano; cor. 4 cent. Siam. lata; fruct. allipsoiseus, utrinque attemuatus.

Hab. Povolic, ad Byram (Messer) at aliki.

Iperies, ut videtur, amodum rariabilis quum Besser fam undecion sarictates gius enumerat.

\section{Crepinia carpathorum Gogr mss.}

tcculei gebiles, parum dilatati, temiter falcati, ad ramos flor.copiosi; fol maxima, amplissime obovata, apice subrotuniata, basi attemucto- cuneata, glauca, is costam eglanivilosa, sentes magni, orato-convergentes, fere sonnes simplices; pet. breviter tomentelli, inermes, eglandulosi; stip. virides, maximae, auriculis divergentibus; pro. 1-2, glabri, 7-10 m. longi; tubus orato ellipticus: sep. pinnelis foliaceis, paulo ontatis instructa, style fere lanati, siseo subplans; cor.......; fruct maximus, ovato-ellipticus, basi rotunsater, apice hais aut vix Depressus.

Hab. Hungaria hr., Sipto': at Fenerpatak (2. Michter).

1493. Orepinia sphaeracalyx Giogr 6 ab. $\mathcal{N}^{\circ}-2212$

ibulei validi, falcato-dilatati, somos flor. Debiliores et geminati; fol. oblongo-lanceslata, fasi breviter attenuata, ai basin costare paulo glansulosa, Dentes convergentes, sup. simplices; pet. copiose glaniaulosi, acculeati, pubescentes ; stip. latac; pes. 1-4, glabri, 12-16 m. longi; tubus globosus; sp......; stagli hirsutissimi, majasculi, gisco subplans; cor......., fruct. magnue, globosus. Hab. Ihone, id Francheville (M. Chabert).

1494. Crepinia patulidens Clige mss.

teculic breves, gilatati, valie falcati, as ramos flor. pauciores, fol. sborats-acuta, basi notundata, as costam eglandulosa, grextes orato - triangulares, aperti, inf biserrati, pet pubescentes, eglanilosi, sus. inames: 
$-126--$

stip.sirions, médioeres, auriculis rectis; ped. 1-2, glabu; 4-5 m. longi; tubus. rotunsatus; sep. pinmulis pubescentibus, ammibus integris, angustis instructa; styli lanati, Siscs plano; cor.........; fruct subglobosus, utrinque rotunisatus. Hab. Sueciz, Halland: at Lallstorp (Y.t. Gabrielsson).

1495. Crepinia lururiosa Gogr mos.

Acculei meciseres, inclinato- dilatati, as ramos flot. ruberrimos stipulares: fol. ample obovata, vis acuta, basi rotuniata, crocosa, pallive virioi glavcescentia, nervosa, at costam glaniulosa; Dentes recti, late triangulares, fere onnes biserrati; pet. rubentes, gense tomentosi, glandulosi, parce aculeati; stip. ruberrimae, maximse, auriculis rectis; per. saepe 2, glabri, $1 / 2$ aret. lonsi; tubus rotundatus, sep. pinmulis integris, latiusculis instructe; styli lanati, sises plano; cor........; puct. sat parwus, globos-comicus, basi late roturiato. umbilicatus, apuice abrupte strangulatus.

Hab. Hungaria bor, gzenes: ad Löse (2. Richter).

t† Disens comicus.

1496. Crepinia robustior Gogr Bab. $N-2213$.

Aculei folcato-dilatati, as ramos flor. plus minus copiosi; fol. oboratoelliptica, breviter attenuata, basi subrotuniata, as costam eglaniulosa; sentes lati, subconvergentes, fere omnes simplices, pet. laxe prebescentes, aculeati, eqlaninlosi; stip. latae, peo. saepe corymbosi, villosissimi, 4-10 m. langi; trubus os oivens, in $1 / 3$ inf. pubescens, sep........ styli villosi, gises canico; $\operatorname{cor} . . . . . .$. ; fruet ovoidens, basi.gepressus, apice attemuatus.

Hab. Dhone, as Chaponost (P Chabert).

1497. Crepinia arnassensis Cgogr mss.

Aculei parum inclinati, subcylinarici, longr; as ramos flor.geminati; fol. oblongo-lanceslata, et acuminata, basi fere rotundata, glavescentia, ad costam eglanaulasa; Dentes fere onnes biserrati, sperto-triangulares; pet. glansulosi, pubescentes, aculeati; stip. angustae, virides, freviusculce, gorso pubescentes, aurriculis semisivergentibus; pes. 1-3, glabri, 6-8 m. longi; tubus obovato-sblongus; sep. pinnulis annibus angustis, 1-3-gentatis instructa, acumine integro; styli villosi, gisco sat conico; cor.........; fuct prarkes, oborato - oblongess, basi subrotundatus, apice longius attonuatus.

Hab. Rhone, ad Aornas (O). Granoger). 
$-127-$

1498. Brepinia Thichterii Goge 8ab. N-2214; Gogr Contr. fl. slar. II, p. 36. teculai brevissimi, falcato-gilatati, as ramos flot. nulli, fol. oblongo- acuta, fasi sensim attenuata, at costam eglansulosa; gentes fere smnes biserrati, onato-covvergentes; pet. villss; aculeati, eglanisulosi; stip. mubentes, latae, nuriculis subsivergentibus; per. 1, glabri, 13-15 m. longi; tubus ovoideus; seps. pinmulis foliaces-lacimiatis instructa, acumine foliaces; styli lanati, gisco comiso; cor.magna, eciliata; fruct. soidens, utrinque gopressus.

Hab. Hangaria, at Ofen (2. Michter); Croatia; Terbix, ad Melgrade.

3.- Pilosiusculace Gogr Essai p. 30; Goger Gxb. p. 168.

Folislis mediis ramsrum floriferorum supro glabris, subtus taxtum as nervos primarios normaliter pubescentibus, caeterum glaberrimis; serroturis plerumque omnins simplicibus.

Ytyli glabri vel vix isppersi pilosi

$1\left\{\begin{array}{l}\text { Styli paulo hirsuti } \\ \text { Sfyli villosi vel lanati }\end{array}\right.$

2

8

Fructus obovato- sblongus vel oblongus

2 Fructus rotuniatus, ovatus vel ovoîdeus.

15

Tesunculi glabri

3. Sesunculi villosi

Fuctus obsvats-oflongus vel suboblongus

ir Fructur oblongus

3

Irecies N-149g-1506.

4

Upecies N:1507-1508.

Discus comiens

5 Discus plannes val subconicus

5

Disens plus minusse coniens

$\sigma\{$ Discus planus

Foliola elliptica rel oborato-oblonga

7 Foliola oblonga

Fructus ovoivens vel obovato-oblongus

Fructus sblongus

6

Qpecies $N^{\circ} 150 g-1511$.

Srecies N $1512-1517$.

7

Irecies $N-1518-1520$.

Species $N-1521-1523$.

Gpecies $\mathcal{N}: 1524-152 \mathrm{~g}$.

9

13

$8\{$ Fructus globosus

Fructus ovatus val ovato-rotundatus

Qpecies $N: 1530-1533$.

Folioke obovata val slovato-sblonga

$9\left\{\begin{array}{l}\text { Foliola oblonga } \\ \text { Toliol }\end{array}\right.$

Species N-1534-1539.

10
12

Foliola ovata rel alliptica.

$\dot{S}_{\text {preces }} N=1540-1544$. 
Foliola inaque biserrata

10 Foliola ommino simpliciter serrata sol prorsus biserrata Ipecies N-1545-1548. Folisla basi rotunidata

11 Foliola basi attenuata

$12\{$ Discus praulo conicus

Dentes omnes simplices

$13\{$ Dentes alii simplices alii biserrati

Pasunculi villosi

$14\{$ Seannculi glabri

Fructus obovato-oblonguis vel oflongus

Fructus globosus

$15\left\{\begin{array}{l}\text { Fructus svato-rotuniatus vel ovatus } \\ \text { Fructus suborsidens }\end{array}\right.$

Pesunculi glabri

$16\{$ Sesunculi villosi sel paulo glandulosi

hecies $\mathcal{N}: 1549-1550$.

Irecies $N^{\circ} 1551-1557$.

Gecies $\mathcal{Y}^{\circ}-1558-1561$.

ipraies $N=1562-1566$.

Species $f: 1567-1571$.

Grecies N:1572-1574,

Grecies $\mathcal{N}=1575-1582$

16

Ipried $1=1583-1586$.

Inecies $N: 1587-1593$.

Species $\sqrt{-1594-1596 .}$

17

Ipecies $\mathcal{N}: 1597-1604$

Foliola onnia simpliciter serracta

$17\left\{\begin{array}{l}\text { Foliola alia simpliciter serrata alia necne } \\ \text { Foliola omnix biserrata }\end{array}\right.$

Foliola ovatx, elliptica, obovata vel obovato-oblonga

18 Foliola sblonga

Ineries N:1605-1606.

Ipecies $\mathcal{S} 1607-1612$.

Ipecies $N: 1613-1617$.

Ipacies N:1618-1622.

1. Ityh glabu sut nix asperse pilosi.

a. Fructus rotandatus, oratus vel ovoidens.

1499. Crepinia avenostephnna Gogr \&ab. N-2223.

teruli inclinato-gilatati, as ramos flor copiosissimi; fol-obosato-acuta, basi attenuata, as costam inferne paulo glaniulosa, gentes triangulari-convergentes, fere omnes biserrati; pet-villosi, rculeati, hinic inde glandulosi; stip. rubentes, auriculis rectis; per. 1, glabri, 6-7 m. longi; trubus orviteus; sep. pismulis smmibus foliaceis, valte gentato-glanoulosis, acumine ample foliaces; stgli glabri, Dises conics; cor - eciliata; fruct. ovoidens, utrinque paule gepressus.

Hab. Peamont., is gelleer Lavioises (E. Rostan).

1500. Crepinia latipetala Gige Lab. N-2224.

Aculei minuti, faleati, a mos flor.nulli;"for umplissime elliptivo- 
$-129-$

obtusinscula, basi rotiunista, costain eglandulasa, Gentes simphies, orato-convergentes, pet. tamentosi, aglaniulosi, inermes; stip. latae, virides. auriculis convergentibus; pès. 2-5, daviri, g-11 m. longi; tubus orsideus; sep. pinnulis latiresculis, 1-2-gentatis instiucta, rcumine foliaces; styli subglaberrimi, ojisco plans; cot. smplissirma, cciliata, fruct. maximus, ovoiseus, basi subrotuñatus, ais spiom breviter attemuatus.

Hab. Hautes- Prénés, à Mavère (H. Mardère).

1501. Erepinia perflata Gogr mos.

Aculei miviores, Dilatati, temiter falcati, ais ramos flor. ruberrimos plerunnque melli; fol amplissime oblongo-reuminata, basi rotuniata, viridir, ad costam eglandulosec; ventes simplices, lanceolato-subeonvergentes, pet. tirrentisi, rubentes, inermes, eglanioulosi, stip. nubentes, maximae, auriciclis sulivirergentious; per. 1-2, glabri, $5-7$ m. longi; tubus ovitens; sep-pinnulis latis, sub-

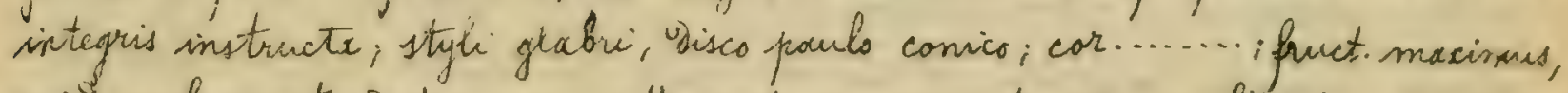

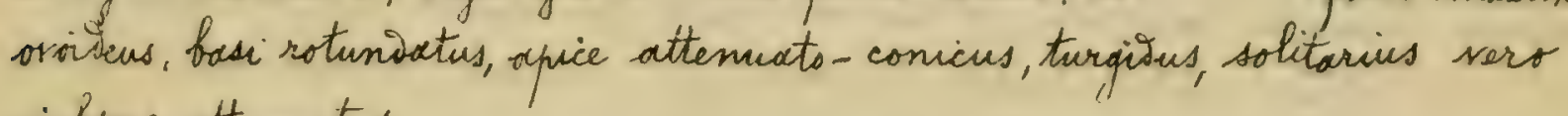
interne attemates.

Hab. Hungarix for, Izenes: ad Lacse (2. Aichter).

1502. Crepinia anglorum Gigr Lab. No 2225.

taculei breves, parvi, temiter falcati, as ramos flor gaminati, fit olovate-

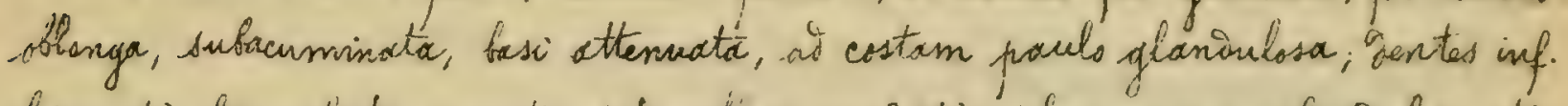
fiserrati, linceolato-aperti, pet, vilioni, cenleati, sinferne parse glansulosi; stip. basi rubentes, latie, auriculis divergentibus, per. 1. glabre; crassi, $12-14 \mathrm{~m}$. Congi;

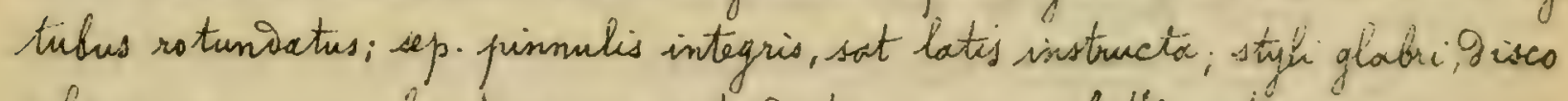
plans; cor....... finct parvus, rotundaties, aprice sufattionuatus.

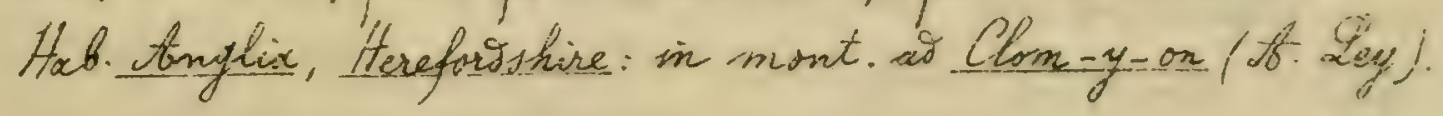

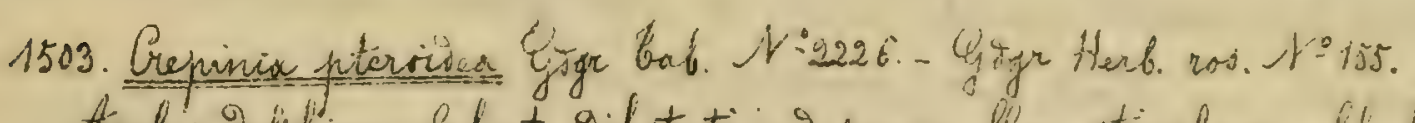

ticulei Defiliones, falcato-gilatati, as ramos flor. stipulares; fol firma, atrovirentia, late obovata, utrinque punlation altemasta, sit costam eglariou-

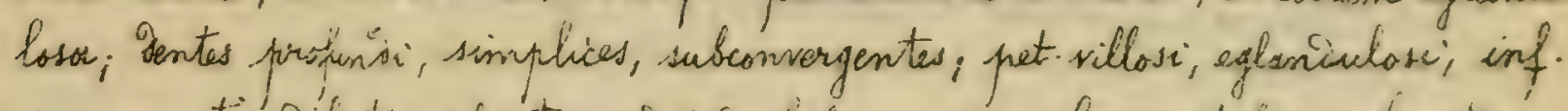

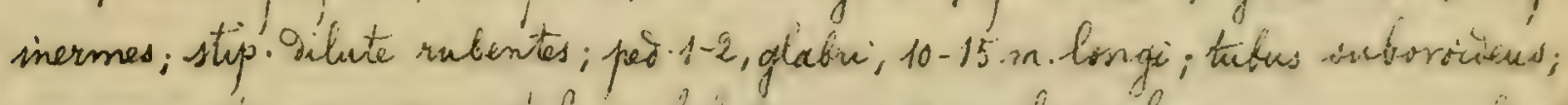

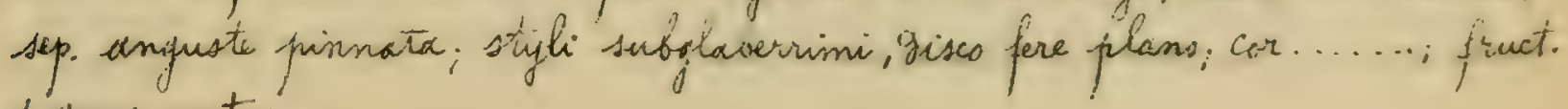
parsus, avatus.

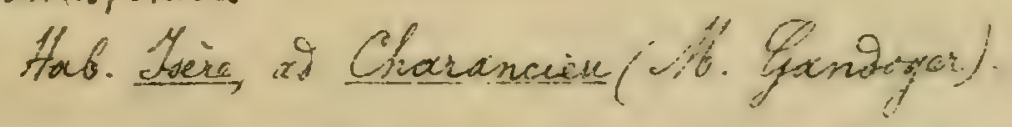


$-130-$

1504. Prepinia carpinvides Goger mss.

teculei breves, falcato- Lilatati, as ramos - flor nulli; fol ample oblongs, breviter acuta, basi rotuniate, id costam eglamivilosa; dentes omnes biscrrati, late subpatuls - triangaloaes; pet. puebescentes, glainoulosi, inermes; stip. latas, brests, bai mubentes, auriculis rectis; pes. 1-2, glubri, 14-16 m.longi; tubus el lipticus; sep. pinmulis onnibus foliaceis, 3-4-gentato-glaniuclosis instructa, acumine dentato, styli glabri, pouciores, laxi, disco longe conics; cor. fruct. asato-ellipticus, utringque subroturioatus.

Hab. Hizrania bor. Navarra: ad gertis (\%. H. Lacoizqueta).

1505. Creminia folïgera Googr Eab. N-2227.

teculei gebiles, parm dilatati, falcati, as ramos flor. valie foliosos geminati, fol. parva, anguste obovato-sblonga, utrinque attenuata, supra pallide niribix, subtus albisa et as nervos villaso-farinosa; gentes simplices, conrergentes; pat. cinores-tomentosi, aculeati, eglanoulasi, stip. latiusculae; pes.1-3, glabri:, 7-10 m. longi; tubus breviter svoidens; sep. pirsmulis pubscentibus, brevibus, subfoliacris copiose instructa; styli subflaberrimi, gisco paulo convic; cor palline rosed, hand ciliata; fruct. plerusnque ovatus.

Hab. Duy-je-Dome, ad Montbunbir (M. Iubosclaro).

Speais induments ad nerros albo-farinoss sane curiosa.

1506. Crepinia vasconica Gogr Herb. Pyren. (1884), p.69.

toculei breves, auri, parum falcato - illatati, somos flor geminati, fol. ollonga, freviter linceolata, basi truncata, glaucescentia, do costam eglanioulosa; dentes onses biserrati, lancealato-aperti, triangulares; put. villosi, aculecti, sat eopiose glanioulosi; stip. breves, virives, gorso villosac, auriculis rectis; pad. 2-7, villosi; 17-20 m. longi; tubus ovato-ellipticus; sep. pinnulis brevibus, sillosis, latis, paulo sentatis instructa, styli glabri, pauciores, gises valive comico; cor....... fruct. sat-parrus, ovato-ellipticus, basi sepressus, as apicar breviter attemuatus.

Hab. Sousses - Iyrénies, à Corbares (Mto. Ganooger).

b. Fructus sborato- oblongus vil oblongus.

+ Peanculi sillosi.

1507. Crenimia pilosella Gogr Eab. Ni2228.

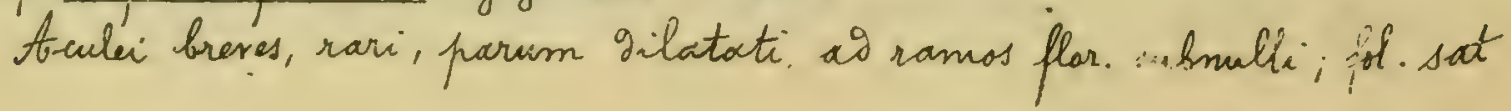


$-131-$

late ovato-elliptica, brevissime attenuata, basi notuniata, lucic atrovirentia, ad costam eglanioulosa; Dentes simplices, subeonvergentes; pet. satlosi, eglariulosi, aculeati, stip. Senticulatre; pas. 2-3, sillasi; 5-8 m. longi; tubus sblongus, basi pubescens, sep. pimmalis latis copiose instructo; styli glabri, disco valix comico; cor.magna, alba, eciliata; fruct. breriter oblongus.

thas. Ahone, at Charbonnieres (I. Chabert).

1508. Crepinia modendron Ggge \&ab. N-222g.

Acculai valioi, falcato-Dilatati, ad ramos flor.capissi; fol. ample obovatooblinga, acuta, bai rotuniata, as costam eglanialosa, ientes simplices lancolato-convergentes; pet-eyínivulosi, tomentosi, aculeati; stip. basi rubentes, latar, auricilis divergentibus; per. 1-2, pubescentes, 5-7 m. longr; tubus oblongrs; scp. pirmulis omnibus integerrimis, foliaceis instructa; styli subjlaberrimi; sisco pilano; cor eciliata, fruct-sblongus, ptrinque longe attenuatus. Hab. Helvetix, at Lehnebelhom prope Lurich, alt. 4000' (K. Lehmann).

t+ Tesurculi glabui.

\$. Fructus abovato-oblongus vel suboblongus.

$\sim$ Discens consius.

1509. Crepinia Costar Gogr \&ab. N-2230.

Aculei recti, Dilatati, sid ramos flor.coprissi; fol. parro, obovato-subacutta, bresi notuniacta, so costam vix glaniaulosa, gentes inf biserrati, lancedatsconvergentis; pat: prorsus glanduloxi, sillasi, aculeati; stip. virides, auriculis Divergentibus, pes. 1-2, glabii, 14-15 m. longi, tubus sbovato-sblongus; sep. pimnulis smmibus latis, 1-2-gentatis instructa, acumine lato, integro; stygle subglaberrimi, dises conici; car eciliats; fruct. sbovato-oblongus, intrinque depreassus.

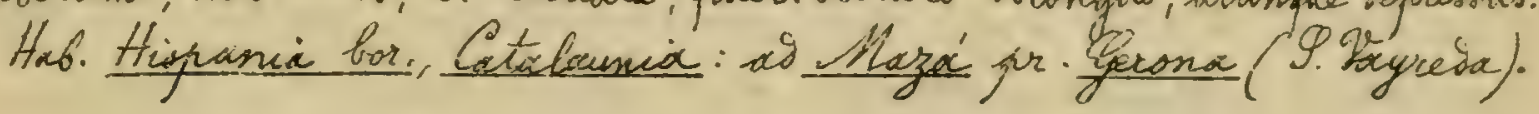

1510. Crexinix racemulosa Gjogr Bexb. N-2231.

Stculei Debiles, parum gilatati, xo rannos flor. recti vel inclisuati; fol obovatooblongk, ucuminata, basi breviter attenuata, as costam eglasinulosa; ientes simplies, resti, pet eglanaulosi, pubescentes, aculeati, stip. majusculor, auriculis

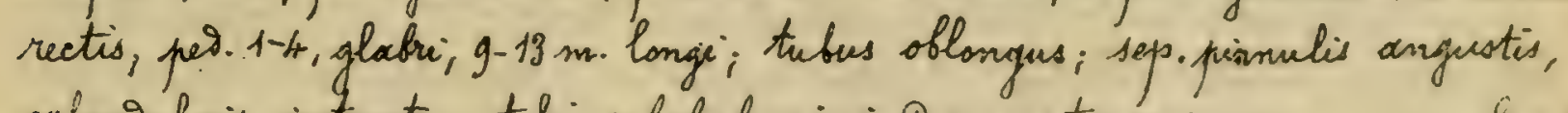
eglaniulosis instructa, style subglaberrimi, desco sat conico; cor....... fruct. parns, brato - oblongus, apia peasertion attenuatus.

Hab. Haute-Sanie, al Mont-Sion (Miget). 
1511. Cremina Groalii yogr bab. N-2232.

Acule tenviter et vald falcati, ad ramos flor. geminati; fol. oblanga, abrupte subacuminata, fasi attenuata, costam eglariulosa; ientes sati, recti, simplices; pot. pubescentes, sglaniclosi, sup. inermes; stip. longar, virides, auriculis rectis; per. 2-4, glabri, 14-16 m.longi; tubus obosatio-ablongus; sip. pinmulis subintegris, longis, parrum latis instructa, acumine integro, lato; styli glabri, laxi; gisco sat conico; con........ fruct. suboblongus, basi Oepressus, apice strangulatus

Hab. Basses - flpes, as Bonvolieres (Proal).

$\sim \sim$ Disus plarns vel subcomicus.

1512. Cresinia camptomorpha Gigr \&ab. N-2233.

faculei falcato-gilatati, id ramos flor. flesuoso-ercuatos sat copiosi; Frl. oborata, breviter acuta, basi rotundata, ad costam eglandulosa; dentes simplises, recti; pot.oglandulosi, villosi, inf inormes; stip. medioeres; pad. 1-2, slabri, 17-22 m. longi; tubus oblongus; sep. elongata, pinnulis onnibus $l_{x-}$ triesulis instructa; styli subalabcrimi, Dises plano; cor.rosea, eccliata; fruct. oforato-oblongus, phus minusve depressus.

Hab. Thane, ad tornas (N. Gandoger).

1513. Givinia subbiserrati yogr bab. N-2234.

toculei breves, cilatati, rectivisuli, as ramos flor geminati, fol acute sforato-shlanga, basi attoncata, intense virisia, as costam eglandulosa; Dentes fire omnes biserrati, aporti, lancerlati, pat. puleseentes, sculeati, sparse plansulosi; stip. anquitae, Darso pubescentes, auriculis civersentibus; ped. 1, glabri; 12-14 m. longi; tubus sforats - oblongus; sep. pinnulis brevikus, latis, 2-3-Oentatis instructa; styli asperse pilosuli, aisco subplano; car....... f fuct. oborato - oblongus, apice strangulatus.

Ha6. Loire, in monte Silat ad la Madeleine, $3800^{\circ}$ (N6. Gandoger).

1514. Brepinia crecta Goar mas.

Aculai valiai, subiliatalo-falcati, as ramos flor pherumque nulli; fol. arnple forvatio - oflmga, utringie paulation attenuata, taute viridia, is costan eglarisulose; Dentes late ovato - convergentes, inf. fiserrati; pet. tomextosi, hine iño glangulosi, inermes; stip. virites, magnae, suriculis subrectis. 
$-133-$

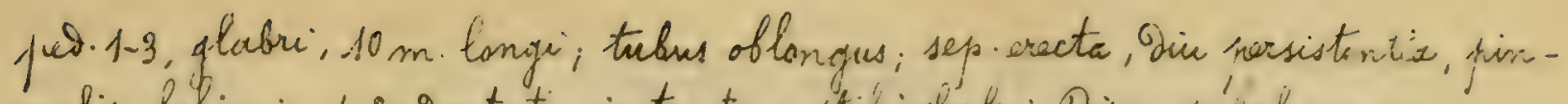
mulis foliaceis, 1-2-gentatis instructa; stigh glabri, Disco subilane; cot....... fruct. magnus, subsiblongus basi paulo atteneratus, apice strangubato-entracties. Hob. Hungaria bor., gyag: ad hovicicac (L. Richter).

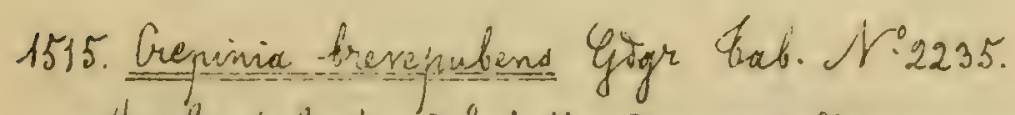

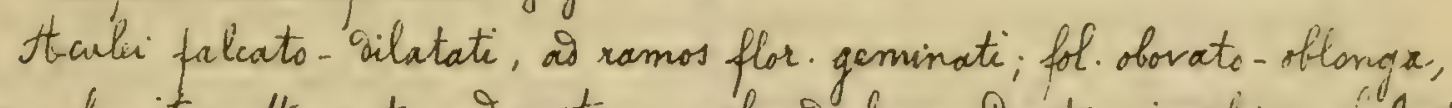
utringue burites atternsata, ad costam eglaniulosa; Dentes simplice, pleatoconverqentes, pet. villasi, eglanioulosi, inermes; stip.magnae, auriculis rectis;

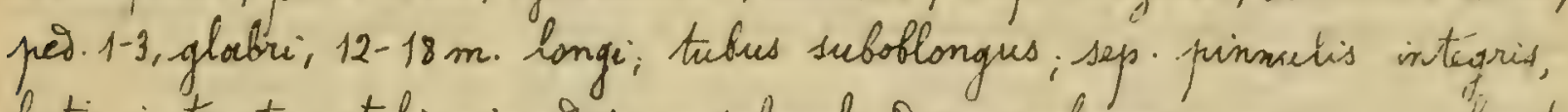
latis instructa; styli vix oissperse pilssuli, Disco subcomico; cor........ fruct. magnus, plerumque subobiongus, utringue Depressus.

Hob. Hrute- Savoie, as Habere- Doche (Miget).

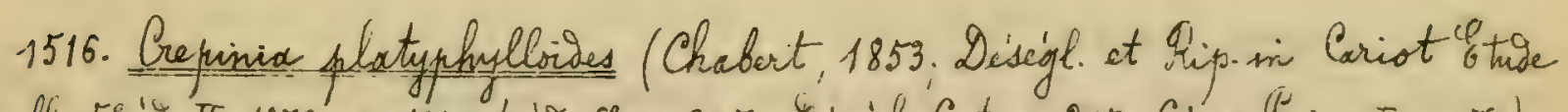

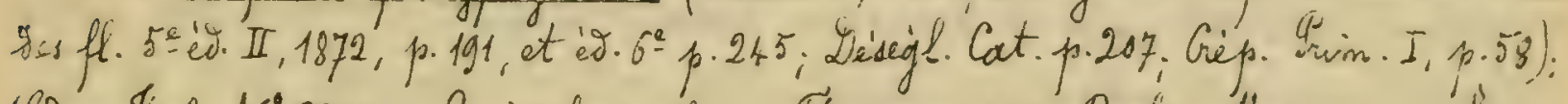
Gogr tab. N: 2236. - C. implexa (Gren. Ft. jur. p. 238; Baker Mon- ros in Lirn. journ. XI, p-235); C. sotstitialis rar Demidata (Gren. FP. jur. I, p. 238). - 93aker Herb. brit: ros. N-17. Gigr Herb. ros. NY-503.

Fonlei pauciores, falcato- Dilatati, ad ramos flor plesumgue rulli; fol. ample oblonga, utringue breviter attemuata, ao costam egitarioulosa, ger tes simplices; pet inermes, eglanioulosi, villosi; stip. angusstac, auriculis Cakis,

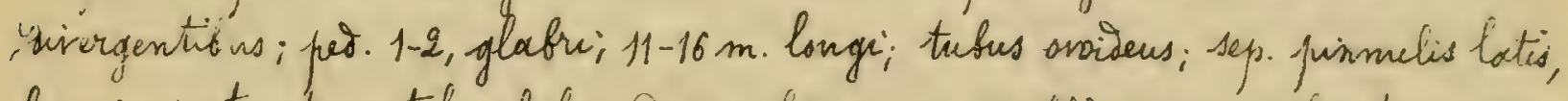
longis instructa; stgli glabri, brisec pliano; cor pallive rosea; fruct. magnus, ovoidens, getressus.

Hab. Rione, wo Charbonmieres (I. Chabert!) t alibi in Envog ax.

1517. Gepinia filopes Gogr tob. N-2237.

taculei breves, parvi, parum falcato-gilatati, ai ranos flor. sat iniosi, jol. oblongs-acuta, basi bresiter attenicata, as costam eglanaulose; Dentes subrectis, simplices; pet aculeatr; in lerne sparse glanioulosi, puberiontes; stip.

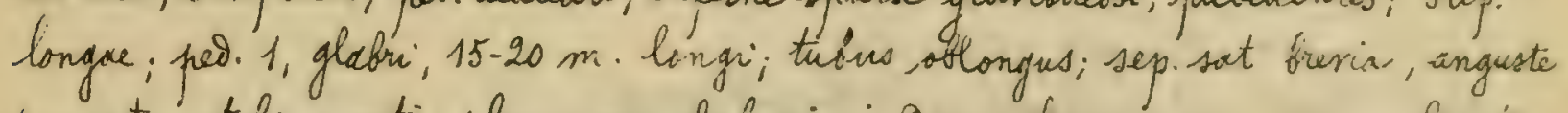
hinnata; styli exserti, plerumque glaberrimi, gises plano; Cor........ fruct. sborato-oblongus, basi saine valie athematius.

Hab. Isere, do Charancien (No. Ganoger). 
$-134 \ldots$

\$\$. Fructus solongues.

$\sim$ Siscies plames.

1518. Crepinia cyanocalya ('Gogr Essai p. 31 nomen solum; Gojgr Ros. nor. II,

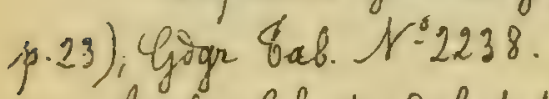

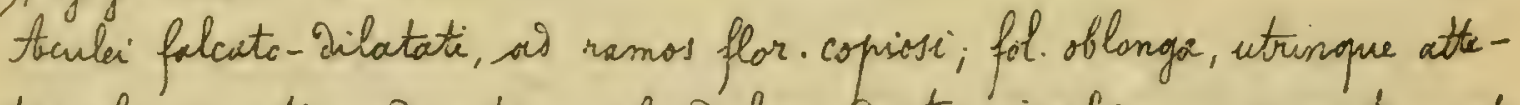
nuata, glaucescentio, is costam sglandulosa; isentes simplices, cosnvergentes; pet.

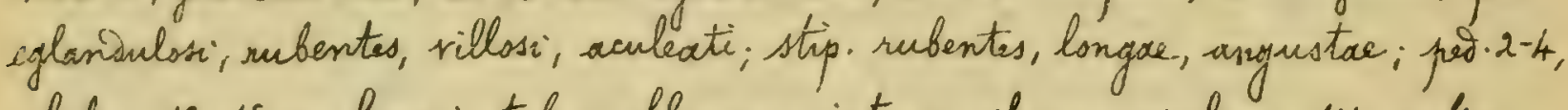
glabri, 13-16 m. longi; thebus sblongus, intense glanco-coeruleus; sep. rubra,

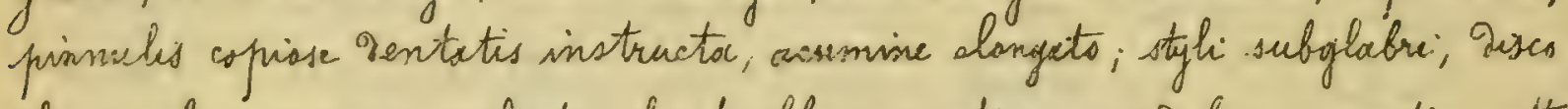
plaminsculb; cor. rosea, eciliata; fruct. oblongus, utrinque ses basi pracerertion atten.

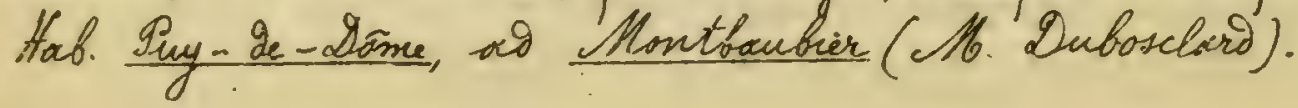

1519. Prepinia hamate Coogir mis.

Acculei silatati, vative et longissine foleato-hamati, ad ramos flor. saepries mulh; fol sat late solongo-subacuto, basi sensim attemuata, lucise et a.moene viridia, at costam eglarimulosa; Dentes simplices, lanceolato-recté; pet. rubentes, tomentosi, inormos, eglanivulasi; stip. rusure, mesiocres, auriculis semidivergentibus; pes. 1, glabui, 1 cent. longs: tubus oblangus; sep. pinnulis latiusculis, subventatis instructa, stigli glabsi, Discs plans; cor........., fruct. magnus, oblongus, basi subrotunisats-umbilicatus, apuce sittenuatus valdeque strangulatus.

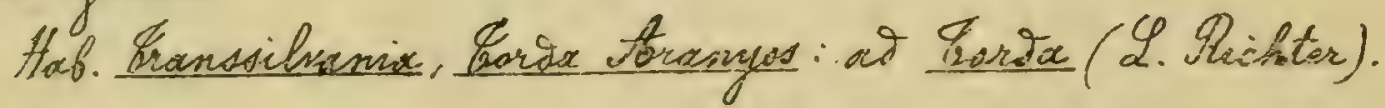

1520. Brenimia calycochlamys Gogr \&ab. N-2239.

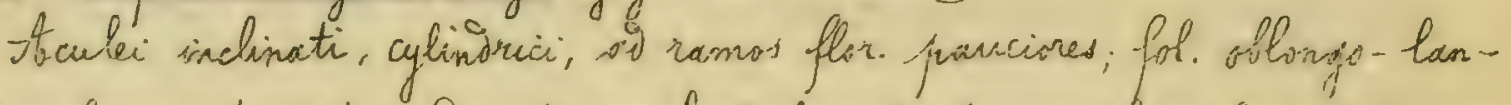

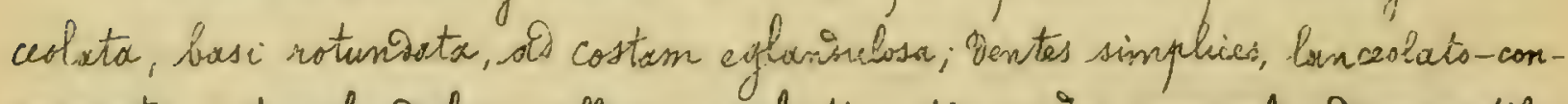
vergentes; pet. eglanacelosi, villasi, aculeati, stip. virites, aurciculis givergentibus, pes.1, glabri, 6-7m. longi; twhus oblongus; sep. pinmulis snmibus laciniatis, Silatatis instructa, ucumine foliaces; styli glabri, Dasco plano; cor eciliata; fruct. oblongus, utrinque attenuatus.

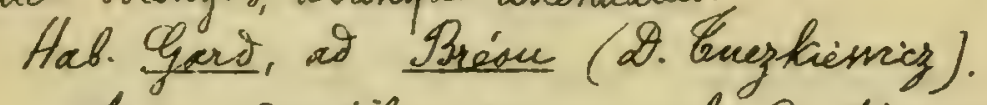

A procecestentibus prims occulo isitinguitur sepalis magis Dilatatis et aculesrum forma. 
$-135-$

$\sim \sim$. Discus plus minusve comicus.

$X$ Foliola cllipitica val obovato-oblonga.

1521. Brepinia glandulasella Gogr Eab. $\mathcal{N}-2240$.

Aculei breves, purum falcato-gilatati, ad ramss flor geminati, fol obovatosolonge, utrinque attenuata, pallide ylauco-virivia, at costam parce glantalosa; dentes lancealats-convergentes, fere onnes biserrati, pert. aculacti, laxe pubsesentes, sparse glantulosi; stip. longace, aurioulis rectis; pres. 1-2, glabii. 11-13 m. longi; turbus oblangus; sep. pinmulis latis, 3-6-gentato-glanidulosis copiose instructa, acumine longissima, lato, gentato; styli glabri; Disco pauls conics; cor......, furct. oblongus, basi attemuatus, apice longissine strangul. Hab. Italia orient, at Faenza ( 2 . Calvesi).

1522. Crepinix amplidens Gogr mss.

tbculei ralivi, falcato- Dilatati, as ramos flor geminati, fol. latiusculc obovoto-oblonga, aenta, basi subrstuniats, so costam parcissime glaniulose; ientes profunive triangulares, subpatuk, inf. biserrati, pet. longe pilosi, aculeate, hine inae glasiaulssi; stip.latissinac, sat breves, vïides, auriculis givergentibus; per. 1-2, glabri, $15 \mathrm{~m}$. longi; ticbus oblongus; sep. pinnnelis ammibus integris, sat latis instructa, styh glabri, giseo paulo conico; cor. magna, eciliata; fruct. anguste oblongus, utrinque attemiatis.

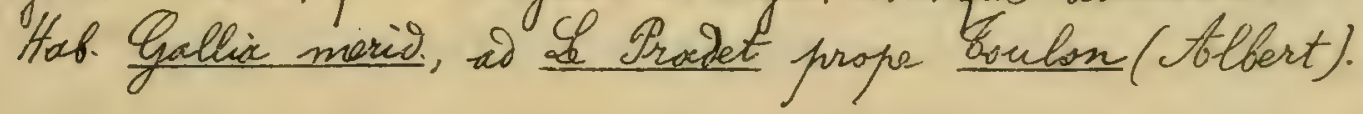

1523. Geninix ellipties Gogr Goub. N-2241.

Aculei falcato-dilatati, as ramos flar panciores; fol. late ellipticorentiuscula, basi rotuniata, as costam eglaniaulosa; gentes simplices lanceolato-aperti; pet-tomentosi, eglanioulosi, parce aculeati; stip. latissimae, sinides, auriculis givergentibus; par.1, glabri; 13-15 m. longi; tubus obovato-sblongus; sep. pinnulis smibus latis, lacimitis instructa, acumine late foliaceo; styli glabri, Disco comio, cor. eciliata, fruct. oblongers, utringue attermatus.

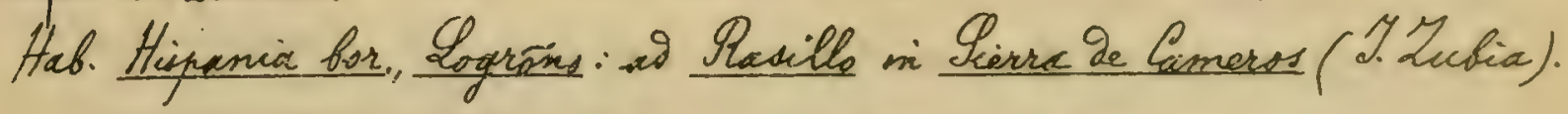

XX Folisla oblanga.

15.24. Greminia Parlatorena Cogr Erb. N-2242.

Acculec parvi, freves, primm folcati, ad ramos flor. pauciores; fil of - 
$-136-$

longi- anceoluta, basi attenuata, a costam eglandulosa; ientes lanceolatorecti, inf. biserrati; pet. glaniulosi, pubescentes, aculeati, stip. virives, auriculis rectis; pes. 1. glabri, 10-11 m. tongi; tréns oblongus; sep. pinnulis Cacinictis, intis instructa, acumine foliaces-laciniato; styli glabri, disco tat conico; cor. sciliata; fruct. breviter oblongus, apice attenuatus.

Hab. Atalia orient, ac Brisighella prope Faensa (2. Calvese).

1525. Crepuina minuta yogr \&ab. N:2243.

Aculai gebiles, inclinato-Dilatati, a ramos flor. copiosi, fol.conferta, prawa, sblongo-acuta, fasi attemuata, glaucescentix, is costam eglandulosa; orentes simplices, lanceoleto-aperti, pet.eglaniulosi, pubsecentes, aculeati; stip. longar, suboilatatae, auriculis rectis; pet. 1, glabri, 5-6 m. longi; tubus gracilis, oblonqus; sep. pirnmlis brevifus, latis, 1-2-bentatis copiose instructa, acumine la tinsculo; sty harvi, sub́glaberrimi, osisco paulo conico; cor parva, paltide roses, eciliata; fruct. parrus, anguste oblengus, entrinque attemiatus.

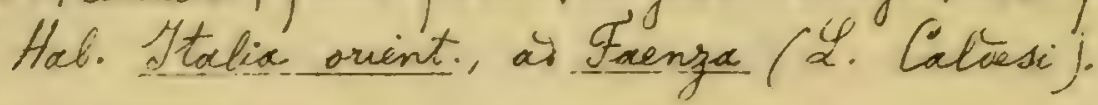

1526. Creninia Corvaliana Gyar mss.

Aculiei parvi, temes, inchisato-sub-eilatati, as ramos fisi. pranciores;

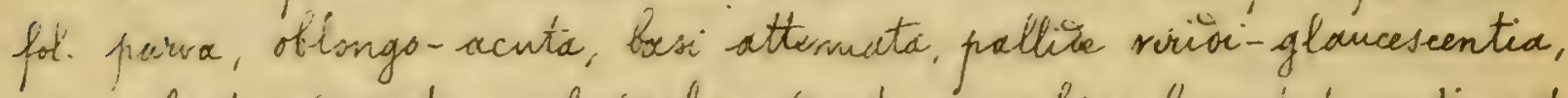
saspe phiata, as costam eglanioulosa, Gentes simphies, lancesiato-recti; pot. glanioulosi, villosi, inf inermes; stip. Latae, sat treves, rubentes, auriculis rectis, per. 1-2, qlabivi, 1 cent. longi; tabus stongess - sep. pinnulis numerosis, imnilus latiusculis, 1-2-gentatis instructa, styli glabie, Gdisco pauls coneics, car. majudcula, rosex, eciliata; fruct sat magnus, oblongus, basi vepressus, oc spicem abrupte attemeatus.

Hab. Damne-et-Loire, at It Laurent-I'Jivionay (Nt. Eranooger).

1527. Crepinia Decurrens Gogr Kab. N-22k4.

tivilie octiles, falceto-ilatati, à ramos flor.sparsi; fol. oblongo-acuta, Husi attenuata, viridix, as costam eglanioulosa. Denites simplices, lanceslato-subconvergentes; pat. sillosi, sup. inermes, inf: hine inje glanisulasi; stip. Latae,

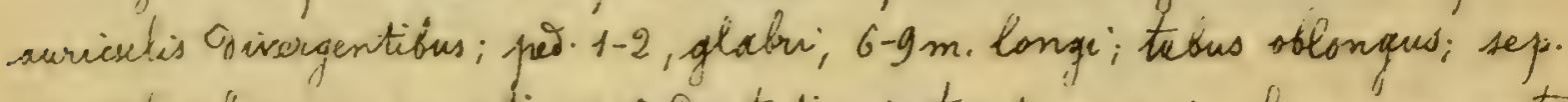
hinmaris longis, angustis, 1-2-dentatis instructa, acumine lorgo, arywisto:

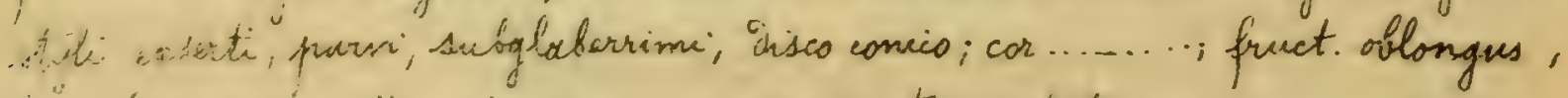
hasi longistime attenuate - oeverrons, apice stranqulatus.

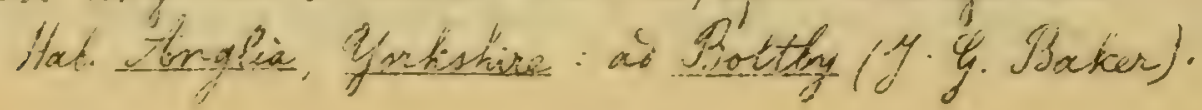


$-137-$

1528. Crepinia psilogyna Gogor mss.

Acculei tenues, subilitatate, minute faleati, aes ramos flor nulli; fol ample

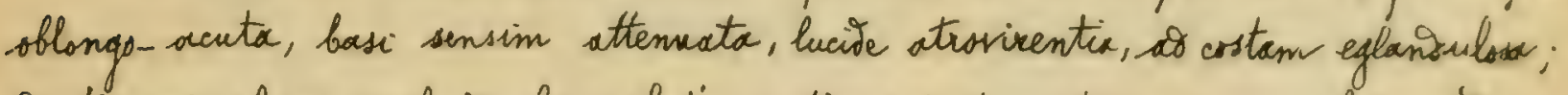
Dentes simphices, frofunise lanceslati, recti; pet. tomentosi, mermes, hinc inde

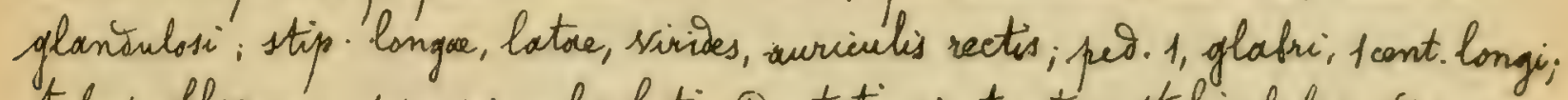
tubus oblangus; sep. pinmulis latis, Dentatis instructa; styli glabri, ciases comis; cor....... fruct. maximus, oblongus, utrinque longe attemeatus, turgiones. Hab. Hungaria, is Leasifalu (2. Richter).

1529. Grepinia cantalica Gigr Lab. N-2245.

Acculei parvi, breves, parmon falcati, is ramos flot panciores; fol. ample oblongs-acuta, basi attemusta, ad costam eghanaulosa, gentes simplices. lanceolato-aperti, pet eglangulosi, tomentosi, subinermes, stip. vinwes, maximoe, aurieulis ivergentibus; per. 2-4, glabui, 13-15 m. longi; tubus oblongus; sep. pirnmalis anmibus integerrimis, latis instructa; styli unblaberrimi, disco convic; cor eciliata; fruct-oblangus, utrinque attenusctus.

Hab. Cantal, in nemore Lioran, alt. 4300' (F. Hécifuend).

2. Ityli paulo hirsuti.

a. Fructur globosus.

1530. Gepinia obesa Gögr 8ab. N22246.

Accule panciores, validissini, falcato-Dilatati, al ramos flor mulli; fol. firma, ample obovato-obtusiuscula, basi breviter truncata, intense virisia, ad costam eglanioulosa: Dentes simplice, lati, triangulari-convergentes, pet. inermes, villosi, eglandulasi; stip. magnde, auriculis divergentibus; ped.2-3, glabri, g-11 m. longi: tubus rotumoatus; sep. pinmulis latis, 2-g-Odentrivis.

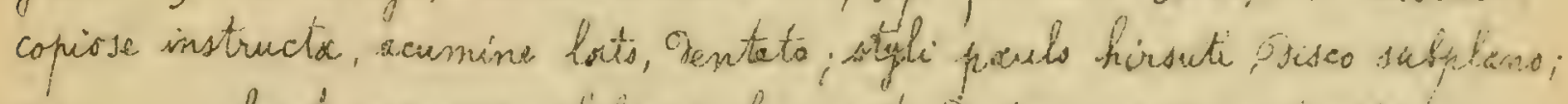
cor....... pruct. magnus, glovosers, basi rotunatus, apice contractus. Has. Haute- Invoie, in valle Leschance prope Itonnew (Moverier).

1531. Brepinia hamiglancs çogr Bab. N224\%.

Aculei parni, mchinati, jilatati, ai ramos flor capioss; fol. Oblongoreuta, basi roturibata, subtus glauce, ad costam eglaniulosa, dentes simplices, triangulari-convergentes, pet inermes, exlanduloss, villoxi; stip. hatre,

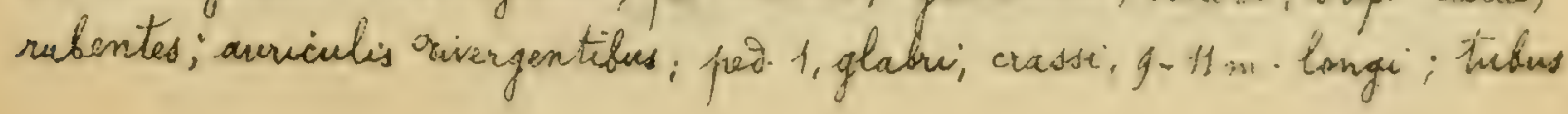


rotuinoatus; sep. pinmulis latis, 1-2-dentatis instructa; styli puebescentes, Disco plano; cor........ fruct. magnus, globosus.

Hab. Anglia, Hereforishire: as toymestry (fo. Ley).

1532. Creninia brevitube Goge \&ab. N-2248.

tculei breves, inclinato-dilatate, as ramos flor. copiosissimi; fol. oflongo-subacuta, fasi fere rotuniata, ad costam eglanidulosa; dentes simplices, sublanceolato - recti; pet. aculeati, eglanaulosi, tomentosi; stip. latac, oilute nubentes, auriculis Sivergentibus; pad 1-3, glabii, 11-12 m.longi; tubus rotunDatus; sep. brevia, pinmulis hinearibus, 1-2-Dentatis instructa, acumine integro; styli pauls villosi, disco plano; cor........; fruct. parsus, globosus, utrinque rotundatus.

Hab. Anglia, Cheshire: as Noreton (Harbord Lewis).

1533. Crepinia micracoccos Gogr Eab. $N-2249$,

Acculei falcato-Dilatati, so ramos flor. geminati, fol. late obovato-acuta, fasi rotunidata, intense viridia, ad costam eglaniulasa; Dentes simplices, lati, aperto-triangulares; pet-aculeati, gglanaulosi, villosi, stip. longace, auricuKis Divergentibus; ped. 1, glabri, 10-11 m. lsngi; tubus globosus; sep. pinnulis integris, brevibus, latis instructa, acumine lato; styli pubeseentes,' gireo subplano; cor........ fruct. minutus (1 cent. lat.). globosus nec attemuatus. Hab. Anghia, Devonshire: is Laira prope Pymouth (M. Archer Mriggs).

6. Fructus ovatus vel ovato-rotuniatus.

1534. Crepinia juncta (Puget) in Gogr Gab. N-2250; Keller in Isiesbaur et Haselferger Rosenfl. Oberaest. p. 16 (var. plusglanioulosa).

Aculei ad ramos flor inclinato-dilatati; fol. obovato-oblonga, utrinque atienuata, is costam eglanialosa; dentes patili, acuti, inf.biserrati; pet. sparse glanimlosi, pubescentes, parum acreleati, stip. saepius glabrac, auriculis reutis, rectis; pes. 2-4, glabi; 14-18 m. longi; tubus breviter obovatus; sep. breviuscule, pinnulis subintegris, angustis instructa; styli breves, pare hirsuti, disco plano; cor. subalba; fruct. parvas, plerumque ovates, basi rotuniaxtus, apuce attemuatus.

Hab. Haute-Lavoie, as Ehonon (Duget). 
$-139-$

1595. Grepinia illecebosse Goger Gab. N=2251.- Googr Herb ros. $N=221$.

Acculei gilatato-subfolcati, à ramos flor. Demiactos coprosissimi; fol. sbovato-oblonga, breviter attemuata, basi parum contracta, pallise viridia, as costam eglaniulosa; Dentes recti, simplices; pet eglanoulosi, villosi, inf. inermes; stip. virides; pes. 1-2, sparse pilssi, 14-17 m. longi; tubus freviter ovoiteus; sep. eglanimlosa, angustius pianata; styli subcolumnares, pauls hirsuti, Disco fere plano; cor........ fruct. plerumque ovatus. Hab. Ysere, as Charancien (N6. Ganioger).

1536. Grepinia burdorum Gogr Eab. N 2252.

Acculei minutissioni, inclinati, ad ramos flor.copiosi, fol. parva, obovato-obtusa, basi corrata, id costam eglanoulosx; dentes simplices, ovatoconvergentes; pet sillosi, eglarioulosi, aculeati; stip. mubentes, auriculis rectis; pid.1-2, ylabri;, 10-11 m. longi; tubus ovato-roturisatus; sep.......... styli

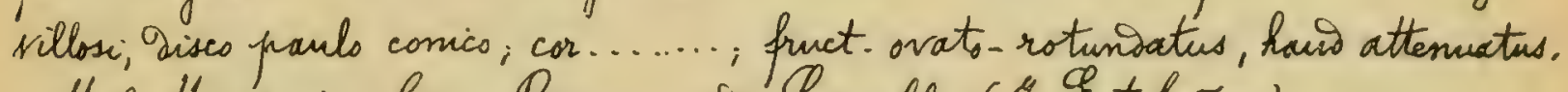
Hab. Hispania bor, Burgos: as g-oncille (J. Estebañey).

1537. Crepinia stigmatophora Googr Gab. N-2253. - Gogr Harb. ros. N:37. Acculei folcato-dilatati, ad ramos flor. saepe geminati, fol. oborato-elliptica, breviter acuta, basi rotuniate, atroverentia, as costam eglanioulosa; Dentes simplices; pet eglanioulosi, longe pilosi, aculeati; stip. virides; ped. 1-2, glabri, 12-15 m. longi; tubus ovatus; sep. pinnulis brevibus sat anguste instructa; stgli subcolumnares, inferne villosi, gisco plano; cor........., fruct. ovatus.

Hab. Ysire, at Charanciew (Nh. Ganooger).

Styli ultax giscum 21/2m. longi; eis Rriparticrum (Stylosear) sat similes, ut occurrit in pluribus Crepimis; set species hue certe aonumeranoa.

1538. Crepinia ulmaria Gogr bab. N-2254. CGogr Herk ros. N-48.

to proxecesente giffert folishis majoribus, ovovato-oblangis, peos. bresiouibus, 7-10 m. longis, sepalis latius pirnatis, stglis subsessilibus nee columnatis siseogue paulo comico.

Hab. Here, at Charancien (N. Gantoger).

1539. Crepinia misera Gogr mss.

Acenlei valior, valde falcato-gilatati, ad ramos floi-copiosi, for. 
$-140-$

obovato-oflonga, abrupte acuminata, basi rotunioata, as costam eglañoulosa; Dentes simplices, triangulari- subconvergentes; pot. eglan'sulosi, villosi; parce aculeati; stip. longar, latissimae, sirides, auriculis valte divergentibus; pet. corymbosi, glabri; $18 \mathrm{~m}$.longi; tubus ovato-ellipticus; sep. pinmulis ommibus integris, latis instructa; styli paulo hirsuti, gises conico; cor....... fruct. sat parmes, ovato-conicus, basi rotuniato-gilatatus, apice breviter attenuartus. Hab. Hispania medix, ad telbarracin (93.2apater).

c. Fructus ovoidens vel obovato-oblongus. + Foliola orata inl elliptica.

1540. Creninia trichoneurioies (Muget) in Gragr Gab. N:2255.

Acculei Dilatati, inclinati vol falcati, io ramos flor. Debiles et sat copiosi; fol.ovato-elliptica, utrinque subrotuniata, firma, crassa, at costam eglanDulosa; Dentes inf. paulo biserrati; pet. adsperse glanioulosi, pubescentes, aculeati; stip. lattae, subtus pubescentes, auriculis Divergentibus; per. 1-4, villosi; 4-10 m. langi; tubus oboratus; sep. pinmulis parum latis, Dentato-glanisulosis instructo, styli pauls villosi, Disco subplans; cor. rosea; funct. macimus, ovoidens, fasi rotunisatus, apice breviter attemuatus.

Hab. Rhóne, as Eassin (Moullu).

1541. Crepinia blepharopoia Gogr Eab. N-2256.

taculei clangati, salos falcati, dilatati, ar namos flor.sat copiosi; fol. late svato-elliptica, six attemusta, basi subrotundata, a costam eglanioulosa, gentes simplices, lati, pet. pubescentes, basi paulo glanioulasi, aculeati; stip. mesiocres; pis. 1-3, willosissimi, $5-7 \mathrm{~m}$. Congi; the bus sovato-oblongus; sep. pinnato-gentata; styli pauls hirsuti, disco sat conico; cor........; fruct. oboratus, apice attemaitus.

Hat. Phane, id Eully (M.Chabert).

1542. Crepinia mutinensis Gogr Eab. N-2257.

toculei falcato- Dilatati, as ramos flor sat copiosi; fol. parva, ovatoelliptica, utrinque rotundata, à costam eglanoulosa; Dentes simplices, apertotriangulares; pet-eglanoulosi, villosi, aculeati; stip. virisos, auriculis devergentibus; pes. 1-3, glabri, 15-17 m. longi; tubus avoideus; sep. pirsmelis latis, 1-2-gentatis instructa, acumine lato, integro; styli hirtelli, Dises conico; 
$-141-$

cor. masima, rosea, eciliata; fruct. parrus, ovoidens, basi subsepressus, is apiom attemuatus.

Hab. Mtalia media, prope Modene (G. Gibelli).

15+3. Greminia Leubolsiana Gogr Lab. N-2258.

Atculei pauciores, falcato- dilatati, ad ramos flor. nulli; fol. emple elliptico-obovata, vix acuta, basi rotuniata, al costam eglanidulosa, gentes simplices, late ovato-subrecti; pot. eglandulosi, tomentosi, sup. inermes; stip. sirides, masimac, suriculis Sivergentibus; pes. 2-3, glabri, 11-12 m. longi; tubus sbovato - blongus; sep. pinnulis dentatis, latis instructa, acumine foliaces, integro; styli praulo villosi, Disco convico; cor prarva, alba, eciliata; fruct. obovato-oblongus, utrinque attenuatus.

Hab. Eyrol, ad Enadenswals prope Hall ( $\%$ Gremblich).

1544. Crepinia lentorhlaea Gogr bab. $N=2259$.

Acculei parvi, temuiter falcati, parum dilatati, ad ramos flor. minutos copiosi; fol. elliptico-obovata, utrinque subrotuniato, as costam eglanioulosa; Dentes simplises, ascendentes; pet. inermes, eglaniulosi, villosi; stip. latissima, auriculis subrectis; pes. 1-2, glabri, 8-12 m. longi; tubus sbovatus; sep. pinnulis annibus integerrimis, eglanoulosis angustius instructa; steli rix pilssi; arseo sat conics; cor. rosea; fruct. obovatus, basi Depressus, apice attenvatus.

Hab. Haute - Savoie, as Mingy (Muget).

Tt Folisla obovata vel obovato-oblonga.

\$. Foliola sminio simpliciter serrata vel prorsus biserrata.

1545. Crepinix remota Gigr Lab. $N: 2260$.

Rami elongati, flexwoss -.. decumbentes; cculei dilatati, minutissime falcati, is ramos flor. geminati; fol. ample obovat oblonga, utringre paulation attenuata, saturate vividia, as costam toto glanoulosa, gentes lect, apento triangulares, ammes fiserrati, pet. glanoulosissini, aculeati, laxe et parce presescentes; stip. Latas; auriculis Sirergentibus; peo. 2-4, praulo villosi, 14-17 m. longi; tubus oborato-oblongus; sep. pinnulis marginibus planioulosis instructa; styli parce pubescentes, dises plans; cor....... fuct. svoiteris, utrainque attenuatus. 
$-142-$

Hab. Gyrol, as Grier ('Jlse).

Fruticulus copiose glemaulosus, habitu Rosam repentem Pcop. referens.

1546. Prepinia similis Cjogr Bab. N-2261.

A culei falcato- Dilatati, id ramos flor. mulli; fol. parra obovato-acuta, basi late coriata, ad costam glamoulosa; Dentes ovato-aperti, inf. biserrati, pet prulo glanisulosi, aculeati, pubescentes; stip. rubentes, auriculis parum dirergentibus; peo. 1-2, glabri, 12-13 m. longi; tubus sboratooblongus; sep. pinnulis onmibus integris, angustis instructa; styli pubescentes, Disco conico; cor eciliata; fruct. obovato-oblongus, utinnque attenuatus. Hab. Garo, ad Le gigan (D. Euezhaimiz).

1547. Prepinia Micheliana Googr mss.

Aculei vatisi, longi, inclinato-Silatati, at ramos flor.copiosi; fol. sat late oborato-acuto, basi sbrupte truncata, ad costam eglanioulose; ientes aperti, lanceolato - triangulares, plerumgue smnes biserrati ; pat. pubescentes, sculeati, paulo glaniaulosi; stip. rubentes, latae, auriculis rectis; peir. 1-2, glabri, 8-10 m. longi; tubus obovato - oblongus; sep. pinnulis onnibus latis, 1-2-Dentatis instructo, acumine integro, styli pubescentes, Disco comics, cot.rosea, cichiats; fruct.obovato-oblongus, basi subotunsatus, apice attenuat. Helb. Htalix orient, at Foenzax (LL. Calsesi).

1548. Crepinia Diversitens Googr $6 a b . \mathcal{K}=2262$

Abculei breves, inchinato-Dilatati, ad ramos flor. plerumgue nulki fol. obovato-oblanga, acuta, basi attemuata, do costam aglarisulosa; dentes plorusnque omnes biserrati, profunise aperts - triangulares, pet. pubescentes, glanisulosi, inarmes; stip. latae, virioes, arso paulo pubescentes; peod-1, glabri, 8-10 m. longi; tubus oroideus; sep. pinmulis latis, copiose gentatis instructa, acumine subfolices; styh pubescentes, aresco paulo conics; car. albo-rosea, eciliata: fruct avoideus, utringue attenuartus.

Hab. byush, in monte Maranja frope Grente (E. Gelmi).

\$. Foliola inoeque biserrata.

〜. Foliola basi rotuniata.

1549. Orepins Sapini Gogr Eab. N-2263. Googr Herb. nos. No154. 
$-143-$

toculei valivi, falcato-gilatati, at ramos flor.copiosi et geminati; fol otovato-oblonga, lanoeolata, basi rotundata, sessilia, as costam eglanisulosa; Dentes aperto-triangulares, inf. poulo biserrati, pot.eglanioulosi, pubescentes, reuleat, stip. angustae, auriculis latis, patulis; pes. 2-4, glab ri, 11-15 m. longi; tirbes oboratus, sep. pinnulis latis, ommibus gentatis instructa; styli parce villosi, gises paulo conics; cor. pallide rosea; fruct. brenter obovatus, utrinque repressus.

Hab. Plhone, at tornas (N. Ganooger).

1550. Crepinix pouncularis Gogr Eab. N-2261t.- Gogr Herb.ros. N-19g. Tcculei validi, inclinato-dilatati, al ramos flor. copiosissimi; fol. late obovato-oblonga, freviter attenuata, basi rotundata, intense virioti-subglauca, oi costam eglandulosa; Dentes simplices, recti; pet. eglanioulosi, villosi, inf inermes; ped. 1-3, glabri, 15-20 m. longi; tubus sbovato-oblongus; scp. pinmulis eglanimlasis, parum latis, sat brevibus instructa, styli caserti, pauto pubescentes, disco subplans; cor....... fruct. ovoidens, basi freviter depressus, apio plus minusve attenuatus.

Hab. Here, is Charancieu (Mb. Ganooger).

$\rightarrow \sim$. Foliola basi attenuata.

1551. Crepinia arcuata Gogr Eab. N-2265.

Aculei faleato-dilatati, as ramos flor. folioso-arcuatos saepins mulli; fol. obovato-oblonga, utrinque attenuata, lacte viridia, od costam eglaniulosa; Dentessubsimphices, lancealato-aperti, pet inermes, eglaniulasi; villosi; stip.latar, breves, aurienlis rectis; pod. 1-2, glabri; 7-g m. lonigi; tubus svoideus; sep. pionnulis mediscribus, 4-6-dentato-glasioulosis instructo, acumine parum lato; styli pubescentes, disco sat conico; cor.rosex, eciliata; fruct-breviter ovoideus, basi rotuniatus, apice attenuato-camicus, centrali oblongo, wtrinque attenuato.

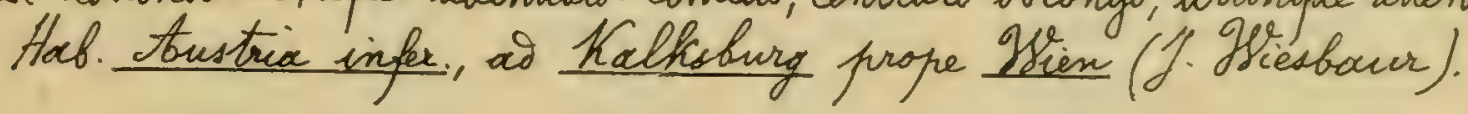

1552. Crepinia laxepilosx Gagr \&ab. N:2266.

Aculei gebiles, inchinati, s ramos flor sat copiose; fol.obovato-acuta, basi attenuata, lucita, is costam eglanioulosa set foliob meisi inferne 1-2aculeolats; Ientessufinplices, recti, fet.reuleati, eglandulosi; lascissime vel sparse pilosi, interdium fere glabri; stip. mesiacres; ped. 1-2, glabri, 10- \# m. longi; 
$-144-$

tubus obovato-oblongus, sep. pinnulis pauls dentato-glanvulosis instructa, acumine latiusculo, styli paulo villosi, Jiseo valise conico; cor. nosex, eciliata, fruct. obovatus, utringue attemuatus.

Hob. Rhone, at Ecully (I. Chabert).

1553. Gupinia lonata Gogr Gol. N:2267.

Acculei obliles, inclinati, vix Dilatati, as ramos flor. sat copiosi; fol. obovata, utrinque paulation attenuata, as costam eglanioulosa; Dentes inaequales pet. copisse glasioulosi, onmes hirsuti, inf. inermes; stip. mesiocres; pes. 1-2, glabui, 10-13 m. longi; tubus ovoidens; sep. pirnmlis sat latis, dentatis instructa; styli paulo villosi, disco parum comio; cor. roser, eciliata; fruct ovoidens.

Hab. Mhone, at Doirdilly (P. Chabert).

1554. Brepinia Forgeoti Gogr Gab. N-2268. - Gigr Harb ros. N= 57.

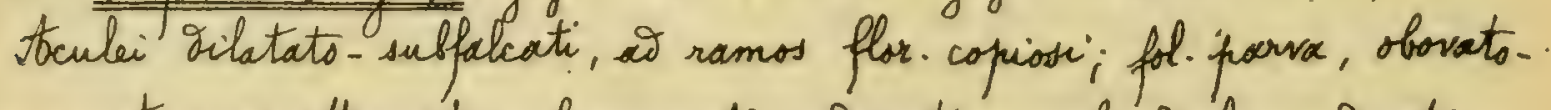
oblonga, utrinque attemuata, glaucescentia, as costam eglandulosa; gentes inf-inaequales; pet-villosi; eglaniulosi, anmes inermes; stip. virides; per. 1-2, glabui, 10-13 m. longi; tubus obavatus; sep. pinmelis glabris, 2-3-gentatis, anguste instructo; styli villosuli, 2isco paulo conics; cor. albo-rosea, parra, inferne subiliata; fruct-or-vous.

Hab. Thoone, ad Mangre' prope gillefranche (M. Gandoger).

1555. Prepimia Oontochlamys Gogr bob. F-2269.

Farlei secwerentes, falcati, as ramos flor. pauciores, fol obovato-oblonga, utrinque sensin attemuata, iv costam eglanivlosa; Bentes recti, inf subinaequales; pet inames, eglanoulosi; villosi; stip. Ratar, medis rubentes, auriculis saluectis; peg. 2-4, glabin, 13-21 m. longr; ticbus sbovatus; sep. pin-

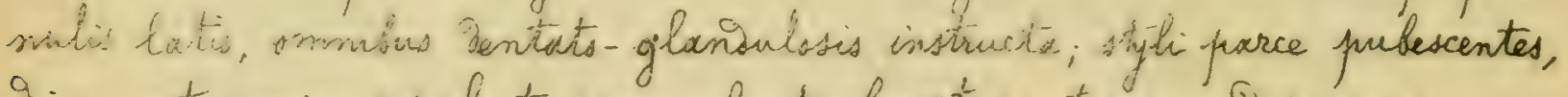
Sisco sat conis; col lacte rosea; fruct. obovatis, itrinque Giepressus. Hab. Haute- Savoie, at Siringy (Muget).

1556. Crepinia fuiburgensis Cogor Tab. N-22\%.

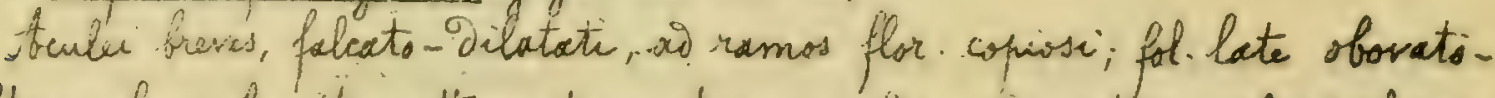
subobtuse, fasi ireviter attemuata, intense virisia, as costam eglanaulosa;

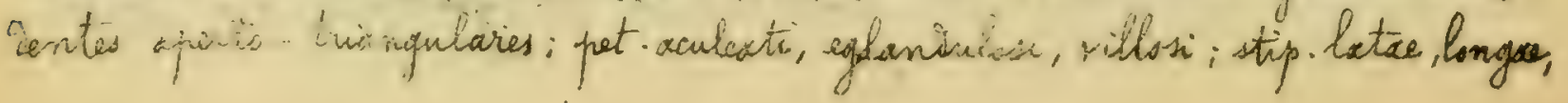


$-145-$

auriculis Dirergentibus; ped-1-3, glabri, 8-11.m. longi; tubus ovoideus, sep. pimulis angustis, eglantulasis instructa, styli villosuli, tiseo proulo conico; cor........ fruct: breviter ovoidens, basi saepius rotundatus, apnice rittenuatus. Hab. Holvetia accid, Fribourg: ad Cases g'Alhieres (L. Tavrat).

1557. Crepinia - Weibreichiana Goar Gab. N-2271.

Aculei falcato-gilatati, ad ramos flor-panciones; fol. obovato-acuta, basi abrupte attenuata, is costam eglaniaulosa; isentes ovato-operti; pat.villosi, eglanimlosi, aculeati; stip. virides, auriculis rectis; pes $2-3$, glabui, 14-16 m. longi; tubus ovoidens; sep. pinmulis amsibus integris, latis instructa; sty $f_{i}$ pubescentes, disco pualo comis; cor.eciliata; fruct. orvidens, utrinque depressus.

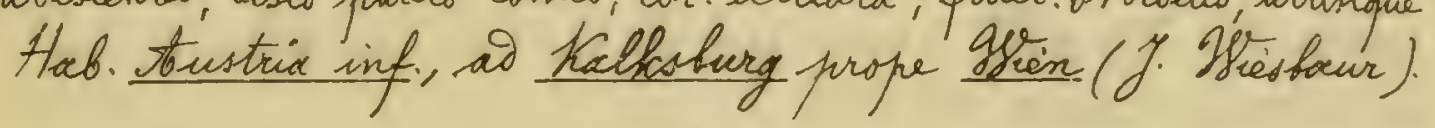

t+t Foliola oblonga.

§. Discius prailo comicus.

1558. Brepinia anglica Gogr bab. N-2272.

toculei breves, falcato- Silatati, as ramos flor-pauciores; fol. prarva, oblongo-lanceolata, basi subrotuniata, at costam eglangulasa; gentes lancolato-apertisimplose sillosi, hinc inde glanoulosi, aculeati; stip. angustre, auviculis rectis; pès. 1-2, glabri, 6-8 m. longi; tubus obovato-oblongus; sep. pirmalis ommibus angustis, eglasioulsis instructa; styli pubescentes, Disco praulo comics; cor......... fruct. parsus, orvidens, busi attenuatus, apice strangul. Hab. Anghia, Yorkshire: ad Boltby (Y. G. Baker).

1559. Grepinic funesta Gogr inss.

teculei breves, falcato-Dilatati, decurrentes, ad ramos flor. sparsi; fol mecivcria, oblango-acuta, basi attemcata, pallidius siridia, edo costem sglangulosa, dentes simplices, trisengulares, recti; pet. tomentosi, salaniulasi; in f-inermes; stip. Longae, latoe, virides, auriculis subsirergentibus; pred.1-3, pinfiescentes, 10-12 m. longr; tubus fovato-oblongus; sep. pinnulis latiusculis, ventatis instructo, styli parce prebescentes, gisco convico, cor...... fruct. majusculus, sbovato-oblongus, basi subrotunaatus, apice longissime attenuato - strangulatus.

Hab. Eransitvamix, Gyolnok Dokoka : as Harajan (2. Richter). 
$-146-$

1560. Prenima uicola Gögr mss.

Arculei longi, rectivseuk, subeylinarici, as ramos flot. geminati; fol. sat late suboblonga, breviter acuminata, basi sensim attenuata, supra glaucescentia, as costam exlasidulosa; dentes simplices, recti, late triangulares; pet.eglanaulosi, tomentosi, sup. inermes; stip. virides, magnae, auriculis divergentibus; ped.1-2, glabri, 6-g m. longi; tubus obovatus; sep. pinmulis amnibus latis, 1-2-Dentatis instructa, reumine integro; styli pribescentes, Sisco vix conico; cos.lacte rosea, sat porva, eciliata; fuct mesioeris, obovatus, basi rotuniatus, apuice attomuato-comicus.

Hab. Larne-et-2oire, It Lawent-2'Ansenay (M. Gonogor).

1561. Crepinia Clavistuina Giger mss.

Aculei flavescentes, breves, bilatato-inchinati, ad ramos flot pauciores; fol. mesiocria, oblong-acuta, basi attenuata, paltide viridia, at costam non glansulosa; gentes aeque simplices et biserrati, ovato-convergentes; pet. tomentasi, inermes, eglanisulasi; stip. longue, latue, virides, auriculis divergentibus; ped.1-2. basi pubeseentes, $5-6 \mathrm{~m}$. longi; tubus orrideus; sep. pinnulis latiusculis, subientatis instructa; styli villosuli, gisco subcomico; cor.......; fruct. sat magnus, ovoideus, basi subrotunbatus, apuce attenuatoconicus.

Hab. Eranosilvania, Isolnok Doboka: a Korajan (2. Tichter).

\$§. Discus planus.

1562. Crefinia iberensis Goger bab. $N=2273$.

Acculei falcato- gilatati, ad ramos flor. copiosissini, fol. oblongoacuta, basi attenuata, ad costam eglanoulosa; dentes simplices, lanceolatoconvergentes; pet. pubesentes, aculeati, hine inde glanoulosi; stip. virioes, auriculis rectis; pes.1-3, glabri, 12-15 m. longi; tubus svideus; sep........; stygli hirtelli, gises subplans; cor ....... fruct. ovoidens, hand ant vix iapressus. Hab. Hispomix bor, ad Coronilla prope Loyroño (J. Lubia).

1563. Brenimia Decidua Gogr mss.

ticulei debiles, parum Silatati, valie et longe falcati, ad ramos flor. geminati; fol mediocria, oblanga, abrupte acuminata, fasi sensim attenrata, glaucescentix, as costam eglanaulasa; Dentes simphice, triangulares, recti; 
$-147-$

pet. aculeari, eglanoulosi, tomentosi; stip. siusses, beves, latiusculae, auricuhis rectis; per. 1-2, glabri, $7 \mathrm{~m}$. longr; tuhus ovoideus; sep. pinmulis subintegris, angustis instructa; styli parce pubescentes, disco plans; cor....; fruct. majisculus, ovoideus, basi depressus, aprice abrupte strangulatus. Hob. Hungaria, ad Subacosi (2. Tichter).

1564. Grepinia Gozza (Gogr. Ros. I. p.11, errore Cozza ); Gogr \&ab. N: 2274 ; Gogr Contr. Pl. slav. II, p. 37. - Gogr Herb. ros. N-183.

Aculei gebiles, falceto-dilatati, romos flor. copiosi; fol sat anguste oblongo-acuta, hasi attenvata, as costam eglaniulosa, Dentes simplices, recti, lanceolati; pot. eglanioulosi, pulescentes, inf inermes; stip. sirides; pes. 1-3, glabri; 10-19m. langi; tubus obovato-oblangus; sep. pisnulis latrisculis, 2-4-Dentatis instructa, acumine lato; Styli parce villosi; isico plano; car. amoene rosea, eciliata; fruct. sat parrus, suboblangus, utringue attemuatis.

Hab. Rhone, a fornas (No.Gandoger).

1565. Crepinia chasonensis Goar Eab. N-2275.

Acculei falcato- cilatati, as ramos flor. phus minus copiosi; fol. oflongo-auta, fasi attenuata, ad costam eglandulosa; dentes simplies, lancolato-recti; pot.eglanoulosi, tomentosi, inermes; stip. virides, auriculis divergentibus; pes.1-3, ylabri, g-10 m. longi; tubus ovoideus; sep. pirnulis integris, latis instivicta, acumine hineare; styli pubescentes, giseo plans; car. eciliaia; fruct. ovoidess, utringre attemeatus.

Hob. Mosia merid., to Nanytsch (Laupmonn).

1566. Crenimia sapiens Goger mss.

Toculei hreves, parri, parum dilatato-falcati, wo ramos flor. sparsi; fot. mediocria, solongo-renta, fasi attenuata, glaucescentia, sicsitasn son glaniulosa; sentes lanceolato-aperti, inf-biserrati; pat-laxe pufescentis, - superne fere glabri, inermes, hinc inde glanoulosi; stip. longre, latae, auriculis subrectis; pos.1-3, glabri, 1 cent. longi; tubus ovoiders; sep. pinmulis angustis, subintegris instructa, styli paulo pubescentas, sisco fore plano; cor........; funct-mediocxis, freviter svoideus, basi subiepressus, apice parum attenuatirs.

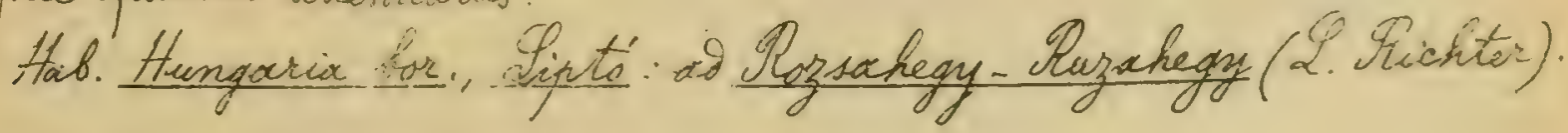


$-148-$

d. Tuctus oblongus.

T Dentes ali simphies alic biserrati.

1567. Crepinix schisticola Gige Eab. N-2270.

tculei falcato-silatati, as ramos flor. copiosi; fol parra, sorato-

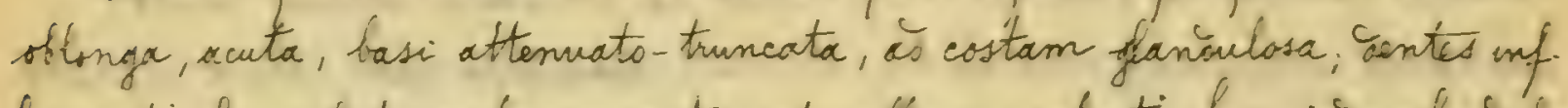
biserrati, lanceolato-subeonsergentes; pet sillosi, aculeati, hine ines glaneablos; stip.rubentes, auriculis isirergentitus; pet. 1, glabri; 8-9m. longi; thbus of longus; sep. pinnulis latis, 1-2-oentatis instructe, acumine integrs, steyli villoss, giseo comis, cor. eciliata; fruct. breviter oblongys, praulo capressus. Hab. Gavi, as Le gigan (2. Eurghiesnion).

568. Crapinia airiatics Gagr Bab. N-2277; Goger Contr. Hl.slar. II, p. 38.

Aculei ailatati, temuiter et parum folcati, ad ramos flor panciores, fol. firma, ample obrats-oblonga, acuta, basi rotuniate, set pallias viriestia, is costam eglanioulosa; isentes aperti, inf. biserrati; pet. eglanioulose; aculeati, villosi; stip. longae, latac, auriculii sivergentifus; pes. 2-3, glabri; 10-12 $m$. longi; tubus oblangus; sep. pinnulis foliaceis, 2-3-Dentatis instructa, acumine late foliaces-dentato; styli pulescentes, Oisec sat conics; car......; fruct.magnus, breviter oblongus, basi plus mimusse Dapressus, apice atteruatus. Hak. Halia orient., as Pesaro (2. Caliesi) et in Dalmatia.

1569. Bepinix coomatc Giogr mss.

Acculei Pebiles, longi, parum folcato-Dilatati, at ramos Plor rarissimi; fol. late oflongo-lancerlata, basi rotunotata, as costam opparse glanioulos: sentes mosni, lancoalato-recti, fere ommes hiserrati; pet-pribescentes, aculeati, rubentes, hine inse glanumlesi; stip. sat pairac, fasi rukentes, auri-

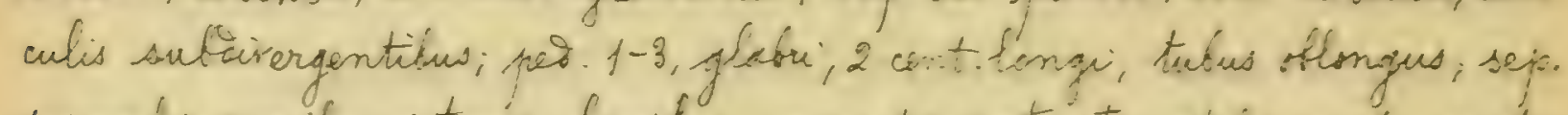

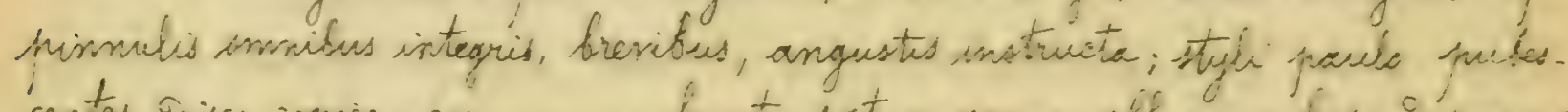

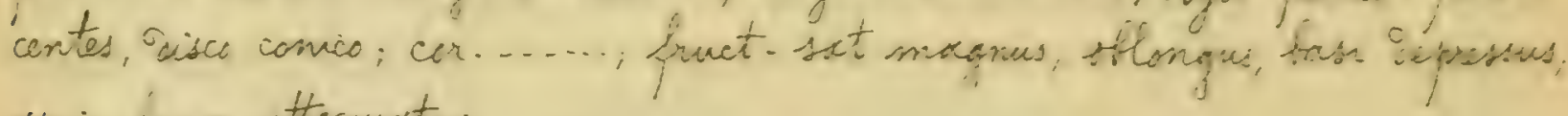
Apric ionge atterniatus.

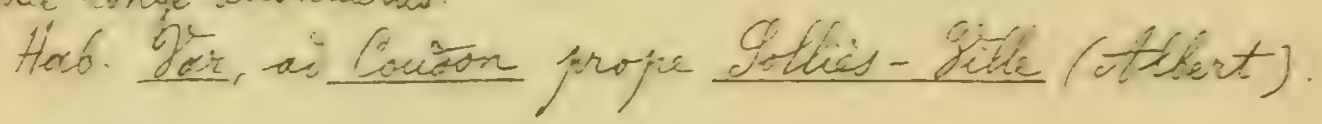

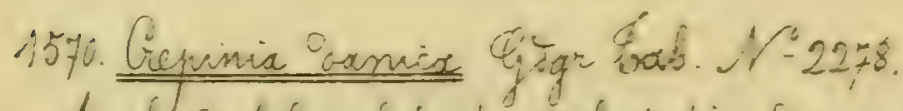

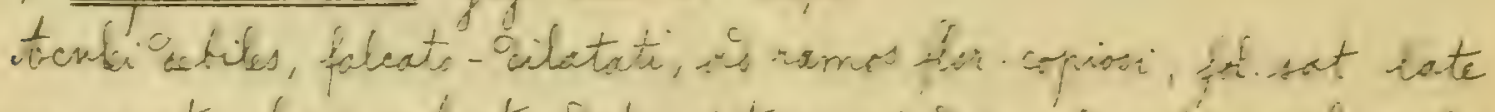

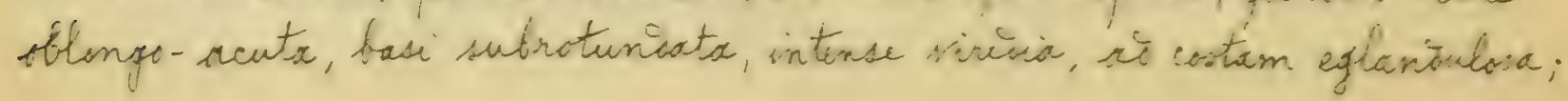


Dentes lanceolati-aperti, fere onnes biserrati; pet. sillosi, aculeati, sparse glanivulosi; stip. rubentes, majusculae, auriculis givargentibus; ped. 2-9, interaum sparse glanauloselli, $7-9 \mathrm{~m}$. longi; tubus oblongus; sep. rubra, pinmulis brevibus, latis, 2-3-bentatis copiose instructa, acumine foliacesDentato; styli pubescentes, Disco comico; cor.rosea, magna, eciliato; fruct. sblongus, utrinque attenuatus.

Hab. Dania, ad Hornalet Mark (Lohrtmann).

1571. Crepinia oreodoxa Gigr mss.

toculei parvi, parum folcato-2ilatati, breves, wasmos flos. rarissimi, fol mediocria, bovato-oblonga, acuta, basi rotuniacta, ad costam eglansuclosa; Dentes lancealato-recti, inf biserrati, pet eglanioulosi, pubescentes, reculeati; stip.latiusculae, longae, basi subentes, auriculis rectis; pes. 1-2, Ylabri, 12 m.langic; tubus oblongus; sep. pirnulis latis, 2-4-gentatis, instructa, acumine fohiace- Dentato; styli paulo villoxi, giseo conics, car......; fruct. maximus, elongatus, basi attenuatus, apice longissime strangulatus. Hab. Yar, in montibus as La Marter (Jblbert).

\section{T† Dentes smmes simplices. §. Pedunculi villosi.}

1572. Crepinia opulenta Gige Gab. N-2281.

Acculci caulinares validissimi, falcati, saliv dilatati, ad ramos flor minores; fol ample obovato-oblonga, utrinque abrupte attemuata, apice acuta, strovirentia; - costam eglanidulosa; Dentes simplices, recti, pet. villosi; eglaniaulasi, aculeati; stip.latac, pas. 2-5, pubescentes, 8-10 m. longi; tubous sblongus, fasi villosus, sep.maxima, pinnulis latis, 2-4-Dentatis instructa; styli paulo villosi; disco camico; cor........; fruct maximus, blongus, utrinque Sepressus.

Hab. Thane, ad Ecully (T. Chabert).

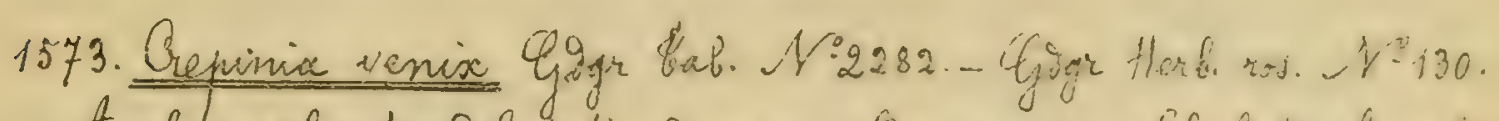

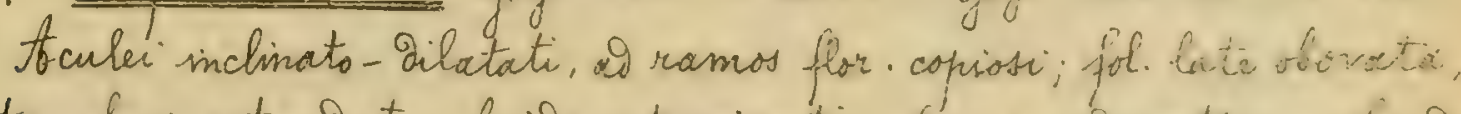
breviter, bosi rotundata, lucise atrovirentix, firma, ab costam cgiansicLosa; rentas simplises, recti; pet. eglandulosi, nermes, villosi; stip. latiusculae; ped. 1-2, sparse pilasi, $16-25 \mathrm{~m}$. longi; tubus oblongis; $-18 p$ 
$-150-$

pinnulis latis, 2-4-Dentatis instructa; styli paulo villosi, Disco subplano; car...... fruct.maximus, oflongus, utringue attemiatus.

Hab. Thane, sornas (M. Gandoger).

1574. Prefinia ramealis (Puget in Disegl. Cat.p. 203); Gogr \&ab. No 2283. - Diségl. Herb. ros. $\mathcal{1}-66$.

tbeculi falcato - Dilatati, a ramos flar. sulli; fol. obovato-ellipAica, breviter attenuata, basi rotunisata, ad costam eglaniulosa, dentes simplices, recti, pet. sparse glanidulosi, villosi, saepius inermes; stip. glabrae, angustae, auriculis rectis; pod-1-4, sparse pilasi, 10-16 m. longi; tubus elongatus; sep pinnulis integrid, latis instrercta; styli paulo sillosi, istco sat conico; cor albo-roses; fruct. anguste oblongus, praecos.

Hab. Haute- Savoie, as Bhonon (Muget) lacs unico et classico!

§. Medunculi glabri.

1575. Creprinia proacutata Gigr bab. N:2279.

Aculie faleato- jilatati, ramos flar. coprosissimi; fol. oblongoncuminata, basi valde acuta, dostam eglandulasa; Dentes simplices, lanceolats-aperti; pet. villosi, eslanidulosi, aculeati; stip. virides, auriculis aivergentibus, p28. 1-3, glabri; 20-22 m. longi; tubus oblangus; sep. igrota; styli pusulo villosi, Bises comico; cor......; fruct. oblongus, basi subjepressus, apice attenuatus.

Hob. Itakia orint, Ionza (2. Caldesi).

1576. Crepinia wrbicoides (Crép.) in Gjogr \&.ab. N-2280.

trculei gebiles, inclinato-subsilatati; fol anguste oblongo-lanceolata, basi atternata, at costam sparse planiulosa; dentes sinplices, ricter; pet-glanaulosi, villosi, aculeati; stip.apice gilatatas, auriculis subwetis; poo. 1-2, glabri, 6-10 m. longe; tribus clongatus; sep. pinmulis subintegerrimis, angustis instructo, stipli parce pilose; Disco conico, cor.......; fiect. Plongus, basi prosertion attematis.

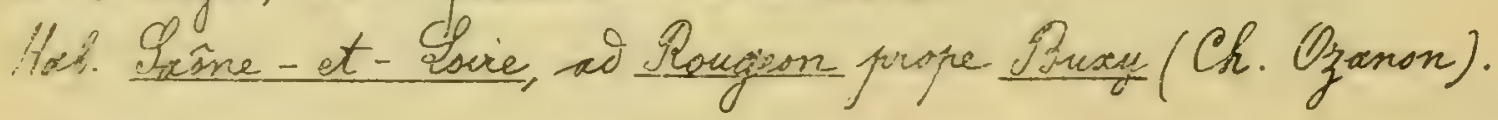

1577. Cupnina Frenmii (Gommasini) in Goge Gas. N-2284, Gogr Conte. H. 
$-151-$

Acculei falcato-Dilatati, as ramos flor nulli; fol amplissime obovatooblanga, subacuta, basi rotundata, glaucescentia, at costam eglandulosa; sentes simplices, recti, triangulares, pot. villssi, inermes, basi pacelulum glanaulosi; stip. virides, arriculis rectis; pes. 1, glabri, 19-22 m. longi; tubus oblongus; sep. pinnulis integris, amplissimis instructa, acumisve foliaces - subintegro, styli mesiocriter villosi, sisco vix conico; cor magna, rosea, eciliata; fruct. maximus, oblongus, basi longe subvecurrenti. attenuatus, apice inflatus.

Hab. Istria, as Mala-Utzka montis Magaiore, alt.3300' (J. Frayn).

1578. Crepinia troperiana Gogr mss.

toculei breves, parn; parum faleato-gilatati, et ramos flor. mulli; fol. sat late sborato- xuta, basi rotuniata, ad costam parce jlaniulose; Dentes simplices, profunde lanceolati, recti; pet. rubentes, villosi, hine inde glanoulosi, sup. inermes; stip. basi rubentes, breves, sat angustac, auriculis apertis, pes. 1-4, glabri, $12 \mathrm{~m}$. longi; tubus oblongus; sep. pinnulis latis instructa, acusmine jubfoliaces; styli paulo villosi, Disco comico; cor. sat pallide rosea, eciliata; fruct. anguste oblongus, utringue longe attenuatus, apice strangulatus.

Hab. Hox, as It Eroper in regione maritima (Mb. Gandoger).

1579. Crepimia vatipetala Gogr \&ab. N-2285. C. Heivigare (Mlacki in Dest. bot. Leits. 1887, p.384).

teculai brenss, folcato-Dilatati, as ramos flor.geminati; fol-mediscria, sfovato-subacuta, basi roturioata, pallide virisia, as costam eglaniulosa; Dentes simplices, triangulari-subconvergentes i pet. eglanisulosi, aculeati, pubescentes; stip.magnae, auriculis nectis; pes. 1-2, glabri,g-11 m.longi; tubus oblongus, sep. pirnulis integris, lates instructa, acumine latiuseuls, stygli hirsuti, Gisco conics; car. rosea, eciliata, petalis snatis; fruer- anple oblongus, basi opuressus, apice longe attenuatus.

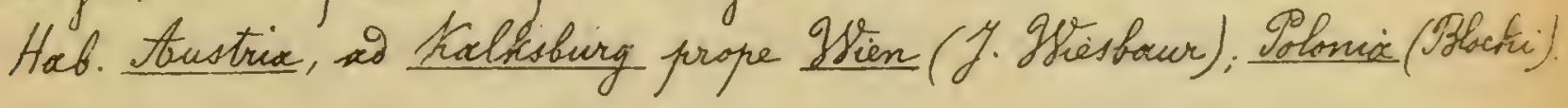

1580. Greninia inflata Gogir mos.

Acculei bur.s, salve falcato-gilatasi, as ramos flor geminati; fol sat late sbovata, breviter acuta, basi rotundata, as costam eglaniulosa; Contes simplices, lanceolato-convergentes, pet. eglansuiss; tomentosi, inf inermes, 
$-152-$

stip. virides, amplissimae, auriculis rectis; pet. 2-4, glabri, 8 m.longi; tubus blongus; sep. pinnulis latis, 1-3-ventatis instructio, acumine foliaces- isentato; stgli paulo vellosi, gisco conico; cor.......; fruct maximus, oblongus, Rasi Bepressus, apice attenuatus, turgiotus.

Hob. gar, in montibus a 2 d Martre (folbert).

1581. Bierinix umonipetala Gogr Bab. N-2286.

Arculei breves, folcato-Gilatati, as ramos flor. mulli; fol. obovato-oblongx, acuta, basi attermata, ai costam eglanioulosa; dentes simplices, ovato-convergentes; pet sparse glanioulasi, nebentes, villasi, inermes; stip. rubentes, auriculis rectis; pes. 1. Glabri, g-10 m.longi; tubus oblangus; 1ep. pinnulis latis, 4-5-ientaits instructo, acumine lato, integro; stigli pauls villosi, Disco corrics; car irrtense rosea, eciliata; fruct. oblongus, utringue attemuatus.

Hab. Hispania bor., Logroño: at Rasillo Lierra de Cameros (I. Lubia).

1582. Creninia iberoum Gür bab. N-2287.

teculei breves, falcato-gilatati, so ramos flor.copiosi, fol. parva, obowato-oblonga, acuta, basi attemeata, so costam cglanículosa; gentes simplies, ovato - convergentes; pet.eglanaulosi, villosi, aculeati, stip.rirides, auriculis ractis; pes. 1, plabrei, g-10 m.longi; tubus oblongus; rep. pirmulis Latis, lrciniatis instrusta, acumine foliaieo osentato; styli villosuli, gisco comice; cor pallisa, esiliata; fruct. oblonous, utrinque attemuatus.

Hab. Histrenia meirix, is Sierra ge Moscueruela toragomia austr. (i). Martion.

3. Ityli ànse villosi vel iansti.

a. Fructus globosus.

1583. Creninia macrostulis Goar Gab. N-2288. - C. opacifoha (Crépin in litt. nox Chabert).

Aculei debiles, inclinato-ielatati; fol parra, oborato-subattenuata, basi rotuniata, coriaces, crassa, ab costam eglarioulosa; Tentes simplices, a perti, pet. sparse glaniulosi, villosi, parce aculeati; stip. meviocres, auriculis parum divergentivus; pet. 1-2, glabri, crassi, epice inflati, $12-15 \mathrm{~m}$. longi; tubus ovatus; sep.......; tyli maximi, Lavati, sisco sat conico, cor.......; fruct.maximus, subolobsus, basi semper rotunivatus.

Hob. Cote-g'Or: is Heursault (Ch. Ozunon). 
$-153-$

1584 . Grepinia Mauksihui (Kit. in Gchultes Oesterr. fl. ed.2- II, p.69, 17-1843; DC. Prodr. II, ros..N-138); Googr \&ab. N-228g.

Ipecias obscura, ab anctore loco cit. sic Descripta: Dre fast kugetigen Fruchtknoten, so wie die Bhumenstiele, unberhaart, Die Blattstiele ariusig Kackerig; Der Ytangel mit zeratreuton etwas zuruck gekrïmmten stackeln; ie Biatter fast unberhoart.

tíab. Hungeriat, aे 2upe (Mauksch).

1585. Crefinia mirania CGogr mss.

A sulai Dilatati, valde faliati, ad ramos flor rubros geminati; fol ample suboblongs-acuta, basi attenuato-cuneata, pallide viridia, à costam eglaniulosa; Dentes simplices, aperto-triangulares; pet. tomentosi, aculeati, in ferme adperse glaniulosi; stip. virives, longae, angustae, auriculis convergen tibus; pos. 1-2, glabri, $3-5 \mathrm{~m}$. losgi; tubus subrotuniatus, sep. pinnulis latiusculis, subsentatis instructa; styli lanati, gises plans; cor.......; fruct. médiocris, subglobosus, basi rotundatus, apice houd attenuatus.

Hab. Hungarix for. Isepas: at Locse (2. Richter).

1586. Prepinia spicant (Gogor Ros. nor. II, p.23), Gogr Gab. N-22g0.-Gogr Herb. ros. M-271.

Aculei parum. falcato-gilatati, id ramos flor pauciores; fol-obovato-attemeata, basi vix contracta, atrovirentix, oo costam eglanaulosa; dentes simp"sices, freves; pet. eglanioulosi, pubescentes, parce aculeati; stip. longac; pro. 1-2, glabri, 4-7m. longi; tubus sratus; sep. pinnulis ommibus $l x-$ trisculis, heribus, Rentats-laciniatis instructa; styli sublanati, gisco plano; cr. ronea, eciliata, fruet. rotuniactus.

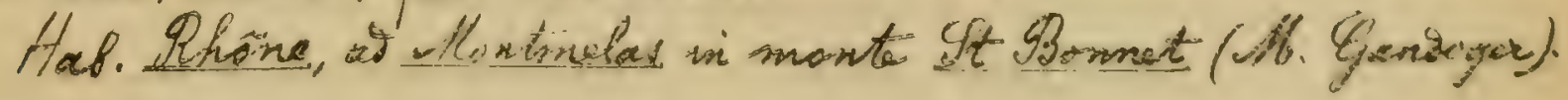

6. Fructus ovato-rotiniatus vel ovatus.

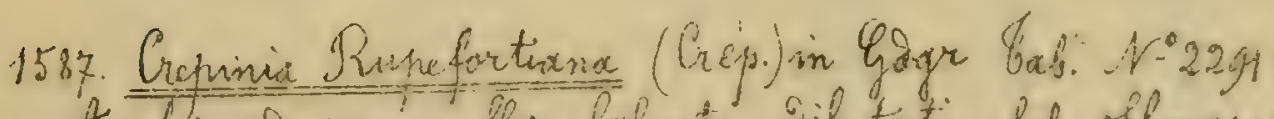

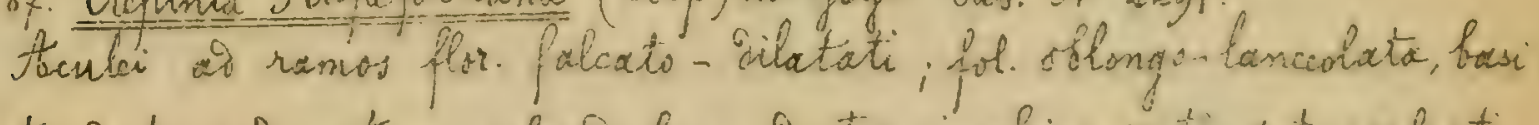

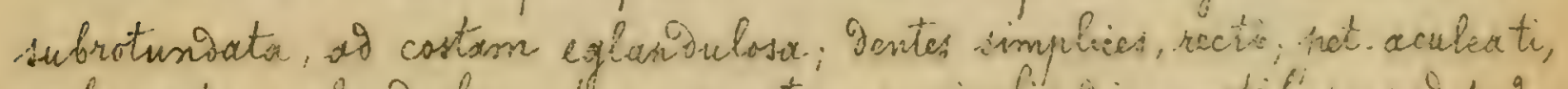

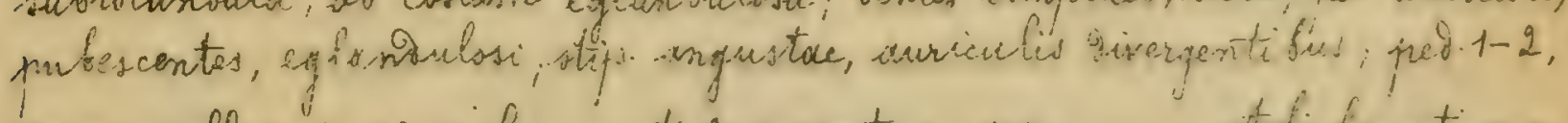
crassi, Nillsti; 4-12.m. longi; tubus ovatus; sep...... ; styli tanati, majusculi, aisco plano; cor........ pruct.maxisnus, ovatus, ac apre whe- 
$-154$

matus, basi roturioatus, centraic vero infune valse Decurrenti. Hab. Belgium, Samux: ad Pochefort (F. Céfin).

1588. Crepimia pinguis (Gip.) in Googr Eab. N-22g2.

-Aculer falcato-gilatati, late oborato-subihomboidalia, fasi fere rotunsater, apice breviter ecuta, ai costam eglanoulosa; rentes convergentes, inf. biserrati; pet-eglanouloss, inermes, sillosi; stip. latar, auriculis givergentibus; pes-1-4, crassi; glabri, 12-17 m.longi; tubus oratus; sep. pinsulis latiusculis, gentatis instructa; styli magni, lanati, giscs plano; cor........ fruct maximus, 2 cent. Diam. latus, ovato-rotuniatus, a pice. attenuato-conicus, basi plerumque rotunisatus, centrali excepto.

Hat Haute-Yavaie, in valle Leschanx (Bouvier).

158g. Crepimix miscens Gogr mss.

Aculei breves, parvi, falcati, parum gilatati, ad ramos flor. geminati; fol. amphinscule oblongo-acuta, basi sensin attenuata, glace-siribix, ad costam eglandulosa, dentes triangulari-subconvergentes, subliverscte, pot. tomentosi, paceuleati, eglanioulosi; stip. sivides, latore, longae, auriculis rectis; per.1-2, glabri, 10 m.longi; tubus ovatus; sep. pirnmulis angustioribus, 2-3sentatis instructa, styli jillosi, gises sat comico; cor....... fruet magnus, arato-conicus, basi roturiatus, apice attemuatus.

Hab. Eranssilvania, Eorda toranyos: ad Eorachko' (2. Richter).

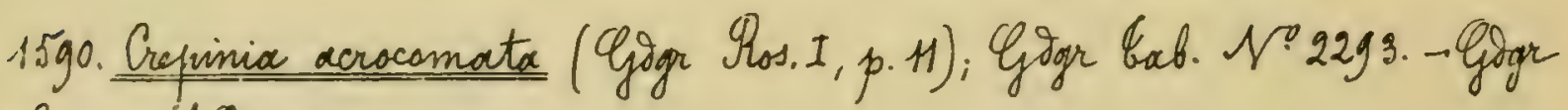
Herb.ros. N= 226.

Acculei faleato-dilatati, sid ramos flor. intersum nulli; fol. ovato-elliptica, basi cordata, apice subrotuniata, amsene virioia, ad costam eglanDulosa; Dentes simplices, subconvergentes; pet. villosi; eglaniulosi, inermes; stip. latae, rubentes; pex. 1-3, glabri, 7-10 m. Longi; tubus suboboratus; sep. pimmulis subintegris, latis coprose instructa, acumine subfoliaces; styli usanse hirsuti, bisco sat canco; cor. rosed, cciliata; fruct. fere ovatus. thab. Rhone, at tomad (M. Ganooger).

1591. Crepinia amoenicorona Gogr 8ab. N-22g4.

A.culei breves, parri, temiter faleati, at ramos flor.copiosi; fol. oborato-subicuta, basi breviter attemuata, sat coriacea, as costam eglanise- 
$-155-$

losa; Gentes simplices, late triangulari-convergentes; pet. Tomentosi, I parse glandulosi, inf inermes; stip. latissinae, sirides, glabrae, auriculis subrectis: ped.1-3, glabri, g-10 m. longi; tubus oratus; sep.maxima, subpersistentia, pionmulis integris, foliaceis, elongatis parce instructa; styli magm; lanati, gisso plans; con....... fruct magnus, oratus, basi rotunidatus, spice haud aut vise attenuatus, centrali autem inferne abupte gecurrenti.

Hab. Iyrol, ai Lavalese in valle di Fimme (G. Gelmi).

1592. Crepinia tomentulosa Gogr Eab. N-2295.

trculei valiai, oi ramos flor. falcato-gilatati; fol. late oforato-rhomboitalia, basi subrotundata, apuice vix attenuata, costam eglaniaulasa: Dentes simplices, aperti, pet. eglansulosi, cinereo-tomentosi, aculeati, stip. latse, auriculis vivergentibus; ped. 1-2, glabre; 7-12 m. longi; tubus ovatus; sep-pinmelis latis, parum gentatis instructa; styli dense villosi, Gisco subplano: $\operatorname{cor} . . . . .$. fruct ovato - subrotuniatus.

Hab. Houte-Lavoie, Habire-Lullin (Muget).

1593. Crepinia brevearmata Gogr mss.

Toculei parvi, brevissimi, inchinato-subsilatati, ad ramos flor. stipulares; folmediacria, obovato-subittemuats, base contracta, intense viridia, id costam cglandulosa; . Jentes simplices, orato-convergentes; pat-eglaniulosi, tomentosi, rculeati; stip. virides, latar, longae, auriculis rectis; pes. 1-2, glabri, 6-8 m-longi, tubus oratus; sep.pinsulis angustioribus, 1-2-gentatis instructe; styli villose', qises plano; con.........; fruct. sat parrus, ovato-comicus, basi rotuniatus, aprice subattemeatus.

Hab. Hungaria bor. Liptó: ao Likanker (L. Richter).

c. Fructus suborviiens.

1594. Orepinia compacta Gogr Gab. N-2296.

to culei as ramos flor compactos folissosque falcato - Silatati, fol obovato-oblanga, utrinque attenuata, ad costam eglandulosa, gentes simphices, subconvergentes; pet. eglanoulosi, aculeati, villasi; stip. longue, auriculis latis, givergentibus; pes.1-3, glabri, 4-10 m.longi; tubus ovatres; sep.pinnulis subintegris, longis, angustioribus instructa; styli sublanati, Driscs plans; cor ......... fruct. subovoideves, basi rotungatus, aprice attemestus. Hab. Haute- Savoie, at Habere- Poche (Muget). 
$-156-$

1595. Greninia lemid Gogr Gab. N:2297.

Rutens, subinermes; aculei gilatato falcati, at ramos flor. mulli; fol. late oblonas-reuta, basi attenuata, coriacca, inferne às costam paulo glan-

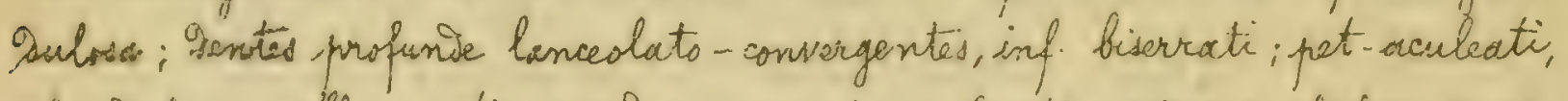
glandulesi, villasi; stip. sirides, ouriculis subrectis; ped-1-2, glabri, 11-12 m. langi; tubus ovoiseus; sep. pinnulis latiusculis, 1-2-2entatis instructa, acumine subfohiaces, integro; styli maximi, hanati, Diseo plare; cor......, fruct. subovoidens, basi gepressus, apuice comio-attenuatus.

Hob. Masses-Alnes, ad Gowphieies (Mioal).

1596. Crepinia ofentephora Gogr Gab. vV:2298.

Acculei minutissini, sparsi, haud aut vix Dilatati, tenuiter falcate, à ramos flor.milli; fol. oborato-sblonga, sensim attennata, basi subrotumiata, as costam glaniulosa; Dentes lati, onnes biserrati; pot copriose glanioulosi, lase pubescentes, fere onnes imermes, stips meiviocres, peid. 1-3, glabri, $17-21 \mathrm{~m}$.

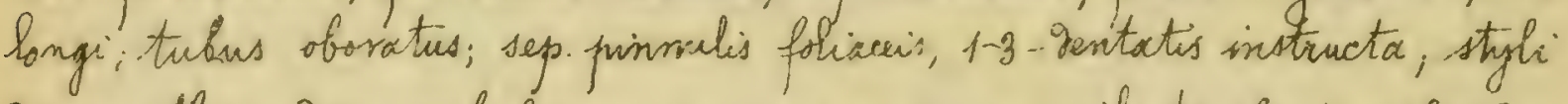
dense villosi, gisco subplano, cor.magna, rosex, eciliata, fruct. iubovoriens. Hab. Thône, at Tranchevilk (9. Chabert).

d. Fructus obovato-oblongues vel oblongrus.

+ Peamenti villosi val subglanimlesi.

1597. Crepinia supramida Gotor tob. F.229g.

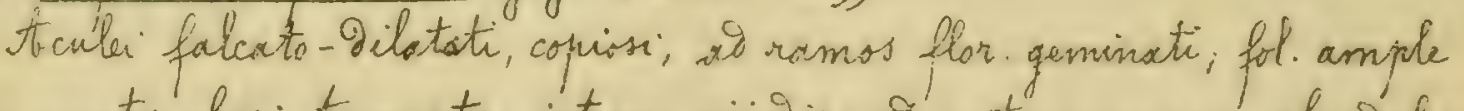
oblongo-ncuta, basi teuncata, intense sivisia, at costam sparse glanouloss besique 1-2-aculeolata, Dentes profunisi: lanceslati, catra-inf. SuAtivisorrati,

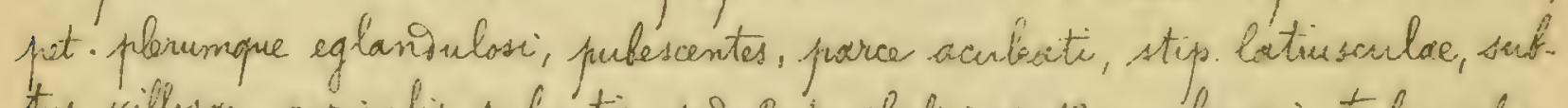

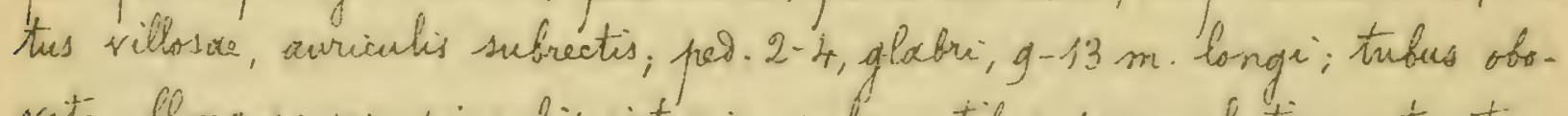

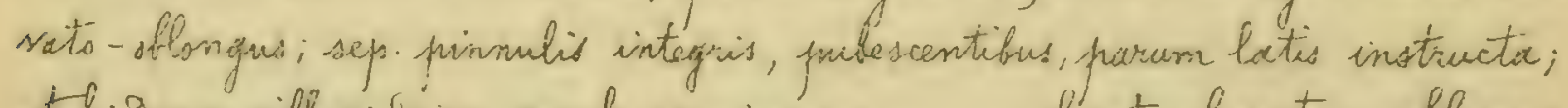

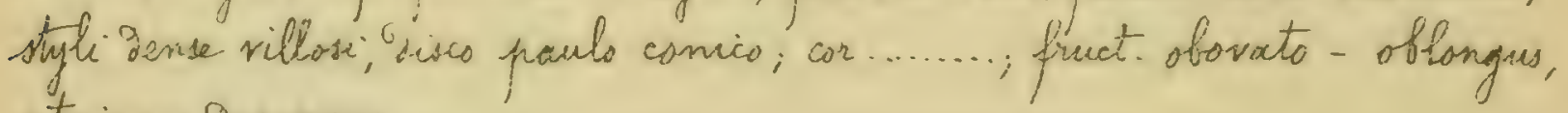
utrinque gepressus.

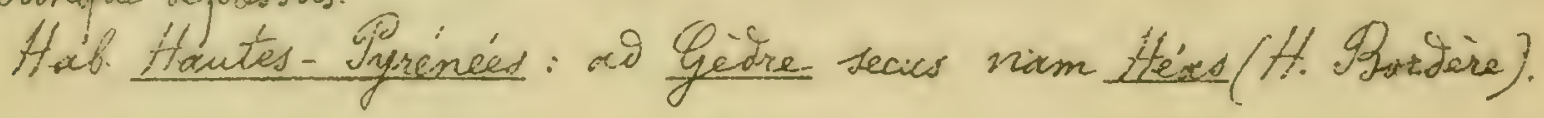

1598. Crenimix mitiramex Gjogr Gab N 2300.

Aculi rarissinn, minuti, frevissimi, ad ramos flor. mulki; fol. subbblonga, utrionque parum atremeata, as costam eglanioulosa; Dentes 
$-157-$

simplices, firlexts-convergentes, pist eglandulosi, nillosi, inf inermes; stip.

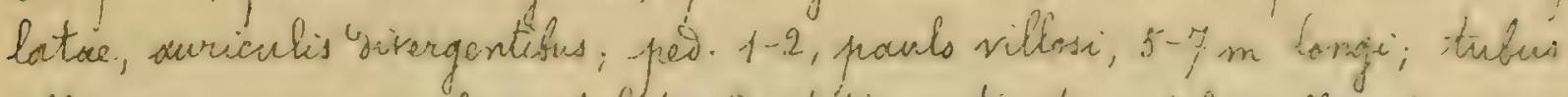
-oblongies; ser. primalis sat latis, dentatis instructor, styli villosi, gisto sai conico; cor. mingsna, roser, fruct. oforato-oblongus, apice aitenuatis.

Hah. Houtes- Pyrénées, od frrend (H. Bordere).

1599. Grepinia cinereosula Gogr Eab. N-2301. Gogr Horb.ros. N.240

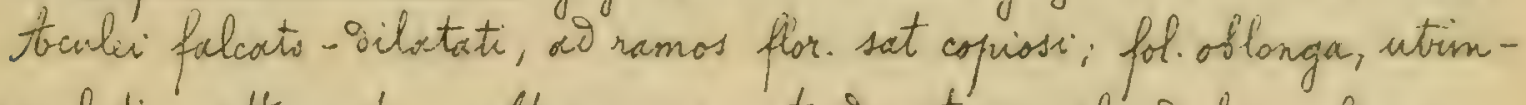
que paulation atternata, subtus cinerex at at costam eglanoulosa, firma, superne intensse rïridix; Dentes simplices, convergentes; pet. tomentosi, eglanDulosi, inermes; stip. latiuscular; pas. 1-3, spocrse pilasuli, 10-13 m.longi; turbus oblangus; sep. pinmolis angustis, 1-4-gentatis copiose instructa; styli gense villosi, gises subplano; cor........; fruct ample oflongus, a's apicem valie attemuatus.

Hob. Thine, th tornas (H. Gandoger).

1600. Crepinia convergens Gior Eab. $N=2302$.

A praceciente diflert foliolis longioribis, Dentibus magis convergentibis, prodenculis longioribus (16-20 m.), magis villosis et insuper hine inde temuiter glanialosis, tubo longiore, basi villoso, sepalorum pinmulis latisnibus, plerromque integris fauctuque longiore.

Hab. Houte- Savoce, Ad Habere- Poche (Guget).

1601. Prepinia operta Gogr Sab. N-2303.

foculai faleato-inlatati, ad ramos flar. copiosi; fol oblongo-acita, basi aitennata, sividi-glaucescentia, ad costam eglaniulosa; dentes simphices, Lonceolat-convergontes; pat. eglaniulosi, aculeati, villosi; s-tip. latar, acoriculis rectis; pad. 2-4, pubascentes, 11-13 m. longi; tubus offongus; sep. pin:malis angustis, 1-2-Dentetis instructs, acumine suffiliformi; stipli fere Ranati, giscs pauls conico; cor........ funct. parsus, oboveto-oblangis, apuice. strangulatus, contiali basi a securrente et flongs.

trab. Inecia, Scamix: ad Sillarog. (Y. to. Gullberg.).

1602. Brepinia Marcailhoui Ggor mss.

toculi tenmes, brevissimi, inclinato-rubilatati, as ramos flor stipubares; fol-mediscria, oblongo-acuta, basi isinge attenuata, sivibi-glau- 
$-158=$

cescentia, crassa, nervosa, aid costam eglaniulosa; Dentes simplices, lanceolatoconvergentes; pet. tomentosi; inermes, eglanioulosi; stip. basi rubentes, longae, Dilatatoe, auriculis rectis; peo. 1-2, intersum 1-2-glanoulis obsiti, 1 cent. longi; tubus oborato - oblongus; sep. pinmulis foliaceis, 1-2-gentatis instructa; styli villosi, gisco comico; cor....... fruct-parrus, obsvato-oblongus, basi attemuatus, apice longe strangulatus, glancescens.

Hab. friège, fox: in nemore Lauret, Gol de Marmare, alt.4000' (A. Narcaithou 2' Anmerie).

1603. Crepimia violascens (Puget) in Googr Eab. $\mathcal{N}^{-}-2304$.

Rami pruinosi, glaneo-violacei; aculei faleats-Dilatati, as ramos fior. sat copiosi; fol obovato - oblonga, subattenuata, basi valde contracta, in -

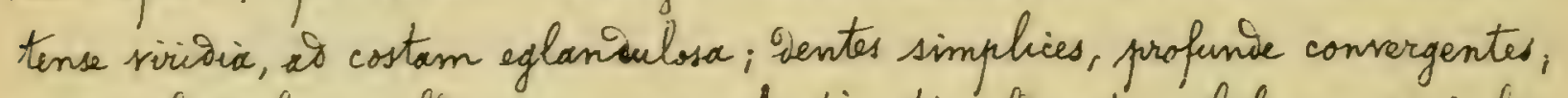
pet-eglaniulosi, villosi, parce aculeati, stip. elongatae, glabrae, auriculis lancealatis; pes. 1-tr, nllasi, 5-10 m. longi; tubus oblongus; sep. pinnulis anqustis, parce ientatis instructa; styli exserti, villosi, Disco comico; cor. rosea; fruct.magnus, oblongus, centrali val solitario basi gecurrenti. Hab. Haute- Iavoic, ad Habère-Poche (Muget).

1604. Prepinia invaions Gogr mss.

Fcculei pareiores, breves, inclinato-Dilatati, parri, aid ramos flor-rari; fol-meisiocria, oblongo-acuta, basi attenuato-cuneata, pallidius virioia, sio costam exlanivilosa; fohislo terminali of basin costae 2-3-aculeolato; Destes simplices, lanceolato-recti; pet. aculeati, plorumque eglanioulosi, laxe et longe pilosi; stivs. virides, sat longae, latiusculae, auriculis rectis; pros. 1-3, rine inde pilosuli, 7-10 m. longi; tubus oblongus; icp. pinnulis latiuscen-

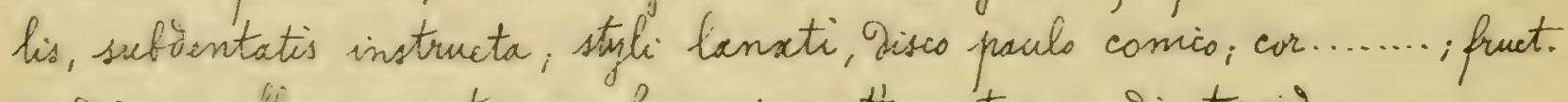
mebricais, oblongus, utringper longissine attemuatus, mesio turgidus.

Hab. Hungaria, as Musaorsi (2. Irichter).

tt Pesunculi glabri.

\$. Foliola alia simpliciter serrata, alia necne.

1605. Grenimia Dichtlii Gigr Zab. N-2305.

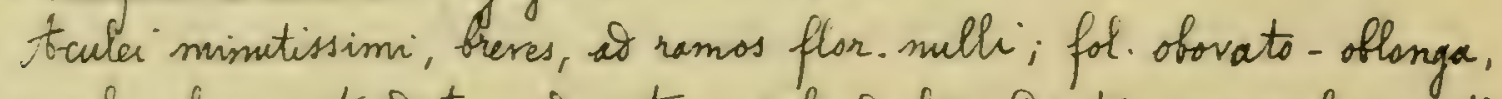
obturiuscula, basi rotuniata, ad costam eglaniulose, Dentes inaequales, srati, 
$-159-$

aperti; pet. inermes, villosi, eglaniulosi; stip. virioses, auriculis aivergentibus; pes. 1-2, glabri, 6-8m. longi, tubus oblangus; sep. pinnulis foliaccis, 1-2ientatis instructo, acumine gentato; Shigli lanati, gisco conico; cor eciliata ; funct. macionus, oblongus, utrinque attenuatus.

Hab. Austria infer., ad Kalksburg prope grien. (Jo. Wichtl).

1606. Prepinia Langeana Gogr Gab. N-2306. - D. Dumetorum (Range Haanis. Dansk. non Qtmill.).

Tculei breves, falcato-gilatati, as ramos flor. panciores; fol ample oblongsacuta, basi attermata, intense virivia, a costam eglaniaulosa; Dentes lanceolatoaperti, inf inarguales; pet eglarioulosi, villosi; reuleati; stip. magnae, ourriculis rectis; pes. 1-3, glabri, 10-12 m. longi; tubus aroideus; sep. pinnulis annibus latiusculis, 1-2-9entatis instructo; sthli lanati, gises conico; cor......, fruct-magsus, ovoidens, apuie attermatus, centrali inferne attemuato-gecurrenti.

Hab. Damia, at Helleboek (9. F. Luniaguist).

\$. Folisla onmia biserrata.

1677. Crepinia pilosiuscula (Opiz in Tresl Iuppl fle cech; Gratt. Ros. mon. I, prolegom. p. 39): Gogr Gab. N-2307, Gjogr Contr. fl. slar. II, p. 37.

toculei compressi, falcato-Dilatati; fol avata, conacea, Dentes fiserrati; pet-villosi, aculeati; stip. subtus pilosube; pes-glabri; tubus oblongus, lacvis; sep. pinmulis eglasioulosis instructa; stigli sublanati; cor.calyce longiot, fruet. oblongus.

Hab. Bohemia, at Kuchelbed (Opis) et alibi.

Fructus a Brattinichio sactus Sictus, certe sot oblongus.

1608. Crepinia rupiola Gogr mss. - Magmier Fp. sel exs. No253.

Acculei minutissimi, inchinato-Dilatati, ad ramos flor nulli; fol parra, coriacea, oborato-subacuta, basi rotuniata, ad costam eglanialoss; gentes onimes biserrati, aperto - triangulares; pet sparse glanoulasi, subinermes, tormentosi; stip. breves, latoe, fasi rubentes, auriculis rectis; pet. 1-2, glabri, 7-8m. longi; tabus oblongus; sep. pinnulis smnibus latis, brevious, 2-3-2entatis instructa, acumine intigro; stagh: gense horsuti, gises subplans; cor. altsa, eciliata; pruct. oblongus, turgiaus, utringne longris attenuatus.

Hab. Lovie, is Gollani- Malleval (Soulla). 
$-160-$

1609. (refuinia phargigpinx Gjogr mss.

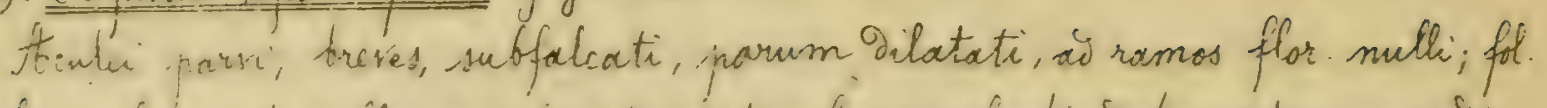

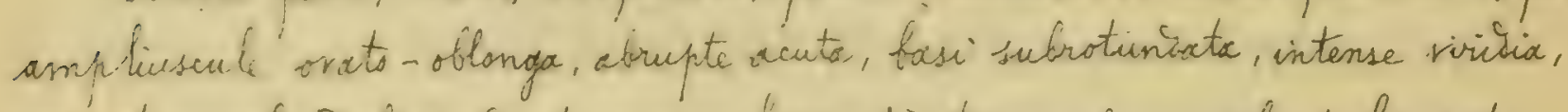
wi costam exfansalosa; ientes anmes bisencati, triangulares, subpatuli; pet-

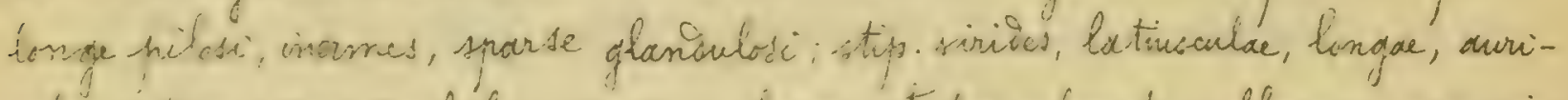

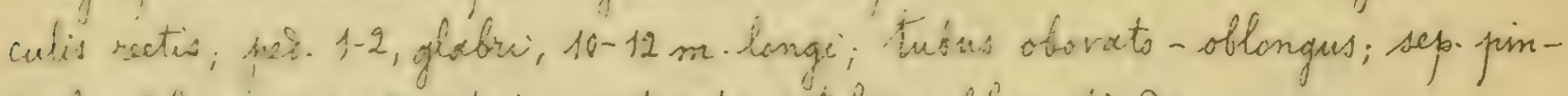
malis folixesis, $2-3$ - rertatis instructa; styli sublanati, giseo conico; cor ……;

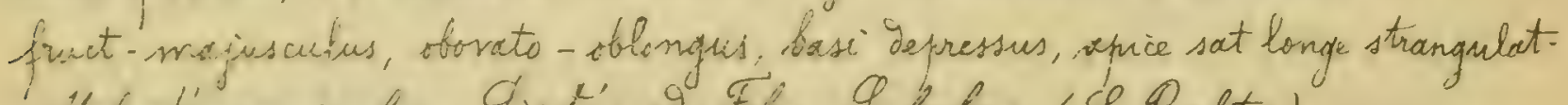

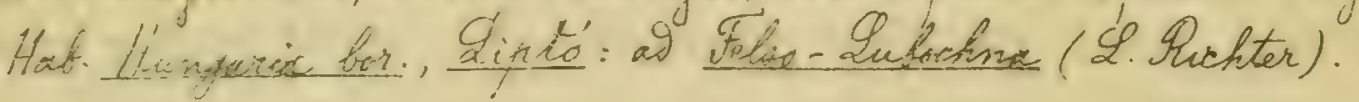

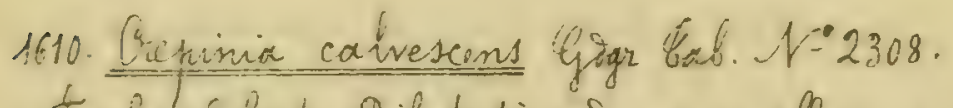

thali fulirto- Tilatati, si ramos flor-copiosi; fol oblongo-lanseolata, aruminata, basi rotuniata, glauca, is costam eglaniulosa, Pentes amnes biserrati, lancolato-wherti; pat-glanimlosissimi, laxe villosi, subinormes; stip. parvere, grso glarioulosoc, ouriculis rectis; peo-1-3, graciles, glabri, 17-19m. langi, tubus elongatis, glaucus; sep. pinnmlis omnibus latiusculis, 2-3- Dentatis, inotructa, acumine elongato; styli lanati, aiseo plans; cor........ fruct. oobongus, angustus, utrinque atternatus.

Hab. Stalix arient., Faenzx (2. Calsesi).

1611. Crepinix infraglauca Ggagr 6.b. $N=2309$.

Acculei breves, falcato-dilntati, ad ramos flor. Densos mulli; fol. parva, oborato-oblonga, wiuta, basi attenuata, subtus glauca et ad costam eglanderlose, sentes honge; lanceolato-aperti, smnes biserrati; pet-subinermes, villoxi, eglanoulosi; stip. brens, latas, auriculis rectis; pre-1-3,glabri, 8-10 m.longi; tubus svoideus; sep. brevia, privmelis integris parum latis pinstructa, acumine foliares, integro; shyli hirsuti, gisco coniss, esr.albx, eciliata; fruct. poerves, ovioidus, aprice ettenuatus.

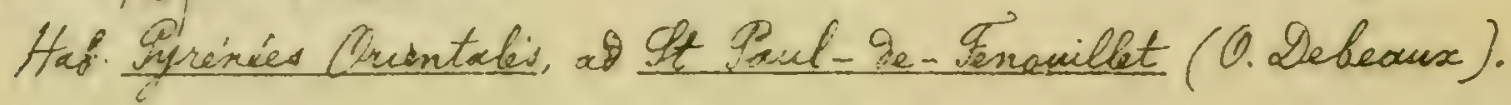

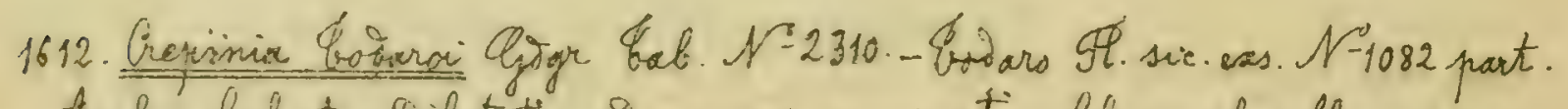

teculei falcato-ilatati, id ramos gominati; fol ample oblongs-acuminata, basi valie attermata, costam glandulosissina, Dentas valse biser-

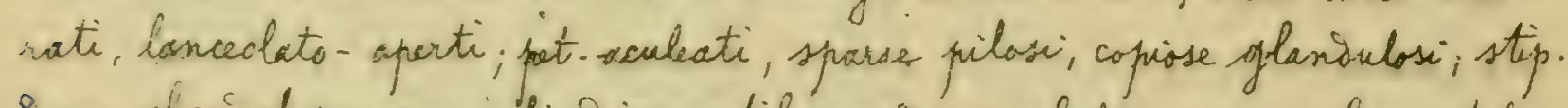

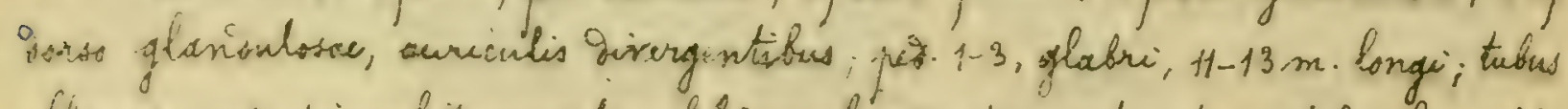
ofisngas; sep pinnulis snnithos folinceo-laciniatis instructa, styli lanati, 
$-161-$

Yiscs comis; car.magna, eciliata, fruct. oblangus, utrinope attien catus. Hab. Sicilia, in montosis ad Nirto (A. Godaro).

Curn 9. sicila Gov. erat mixto. - Srecies folies 2istinotissima, Seabiatios (Chavinia) reforens, sed nervi secunioarii eglaxiulssi, saltem in sicio.

\$§. Foliola ammia simpliciter serrata.

$\rightarrow$ Foliola ovate, ulliptica, obovata vel obavato-oblonga.

1613. Prepunix carwiorhora. (Gogr inos. nov. II,p.24; Gogr decaies plant. mor III, 20 , Gogr bab. N=2311.

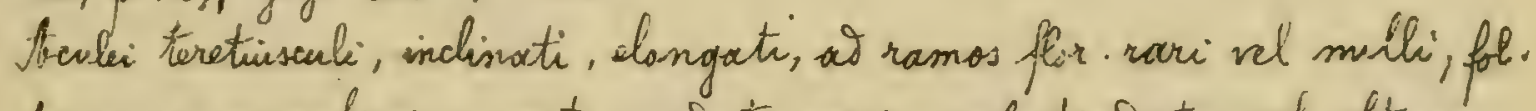
snembranacea, amplissime ovats-coriata, apuse sufiotuniata vel obtusas jrominule neriosa, a costam glanoulosia; Oentes simplices, magni; aperti; pat copisse glomoulosi, pubescentes, inf inurmes; stip. latixe; pes. 1-3, glabri, 16-20 m. Longi; tubus sblangus; sep. magna, apice gorsi interisum asporse glanxulosi, pinmelis foliaceir, copiose dentato-glandulosis instructa; stejki - Hirsute, aiseo subplano; cor.rosea, eciliata; fruct. obovato-oblangus, basi ralie ttemuatis.

Hab. IRône, is Srancheritle (M. Chabert).

Spbilissina precies. Dosim pilophyllam Tau sxocte referens.

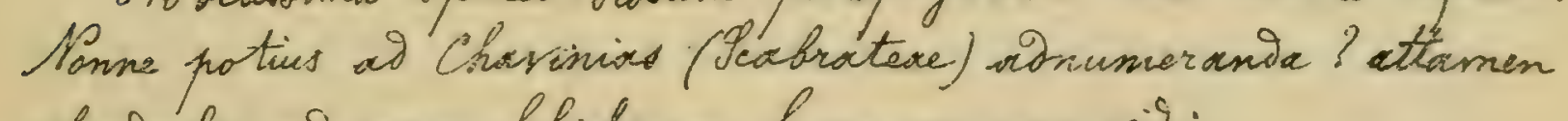
ghandiches ai nervos foliobrem hucusque non sidi.

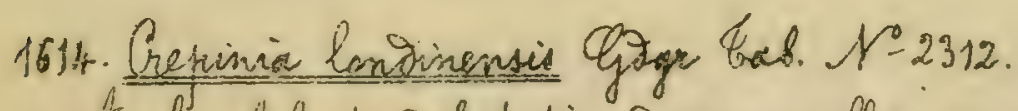

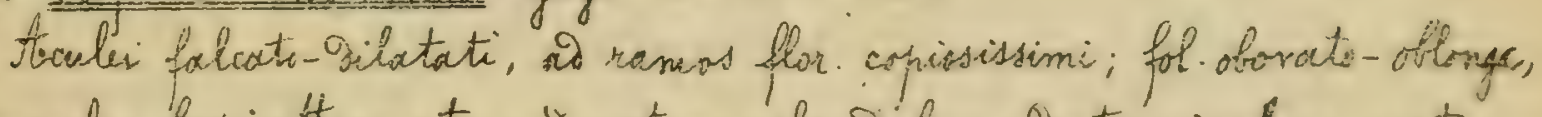

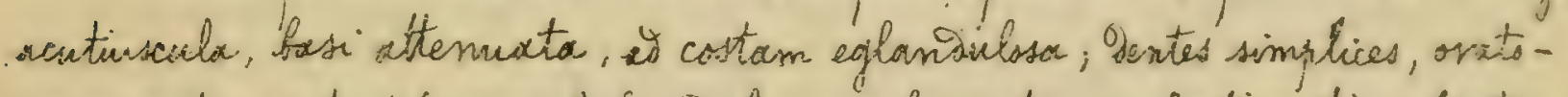

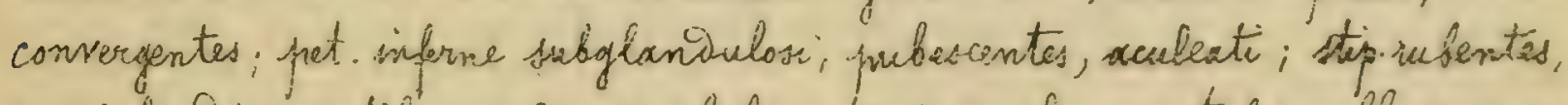
suriculis ivrergentikes; pes.1-2, ghabri, 12-14 m. longi; tisus oblonqus; sep. pimnulis anqustis, 1-2-gentatis instructa, acumine la tiusculo, integro :

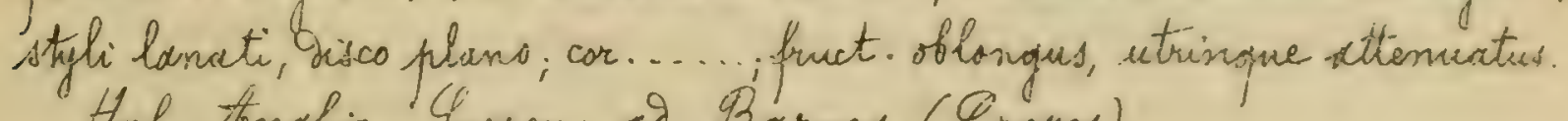
Hab. Ionahia. Serrey: as Marmes (Groves).

1615. Creminia lencotricha Gogr Bab. M-g313.

Foulei pouciores, falcato -. gilatati, ad ramos flor gloues mulli; fol.

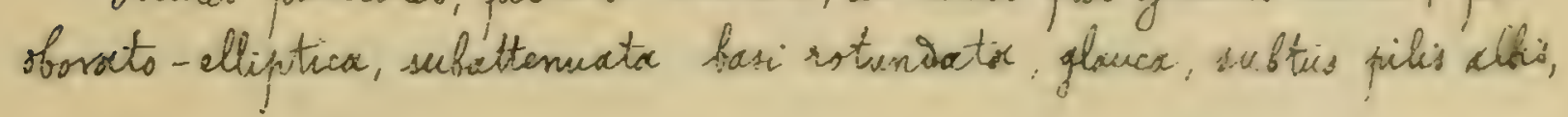


$-162-$

Clongatis ofsita et ad costam eglaniulosa; Dentes simplices, corvergentis; pet eglanimlosi, villosi, inf.incrmes; stip. sirides, latre, auriculis rectis, jeo 1-3, glabri, 5-8 m.langi, tubus obovat-oblongus; sep. prinnulis angustis, prulo gentatis instructo, acumine lato; stifli magni, giense villosi, giser sat comico; car rosea; fruct oforoto-obongus, apice attenuatus. Hab. Haute-Lavrie, as Gingry (Giget).

1616. Greninia lenidota Gagr Gab. M-2314.

teculei falcats - Dilatati, as ramos flor-copiosi; fol elliptics-obovata, utringue poulation attemuata, os costem eglanaulase; Dentes simplics, recti, pet. Nillosi, eglanisulosi, inf paulo aculeati, sup inermes; stip. meDiscres, auriculis rectis; pei. 1-3, glabri, 10-15 m. longi; tubus solongus, glaucrs; sep.pisnulis angustis, terneitor dentatis instructa; styk hirsuit, sises subplano; cons. sea; fuect. anguste oblongus.

Hab. Haute-Sarvie, id Sringy (Muget).

1617. Creninia nigividenta Gogr \&ab. $\mathcal{Y}=2315$.

treulei faleato-Silatati, as ramos flot copiosissimi et geminati; fol. miciacria, obovato-elliptica, freviter attemuata, basi rotunisata, at costam eglaniulosx; ientes magni, rigios; acuti, sperti, simplices; pet. nubentes, inf: inermes, sparse planoulosi; stip. meiv rubentes, apric gilatata, auricilis rectis, pred. 1-5, glabri, 12-21 m. longi; tubus soborato-oblaxgus; sep. pinmules latiuseulis, parum dentates instructa; stygli dense rillosi,

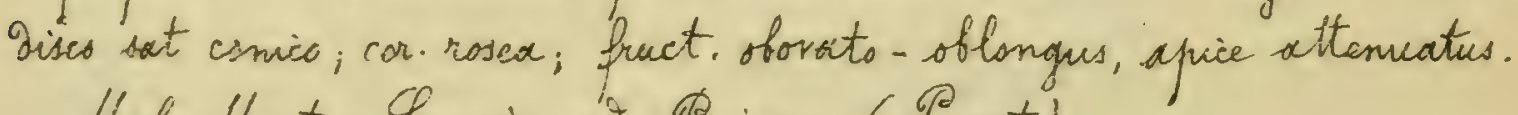

Hab. Haute-Lavoie, is Pingy (Duget).

$\sim \sim$ Foliska oblonga.

1618. Crepinia Rrtani Gogr Gab. N-2316.

Fculei faleato- dilatati, as ramos flo. copriosi; fol. sat hate oblongo-acuta, fasi subattemuato; ad costam eglanivulosa, dentes simplices, apertolinceolati; pet. glanaulssissimi, pubescentes, aculeati; stip. rubentes, suriculis parum givergentibus; pet. 1-3, glabri, 8-gm. longi; tubus oblongus; sep. pinnulis onnibus integris, foliaccis instructo, styli lanate, disco plano; cor, rosea, eciliata, fruct oblongus, apice proesertim attematis.

Hab. Pevemont., in mantibus ag Mral (\&. Rostan). 
$-163-$

1619. Crepinia megalocarpa Gagr baf. N-2317, non Disiget.

Acculi gebiles, inclinati, at ramos flor. flexuosos subnulli; fol. oblongo-lanceolata, basi attemacta, petiolulata, lucioa, ad costam eglaniulosa; gentes simplices, magni; pot. sparse glanisulssi, pubescentes, parce aculeati, stip-glabrae, mediscres, acriculis suboiraricatis; ped-1-3, crasse; glabri, 1 cent. langi; tubus sblongus; sep. mesiocriter pirnata; styli sonse villosi, Oisco subanico; cor pallide rosex, fruct: maximus, elangatus, basi attenuates.

Hab. Ihoñe, at fornat (M. Gandogir).

C.megalocapra (Désigl. in Maim. acad. Le Mlaine-et-Loire,

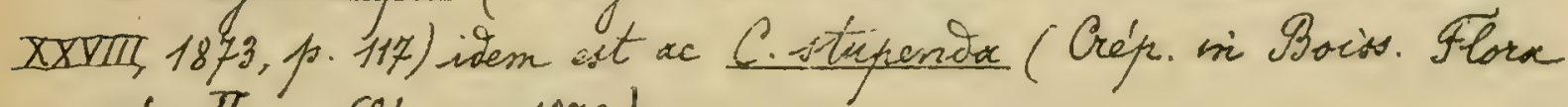
orient. II, p. 684, 1872).

1620. Grepinid manens Gogr mss.

Acculei subylingrici, vix. Dilatati, lange et valie fulcati, ad ramos flar. stipulares; fol. latiuscule oblong-acuta, basi attenuato, lacte viridia, ad costam eylanioulosa; dertas simplices, frofunid, lanceolato-recti; pet. longe pilosi, aculeati, hine inde glandarlosi, stip. virides, latiusculac, parmess longae, auriculis subsivergentitues, per. 2-5, glabri, 12-14 m.longi; tubus of longus; sep. pinmulis latiusculis, 1-3-Dentatis instructa, styli villsi; gises comico; cor......., fruct. magnus, blongus, basi gepressus, apuice longissime attenuato-strangulatas, centrali val solitario inforne attenuato.

Hab. Transsilvania, Korys: ad Magyar o' Kereke (2. Richtes).

1621. Orepinia schistocarpa Gogr Bab. N-2318. - Goger Herb ros. N-366.

Accuki gebiliores, faleato- isilatati, as ramos flor copiosi; fol. oblongo- icuta, basi rotuniata, costam eglan sulosa; gentes simplices, recti; pet. infeine paulo glanialosi, pubessentes, aculeati, stip.mesiacres; poo. 2-4, glabri, 11-15.m. longic; tubus elongatus; sep......... otyli gense villosi, gisco subplano; car....... fruct. ample elongates, utrinopue valts attemuatus, turgitus. Hab. Rhove, It finas (H. Ganoger).

1622. Crepinix jidicabilis Googr ms.

Aculei breves, falcato-gilatati. ad ramos flor.copiosi, fol. sat ample oblong-acuta, basi rotundata, crassa, is costam eglanaulosa; arentes simplias, ovats-convergentes; pet.eglaniulosi, tomentosi, aculeati; stip. longac, 
latissinge, virises, auriculis direcgentibus; pèd 1-2, glabri, $15 \mathrm{~m}$. langi, trious

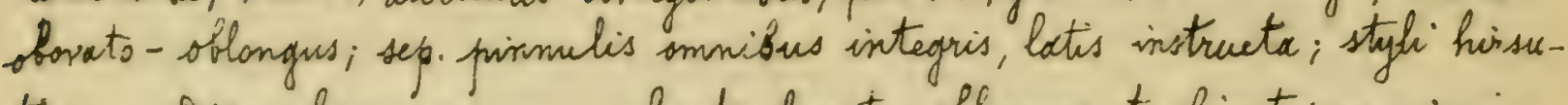
tissimi, Dises plano, cor........, pruct. obovato- oblongus, turbinatus, apice inAlatus, basi longe attenuatres et subsecurrens.

Hab. Soroogne, io Sayzec (No. Ganooger).

\section{4.- Mrbicare Gogr Essai p. 3:"Gig. Eab. p.173.}

Foliolis mesius ramorum floriferorum supra glabris, subtus ad nere. vos pubescentibus, cum pilis raris inter norss hinc inse sparsis; sevreturis simplicilus; petishis villosis rel tomentosis.

1 Styti glabri vel sparse pilosuli

1 S Styli hirsuti vel lanati Sisunculi glabri

2 Sesunculi villosi Foliola basi rotundata

Ipicies N-1623-1627.

3 Foliola basi ettemuata

Fructus ovoideus, vel obisato-sblangus

$4\{$ Encetus oblengus

Fructus globosus vel oratus Petioli inf- vel onnes mermes

5 Setioli annes aculeati

$6\left\{\begin{array}{l}\text { Fructus woideus sel obovato-oblongus } \\ \text { Fructus globosus, rotuniatus vel ovatus. } \\ \text { Fructus oblongus }\end{array}\right.$ Fructus oblongus

Toliola annia simpliciter serrata

Irecies N $-163 \mathrm{~g}-16+ \pm$ Irecies $N=1628-1620$.

Inecies $N=1630-1634$. Irecies N: $1635-1635$

Qnecies $\mathcal{N}^{\prime}=1645-1643$ Grecies N-1662-1667

7 Foliola inf. biserrata Setioli inarmes

$8\{$ Setioli aculeati

$\{$ Pedunculi villose

Theaies $\mathcal{N}^{-1649-1650 .}$ Inecied N-1651-1655. Shecoss N'1656-1661.

$9\{$ Seanculi glabri

Fructur rotuniactus, plobosus rel astus

Inecios 16-1668-1670.

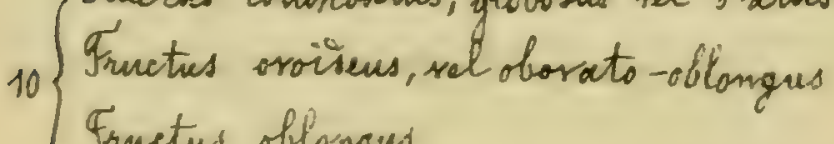

Inemis $19-1671-1674$

Shecies $f: 1675-1681$. 
Fructus globosus vil roturidatus

Fructus ovatus vel sato-rotuniatus

11 Eructus avoideus vel obsrato-oblongus

Fructus stlongus

Toliola inaeque vel biserrata

12 Toliola oninino simpliciter sarrata Petroli inf. vel omes inermes

$13\{$ Setioli annes aculeati

Foliola basi attomeata vel cuneata

Inevies N:1682-1685.

Epecies $N=1686-1690$.

Snecied $\mathcal{N}-1691-1696$.

Stecies $f: 1697-1702$.

$14\{$ Foliola fasi rotumiata

15 Setioli ammes aculeati

Ypesies $\mathcal{N}=1703-1709$.
Yreaies $N=1710-1712$.

Petioli saltem inf. inermes

Petioli onnes aculeati

$16\left\{\begin{array}{l}\text { Petioli onnes inermes } \\ \text { Petioli inf-inermes }\end{array}\right.$

Discus planus

17 Discus plas minsesse conicus

Foliola ammino simplicitex serrata

18 Foliola inaeque vel fiserrata

Foliola basi attenuata

$19\{$ Foliola fasi uturitata

Setioli annes inermes

$20\{$ Getisli annes plus minusse aculeati

Inecris N:1713-1716.

Inecies N-1717-1719

Ppeies $N=1720-1722$

Ipecies 1:4723-1728.

Ipecies fro 1729-173.

Species $N=1732-1737$.

कpecies $N=1738-1720$.

Grecies $N \cdot 1741-1746$.

1. Otyh glabri val sparse pilosuli

a. Sesunculi villosi.

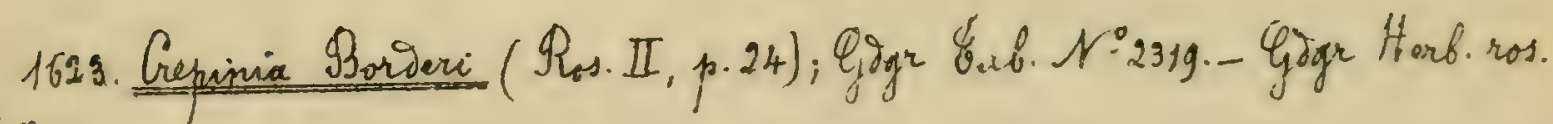
$\mathcal{N}-336$.

ftenlei Debiles, longi, as ramos flos. gracili-flexuosos-sacpins gemirati; fol. vato-acuti, basi rotuniats, intense siridia, as costam eglanisulosa; "dentes simplices, pot eglansulosi, subinermes; stip. angustar, subtus pilosie, auriculis Sivergentibus; pet. 1-3, gracites, pparse pilosi; trbus ovösens; se p. pirnnulis ientatis, bresibus, late instructa, styli glabui, qusco conics; cor. alba, sal'subalba, parva, eciliata; fruct. parves, onoidens.

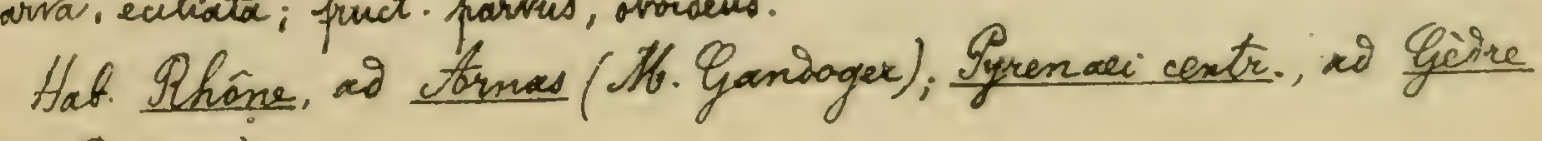
(H. Sorgere). 
1624. Grepina becurtata Gogr Gob. N=2320.

Feculei falcato-Dilatati, eg ramos flot. stipulares; fol. ample obovatooblonga, strinque attemuata, mevis Dilatata, firma, intense sivioia; gentes convergentes, simplices; pot aculeati, inferne sparse glanidulosi; stip. latae, suriculis parum divergentibus; peo. 2-7, villosi, 5-11 m. langi; trebus breviter sbovatus; sep. pirmelis pancioribus, angustis instructa, acemine gilatato; styli ghabri, gisco sat comis; cos......... fruct. Greviter aroidens, apice ettenuatus.

Hab. Rhane, ai Chaponost (Moulle).

1625. Crepinia cincinnata Gagr Eab. N-2321. - Gogre Horb. ros. N-7.

Acculei Debiles, temiter falcati, copiosi, as ramos flot. sacpe geminati, fol. tennia, ovato-auta, basi rotuniata, intense viricia, Dentes simplices, ciliati; pet.eglaninlosi, inesnes; sijp. meisoeres; ped.1-9, sparse pilosuli, 14-18 m. Longi; tubus oblongus; spp. punmulis latiusculis, 1-4-gentatis instructa; styli subglaberrimi, Disco paulo comics; cir. pallide rosea, eciliata; fruct magnes, oblangus, utsingue attemuatus, mestis turgions.

Hab. Olhine, at tornas (M. Ganooger).

1626. Crepinia Gury.ale Goar Gab. N'2322. Goor Herb. ros. N-148.

teculei inclinato-gecurrontes, a? ramos flor. flexusos sage nulli; fol. sblonga, utringue pioulation witennata, temior; dentes simplices, patuli; pet. inermes, eglanonlosi; stip. latinsculae, auriculis rectis; pes. 1-2, villosi, :-12m.longi; tubus elangatus; sep. brexiter pinnato, acumine gilatato; cor prallive rosea; fruct. oblangus, utrinque attenuatus.

Hab. Qhoñe, ad timas (V. Gansoger).

1627. Crepinia latifolia Govgr Gors N'2323.

taculei rarissimi, falcato G dihatati, ad ramos flor. melli; fol amplissime -blongo-acuta, basi breviter attenuata, pallide viridia, costam eglanianlos.; Dentes lanceolorto-aperti; pet. hinc inde glanivulose; aculeati, stip. ruhentes, magnare, auriculis rectis; por. 2-5, villosi; 6-8m.longi; tubus oflon. gus; sep. pinmulis foliaceis, 3-4-ientatis instructa, acumine longs, folinces, integro; stafli atsperse pilosuli, gisco canico, cor.magna, frallide rosex, culiata; fruct. oblangus, basi Songe attenuatus, apice inflatus.

Hab. Hacte- Savoie, ad La Inya prope tomecy (Bousier). 
$-167-$

6. Sènnculi glabri.

+ Folisla basi rotuniata.

\$. Fructus globosus vel vartes.

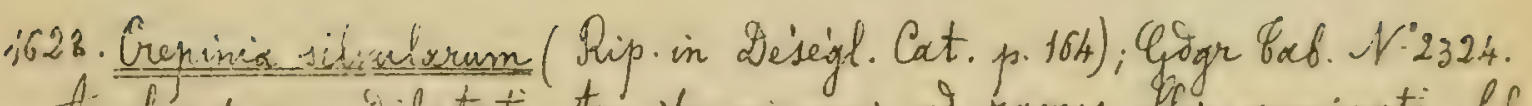
foculei parusm cillatati, temiter nicurve; as ramos flor geneinati; fol. - tovertia, painlo attemuata, pallide viridia, gentes acuti, aperti; pet acculeati, egtanionlosi; stip. parvas, surviculis givergentikus; pas. 1, glabri, 7-10 m. longi; tufus brositer avoideus; sap. parva, pinmulis frevibus, angustis ino-

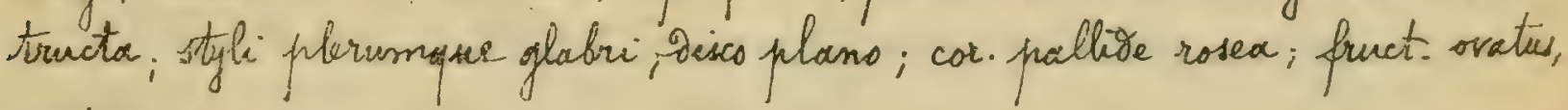
minot.

Hab. Haute-Saraie, ad Pringy (Duget), unie Mipart habuit atoue Descripsit, sicut at ego.

Grrarit, ut nimium frequens, Déseghlise hane steciem in Canineis

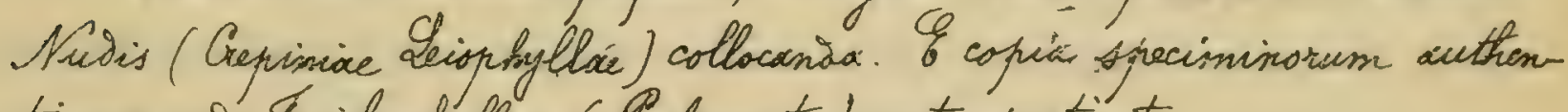
ticoum, os Erichophyllas (Mubescentes) certe pertinet.

1629. Crepinia montanx Gogr \&ab. N-2325.

Aoculei falcato-Dilatati, ai ramos flor copiosissimi; fol obovate, freviter acita, basi rotuniata, atrosirentia, costam eglasioulosa; bentes aperti, triangulares; pet eglanioulosi, aculeati; stip. masnar, longae, auriculis disorgentibus; pas-2-3, glabri; $16-18 \mathrm{~m}$. longi; thibus roturioatus; sep-pinmulis foliaceis, 2-9-Dentatis sopiosissime inotructo, accumine folices-Dontate; stekli sparse pilosuli; grides plano; cor........, fruct maximus, flobosus, nec attenuatus.

Hab. Houte-Savoie, at Mont Sion (Mouvier).

\$5. Fruetus ovrideus vel shovato-oblongus.

$\sim$. Petroli inferioies vel annes inermes.

1630. Crepinia Dentisenala Goar bab. N'2326.

Acculei falcato- Cilatati, as ramos flor. saepe melli; fol. oforato-oblonga sel oblengs-acuta, basi subrotuniata, atrovirentia; Dentes recti; pat. eglanDulosi, sape fere onnes inermes; stip. longae, pes. 1-3, glabri, 14-16m. longi. tubus oblongus, sep. primnulis elongatis, latis, profunde sentats - glanialosis instructo; styli assparse pilosuli, deses paulo conics, car. rosea, eciliato; 


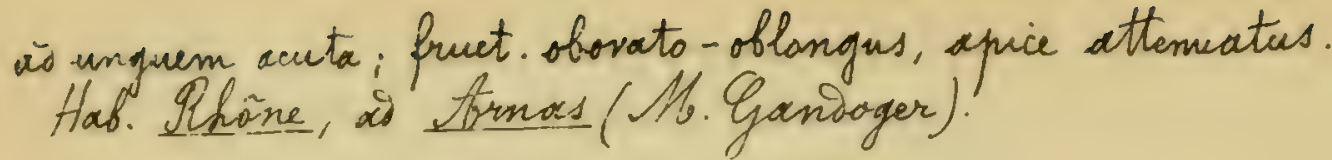

1831. Crepinia alligata Giar tab. N-2327.

toculai gebites, necti, hans dilatati, id ramos flor pauciores; fol ample oflong-lanceolata, basi subrotienata, of costam eglanaulosa; gentés remoti, lati, subconvergentes; pet eglanimlosi, sinermes, cineres; stip. virioss, latas, ourciculis divergentibus; sat 1-2, glabri, 11-14 m. longi; tubus ovoideus; sep........ styli sparse pilosuli, disco subplans; cor.......; pruet. ovoideus.

Hab. Iypenai centr., prope Giere of viam Héas (H. Baraie).

1632. Crepinia geraphylla ligar val. P.2329.

Tubens, inferne denvidata; acuici harvi, inchirato-suboilatati, of namos flor-subnulli; fol parwa, ob:vato. blenga, breviter attemuata, basi rotunSata et plicata, coriacea, nervosa, aentes sat freves; pat. eglandulosi, extrainf. incrmes; stip. rubentes; pej. i-2, glabre; 7-10 m. tongi; tubus oroioens; sep. rubentia, pinmulis ammibus 'ativisulis, proulo dentatis instructa, styli parce pilssuli, dises paulo conico; cor. lacte rosed, eciliata; fruct. oroideus. Hois. Rhone, w Nontonelas in monte It Isonnet (M. Gandoger).

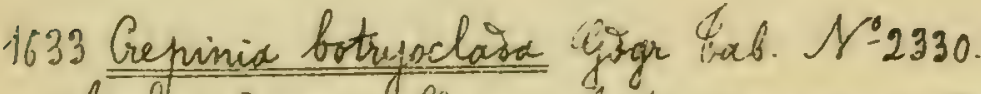

foculei so ramos flor. esnferto-racemosos gebles, inchinato-gilatati fol. parra, obasato-subácuta, basi rotusivata; gentes recti, pet eglandulosi, inf inemes, stip. sinides; pet. 1-2, gik bri: 17-20 m. Congi; tubus oflongus; sep. pinnulis latis, larugis, 1-3-Dentatis instructa, styli vix prilosuli, disco

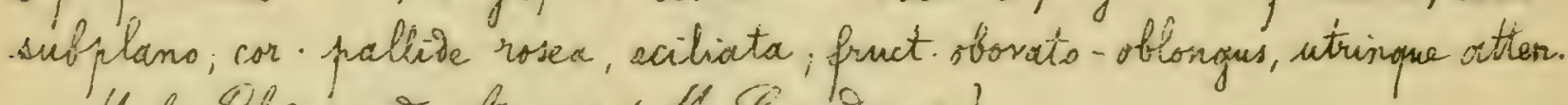

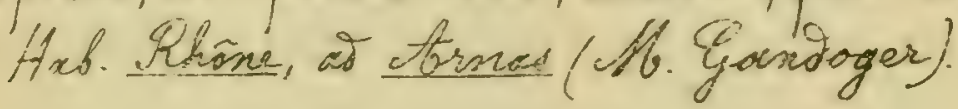

\section{Crepinia erratica Cogr ms.}

foculei breves, ojilatats - subfelcat, ad ramos flor. sparsi; fol. ellipticosubacuta, basi rotunisata, wo costam eglaniulosa; Dentes lanceolato-conver-gentes; pet. eqlaniulosi, inf-inermes; stip. virides, latissimar, aurciculis

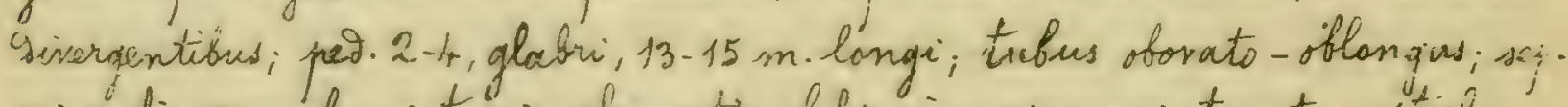

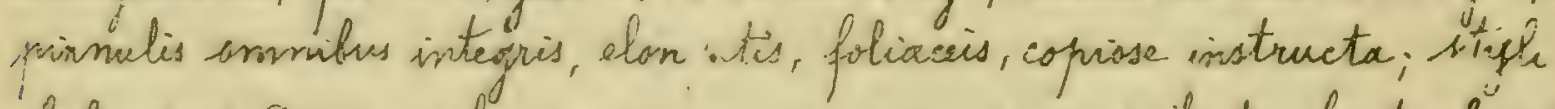
Afaberrimi, grises paulo conico; a mana, hrsex, eciliata, fruct abovaton chlongus, setrincere sufatitemuate:

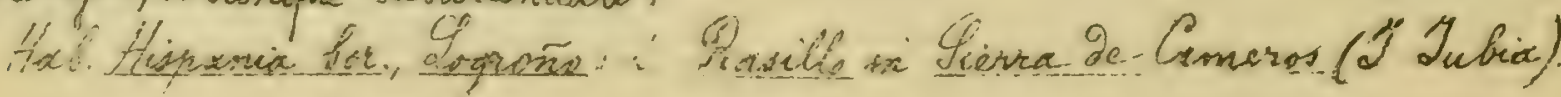




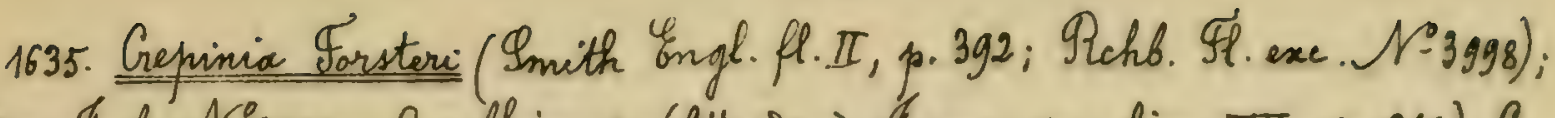

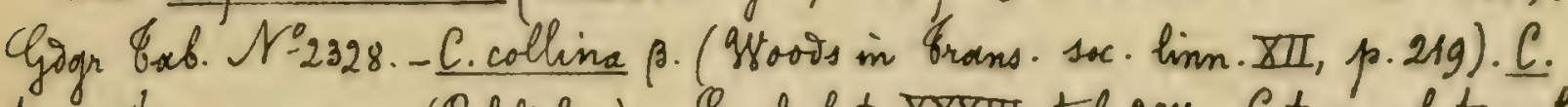

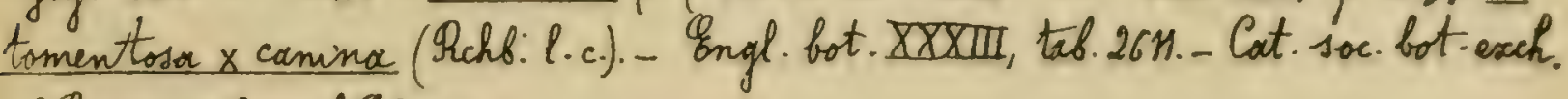
of Lonio. 28. 3a, N-351e.

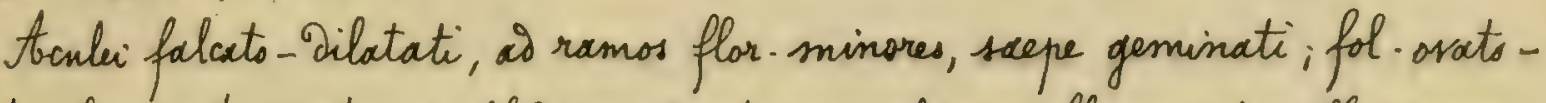

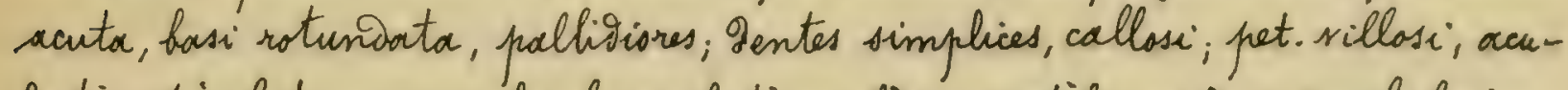

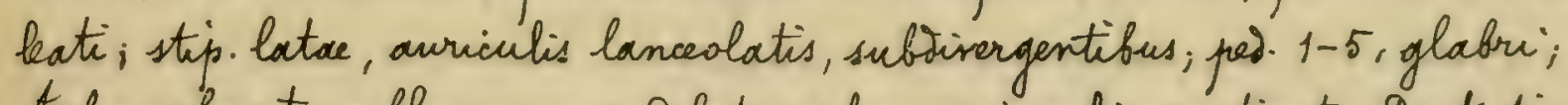

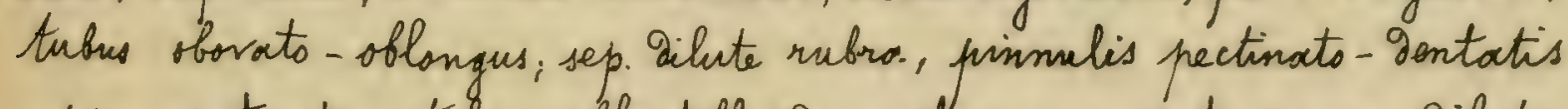

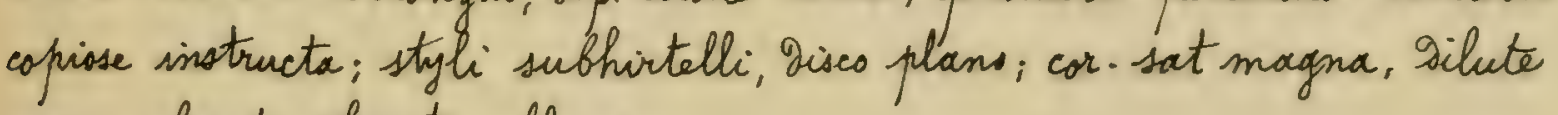
nosea, fruct. oborato-stlongus.

Hab. Anglix. - Deseripsis ox cione citata.

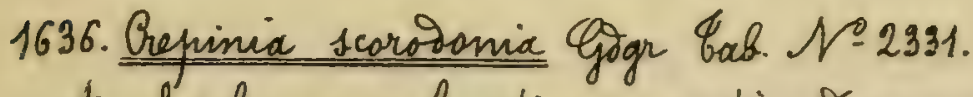

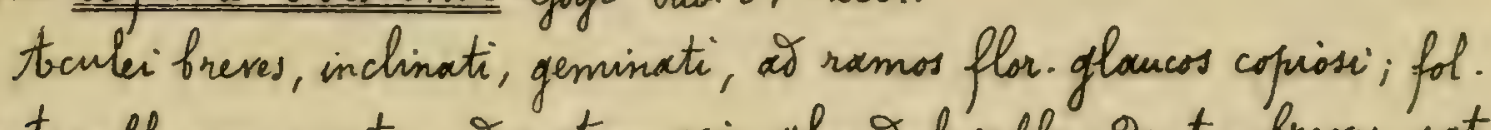

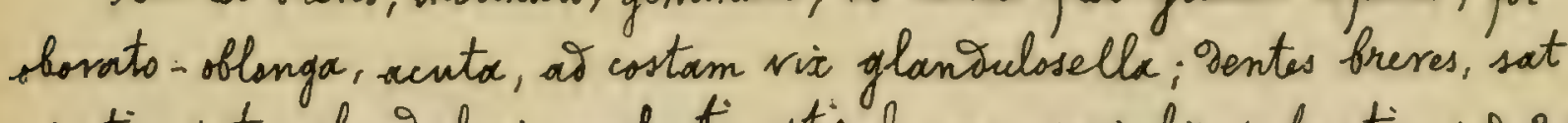

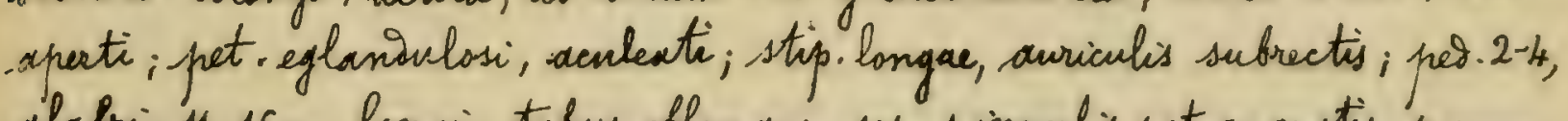

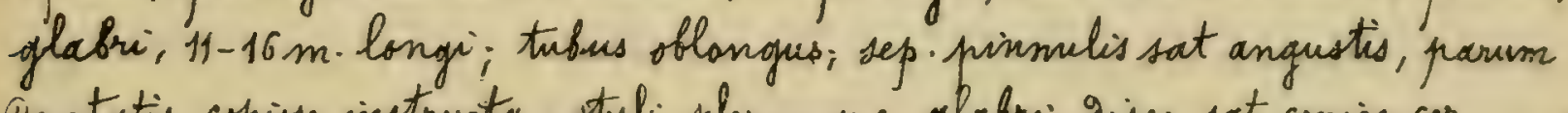

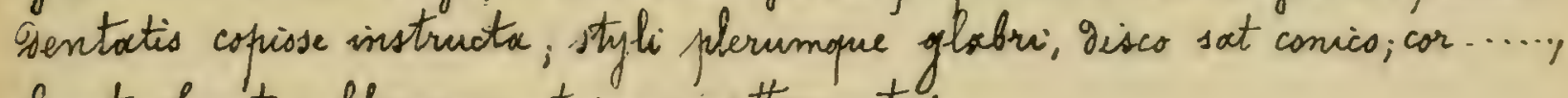

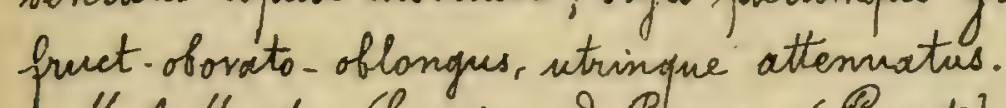

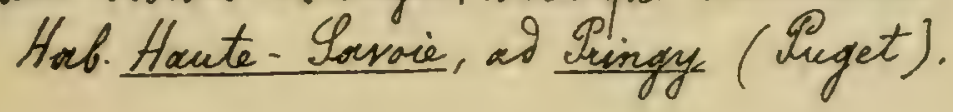

1637. Geppimia pampera Gogr bab. NNo2332.

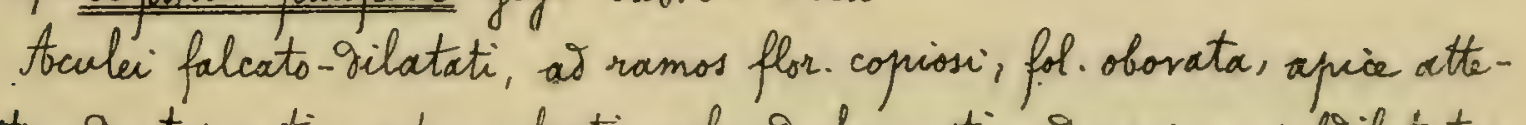

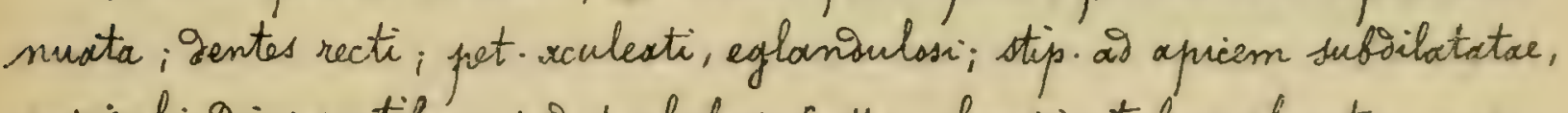

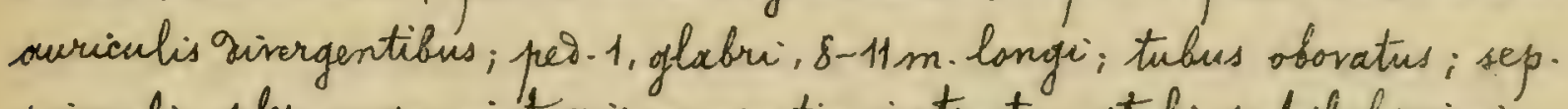

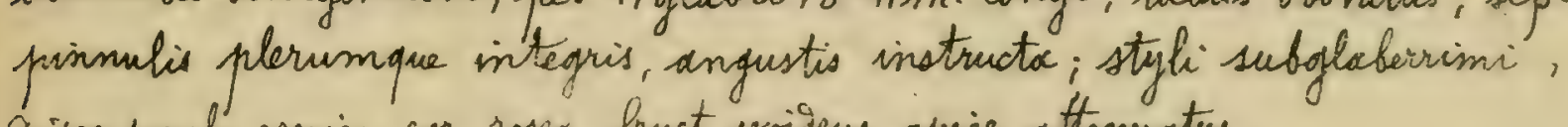

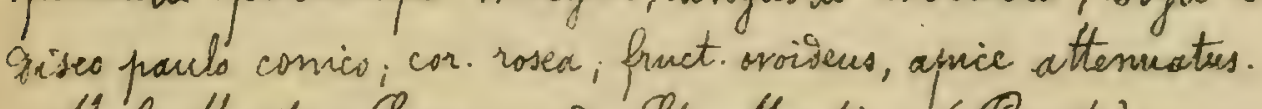

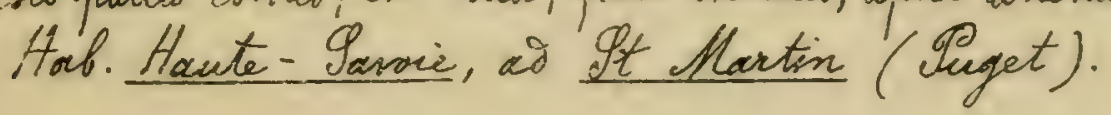

1638. Crepinia pallidinctala Googr Gab. No2333.

Aculei sat bebiles, falcato - vilatati, as ramos flor coprossi, fol obovato-

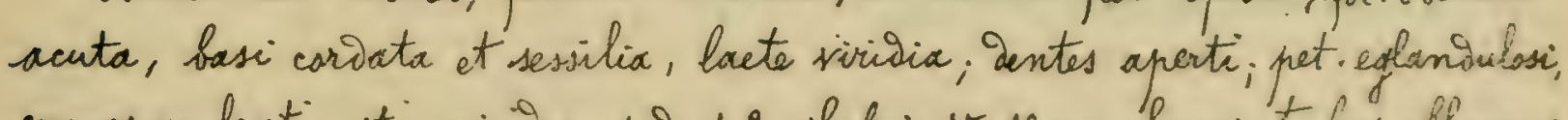
connes sureleati; stip. virives; pes-1-3, glabri, 15-18 m. Longi; tubues oflonosus; sep. pinmulis sat latis, vix gentatis instructa, Stafli sparse hirteili, Bisco 
$-170-$

subrlano; cor pallise rosea, eciliata; fuect. obovato-sblongus.

Hob. Mháne, as tornas (Nh. Ganiager).

$\S \S$. Fructus oblangus.

1639. Cresinia Notarisiana Gogr \&ab. N-2334.

Aculei temiter falcati, io ramos flor. geminati; fol obovato-oblonga, acuta, bass rotuniata, as costam eglanioulosa, jentes lanceolato-convergen-

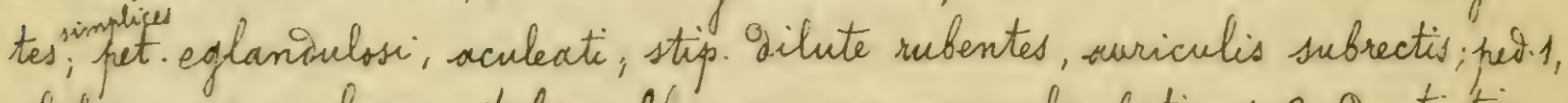
glabiri, g-10 m. longi; tubus oblongus; sep. pinnulis latis, 1-2-dentatis instructa, aciemine integro, sthli glabri, gisco plano; cor. eciliata; fruct. oblongus, utrinque oepressus.

Hab. Atalia orient. Iod Fenza (2. Caliesi).

1640. Crepinia trusca Gogr Gab. N-2335.

Acculei debiles, falcato-Dilatati, ad ramos flor. copiosi, fol obovatssblinga, acuta, basi rotuniata, ad costam eglanioulosa, dentes briangulari-convergentes; pet. eglanioulosi, parce aculeati; stip. mediocres, auriculis givergentibus; per. 2- 5, glabri, graciles, $14-16 \mathrm{~m}$. longi; tubus elongatus; sep. pirnmulis latis, eglaniulosis instructa, acumine foliaces, elongato, styli subglaterrimi, Disco paulo comics, cor. pallise rosea, eciliata; fruct. oblongus, utrinque ses basi pracesertion atternuatus.

Hab. Ytalia meirix, as Sisa in Selva Pisana (G. Lavi).

1641. Crepinia parcearmata Ǵggr mss.

Aculei minutissini, parum folcato-gilatati, as ramos flor nulli; fot. sat late oborato-oblonga, acuminata, basi rotunoata, as costam non glaniulasa; Dentes orati, recti, inaeque biserrati, pet. inforne subglañoulosi, onnes inernes; stip. rubrse, mesiocres, auricalis rectis; fes. 1-2, glabri, $12 \mathrm{~m}$. longi, tabus oblonges; sep. pinnulis latis, paulo dentatis instructa, acumine foliaceo, integro; styli glabri; gisco prauls comico; car mesiocris, eciliata; fruct. oblangus, fasi gecurrens, aprice longe sthematus. Hoab. Far, ad Golkis-boucas (Stbert).

1642. Crepinia devata Gage bab. N-2336.

ibculei falcato-gilatati, "g ramos flor-panciores, fol-obovato-suba- 
$-171-$

cuta, basi rotuniata, io costam eglanioulosa; gentes simplices, ovato-aperti; pet eglanioulosi, aculeati; stip. virides, auriculis Sirergentibus; pes. 1-3, glebri, 7-8m.longi; tubus oblangus; sep. pinnulis onmibus integerrimis, foliaceis instructa; styli glabri, Disco plano; cor.rosea, eciliata; fruct. parvus, oblongus, utringue attemeatus.

Hab. Garo, at Bréau prope Le Gigan, alt. 3000' (D. Eneskiewicy).

1643. Crepinia rigidifolia Googr \&ab. N-2337.

Acculei folcato-dilatati, ad ramos flor-geminati; fol. ampliuscule oblang-acuta, basi rotunianta, rigida, at costam eglanioulosa; Dentes simplices, lanceslato-aperti, pet eghandulosi, cueuleati, stip.magnae, auriculis Divergentibus; pes. 2-9, glabri, 8-10 m.lengi; tubus oblongus; sep. pinnulis eglanioulosis instructa; styli subhirtelli, Disco plans; cor.........; fruct maximus, oblongus, apice breviter attenuatus et inflatus, fasi valoe attenuato- vecurrens.

thab. Helvetia, at $2 a$ Sarray (Favrat).

1644. Crepinia megastula Goger mss.

teculei valivenli, Silatati, faleati, a ramos flor. geminati; fol. amphinscule oblonga, abrupte et breviter acuminata, basi rotumata, wo costam eglam gulosa; gentes simplices, recte, late rvato-lancerlati; pet. hinc inge glanioulosi, aculeati; stip. longae, sat angustar, Dorso pribescentes, auriculis subrectis; per.1-2, glabri, $10 \mathrm{~m}$. longi; tuhus solongus, sep. pinnmlis annibus integris, foliaceis instructa; styli glabri, subcolumnares, disco valse comico; cor. albs, inferme paulo ciliata; fruct. oblongus, basi gepressus, apice longe attemuatus. Hab Gironie, as Montferranos (2. Motelay).

It Foliola basi attemuata.

5. Sinctues globosus, notieniatus vel ovatus.

1645. Crepinia 2abelii Gjogr bab. N-2338.

Acculei parn; gilatati, parum falcati, ad ramos flor.2-3-ti; fol. parva, oborato-subattemata vel obtusiuscula, basi trumcata, atrovirentia, co costam eglaniulosa, gentes simphies, triangular:- convergentes; pet. eglaniulosi, sculeati, stip. Dilatatare, auriculis sat Tivergentibus; peo. 1-2, glabri, 10-12 m. longi; tubus ovatus; sep. pinmulis latiusculis, 1-2-gentatis instructa, ace- 
mine foliaces- Dentato; styl vix pilasuli, 'isco subplano; cor. prallide rosea, eciliata, fruct. oveatus, fasi subrotuniatus, apuice breviter attenuatus.

Hab. Hanorre, as Heiligenstadt (Label).

1646. Crepinia frachycarpa Googr Gat. N-233g.

Aculei caulinares validi, late falcato-dilatati, ad ramos flor.minores; fol. oborata, utringue breviter attenuata, ai costam eglanaulosa; Bentes simphices, convergentes; pet. hirc inje glanioulosi, inf inermes, stip. latae, auriculis rectis; per. 1, glabri, 5-7 m. longi; tubus orato-rotuniatus, sep. pinmulis inte. gris, brevibus, angustis instructa; styli subglaberrimi, Dises paulo comico; cor. prallide rosea; fruct. parrus, rotuniatus.

Hab. Basses-Pyrénés, as Biarrity (H. Bordère).

1647. Grepinia fortuito Gagr mss.

Atculei parwi, inchinato-gilatati, ai ramos flor. stipulares, fol parva, obovato-oblonga, acuta, basi praulo aitenuata, as basin costa glanioulosa; gentes simplices, ovato-recti; pet. eglangulosi, parce aculeati; stip. angustae, longuisculae, gorss galute rubentes, auriculis divergentibus; ped.2-4, glabri, 5-7 m.longi; tubus ovato-rotuniatus; sep. frevia, pinsulis integris, sat latis instructa; styli paulo puebesentes, Disco plans; cor........, fruct. parrus, subgloboso-comicus, basi rotunaatus, apice strangulatus.

Hab. Iranssilvama, Kolozs: ad Lontelke (2. Michter).

1648. Gepinia Terroris Gogr \$ab. N=2340.

Acculei Dilatati, prorum falcati, à ramos flor. sat copiosi; fol. oblonga, utrinque sed aprice praesertion sension attenuata, al costarn eglanioulosa; Dentes simplices; pet eglaniulosi, inf inarmes, stip. virides; pes. 1-2, glabri, 10-15 m.longi; tubus ovatus; sep. erecta, pinmulis latis, longis, gentatis copuose instructa; styli subglaberrimi, giseo plano; cor.........; fruct. rotundarto-subglobosus.

Hab. Isire, at thermas It-Prire-2e-Salatru prope lacum (N. Ganiogy.).

\$§. Fructus ovoivens rel soorato-oblongues.

$\sim$ Foliola in Eriosa hiserrata.

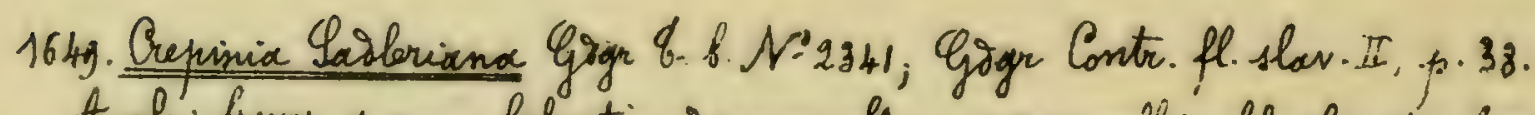
Acculei freves, parum falcati, as ramos flor. saepe muili; fol. oborata, becriter 
$-173-$

acuta, basi subattenuata, as costam eglanderlosa; dentes ovats-convergentes, inf. biserrati; pet eglanimlosi, aculeati; stip. parva, Silute rubentes, auriculis rectis; pes.1, glabri, 10-12 m. longi; tubus ovoidens; sep. pinnulis latis, 2-3Destatis instructo, rcumine foliaceo-dentato; styli subglaberrims; gisco sat conics, cormagna, eciliata, fruct. ovoideus, base Depressus, apice attemeatus. Hab. Hungaria, at Brocksberg prope Budapest (L. Hichter); Derbie, firope Beloprede (Pancic).

1650. Crepinia Hexiramea Gogr Gob. N-2342.

Acculi Debiles, rectiusculi, parum gilatati, as ramos flor. flecuosos nulli; fol. oblongo-acuta, basi breviter attenuata, tenuia, pallide vivivia, as cosAam eglanoulosa; Dentes late aperto-triangulares, inf. biserrati; pet-laxe pubescentes, aculeati, hine inde glanioulosi; stip. angustore, auriculis givergentikus; pes. 1. glabri, 8-10 m. longi; tufus ovoideus; sep.......... stygh glabri, disco plano; cor........... fruct. parrus, ovoidens, basi subrotuniactus. apice attemuatus.

Hab. Anstrix inif, ad Kalksburg (\%. Hiesfaur).

$\sim \sim$ Foliola ammia simpliciter serrata.

$X$ Petioli inermes.

1651. Crepinia minutula Gogr \&ab. N-23k3.

toculei copiosi, gebiles, falcato-Dilatati, as ramos flar-geminati; fol. oflonga, utrinque breviter attenuato, as costam eglanivulosa; gentes apartstriangulares, plerumpue simplices; pet eglandulosi, inermes; stip. sirides, subtur proulo probescentes, auriculis rectis, per.1-3, ylabri, 13-20 m. longi; tubus ovoiteus; sep. pirmulis latinscalis, longis, villosis, paulo dentatis instructa, acumine snbfoliaces; styli subhirtelli, cis-co plano; cor......., fruct - freviter ovoideus, basi subrotuniatres, apice abrupte strangulatus.

Hab. Hautes- Iyrénées, ad Gietre secus viam Suy (H. Bordère).

1652. Grepinia sepium Gogr \&ab. N-2344.

Aculer falcati, is ramos flor. sparsi; fol ample obovato-obtusuiscula, basi attemuata, lacte viridia, costam eglansulosa, dontes simplices, lancerlato - convergentes; pet basi paule glantulase; inermes; atip. longre, sat lator, nuriculis rectis; per. 1-3, glabri, $12-15 \mathrm{~m}$.langi; tubus ovoidens; sep. 
$-174-$

prinmulis latis, 2-3-Dentatis copiose instructa, ccumine foliaceo-Oentato; styli pilosuli, Disco prauls comico; cor rosea, eciliata; fruct. ovoideus, basi plus minusse Depressus, aprice attenuatus.

Hob. Tustria infer, ad Kalksburg prope gsien (J. Hiesbaver).

1653. Crepinia pallidevirens Gigr Gab. N:2345.

Acculei Debiles, subcylinarici, minute faleati, ais nomos flar. rubros coprosi; fol. obovato-elliptica, utrinque breviter ettemuata, mesis dilatata, pallive rividia, as costam eglanoulosa; ientes recti, simplices; pet. eglandulosi; sup vix aculeati; stip. latar, longae, dilute rubentes, auriculis sivergentibus; pes. 1-2, glabri, 9-12 m. longi; tubus obovatus; sep. angustioribus, smmibus integris, copiose instructa, styli subglaberrimi, gisco paulo conico; car. sat parva, lacte rosea; fruct. breviter ovoidens.

Hab. Haute-Savoie, ad It Martin (Suget).

1654. Grepinia resecta Gonr Sab. V:2346.

toculei gebiles, as ramos flor. Silatati, sat folcati; fol. obovato-oblonga, utrinque attenuata, ad basi costace folioli medii paulo glaninlosa, Tentes simplices, lanceolato-aperti; pet. hine inde glandulosi, inermes; stip. minutae, angustae, auriculis oivergentibus; pot. 1, glabri, 11-13 m. longi; itubus obovato-oblongus; sep. pinnulis angustis, parce dentactis instructa, styli glabrescentes, Disco paulo conico; cor .......; fruct. sfovatosblangus, basi rotungatus, aprice attenuatus.

Hob. Lavoie, ad Lalins prope Moütiers (Muget).

1655. Grepinia randensis Gogr Gab. N-2347.

toulei auri, gilatato - subfaleati, ad ramos flor-copiosi; fol.oboratooblonga, ainta, basi subtruncata, pallide viridia, ad costam eglanduloso, Dentes simplices, aperto-triangulares; pet. eglandulose, inermes; stip. Latae, longae, auriculis givergentibus; per. 1-2, glabui, g-11 m.longi; tubus orriDeus; sep........, styli parre pilosuli, giscs sat camico; cor......... ifruct. ovoidens, utrinque parum gepressus.

Hab. Helvetia, Gaud: ad Eartegnins supra Rolle (Farrat). 


\section{Petioli aculeati.}

1656. Crepinia perserutata Gogr Gab. $N-2348$.

toculei falcato-Dilatati, as ramos flor. copiosi; fol. late oblongo-rhomboidalia, attemuata, basi arrupte contracta, as costam eglanisulosa; contes simplices, lati, recti; pes. 1-2, glabri, 8-10 m.longi; tubus oborato - oflonJis; sep. pinnulis integris, parum latis instructa; styli sparse pilosi, cisec conico; cor........ f fruct. magnus, svoteus, utrinque ospressus.

Hab. Houte-Lavoie, at Habere-Lullin (Suget).

\section{Crepinia proclivis Gogr mss.}

Aculei breves, parum silatati, tenuiter falcati, ad ramos flor. coprosi, fol-late obovato-oflonga, basi sensin attenuata, al costam eglandachosa; Dentes simplices, lanceolato-convergentos; pet. aculeati, hine inde glandulosi; stip. breves, latissimae, virites, auriculis givergentibus; ped-1-3, glabri, $18 \mathrm{~m}$. longi; tubus obovato - oblongus; sep.........; styli glabri, gisco valie conico; cor......... fruct mediocris, obovato-oblongus, basi subrotuniatus, of apicem sat attenuaters.

Hab. Hispania medix, of fblbarracin (M. Mapatar).

\section{Grepinia vagensis Goor mss.}

Aculei tennes, subsilatato-inclinati, os ramos flor. sape nulli; fol. mediveria, oblong-acuta, fasi subtruncata, as basin costas glaniulosa; Dentes simplices, breves, ovecto-xperti, pet. basi pauls glasioulosi, parce ocenleati, stip. freves, virides, duriculis subrectis; ped.1-2, glabri, $5 \mathrm{~m}$. langi; tebus oboverto-oblongus; sep.......... styli subglaberrimi, gisco bre plans; cor. ignota; fruct. mediveris, oborato-oblangus, utrinopue attemuatus, sat turajians. Hob. Hungaria bor., gerag ad Kovacsi ('L. Thichter).

\section{5g. Orepinia attemeatifolia Gogr toab. $N=234 \mathrm{~g}$.}

Aculei auri, breves, falcato-gilatati, as ramos flor.geminati; fol obovato-oblonga, utringue attenuata, glaucescentix, as costam eglaniulosa; iontes simplices, lanceolato-aperti, pet. eglansulosi, aculeati; stip. latio, arriculis givergentibus; per. 1-2, glabri, 8-10 m. longi; tibus obovato- of longus, sep.pinnulis Congis, parum latis, 1-3-dontatis instructa, acumine longo, parum lato, styli villosi, aisco subplans, cor......., fruct. ovoideus, 
$-176-$

basi gepressur, apice strangulatus.

Hab. Yurcia, insula Oelans: inter Eveta et Farjestaden (Y. M. Schentz).

1660. Grepinia continens Goger mss.

Acculei longi, temiter falcati, parum silatatr, ad ramos flor. mulli; fol. amplissime elliptica, subacuta, basi vix attenuata, ai costam eglanioulosa, Dentes simplices, late triangulari-subconvergentes; pet-eglaniaulosi, subinermes, stip. magnace, virites, auriculis Sivergentibus; peot. 2-4, globri;, 10-12 m. longi; tubus oborato - oblongus; sep..........; styli glabui, Disco conics; cor.....; fruct-magnus, obovato-oblongus, basi roturivatus, apice attemuato-comicus, centrali vor inferne oepresso.

Hab. Iranssilvamia, Golnok Doboka: ai Csernefalu (2. Sichter).

1661. Crenimia breviplia Goge Bob. N-2350.

A culei gebiles, at ramos flor. falcato- Dilatati; fol praiva, obovato-elliptica, subacuta, basi paulo atternata, intense viriaia, ad costam eglanauloso; Dentes simplices, apertic; pet. eglarioulosi, odclute mubentes, inf. inermes; stip. sat magnae, auriculis subrectis; per. 1, glabri, 9-16 m. longi; tubus obovatus; sep pinnulis vix dentatis, copiose instructa, styli glabei, gisco subplans; cor. pallive rosea; fruct-ovoìiens, aprice atterniatus. Hab. Haute- Savoie, ad Pringy ( Pinget).

$\S \S \S$. Fructus oblongus.

1662. Crenimia vallesinca Goger Tab. N-2351.

Acculei Debiles, gilatati, temiter falcati, as ramos flor. mulli; fol. sat parva, obovata, utrinque sensim attenuata, is costarm eglaxiulosa; Dentes simplices, triangulares, aperti; pet. sparse glanisulosi, inermes; stip. meoiocres, latse, suriculis isirergentibus; prov-1-9, glabri, 6-7 m. longi; trubus oblongus; sep........; styli parvi, glabri, Qisco subplans; cor....... fonct.parves, oblongus, utringue attenuatus.

Hob. Helvetia, galais: at gramsis (2. Tovrat).

1663. Geninia amphirhyncha Gjog tab. N-2352.

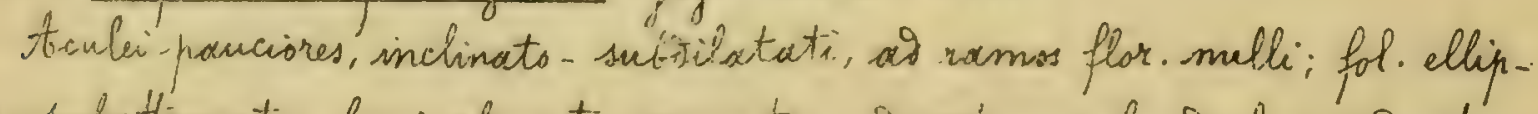
tico-subattemusta, basi abrupte crs:ata, ad costarn glaniuloss; dentes 
$-177-$

simplices, necti, valie profunisi; pet- eglanisulssi, parce aculeati; stip. auricalis Qirergentibus, pes. 1, glabri, 11-13 m. longi; tubus elongatus; sep. pinnulis integris, anguotis, pancioribus inotructa; styli asperse pilosi, vises paulo conics; cor........ fruct. elongatus, basi depressus, apioe longissime et anguste strangulatrus.

Hab. Ihone, a Limonest (G. Chabert).

1664. Crepinia obsecrans Goor mss.

toculei tenmes, inchinato-subsilatati, at ramos flon. nulli; fol. meiocria, obsvato-oblonga, acuta, basi attenuata, id costam eglanisulosa; Dentes simphices, late triangulares, aperti; pet. eglanoulosi, inf. inermes; stip. breves, latar, sirioses, acuriculis subrectis; pes. 1-2, glabri, 12-14 m. longi; tubus oblongus; sep. pinmulis latis, 1-2-Ientatis instructo, acumine foliaceo; styli adsperse pilosuli, aises subplans; cor........, fruct. sat parvus, breviter oflongus, basi attenuatus, apice contracto-substrangulatus.

Hob. Hungarix bor. Liptó ad Itrakx (2. Rickter).

1665. Greninix clavoides (Gogor Ros. nor. I, p. 11), Ggogr Gab. N.2353. Gogr Herb.ros. N:76.

toculei Debiles, falcato-gilatati, as ramos flor. inermes; fol. oblonga, utringue attenuata, as costam eglandulosa; gentes simplices, pratuli; pet. eglaniulosi, parce aculeati; stip. virides; pes. 1-4, glabri, 12-20 m. longi; tubus oblongus; sep. pinnmlis eglanoulosis, mediocribus instructe; styli susglabri, Dises comico; cor. pallide rosea, eciliata; fruct. clavatus, ample sblongus, apice inflatus, basi longissime sttemuatus, 16-18 m. Fiam. latus.

Hab. Ihone, ad fornas (No. Gandorer).

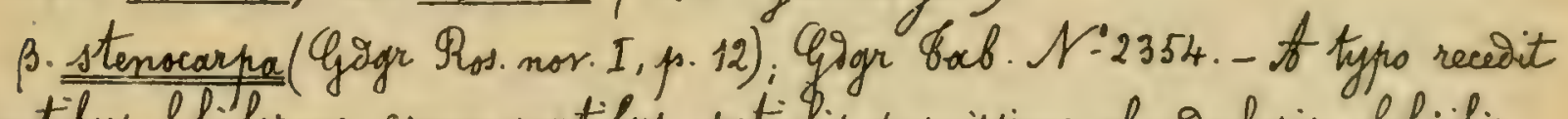
Dentibus foliolorum convergentibus, petiolis parcissime glanivelosis, foliolis anguotioribus et longioribus, fructuque angustiore, $10-11 \mathrm{~m}$. Siam. lato. Hob. Rhone, as fornas (Mt. Ganaoger).

1666. Gepinia celtiberica Gogr \&ab. N:2355.

stculai breves, faleato - Silatati, as ramos flor. geminati; fol. obovatooblonga, auta, fasi sensim attenuata, as costam eglaniulosx; dentes simplices, lanceslato-recti; pet eglandulosi, inermes, stip. latissimac, virides, auriculis divergentibus; per. 1-2, glabri, 6-8m. longi; tubus shongus; sep. 
$-178-$

finnulis parum latis, 1-3-gentatis instructa, acumine integrs; styli subglaberrimi, dises plano; cor parva, eciliata; fruct.oblongus, basi attenuatus, aprie longe constrictus.

Hat. Hispamia media, in Tierra os Mosqueruela, Geruel (Mr. Martin).

1667. Gepinia erythropetala Gogre toab. N-2356.

Acculei parum Dilatati, minutissime falcati, aD ramos flor. copiosi; fol. oforato-oflanga, basi breviter attenuata, apice rotundata, à costam eglaniulosa; Pentes simplices, breves, pauls convergentes; pet. inferne spaise glaniulosi, inf.inermes, stip.longae, latae, mesio rubentes, auriculis givergentibus; peo. 2-4, glabii, 17-21 m. longi; tubus oblongus; sep. pirmilis latiusculis, numerosis, copiose gentatis instructa; styli subglaberrimi, "visco praulo comico, cor.magna, intense rosea, aì unguem nitive albioa; fruct. oblangus, spuice atternuatus.

Hah. Houte- Savie, at Pringy (Suget).

2. Ytyli hirsuti val lanati.

a. Peannculi villosi.

† Fructus roturiatus, globosus vel ovatus.

1668. Creninia myacentha Gogr \&ab. N-2357. - Gogou Herb.ros. N-151. Foculei breves, parri, as ramos flor. sape nulli; fol. ovato-acuta; basi notreniata, at costam eglanioulosa; Pentes simplices, ovati; pet. sparse. glanioulosi, aculeati; stip. subtus pubescentes, auriculis rectis; pod.1-2, graciles, sparse pilosuli, 1 cent. longi; tubus breriter ovoideus; sep. latiuscule fimnata; styli hirsuti, gisco plano; cor intense rosex, eciliata; fruet. parvus, ovatus, fasi regressus, apuce fieviter attomuatus.

Houb. Thone, ai tornas (M. Ganooger).

1669. Geninia Jimidiend Gogr Gab. N-2358.

Aculei falcato-Dilatati, as namos flor. Stifulares; fol-obovocto-oblanga, freviter acuta, basi. subrotuniata, lacte viridia, ad costam eglanimilosa; Dentes simplices, lanceslato-convergentes; pat. eglaniaviosi, aculeati; stip. latae, ouriculis subrectis, peo. 2-9, sparse pilosi, 6-g m.longi; tubus rotuniatus, sep. pinmulis brevions, eglanivulosis instructa, acumisine angusto;

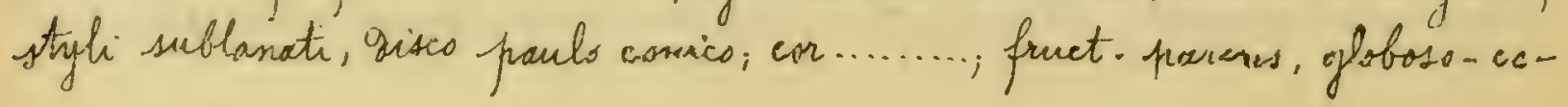


- 179-

sicus, basi rotuniains, apice atternatus.

Hab. Helvetix, gaid: as Signal ge Chexbres (L. Favrat).

1670. Grepinia brachyovon Gogr Gab. N:235g.

Aculei parri, inclinati, as ramos flor. gensos mulli; fol parra, obovatosubacuta, fasi sotundata, rugosa, coriacea, ad costam glasiaulosa; 'dentes simphices, brevissimi, ovato-obtusi; pet. aculeati, eglanisulosi; stip. rubentes, auriculis rectis; pel. 1-2, villosi, 6-8m. longi; tubus roturiatus; sep. brerissimas, pinnulis integris, angustis instructa; styli villosi; dises concio; cor. eciliata; fruct. parrus, globosus, basi rotuniato-umbilicatus, apice subrotuno. Hob. Mouchev-iu-Mhone, io Martignes (to. touthoman).

T+ Fructus oroiveus vel oborato-oblangus.

1671. Crepinia formicata Gogr bab. $\mathcal{N}=2360$.

Afculei valioi, ad ramos flor. falcati, late gilatorto- decurrentes; fol.obovato-oblonga, vix attemuata, basi subrotuniata, hucide et ansene virentia, at costam eglanivelosa; dentes sinphices, apertic; pet. incrmes, eglanivelosi; stip. longae, latar, auriculis sivergentibus; per.1-4, pilosuli, 9-20 m. Longi: tribus oforato-oblongues; sep. pirnulis integris, pancioribus, sat latis instructa; styli moymi, sisco subplano; cor......; finct, obovato-oblengus, as apriem fracestion attemuatus.

Hab. Haute-Savoic, as Haboire-Poche (Tuget).

1672. Grepima graniciconana Giogr mos.

Ficulei breves, dilatati, temuitor falcati, as ramos flax.copissi; fol ample sborato. subacuta, basi sensin roturiata, atrorirentio, as costam glaniulosa, Dentes simplices, late orato-subeonvergentes; pot-eglarioulosi, acculeati;

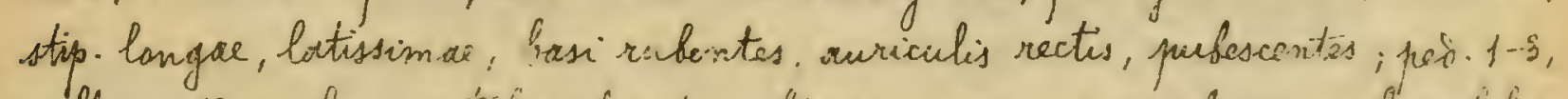

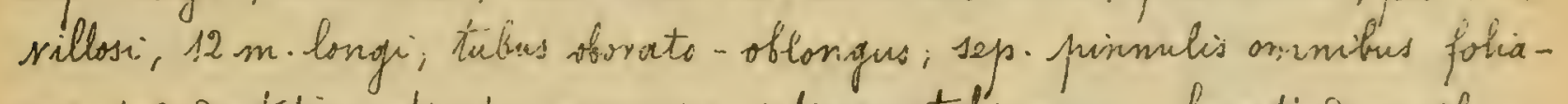
ceis, 1-2-Dentatis instructa, Acrumine integro; stghli magni, lanati, lisco flano;

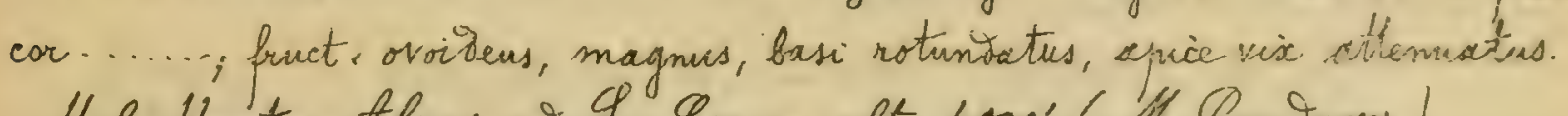
Hab. Hawtes folpes, ad La Grave, alt. 4000' (M. Ganooger).

1673. Crepinia laxipila Gogr Gab. Yo-2361.

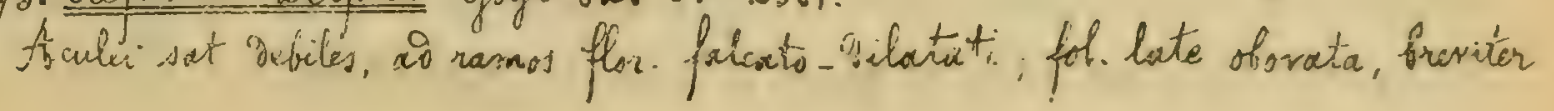


$-180-$

ettemecta, fasi rotaniata, as costan eglanoulosa; Dentes simplices, apertic; pet. parce oculeati, inf. sparse glansulosi, laxius pubescentes; stip. longas, auriculis givergentibus; pes. 1-2, hirtelli, g-12m. longi; tubus oblongus; sep. pinme his angustes, temiter dentatis instructa, acumine saepe foliaces; styli villosi; Dises sat comico; cor....... fruct. obovato-oblongus, utrinque repressus. Hab Lavoie, to Salins prope Noütiers (Muget).

1674. Crepinia sphoenoclada Gogr bab. N=2362.

Acculei faleato-Dilatati, ai ramos flor. remotos, copiosi, 4-6-ti; fol. late srato-elliptica, utrinque subrotuniatoc vel of tusa, lucise at amoene viriaia, at costam eglandulosa; sentes simplices, lati, recti; pet. eglaninlosi, inf. inermes; stip.magnac; per. 1-3, villosissini, 12-15 m. longi; tubus oroidens; sep-pinnulis latiusculis, dentatis instructa; styli lanati, Disco paulo conico; cor. magna, eciliata; fruct. breviter ovoivens, sepalis siu coranatus. Hab. Ihone, is Chaponost (M. Chabart).

tft. Fructus oblongus.

1675. Orepimia poototricha Gogr Eal. N-2363.

foculei sat debiles, falcato-Dilatati, as ramos flor. plerumque melli; fol. oborato-oblonga, utrinque paulation subrotuniata, at costam eglanoulosa; Dentes simplices, convergentes; pet eglandulssi, fore onnes inermes; stip. sirides; per.1-3, villosi; 10-13 m. longi; tubus oblongas; sep. pinmelis gontatis, anguestis copisse instructa, stighi villosi, disco praulo camico; cor.......; fruet. Oblongus, basi gapressus, apice valis attennatus.

Hab. Qhane, as Limonest (PChabert).

1676. Crepinia Gyrus Gogr Gab. N-2364.- C.pyriformis (Dèségl. in Christ Rosen ger Schweiz p.185 non Isvartz). C. imitata (Déségl. in Mém. aced. De Maine - et-Loire XXVIII, 1873, 13.120; actr.p.24); C. Dumetorum f. Ehuillieri: (Christ. l.c.). Billot axs. $N=3588$.

Abculer à ramos flot. flexuosos falcato-gilatati; fol. anguste oblongo-acuta, basi subcuneata, at costam eglanaulasa, dentes convergentes, simplices; pet-eglaniulosi, sacpe inermes, stip. elongatre, subtus pubescentes, auriculis rectis; pet. 1-i. pparse pilosi; 6-10 m. longi; tubus .elongatus; sep. pinmulis integris, la ivesculis, pancioribus instructa; styli 
$-181-$

villosi; Dises sat conico; cor.magna, alba; fruct. elongatus, pyriformis, basi oecurrens:

Hab. Houte-Savoie, at Habire-Toche (Suget) nec alib:

1677. Breminia. Threha Gogr Bab. N-2365.

Aculei inchinati, ad ramos flor. geminati; fol amphisscule oblongeacuminata, basi atternuata, ai costam eglasioulosa; gentes simplices, lanceolato-convergentes; pet. eglansulosi, inermes; stip. virides, auriculis Divergentibus; peo. 1-3, villosi, 11-19 m. longi; tubus sólongus; sep. pinmulis latis, 1-2-dentatis instructa, acumine elongats saldeque foliaces-dentats; styli villosi, isses subplans; con. eciliata; fruct. breviter oblangus. Hab. Gart, at Le higan (O). Gueshiemion).

1678. Greninia ilicetorum Cojogr 6ab. N-2366.

Acculei valici, solis falcato-2ilatati, ad ramos flor. sat copiosi; fol. elliptica, viritia: al costam eglanouloss; Dentes simplices, callosi; pet. a culeate, sparse glanioulosi, stip. Gilatatae, auriculis givergentibus, peod. 1-4, villssi, brevessimi; tubus oblongus; sep. pinnulis Dentatis, latis longe instructa, styli panciores, villosi, disso comico; car. pallide rosea; fruct. oblangus, fasi rotuniatus, centrali excepto.

Hab. Rhone, as Ecully et Chaponost (9. Chabert).

1679. Prepinia convensula Gogr \&ab. N-2367.

Arenlei faleato-Dilatate, as ramos flor. pranciores val nulli; fol. sorato-elliptica, attenuata, basi vise contracta, medis gilatata, ad costam eglanioulosa; Pentes simplices, aperti; pet. aculeati, inf proesertion glanduelosi; stip. sat breres, auriculis rectis; pes.1-2, villosi; $7-10 \mathrm{~m}$. longi; tubus elongatus, sep. pinmulis integris, paucioribus, latiusculis instructa, styli villosi, gisco subplano; cor....... fruct magnus, elongato-pyriformis, utrinque attemuatus.

Hrob. Frieche, ad Daveysicux prope Annonay (Moullu).

1680. Crepinia vinosfonensis Gotgr 8ab. N-2368.

teculei falcato-Dilatate, a ramos flor. plerumque nulli; fol oforata, utrinque attemuato, late viriain, as costam eglandulosa; bentes simplices, aperti, sublanceolato-triangulares; pet parce aculeati, eglanioulosi; 
$-182-$

stip. Congae, auriculis rectes; pes. 1-3, sparse pilosi, 10-13 m. longi; tutus ollongus; sep....... styli parvi, villosi, gisco sat comics; cor....... f fruct. magnus, oblangus, utrinque ser besi praesertion attennatus. Hab. Anstric inf, ai Kalksburg prope glien (Y. Hsiesbour).

1681. Crepinia cuncibasis Giagr mss.

Acculei temiter faleate, parum silatati, as ramos flor. rari; fol. ample sblongo-acuta, basi cuneata, subtus albo-glauca, cos costam non glanioulosa; Dentes simplices, lanceolato-convergentes; pet eglanisulosi, parce aculeati, stip. magnae, glanco-rivides, auriculis givergentibus; ped. 1-2, villosi, 5-7 m. longi; tubus oblongus; sep. pinmelis integris, sat latis, acumine foliaceis instructa; stagli villosi, gises conico; cor........, fruct-masimus, oflangus, utrinque longe attenuatus, medis turgious.

Hab. Hungaria bor. Liptó : ad Teherpatak (2. Michter).

6. Pesunculi glabri.

T Fructus globosus vel rotuniatus.

§. Folisla inaeque vel biserrata.

1682. Grepinia Carioni (Deiégl. et Gillot in Bull soc. bot. De Delgigne XIX, 1880, p. 34); Goge tab. S T:2369.

Aculei falcato-ailatati, as ramos flor, panciores; fol-orato-elliptica, oftusa, Sentes biserrati, patuli; pet. poulo glanaulosi, sup. inermes; stip. glabrae, auriculis Sivergentibus, pet. 2-5, glabri, breves; tuchs oroideus; sep.riritia, pinmulis paulo dentato-glanoulosis instructa, acumine foliaces; style hirsuti, sisco subplano; cor. albo-rosea; fruct. rotuniatus.

Hat. Saone-et-Lire, toutun, etc. Gtiam Bolgium, tonglix meris.

1683. Orepinia caloshhaera Gigr Gab. N:2370.

Ficutei breves, falcato-Silatati, purpureri, à ramos flor-copiosi; fol obosato-subobtusa, basi roturioata, coriacea, so costam eglanoulosa; dentes ovatoaperti, inf. biserrati; pet. glaniulosi, aculeati; stip. virides, auriculis rectis; pes.2-4, glabii, 7-g m.hongi; thines globosus, sep. pinnulis latis, 1-2-3entatis instructa, acumine integro; stylli lanati, gisco plano; cor eciliata; fiuct. grobose-gepressus.

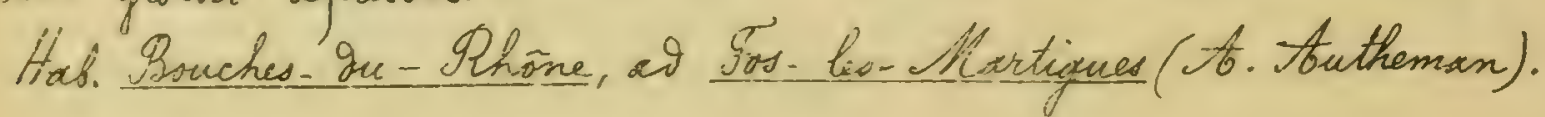


$-183-$

1684. Grepinia longigpina Gogr \&ab. N:2371.

ticulei elongati, delatati, necti aut paulo inclinati, oi ramos flor copiosi. fol oblongo-acuta, basi attenuata, visidi-glaucescentia, ad costam eglanoulosa; Sentes fere omnes biserrati, pperto- triangulares; pet glandulosi, aculeati, lase pubescentas; stip. magnac, latae, suriculis rectis; pes. 1-2, glabri, 5-6 m. longi; tubus rotunisatus; sep........; styli Dense lanait, Dises plano; coi...... fruct. giobosus, basi rotunsatus, apice brevissime attemuatus. Hab. Envingia, of Osterplo propre Heimar (C. Haussbnecht).

1685. Crepinia cuneifolia Gogr bab. N-2372.

Acculei falcato-gilatati, as ramos flot.copiosi; fol. oblang-acuta, basi longissime valreque attemuato-cuneata, newrsa, as costam eglanivulosa; dentes inaequales, lanceolato-convergentes; pet eglangulosi, aculeati; stip. virives, latae, auriculis Divergentibus; per. 1-3, glabri; $12-13 \mathrm{~m}$.longi; tubus ovato-rotuniatus; sep. pinmulis param latis, lociniatis instructa, acumine integro, parum lato; styli villosi, dises plano; cor....... fruct ample subglobosus.

Hoc. Anglia, at Codsall (j Fraser).

\$. Foliola ommino simpliciter serrata.

1686. Crepinia Desyphora: Gogr Eab. N-2373. - Gogr Herb-ros. Ne 308.

ticulei validi, falcato-Dilatati, ad ramos flor.melli; fol. late oblongorhomboidalia, subacuto, basi vis attemuata, ar costam eglandalose; Dentes simplices, lanceolato - sub́convergentes, pet. eglaniulosi, inermes; stip. latissinae, subtus glabrescentes, auriculis subrectis; fot. 1-3, glabri; 10-13 m.longi; trebus svatus; sep......... styli horsuti, gisco praulo comico; cor........ fruset. maximus, crassus, bubglobosus.

Hab. Hautes- Tyrénés: ad Gère secus viam Lur (H. Mardere).

1687. Crepinia orogenes Goger Gab. N'2974.

Aculei gilatati, tenmiter falcuti vel inclinati, at ramos flor.copriosi; fol. sovato-subattemuata, fasi fere rotuniata, virisi-glaucescentia, as costam eqlaniaulosa; Dentes simphices, lanceslato-aperti; pet eglanisulost, inermes; stip magnar, auriculis rectis; ped. 1-2, glabri, 4-6 m.longi; tubres rotunoretiw; sep.pismelis filiformibus, 2-3-2entatis instructa, acumine angusto; 
$-184-$

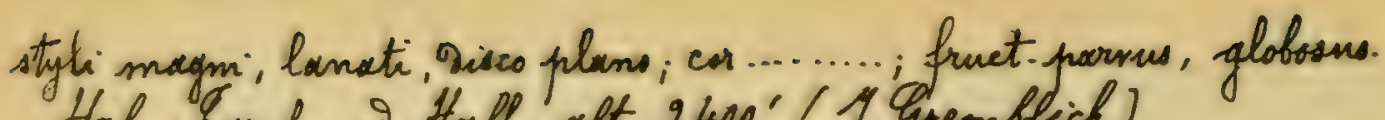
Hab. Eyprt, ad Hall, alt. $2400^{\prime}$ ( Y.'Gremblich).

1688. Orenimia nithyophila (Grogr Essai p. 31; Ros. II, p.25); Gogr Zab. N: 2375. - C. opaca (Gren. in Billot forchiv. $p .332$; Crep. in Bull. soc.bot. Balg. $V, p .21$ non $F_{\text {ries }}$.. - Millot exs. N 1478 .

toculei at ramos flor. falcato-Dilatatis fol. ovata, acuta, basi rotunisata, as costam eglaniulosa, superne viridi-opaca; Dentes simplices, ciliati; pot. eglandulosi, tenniter aculeati; stip. subtus pubescentes, suriculis subivireryentibus ; pes. 1-4, glabri; tubus globosus; sep. mèriacria, pirnata, eglanaculosa, styhi gense villosi, gisco plans; cor. pallesa; fruct. sphaericus.

Hab. Jurk, in pinetis (Girenier).

C. opace (Fris Il. hall. p. 37) al Crepinia Dinymoniontas pertinet; Gogr Mon. ros. II,p.398.

1689. Orepinia Warwichiana Gogr bab. N-2376.

treulei breves, falcato-gilatati, as ramos flor. copiosi; fol. oblongs-acutriscula, basi subrotundata, ad costam eglanaulosa; dentes simphies, aperto-lanceolati; pet. eglandulosi, aculeati, stip. sirides, auriculis subirivergentibus; ped.1, glabri, 8-10 m. longi; tubus orato-rotuniatus; sep..........; styli villosi, gisco sat comico; cor.......; fruct. parvens, globosus. Has. Anglia, as Yoarwick (Y. Sraser).

16g0. Crepinia festinata Gagr snss.

Acculei breves, parri, inclinato-subilatati, as ramos flot-geminati; fol. medicria, anguste oflongo-lanceolata, fasi attenuato, as costam eglania-

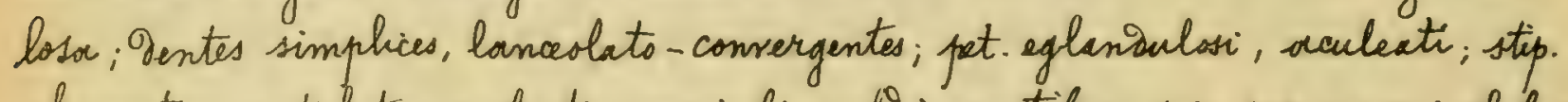
elongatae, sat latae, rubentes, auriculis subiviergentibus; pes. 1-2, crassi; glabri; 1 cent. longi; tubus subrotundatus; sep. pinnulis annibus integris, latis iss truetor; styli magmi, lanati, aisco plano; cor........ fruct. sat magnus, subglobosocomicus, basi rotunsatus, aprice attenuatus.

Hab. Hungaria bor., Liptó :as Mozsaheyy Muzahegy (2. Richtor).

to Fructus oratus vel ovato-rotuniatus.

\$. Tetioli infer. vel asmines inermes.

1691. Crepina amphisboena Gogr Bab. N-2377. - Gogr Herb.ros. N-250. 
$-185-$

t-culei ramos flor. falcati, parum Dilatati; fol. tate ovato-acuta, hasi rotunista, firma, intense viridia, ad costam eglanaulosa; dentes simplices, convergentes; pet. hine inde glanidulosi, inermes; stip. sivises; ped. 1-3, glabi, mesiocres; tubus svoidens; sep. pinnulis latiuseulis instructa; styh villosi, isco subplano; cor. rosea; funct. late ovatus, basi gepressus, striatus. Hob. Rhone, at Drinss (M. Gandoger).

1692. Brepinia Yteiniana Gogr Hat. N'2378.

Aculei breves, tenuiter et parum falcati, as ramos flor. copiosi; fol. obovatoacuta, basi subtruncator, glausa, at costann eglanioulosa; Oentes simplices, triangulares, recti; pat. inermes, eglanoulosi, stip. rubentes, auriculis sat $\partial i-$ vergantibus; jed. 1-3, glabri, g-10 m. longi; tuhus ovatus; sep. pinnulis smmibus integerrimis, farum latis instructa; styli lanati, aisco plans, cor. rosea, parva, eciliata; fruct. parsus, avato-rotuniastus.

Hab. Gyrol, ad toichat prope Hall ( $y$. Gremblich).

1693. Crewinia ludens Gogr mss.

toculei faleato-bilatati, ad ramos flor. nulli; fol ample shorato-oblonga, subacuta, fasi sension attenuata, av costam eglandulasor; dentes umnes biserrati, late triangulares, aperti; pet. inermes, hine inde glanialosi; stip. longae, latio, virioses, auriciulis sat divergentibus; per. 1-3, glabri, 1 cent. longi; tutus ellinticus; sep. pirnulis ansibus foliaceis, 1-3- Sentatis instructa; styh magm; lanati, gises sat comico; cor...... ; fruct. arato-ellipticus, etrinque subrotuniatus.

Hab. Ier, as La Martre (folbert).

1694. Rreninia sungenoìes Gotr taf. No2379.

Acculi falcato-Dilotati, à ramos flor. interdum mulli; fol. obrato slonga, utrinque sensim attenuata, lacte virisix, so costam eglanichose; Dentes simplices, lancealato-sufoonvergontes; pet eglandulose, inermes; stip. silute rubentes, auriculis parum Divergentibus; ped. 1-3, glabri, 12-15 m. Congi: tubus oroideus; sep....... Ftyli sublanati, bisco fore plano; cor......; fuct. parvus, ovatus, basi plerumque rotundatus, apice subdepressus.

Hab. Hautes - Tyrínés: as Piedre secus riam Luz (H. Sordère). 
$-186-$

1695. Crepinia Cilaromelanos Gogr Gab. N-2380. - Gogr Herb. ros. N-351. trculei gabiles, inclinato-Dilatati, as ramos flor. saepe nulli; fol. oblonga, utringue sensim attemuata, as costam eglaniulosa, atrosirentia; ientes rect, simplices; pet. eglandulosi, inermes; stip. sirides; ped. 2-3, glocbri, g-13 m. longi; tulus freviter ovsidens; sep. pinmulis sape integris, longis, wngustis instructa; styli villosi, giseo paulo comico; cor.......; fruct ovato-ro. tunisatus, utringue parum depressus.

Hab. Hère, at thermas It Sierre-De-Salatru (N.Gandoger).

1696. Creninia Labrellata (Gogr Ros. nor. II p. 25); Gogr \%ab. N=2381. Googr Herb. ros. $N: 132$.

Abculer debiles, inchinati, parum oilatati, as ramos flor. sat copiosi; fol. obovato-oblonga, freviter attenuata, basi cordata, sio costam eglaniulosa; rentes simplices, eperti; pet. eglaniulosi; inf. inersnes; stip. megiseres; pet. 1-2, glabri, 13-15 m. longi; tubus breviter ovoideus; sep. pinmulis angustis, 2-4-gontatis instructa; styli hirsuti, diseo subconico; car. pallide rosea; fruct. parrus, svatus, apice attenuatus. thab. Phone, of tornas (M. Canoger).

\$. Setioli onines aculeati.

169\%. Grepinia Colkini (Gogr Mas. nov. I. p.12); Goor Gab. N:2382. - Gogr Herb. ros. Nं346.

Acculei breves, parvi, inchinato-gilatati, ad ramos flor areuatos sat copiasi; fol. oforato-oblonga, utrinque sensin attennata, as costarn non - glaniulosa; ientes breves, simplices, aprerti; pet. eglandulosi, aculeati; stip. sat Gilatatac, auriculis rectis; pes. sacpe 2-3, glabri, 6-9 m. longi; tifus ovatus; sep. pinnulis vix gentatis instructa, acumvire elongato; styli villosi, disis jubplans; cor.rosea, eciliata; fruct. sat parsus, subrotuns. Hab. Ithone, a tornos (M. Gandoger).

1698. Grepinia gravis Goge mss.

Atulei valiai, palcato-dilatati, as ramos flor. copiasi; fol. sat iate shoseto-ofenga, subacuta, basi sensim attenuata, as costam eglasiau-

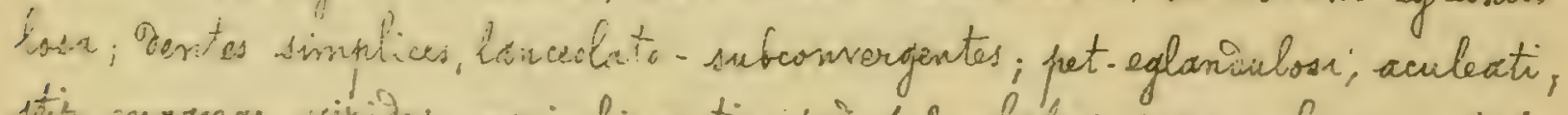

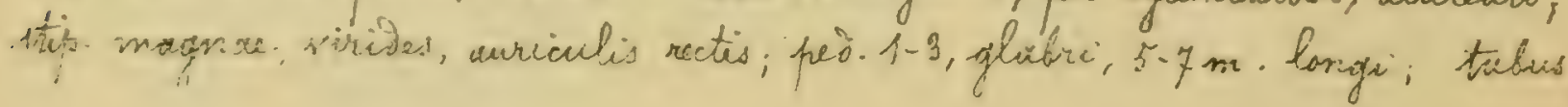


$-187-$

vvatus, sep. pinnulis sat angustis, onnibus 1-3-gentatis instructa; styli villasi, gisco subplano; cor ........; fruct maximus, ovatus, basi gepressus, apric attemeato-comicus.

Hob. Hungaria, Bubapest at Istenhegy (A. Steffeck).

1699. Crenimia uncinelloides (Muget) in Gjogr bab. N-2387. Diseglise herb. ros. N:67.

Feculei panciores, faleato-gilatati, à ramos flor.rari; fol. late ovatoacuta, firma, pallide viridia, io costam eglanialosa; Dentes inoeguales, late. triangulares; pet eglangulosi, parce aculeati; stip. batae, auriculis rectis; peo-1-3, glabri, mesiocres; tubus ovoideus; sep. pinmulis integris, latis instructa; styli villosi, Dises subplans; car alba; fruct. saepius ov xtis. Hab. Houte- Savori, as Habire-Lullin (Buget), Helvetia, frope Fribourg (Lagger).

1700. Crepinia orthachlamys Gogr Gab. N-2384.

Itculei minuti, gilatocti, temiter folcati, to ramos flor. glaucos copisosi, fol ovato-elliptica, lacte viridia, nervosa, valde cariacea; Dentes simplices, Durii, operth; stip. sirìdes, basi excepto rublra, auriculis givergenthiss; pet aculeati, eglanaulosi; per. saepius 2, plabri, 3-5 m. longi; tubus ovato-ellipticus; sep.crassa, arecta, giv persistentia, pinnulis integris, angustis, parce instructa; styli magni, lanati, bisco plano; cor.......; fruct magnus, svatus, basi rotungatus, apice strangulatus.

Hab. Helvetio, Gelais: at Loneiche-les-Sains (Lorenti).

1701. Crepinia heteromorphac Gögr \%ab. א-2385.

toculei longi, parum faleato-Dilatati, ad ramos flor interoum nulli; fol. oforato-oblonga, attenuata, basi noturisecta, at costam eglanionloser; Dentes simplices, recti, acuti; pet. eglanoulasi, aculeati, stip. sirides; ped 1-2, glabri, 10-11 m. longi; tubus ovato-ellipticus; sep......... stigh villosi, giscs paulo conico; cor........, fruct. nume rotuniatus, munc breviter ovorideus. Sasi paulo depressus, aprice attenuatus.

Hab. Mhome, Mont-Cingre prope Lyon (P.Chabert).

1702. Crefinia syngenes Ggogr Gab. No 2386. Gogor. Herb. ros. 11-289.

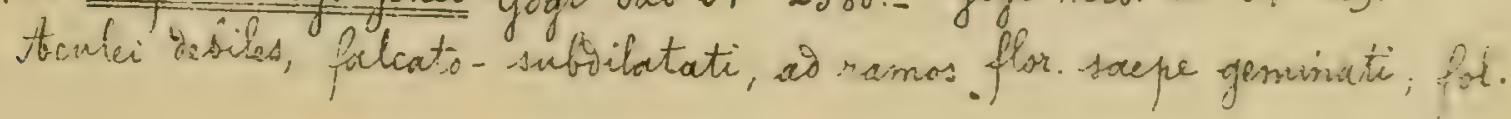


$-188-$

late oborato-oblonga, frevissime attemuata, basi subrotunsata, intense viriGia, a costam eglansulosa; gentes simplices, aperti; pet-eglanioulosi, aculexte; stip. latiuscular; pes. 2-4, glabri, 10-13 m. lange; tubus aroideus; sep. pinmulis gentatis copiose instructa, acumine elongato; styli villosi, gisco patilo comics; cor.......; pruct. ovatus, apice attenuato-strangulatus.

Hab. Phone, at Montmelas in monte It Sonnet (H. Ganooger).

$t+T$ Fructers oviseus vel oborato-oblongus.

§. Foliala basi attenuata vel cuneata.

$\sim$ Setioli onnes aculeati.

1703. Crepinia acanthoclada Gagr Gab. N-238\%. - Gogr Hert. ros. N-265. Treulei inclinato-subsilatati, as ramos flor. Densos copiosissimi; fol. oblongo-acuta, basi breviter attenuata, atrovirentia, as basin costae folioli medii sacpe 1-3-aculeolata; itntes simplices, lanceolati; pet eglanaulosi; aculeatissimi; stip. lata, auriculis rectis; per. 1, glabri, 6-13 m. longi; tubus obovatus; sep. pinnulis latis, longis, integerrimis copiose inotructa; siyli innati, aisco valse conico; cor. albo-rosea; fruct obovatus, is apriem attenuatus.

Hab. Herenaci cente., ad Gedre (A. Borgère).

1704. Crepinia xojectiva Cogr mss.

Aculei breves, subfalcato-jilatati, parvi, ad ramos flor saepuis mulk: fil.majuscula, oblongo-acuta, basi atternata, ra costam eglandulosa; oontes simplices, lancealato - aperti; pet. eglanaulosi, aculeati; stip. virides, lingar, sat latas, auriculis sivergentibus; -jes. 2-4, glabri, 1 cent. longi; tebus oborato-oblongus; sep. pinnulis onnibus latis, 1-2-gentatis instructa; stughi lanati, siseo comico; cor........, fuct. médiacris, obovats-oblongus, basi rstuniatus, aprice attenuato-strangulatus, comicus, centrali inferne attenuato. Hab. Eransilvania, Corda toranyos: as Gorix Hosadék (Q. Aićnter).

1705. Crepinia pyenodes Gogr mss.

Acculei médiocres, falcato-gilatati, as ramos flor-nulli, fol conferta, sat parva, iborato-oblonga, six acuta, basi attenuata, à costam eglan-

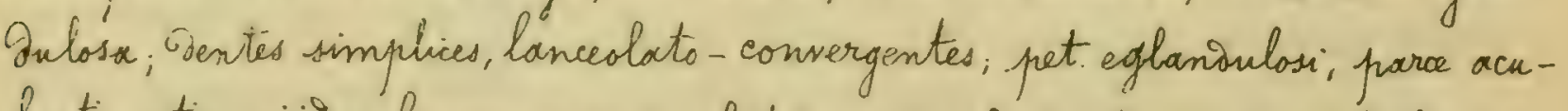
veati, stip. virives, longae, parum latae, auriculis nectis; ped. 1, glabri, 3-4 
$-189-$

m.longi; tubus ovoideus; sep. pinnulis integris, parum latis instructa; styh lanati, disco sat conics; cor ..........; fruct. mediveris, ovoideus, basi gepressus, apice ottenuato-conicus, strangulatus.

Hab. Eransilvania, Izolnok Dobokx. as Iinfalva (2. Michter).

1706. Grepinia oxyrhaphis Gogr mss.

Aculei vativi, falcato-Dilatati, samos flor. verticillati, fol ample sblongs-acuta, basi sensim attenuata, ai costam eglanaulose; gentes simplices, late triangulari-subconvergentes; pet. parce pubescentes, eglariaulesi, aculeati, stip. maximac, Dilute rubentes, auricalis givergentibus, per. 2-4, glabri, 12-13 m. longi, crassi; tubus ovoivens; fo p. prinnulis subintegris, latis instructa; styli villosi, disco plino; fruct.magnus, breviter ovoideus, utrinque iepressus, meaio valse turgisus.

Hab. Hungarix, ad Kokukhegy prope Muaxpest (A. Steffeck).

1707. Creninia placida Gagr Gab. N-2388.

Fculei falcato-gilatati, wo ramos flor. nulli; fol-obovato-elliptica. utrinque attenuata ses basi cuneata, is costam eglaniulosa; dentes recti, simplices; pet-aculeati, inferne paulo glandulosi; stip. sivives; pet. 2-3, Alabri, g-11 m.longi; tubus svoivens; sep........... styli villosi; discs paulo comico; cor.........; fuet.maximus, ovoideus, basi sensim attermatus, apice inflatus.

Hab. Inoñe, at Ecully (P. Chabert).

1708. Crepinia araborum Gogr smss.

teculie: icbiles, parum folcato-Dilatati, as ramos flor. nulli; fol mediocria, sfovato-sblonga, subacuta, basi attenuata, at costam glanisulosa; gentes onnes biserrati, lanceolato-recti; pet. villosi, glanderlosi, aculeati; stip. fres'es, latar, virives, auriculis sat divergentifus; pes.1-3, glabir; $10 \mathrm{~m}$. longi; tubus ob-ovato-oblongus; sep. pinnulis latis, 1-6-gentatis instructa, aicumine foliaceo, integro; styli villosi, Disco paulo conico; cor-sat magna, ecir liata; fruct. obovato- oblongus, utrinque attenuatus.

Hab. Algeria arient., pirope Constantine (ge Rebour).

1709. Prepinia pallivior Gogr imss.

Arulei pauciores, valionli, falcato- Dilatati, at ramos flor nulli; fol. 
$-190-$

ampliuscule oblongo-acuta, basi attenuata, à costam exlandulosa; Dentos sato-cuspivati, aperti, inf paulo inaequales; pet. villosi, eglanioulosi, aculeati, stip. virides, longae, angustae, auriculis oivergentibus; pes. 1-2, glabri, 7-10 in. longi; tubus obovato-oblongus; sep. pinnulis parum latis, 1-3dentatis instructa, acumine foliaces-Dentato; styjl sillosi, gisco plano; cor......; fruct.mésiocris, obovato-oblongus, basi gepressus, apice longe attenuatostrangulatus.

Hab. Iransilvania, Kologs: of Magyar i Kereke (2. Richter).

$\sim \sim$. Petioli saltem inferiores inermes.

1710. Crepinia Obaria Gogr Gab. N-2389. - Millot exs. N-3724.

Acenlei mevioeres, falcato- Vilatati, as ramos flot. saepe nulli; fol. ellipticeslovata, utrinque attenuata, as costam eglanoulosa: Ventes simplices, resti; pet. eglaniulasi, pilerumgue annes inermes; stip. mediscres, auriculis rectis; peis.1-3, glabri, 8-12 m. langi; tubus oboratus, sep. pinnulis integris, langis, angustis instructa; styli hissuti, gises paulo canico; cor. rosea; fruct. obovatus. Hat. Gard, at Aulas prope Le gigan (D. Enezkiewriz).

1710 bis. Creninia tragacanthina Gogr mss.

toculai bress, parvi, inchinato-suboilatati, as ramos flor. sparsi; fol. parva, obovato-oblonga, acuta, basi truncata, ai costam eglanisulosa; dentes simplices, profunisi, orreto-convergentes; pet.eglaniclosi, inf. inermes; stip. latae, sat breves, virides, aurriculis rectis; pos-1-2, glabri, $1 / 2$ cent. longi; tubus oforato-oblongus; sej........; styli lanate, giser concic; cor.....; fruct. sat parrens, obovato-sblongus, basi subrotuniatus, apice attenuatostrangulatis.

Hab. Hungaria, at Magi fürdo (2. Michter).

1711. Grepinix platynetala Gigr Lab. N-23g0.

ticulei inchinati, parum Dilatati, as ranos flor. tennes, rectiusculi; fot. sborato-subattemeato, basi fere rotursata, of costam eglandulosa; gentes simplices, paulo convergentes; pet. eglanieulosi, inermes; shp. virises; pes. 1-2, glabri, 5-7 m.longi; tubses ovoidens; sep. pinssulis integris, pubescentibus, lativeculis, lonse instructa; styli viliosi, Disco plano; cor major, 7 cont. Siam. itia, ecelinta; frect. ovoideus.

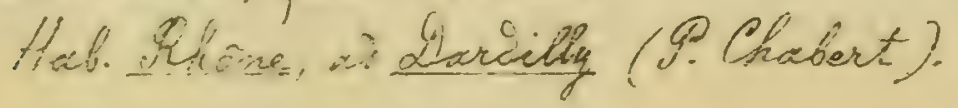


$-191-$

1712. Crepinia pluriaps Gigr Eacb. N-2391. - C. Herbichiana (Mlocki in Oast. bot. Leits. 1887, p. 419).

Abculei debiles, parum bilatati, tenuiter foleati, at ramos flor sat copiosi; fol. sat axguste obovato-oblonga, utrinque attenuata, as costam eglanQulosa; gentes simplices, aperti; pet.eglanivlosi, inf. inermes; stip. mediocres, auriculis givergentibus; ped. 1-2, glabri, 3-8 m. longi; tubus oboratus; sep. pinmulis integris instructa, acumine dilatato; styli villosi, gisco subplano, cor. ; fruct ovoivens, basi rotunisatus.

Hab. Hante- Savoie, Habie-Lullin (Suget); Bukoswina (Mlocki).

\$. Foliola basi notuniata.

$\sim$ Petioli annes inermes.

1713. Brepinia brachyacentha (Gogr in Mull soc. Dauph. 1874, p. 15), Gogr Garb. N:23g2; Gogr Contr. fl. slav.II, p. 3g. - Googr Herb. ros. N-252; Yociéte gauph. exs. N-111.

Aculei gebiles, freves, sichinato-subsilatati, ad ramos flor. pauciores; fol obovato-oblanga, attenuata, basi rotuniata, à costam tglaniulosa; Dentes simplies, aperte; pet. eglaniulosi, inermes; stip. sisides; pet. 1-2, glabri, 12-14 m.longi; tubus ovoideus; sep. pinnulis langis, latis, 1-4-gentatis instructa; styli hirtelli, bisco subplano; cor parre, pallide rosea, eciliata; fruct. ovoidens, utrinque sed apice prassertion attenuatus.

Hab. Thane, Trnas (M. Gorndoger), Dalmatio et Megi dx nubialis.

1714. Orepinia anconitana Gogr mss.

teculei Congi, folcato-ivilatati, is ramos flor. stipulares, fol late ovatoelliptica, abripte et parum acuta, basi rotunisata, pectiolulata, cos costam eglanisulosa, gentes simplices, late orato-convergentes; pet. eglandulosi, subinernes; stip. longae, virixes, auriculis givergentifues; per. 1, glabe: 13-15 m. longi, tubus obovato - oblongus, sep. pinnulis comnibus fohiaceis, 2-3-gentatis instructa, deumine integro; styi hirsuti, Cisco comico; cor-magna, rosex, eceliata; fruct-obovats-oblangus, utrinope attenuatus. Hab. Malia arient., ad soncone (2. Goolucci). 
$-192-$

1715. Brepinia famtiaris Cogogr mss.

Acculei tennes, rectiusculi, vix Silatati, as ramos floc. stipulares; fol. late obovata, utringue sensim notuniata, atrovirentia, as costasn eglanioulosa; Dentes simplices, breves, subconvergentes; pet. inermes, eglanduloss; stip. virives, meidiocres, auriculis subrectis, pes. 1, glabri, 5m. longi; tuhus oroi-. rens; sap. vise pinnata, pinmulis latis, subintegris instructa; styli magmi, lanati, gisso plano; cor …… fruct maximus, glaucus, ovoveres, hasi abrupte gecurrens, apric Dilatatus.

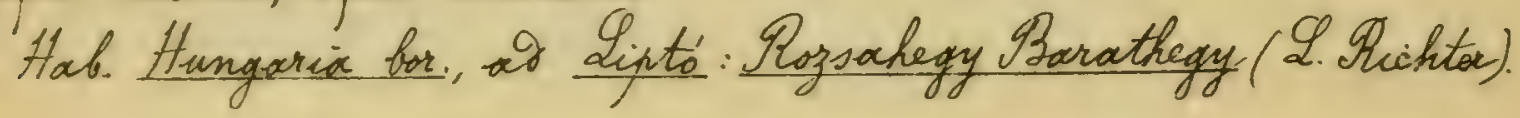

1716. Crepinia rubristimula Gogr bab. N-2393.

Gota, propurea; aculei valide; falcato-Dilatati, ad ramos flor.minores; fol. oborato-oblonga, paulo attenuata, fasi rotuniata, ni costam eglaniulosa; dentes simplices, aperti; pet. inermes, eglandulosi; stip. rubentes, longar, auriculis subrectis; per. 2-4, élabri, $12-21 \mathrm{~m}$. longri; tubus sbovatus; sep. pinnulis brevibus, Dentatis, set angustis instructa, styli villosi, Disco sat comic; con.magna, rosea, aciliato; fruct. parros, oboratus.

Hab. Haute-Savoie, ad It Martin (Muget).

$\sim \sim$. Petioli inferiores inermes.

1717. Crepinia icoglossa Gager Eab. N-2394.

Aculei Debiles, freves, inchinato-gilatate, as ramos flot.panciores et minuti; fol obovato-oblonga, sensin attenuata, basi rotunoata, ad costarm eglanioulosa; Dentes simplices, aperti, pet. eglaniulosi, inf. inormes; stip. virides; ped.1-2, glabri, $12-15 \mathrm{~m}$. longi; ticus obovato - oblongus; sep. pinnulis latiusculis, 1-2-Dentatis instructa; styli villosi, Disco subplans; cor pallide roses, acute unguiculato, eciliatris; fruct. obovats-solongus, utrinque atternatus. Hob. Phone, as Duilly prope gíllepranche (N6. Ganooger).

1718. Freninia lencostions Gogr \&ab. N:23y5.

foculei prarsi, falcatos. Dilatati, as ramos flor nulli; fol oborato - ollon-

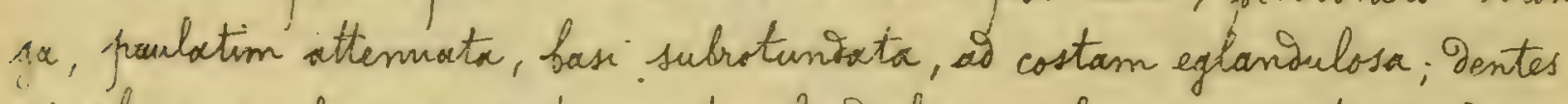
sinphices, paulo convargentes; pet. eglandulosi, inf inermes; stip. mediores;

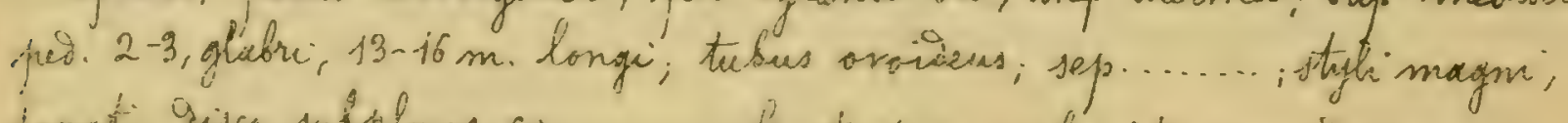

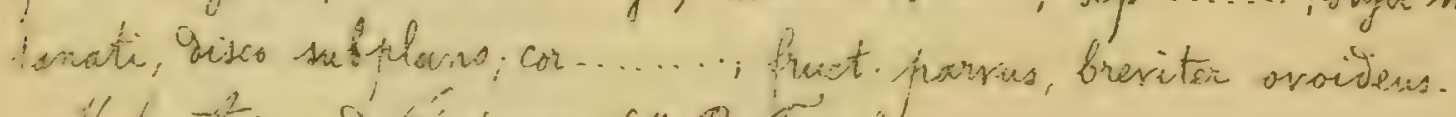

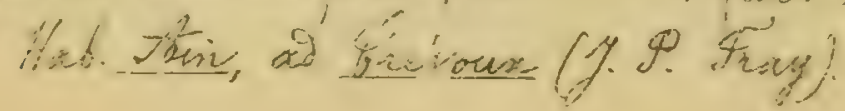


$-193-$

1719. Reninia stenphetala (Gogre Ros.nor. Ii p. 12)i Gogr Lab. N-23g6. - Gogor Herb.ros. $\mathcal{N}: 343$.

toculei panciores, parrri, inclinato-subsilatati, ad ramos flar. flexuosos mulli; fol. oborats-oblonga, scuta, basi subrotumiata, lacte vivioix, ad costam eglanaulosa; Dentes simplices, lanceolati; pet eglanialosi, inf. inermes; stip. sat angustac; peos. 1-3, glabri, 15-20 m. longi; tubus obovato-oblongus; sep.pinmulis sabintegris, latiusculis instructo; styli villosi, Disco subplano, cor pallide rosed, petalis gisjunctis, anguste unguiculatis, eciliatis; fruct parres, obovato - oblongus, basi gepressus, apuce valie contractus. Hab. Rhone, to trnas (M. Ganonoger).

1719 bis. Bepinia zagrabiensis Gogr mos.

Accular gebiles, inclinato-Dilatati, io ramos flor. nulli; fol-obovata,

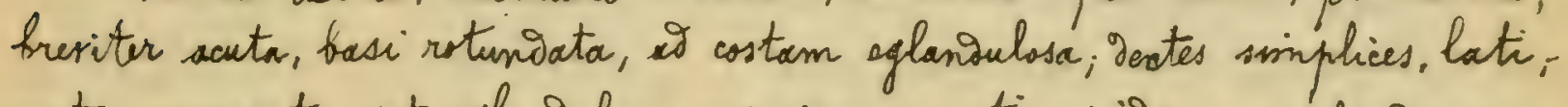
vato-convergentes; pet eglanioulosi, sup. inermes; stip. virises, auriculis bivergentibus; pes. 1, glabri, 13-14 m. longri; trebus obovato-oblongres; sep. purnulis anmibus foliaceio, 1-3-gentatis instructa, acumine integro; styli hirsuti, Pisco valide comico; cor rosea, eciliata; fruct. ovoidens, basi rotuniatus, spice atten.

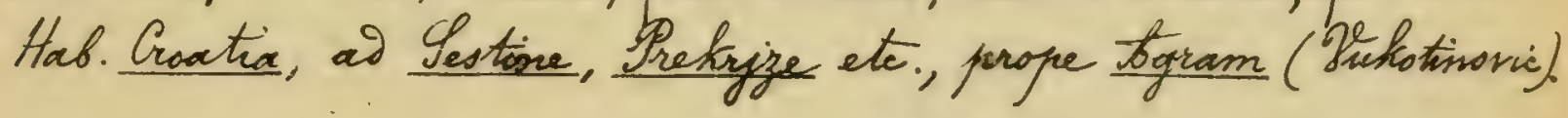

$\sim \sim \sim$. Petioli onmes aculeati.

$X$ Discus planus.

1720. Creninia tonnomiana (Muget) in Gagr Oob. N.2397.

Foculei à ramos flor. Debiles, siehinati sel rectisisculi, fol orato-dliptica, breviter attermatto, basi rotuniata, al costam eglandulosa; Destes inf. subbiserrati; pet. glanoulosi, parce weuleati; stip. angustar, ad auriculas subtes poulo pubesientes; per. 1-4, glabri; $11-14 \mathrm{~m}$.longi; tulus sroideus; sep. finmulis brevibus, villosis instructa; styli villosi, discs plano; cor. rosea; fruct. sat parrus, ovoidens.

Hab. Arriche, as Kitallon prope Annonay (Boullu).

1721. Gepinia Vukstinsvieü Gigr Eab. N'23g8; Gogr Contr. fl. slav merid, II, $p \cdot 3 g$.

Accules breves, falcats - Dilatati, ad ramos flor-geminati; fol late boseta, abrupte acuta, basi rotunoata, as costam eglanidulosa; dentes sim- 
$-194-$

plices, avato-convergentes; pet. parce aculacti, inferne paulo glanioulosi:; stip. vivides, auriculis Divergentibus; pes. 1-3, glabri; $12-14 \mathrm{~m}$. longi; tutus ovoisens; sep. pinnulis latis, 1-2-gentatis instructa, acumine integro; styli villosi, Diseo plans; cor eciliata; fruct. ovoidens, basi praulo sepressus, apicem attemiatus.

Hab. Croatia, ad Prebrize (T. Gukotinovic).

1722. Crepinia vireticola Googr Gab. N-23gg.

toculei as ramos flor. Febiles, Silatato-subfalcatic; fol. sat parrea, oflongs-acuta, basi subroturiata, lacte virisia, ai costam eglaniaulosa; ientes simphices, lanceolato-aperti; pet. eglansulosi, aculexti, stip. longare, Angustae, auriculis Sirargentibus; pes. 1, glabri, g-11 m. longi; tubus ovoidens; sep. pinnulis latinseulis, 2-3-Dentatis instructa, acumine longo, sat lato; styli panciores, villosi, 2eiso plano; cor ........; fruct. frarrus, orsidens, utionque gepressus.

Hat. Suecia, ad Earup Seamiae (I. Af. Eullberg).

$X X$ Discus phow minusse comicus.

1723. Chepinia Nisus Giogr \&ab. N:2400.

toculei falcato-gilatate, as ramos flor. saepins mulli; fol. amplissime, oblongo-rhombiratia, acuta, basi rotumiata, as costam eglanioulosa, gentes simplies, remoti, rotuniati, pet eglanialosi, aulenti; step. magnace, auriculis Divergentibus; pes. 1-3, glabri, 5-10 m. longi; tubus obovatus; sep. plerumgue integris, latiusculis instructa, acumine saepe foliaces-lacimiato; styli villosi, gisco canis; cor. subalba; fruct. ofovatus, oypice valie attenuaters.

Hab. Iyrenoei centr, as Gière (H. Bordere).

1724. Crepinia belonivimarpha Gogr bab. N-2401.

Acculei minutissinn, recti, parum gilatati, ad ramos floz munc panciores, nune nulli; fol parva, obovato-oblonga vel oblongre, basi noturidata, aid costam eglaniulosa; oentes simphies, valor profunsi, lanceolato-recti; pet. aculeati, eglanioulosi, laxissime pubescentes rel spasse pilosi, stip. parvac; per. 1-2, glabu; $5 . \mathrm{gm}$. longi; tubus ovoideus; sep. pinnulis latrisculis, 1-2-Dentatis instructe; styli villosi, diseo sat comico, car frarra, eciliata; fruct. parrus.

Hab. Mhone, ad Chaponost (P. Chabert). 
$-195-$

1725. Crepisia gracilenta Gogr Gab. N=2402. Gigr Herb. ros. No65.

foculei id ramos flor. graciles parvi, inclinati vel rectusculi, ad ramos vetustos vero majores; fol ovato-elliptica, breviter attemuata, basi coroata, sessilia, ad costam eglanaulosa; Dentes simplices; pet. inferne paulo glansulosi, parce aculeati, stip. angustiones, auriculis divergentibus; peo. 1-3, glabri, 14-20 m.longi; tubus obovotus; sep. pinnulis latis, longis, dentatis instructa; styli villosi, dises comico; cor. pallide rosea; funct. sat parrus, sbovatus. Hob. Ihone, ad Eleigé (M. Ganooger).

1726. Crepinia tinctilis Googe Eab. N:2403.

Acculei inchinati, parum Dilatati, wa ramos flor-copiosi; fol. obovato-subobtusa, basi cosdata, ad costam paulo glanoulosa, gentes simplices, apartotriangulares; pet. copiose glanoulasi, aculeati; stip. parvac, virides, auriculis givergentibus; pet. 1-2, glabri, 6-7 m.langi; tudus ovoideus; sep. pinmelis subintegris, parum latis instructa, styli villosi, gisco comico; cor........; fruct. breviter assidens, utringue subiepressus.

Hab. Bouches - du - Mhone, ad Martigues (Jo. Autheman).

1727. Crepinia trichonewra (Mipart in Crip. Prim. I, p.59; Dejsegl. Cat.p.

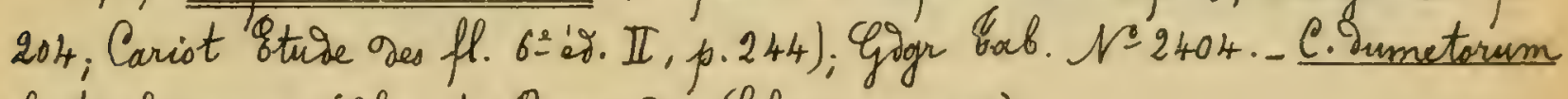
f. trichoneura (Christ Iosen ger Schweiz p. 185).

toculei copiosi, verticillati, ad ramos flor. inclinati vel rectiusculi; fol. late obovata, fasi rotundata, as costam aglasioulosa; Dentes simplices, acuti, pet. eqlandulosi, vculeati; stip. latiusculae, auriculis givergentibus; - per. 1-3, glabri, 10-22 m. longi, tubus oroidens; sep. pinmulis latis, parce dentatis instructa; styli villosi, Diseo paulo comico; cor. mayna, subalba, fruct magnus, svoidens.

Haf. Cher, at Eurly (Ihifart): Haute-Saroie, at Ehonon; Singy ( Duget), etc.

1728. Crepinia protecta Gogr mss.

Acculai freves, sat parsi; subailatati, tenuiter falcati, ad ramos flor. prorsi; fol late oblongo-acuta, basi rotundacta, as costam egiandulosa, dentes simplices, lanceolato-convergentes; pat. eglaniulosi, iculeati; stip. longar, latinsculac, virises, auriculis subrectis; pes.1-2, glabri, $11 / 2$ cent. Congi; tubus obovato-oblangus; sep. pinnmlis angustis, integris instructa; styli 
$-196-$

villosi, giseo sat comio; cor.......; fruct. oborato - oblongus, basi totundatoSilatatus, apure ratse attenuato-comicus, strangulatus.

Hab. Eransilvanix, Kolozs: at Kalote Iy. Kivaly (2. Richter).

it t+ Fructus oblongus.

\$oliola inaegue vel biserrata.

1729. Crepinia brochystylis Goor Lah. $N=2405$.

Acculei breves, parvi; gilatato-inclinati, as ramor flor. mulli; fol. late oborato-subattenuata, basi rotunsato-covata, viviid-glaucescentia, as costam eglanisulosa; Dentes aperto - triangulares, inf. biserrati; pet. eglandulosi, subinermes; stip. magnae, auriculis Sivergentibus; pes. 1-3, glabri, 4-7 m. longi; tubus oblangus; sep. pinnulis brevibus, latis, 3-4-gentatis instructa, acusnine lato; styli brevissimi, sublanati, gisco plano; cor. parva, pallise rosea, ociliata, fruct. maximu, oblongus, basi longe attenuatis, aprice contracto- inflatus.

Hab. Toustria infer., as Kalksburg frope gien (Y. Hoisbaur).

1730. Crepinia militans Gigr mss.

Aculei rarissimi, falcato-Dilatati, as ramos flor mulli, fol. parra, oblonga, utrinque sensim attenuata, ad basin costae glaniulosa; dentes ovatoconvergentes, inf. biserrati, pet. inermes, inferne paulo glandulosi; stip. frarva, breves, virides, auriculis sat givargentibus, pet. 1-2, glabii, 3-4 m. Longi; tubus sblangus; sep. pinnulis integris, angrestis instructa; stylis lanati, Sises subplano; cor......... fruct mediscris, anguste oblongses, utim: que attemuatus, apuice strangulatis.

Hab. Hunavia, ad Sudxorsi (2. Richter).

1731. Crenimia Maasï Gogr Gab. N:2406. - Maenitz Herb. europ. N:2234.

toculer silatati, tenviter falcati, wa ramos flor. flexnosos subnulk: fol. ovato-elliptica, subacuta, fresi breviter truncato-attenuata, as costam eglaniulosa; Oentes lanceolati, fere omner fiserrati; pet-eglandulosi, acculeati, stip.magnxe, subtus purbsentes, suriculis givergentibus; pes-1-2, glabri, 10-13 m. longi; tubus oblongus; sep.pinnulis breribus, latis, 1-3-gentaitis instructa; styli villasi, gisec plano; cor. amplissima, eciliata; fruct. oflangus, utrinque depressus.

Hab. Saxamix, at Neuhatsenseben (Y. Maas). 
$-197-$

\$§. Foliola ammino simpliciter serrata. ح. Foliola basi rotuniata.

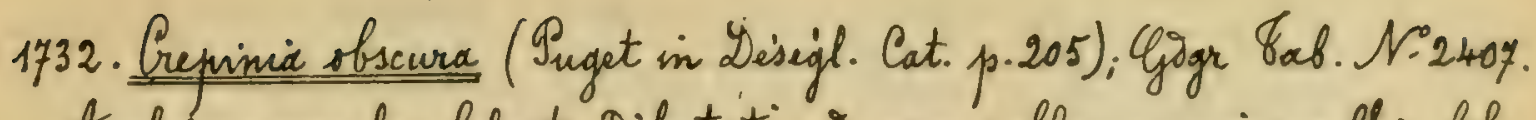
Acculer inarquales, falcato - Dilatati, as ramos flor. sacpins mulli; fol. sovats-elliptica, inatense siridiax, ad costam eglanioulosa; Destes simphices, aperti, pet. hinc inde glarioulosi, aculeati, stip. subtus pubeseentes, auriculis rectis; per.1-9, glabri, 6-10 m. longi; tubus sblongus; sep. anguste pinnata, acumine dilatato: styli fesciculati, riseo valoe comis; cor. magna, subalba: fruct. elongatus, utininge attonuatus.

Hab. Haute- Savoie, as Trogonnes et Pingy (Muget).

1733. Crepinia platystephana Gogr bab. N-2408. CGogr Herb ros. N=10. toculei falcato-2ilatati, ramos floi. mulli; fol. late oblongo-acuta, basi rotunioata, ai costam eglanioulosa; Dentes simphies; pet. eglarioulosi; extra-info inermes; stip. angustae, auriculis rectis; pes. 1-3, glabri; 7-12 m.longi; tubus oflongus; sep. pinnalis smnibus latis, elongatis, haut aut nix gentatis instructa, acumine longo, foliaces; styli villosi; gises sat conico; cor.magna, pallide rosea, fruct. oblangis, apice attenuatus. Hab. Houtes-Pyrineis : ad Gavainie (H. Sortère).

1734. Crepinia oxypetala Goge \&ab. N-240g.

Acculai gebiles, gilatato-minati, ramos flor. foliosos mulli; fol. shovato-subattenuata, basi rotunvato-coroata, glaucescentia, at costam eglariaulosa; gentes simphices, aperts - triangulares; pet. eglanivulosi, inf soli paulo aculeati; stip. longae, gilatatar, awriculis Divergentibus: per. 1-3, glabri, 6-8 m. longi, tubus oblongus; sep. prinnulis brevibus, foliaceis, 2-3-gentatis instructa, acumine foliaces-gentato; styli sublarseti, Disco conics, cor-rosea, petalis acutissimis; fuct. anguste oblongus, basi becurrens, apice attemiato-strangulatus.

Hab. Austria infer, ad Kalksburg prope ghin (Y. Hristant).

1735. Crepinia Gauitiniana Gogr Sab. N-2410.

Foculai falcato-gilatati, a ramos flor-copiosi; fol. late oboratostusiuscula, fasi rotungata, a costam eglanoulosor; ontes simplices, ovato-aperti, pet. eglanoulosi, aculeati, stip. longae, pirides, auriculis 
$-198-$

Divergentitus; per. 1-5, glabri, 12-15 m. langi; tubus ollongus; sep. prinnulis integris, latis instructa, tcumine valise foliaces; Stygli villosi; Disco comico; cor eciliata; fruct. oblongus, utrinque attenuates.

Hab. Helvetia, as Lurich, alt. 2000' (K. Lehmann).

1736. Crepinia nersigera Gogr bab. N-2411.

Acculei minuti, necti vel inclinati, teretes; fol ovato-elliptica, utrinque breviter rotuniata, firma, coriacea, prominule nervosa, ad costam eglanoulosa; Dentes simphices; pet. eglanoulosi, subinermes; stip. latae, auriculis Sivergentibus; per. 1-2, glabri; 8-10 m. longi; tubus elangatus; sep. sat late pinnata', eglanoulosa; styli villosi, gises subplans; can.....; fruct maximus, elongatus, utrinque vix attemuatus.

Hab. Ardeche, at Daveyzieux frope tomonay (Doullu).

1737. Crepinia amphicarpa Gigr fab. N:2412.

Acculei gilatato-inclinati, longinsculi, ad ramos flor saepuis mulli; fol. elliptico-subattennata, basi breviter rotuniata, lucioa, as costam non glanoulosa; Dentes simplices; pet. hine inse glanioulosi, aculeati, stip. latae, subtus pubescentes; pes.1-2, glabri, 10-14 m.longi; tubus oblongus; sep. parra, eglanioulosa; styli hirsuti, Diseo plano, cor........ fruct. anguste oblongus, basi gepressus, apice attenuatus.

Hab. Rhōe, at tomas (M. Ganooger).

$\sim \sim$ Foliola basi attemuata.

$X$ Petioli onnes inermes.

1738. Crepinia approximata Gogre bab. N-2414.

Aculei gebiles, falcato-Dilatati, as ramos flor. panciores; fol. oblongoacuta, basi attemiata, intense siridi-subglauca, at costam eglanoulosa; rentes simplices, lanceolato - subconvergentes, pet. eglanoulosi; omnes inermes; - stip. longuisculac, auriculis rectis; pes. 1-2, glabri, g-13 m. longi; tubus sblonqus; sep. pirnulis latiusculis, 1-2-Dentatis instructa, acumine magno; styli sublanati, breves, gisco sat comiso; cor. pallise roses, petalis acutis, ecitiatis; fruct.magnus, oblongus, aprice attemuatus, hasi subrotunartus, centrali excepto.

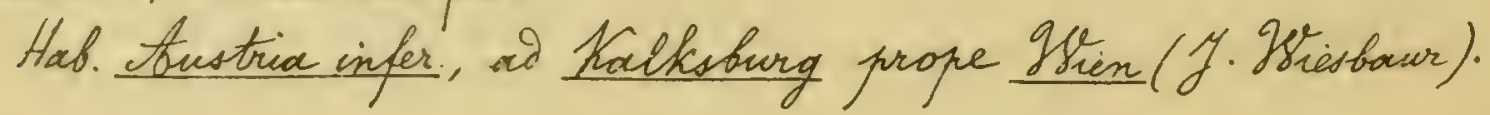


$-199-$

1799. Crepinia georgica Gogr mss. - Brotherus Pl cauc an. 1877. Glanca; tenler Dilatati, elongati, temuiter et valde falcati, as ramos flor. Sparsi; fol-mésiscria, oblongo-acuminata, basi breviter attemuato, ad costam eglansulosa; Dentes simplices, anguste lanceolati, recti; pet eglansulosi, inermes; stip. virides, longae, awriculis semi-givergentibus; peó. 1, glabri, 16-18 m.longi; tubus oblongus; sep. pinnulis subintegris, parum latis instructa; styli hirsuti, sisco valde comico; cor........ fruct. oblangus, busi longe atternatus, apice attemuatus et in $1 / 3$ sup. turgiases.

Hab. Coucasus, Georgia : ai Borshom prope fluv. Kura (Brotherus).

1740. Crepinia misella Gogr mss.

toculei termes, inclinati, vise Dilatati, wi ramos flor.minctos nulki; fol. parra, oblonga, breviter acuta, basi attemuata, ais costam eglanisulosa; dentes simplices, ovato-subconvergentes; pet. eglanialosi, onnes inermes; stip. sivides, breves, latinsculae, auriculis subrectis, pet. 1, glabri, 6-8m. longi; tubus oblongus; sep. pinnulis integris, angustis instructa; styli villosi, gisco subearnics, cor ……, fruct. parvus, oblongres, basi attemuatus, aprice longe cantracto - substrangulatus.

Hab. Hangaria, Solowisi (L. Sichter).

XX Petioli plus minusve aculeati.

1741. Crepinia urbica (Leman in Bull phils.n. 1118, extr.p.9, 19-5; 30 -

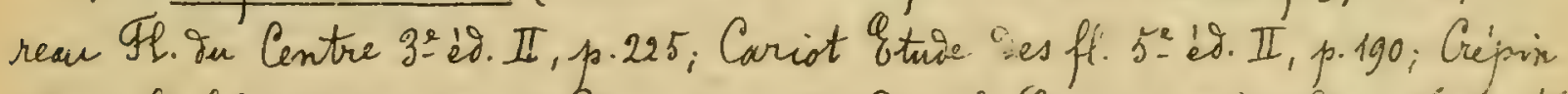

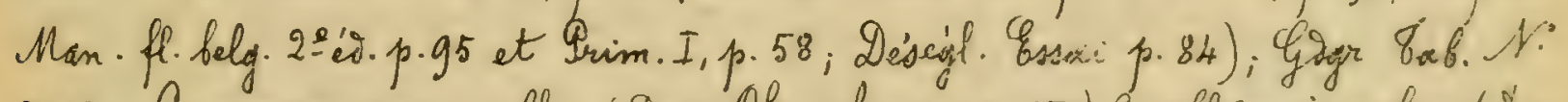

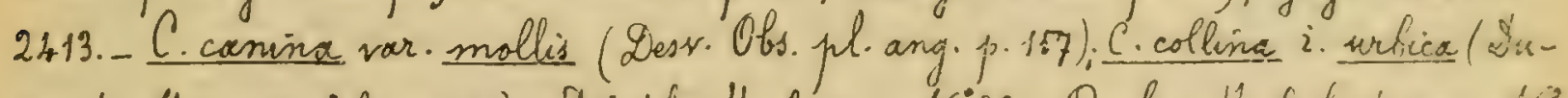

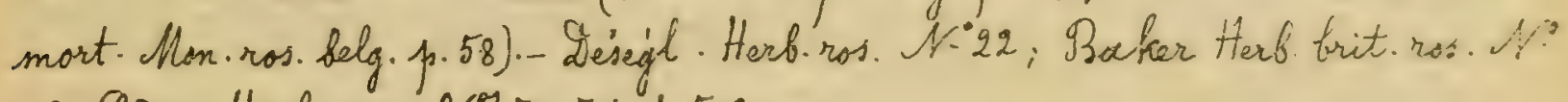
16. Gogr Herb. ros. N959, 501 et 502 var.

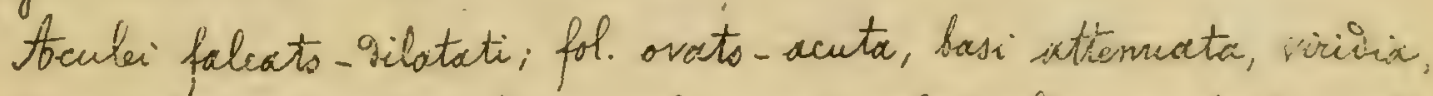

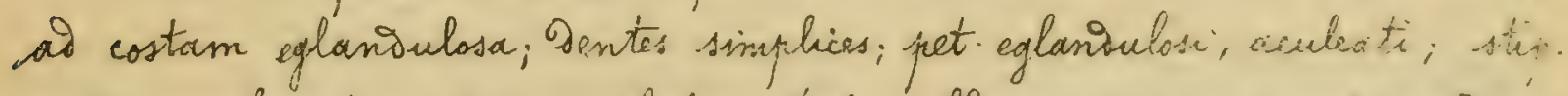
subtus purfescentes; pet. 2-4, glabri; tubus sblongus; sej. punnulis mestiscribus, Bentatis instructa, styti villosi; gises subplans, cor. pallide rosen; fruct. oblongus, laevis.

Hab. Guropa plewr.

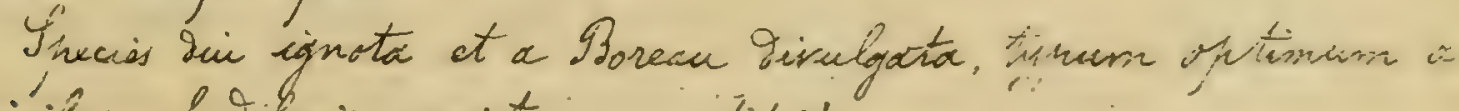

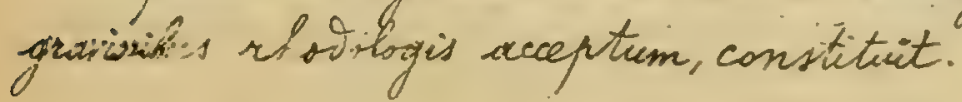


$-200-$

1742. Cropinia elisophylla G2ogr \&ab. N:2415.

toculei rarissimi, falcato - gilectati, as ramos flor nulli; fol oblongolancerlata, fasi valde ittenuata, nervosa, al costam eglandulosa; Dentes simplices, lanceolato-aperti; pet. eglanisulosi, parce aculeati; stip. breves, auriculis Sirergentibus; pes. 1, glabri, g-11 m. longi; tubus obovatus; sep. finnulis integris, longis, angustis instructo; styli pillosi; Sisco paulo comico: cor........., fruct. breviter oblongus, utrinque prarum Depressus.

Hab. Haute-Lavoie, at Habeire-Lullin (Muget).

1743. Prepinia anacantha Gogemss.

Acculei rari, breves, gilatato-subfalcati, parri, as ramos flor. nulki, fol. latiuscule oflango-acuta, basi ttenuata, as costarn eglandulosa; ientes simplices, aperto - triangulares, pet. basi subglaniulosi, inf. inermes; stip. virides, angustar, breviuscular, surveulis subsirergentibus, pat. 1-2, glabri, 10-12 m. Congi; telous oblongus; sep. primulis integris, angustis instructa styli sublanati, giseo plano; car........; fruct majuseulus, oblongos, $\mu-$ trinque lange attenuatus, meis sat turaious.

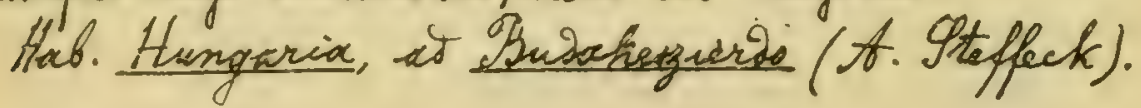

1744. Gepinix macracalux Ggog \&ab. N:2416.

toculei valivi, falcato-Dilatati, ar ramos flor glancos plus misnesse copiotr; fol ample oblonga, eitrinquie sed apuice priaesertion attenuata,

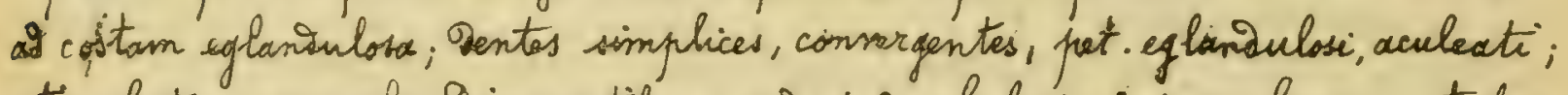
stip latae, anriculis Sivergentibus; pes. 1-3, glabri, 6-10 m.longi; tubus madinus, elongatas; sep. pionulis integris, puram latis instructa, styli villosi, Disco sat comio; cor....... pruct maxionus, elongatus, basi saepe gecurrens, apice atternuatus.

Hab. Hante-Savoie, at Fessy ( Duget).

1745. Crepinia nubroviens Gogr Eab. No2417.

Rubens; aculei silatato-subfalcate, nit ramos flot. intersum mulh; fol. oblnga, abrupte acuminata, basi valse attenuata, pallise viridia, as costam eglanaulasa; Dentes simphices, lanceshato-aperti, pet. sparse glaviaulosi, aculeati; stip. longae, auriculis acutissionis; subirivergentibus; pes-1-2, glabri, 14-17 m. longi; tubus oblongus; sep. pinnulis latis, 3-4-9entatis insiructa, acumine longissimo, lato, integro; staghi villose, giseo plasso; car......; 
$-201-$

funct. oblongus, basi repressus, apice attenuatus. Hab. Suecix, ins. Celand: at folbrunax (Y. N. Schentz).

1746. Crepinia Dicthyaphors Gogr mss.

Acculei minuti, recti, vix Silatati, brevissini, as ramos flor. nulli; fol ample oblango-lanceolato, basi attenuata, pallida, as costam eglan2ulosa; Dentes simphices, late triangulares, recti; per. lase eglanioulosi, laxe pubescentes, rculeati, stip. virisies, langre, angustae, ourciculis oivergentihus; per. 1-3, glabri; $15-18 \mathrm{~m}$. longi; tubus oblongus; sep..........; styli villosi, disco plano, car....... fuct. mestiocris, suboblongo - comices, basi rotumiatus, apice attemuato-strangulatus.

Hab. Hungarix, as Buackeszierdo ( At. Ateffeck).

5- Pilosace Googr Essai p. 31; ej: Gab.p.179.

Foliolis medianis ramorum floriferoum supra pilasis Devx glabris, subtus vers, etiam adultis, tots pubescentibus plerumque simplicita serratis; potiolis villosis, sel tomentasis.

Peaunculi glaberrimi $1\left\{\begin{array}{l}\text { Pesunculi glanaulosi at simul paulo hirsuti } \\ \text { Peaunculi tantum villosi }\end{array}\right.$ Styli glabri vel sparse pilosuli

2 Stuli nillosi vel lanati Soliola basi noturiacta

$3\{$ Foliola basi plas minus attemiata Fruectus ovatus, ovoidens vel phovato-sblonigus

$4\{$ Fructus oflongus

Rami flarales inermes

5 Tami llorales acceleati

Fructus ovatus, svoiveur vel obovato-oblongus

$6\{$ Fructus oblongus

Petioli inf vel superiores inarmes

$7\{$ Getioli annes inermes vel onnes aculeati
2

Species $\mathcal{N}^{2}: 1846-1848$.

18

3

8

4

6

5

Ipreies N-1756-1763.

Ipares $N^{0}-1747-1748$.

Iprecies N-1749-1755

7

Yrecies N-1777-1782.

Trecies $191764-1769$.

Ipecies N.1770-1776. 
Fructus dohosus, rotuniatus vel ovatis

$8\{$ Fructus sreviter ovoidens

Iruetus obovato-oblongus vel oblongus

Fotiola simplicitex serrata

$9\{$ Foliola inacque vel biserrata

Fructis globosses vel rotunisatus

$10\{$ gructus ovatios

Petroli inferiores vel orrmes inermes

$11\{$ Petioli ommas arulexti

$12\left\{\begin{array}{l}\text { Petioli onnes aculeati } \\ \text { Petioli inf. inermes } \\ \text { Petioli onnes inermes }\end{array}\right.$

Foliotic inareque vel biserrata

$13\{$ Goliola omnino simpliciter serrata

9. pecies $15-1783-1786$

Grecies $N: 1797-1792$

Q neries $V_{-17} 8-1785$ yecris $N-1730-1796$ Sinecis $N^{\circ}-1800-i 802$ Iprecies $\sqrt{2}-1803-1806$. grecies $1907-1814$ $14\left\{\begin{array}{l}\text { Rami florales inermes } \\ \text { Rami Horale' aculea }\end{array}\right.$

14 Tami florales aculeati

$15\left\{\begin{array}{l}\text { Tetioli amnes inermes } \\ \text { Getioli annes sculeati } \\ \text { Metioki inkricres inarnes }\end{array}\right.$ Fliola basi notuniata vel subrotuniacta

16 Foliola base plus minusve attemata Foliola basi notuniata

17 Foliola frasi phus minusse attenuate Styli glabwi rel susperse pilose

18 ? Styli villosi vel larati Foliola elliptica sel. obovata

19 Foliola obovato-oblonga sel shlongr

Iperis $\mathcal{N}^{\circ} 1812-1813$.

Ipecies $N^{0}-1814-1820$.

Yhecies $\mathcal{Y}^{3} i 821-1825$ Species $1 / 1826-182 \mathrm{~g}$ Qpecies $1: 1830-1833$. Sirecris $N-1834-1839$ Ineciss $x^{\circ}: 1840-1845$. Fructus sblonsous

$20\{$ Fructus oratris, ovoideus vel obovato-sblongus

$21\{$ Rani florales aculeati

21 Sami forabes inerr -1

Fructus ovioidens

$22\{$ Fructus oblongus

Fructus globosus, ovatus siel sbovato-obiongus

Thecies $N=1849-1852$. Shecies N $-1853-1854$. Incies $N-1955-1860$. Ipecies N:1861-1863. Enecied Y $^{3} 1864-1869$ $23\{$ Fructus oblongus 
Fruetus amries ovoidei vel obovato-sblonge

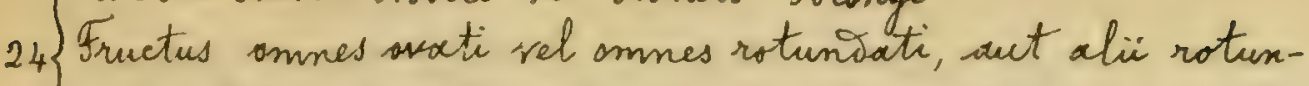

Dati, alii ovati, alii ovoitei in codem specimine Foliala basi roturisatio

25 Folioh hasi attemuato

$26\left\{\begin{array}{l}\text { Discus planus } \\ \text { Discus plas minus }\end{array}\right.$

20 Discus plus minusse conicus

Ihecies N $1870-1877$. Ypecies $\mathcal{Y}-1878-1880$. Ipecies $\mathcal{N}: 1881-1887$.

Tami florales inarmes

27 Tami florales aculeati

Tlami florales inermes

28 Thami florabes aculecti

Yhecies $N^{-1888-1889 .}$

Ihrexies NV:1890-1895.

Ipecies 1-1896-1897.

ipecies $x-1898-1904$

A. Sesunach glaberrimi.

a. Tyli glabri vel sparse pilosili.

+ Foliola basi notuniacto.

§. Fructus ovatus, svoitens vel obovato-oblongus.

$\sim$ Rami florales inermes.

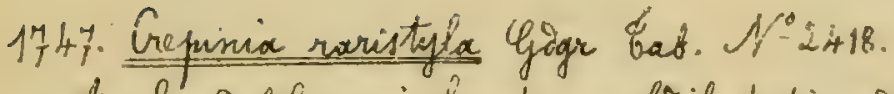

Fculei gebiles, inchinato-subilatati, as rumos flor. Tirtuosos nulli; fol. parra, oborato-oblonga, apice subrotuniata, fasi cordata; jentes simplices, aperti; pet. inferne paulo glanimlosi, parce aichleat:; costa meivi fol. eglandulosu; stip. parrac; pet. 1-2, glabri, 8-10 m. longi; tubers cbovato-oblongus; sep........ styli glabri, pauciores (2-5), giseo comico; cor....... pinct. obovato-oblongus, utrinque paulo gepressues.

Hab. Ihane, ao tornas (M. Ganioger).

1748. Grepinia coesaraugustana Googr Eab. M:2419.

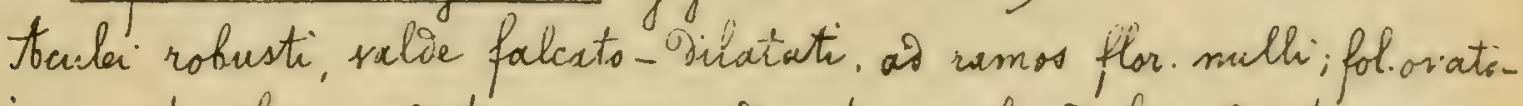
elliptica, acuta, basi corsata, crassa, à costam eglansulosa; jentes simephices, lanceolato-aperti, pet. parce aculeati, hinc inde glasioulosi; stip. rubentes, latae, ourciculis subdiverdentibus; ped. 1-2, glabri; 13-14 m. lsngi; terbus obovato - oblongus, sep. pinmulis integerrimis, latiusculis instructo,, acumine lato, styli aspperse pilosuli, gisco comico; cor-eciliata; fruct. obovato-oblongus, busi rotunaatus, apice valde strangulatus.

Hob. Hispania bor., di Logroñ (c. Lubia) et Saradosse (De Suon). 
$-204-$

$\sim$ Rami florales aculeati.

1749. Crepinia Massotiana (Gogogr et Debeaux) in Gogor qab. N=2120. Aculei inclinato-gilatati, at ramos flor. pauciores; fol obovato -oblonga, utrinque subroturdata, à costam eglandulosa; Dentes simplices, ovato-convergentes; pet-inermes, eglanoulosi; stip. sirides, auriculis rectis; peg.1, glabri, 10-11 m.longi; tubus svoidens; sep. pinmulis latis, 2-3Dentatis instructa, acumine integro; styli glabri, Jisco praulo comico; cor. eciliata; fruct. svoisens, utrinque attemuatus.

Hab. Iyrinées Orientales:- ad Le Boulou (Massot; Sellet; Debeaux).

1750. Crepinia ratarginaria Googr Gab. N-2421. Gogr Herb. ros. N-973. Acculei falcati, mosice dilatati, as ramos flor.copiosi; fol ample oblongs-acuta, basi rotueniata, à costam eglaniulosa; sentes simplices, aperti, pet. eglanoulosi, aculeati; stip. latac; pers. 1-2, glabri, 15-18 m. longi; tubus sbovato oblongus; sep. pinnulis latis, 1-2-gentatis instructa, acumine subfolizes; styli aspperse pilosuli, gisco subplano; cor .......; fruct. ovoideis, aprie froesertion attenuatus.

Hab. Hsire, as thermas It-Tierre-De-Paladou (No. Ganooger).

1751. Crepinia colummifera (Fries Novit. fl. suec. ed. 1.; Trchb. Fl excurs. $\mathcal{N}$-39g8); Gogr 8ab. N-2422.

Aculei faleato- Dilatati, compressi, ao ramos flor. sparsi; fol. Late svato-rotuniata, acutriscula, basi rotundata, ad costarn eglanisulosi, Ientes simphices; - pet eglanisulosi, subincrmes; stip. sultus villosar, peid saepe 3-g, glabri; tubus oroidens; sep. firmulis Dentatis, ciliatis instructo, acumine lato; styli subglabri; cor pallise incarnata, basi alba; fruet. ovoidens.

Hab. Srecia (Fries); Lamia (Meyer); Germamia centr. (Meichenbach).

1752. Crepimia heterostigma Goger Gab. N-2423.

toculei falcato-ailatati, ai ramos flor. sat copiosi; fol late obovata, sapins utrinque subrotuniata, wi costam eglanioulosa; dentes simphies, recti, pet. inferne sparse glanoulosi, inf inermes; stip magnoe, latae, auriculis oivergentibus, pes. 1-4, glabii, 6-1im. longi; tubus sbovato- oblongus; sep. prinmulis latirisculis, parem sentatis instructa; stygli alie glabri, 
$-205--$

alï sparse pilosuli, Disco acuto, salde conico; cor........ fruct mognues, ovoidens, basi gepressus, in $1 / 3$ sup. Turgidus, apice breviter attemuatres. Hab. Haute-Savroie, ad Habire- Soche (Suget).

1753. Crepinia turgeseens Gogr mss.

Aculer Dilatati, valde falcati, ao ramos flor. stipulares; fol. obovatosubobtusa, basi noturiasta, coriacea, do costam eglaniulosa; Dentes ovati, simplies, recti; pet. inferme subglaniulosi, aculeati; stip. latissimace, virioses, Dorss vix pubescentes, auriculis rectis; peo. 1-2, glabri, 10-11 m.longi; tubus oroivens; sep. pinnulis onnibus integris, latis, copiose instructa; stygh paulo villosi, Viseo subcomico; cor...... fruct magnes, turgians, oroideres, bosi apressus, apice abrupte attensatus.

Hab. Hispania bor., ad Logroño (Y. Zubia).

1754. Crepinia australis Gogr Gab. N-2424.

teculei debiles, falcato- dilatati, ad ramos flor. sparsi; fol. obovatooblonga, acuta, basi rotuniata, ai costam eglañoulosa; Ientes simplices, ovato- subconvergentes; pet. eglanimlosi, subinermes; stip. Latae, auriculis aivergentifus; pet. 2-3, glabri, 14-16 m. longi; tubus ovoideus; sep. pin. mulis parum latis, 1-2-rentatis instructa, acumine lato, integro, styli subglaberrimi, Dises conics, cor........ fruct. avoides-canicus, basi rotunDatus, apice atternuatus.

Hob. Bouches-2u-Rhane, at Merre (Jt. toutheman).

1755. Crepinia Bivoneana Gogr mss.

Aculei breves, Dilatati, rectuisculi, oo ramos flor articulatos sat copiosi; fol. sat parva, obovato - subacuta, basi rotuniata, ai costam non glaniaulosa; Dentes simphices, svato - subconvergentes; pet. basi subglanzulosi; aculeati; stip. breves, subtus villosac, viriedes, auriculis rectis; pes. 1, glabri; 11-13 m. longi; tubus ovactus; sep. pinnalis vix hirsutis, amibus subfoliaceis, 2-3-2entatis instructa, acumene in te gro; stygh subglaberrimi, Diseo camico; cor. magna, nosea, eciliata; fruct. avatus, utrinque noturidatus.

Hab. Sicilia, m. Nébrodes: ad. Castelbuono (M. Lojacono). 
$-206-$

$\S \S . F_{\text {ructus oblongus. }}$

1756. Creninia paucismina Goer Gab. M-2425.

toculai gesiles, falcato - gilatati, as ramos flar. nulhi, fol obovatooblonga, utrinque rotuniata, as costam eglaniculosa; gentes simplices, lati, svati; pet inermes, inferne paulo glanioulosi; stip. latissimac, anriculis givergentibus; ped. 1-2, glabri, 12-19 m. longi; tiebus elongatus; sep. frimulis invitegris, angustis instructa; styli glabii, isisco subplans; cor. sidnota; fruit. angueste oblongus.

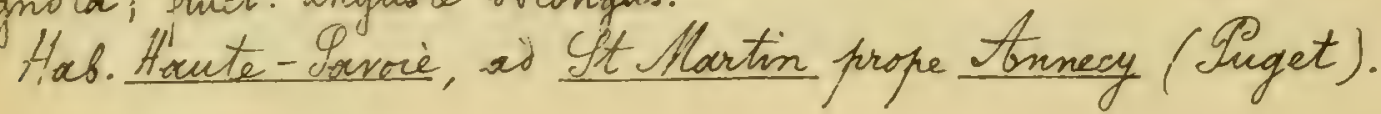

1757. Crepinia Guavalajarae Gogr inss.

Aculei falcato-Dilatati, ad ramos flar. nulli; fol. late obovato-obinsa, basi rotuniata, crassa, ad costam eglaniulosa; jentes simplices, svato-eperti; pet. inferne paululum glandulasi, inermes; stip. viribes, latae, subtus toto pubescentes, auriculis givergentibus; pet. 1-2, glabri, $20-22$ $m$. longi; trbus oblongus; sep. pinnulis pubescentibus, anmibus foliaceo-dentatis copiosissime instructa; styli glabir, disco valse comico; cor. rosea. sciliata; fruct. oblongus, utrinque longe attemuatus.

Hab. Hispania centr., at Guadalojara (F. Fernander).

1758. Crepinia stauroclása Gogr Gab. N-2426.

Acculei falcato- silatate, a ramos flor patulos mulli; fol parva, obovato-acutriscula, bosi notuniata, do costam eglandulasa, Centes simplices, aperto - triangulares, pet. aculeati, glanoulosi; stip.rubentes, auriculis rectis; pes. 1-2, glabri, g-10 m. longi; tubus sblongus; sep.........; styli glabri, gises parvo, sat comics; cor........, fruct oblongus, basi rotuniatis, aprice attenuatus.

Hab. Hispania bor, of Logroño (I. Lubia).

1759. Creminia anirosacea Gogr Gab. N-2427.- Gigr Herb.ros. N-166. Aculei parum vilatati, tenuiter falcati, oi ramos mox mulli; fol. obovato-subacuta, basi coriata, ad costam eglaniulosa; dentes simplices, aperti, pet.bosi paclo glanioulosi, inf. inermes; stip. mesiseres; pos. 1-2, interoum 1-3-glanoulosi, 11-14 m. longi; tubus oblongus; sep..........; stuli asprerse pilosuli, disco conics; cor.......; fruct. oblongus, basi atte. 
$-207-$

innatus, apice Dilatatus.

Hab. IRhone, at Gleizé (M. Ganooger).

1760. Gepinix infracoroata Gogr \&ab. $1 /-2428$.

Acculei vebiles, oilatats-subfalcati, as ramos flor. panciores; fol. sat late obovata, paruem acuta, basi corisato-emarginata, atrovirentio, ai costam eglanioulosa; Jentes simplices, ovati, recti, pet. inermes, eglanoulosi; stip. Longac, virides, auriculis sivergentithes; ped. 1-2, glabori, 10-11 $\mathrm{m}$. longi; tubus sblongus, sep. elongata, prinnulis omnibus foliaicis, paulo sentatis, acumine integrs; style glaberrimi; , Pises conico; cor. abo-rosex., sciliata: fruct. obiongus, utrinopue valds altenuatus.

Hab. Eyzol, as So Antonio prope Brente. (Gilmi).

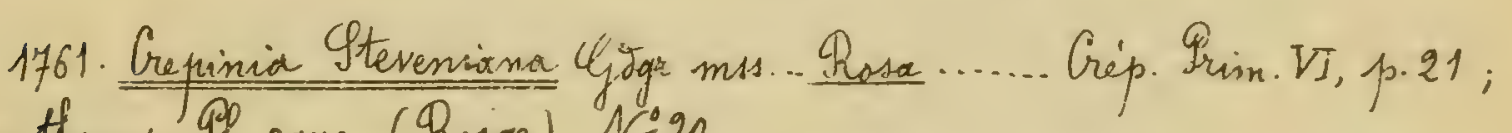
Protherus Pl. canc. (Rosac) N-20.

Areculei breves, tenuiter et parum falcati, parvi, ad ramos flor. - hanciores; fol ample obovato-oblonga, acuta, frasi roturiasta, viricia-

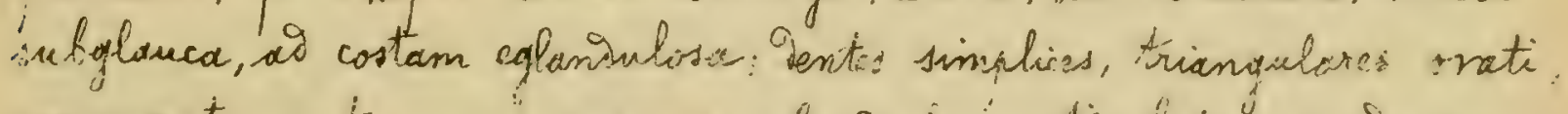
onvergentes; pet. amnes inermes, eglanicilosi; stip. latae, virives, acuriculis parusn Yirengentibus; per. 2-4, glabri, $14-16 \mathrm{~m}$. longi; tubus oblongus; sep. nimmulis glabris, latis, 2-3-Dentatis instructa, acumine integro, styli glabri, aisco canico; cor.magna, alba, eciliata; fruct frobabilius oblongus, utringue attemeatus.

Hob. Caucasus, Carthalinia: as Borshons frope fhur huvai (Brotherus).

1762. Crepinia probata Gogr \&ab. N:2429.

Acula breves, falcato - bilatati, do ramos flor. pauciores; fol parva, ovato-elliptica, utrinque rotuniata, ai costim eglaniulosa; Dentes simphices, ovato-subconvergentes, pet. eglanaulosi; aculeati; stips. rubentes, latae, auriculis Sivergentibus, peo. 1, glabri; g-10 m. Longi; tuhus ofCongus; sep. pinnmlis angis tis, 1-2-Dentatis instructa, accumine hatiusculo, integro; styli glabri, gisso conics, cor eciliata, fruct. oblongus, as apicem prasertion attemuatus.

Hab. Hispania bor. Logroño: ad El Pasillo in Seirra De Cameros (C. Zubia). 
$-208-$

1763. Cresinia aidaucta Gogr mss.

Aculei breves, parum gitatati, tenciter et valde falcati, os ramos flor. feminati, fol. parva, elliptico-acuta, basi rotunoata, ai costam eglenaulosa; gentes simplices, profunise lanceolato-convergentes; pet. rubentes, aculeati, eglandulosi; stip. longar, basi rubentes, sat angustac, price pu bescentes, auriculis semiaivergentibus; ped. 1-3, glabir, $8 \mathrm{~m}$. longi; tubus oblongus; sep. rubra, pinnulis ammibus integris, linearibus instructa., acumine latisre; styli assperse pilosuli, Disco canico; cor-rosea, parva, eciliata; fruct. oblongris, utrinque, set basi prasertion, longe attenuaties. Hab. Hispania mesia, ad Aflbarracin (9. Lapater).

T+ Foliola basi plus minusve attemuata.

§. Fructus ovatus, avoidens rel obovato-sblongus.

$\sim$. Fetioli inferiores vol superiores inermes.

1764. Grehinia improvioa Goor \&ab. N:2430.

Gracilis; aculei cylinarici; tenues, inclinati, as ramos flor nulli; fol. obovato-oblonga, subacuta, basi sension attenuata, a costam eglandulosa; Jentes simplices, sublanccolati, recti; pet. sup. inermes, hirce inde glangulosi; stip. ririoses, aurrinis givergentibus; pes. 1, glabri, 13-14 m. longi; tubus suboblongus; sep. brevia, pinnulis linearibus, 1-2-2entatis instructa, acumine subfoliaces, integro; styli exserti, glabri, Wisco comico; car........ fruct. parwus, obovato - oblongus, basi lange attenuatus, in 1/3 sup. turgives, dein abrupte strangulatus.

Hab. Inpol, of Baumbirchen prope Hall (Y. Gremblich).

1765. Crepinia stille Gogr bab. N-2431.

Acculer cylinorici, breves, rectiusculi, ar ramos flar. sparsi; fol. obovatoscuta, basi atternato, truncata, lacte siridia, is costam eglaniulara; gentes simplices, sublanceolato - corvergentes; pet. eglandulosi, sup-inermes; stip. angustae, virides, auriculis sot Divergentibus; pè. 1-2, glabri, 10-12 m. longi; tubus oroideus; sep. pinnulis onnibus integris, latis instructa, acumine foliaces; styli glaberrimi, gisco paulo conics; cor. rosed, eciliata; fruct. breviter ovoioens, utrinque parum attenuatus.

Hab. Gyrol, is Orente (G. Gelmi). 
$-209-$

1766. Geninia tithymaloides Ggyr bab. N-2436.

Fcculei pranciores, falcato-Silatati, at ramos flot. foliosos mulli; fol. sobovato-oblonga, utringue attenuata, amoene et hicive virioia, as basin costace folioli mediu paulo glanoulosa; Dentes simplices, convergentes; pet. eglanioulosi, inf inermes; stip. mediceres; pes. 1-3, glabri, 10-13 m. longi; tubus obovato-oblangus; sep. pinnulis latiusculis, vix dentatis instructa; styli subglaberrimi, bises sat conico; con........; fruct. obovato-oblongus, utrinque paulo oepressus.

Hab. Rhöne, ad tornas (M. Ganooger).

1767. Crequinia gymnostigma Goge Gab. N'2437.

teculei falcato- Dilatate, as ramos flor. geminatic fol elliptico-obovorta, utrinque breviter attemuata, al costam eglanioulosa; Dentes simphices, recti; pet. eglanialosi, inf. inermes; stip. breves, latissimae, auriculis ree tis; pos. 1-3, glabri, 10-14 m. longi; theus obovato-oblongus; sep. pinmulis latis, paulo oentatis instructa, styli glabri, Dises sat conico; cor. pollide rosea, magna, inferne paulo ciliata, fruct. svoidens, apice attemuat. Hab. Nanche, as Gretot (G. Lebel).

1768. Creminia hypotricha Gigr Eab. N-2438. C Googr Herb. ros. N-74. toculei gabiles, parem falcato - Dilatati, ad ramos flor. sat copiosi, fol. late obovato-oblonga, subattemuata, basi truncata, atrovirentia, ad costam eglandulosa; Pentes simplices; pet eglanioulosi; inf. inermes; stip. latae; poo. 1-2, ylabri, 7-10 m-langj; tubus oborato - oblongus; sep. pinnulis subintegris, pubescentibus, latinsculis instructa; sty $h$ : aos. perse pilosuli, Dises conics, cor ........, fruct. ovoideus, utrinque sttennatus, mesio turgidus.

Hob. Isere, ad Charancieu (M. Ganoger).

1769. Crepinia plesiotricha Gjogr mss. CRosa ....... Crepin Prim. II, p. 21. - Mrotherus Plant. cauc. (Mosae) N=25.

tewlei longi, tenuiter inclinato-subsilatati, ad ramos flor. sat copiosi; fol valda glanca, obovato-acuta, basi breviter atternata, as costam eglanioulosa; dentes lanceolato-subconvergentes, inf. paulo biserrati; pet. hine inse glaniulasi, inf. inermes; stip. virives, longoe, parum latac, auriculis divergentibus; per. 2-3, glabri, 8-10 m. langi; trubus 
$-210-$

ovxto-ellipticus; sep. purpurea, pinnulis latiusculis, 1-3-gentatis isstructa, acumine lato, integro; styli paulo villosi, gisco camico, cors. rosea, eciliata; fruct. probabilius ovato-ellipticus, fasi pracsertion gepressus. Hab. Caucasus, Carthalinia: is tochabich prope fiurium gosko (Brotherus).

Foliolis valte glaucis, pube araneosa, pichis crispis basi bulbosulis, sone curiosx.

$\sim \sim$. Setioli onnes inermes vel omnes aculeati.

1770. Crepinia Arcturus Gogr Eab. $N-2432$. Gogr Herb. ros. $N=258$. Acculei debiles, ad ramos flor. Densos falcato- Dilatati; fol. latiuscule oblongs-acuta, basi sensim attenuata, oc costam eglandulosa; Dentes fiserrati, recti; pet onnes aculati, eglansulosi; stip angustae, auriculis parum girergentibus; per. sage 2-3, glabri, 5-13 m. longi; tubus. oblangus; sep. pinnulis integris, pauciaribus, parum latis instructa; styhi subglaberrism; gises sat comico; cor. albo - subrosea, eciliata; fruct. ovoidens, apice attenuatus.

Hab. Ihone, ad tornos (M. Ganooger).

1771. Creninia axensis Goor ms:

Aculei breves, parvi, Dilatato-falcati, ad ramos flor geminati; fol. sat late oblonga, utrinque attenuata, mesio gilatata, ai costam eglanDulosa; Dentes simpilices, late apento-triangulares; pet eglanoulosi, smnes aculeati; stip. basi rubentes, longae, latae, auriculis givergentibus; pes. 1-3, glabri, 15-17 m. longi; tubus suboblargus; sep. pinnulis ommibus subintegerrismis, latis instructa; styh hine inde pilosuli, isisco comico; cor.......; fruct. latursenk obovato- blongus, basi acpressus, apice valie strangulatus. Hob. Itriege, ac Atson prope $\frac{f}{6 x}$ (At. Maracithon J'toyméric).

1772. Gepinia Fontonesii Goar mss.

Aculei parmm falcato-gilatati, sat longi, as rainos flor. copriosiores; fol ampliuscule oborato subacuta, basi attenuato, ar costam paulo glanSulosa; ientes simplices, lanceolato-convergentes; pet. onnes aculeati, hinc. ino glaniulosi, lasa pubescentes; stip. Congae, latae, nubentes, gorso glabraes ruriculis ivergentibus; per. 1-3, glabri, $10 \mathrm{~m}$. longi; tubus obovato- of - 
$-211-$

Congus; sep. pinnulis longis, omsibus parum tatis, 1-3-gentatis instructa, acusmine integro; stegli rosperse pilosuli, Disco plano; cor....... fruct. ovoiteus, utrinque longe attemuatus, basi sat jecurrens.

Hab. Atgeria, Constantine: at Bow Shaleb (Go Reboid).

1773. Grepinia Briggsii Gogr Gab. N-2433.

Aculei breves, falcato-gilatati, à ramos flor plerumgne mulli; fol. oborecto- oblonga, obtusiuscula, basi subattenuata, vivioi-glancescentia, wi costam eglaniulosa; Dentes simphies, lanceolato - aperti, pet. hine inde glanioulosi, onnes parce aculeati; stip. parvar, angustac, suriculis of vergentisus; pers. 1-3, glabri, 6-8 m. Congi; trubus ovorieus; sep. pinnulis sat latis, eglanaulosis instructa, xcumine lato, exserto; styli, saepiis glabri, sises plano; cor. pallire rosen, eciliato; funct ovorinens, apice attomuatus. Hob. Anglia merid. Comwallis: as Grevol, Hessenforo et Peaton (Aorcher Briggs).

1774. Crepinia salicetorum Googr : Vab. Ne2434.

Feculei is ramos flexussos, elongatos inclinato - gilatati; fol obovatooblonga, à costam eglandulosa; Dentes simplies; pet. inermes, cglanivulosi; stip. latae, subtus pubescentes, auriculis brevibus, Sivaricatis; pes. 1-s, longi, glabui; tubus obovato-oblongus, sep. angueste pinnata; styh breves, glabri, Sises plano; cor......., pruct. obovato- oblengus.

Hak Rhone, in salicetis iois toraris tornas (M. Ganooger).

1775. Crepinia ancylacantha Gogr mss.

Acculei heves, subilatati, temiter folcati, at ramos flor stipulares; fol. sat parva, obovato-oblonga, acuta, basi attenuata, ai costam eglansulosa; gentes simplices, ovato-aperti; pet. inermes, eglanioulosi; stip. virises, latae, longuissculac, auriculis Sivergentibus; peo.1, glabri, 1/2 cent. longi; tubus oblongus; sep. firmulis brevitus, latis, omnibus 3-4-gentatis instructa; styli proulo hirtelli, gises plano; cor........ fruct. magnus, obovato-oblongus, basi' Sepressus, apice attennato-subcomicus.

Hab. Hungaria bor., Siptó: at Rozsaheyy Barathegy (2. Richter).

1776. Crepinia trichosenala Gogr bab. N:2435.

Aoculei folcato-gilatati, at ramos flor. Denuibatos panciores; for. elliptics-oborato, parum acuta, brsi breviter tuncata, atrovirentia, of 
$-212-$

cottam eglanioulosa; ientes simplices; pet eglaniulosi, onnes inermes; stip. meviocres; peit. 1-2, glabri, 8-11 m. longi; trbus oblongus; sep. hirsuta., pinnuhis integris, sillosis, latiusculis instructa; styli asoperse pilesuli, Disco pauto comis; cor. alba, eciliata; fruct. obovato- blongus. Hab. Phône, ai Mongré prope Pillefranche (N. Ganooger).

§. Fruetus oblongus.

1777. Crepinia elisophora (Gigi- Ros. II, p. 25); Gogr bab. N:2431. Gigr Herb.ros. N-g3.

teculi falcato-subilatate; oecurrentes, at ramos flor. foliosos subnull; fol. elliptio-obovata, utrinque attemeata, atrovientia, nervosa, ag costam eglanoulosa; gentes simplices; pet. eglanoulosi, inermes; stip. sub ins hirtellac; pes. 1-3, glabri, 16-20 m. longi; tubus anguste sblangus; sep. pubescentia, pinmulis integris, latis instructa, styli panciores, sparse pilosuli, sisco conico; cor. sat magna, pallide rosea, eciliata; fruct. oblong-clavatus, aprice turgitus, hasi longe attemeatus et jecurrens. Hab. Rhane, of tornas (No. Gantoger).

1778. Crepinia caloxys Googr inss.

trculei meviocres, silatati, temuiter faleati, as ramos flor.nulli; fol. latiuscule oblang-lancerlata, acuminata, basi acuta, ai costam non glanoulosa; dentes simplices, langi; ovato-subconverojentes; pet. eglaniaulasi; parce sculeati; stip. virives, longae, latae, auriculis givergentisus; peo. 1-2, glabri, 7-10 m. longi; tubus oblangus; dises plano; cor....... pruct. magnus, oblongus, base attemuatus, as apicem valie attenuato-strangulatus.

Hab Hungaria, Muiapest: at Mstenkegy (L. Michter).

1779. Grepinia lencochlora Gogr qab. N-2240.

toculi falcats-gilatati, as ramos flor.nulh; fol. obrvato-acuta, basi. attenuata, valde glaues-rerescentia, subtus grisea et ad costam eglanaulose; ientes simplices, perto - triangulares, pot. subglanoubsi, iscermes; stip. virives, anriculis divergentibus; pet. 1-3, glabri, 7-8 m. longi; tubus oblongus; sep. pinnulis angustis, 1-2- Tentatis instructa, acumine integro, angruto; styli glaberrimi, aisco conico; cor. eciliata; fruct. oblongus, utrinque valse attemuatus.

Hab. Hatia centr, Moiene as Saxoli (G.Gibelli). 
$-213--$

1750. Crepinia stenocarna Gogr bab. N-2441 (non Disegl.).

Aiulei Dilatati, elongati, recti vel inchinati, ad ramos flor. sat copiosi; fol obovato- oflonga, acuta, basi breviter atternata, lacte viriaia, as costam eglanoulosa; Dentes simphies, lanceolato-aperti; pot. eglandulosi, aculeati. stip.longae, angustiores, auriculis rectis; pes.1-2, glabir, $14-16 \mathrm{~m}$. longi; tubus elongatus; sep. pinnulis angustis, 2-3-ientatis instructa, acumine foliaces, laciniats; styli glabri, disco conics; cor........ fruct. anguste oblongus, basi rotuniatus, aprice longe attemuatus.

Hab. Haute-Lavoie, as Annecy-le- hienx (Gouvier).

C. stenocarpa (Déseigl. in Ném acas. Je Maine- At- Poire XXVIII. 1879, p. 114) idem est ac C.leptocarpa Gogr, 1871.

1781. Crepinia pontificalis Gogr mss.

teculei inchinato- gilatati, ar ramos flor-geminati; fol. oblongoauto, basi attenuata, costam eglansulosa; dentes simplices, lanceslato - subcanvergentes; pet. eglanoulosi, aculeati, stip. virides, angustac, subtus toto pubescentes, auriculis Sivergentibus; pes. 1, glabri, 12-14 m. longi; tubus oblongus; sep. pinmulis longis, onnibus integris, parum latis instructa, styli subylaberrimi, gisco parum conico; cor........; fruct. freviter oblongu, utringue attemiatus.

Hab. Halia orient, ai Castelfivardo pr. Incine (2. Paolucci).

1782. Crepinia strangulituba Gogr oxf. N:2442.

Aculei falcato-gilatati, as ramos flor. copiosi et geminati; fol. oblongo-acuta, fasi attenuata, at costam eglanialosa; Dentes simphices; pet. egloniulosi, parce aculeati, stip. virides; pet. 2-4, midiscres, glabri; tubus oblongus, apuice valde strangulatus; sep. nubentia, pinnulis angustis, 2-4-dentatis, subglabris instructa, acumine lato, laciniato; styli subglaberrimi, gises conico; cor. pallide rosea, cciliata; fruct. oblangus, basi depressus, aprie valie attemeato-strangulatus. Hab. Phone, ad Soucueu (IP. Chabert).

6. Styli villosi vel lanati.

Fructus globosus, notuniatus val ovatus.

\$. Foliola inacque vel biserrata.

1783. Crepinia funtetorum (Mess. Quppl. III as Cat. erem. p.20; si. Enum. 
Toiol. p. 18,61 et 595; Ipreng. Syst. II, p. 548; Tehb. Fl. exc. N-3976; Level. TP. ross. II, p.78; Evichw. Schizzze p. 164; Mor. Fl. au Centre 3-id. II, p.225); Gogr bab. N.2443; Gogr Contr. Ff. slav. II, p. 39. - C. solstitiolis var.b. sphaeracarpa (Cariot Etude des \&l. 5- è. II, p. 191). C. conifolia $\beta$. fruitetorum (Dumart. Mon. ros. belg. p. 62; Goret F1. jut. p. 213; Christ Ros. Der Ychweiz p. 18g). treulei falcato- Oilatati, ramos flor. ternati sel stipulares; fol. elliptica, intense visitia, subtus cinerea; Sentes subbiserrati; pet.rubentes, aculeati, sparse glanivulosi; stip. villosae, serrulatae; peo. 1-2, glabri, brevissimi, tubus globosus; sep. pinnulis gentatis instructa; stagli hirsuti; cor. rosea, basi pallivior; funct. magnus, subglobosus.

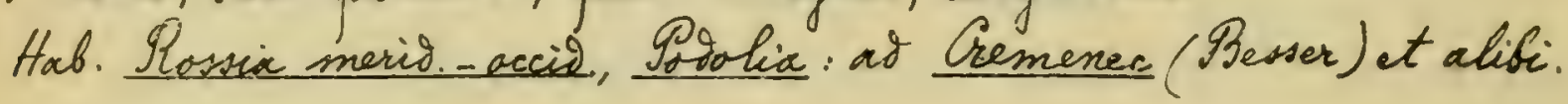

1783 bis. Crepinia puroides Gogr mss.

to-culei breves, falcato-rilatati, as ramos flor mulli; fol. amplissine oblongo-acuminata, hasi attemuata, crassa, wo costam eglaniulosa; Dentes inf biserrati, late ovats-convergentes; pet. paulo gianoulosi, aculeati, lase pubescentes; stip.magnac, sirides, auriculis subsivergentibus; ped. 1-3, glabri, 10-13 m.longi; tubus subglobosus; sep. pinnulis latis, omsibus 2-3-ventatis instructa; styli lanati, gisco plans; cor......... fruct magnus, globoss - Vepressus, umbilicatus.

Hab. Hungaria, at Msidaorsi (L. Richter).

1784. Grepinia Didymoxys (Googr et Debeaux in Debeause Mater. ros. Syren.1878,p.21); Grag Gaf. N-2444.

Aculei breves, inclinats- jilatati, as ramos flor.copiosi; fol. ample oborato-acuto, nerrosa, a costam interoum glaniulosa, Oentes biserrati; pot. aculeati, eglanialosi; stip. latar; pel.1-3, glabri, mesiscres; tubus rotusiatus, sep. pinmulis lats, gentatis sat longe instructa; styli villosi, Qiscs plans; cor.......; funct. parrus, globosus.

Hab. Myrenés Orient, as Mont-Iovis (P. Pellet).

1785. Crepinia Boorevi (Hoois in Erans. of Limn. soc. XII, p.210; Bratt. Ros.mon.II, p. 237; Dumort. Ros. belg p. 10); Gogr Gab. No.2445. - C. inovore a. (Morrer Brit. Hl $2.3 \leqslant$ p. 235 non Fries, Im. Engl. fl. II, p. 388; MaKer Rev. p.26; g. Grit. ros. in Limn. Journ. XI, p. 236); C. tornentella f. Borreri (Christ Rosen ver Petweiz p. 131). - Engl. bot. tab.2579 et es. 3 a tab. 471 . 
$-215-$

teulei falcato- Dilatati, is ramos flor. stipulares; fat ovata vel el liptica, intense virivia; Dentes subbiserrate; pet. glansulosi, aculeati; stip. subtus pubescentes; pes. 1-3, glabri; trbus oratus; sep. pisnatis, pinmulis Dentato-glanoulosis instructa; styli villosi, tisco subplano; cor. laute rosea; bunct. satus vel subglobosus.

Hab. Scotia, ai Goimboury, et alibi:

1786. Creninia paradoxa Gogr Gab. M-2446. C.leopoliensis (Blacki in Oest. bot. 2euts. 1887, p.269).

toculei iebiles, parvi, parum fockato-gilatati, as ramos flor prouciores; fol elliptico-acuta, at coscam pauls glanioulosa; Dentes acuti, inaequales; pet. subinermes, hine inde glasioulose; stip. angustac, auriculis parum rivergentibus; peg. 1-3, giabri, longi; tubus ovato-élipticus; sep. pinnulis latiusoulis instructa, styli villosi, Disco poulc comies, cor.....; fruct parrus, ovatus.

Hab. Rhone, ad Gleigé (M. Ganooger); Polonia (Mlocki). No1791 hue fortimet.

\$. Folisla simpliciter serrata.

$\sim$ Fractus globosus vel rotuniatus.

$X$ Petioli inferiores vel onnes inermes.

1787. Gepinia platysenala Gigr Gab. N-2447. - Gogr Herb. ros. N-377. teculei rarissini, dilatato - faleati, à ramos flor. rubros rulli; fol. elliptica, breviter attemuata, basi rotaindata, as costam eglaninlosa; zentes simplices, aperti; pet. eglaniulosi, inf. inermes; stip. latac, auriculis Divergentibus; pes. 1-3, glabri, 5-g m. longi; tubus orato- subrotuniotus; sep. pimmulis latis, villosis, parce dentatis, copiose instructa, acumine scepe folixer-laciniato; styli lanati, disco subplano; cor laete rosea; fruct. aubglobosus, apice atternatus.

Hab. Hrenai cente, ad Geire (H. Bordère).

1788. Crepinia Jsebsterii Gogr 8ab. N-2448.

Aculei dilatati, temunter falcati, ad ramos flor copiosissimi; fol. ample oblongs-acuta, basi longe cureata, subtres glaucs-coerclea, prominule nervosa et al costam eglandulosa; Dentes simplices, apertolanceolati, pet, paulo glanivulosi, inermes; stip. viriàs, latae, auriculis 
$-216-$

givergentifus; ped.1-4, glabri, 6-7 m. longi; tubus roturiatus; sep.erecta, Diu persistentia, pinnulis smibus integerrimis, parum latis; styli magmi. lanati, Sisco plano; cor eciliata, fruct. magnus, globosus. Hab. Anglia, frope York (G. Grebster).

1789. Greninia Dasycarpa Gogr Gab. M-2449.

Aculei falcato-Dilatati, à ramos flor. panciores, fol firma, anguste oblongo-acuta, basi attenuata, al costam eglarisulosa; gentes simplices; pet. plerumque eglanoulosi, onnes inermes, stip. angustoe, rubentes; pes. 1-2, flabui, $12-15 \mathrm{~m}$. longi; tubus oratus; sep........, styli hirsuti, gisco plano; cor.......; fruct. magnus, (16. m. Piam latus), turgious, oratoroturivatus vel rotunioatus, utrinque aepressus.

Hab. Rhône, ad Trancheville (9. Chabert).

XX Petioli onnes aculeati.

1790. Crepinia Moraci (Grip.) in Ggogr Eab. Ni2450

Acculei Dilatati, rectiusculi, ad ramos flor. copriosi, fol anguste obovato-oblonga, utrinque attenuata, ad costam eglansulosa; Dentes simplices, patulo-erect;; pet. eglanoulosi, temicter aculcati; stip. latae, aurieulis rectis; pes. 1-3, glabri, 4-8 m. longi; tubus rotuniatus, sep. pinnulis integris, angustis instructa, stgli magni, lanate, Dises plano; cor rosea; fruct. globrsus, apice haid out rice attennatus.

Hob. Selgium, Luxembourg: at It Hubert (Nooreau).

1791. Crepinia notata Gogr mss.

Acculei breves, falcato - Silatati, ar ramos flor copiosissimi; fol. oflongo-lanceolata, fasi attemuato-cuneata, intense virivia, ao costam -glaniulasa; Gentes lanceslato-aperti, onnes copiose biserrato-glanioulosi; pet. aculeati, glanoulosissimi, stip. latissimac, longae, glancescentes, gorso glaniulosae, auriculis sat divergentibus; pes. 1-3, glabri, $15 \mathrm{~m}$.longi; tubus oblongus; sep. pinnulis snnibus latis, marginibus valde bentato-glanioulosis instructa, styli villosi, gises comico; cor.magna, eciliata; fruct. of longus, basi attemuatus, apice valie strangulatus.

Hob. Halia ovient: prope Aoncone (2. Paolucci).

to: Chavinias (Scabratexe) forsan pertinet. to gregulum proces. spectat et post N'1786 nunc collocania est. 
$-217-$

1792. Grepinia sclerostephana Gogr Gab. N:2451.

tralei valisi, recti, gilatati, as ramos flor sat copiosi; fol oborato blonga, acuta, basi breviter attenuata; Dentes simplices, acuti; costa meDia paulo glanioulosa; pot. aculeati, glanioulosi, stip. longre, apice Dilatata, auriculis givergentes; pe $9.1-3$, glabri, 3-6 m.langi; tubus ovatus; sep. crassa, coniacea, erecto, give porsistentic, pinnulis integris, latiusculis instructa; styli magni, lanati, gives plano; cor......... fruct. rotuniartus, apice beviter attemuatro.

Hab. Helvetia, Jalais: I Ioniche-les-Mains (Lorenti).

1793. Creminia helvetica Gogr bas. N294.52.

foculei validi, falcato - giloticti, ai ramos flor geminati; for ample obovato-obtusiuscula, fasi attemuato-cuneata, crassa, lacte siridia, at costam eglanimlosa; Dentes simplices, late lanceolato-convergentes; pet. eglanioulosi, aculeate; stip. amplissimac, longae, auriculis sat sivergentibus; pes.1-3, glabri, 5-8 m. longi; thebus rotundactus; sep. pinnulis prouciorilus, angustis, eglaniulosis instructa, acusnine longo, lato; styli magni, lanati, oises plano; cor......, fruct. sncognus, globosus, apice breviter strangulatus, centrali vero inferne oacurrente.

Hab. Helvetia, galais: at Mayens ge Sion (Mgoff; L. Forrat).

179it. Crepinia cerasoides Googr Gab. N:2453.

Itculei Debiles, folcarto-Silatati, à ramos flor panciores; fol. oboratoacuta, basi rotuniata, at costam eglaniulosa, 2ientes simplices, ovatoaperti; pet. eglaniulosi, aculeati; stip. virides, latissimae, auriculis givergentibus; per. 2-3, glabiri, 5-6 m. longi; tulus rotunaatus; sep. pinnulis integerrimis, onsibus latiusculis instructa, styli villosi, Disco subplans; con....... fruct, parrus, globosus, apric poulo attennatus. Hab Anglix. Hereforshire: ad Gormbrigge (t.Ley).

1795. (reninia Companyoix (Debeaux Mater ros. Iyren., 1878, p. 22); Gogr Gob. N: 2454 .

Aceulei falcato-gilatati, ai ramos flor. saepe nutli; fol parva, obovato. spathulata, acuta, id costam eglandulosa, pallice virisia; dentes simplices, acuti; pet. eglaninlosi, onnes aculeati; stip. parvar; ped. 1-2, glabri, 10-12 m. langi; tubus rotundatus; sep. sat fursistentia, pinmulis latis, gentatis 
$-218-$

villosis instructa; stygli hissuti, diseo plans; cor........ puct. notuniatui. Hab. Pyréncés-Orientales, as Mont-Lomis, alt.4000"' P. Mallet).

17y6. Crepinia accinitina (Debeaux Mater ros. Iyren, 1878, p. 22)i Gigic dab. Ni2455.

Aculei valivi, gilatati, valos falcati, ad ramos flor. gemiseati; fol.

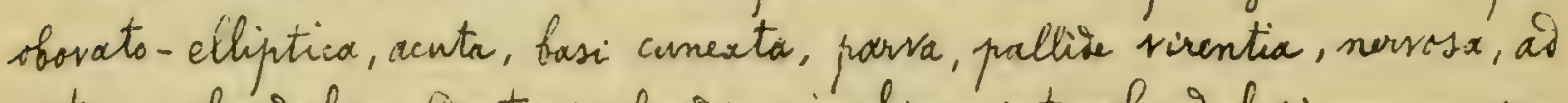
costam eglaniulosa; isentes profunsi, simphices; pet. eglanioulosi, omnes aculeati; stip. mevieress; pes. 1-2, alabri, 10-12 m. longi; tubus ovato-notuniatius; sep. pimmulis in persistentibus, lanceolatis instructo, styli villosi; Disco plans; cor........ Bruct. majusculus, globosus.

Hab. Iyrénés- Orientales, Mont-2ovis, alt.4000' (P. Pellet).

$\sim \sim$ Fructus avatus.

1797. Creminia lencella Gogr mss. - C. lencantha Gogr \&ab. N-2456 non Losis. ticulei parvi, gilatati, temuitor falcati, as ramos flor. 2-3. ti i fol. latiuscule oforato-obtusiuscula, basi subtruncata, pallive virioi-glaucescentia, at costam eglasioulosa; Dentes simphies, triangulari-convergentes; pet-eglaniulosi, inermes; stip. latissinae, auriculis givergentibus; ped.1-3, olabri, 7-9m. longi; tubus ovatus; sep. pinnulis integris, latis instructa, acumine longo, suffoliaces; styli magmi, lanati, disco subplans; cor. alba, eciliata; fucct. ovatus, basi rotuniatus, apuice breviter attenuatus, centrali vero decworente.

Hab. Inecia, Hestrogothix : in monte Kinnehulle of Hesterplana (1. C. Fetterstedt).

1798. Prepinia auctior Gogr mss.

Foule valiauli, gilatati, temiter falcati, as ramos flor geminati; fol ample oforato-oblonga, abrupte acuta, basi rotuniata, ad costam egianiulosa; Dentes simplices, ovato-convergentes; pot inames, eglaniulasi; stip. maximace, siribses, auriculis sat Sivergentibus; pet. 1-2, glabri, 2-4 m. longi; tubus ovatus; sep. pirnmulis subintegris, latis instructa; styli vilhosi, Diseo plano; cor........, fruct.magnus, ovato-canicus, basi $2 i$ latato - unbiticatus.

Hab. Hungaria for., Szepes: Lo Lacse (L. Richter). 
$-219-$

1799. Crepinia longistyla Gogr Gab. N'2457.

Acculei Jebiles, inclinato-subsilatati, as ramos flor. socepiis nulli; fol. sat late obovata, utrinque abrupte attenuata, mesio gilatata, pallide viridia, ad costam eglanaulosa, Dentes simplies, pet. glaniaulssi, inf. inermes; stip. longre; per. 1-3, glabri, 7-10 m. longi; tubus ovoides-subturbinatus, medis Silatatus; sep. pinmulis integris, latis instructa, acumine foliaces, laciniato, styli lanati, columnares, $5 \mathrm{~m}$. longi, $3 \mathrm{~m}$. lati, gisio subplano; cor.magna, rosea, eciliata; fruct. ovatus.

Hab. Thone: ad aquaciuct raman. Chaponost (P. Chabert).

Of stylos columnares at Synstyleas (Mipartice Bomentelloidac) accedere videtur; sed Liscus fere planus et habitus Crepiniarum.

t Fructus breviter ovoidens.

\$. Petioli onnes aculeati.

1800. Crepinia eglanianlosa (Gogr Essai p. 33; Gigr Ros. II, p. 32); Gigr Sab. N=2458. - C. Friesii (Lagger et Puget in Christ Posen ver Ychweiz p. 137, non Schentz, C. rugasse (Dematra \&ssai monogr. PRos. 2e Frib. 1818, p.4 non Shumb., nec Gratt.). C. abietina f. eghenioulosa (Crist l.c.).

tenlei falcato-gilatati, ad ramos flor opiosi; fol ovata, basi abrupte cureata, apice rotumiata, sbscure virioia, promisnule nervosa, subtus grises-rugasa, it costam glaniulosa; dentes inf. biserratic; pet. glandoulosi, innes aculeati; stip. marginibus glaniulosae, aurionlis rectis; pes. 1-4, glabri, g-15 m. longi; terbus ovoirens; sep. erecta, Die persistentia, pinmulis latis, folinces-gentatis instructo; stygh villosi, gisto plano; cor lacte rosea; fuct. breviter ovoidens, basi rotuxiatus, apice attenuatus.

Hab. Hehotia, ad Fribawry (Dematra, sec. Lagger), Uri, in montass prope Ysasen et inter Wasen et Goschnen, Intschi; Schattorf (Gisler); Graub, gorberrheinthal Pims (Riggenbach). Rosa mugase Ghunb. Fi. jap. p. 213, DC. Protr. II, Ros. N: 48, at Chinenves pertinet. Mosa Friesiu Schent Stuid shand. Rosx p.39 (18\%2) is Sugetiis (Gomentosecce) collocanio est.

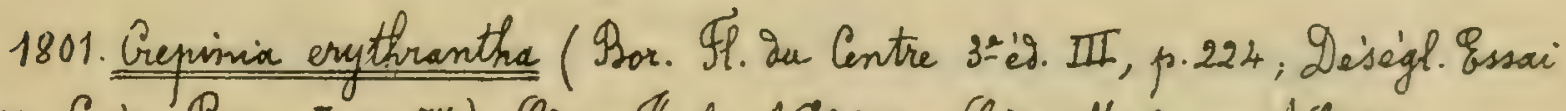
p. 81; Grip. Prim. I. p. 57); Gogr Eab. N:2460. - Gogre Herb. nos. N:557 var. Aculei salisi, falcato. Silatati; fol parva, ovata val elliptica, acuto, 
$-220-$

ai costam eglanidulosa; gentes simplices; pet. eglaniaulosi, annes aculeati; stip. angustae, subtus pulescentes; por. 1-2, glabui, breves; tubus ovoideus; sep. pinmulis longis, eglanônlosis instructo; styli villose; gisco subcomico; cor. intense rosea, eciliata; fruct. breviter ovoidens.

Hab. Gallia occid., prope Angers, te.

1802. Crepinia bayonnensis Gigr Cab. N:2461.

th-culei sat gebiles, falcato-gilatati, à nanzos Glor. copriosi, fol. ollonga, utrinque, sed apice prosertion attenuata, a costam eglangulosx; gentes simplices, aperti; pet.eglanoulosi, smmes parce sculeati; stip. breves, parum latore, ouriculis rectis; pes.1-3, glabii; 8-12 m. longi; tubus ovoiseus; sep. brevia, pinnulis integris, angustis instructa; styli exserti, lanati, gisco praulo conico; $\cos$.......; frusct. breviter ovoideus, basi rotuniatus, apice attemuatus, centrali suboblongo t utrinque attenuato.

Hab. Basses- Pyrénés, at Bayonse (H. Bordere).

\$. Petioli inferiores inermes.

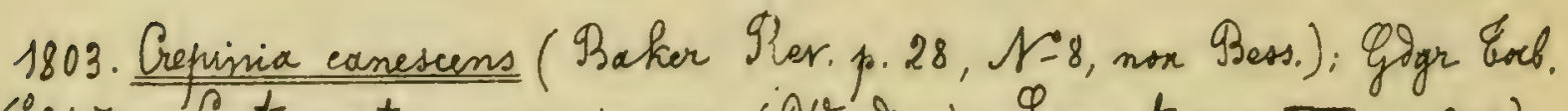
$N^{0}-245 \mathrm{~g}$ - C. tomentose var. incana (Yooss in Lim. trans. XII, p.203); C. casia var. incana (Gorrer in Hook. Mrit. fl. 32 és. p 242), C. canina var. incana (Maker Mon. brit. nos. in Limn. Journ. XI, p.230). - Baker, Herb. ros. 1.20 .

Fculei falcato-Dilatati; fol orata, villoso-canescentia, ad costam glanivulosa; dentes arguti, fiserrati; pet. glanoulosi, omnes parce aculeati, stip. Denticulatae, subtus pubescentes; pes. 1-3, glabri; tubus ovoideus; sep. decisua, pinnulis ciliatis, bentatis instructa; shyli villosi; cor. rosea; fruct. magnus, breviter ovicieus.

Hab. Anglia, Oforkshire, Devonshire; t alibi (Diseglise Cat.p.211). Futex oforem resinosam eximie redolens, secunoum acutorem (sed an recte?) quo charactere cum Pugetiis (Eomentoseae) sot congruit, peounculi vero loevs nec hispidi.

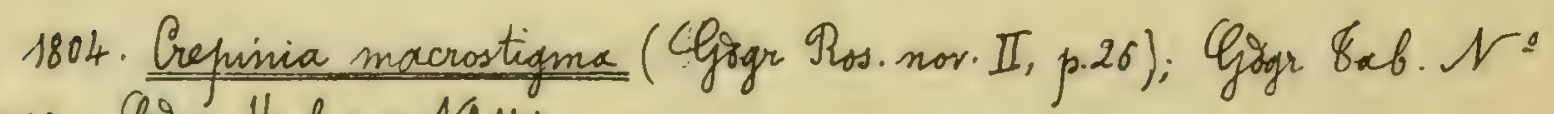
2462. Gogr Herb.ros. N: 144.

foculei falcato- Iilatati, ai ramos flor. copiosi; fol late oborato-oblon- 
$-221-$

ga, utrinque attemucta, saturate viridia, so costam eglasioulosa, dentes simphices; pet.eglisioulasi, inf. inermes; stip. Dilatota, ped. 2-4, glabu; 10-15 m.longi; tubus ovoiders; sep. pinnulis integris, latis instructa, acumine elongato; styli magmi, lanati, gisco subplano; cor. sat pallide rosea, eciliata; fruct. breviter ov oidens, havio ant vix defressus.

Hab. Phone, ad tornas (Mb. Ganoger).

1805. Crepinia cencisia Egogr mss.

A.culei minutissimi, jarwm Dilatati, tenuiter falcati, so ramos flst. copsiosi; fol ampliescerle sorato-subacuta, basi attenuata, intense viridia, à costam eglaniulosa; dentes simplices, late ovato-convergentes; pet. eglandulosi, sup.inermes, stip. hreves, latissimae, viriues, subtus toto villosac, auriculis divergentibus; ped. 1, glabri, $8 \mathrm{~m}$. Longr; tuhus ovoideus; sep. pinnulis pancioribus, onnibus integerrimis, parum latis instructa; styli lanati, gisco subplans; cor....... fruct. ovoidens, utrinque attennat,

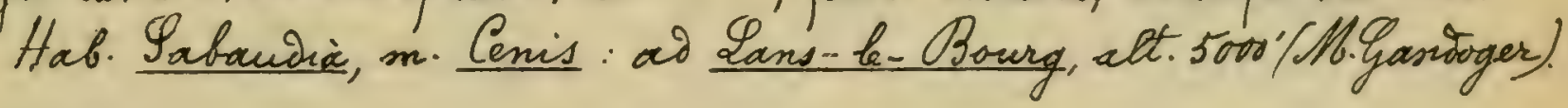

1806. Crepinia orophila (Rip. an Gren.?) m Ggogr Sab. N'2463.

tculei falcato- dilatati, vel subirchinati, ai ramos flor.copiosi; fol. ample obovato-oblonga, utrinque atternatc, superme grisescentia, nervosa, at costam oglanialosa, gentes simplices, recti; pet eglaniulosi, inf. inerines; stip. glabiac, longoe, latissimae, mbentes; pes. 2-5, glabri, 7-14 $m$ langi; tubus ovoibeus; sep. pinnulis angustis, parum gontatis instructa, acumine lato; styli villosi, disco sat conico; cor. lacte rosea; fruct. ovoidens, aprice attenuatus.

Hab. Houte-Savoie, ad Mont-Sion (Mrget).

$\oint \oint \oint$. Petioli omnes inermes.

1807. Crepinia mesomorpha (Gogr in Bull soc. Murith, IV, 1876, p. 33); Gigr bab. N:2464.

Aculei breves, falcato- dilatati, as ramos flor. plerumopue nulli; fol. ample oblongo-subacuta, basi rotuniata, â costam eglaniulosa; Dentes simplices, ovati; pet. inermes, eglandulosi; stip. latae, subtus pubescentes, suriculis rectis; per.3-6, ghibri, 18-21 m. longi; thibus ovoidens; sep. pinmilis latis, 1-2-Dentritis instncta; styh villosi, sisco pauls comico; cor......; funct. breviter ovoideus, base rotundatus, aquice strangul. Hob. Helvetia, galais, in vinetis prope Sion (\&.C. Ifolf). 
$-2.22-$

1808. Crepinia angustifolia Gogr Eab. N-2465.

toculei freves, dilatati, tenuiter falcati, ai ramos flor minutissimi et stipulares; fol. anguste oblonga, utrinque freviter attemuata, condensata, to costam eglanidilosa, Dentes simplices, lanceolato-aperti, pet. eglanialosi, inermes; stip. mediseres, auriculis subairergentibus; pes. 1, glabri, 5-7 m. langi; tubus ovoitens; sep. pinnulis brevitus, parum latis, 1-2-Dentatis instructa, acumine sat angusto; styk lanati, Disco plano; cor-pallise rosea; fruct. breviter ovoidens, utrinope seo apice praesertion gepressus.

Hab. Lucia, Blekingia: ad Lyckeby (T.T. Lunioquist).

180g. Grepinia calochlamys Ggor bab. $\mathcal{N}=2466$.

A-culei gebiles, falcato-gilatati, a ramos flar. foliosos geminati; fol. late obovato-obtusiuscula, basi subtruncata, sat pallide viridia, as costam eglasidulaso; Dentas simphices, late lanceolato-convergentes; pet. eglangulosi, inermes; stip. maxinace, auriculis divergentifus; pes.1-3, glabii, 9-11m. longi; tubris ovoideus, sep. ubra, pirmulis angustis, eglandulosis instructa, acumine maximo, late foliaceo, sentato; styli lanati, gisco subplano; cor.magna, rosea, eciliata, fruct. freviter ovoideus.

Hab. Inecia, gestrogothia : Kestad montis Kinnokulle (J.\&. Zetterstegt).

1810. Crepinia callosa Gogr mss.

Aculei gilatati, tenniter falcati, ai ramos flor. sat copiosi; fol. late ofovota, abrupte et vis acuta, basi truncata, intense viribia, as costam non - laninlosa; dentes simplices, late subpatulo - triangulares: pet eglaniu Losi, inermes, stip. latac, sivides, auriculis parum Sivergentibus; peo. 1-2, glabri, 11-13 m. longi; tubus ovoidens; sep. pinnulis pancioritus, vix hirsutis, onnibus integris, lates instructa; styh lanati, Disco conico; cor. ignota; fruct. ovoidens, basi abrupte truncato-attenuatus, apice strangulatus, metio inflatus.

Hab. Suevix, Halland: gl gabery (y. To. Gabrilsson).

1811. Crepinia epimedium Gogr $\$$ ab. $N^{\circ}=2467$.

Aculei falcato-dilatati, ad ramos flar-copiosi; fol. obovats-acuta, basi rotuniata, a costam egl rioulose, gentes simplices, lati, falcati; pet. basi paulo glanoulosi, incermes, stip. unbentes, longae, latac, auriculis $g_{i}-$ 
$-223-$

vergentibus; peo. 1-3, glabri, graciles, 2-21/2 cent.longi; tubus ovoitens; sep. pinnulis latiuseulis, Dentatis instructa; styh exserti, discs sat conico; cor magna, lacte rosea; fruct. ovoideus, basi rotunidatus, apice atten. Hab. Haute-Savoic, as Pringy (Puget).

T+Fructus sbovato-oblangus vel oblongus.

§. Foliola inaegue vel biserrata.

$\sim$ Rami florales inermes.

1812. Cripinia Blyttii (Goger Essai p. 31, Gogr Ros. nov. II, p.26), Gogr \&.eb. N:2468. - C. pubescens (to. Mlytt FP. Gogn., non Fisch, nec Lem., nec

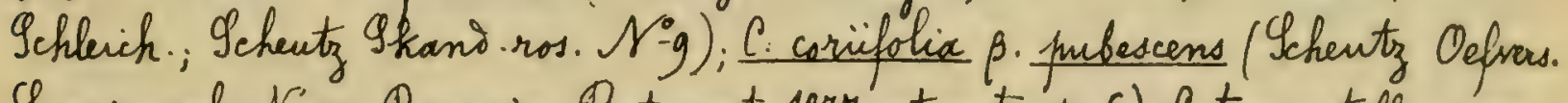
Irenge och Norge Rosa in Pot. not. 1877, et extr.p. 6). C. tomentella var. vexioniensis (Schentz exs.).

toculei coulinares, valioi, falcato-Silatati, as ramos flor. mulli; fol. lite oblongo-rhomboex, utringue sensim attenuata, as costam glanoulosa; dentes crebre biserrati; pet. inermes, temiter glanioulosi; stip. latae, Forso glanaulosae, auriculis divergentibus; pes.1-2, glabri, 4-6 m. longi; tubus oforato-oblongus, sep. erecta, serius decibua, pinnulis integris, praucioribus instructa, acumine letto, dentato; styli lanati, sises plano; con. ignota; fruct magnus, obovato-oblongus, subturbinatus, basi valde attemuatus, aprice turgidus.

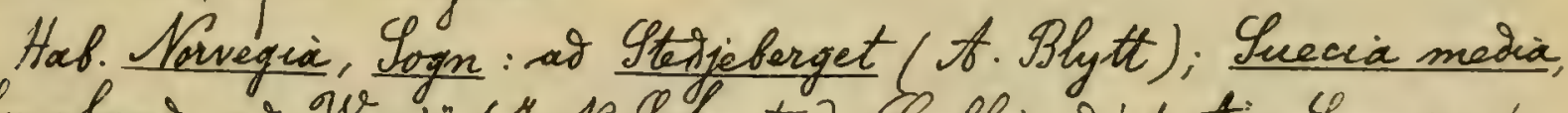
Imoland. at Isexis (\%. M. Icheutz); Gallix, oept. tin, Savoie, ete.

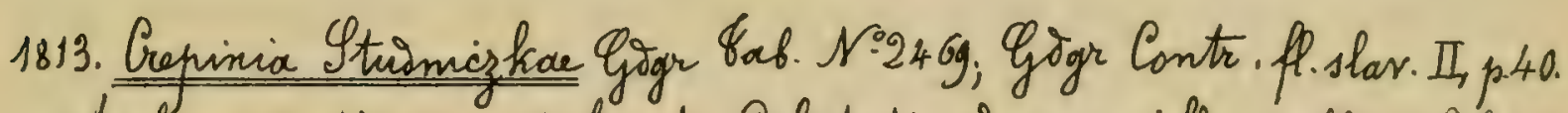
Aculei minutissimi, inclinato-gilatati, ar ramos flor mulli; fol. obovato-obtura, basi rotuniata, id costam eglaniulosa; ventes a pertotriangulares, argute biserrato-glanoulosi, pet. unbentes, aculeati, sparse glaniulosi; stip. rubrac, auriculis sivergentibus; ped. 1-2, glabri, 10-12 m. longi; tubus Blongus; sep. pinnulis omnifus foliaceis, dontatis ionstructa; styli lanati, sises plano; cor.eciliata; fruct. oblangus, basi praesertim attenuatus.

Hab. Lalmatix, prope Giovanni (K. Stionizha)

Species fohisis creberrime biserrat:-ylanoulosis, inter affines conspicua. 
$\sim \sim$ Rami florales aculeati.

1814. Crepinia sapita Goar Gab. N:2470.

tbculei ad ramos flot. faleato-Silatati, fol late obovato-oblonga, attenuata, basi rotundata, ad costam eglaniulosa, gentes aperti, cuspiouti, extra - infer paulo biserrati; pet. inermes, eglandulosi; stip. latae, longas, auriculis parum sivergentibus; per. 1-3, glabri, 7-11 m. longi; tubus clongatus; sep. pirmulis parum latis, via dentatis instructa; styli villosi, Pisco acute comico; con.......; fruct. anguste elongatus, utrinque salte attemuatus, basi decurrens.

$$
\text { Hab. Hante- Lavoie, at St Gervais (Puget). }
$$

1815. Crepinia pilosa (Opiz is Prese Suppl. as fl. cech. Eratt. Rosac. mon. I, prolegom. p. 38); Goge Gab. N.2471; Goar Contr. H. slar. II, p.40.

Acculei compressi, as ramos flor. falcato-Dilatati; fol. late ovata, ad costam sparse glandulosa; dentes subfiserrati; pet. aculeati, vix glansulosi; stip.ciliatae, subtus pubescentes; peo. 1-3, glabri; tubus oblongus; sep. pinmulis mediocribus, eglandulosis instructa; styli villosi, gisco pulano: cor......, fruct, oblorgus.

Hab. Bohemix, ad Kuchelbad (ifiz, etc.).

1816. Crepinia bosniaca Gogr mss.

Abculei Dilatato-subfalcati, breves, as ramos flor geminati; fol. amplissime oblongo-subacuta, basi rotundata, ab basin costice sparse jlandulosa; gentes late ovato-aperti, inf hiserrati; pet. tomentosi, inf. inermes, fasi adsperse glanoulosi; stip. i irioss, maximae, auriculis rectis; per. 1-2. glabri; $7-9 m$. longi; thbers obovato - oblongus; sep........, styli lanati, Gises plano; cor........, fruct. obovato - oblongus, basi rotionTatus, apice value attennato-strangulatus, centrali vero gecurrente.

Hab. Mosnix, ad 2obaj (2. Richter).

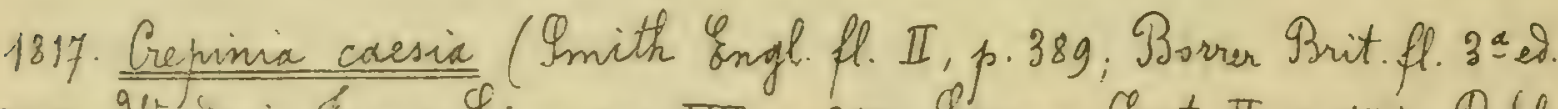
p. 242, grovis in Grans. Lim sac. XII, p. 212, 9 preng. Oyst. II, p. 554, Reht.

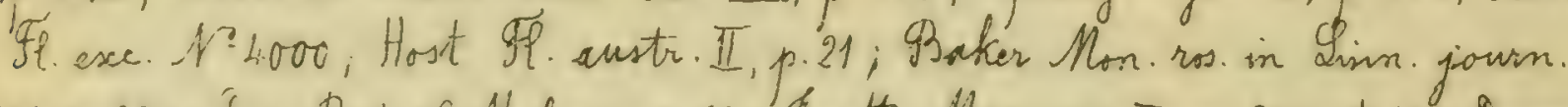
II, p. 232; Les Bot. of Malvern p.66; Eratt. Mon. ros. II, p. 236 et 14; Dumort.

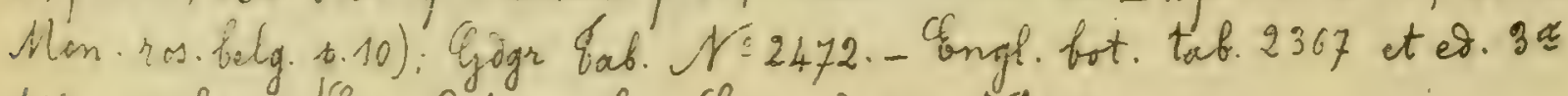
tab. 473 bis. - Exes. Cat. soc. bot. Loni. ed. $3=\mathscr{N}=351 \mathrm{~g}$. 
$-225-$

tculei sufinouguales, falcato- Dilatati, ag ramos flor fore geminati; fol. elliptica, caesia, nervosa, à costam glanioulosa; gentes biserrati; pet. inermes, glanoulosi; stip. pilosae, denticulatas; peo. breves, glabri, tubus oblongus, glaucus; sep. pinnulis pancioribus, gentato-glanioulosis insitructa; styli villosi; cor. laete rosea, funct. oflangus.

Hab. Itnglix, ete.

1818. Creminia exerrans Gogr bab. N-2473.

taculie subrecti, longi, vix bilatati, of namos flor copriosi; fol. oblongolanceolata, basi atternuata, ic costam eglanianlosow; dentes lanceolatovecti, fore onnes biserrati; pet. inermes, hinc inde glanaulosi; stip. vinias, auriculis Divergentibus; ped. 1-2, glabri, 8-g m. longi; tubus oblongus; sep. pinmelis srmmibus integerrimis, latis instructa, styh hissuti, Opiseo plano; cor......, frucit. maynus, oblongus, besi attenuatus, aprice strangulatus. Hob. Anglix, Cheshire: as Jillaston (Y. Harbord-Lewis).

1819. Brepinix angoris Gogr mss.

F-culei tennes, sufisilatato falcati, as ramos flor sparsi; fol. ample - fovato- oflonga, utringue sensin thensata, io costion eglanisulosa; Sientes annes fiserrati, a perto - triangulores; pet. egianiulosi, inermes; stip. virides, beres, latinsculac, auriculis subrectis; prod. 1-2, glabri, 1 cent. longi; tubus obovato - oblongus; sep. pinnulis smnibus folixceis, 2-4rentatis instructa; styli lan ati, gisco subconics; cor......... funct. sat magnus, frovato-sblongus, utrinque attenuatus, apice strangulatus.

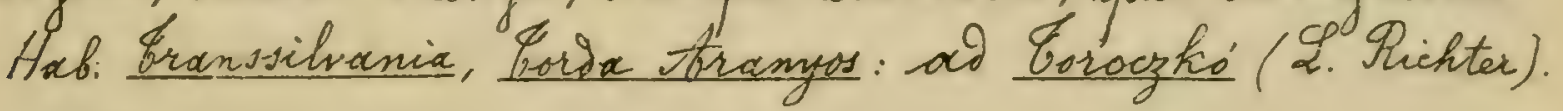

1820. Cres inia Dentifira CGogr \&ab. No-2474.

fo-culei valisi, Dilatati, valiv falcati, as ramos flor. gemmati, fot. amplissime cbovata, utringue breviter acuta, intense viridia, at costam eglanisulosa; ientes latissime triangulares, aperti, inacquales; pet-inermes, eglaninioti; stip. latae, auriculis sat Sivergentibus; pè. 1-2, glabri, $7-g m$. longi; thebus oroideus; sep. pinnulis angustis, 1-2-gentatis instructi, acumine brevi, latiisculo; styli lanati, dises paulo conico; cor. roser, eciliativ; fruct, oborato- oblongus.

Hab. Sucia, Mlekingia: as Nattraly (P. F. Lunsopuist). 
$-226-$

§§. Foliola ammino simpliciter serrata.

$\sim$ Setioli inferiores inermes.

1821. Creminia carneipetala Gogr \&ab. N:2475.

tanlei falcato- dilatati, a ramos flor.copiosi; fol. obovata, utimque breviter attesmata, ad costam eglaniulosa; dentes simplices; pot. eglasioulosi, inf. inermes: stip. sirides; ped. 2-3, glabri, 8-10 m. longi; tubus oblongus; sep. pinnulis integris, latis instructa; styli magni, lanati, Disco subplano; cor.carnea, eciliata, potalis acute unguiculatis; funct sovato-oblongus. Hab. Rhone, in petrosis montis Buisante as Pommiers (M. Ganoger).

1822. Crehinia satthica Gogr mss. - Brotherus 91. cauc exs. (1881) sine num. feculei tenues, breves, inclinati, as ramos flor. stipulares; fol. Obovatoblonga, acuta, basi subrotuniata, lacte rividia, as costam eglanoulosa, gentes simplices, lanceolato - subconvergentes; pet eglanoulosi, inf. inames; stip. breves, sat latar, auriculis rectis; per. 1-2. glabu; 1:-16 m. Longi; tubus oblongus; sep. pinmulis onnibus integris, latis, instructa; styli sublanati, Dises comico; cor......; fruct. oblongus, basi depressus, apice attenuatus.

Hab. Coucasus, Carthahinia: at Gori prope H. Kurà (Meothous).

1823. Crepinia turitella Gogr mss.

Arculei freves, falcato- Dilatati, at ramos flor. panciores; fol ample sblong-acuta, basi attenuata, subtus abbiso-glauca et al costam eglandulosa, Dentes simphices, ovato-aperti; pet eglanioulosi, sup. inermes; stip. virides, latae, longiusculae, auriculis givergentibus; pes. 1, glabri, 1/2 cent.longi; tubus oblongus; sep. pinmulis villosis, onnes integris, latis instructa; styli lanati, aisco plans; cor........; fruct. maximus, elongatus, basi decurrens, in $1 / 3$ sup. turgidus, apice contractus.

Hab. Hangaria bor., Liptó: as Rozsahegy Muzahegy (2. Richter).

1824. Gepinia synearpa Gogr \&ab. N:2476.

treulei falcato- Pilatati, as ramos flor. rubros copiosi; fol. Ofovato-oblonga, acuta, fasi vis attemata, is costam eglanaclosa; dentes simphices, pet. eglaniulosi, inf.inermes; stip. rubentes; ped. 3-5, glabri, 9-14 m.lmgi; tubus oblongus; sep. longa, pinnulis angustis, gentatis, pubescentibus instructa, styli villsi, giseo paulo coniso; cor.magna, rosea, eciliata, oftuse unguiculata; fruct. magnus, oflongus, utrinque subattenuatus. Hab. Mhine, ao Charbonmieres at Fancheville (P. Chabort). 
$-227-$

1825. Crepinia rivularis Gogr mss.

toculei tennes, breves, inchinati, vix Jilatati, à ramos flor sparsi, fol. mesiocria, oblongo-acuta, basi rotundata, al costam eglandulosa; dentes simphies, triangulares, aperti; pet. eglanaulosi, inf. inermes; stip. angustae, breves, virides, auriculis sat divergentibus; pres.1-3, glabri; 1 cent. longi; tubus suboblongus; sop.......... styli villose; rises paulo conico; cor....... fruct. sat parvus, suboblongus, basi gepressus, aprice longe attenuatus.

Hab. Hungaria for" Haag: ad Kovaicsi ( L. Richter).

$\sim \sim$ Petioli annes inermes.

X Foliola basi rotuniata vel subrotumiata.

1826. Crepinia Leontomyx (Gogr in Mull. soc. Murith. IV, 1876, p. 32); Gogr Eab. N:2477. - Saenity Herb. enrop. N:1866.

Fculei Defiles, inclinato-suboilatati, as ramos flor. panciores; fol obovatoacuta, basi rotuniata, costam eglanoulosa; gentes simplices, aperti; pet. eglaniculosi, inermes; stip. subtus villosac, auriculis givergentibus; ped-1, glabri; 10-12 m. longi; tubus oblongus; sep. arecta, pinnulis latis, longis, eglaniulosis instructa; styli villosi, aisco subplano; cor. lacte rosea, eciliata; pruct. oflongus, apice strangulatus.

Hob. Helvetix, Yura: af Couvet, alt. $3000^{\prime}$ (Lerch).

1827. Crepinia agrivaga Gogr mss.

foculei breves, parri, foicato- Dilatati, as ramos flor. interium verticiblati, fol.amplissime sbovats-oblonga, abrupte acuta, basi rotuniata, is costam eglansulosa; Dentes simplices, ovats - aperti, pet. eglaniulosi, inermes, stip. latae, elongatae, sirides, auriculis givergentibus; pet. 2-4, glafri, 1 cent. longi; tubus obovato - oblongus; sep......... styli lanati, viseo plano; cor.........; fruct. meviocris, obovato-oblongus, basi depressus, at apicas attenuatus.

Hab. Gransilvamia, Kologs: as Frékahr (L. Richter).

1828. Creninia mierobasis Gogr Eab. $N=2478$.

toculei deliles, inclinato bilatati, as ramos flor saepies mulli, fol. ovato- lliptica, freviter attenuata, basi subrotuniata, ad costam eglanaulosa; ventes simplices, aperti, pet. inermes, eglansulosi, stip. breves, auri- 
$-228-$

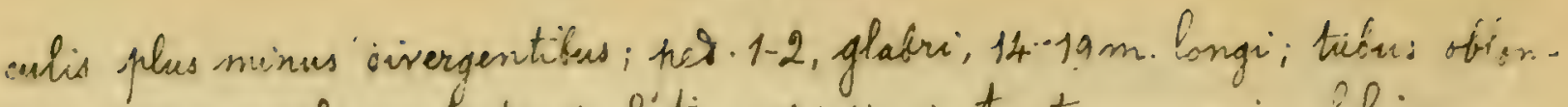
qu: ; sep. pinmulis subintegris, litis, copiosis instructa, acumine foliaces; styh brevissimi, villosi, jisco subcomico; cor. ; fruct. oftongus, a $2 / 3$ sup. usque aे basin valie atienuato- gecurrems, apice Aurjibus et attemuatis. Hab. Haute- Pavoic, at Iingy (Puget).

1829. Creninia paunercula Gijor Gob. N:2479.

foculei defiles inchinats silatati, xs ramos for coniosi, fol obovate-

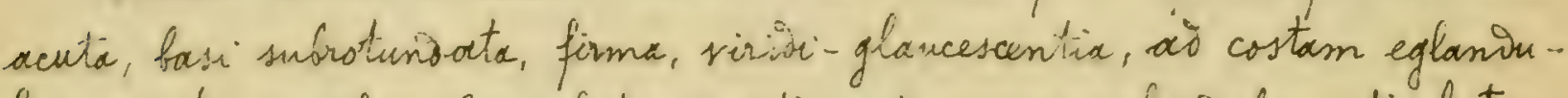
los $x$; Dentes simplicis, lanceolato-aperti; pet. inermes, eglaniulosi; stip. latue, auriculis suboivergentibus; pes 1-2, glabri, $14-16 \mathrm{~m}$. longi; tubus obovatoflongus; sep........ styli villosi, gises sat conico; cor........; fruct oboratooilongus, fasi rotunocitus, apice attenuatus.

Hab. Halia crient, do Focenza (2. Caldesi).

XX Foliola basi plus minusse attemuata.

1830. Creprinia stenotuba Gogr Gob. N:2480.

Fichei falceto- Jilatati, as ramos flor. subnulli; fol obovato-oblonga, utrinque paulation attenuata, ai costam eglaniulosa; dentes simplices, a perto-lanceolate; pet inermes, basi hine inde glandulosi; stip. látiusculare, anviculis sivergentibus; pes. 1-2, glabri, 5-11 m. longi; tabus anguste elongatus; sep-pinnulis gontatis, sat latis instructa; styli sillosi, Hisco conico; cor........ fruct. elongatus, uting ue valde attenvatus, Decurrens. Hab. Houte- Savoic, thabeire - Poche (Inget).

1831. Crepinia subtubulosa Gogr mss.

Aculei rari, breves, parri, Dilatato-subfalcati, as ramos flor mulli; fol. sat late oflongo-acuta, basi attenuata; ad costam eglanioulosa; dentes simphices, ovato-convergentes; pet-eglaniulosi, inermes; stip. brevisialae, sect anguestae, virides, auriculis rectis; ped. 1-2, glabri, 1 cent.longi; tubus of. longus; ses........ styli villosi; gisco plano; cor ....... fruct. sat magmus, oblongus, utringne longe attemuatus, medis turgious.

Hab. Hengaria, as Sudakersierdo (Af. Iteffeck). 
$-229-$

1832. Crepinia mobrinetala Gogr \&ab. N.2481.

tbeulei breves, gilatati, tenniter falceti, a ramos flor. stipulares; fol. anguste oflongo, intense virioia, utrinque attenuate, at costam eglasioulosa; sentes simplices, triangulares, sperti, pet. sxermes, eglanoulosi; stip.magnae, awriculis givergentilus; pè. 1, glabri, g-11 m. lmgi; tubus obovato-oblongus; scp-pinnulis intigris, brevibus parce instructa, acumine longo, lato; styli lanati, gisco paulo comico; cor. rubella, eciliata; fruct. obovato - oblongus, apice attenuatus.

Hat. Suecia, Blekingia: ad Vàttraby (P. F. Lungquist).

1833. Crepinia lagenaria Gogr Gab. $\mathcal{N}-2482$.

Acculei breves, parvi, gilatato-suffalcati, ad ramos flor.geminati; fol. late obovatos, abrupte acuminata, basi subtrencata, pallide virioia, ad costam eglassoulosa, gentes simplices, aperto-briangulares; pat. inermes, eglansulosi; stip. rubentes, magnae, auriculis subrectis; per. 1-2, glabri, 4-6 m. longi, tubus avoideus; sep. pinnulis parum latis, 1-2-gentatis instructa, acumine longo sat lato; sighi lanati, magmi, aiseo subplano; cor. lacte rosea, eciliata, fruct. ovoideus, basi rotunoato-gilatatus, af apicem longissine et valde attemeato-strangulatus, centrali decurrente. Hab. Suecia, gestrogothia: ad Irollmen montis Kinnekulle (\%. \&. Letterstegt).

$\sim \sim \sim$ Setioli amnes aculeati.

X Foliola basi rotuniata.

1834. Crepinia submitis (Gren. in Ichnlt Arch.p.332, Millot tonnot. ft. Fr.p.8). Goar bab. N 2483 . Fillot exs. N:1475? et 1475 bis.

Aculei panciores, falcato. Dilatati, subgeminati, oc ramos flor. saepe nulli; fol. ovata, à costam eglañalosa; dentes simphies; pet. omnes rculeati, eglaniulosi; stip. latae, pubescentes, auriculis givergentibus; per. 2-5, glabri, mesiscres; tubus oroidens; sep. reflexa, eglaniulosa; styli rillosi; cor. majuscula, pallite rosex, eciliata, fruct-obovato-oblongus. Hab. Gallia m Dia, ete.

1835. Crepinia emiliensis Gagr mss.

toculie elongati, slatati, parum falcati, ad ramos flor. mulli; 
$-230-$

fol. ample ovata, abrupte acuta, basi corsata, lacte virisia, as basin costare aculeolato; dentes simplices, lete ovato-aperti; pet. eglansulosi, om. nes aculeati; stip. longae, virises, subtus toto pubescentes, auriculis rectis; piè. 1-2, plabri, $13 \mathrm{~m}$. longi; tubus oblongus; sep. pinnulis temiter sillosis, omsibus foliaceis, integris copiosissime instructa; styli lanati, Gises conico; cor.magna, rosea, eciliata; fruct. oblongus, utrinque longe attemuatus. Hab. Italia orient, as Fonza Emiliae (L. Caldesi).

1836. Creminix eppendiculata Ggar \&ab. 132484.

teculei debiles, falcato-gilatate, as ramos flor. pauciores; fol amplissime elliptico-rhomboidalia, utrinque subrotuniata, ad costam eglanou. loda; yentes simplices, profunai; pat copiose glansulosi; smmes aculeati; stip. magnae; per. 1-3, glabri, 15-18 m. longi; tubus oblongus; sep. longis. sima, pinmulis integris, latis, spathulatis instructa; styli lanati, subcolumnares, Disco pouilo comico; cor.......... fruct. oblongus.

Itab. Itin, of St-Digir-sur-Chalarome ( I. P. Fray).

1837. Crepinia efficax Gygr mss.

tocrlei valiai, gilatati, tenviter falcati, as ramos flor.copiosi; fol. latiriscule oborato-oblonga, vix acuta, basi rotunjata, ai costam eglanDulosa; Iontes simplices, triangulari-convergentes; pet. eglanoulosi, onnes aruleati; stip. latae, frevinsculac, sirises, auriculis givergentibus; prod.1-2, glabri; 7-8 m.longi; tubus obovato - oflongus; sep........... sthgli lanati, sise plano; cor.........; fruct. sat parrus, oforato-oblongus, fasi subiepressus, aprice attenuato-conicus, strangulatus.

Hob. Eransilvania, Korozs: od Hagyar o'kereke (2. Richter).

1838. Crepinia panounitana Gogr bab. N-2485.

taculei breves, falcato-Dilatati, io ramos flor.copiosi; fol. late oratorotuniata, oftusiuscila, basi cordata, ai costarn eglandulasa; dentes simphices, ovato-aperti; pet. eglanaulose; laxe pubescentes, onnes aculaati; stip. rubentes, auriculisgivergentifus; pet. 2-3, glabri, 8-g m. longi; tubus sblongus; sep. pinnulis sarfins integerrimis, latiusculis instructa, acumine anyusto; styli villosi, diseo paulo conico; cor.magna, eciliata; fruct. magnus, freviter oflongus, basi proesertim attematis, apice inflatus. Hab. Dicilia, hrope Talarme (Gussone!). 
$-231-$

1839. Crepinia leucantha (Lois. Notes, 1810, p. 82, 2C. Fe. frane. IV, p. 535; Merat FP. par., 1812, p. 193; Bast. Suppl. fl. Maine -et-Loire p. 32; Bor. Fl. an Centre 3a'r. II. p.216; De'se'gl. Cat. p. 198; Gratt. Ros. mon. II, $p$.

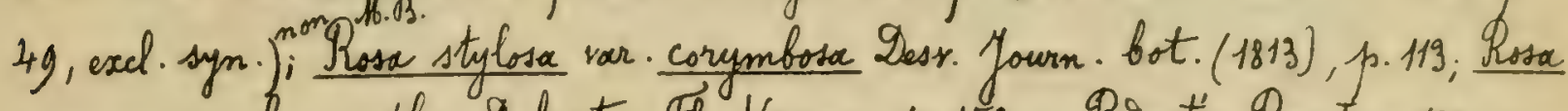

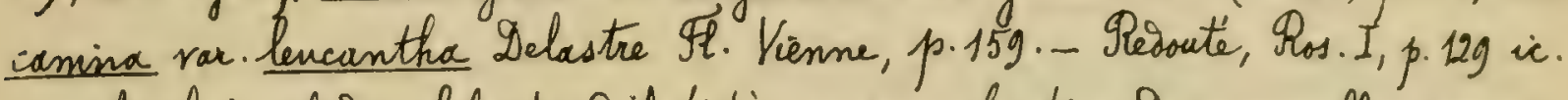
teculei valisi, falcato-Dilatati, saepe rubentes, as ramos flor. geminati aut verticillati; fol ovata, basi rotungata, sup. acuminata, inf. vero obtusa; Dentes simphies; pet. eglanivulosi; annes aculeati; stip. latace, pu. fescentes; per. Tense corymbosi, glabri, brerissini; tubus oblongus; sep. glabro, pinnulis et acumine fliaceis instructa; styli exserti, subcolumnares, villosi; cor.majuscula, alba, cciliata; fruct. oblangus.

Hab Eure-et-Loir, at Dreux; Mewdon; Cher; Gonne; tonglia mor. Sprecies a me in Babulis rhobologicis casu fortwito nuper omissa, certe ad Crepinias (Canineae) pertinet, voversantitus tamen Desreau et Bareace. - Descriptis Brattinichii ad C. obtusifoliam (Desr.) potius spectare visetur. (Pive Gige Monogr. ros. I, p. 135).

Koss lencantha MSieb. IP. Taur-canc. suppl. III, p. 352, e Cancaso, munc R. Brebersteiniu Gratt. Ros. mon. II. p. 5, constituit.

XX Folisla basi plus minusve attenuata.

1840. Crepinia confinis Gogr Tab. N-2486. - Gogr Herb. ros. N-52.

fonkei dilatati, rectiusculi vel subinclinati, io ramos flor. glancos sat copissi; fol. late elliptico-acuta, basi attemuata, al costarm eglanianlosa; Dentes simplices, acuti, subpatuli; pet. eglandulosi, annes aculeati, stip. latac, subtes pubescentes, auriculis divergentibus; pat. 1-4, glebri; 10-14 m. longi; tubus oblongres; sep. pinnulis mediocribus, villosis instructa; stgli villosi, Disco plano; cor ......... fruct. obovato- oflongus, utrinque attenuatus.

Hab. Mhone, ad Aornas (M. Ganooger).

1841. Crepinia acioula Giger 8ab. N:2487.

Aculei falcato-2ilatati, ad ramos flor. foliosos copiosissimi; fol. of longo-lanceslata, basi poulation attemuata, lacte viridia, as costam non glaniulosa; ientes sinplices, recti, lanceolati; pet. eglaniulosi; onnes acceleati, stip. latre, virives, auriculis subsivergentitus, pes. 2-4. glabri, 12-16 m. hongi; tubus oblongus; sep. pinnulis subintegris, longis, Cativeculis ins- 
$-232-$

tructa, styli breves, lanati, Disco plano; cor..........; fruct. breviter oblongus, utrinque depressus.

- Hab. Gyrenaci centr., Geidre: ay viam Héas (H. Bordère).

1842. Grepinia sorrentina Gigr mss.

torulei valiai, faleato-jilatati, ad ramos flor.copiosi; fol late elliptico-obovata, abrupte acuta, basi cuneata, pallise virivia, as costam eglansulosa, Dentes simphies, valise profunisi, triangulari-subonvergentes; pat. onnes aculeati, eglan oulosi, lase pubescentes, stip. rubentes, longae, parum latar, gorso pubescentes, auriculis givergentibus; peor.1-3, glabri, $18 \mathrm{~m}$. longi; tubus oblongus; sep pinsulis snnsibus parum lates, 1-3-Dentatis, instructa, acurrvine integro; styli lanati, Disco flano; cor eciliata; fruct. oblongus, basi iccurrens, apuice attenuatus.

Hab Saples, ad Gragnano in Lorrentinis (F Attoselli).

1813. Creninia lagenoives Giogr mss.

Acalki rari, freves, parvi; Dilatato-falcati, ad ramos flor. mulk; foll parwa,

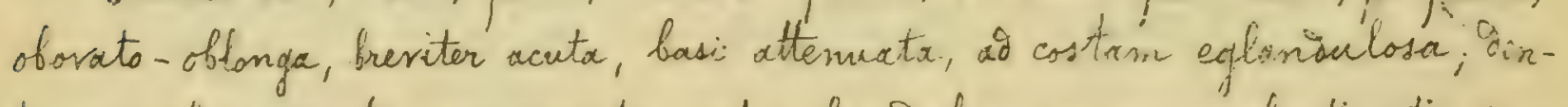
tes simplices, evato-convergentes; pet. eglanoulosi, amnes acculeati; stip. parvoe, virides, breves, angustae, awriculis subsirargentibus; has. 1-2, glabri, 12-13 m. longi; t tabus oblongus;, sep. pinnukis subintogris, angustis instructa, styli vilhse; Gisco plano; cor........., fruct. sat parsus, oblangus, fasic Depressus, aprice longe attenuatus, valiveque strangulatus.

Hob. Gransilvanix, Isolnok Dobokx: Koyselyfalu (2. Richtor).

1844. Crepinia anthophylla Goge Gab. N:2488.

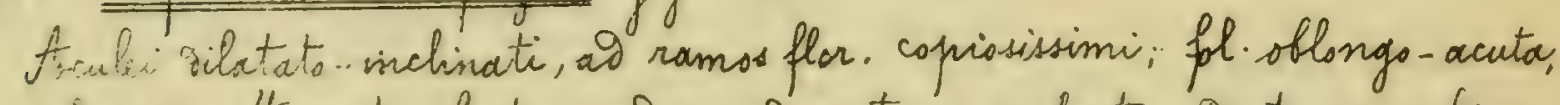
basi abmpte atternuata, lacte virisia, as costam aculeata; gentes siorphlias, recti, put. eglanioulosi, onnes aculeatissinn;; stip. longiusculae, latoa, subtus glabroc; peas 1-3, grabir, 7-15 m. longi; tubus oblongus; sep. pinsulis integris, sect latis instructa, styli dense villosi, gisco comico, cor.......... bunt. magnan, sblangus, itrinque paulation attenuatus.

Hab. Saxses- Pyizéés, al Siviority (H. Pordère).

1845. Grepinia cretica Gigr mss. - Reverchon, Pl. ore Giete 1884, N-280. Aculei bre, issimi, parum vilatati, tenuiter falcati, at ramos flor. sat 
$-233-$

copiose; fol mediscria, sbovato-oblonga, subacuta, basi attenuata, pallide virioia, as costam eglanisulosa, Dentes simplices, late ovati, recti; pet eglanaulosi, onmes aculeati, stip. brevissimae, parum latoe, virides, apuice oorsi pubescentes, auriculis Sivergentibus: per.1, glabri, $12 \mathrm{~m}$. longi; tubus oblongus; sep. pinnulis annibus integris, parum latis instructa; styli subla nati, sisco concic; cor. sat magna, eciliata; fruct. oflongus, utirinque longins attermatus.

Hab. Creta, at tomalos (6. Reverchon).

2. Pesunculi glasioulosi et simul paulo hirsuti.

1846. Crepinia elegans Gogr Sob. N:2489.

Aceuli minutissini, inchinato-subsilatati, ait ramos fhor plus minusse copissi; fol. obovata, utringue subrotungata, pallive virioio, as costam eglaniulose; Dentes simplices, aperti, breviter triangulares; pet. eglansulosi, inermes; stip. saepe rubentes, maximae, acriculis givergentibus; pad. 1-3, alii grabui, alu hirtello-subglanioulosi, 4-6 m. longi; tubus ovoideus; sep. pinmulis latis, eglanioulosis instructa, acumine ample foliaces-lobata; styli lanati, giscs plans; cor magna, lacte rosea, eciliata, funct. ovoidens. Hab. Inecia, Destroyothix: in monte Kinnchulle, as Kestad C.\&. Lettorstert].

1847. Crepinia curtispina Gogr Gab. N'2490- Gogr Herb ros. N-558 var. Aculei debiles, inchinato-subilateti, heves, as ramos Plor. submulli; fol. obovata, utrinque brevissime attemuata, al costam eglanianlosa; Pentes simplices; pet. eglanioulosi, inf inf. incermes, stip. meiroeres, virides; peod. 1-3, hirtelli at assperse glanionloselli, $10-16 \mathrm{~m}$. longi; tuhus oblongus, sep. piinmulis subintegris, latiusculis instructa, styli glabi', axserti, Disco connco; cor......, frust. oblongus, etrinque attenuaties.

Hab. Phóne, ai Chapanost (P. Chabert) at ad tornas (Ho. Ganioger).

1848. Cerisia integrata Gogr \&ab. N:2491.

Aculeci psum gilatati, minutissine falcati, as rames flor geminati; fol ampke to-oblonga, acutiuscula, basi subrotunoata, ai costam

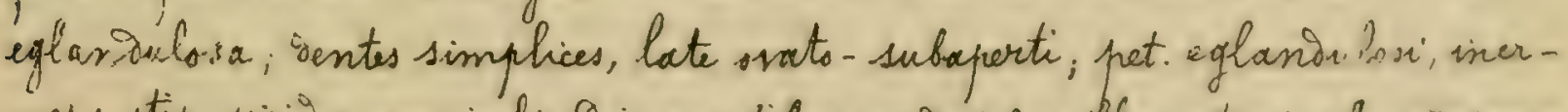
mes; stip. sirives, auriculis givergentifus; per. 1-3, villosi et simne? axporse 
$-234-$

glaniouloselli, 15-17 m. longr; tubus oblongus; sep. primulis integris, latisimis instructa; styli glaberrimi, gisco comico; cor. eciliata; fruct. ample oblongus, basi valie attenuato-Decurrens, apiae subcontractus. Hab. Gavo, at brphy prope Le gigan (D. Euejkieiming).

3. Seaunculi taxtum villosi.

a. Ttali glabi scl adsperse pilosi.

f Foliola clliptica val obovata.

§. Fructus ovatus, svoivens val oborato-oblongus.

1849. Greminia inconcessa Gogr Gab. N-2492.

Freulei maximi, falcato-gilatati, at ramos flor saepe nulli; fol. orata, utringue attemuato, mesio dilatata, nerrosa, firma, as costam eglanDulose, Dentes simphies, aperti, pet. eglanioulosi, laxe pubescentes, onnes parce aculeati, stip. Longae, auriculis aivergentibus; poo. 1-3, villoss, 6-10 m. longi; tubus ovatus, in $1 / 8$ inf. pubescens; sep. brevia, pinnulis linearibus instructor; styli parri, glabri, gises sat comico; cor parva, subatba, eciliata; funct. ovatus, utivingue panlo repressus.

Hab. Hanche, ad Ofretot (\&. Clebel).

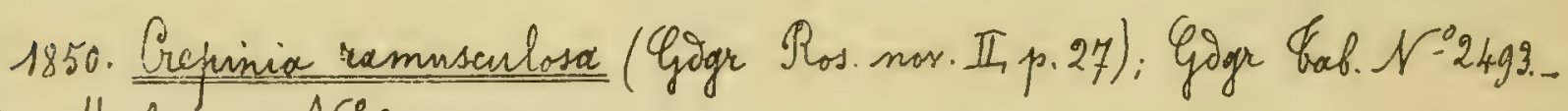
Gagre Herb. ros. N'244.

teculer Mebiles faleato-gilatati, as ramos flor pauciores; fol. ovatoacuta, nitiva, at costam eglanidelosa; dentes simplices; pet. inermes, hine inde glanioulosi; stip. latiusculae, subtus pubescentes, auriaclis parum divergentibus; ped. 2-4, villasi, mediocres; tubus obovato-sblongus; sep. pinnulis eglanisulosis, medicoribus instruceto, styli glabri, gises subconico; car. rosea, eciliata; fruct. obovoctus.

Hab. Ithone, as thrmas (N.Ganooger).

1851. Crepinia commiserata Gogre Gab. N-24y4.

Foculei minutission, termiter falcato-gilatati, ai ramos flor. nulli; fol. elliptico-oftusiuscula, fasi rotuniacta, at costam eglanaulosa; gentes sinphices, prote-porti, pot. inormes, eglansulosi; stip. sirites, aurriculis rectis; pè. 1, killosi, 7-8 m. longi; tubus ovoidens; sep. pismulis integris, latissimis instruct a, acumine late folinces, laciniato; styli aspperse pi- 
$-235-$

losuli, Siscs conics; $\operatorname{cor} \ldots . . .$. ; frect. ovoidens, utringue depressus. Hob. Hispania bor., Catal, as Giodemiras pror. Gorona (9. Yayresa).

1852. Crepinia glauciramex Gogr Eab. N-2495. - Gogr Herb. ros. N-149. toculer gebiles, inchinato-subilibtati, ad ramos flor. sacpe mulli, fol. elliptico- subacuta, basi cordata, at costam eglanaulosx; Pentes simplices; pet. eglanaulosi, omnes inermes; stip. virides; per. 1-3, villasi., $10-14 \mathrm{~m}$. longi; tubus obovato- sblongus; sep.........., styli subgla berrimi, gisco paulo canico; cor......... fruct. maximus, ovoidens, basi semper valde ettenuatus.

Hat. Thone, at Denie' (Mb. Ganooger).

\$. Fructus oflongus.

$\sim$ Rami florales aculeati.

1853. Cresinia mitoclada Gigr Gab. N:24g6.

fecclei falcato - subsilatati, as ramos flor. subgeminati; fol. oborate, eitrinque paulation attenuata, os costam eglandulosa; sentes simplices; ift. eglundulosi, inermes; stip. mexiseres; ped.2-3, villosi, 16-18 m. longi; tubus elongatus; sep. pinnulis subintegris, pubescentibus, latis instructa; styli subglaberrimi, sisco comico; cor. pallide rosea, eciliata; fruct. oflongus, utrinque depressus.

Hab. Rhone, at Chaponost (G. Chabert).

1854. Crepinia anacampseros Gogr tos. N-2497.

Acculei parvi, falcato-Silatati, ad ramos flor. panciores; fol ample oborata, firma, crassa, breviter attenuato, basi roturiacta, intense viriaix, ad costam eglandulosa, Dentes simplices; pet. eglaninlosi, mbentes, plerumque omnes inermes, stip. latos, Dorso villosac, medio rubentes, auriculis Sivergentibus; pes. 1-5, villosi, 10-16 m. longi; tubus oblangies; sep. pinnulis latis, Dentatis instructa, acumine foliaces; styh breves, glabri, Sises paulo cimic; cor.magna, subalba, cciliata; fruct. oblongus. Hab. Rhone, dille-sur- Jarnioux (Nb. Cangrager).

At pracedente recerit filiolis crassioribis, stip. me his rubentibus notioque supra enumeratis. 
$-236-$

$\sim$ Rami florales inermes.

1855. Grepinia lonchitis Gogr Bab. N:2498.

toculei tenues, falcato - subailatati, as ramos flar. mulli; fol. obovatosubacuta, basi paulo attenuata, intense viridia, as costam eglanoulosa, Dentes simplices; pet. eglandulosi; annes inermes; stip. virites; ped.1 3 , villosi-5-7m. longi; tubus obiongus; sep. pinnulis saepe integris, pubes centibus, latis instructa; styli assperse pilosuli, disco subplano; cor. pallive roses, eciliata; fruct. oblongus, utringue attenuatus. Hob. Rhone, ad tornas (M. Ganooger).

1856. Grepinia hanonensis Gogr mss.

Frulei pancisres, breves, valoe falcato-gilatati, as ramos flor-nulli; fol. obovato-acuta, basi notuniata, intense viribia, at costam eglanaulosa; gentes simplices, sublanceslato-convergentes; pot. eglansulosi, prorseus inermes; stip. latae, virides, aurculis divergentibus, pai.1-3, villosissimi, 1-2-qlaniulosi, 14-16 m. longi; tubus oflongus; sep. pinnulis integris, paulo pubescentibus, onnibus foliaceis, styli oisperse pilosuli, disco sat conico; cor-fuallide rosea, eciliata; fruct. magnus, oblongo-fyriformis, in $1 / 3$ sup. turgidus, et inise as basin usque longe attenuato-gecurrens. Hab. Begium. Hoinout: ad Harvengt (M. Gandoger).

1857. Orepinia Guxdarramae Gögr fab. N-2499.

Acculei pouciores, falcato- Dilatati, breves, ag ramos flor milli; fol. slliptico-oborata, breviter acuta, basi rotunsato-truncata, coriacea, as costam eglanisulosa; Ientes simplices, ovato-aperti; pet. eglaniulosi; sup. inermes; stip. virides, auriculis rectis, ped. 2-4, sillosi, 9-11 m. longi; tubus oblongus; sep. pinnulis meviseribus, 1-2-gentatis instructa, acumine integro; styli glabri, giseo conics; cor magna, alba, eciliata; fruct. oflongus, utrinque longe attenuatus.

Hoh. Highania centr. Gierra ge Guxarrama: at El Escorial (N). Lagura).

1858. Creminia seminuida Gogr \&ab. N-2500.

Acculei falcato-ieilatati, as ramos flor. Semiaatos nulli; fol. puarra, obovato-elliptica, utringue subotuniata, a costam colandeilosa; sentes 
$-237--$

simplices, lanceslato- patuli i pet. inf inermes, basi dosprerse glanoulosi, stip. latae, brevsiscular, auriculis parum Divergentitus; pes. 1-3, parcins pilosi, g-12m. longi; tubus anguste oblongus, sep. purnulis angustis, dentatis instructo; styli subglaberrimi, giseo plano; cor. subalba, eciliata; fruct. anguste solongus, utringue attenuatus.

Hab. Houte-Savore, at Sringy (Suget).

1859. Crepinia intenta Googr Gob. N-2501.

Acculer falcato- Dilatati, Defiles, as namos flor. nulli, foll late sboratosubacuta, busi rotuniata, as costam eglandulosa, Dentes simplices, ovato-aperti; pet. aculeati, eglaniulosi; stip. virides, latissimae, auriculis subivergentibus; pet. 2-3, villosi; 6-7 m. longi; tubus oblongus; sep. pinnulis onmibus integris, latis instructa; styli glabri, gisco subplans, cor eciliata; fruct. oblongus, apice aittenuatres.

Hab. Hisnanic bor., as Logroño ('D. Lubia).

1860. Crepinix barcinonensis Gogr \&ab. N-2502.

toculii breves, Dilatato-sublalcati, wiomos Plor nulki; fol elliptico-obtusisscula, basi roturisata, ai costam eglanoulosix; gentes simplices, orato-aperti, pet, sculeati, hine inve glanianbsi, stip. virides, rurriculis at givergentibus; pet. 1-2, iillosi, 10-12 m. longi; tubus oblongus; sep. pinnulis snnibus foliaces-laciniatis instructa, styli glaberrimi, aisco plano; cor. eciriata; fruct. oblongus, utrinque de pressus. Hab. Hispania bor-arient, as Hallvidrera prope Barcelone (9. Sayreda).

t Foliola brovato-sblonga vel oblonga.

\$. Fructus ovoidens.

1861. Grenimia Holubyi Gogr Gob. N-2503.

teculii breves, inchinato- dilatati, as ramos flor. flexuosos nulli; fol. ample oblanga, utringue sed basi prasertion attenuata, glaucescentia, ad costam eglanioulosa; Dentes simplices, cuspinato - triangulares, subconvergentes, pet.inermes, eglaniaulasi; stip.angustae, elongatae, avriculis rectis; per 1-3, sparse filosi, 7-8 m. longi; tubus svoidens; sep. pinnukis le.tinsculis, 2-9sentatis instructa, acumine lato, benticulato; styli glabse; gisco paulo co-

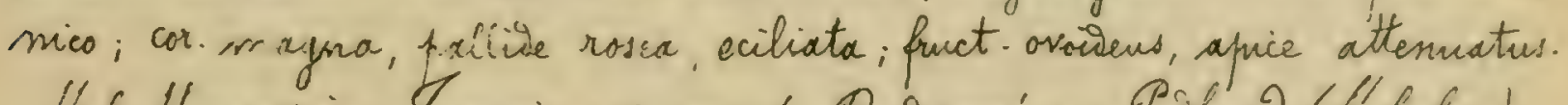

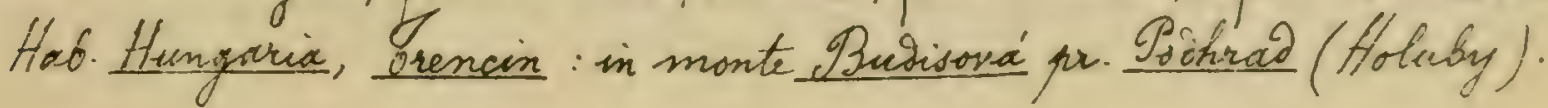


$-238-$

1862. Grepinia fatula Googr Zab. N-2504.

taculei sat Tebiles, a ramos flor. falcato-gilatati ifol. ample oblonga, utringue sensim et parum attenuata, ad costam eglaniulosa; ientes simplices, magni, aperti; pet. eglarioulosi, aculeati; stip. angustac, longae, Tarso pubescentes, auriculis Tivergentibus, per. 1-4, villosi, 13-20 m. longi; tubus oboratus, sep. pinnulis subintegris, brevibus, parum latis, instructa, styli alii glabri, alie assperse pilosuli, gisco paulo comico; cor. ignota; fruct. sat parpus, breviter ovsidens, basi rotuniatus, apuice valie attemuatus.

Hab. Houte-Savie, as Atrenthon (Duget).

1863. Crepinis nemocharis Gogr mss.

Aculei tennes, longi, Vilatati, valde falcati, aid ramos flor panciores; fol. amplissime obovato-oblonga, breviter acuta, basi longe attenuata, pallide viribia, costam eglanoulosa; gentes simplices, late ovato-convergentes; pot. eglanioulose, parce aculeati; stip.longoce, latae, virioses, appice pubescentes, auriculis magnis, givergentibus; pat. 1, paulo villosi, $12 \mathrm{~m}$.longi, tubus obovato-oblongus; sep. pinnulis brevissimis, 3-6-gentatis instructa, acumine foliace- Ventato; styli glabri, Disco plano; car. sat magna, eciliata, fruct. avoidens, utrinque attemuatus.

Hab. Gear, in monte Margè supra foiguines (Jotbert).

$\S \S$. Fructus oflongus.

1864. Creninia remissa Goar Gab. N-2505. - Gogr Herh.ros. N=333. Aculei validi, falcato- Dilatati, ad ramos flor sat copiosi; fol. oblongolanceolata, basi corsata, lucida, ai costam eglanisulosa; ventes simplices; pet-eglaniulosi, aculeati; stip. latiusculae, anriculis givergentibus; pes. 1-3, villosis, meinicres, interium subghaniublosi; tubus sllongus; sep. pinnulis sat angustis, meariocribus instructo, styli glabri, giseo pauls comico; cor. pallise rosea, eciliato, fruct. magnus, oblangus. Hab. Mhone, at Demice' (No. Gantoger).

1865. Crenimia lampophulla Gige Gab. N:2506.

Aculei salive, fatcato-Dilatat, as ramos flor. Debiliores; fol. oblanga, lata, acutiuscula, lucisa, as costam eglaniulosa, Dentes simphices; put. 
$-239-$

parce aculeati, hime inde glanoulasi; stip. latae, auriculis latis, rectis : ped.2-3, villosi; tubus oblongus, hase pubescens; sep. angustries prinata, eglanioulosa; styli glabsi, gises comics; cor. pallide rosea; fruct. sat parrus, anguste ses breviter oblongus, apice attenuatus.

Hab. Rhone, at fornas (M. Ganooger).

1856. Gepinia plagiophylla (Gogr Mos. nov. II. p.27); Gogr 8at. N-2507.Gogar Horb. ros. $X^{\prime}=376$.

It-culei falcato- Silatati, a ramos flor sat copiosi; fol ample oblongoLarceolata, fasi attenuata, atrovirentia, ad costam eglasivilosa; Dentes simphices; pet. inermes, eglanioulosi; stip. sat latar; per. 2-3, pilssi, 11-15 m. longi; tubus anguste oblongus, glaber; sep. pinnulis integris, latis instructa; styli glabri, gices subeonico; cor. subalba, eciliata; fuct. oblongus, basi valie attenuatus.

Hat. Rhone, ad Jornes (No. Ganioger).

1867. Crepinia arimonoyon (Gogr Cossai p. 31 nomen; Gage Ros nor. I,

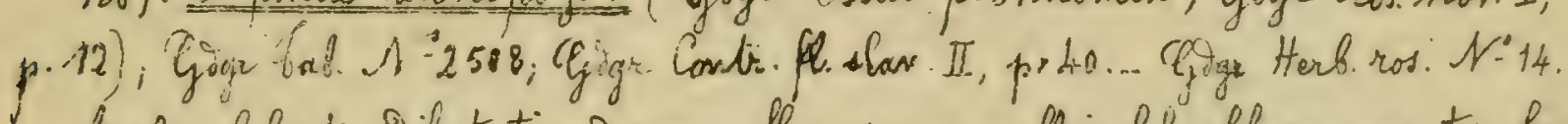
Bculei falcato-Silatati, as ramos flor, saepe nulli; fol. oblongo-acuta, hasi attenuata, at costam eglanivulosa; Dentes simplices, pet eglanioulosi, inermes; pes. 1-3, pilosuli, $17-18 \mathrm{~m}$. longi; tubus oblongus; sep. pinmulis integris, parum gilatatis, Nillosulis instructo; styli subglabic, Disco valie comis; cor. subalbr, meisicris, eciliata; funct. oblongo-subclavatus, basi longe superne. vero freviter attesmatus.

Hab. Prhone, at tornas (No. Ganooger); Croatiox; Dalmatio, Serbix, at Belgrade (Mancie).

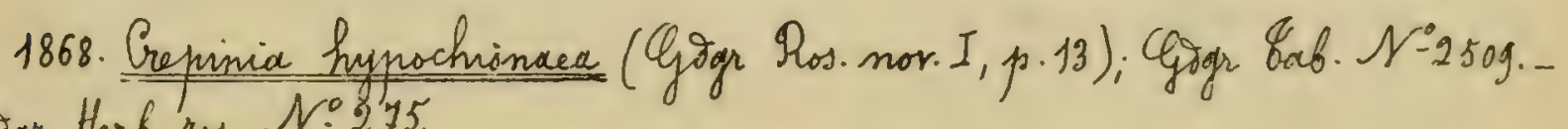
Googr Herb. ros. N:275.

Aculei breviter falcati, Silatato-gecurrentes, amos flor. Plexuosos nulli; fol oblongo-acuta, basi attennata, à costam eglandulosa.; Dentes simplices; pet eglanaulosi, sinersnes; stip. mediceres; pes. 1-2, villosi, 7 -10 m. longi; tubus elongatus; sep. pinnulis integris, latis, villosis instructa; styli paulo villosuli, Disco comico; cor. subalba, aciliata; fruct. magnus, oblongus, utringrive attemuatus.

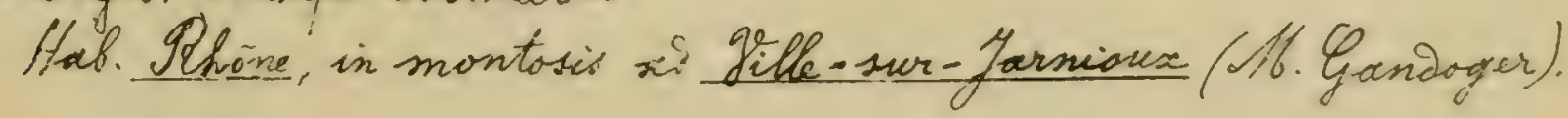


$-240-$

1869. Creminia agricola Gogr Gab. N:2510.

Aculei prarm Silatate, temuiter falcati, a ramos flor. rubellos plerumque nulli; fol. oblonga, utrinque paulation attenuata, atrovirentia, os costam eglanimlosa: Sentes simplices; pet. inermes, glanialosi; stip. latcisculare; pes. 1.3, villosi, 10-13 m. longi; tubus oblongus; sep. pinnulis set latis, parce dentatis instructa, styli subglaberrimi, giseo conico; car. ex albo rosea, eciliata; fruct. oblongus, utrinque attenuatus.

Hab. Rhone, ad tornas (M. Ganooger).

b. Ityli villosi vel lanati.

+ Fructus qlobosus, ovatus vel obovocto-oblongus.

§. Eructus rotuniatus vel ovatus.

1870. Gepinia cycloosenta Goay- Gab. $1: 2511$

Ftuili harvi, Sierissimi, at ramos flor mulli; fol. oborato-subacuta, basi rotuniatia, coriacea, wo costam eglaniulosa; gontes freves, obtuse rotun ixt, inf. biserrati; pet-eglanoulosi, inerms; stip. rubentes, auriculis rectis; neis. 1-3, villosi, 4-5 m. longi; tubus ovatus; sep. firmulis engustis, 1-2-bentatis instructa, acumine frevissinno, integro; styli villosi, gisco valie comio; cor. eciliata; fuct. ovato-subrotundatus, fasi rotuniatus. Hab. Bouches-2u-Khine, at Martioned (Southeman).

1871. Crepinia conglobata Gigr \&ab. N-2513.

tivlie sparsi, guri, falcexto-dilatati, al ramos flor. sacpe nulli; fol. ovata vel elliptica, breviter attenuata, basi rotuniouta, firma, ai costam egloniontsor; grentes implices, recti, pet. oglaniulosi, aculeati; stip.latas, ansiculis subrectis; per.1-2, villosi, 8-10 m. longi; tubus ovatus; sep. ignota; styli panciores, lanati, gisco subplano; cor......., fruct-parvus, rotisndatus.

I'us. Cher, ad Conet frope thenem (Jt. Dideglise).

1872. Crepinix ampla Gögr mos.

thenbi breves, vationli, foleato-gilatati, ad ramos flor geminati; fol. amplissime oborato-oblongr, subacuta, fasi fere roturidata, ab costam non foriniosa; dentes simplices, late svate-convergentcs; prot inermes, basi "glanioulosi, stip. virides, maxinae, auriculis seat givergentibus; 
pet. saepe 4, villosi, 3-6 m. longi; tulus rotuniatur, sep...........; styli lanati, giscs subplans; cor...... fruct. maximus, globosus, haur attemuates. Hab. Hangarix bor., Lipto: a Likenka (2. Michter).

1873. Prepinix Dimorphocarpo Gogr Gab. $\mathcal{N}=2514$.

tbulei at ramos flor. falcato-gilatati; fol obovata vel sborato-oblongr, breviter attemuata, basi corvacta, ai costam eglanioulosa, Dentes simplices, late aperti, pet. eglandulosi, onnes inermes, stip. longae, latiusculos, auriculis givergentibus; pes. 1-3, glabri, 13-15 m. longi; tubus saepins ovoidaus; sep. pirmalis parum latis, Dentatis instructa, acumine lato; styli exserti, sillosi, Disco subflano; cor......; fuet. magnus, plerumare ovatus, basi rotuniactus vel aepressus, apice semper contractus, sed in cosem specimine asunt aligust fructus ovoivei vel etian oblongi.

Hal. Haute-Savoie, at torenthon (Juget).

1874. Crepinia carymbifera (Morch. Hob. p. 319; Rchb. Ft. exc. N-39g8;

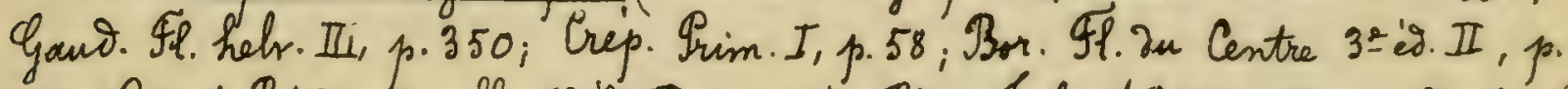
294; Corrist Etive ges fl. 62-iv. II, p.245), Gogr Eah. N-2515; Gigr Contr. fl.

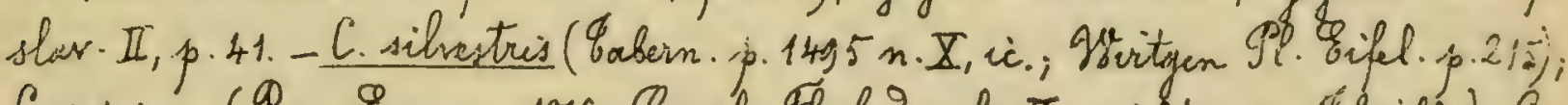
C-sepium (Ran Inum. 1816 ; Gmel. Fl. bas.-als. II, p.424 non 8 himile). C. collina E. silvestris (2umort. Mon. ros. belg. p. 58), C. Tamentosa x canisra (Rchb. Fl. saxon. p. 345; Hirtg. Phein. fl. p. 86, N:701). - Roessig Ros.tab. 27. - Goge Herb. ros. $\mathcal{N}-578$.

Acenlei falcats-Silatati, ar ramos flor. saepius aeminati; fol. ample svato-rotuinata val orficulata, intense viridia; Dentes simphices; pet. aculeati, sporse glangulssi; stip. latal, pubescentes; peos corymbosi, villasi, thour ovoivens; sep. pinmulis Dentatis, latiusculis instructa; stygi villosi, isisco subplano; cor. pallias rosea, fuct. avoidens.

Hab. Euroja media.

1875. Grequinia stephanocalyx Gogr Eab. N-2516.

toculii velidi, faleati, late gilatati, ad ramos flor. copiossi fol. lete elliptica, frevissine attenciata, basi subrotuniata, crassa, atrovirentio, ai costam isterioum paulo glanioulosa; Dentes simplices; pet. eglaniulosi, amnes aculeati, stip. latar; pes. 2-6, villosissinni, sat longi; tubus ovatus; sep. Brevia, prinulis latis, gentatis copiosissime instructa; styli 
$-242-$

hirsuti, 2ises poulo conico; cor. albo-rosea, eciliata, funct subrotuniatus. Hab. Atin, ad It Didier-sur - Chalaronne (Y. Go. Tray).

1876. Creninia virgosa Googr Gos. N-2517.

froulei faicato-gilatati, as ramos flor sat copiosi, fol oforata, utrinque paulation attenuata, ad costarn eglanioulosa; Dentes simplices, aperti, pet. hins inde jlanionlosi, inf inermes, stip. latar, gorso pubescentes, ourriculis givergentibus; ped. 1-3, villosi, $1-2$ cent. longi; tubus svatus; sep. pinmulis latis, ientotis instructa, acumine foliaces; styli villosi, exserti, gisec paulo comis, cor. sorside alba; fruct. parrus, ovatus.

Hab. Phone, at Charbonnieres (Moullu).

1877. Gepinia Luniquistui Giag Gab. N-2518.

Aculei falcato-gilatati, a ramos flor-geminati; fol glaucescentia, olongo-acuta, fasi beriter attienuata, a costam eglanimlosa; ientes simplices, aperti, triangulores; pet. eglanioulosi, eculeati; stip. magnae, longac, eviriculis sivergentibus; fas. 1-2, sparse pilosi, 10-11 m. longi; tubus ovatus, sep. pinnulis brevibus, latis, 1-2-Dentatis instructar acumine

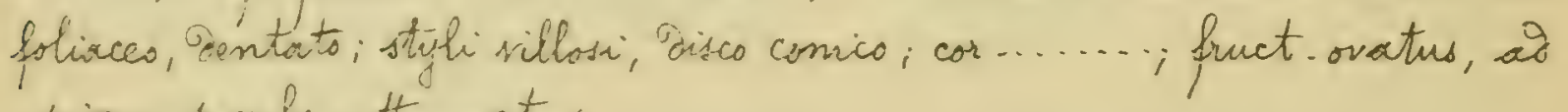
apiam pacho atternatus.

Hob. Inecia, Scania: wo Pinehierna (T. T. Lunipuist).

\$. Fructus ovideus vel sfovato-oblangus.

$\sim$ Foliola basi rotuniata.

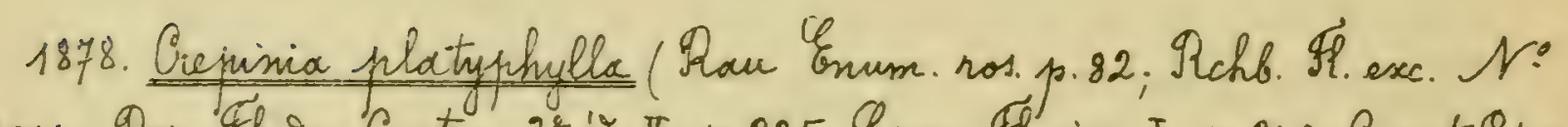

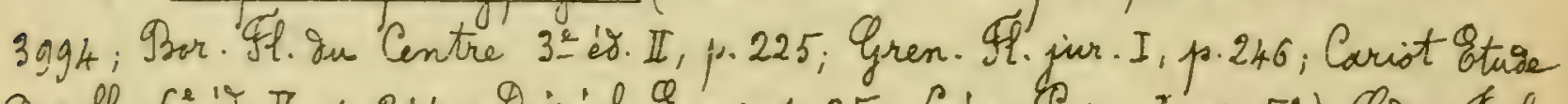

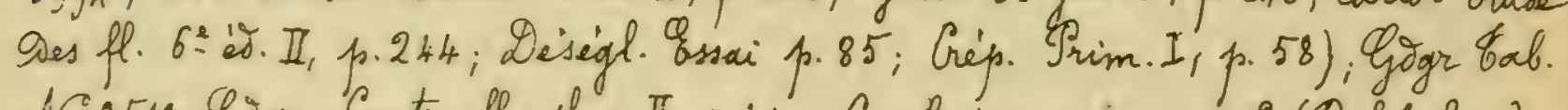

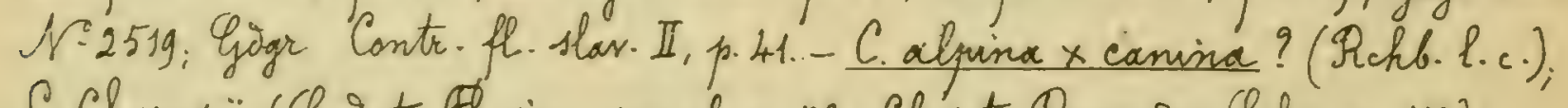
C. Chapusii (Gooet Fl. jur. suppl.p. 73; Christ Tosen oer Ychw. p. 185). C. canina $\theta$. platyshylla (Drimart. Mon.ros. Felg. p. 58; Christ. l.c. p. 184). Billot exs. Nos 1478 at 2261; G3aker herb. ros. N-18? Giger Herb. ros. N: 506 .

Abculer valioi, late foleato-gilatati, fol ample ovato-notundata val orbiculata, subacuta, fasi rotuniata, intense sirivia, Dentes simplices; pet. sparse glanioulosi, aculcati; stip. latae, pubescentes; ped. 2-3, villosi; tubus svoivens; sep. pinnulis latis, gentatis instructa; stagli villosi, disco 
$-243-$

subplans; cor. prallide rosex; fruct. oroidens.

Hab. Europa medix, ete.

1879. Crepinia subpyrensice Gogr Gab. M. 2520.

Fculei breves, falcato-Vilatait, ac ramos flor.mulli; fol obovato-subreuta, basi rotuniata, ad costam eglanderlosa; dentes simplices, ovatoaperti, pet. eglanoulosi, parce aculeati; stip.rirides, auriculis subrectis; poo. 1, villosi, g-10 m. longi; tubus ohovato-oblongess; sep. pinnulis la tis, 1-2-gentatis instructs, acumine foliaces, parce dentato; styli villosi, Discs subplans; cor........; fruct. sbovato-oblongus, apice attenuatus.

Hob. Hispania for., Catalounix: Olot (S. Yayresa).

1880. Gepinia sorbifolix Gogr ofab. p.307 (nomen solum).

ficcie breves, parvi, decurrentes, tenciter et valoe falcati, ac ramss flor. stipulares; fol. amplirescule oflongo-acuta, brsi rotunidata, superne siridi-glauca, at esitam eglanbulosa; Dentes simplices, lats ovatio-convagentes; pet.eglanoulosi, inermes, stip. viribes, longissirna, latue, sorso pu bescentes, auriculis rectis; pies.1-2, villosissimi, 7-8 m. lonofi; tubus obovato-oblongus; sep. primnlis integiris, porrem latis, ut tot acumen, paucioribus, instructa; styh hirsuti, grises paulo camic, cor......, fruct: of avato-oblongus, utrinque vix attomuatus.

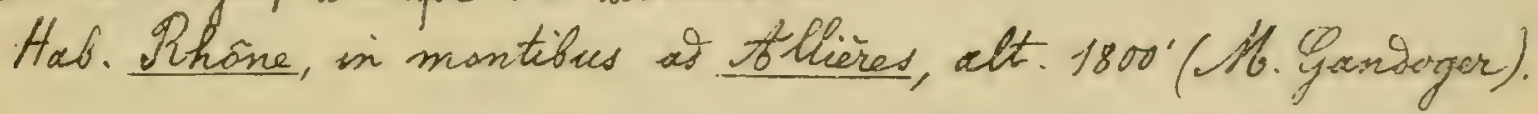

$\sim \sim$ Folisha basi attemeata.

1881. Prepinia pawians Gogr Gab. M-2512.

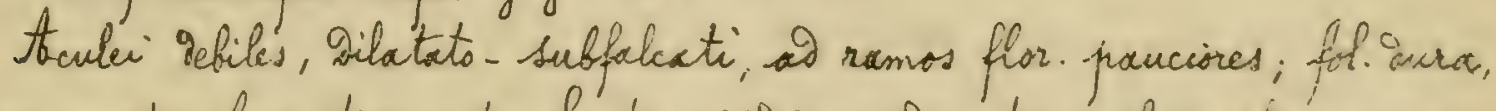
oblang-scuta, fasi trumcata, locte virivio, ad costam glaniausis sima; gentes triengulari subconvergentes, inf. biserrati; fet-aculati, glaniorlosi, inaeque pubescentes; stip. angustase, aurviculis divergentibus; pes-1-2. iillosi, 7-g m. longi; tubus ovato - rotunsatus; sep. pismelis frevious, 3-5-Dentatis instructix, acumine dentats, subfoliaces; styli breses, rilloxi, Sisco plano; cor....... fruct. parvus, subglobosus, fadi rotundactus, aprice. paulo attenviatus, contrali wis oroitso, utrinque contracto.

Had. Helvetia, Hahois: at Bramsis (2. Fastat). 
$-244-$

1882. Crepinia Difficili Gogr \&ab. N:2521.

toculei tenues, parum falcati, id ramos flor pauciores, fol oblongoattenuata, basi cuncate, laete et nitide siridia, as costam eglansulssa; Sentes simplices; pet-aculeati, eglanoulosi, breviter pubescentes; stip. angustac; ped-1-3, villosi, mediocres; tubus ovoiseus; sep. vireseentia, pinnulis set angustis instructa; styli sillosi; Diseo conico; cor. pallice rosea; fruct ovoideres.

Hab. Tlhöne, ad Trnas (M. Ganooger).

1883. Crepinia pachyphylla Gogr Gab. N-2522.

Arculei validi, Decurrentes, falcats - Silatati, as ramos flor.folioss sat copiosi; fol amplissime oblongo-rhomboidalic, utrinque breviter attenuato, atrovirentia, crassa, coriacca, as costam eglansulasa; Dentes simplices; pet. eglas aulosi, inf.inermes, stip. latae, auriculis bivergentibus; pes. 1-4, villosi, 5-12 m.longi; tubus sbovatus, sep. pirnulis latis, gentatis instructo; styli maymi, hirsuti, gisco comics; cor........; fruct. breviter ovoidens, utrinque valde attemuatus.

Hab. Savoie, at Gessons prope Moutiers (Muget).

1884. Crepinia Papillonii (Gogr Ros.nov. I, p. 13); Gogr Eab. N-2523.Gigir Herb. ros. N: 70 .

Acculei à ramos flor. subglaucos falcato-gilatati, fol. suboblonga, acuta, basi attesnuata, obscure mirisia, nervosa, rigitiuscula, as costam parcissime glaviaulosa; Dentes simplices; pet. inermes, eglanaclosi; stip. mediacres, auriculis rectis; ped-1-3, breves, villosi; tubus oroidens; sep. oglanisulsis, sat brevibus instructa, styli subhirsuti, gisco plans; cor. palliore rosea, eciliate; fruct. parrus, ovoidens.

Hab. Rhone at tornas (H. Ganooger).

1885. Crepinia Ge Gllarsiana Gogr Eab. N-2524.

Aculei temes, elongati, falcati, or ramos flor sat copiosi; fol. obovato-oblonga, acutius-cula, basi sensin attenuata, as costam non glominlosa; Dentes simplices, lanceslato-convergentes; pet eglaniulosi, aculeati; stip. virivies, aurieulis rectis; pes. 1-2, villosi, 8-10 m. longi; tubus frovxito-blongess, sep. pinnulis latis, 2-3-rentatis instructa; ccumine folimes- subsentato; styli villosi, Disco subplano, cor eciliata, 
$-245-$

fruct. oborato-oblangrus, utringue attenuatus.

Hab. Basses-Afyes, of La Condamine (Trool).

1886. Crepinia lampocoeca Gogr Gab. N:2525.

Acculer falcato-2ilatati, ar ramos flor. sat copiassi fol. oblonga, utrinque poulation attenuata, as costam eglanoulosa; Dentes simplices; pet. eglaniolosi, inf.inermes, stip. virises; pet.2-3, villosi; 8-14 m. longi; tubus sbovatus; sep. prinnulis laticisculis, proulo dentatis parce instructa, styli lanate, Disco plano, cor......... fruct. ample ovoitens, nitious, fasi rotumiatus, centrali excepto.

Hab. Ihoine, as Ecully (IP. Chabert).

1887. Bresinia albioula Gogr mss.

trarler breves, vise dilatati, tenuissime folleati, al ramss flor. con piosi; fol-ample oblongo-acuta, basi attenuata, subtus albida et as costam -glasiaulosa; dentes simplices, late ovats - convergentes; pet eglanqulasi; aculeati; stip. virides, lator, breves, auriaulis givergentibus; pes. 1-2, basi pubeseentes, 12-14 m. longi; tubus obovato- ollongus; sep. ignota; styli lanati, gisco plano; cor......., fruct. magnnes, poovatcoblongus, utrinque attemuatus, medis turgidus, apice strangulatus.

Hob. Fransilvania, Gorda torampos: ai Forda Hasadék (2. Richter).

it Fructus oblongus.

\$. Diseus planus.

$\sim$ Rami florales inermes.

1888. Orepinia trumeatula Gơgr Gob. N-2526. - Gogr Herb.ros. N-21. Foculei panciores, faleato- Vilatati, ai ramos flot plorumgue mulli; fol-obovata, breviter acuta, basi rotuniata, firma, as costam parce glanoulosa; Sentes simplices, patuli; pot. sparse glanoulosi, paulo aculeati; stip. angustac, rubentes; peet 1-3, villosi; 5-10 m. longi; tubus Slongus; sep........, styli lanati, giseo subplano; cor........; fruct. slongus, friss roturisatus, apice valde attemiatus, centrali autem utrinque contractus.

Hab. Rhane, at tomas (No. Ganooger). 
$-246-$

1889. Crepinia Dalmatice Gisgr bab. N.2527, Goger Contr. Hl. slav. II, p. 41. Acculer inchinats-2ilatati, at ramos flor.nulli; fol ample oblangor, utrinque subrotunoata, ad costam eglanoulosa; acntis simplices, oratospertic; pet-inermes, eglasioulosi; stip. Oorso pubescentes, curiculis rectis; ped. 1-3, graciles, tenviter villosi, 12-14 m. longi; tubus oflangus; sep.pinnulis latiusculis, brevibus, 1-2-Dentatis parce instructar; styh mediscriter pubes. centes, gisco subplano; cor.rosed, eciliata; funct.maximus, oblongus, basi longissime gecurrens.

Hob. Dalmatia. (Pchalch).

$\sim \sim$ Rami florales aculecti.

1890. Crepinia infundibuliformis Gogregr Eab. N:2528. Gogr Herb.ros. N.257. toculei robusti, wi ramos flor. falcato- dilatati, fol. late oboratoc vel elliptica, crasso, firma, at costam eylanoulosa; ientes simplices, lati, convergentas; pet. eglandulosi, parce aculeati; stip. pracsertisn superiores latissimar, auriculis rectis; pes. 2-3, sparse villose; longrisculi; tubus sllangers; sep. pirmmlis pubescentibus, latis instructa, styli hirsuti, aisco plano; cor. rosea; fruct. ample oblongus, turbinato-infundibuliformis, basi imprimis attemuatus.

Hab. Ithone, at Dencié (No. Ganooger).

1891. Cremini bifornis Gagr Gab. N-2529. Giger Herb. ros. N-330.

toculei debiles', tenues, valve falcati, as ramos flor. flexuosos Dilatati; Fl. elliptico-lanceslata, ad costam eglandulasa; dentas simphies; pit. eglanimbsi, inaeque pubescentes, Dein laxe villosi, salie acreleati, stip. angustae, aurciculis girergentes; pet. 1-3, villosi; 3-5 m.longi; tubus of longus; sep. pimmulis elangectis, latis instructa; sty i villosi, disco plano; cor-rosed; puct. ollongus, angustus.

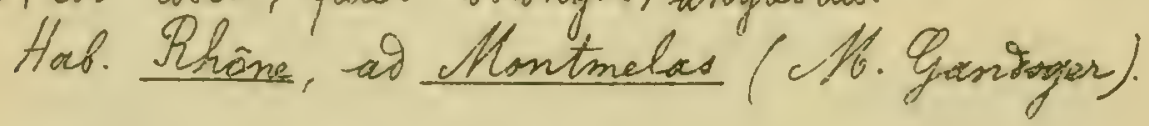

18g2. Gepinia Derispina Gogr mss.

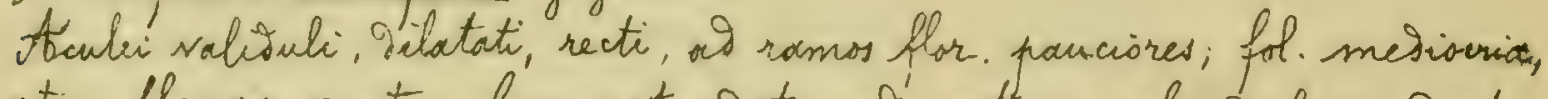
shrsetor oblonga, acuta, fasi rotuniata, wo costam eglonoulosa; dentes inf. biserrati, ovato convergentes; pet siferme subglananlosi, aculeati, stip. virives, latar, breves, auriculis subiavergertitus; pes-1-2, inferne rillosi, 
$-247-$

7-8 m. Longi; tubus oflongus; sep.........., styli lanati, gises subplano; cor.......; fruct. Slongus, basi aepressus, aprice longe attennatus.

Hab. Eranssitwania, Kolngs: as Magyar o Kereke (2. Michter).

1893. Creteinia vichs (Disegh at Mip.) in Giogr Gab. N-2530.

toculei à ramos flor. flexuosos falcato-dilatacti fol amplissime ovato-acuta, as costam eglanioulasa; Dentes simplices; pet. eglasioulosi, fere -onnes inermes; stip. glabrae, auriculis bivergentibus; pes. 2-3, elongati, spar se pilosi; tubus elongatus; sep. lote puinata; styli hirsuti, grisco plano; con pallide rosea, funct alongatus, basi vatio attennatus. Hab. Cher, in silva gienzon prope grieilfont (to. Desighise).

1894. Grepinia emoenipes Gogr bas. N-2531.

toculei robusti, falcato-dilatati, ai ramos flor.copiosissioni; fol. late rbovato-oblonga, acuminata, basi sensim attenuata, os costarn eglanorulosar; Dentes simphices; pet. eglaniulosi; aculeati; stip. Sulat tatac; peo. Dense corymbosi, villosissioni, 8-13 m.longi; tubus oblongus, basi pubescens; sep.brevia, pinmalis angustis, pruciorifus instructa; styli magni, lanati, gises subilano, cor resea, eciliata, fruct oblonges, utrinque atton.

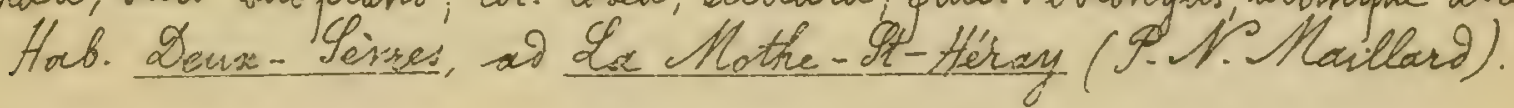

1895. Crepinia lencoacanthax (Debeaws Mater. sos. Tyren, 1878, p.29). Gogor

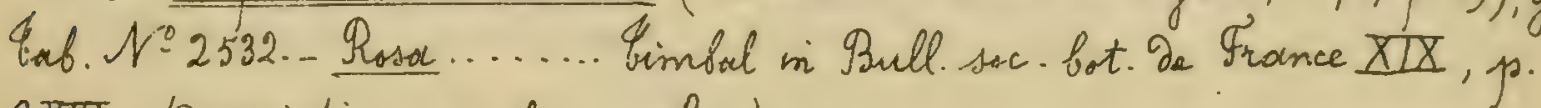
CXXI. (Descriptiones ambac maloce).

Tculei subovilatati, elongati, temmiter falcati, ad ramos flar. rubros copiose, albiar; fol oborato-oblonga, acutinscula, basi sensim atternuata, as costam eglanioulosa; Dentes simplices, triangulari-sulconvergentes; pet. sculeati, hinc inve ylandulosi, stip. latae, awriculis subrectis; pes. 1-2, siflosi, 6-8m. longi, tubus clongatus, sep. crecta, eglanoulosa, pismulis integris, latiusenlis instructa, acurnine subfolixces; styli lanati, Dises flans; cor........ finct-maximus, oblongus, utringue sat attemuatus, Hoch. Pyrinés Qrientales, prope Mont-Louis ( Mimbal; S. Seallet). Q copia speciminorum of amico Debeaus acceptorum, species ad Crepinias nec ad Augetios (Gomentoseac), it bicit auctor, exsidenter fortinet. Insuper, Descriptiones Simbah et Dabexuaci sunt nimis incompletoe, et ut ita dicam, falsac xec pessimas. 
$-248$

§\$. Diocus plus minusse comicus.

s. Tami florales inermes.

1896. Grevimia histines Gogr Eas. $N=2533$.

teculei falcato- Dilatati, ad ramos flor. mulli; fol late obovato-oblonga vel oflanga, utrinque subrotunixta, as costam eglaniulosa; Dentes simplices, recti, pet. inermes, eglaniulosi; stip. latre, longrisculac, auriculi: Divergentibur; ped. 1-9, villosi; $14-22 \mathrm{~m}$. longi; tubus elongatus, quice contractus; sep. Congatas, pinnulis latis, pubescentibus, parce gentatis instructa ; styli hirsuti, diseo conico, car ......., fruct. oblongus, basi gapressus, apice atten.

Hab. Houte-Saroie, at trenthon (Buget).

1897. Crepinia lentostephana Gogr Bab. N:2534. - Billot ass. N-358g bis. Acculci debiles, falcato-gilateti, ad ramos flor.mulh; fol anguste obosato-oblonga, utrinque attenuata, do costam eglanoulssa; gentes simplicas, recti; pat. inermes, eglanbulosi; stip. angustae, auriculis parum givergenAbus, par. 1-2, villosi, 4-8m. longi; tubus oblongus; sep. brevissimae, pinnulis integris, angustis instructe, styli villosi, Disco tat conico; cor-pallive rosea; fruct. anguste oblongus, basi prosertion sttenuatur.

Hob! Thane, at Kassin (Moulle).

Inecirnina florifera ejustem schedulae of stylos glabros, peinnculisome sisperse pilsso-glanoulosis, alteram speciem constitumt.

$\sim \sim$ Rami floriferi áculeati.

1898. Crewinia vejrecularum Gogr Tab. N-2535. - Gogre Herb. ros. $\mathcal{N}_{184 .}$ Acculei robuste, falcato-silatati, as ramos flor-copiosi; fol obovatooblonga, acenta, basi paulation rotuñata, ab costam eglanaulosa; gentes simplices; pet. egfandulosi, annes inermes; stip. measiscres; pet. 1-3, sillosi, 7-11 m. lingi; tukes oflongres; sep........ stoghi firsuti, Gisco comio,

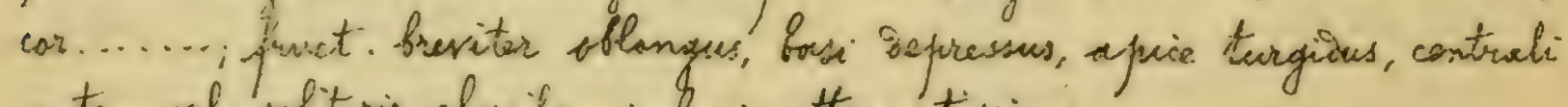
autem vel solitaris Clanformi, basi attencatissimo.

Hab. Tikene, id Ttrmas (M. Ganoger).

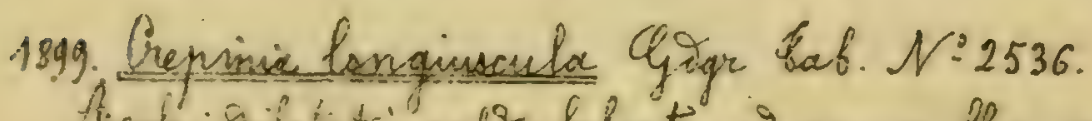

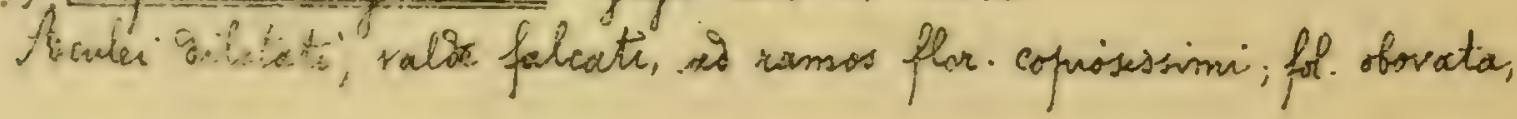


$-249-$

utrinque subrotusinata, sid costam eglaniulosa, dentes simplises, recte;

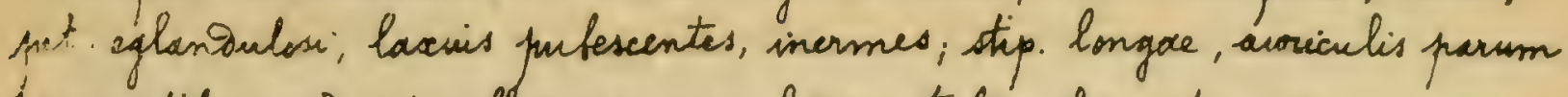
Givergentibus; pers. 1-4, villasi; $14-19 \mathrm{~m}$. longi; tubus elongatus, sep......... singli hirsuti, Sisco valoe conics, cor........ funct elongatus, utrinque valge attenuactus.

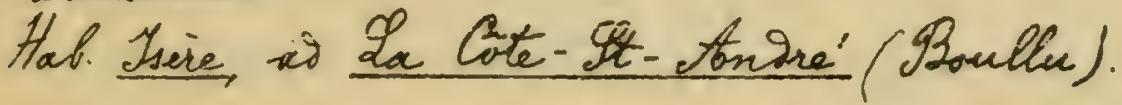

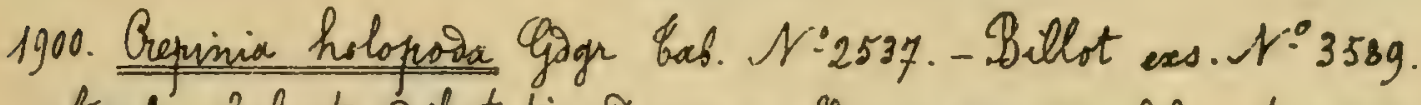
toculei falcato-gilatati, ao ramos flor pranciones, fol. sat prowse, obovata, utrinque roturiata, wi costam eglaniaulasa; Dentes simplices, necte; pet-eglanauloss; inermes, otip. latae, Longae, inf subialatatoe, auriculis vivergentibus; preq. 1-3, glabui, 11-21 m-longi; tubus oblor qus; sep. longa,

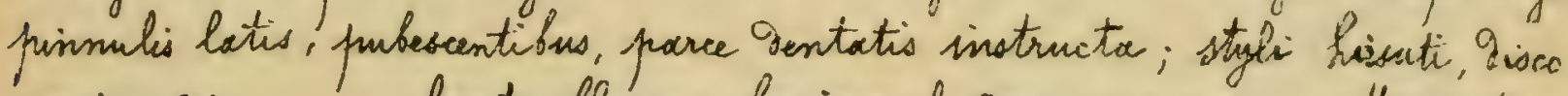

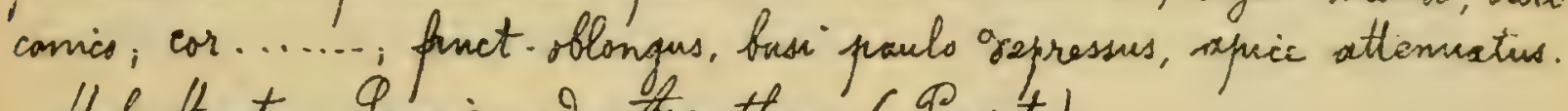
Hab. Haute. Saxrie, of Frenthon (Peuget).

1901. Crepimia anrite Gogre Sab. N-2538.

to-celei Pebiles, falcato-subiliatati, ad ramos flor. sat copriss; fol. Bete

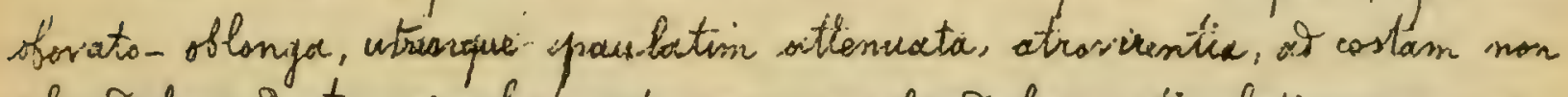
olanoulosa, sentes simphices; pet inermes, eglanioulosi; stip. latissimar, peod. 2-3, villosi, 13-18 m. longi; t tubus oblongues; sep. styki villosi. Sis-co paulo comic; cor....... funct magnus, oblengus, utringue attennaties. Hoo. IRkane, at thix (16. Ganooger).

1902. Crepinia basilacensis Gagre Gab. N-253g.

F culei gilatati, valde faleati, a ramos flor plus minus copiosi; fol. obovato-solanga, utrinque breviter attenuato, ay costam eglanionlosa, intense viridia, Dentes simplices, lanceolato - aperti, pet. inermes, eglanoulosi, stip. longae, aurriculis inergentibus; pes. 2-4, molliter villosi, 14-16 - m. longi, tubus oblongus; sep. pinnulis angustis, eglanoulosis instructa, acumnine angusto, styli villosi, visco paulo conico; cor-pallide nosea, eciliator, fruct. ollongus, basi longe decurrens, apice attenuatus.

Hab. Helvetix, prope Bêle (H. Chriot).

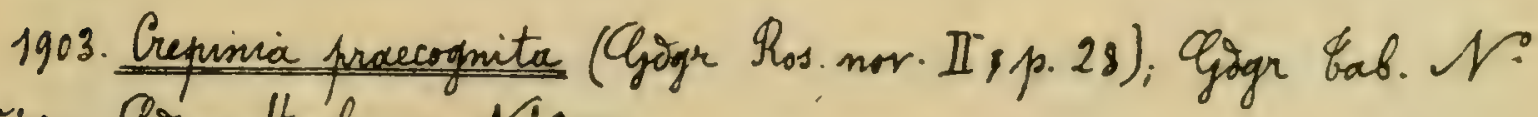
2540. - Goger Herb. nos. N-302. 
$-250-$

tocilci robusti, a ramos flor. Denvidatos falcato- Dilatati; fol anguste oblongo-acuta, basi attemata, nervosa, at costam eglanivulosa; gentes simplices; pet-inermes, eglanoulosi; stip. latiuseulac, auriculis sont givergentibus; pes. 1-4, villasi, mediacres; tubus oblangus; sep. pinmulis latis, onsmibus foliaces. Dentatis coprise instructa; styli villosi, pisco comico; cor parva, soviste alba, eciliata; fruct. magnus, bresiter oblangus.

Hab. Chane, ai Itlix (H. Ganooger).

1904. Grepinia parsimilis (Goor Ros. nor. I, p. 13); Gogr. Eab. N:2541. Goger Herf. ros. N-287.

Acenlei validi, aure; falcato-rilatate, ai ramos flor farinosos copiosi; fol. oblongo-acuta, basi rotuniata, saturate viridi- subcoerulea, as costam sparse glaniulose; Sentes simphices; pet. eglanoulosi, fre inormes; stip. latae, auriculis rectis; per. 2-4, villose; sat longi; tubus oblongus; sep. piomulis

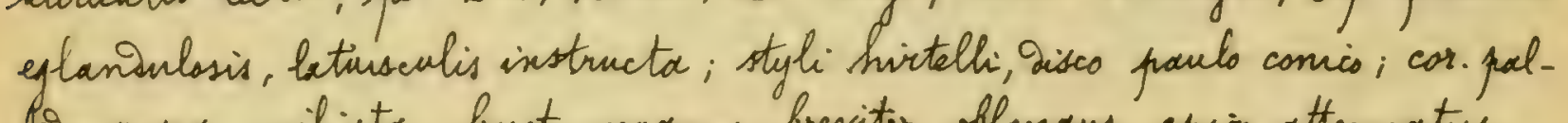
live rosea, eciliata; fuct. magmus, breviter oblangus, apiae attemuatics. Hab. Thone, Nontmelas (No. Ganooger).

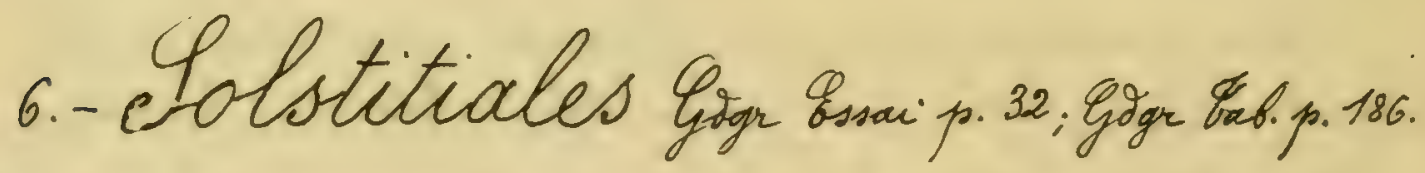

Folishi, stiam abultis, utrinque dense et mollitar hiroutis rel subtomentosis, simpliciter (raro bi-) serratis; petiolis filerumque tomentosis. - Focis fere Sigetionum (Bomentoseae), seo pecumenli et sepala lovia, nee normaliter glanoulosa vel hispiaa.

Peimmenti glafri

1 Sedunculi villosi vel parcissine glanoulosi Fructus globesus

Gructus rotumiaters

Fructus ovato- sotuniatus

$2\{$ Fructis svatus

Fructus avoivens vel sorato oblangus

Fructus oblongus

Fohiola obovato, obovato-oblonge vel ollonga

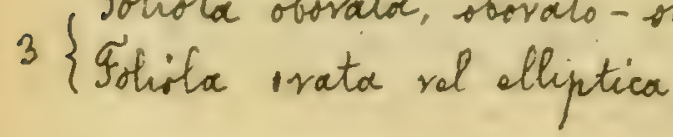

2

22

3

5

6

7

8

16

4

Ypecies $19605-4906$. 
Foliola basi attenuata

4 Foliola basi notuniata

Foliola biserrata

5 F Foliola simpliciter serrata

Mami floriferi inermes

6 Trami florifri aculeati Discus comicus

7 Discus planus Foliola basi rotuniata

8 Goliola basi attumuata Styli lanati

$9\left\{\begin{array}{l}\text { Ityli glabri vel cosperse hirtelli } \\ \text { Gtyli }\end{array}\right.$ Ttyli pubescentes 10 $\left\{\begin{array}{l}\text { Rami floriferi aculeati } \\ \text { Rami floriferi inermes }\end{array}\right.$ Petioli onnes aculeati $11\left\{\begin{array}{l}\text { Petioli subinermes } \\ \text { Setioli anves inermes }\end{array}\right.$ Foliola oforato - oblonga

12\{ Foliola obovata vel oflonga

Getioli annes inermes

Getioh omnes acuíereti

13

Petioli inf-aculeati

Getiohi sup. aculeati

Stali lanati

$14\{$ Gtyli slabri, sparse pilasi, vel villosi Folioba obovata val sbovato-oblonga 15 \{ Goliola oflonga

Strli paulo rillosi

Gty pi hiss

16 \{

Styli hirsuti

Styli lanati

Ptyli glabri aut subolaberrim

Isliola basi rotuniata

Foliola annia simphiciter serrata

18 Folicha inaque vel annia biserrata

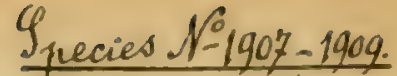

$\varphi_{\text {precies }} N_{0} 1910-1915$

$y_{\text {precics }} \mathcal{N}-19161917$.

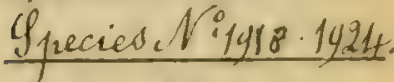

Q inecied $t_{0}$ 1925-1926

$g_{\text {pucies }}^{\prime} N^{\prime} 1927-1935$

9 frecies $\mathcal{N}-1936-1439$

grecies $\mathcal{N}^{\circ} 1940-1949$.

Inecies $N-1950-1957$ 9zecied No1953-1965.

Species N:1966-1971.

12

$\varphi_{\text {pecies }} N^{0}-1972-1973$.

9pecies $\mathscr{N}^{\circ} 1974-1981$.

Grecies No1982-198h.

Grecies N-1985-1993.

14

Yrecies $N^{\circ}$;016-2028.

Species N:19gh-19g5.

Grecies $N$-1996-2000.

Grecies N-2001-2004.

Quecies N:2005-2010. Sprecies N $92011-2015$.

$\varphi_{\text {pecies }} N_{0.9051-2060}$

Yhecies N-9029-203k. 9 pecies N-8035. 2062 Yrecies No2043-2050.

Yprecies N-2061-2066. 
$19\left\{\begin{array}{l}\text { Rami floriferi aculeati } \\ \text { Rami floriferi inermes }\end{array}\right.$

Inecios N:2067-2076

21

$20\{$ Setioli annes aculeati

Foliola basi rotuniata

21 Foliola basi attenuata

Fructur vivieus rel sovato-oblongus

Sructis oblongus

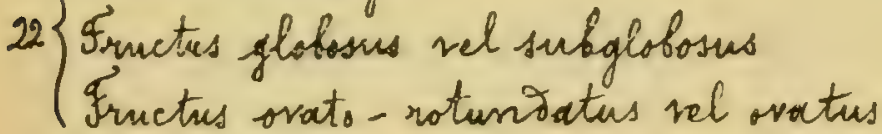

Qtyli glabui vel sparse pilosi

23 Styki villosi

Styli lanate

Ityli glabri, glabrescentes sel pauto hirsuti

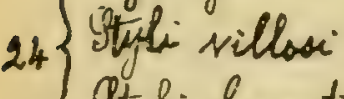

Ityli lanati

Species N. 20\%-2081.

Spevies N. 2082-2087.

Precies $N^{0}$ 2088-2092.

$2 \%$

24

Sprcies N.2093-2095.

Irecies N.2090-9106.

grecies No210\%-2113.

Grecies N.2114-2118.

Grecies No 2119-2128.

Enecies N. 2129-2137.

Inecres No 2138-2144.

Ipresies N.2145-2157

1. Pesnnculi glabri.

a. Fructus globosus.

+ Foliola ovata vel elliptica.

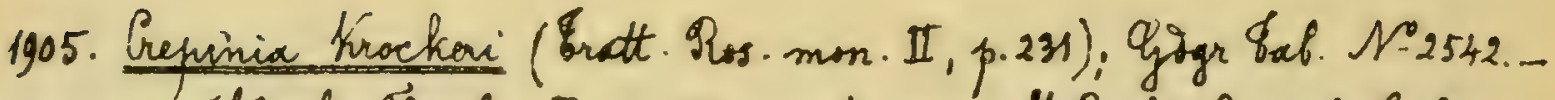

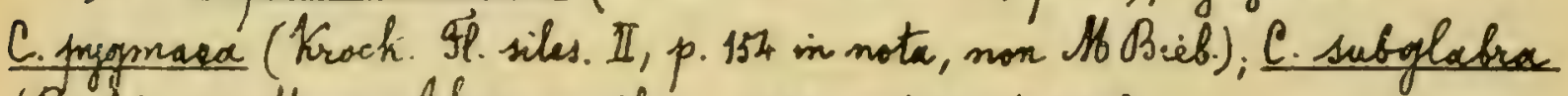
(Borbais in 1lagy. fikad. mathem. 118q. p. 426 et 435).

Nana, prorsus inermis; fol. misucta, elliptics-oftusa, supra livita, sessilia, base integra; Dentes simplices, pet breves, villosi; stip. Dilatatae, rubentes, villow, genticulatae; pes. 1, glabri; tubus laevis, ovato-rotungatur; sep. cinerea, Darso prurpurea, pinnata; styli villosi; car. rosea, calycem nic superans; funct. subglobosus.

Hab. Silesic (Krocter): thengaria (Borbais).

Erecies curiosissina, hucusqua male cogrita.

Ylosa pygmaea M. Bieh. At. taur.-caue. I, p. 393; Ggar Mon.ros. $1, p .183$, as Eurosas (Gallicinear) pertiret.

1906. Gepinia coriifolia (Fries Novit. fl. suec. ed.12,1814, p. 33, D.C. Protr. II, p. 623; Gureng. Iyst.II, p. 554, Rckb. Fl. exc. N-4014; Hartm. Ikand. fl.

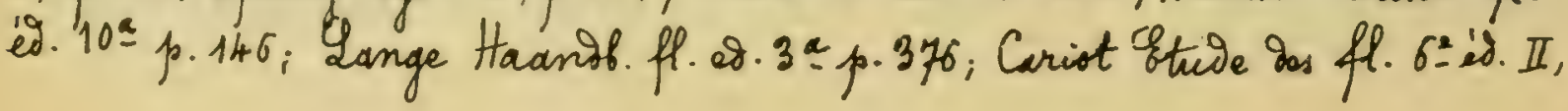


p.245; Desigh. Essai p. 86; Crejp. Mrim. I. p. 57, Dumart. Mon. ros. belg. p. 61; Schent Ykano.ros. p. 29); Googr Eab. N.2543. C. curvula (Fimbal mss. 1864).

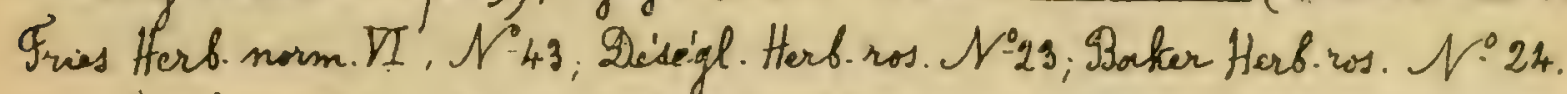

Aculei valide; falcate-Silatati, fol. ovato-acuta, cariacea, nersosa,

gentes subbiserrati; pet. inermes, eglantulosi; stip. valde gilatatice, villosas, pe's ylabu; breves; tubus subotunsatis, sep. erecta, sie parsistentia, pinsulis latis, instructo, styli lanati; car. rosea; fruct. subglobosus.

Hab. Cuecia, etc.

Hucuaque parum nota esset, cam frequenter of C.fuctotorum. (Bess.) sal potiis C. 2umetorum (Ohiill.) furmiseta.

\section{T† Foliola obovata, obovato-oblonga vel oblonga.}

\$. Foliola basi attenuata.

\section{Crepinia crassifolia (Hallm. in Eiljebl. Wrer. FP. III, p. 268); Gogr Gab. N-2544.}

Aculei mèricres, folcato-gilatati, as ramos flor. sparsi, fol oborata, utringue attermata, coriacex, opaer; sentes inaquales; pet. inermes; stip.

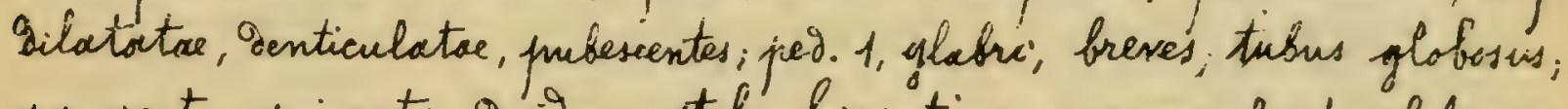
seps erecta, pinnate, geciove, styh hirsuti, con.carnex, fruct. globosus.

Hob. Serecia.

1908. Crepinix cristulata Goor mss.

focutei tenmes, subrilatati, tenuiter faleati, as ramos flor gesminati; fol. parsa, obovato-blonge, utrinque breviter attenuata, as costam eglanaulosa; gentes wato-convergentes, inf. biserrati; pet, inermes, basi paulo glangulosi; stip. Lactac, vivides, breves, auriculis givergentibus; ped.1. glabri, $8 \mathrm{~m}$. longi; tubus rotundatus; sep........ styli lanati, hisco plano; cor........; fruct. subglobosus, utringue notunaatus.

Hab. Eranssilvania, Syolnok Dobkas: Deces (2. Richter).

190g. Grepinia 2epressa Ggar Gab. N.2545.

Acrei robusti, falcato- Dilatati, as ramos flar. 2-3-ti; fol. sat late obovata, reutiuscula, basi breviter atteruata, glaucescentia, at costam eglangulssa, Dentes simplices, lanceslato-perti, pet. hine inde glansulosi, parce aculeati; stip. magnae, longae, auriculis divergentibus; pes. 2-3, 
$-254-$

glabri, g-10 m. longi; tubus rotuniatus; sep.erecta, finmulis integris, latirisculis parce instructa, acumine sat aigusto; styli magni, lanacte, Disco plans; cor......., fruct.magnus, glaboso-Depressus, base umbilicatus, apice fireviter attenuatus.

Hab. Inecia, destragothic: in mante himeh sle od Rustsacker et inter Blomberg et glinosater (7. \%. Letterstest).

§. Foliola basi rotunaata.

1910. Brepinia campicala (Gogr in Mull. sac. Murith.1870, p. 33). Gogor Gab. N:2546. - C. corifolia f. oblonga (Christ Rosen ver Ychweiz p.191). taculei dilatati, valde falcati, ad ramos flor copiosi; fol ample oblonga, utrinque sensim subrotuniata, nervosa, as costam eglaniulosa; sentes simphies, orato - convergentes; pat. inferne paulo ylaniaulosi; inermes; stip.magnae, subtus pubescentes, auriculis breribus, givaricatis. ped. 1-3, glabri; 15-16 m. longi; tubur onatus; sesp. erecta, pisnulis foliaceis, granoulis paucioribus at margines instructa; stýli lanate, aisco plans; cor. alborosea, eciliata; fruct.magnus, globosus.

Hob. Helvetia, galais: as Mayens ge Sion (G. O. Holf).

1911. Crepinia neurophylla Gagr mss.

Acculei breves, parvi, folcato-silatati, as ramos flor copiosi; fol. ample oblongo-acuta, basi rotandata, atrovirentia, framincle narsosa, ao costam eglandulosa; Dentes simplices, breves, ovato-convergentes; pet. eglanimlosi, inermes, auriculis rectis; pes. 2-3, glabri, 3-5 m. longi; tubus rotundatus, sep. pinmelis smnibus integris, latis instructa; styli lanat, Dises flano; cor.........., fruet. maxisnus, globoso-comius, basi Dilatatus.

Hab. Hungaria bor., Liptó. I Orada (2. Richter).

1912. Crepinia coronata Gogr \&ab. N-2547.

Abeulei falcato-vilatati, at ramos flor. panciores; fol late sbovato - subattenuata, basi paulo sotuniata, at costan eglandulosa; ientes simplices, lanceolato - convergentes; pet sparss glansulosi, parce eculeati; stip.magnae, latoe, auriculis Iivergentibus; pet-1-3, glabri, 7-10 m. longi; tubus globosus; sep oreci. pinmulis latis, 1-3-gentatis instructa, acumine tongt, 
$-255-$

ample foliaceo-laciniati, styli magni, lanati, Disco plano; con........; pruct. globosus, hain attemiatus.

Hab. Hehetia, galais: ad Herbriggen (G. O. Wolf).

1913. Gepinia pruinosa (Maker Review p. 27; gj. Mon. but. ros. in Simn. journ. II. p.230, non Linst nec Halle.). Gogr bab. N-2548. C. Caesix (Borrer Brit. He ed. $3 x$ p.242 ex parte).

toculei Dilatati, parum folcatio; fol obovato-atternuate, basi rotungrata, priminosa, à costam glaniulosa; rentes biserrati; pet. sparse glania Cosi, parum aculeati; stip. ciliatae, subtus pubescentes; pes.1-3, glabri; tubus subglobosus; sep. ciliata, pinnata, Deciana; styli hirsuti; cor-rosea; funct. subglabosus.

Hab. Anglia.

Rosa puinosa Lingl. Mos, e Sifiria et Mosa pruinosa Hellr.

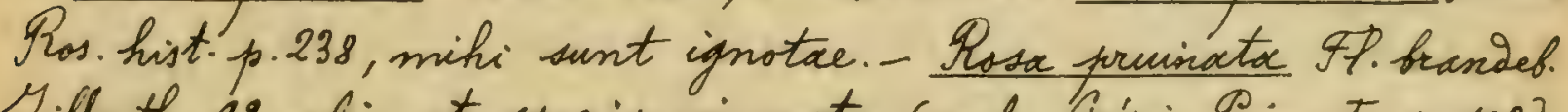

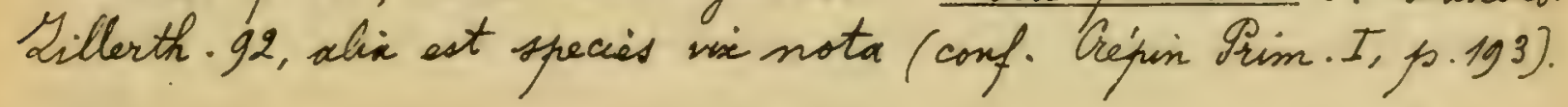

1914. Crepimia privinoviens Goger Eab. N=2549.

Truno-rirens, culei Debiles, parum foleato-Pilatati, as ramos flor. geminati, fol. late obovato-oblonga, vix acuta, basi rotuniosta, acssa, is costarn eglaniulosa; jentes simplices, svato-perti; pet-eglaniulosi; subinermes; stip. maximae, virides, auriculis farum girargentitus; ped. 2-3, glabri; 6-8m. longi; tubus rotindatus; sep. erecta, pinnulis integarimis, pancioribus, smmifus foliaceis instructa; styli albo-lanati, dises plano; cor. parve, interse rosea, fruct. subglobosus, utringue rotuniatus. Hob. Gyrol in monte Gasone prope Brente (G. Gelmi).

1915. Grepinia platycarpa Gogre Gab. N:2550.

Aculei validi, falcato- Dilatcti, à ramos flor. 3-4-mi; fol. oblongscuta, basi cordata, lacte visioi-glaucescentix, ad costam eglansulosa; Dentes simphices; pet. exlanoulosi, subinermes, stip. mesiacres, auriculis di: vergentibus; ped. 1-2, glabri, $5-7 \mathrm{~m}$.longi; trbus rotuniatus; sep. erecta, Dorss set copiose glandulaso-muricata, subintegra, pirmulis filformisus, integris instructa, cusnine lato, stigh lanati, aiseo plans; cor.......; funct. maximus, globoso-ge pressus, hair attenuatus.

Hab. Inecia. Gestrogothis, in monte finnehulle; inter \$slomberg. et Heinisater (\%. \&. Letterotegt). 
$-256-$

f. Iructus rotunsatud.

f Foliola biserrata.

1916. Crepini sambuciblix Gogr Lal. $N: 2551$.

Abculer falcato - Dilatati, as ramos flor. phus minus re copiosi; fol ample oblong-acuta, fasi subattenusta, tenniri al costam eglanioulasa; dentes oratoaperti, ommes fiserrato-glanaulosi; pet. irsermes, inferne pacelo glansulasi; stip. sirides, subtus valde tomentosoc, auricuhis subiverger.tibus; ped.1, glabri, 5-7m. longi; tubus rotungatus; sep. prisnulis integris, pancioribus, angustis instructa, dcumine integro, lato, styli lonati, gisco ilano; cor. eciliacta; funct. notuniatus, apice paulo attenuatus.

Hab. Silenia, as Goerbersiorf (Mtroekleir).

1917. Crepinia cribrosa Gogr Eab. $\mathcal{N}=2552$.

Fculi falcato - Dilatati, at ramos flor. copiosi; fol angur to onga, utrinque attenuato-acuta, as costam gloniaulosa, foliolo meivo fasi aculeolato; gentes apuite - triangulares, onnes biserrate; pet-aculexti, glasia losi;

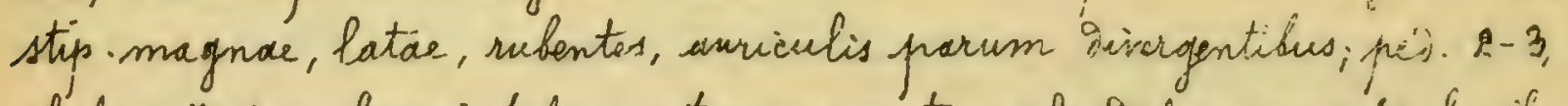
glabui, 7-gm.longr; tubus sratus; sep. erecta, eglangulosa, pismui is fevious, sat angustis, 2-3-gontatis instructa, acumine integre, latiusculo; styli la-

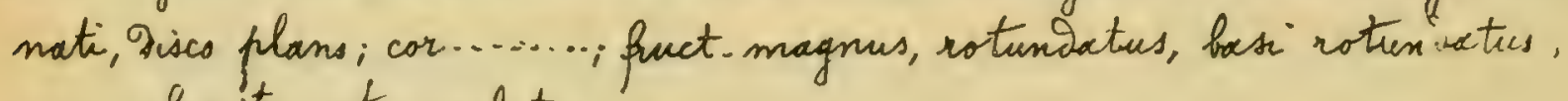
apice breviter strangulatus.

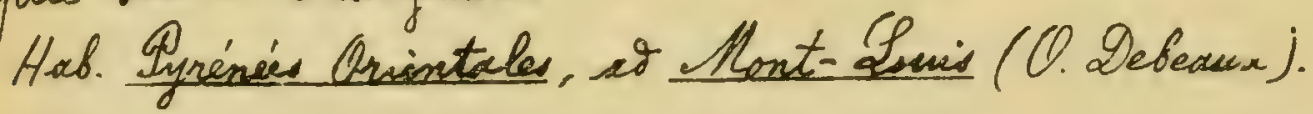

t Foliola simpliciter sarrata.

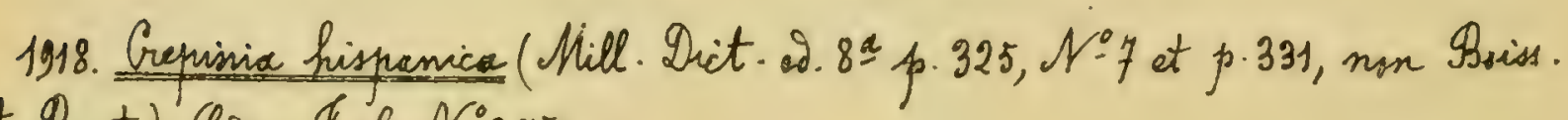
at Reut.), Gige \&ab. N:2553.

trenlei valide; falcati, late gilatati; fol-ovato-rotundata; contes simplices; pat-aculeati; stip. Latiuscular, pubescentes; piet glabri, tubus vatus, sep. Decitur, punmelis acutis, dentatis insìiucta, styli villori; car. prallioe roser, fruct. magnus, sufrotunastus.

Hab. Hiopania (Millar).

C. hispanica (Mriss. Meut. Mug. plant. nov. p. 4ti) sub nomine C.

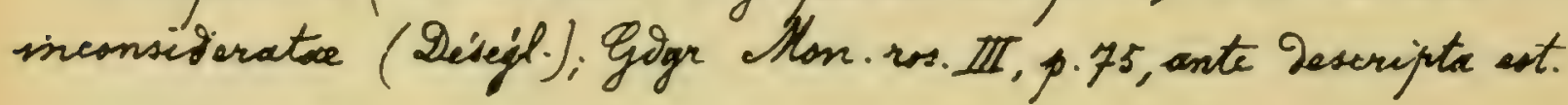


$-257-$

1919. Crepina wimariensis Gogr mss.

Acenlei longi, Dilatati, ternicter ot valie faleate, à ramos flor.copiosi; fol. late oblongo-acuta, basi roturioata, inferne as costam paulo glarioulosac; Dentes simplices, ovato-aperti; pet. eglaniulosi, aculeati; stip. angustac, virives, at auriculas girergentes tantum villosac; pe.s. 2-3, glabri, 4-7 m. Congivi thelus svato-rotuniatus, sep. pisnulis ammifus integris, lativisculis instructa, styli lanati, gisco plano; cor .......... fruct. rotungatus, nec attennatus. Hab. Shuringia, at Hallendorf prope Heimar (C. Houssknecht).

1920. Crepinia rhenana Gogr Gab. N-2554.

A-culei falcato-Dilatati, ad ramss flor. copiosic; fol. latiusonle obovatooblonga, acuta, fosi rotuniacto, at costam eglariaulosa; gentes simplices, ovats-convergentes; pet eglanaulosi; inf-inermes; stip. virioes, auriculis valie Sivergentibus; pet-1-2, glabri, 9-10 m. longri; tubus roturiatus; sep. pinnulis latis, 2-9-gentatis instructa, acumine lato, Jentato; styhi hirsuti, Hiteo plano; cor. lacte rosea, eciliata; furct. rotuniatus. Hab. Mosussir rhenana, at Hechen (Mraeminar).

1921. Crepinia pyrenaeicalo Goge Gab. N:2555.

toculer panciores, falcato-irlatate, aid ramos flor.nulli; fol. obovata, utringue rotuniata, ao costam eglasinulosa, Dentes simplices, late, aperti, pret.egleninlosi, snmes aculeati; stip. subtus glabror, latar; ped-1-3, glabri, 5-11 m. langi; tubus ovatus, sep. brevia, anguste pinnata, acumine lats; styli villosissimi, giseo plano; con. laete rosen; pruct. rotunostus, apriem sthemeatus.

Hob. Fyrenari centr., al Gièdre (H. Sartière).

1922. Crepinia globulo-sa Gogye Gab. N-2556.

Aculei nobusti, falcato-Dilatati, as ramos flor. Debiles; fol obovatooblonga, utringue paulo attennata, al costam eglanioulosa; ientes sinpices, rotunoati, aperti, pet. eglanoulosi, onnes aculeati; stip. subtus villosae, sout breves, Dilatatae, auriculis givergentibus; peid-1-2, glabri, 6-10 m. longi; tuhus ovatus, sep. pinnulis integris, angustis instructa; styhi lanati, isses plans; cor...... puct. rotunioatus, apice freviter strangulatus. Hab. Hehetia, Halais: ad Les Gaves in valle Champea (He La. Soie).

1923. Crepinia sarevitea Gogr mos.

Aculei rativi, folcato-Silateti, no ramos flor. verticillati; fol. 
$258-$

ampliosime shovato-oblonga, vix acuta, basi votuniacta, crassa, do costam eglanialose; Tentes simphices, late ovato-convergentes; pet. eoglanidulasi; inermss; stip. sinides, latissimae, elongatae, suriculis subrectis; pes. 1-2, glabri, $6.7 \mathrm{~m}$. Ingi; tubus avatus; sep...........; styli villosi, Giseo subplans; cor. ignota; fruct.majusculus, subrotunia atres, havi attematus.

Hab. Hungiria bor., Mrepes: ai 2icse (2. Michter).

1924. Grepinio Oumetorum (Shuill. TH pari; 1799, p. 250; Ipreng. Iyst. II,

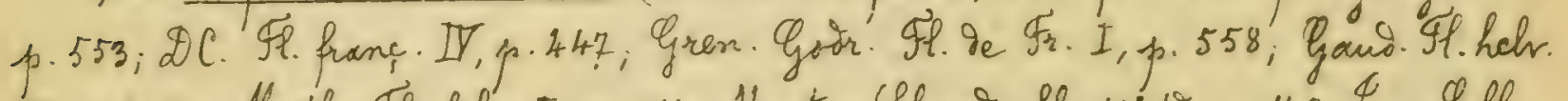
III, p.352, Math. Fe belg. I, p. 174; Hartm. Ykant. Pl. 102éd p. 146; ben. Iyll.

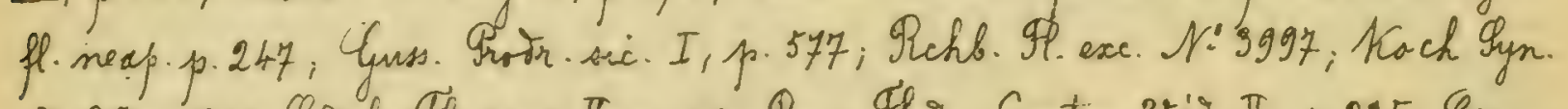

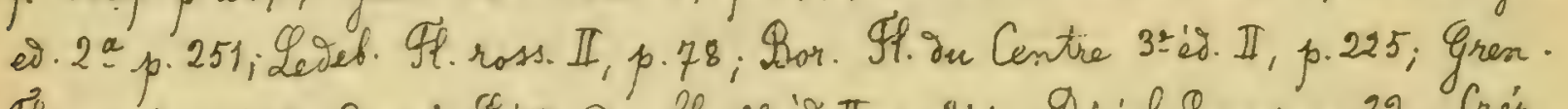
Fi. jur.I, p247; Carior Etude Fes Pl. G- ì. II, p. 244; Déségl. Bssai p. 32 ; Crép. Srim. I, p. 57; Cchenitg Skano. ros. p. 26): Goor \&ab. N-2537; y: Contr. fl.

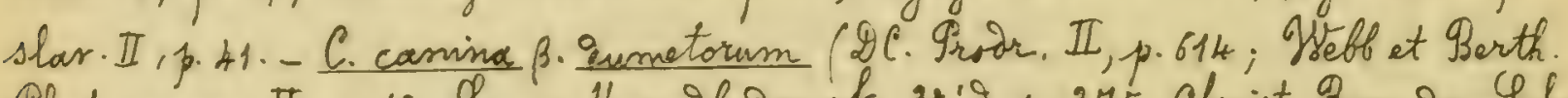

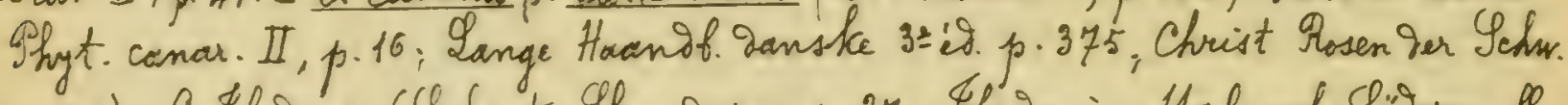
p. 181). - C. Ghedenü (Ychentz Tkand. ros. p. 27; Gherenius Upl. och Go'serm. fl. p.227). - Engl. bot. tab.257g (Imithii) At tab.2610; Fl. adan. tab.2718; Red. at Ghory Mos. 100.- Millot exs. N-1475 et bis; Bater Brit, nos. N: 19; Giger Herb. ros. $N^{M} 352,585$ et 586. - C canmax tomentose (Nitschke in Flara, 1857, p. 459 ).

Fculei valioi; foleato-gilatati; fol ovato-rotuniata, rigida, gentes simphices; pet. eglanoulosi, inermes, stip. latae, pubescentes; per-glabri, tubus svatus; seperectr, deciaua, glandulosa, pinnata; styli hirsnti, isses subconic; cor pallise nosea; fruct. rotunidatus. Hat. Europe.

c. Fructus ovato- rotundatus.

f Tiami florales inermes.

1925. Crennia secunivaria Göar Eab. N-2558.

toculei debiles, falcato- delatati, à ramos flor-nulh; fol-obovato-oblonga, acuta, basi subrotuniata, ai costan eglanaulasa; ientes simplies, ovato-convergentes; pet. eglandulosi, annes inermes; stip. recbentes, awoiculis divergentifis; peo. 1-2, glabri, 7 -9m.longi; tubus ovatus, sep. pirmulis integris, latie instructa, occamine foliaces, Ientato; styli villosi, visco conics; cor sciliata; fruet. ovato-rotuniontus.

Hab. Hispania bor. Logroño: \&l Rasitle in Sierra ge Cameros (J. Lubia). 
$-259-$

1926. Greninia tondersomiana Gogr bors. N:2559.

toculei mimetisiomi, tenniter inclinati, subsilatati, ar ramos flor. milli; for. obovato-oblonga, acuta, base rotundata, al costan eglanarlosa; Dentes simplices, lancedato-pperti; pat. eglanaulasi, inermes; stip. virides, aurisulis parum Sivergentibus; ped-1, glabri, 5-6m. longi; tubus prato - roterndatus, sep. pinnuhis integris, pancioribus, paum latis instructa; styli lansti, risco plano; cor. rosea, eciliata, fruct. ocato-rotundatus, apice attenuatus.

Hab. Luecia, Blekingix: à "grami" (F" Ivanlund).

t Rami florales aculeate.

1927. Crepinix orthosepola Givar Goob. N:2560.

A culei gilatati, temiter falcati, ar ramos flor. 2-3-ni; fol. obovacta, utrinque rotundata, intense viridia, subtus glauca ct as costam eglansulose; ventes simphices, triangulares, aperti; pet eglaninclosi, inermes; stip. breves, latissinar, auriculis Divergentious; pes.1-2, glabui, is-6 m. isngi; stufus ovetus; sep. erecto-conniventia, aprie aliniulosea, pinnelis latis, Ienticublis instructs, scumine suofoliaces, intagro; styli lanati, gises plano; cor......; fucct. magnus, ovouto-rotuniactus, basi abupte securrens, afice breviter attenuatus.

Hab. Luecix, Scanix:- Ortofta (I. to. Eullbery).

1928. Berinia verticillaris Cgogr mss.

Feculei verticillati, maximi, falcato-gilatati, a ramos flor. copissi; fol. ampile obovato - suboblonga, acuta, basi rotundata, lacte siridia, of costam eglasidulosa; Jentes simplices, late orato-subconvergentes; pet inermss, eglanoulosi; stip. virives, Longac, latissimac, subtus pubescentes, nuriulis dact divergentibus; ped. 2-4, glabri, $8 \mathrm{~m}$. longi; tubus ovato - subrotuniatus; sep. pimnulis anmibus foliaceis, brevifus, 1-3Dentatis instructs, acumine integro; styli maximi, laxati, Fisco plass; cor.intense rosex, eciliata; fruct.ovato-subroturiatus, basi rotundatus, apice abrupte strangulatus.

Hab. Hautes - thpes, cinca vicum 2 Grave, in Declivifus alpestribus, alt. $4500^{\circ}$ (M. Cyanooger). 
$-260-$

1929. Crepinia Janses Gogr Eas. N'2561. Gogr Herb.ros. N'114. ticulei ai ramos flor, gefiliores, faleato-silatati, fol ample oblongan utrinque paulatim attenuata, at costam eglandulasa, Dentes simplices, lati, recti; pat. hise inse glanbulosi, inf inermes; stip. subtres pubescentes, acusiculis parum Vivergentibus; - pe soepius 3-4, glabri, 7-12 m. longi; tubus ovatus; sep.erecta, decioua, usmulis integris, latis, elongatis instructa; styli maximi, lanati, gisw plano; cor. laete rosea, eciliata; fruct. pars. vis, orato-rotunsatus, apice paulo attennatus.

Hab. Ihône, of tornas (N. Gansoger).

1930. Crepinix iovophylla Gogr Bab. N:2562.

foculei a ramos flor. folcato-gilatati; fol. ample oblonga, utionque sensin attencuata, is costam eglaniulosa et unitique coerulescentia; jentes simphies, magni, paulo convergentes, pet. eglaniulosi, meíani soli aculeati; stip.magnae, subtes pubescentes, auriculis givergentibus; per. soepius 3-4, glabic, 4-12 m.longi; tubus obovatus, pruinosus; sep. pinmelis integris, angustis, pauciorifus instructa, acumine Congissimo, sat lato; styli lanati, gisto plano; cor. loete rosea, finct. majusaulus, svato-notuniotus, aprice strangulatus.

Hab. Hehretia, Grifourg: ad Nontsoron (Cottet).

1931. Crepinia cyanopota Gogr Eab. N:2563.

Acielei faleato-dilatate, at ramos flot.glaucos sot copiosi, fol. obovato-oblanga vel sblonga, utrinque attenuata vel acuta, viridicerculescentia, is costam eglandulosa; Dentes simplices; pet. eglasiulosi; annes inermes; stip. latar; pes. 2-3, glabri, glauco-coerulei, 12-15 m.longi; tubus vatus, caesius; sep. rellexa, sot anguste pismata, acumine longo, lato, styli lanati, gisco subplans; cor.......; fruet. late ovato-notuniatus. Hab. Dhone, ad Crapme (J. Cariot).

1932. Gre finia rhitridoclada Gogr Hab. N-2564.

Acculei longi, gilatati, inchinato-subfalcati, as ramos flor. rairiatos sat copiosi; fol. obovato-sblonga, acuta, basi attenuata, viridi-subglauca, as costasm eglandulasa; Pentes simplices, aperti, triangulares; pet. eglanoulasi, incrmes; stip.magnae, auriculis sivergentibus; pè. 1-2, glabri, 6.7 m. longi; tubus ssatus; sep.pinnulis integris, angustis, paucioibus 
$-261-$

instructa, acusmine foliaceo, integre, styhi lanati, gisco plano; cor........; fruct. parrus, ovato-rotuniatus, basi abrupte decurrens, apice nec atten.

Hab. Suecir, Yestrogothia, ad Sattra, Itnin (9. F. Lunaquist).

1933. Crepinia marillama (Prorbas Ros. hung. 1880).

tculei Dabilss, falcato dilatati, breves, ad ramos flor stipulares; fol. late obovata, basi rotunsorta, apice vise scuta, crassa, superne pallivins siniaia, subtus albiva, ad costam eglaniulosa; Dentes simplices, late ovatis. subconvergentes; pet. eglandulosi, sup. inermes; stip. virides, dilatatae, lonquisurlac, auriculis Qivergentes; pres. 1-3, glabri; 8-10 m. longi; tubus ovato - stuniatus; sep. frevia, pinnulis ammisus parris, binearibus, integris instructa; styli lanati, disco plano; cor......'; fuct. sat parrus, oratorotunisaties, basi rotunatus, apice vix strangulatus.

Hab. Hungarix, Krasso'- Gorény: D Marilla (Motbás; Lichter).

1934. Crepinia trenesenensis Gojer mss.

tculei dafiles, brevissimi, subfalcato-Dilatati, ramos flor. sparsi, fol. ample sbovato-suboblongx, vix acutiuscula, basi notuniata, intense viriDia, subtus glaucescentia, is costam ealaniulasa; Dentes simphices, breves, ovato-aperti; pot-eglanisulasi, subinermes; stip. Dilatata, longrisculae, auriculis subsirergentifus; pes-2-4, glabri, 3-5 m. longi; tubus ovato- totundatus, sep. pinmmlis subintegris amnibus late folixceis instructa; stypli vellosi, subfasciculati, disc paulo consico; cor. médiocris, rosea, eciliata; fruct. orato-rotuniatus, basi trusicatus.

Hab. Hungarix Erencién: ad Ryej (2. Richter).

1935. Crepinia evecta Goor mss.

Acculai mediores, Onri, Nilatato - subfalcat, ad ramos flor. saepe verticillati; fol parve, oblonge, utrinque sensin attenuato, coriacex, supra nitide atrovirentic, subtus virescertic et ag costam eglandulosa; dentes simplices, profingi, lanceslato - suffalcati; pet. hise inse glaniulosi, omnes rculacti: stip. elongatae, apice poulo dilatata, cacterum angustiones, auriculis Diver. gentibus; ped. 1-2, glabri, 1 cent. longi; turbus svato-rotunoatus; sep. brevia, pinmulis integris, latiusculis instructa; styhi lanati; gives plano; cor.......; fruct. sot parvus, ovato - subglobosus, basi rotundatus, apice depressus. Hab. Hungaria; Fehér: ad Biake (L. Michter). 
$-262-$

d. Fructus ovatus.

$f$ Diseres comions.

1536. Crepinia apertidens Gogr bob. F:2566.

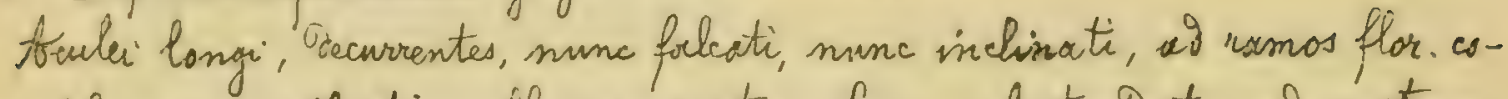
pirsi, fol. parva, elliptico-oblongre, renta, basi subrotumiata, at costorm eglaniaulosa; Dentes simphices, lanceolato-aperti,; pet. saepius eglandulosi, inf. inermes; stip. parvac, angustac, subtus glabara, avriculis sivergentifus. pes 1 2, laeves, 9-17 m. longi; thems breviter ovoivens; sep. pionmulis integris, latrisculis instructo; styh exserti, villosi, Sisco comico; cor......., fruct. ovatus, apice strangulatus.

Hob. Houtes- Prénés, aD Lus-St- Sauveur (H. Borière).

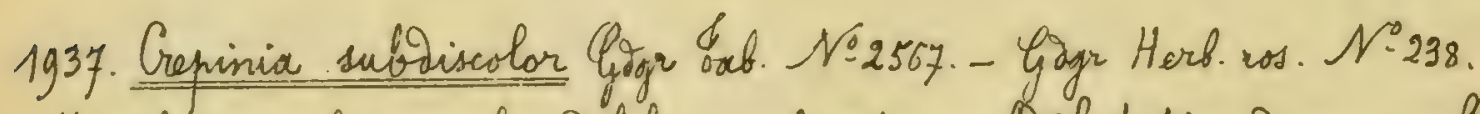
Humilis, gracilis; aculei defiles, inchinato-subsilatati, aio ramos flor. ponciores; follovats-elliptica, utrinque subrotuniata, subtus albiso- is colorix, à costam, eglandaulosa; Dentes simplices; pet inernes, eglaniulosi; sinp. breves, subtus villosae, auriculis latis, rectis, ped. 1-3, glabri, 1 cent. longi; tubus ovoiteus; sep. pinnulis onsibus latis instructa, acumine elongato; styli breves, glabrescentes, Disco pauls consico; cor parva, pallide nosea, fruct. minor, ovatus.

Hab. Mhone, ad trows (M. Ganooger).

1938. Crepinia verbascovies Govor mis.

Focule: breves, inchinato- Dilatati, ab ramos flor. stipulares; fol set parra, sllango-acuta, basi rsturivata, supra rivivi-glancescentia, subtus canescentia, at costam eglanioulosa; Dentes simplices, late patulo_triangulares; pet. echaniulosi, vix aculeati; stip. angustissimae, gorso tanterm villosae, auriculis aivergentibus; pes. 1. glabri, 7-10 m. longi; tubus ovatus; sep.......; styli parce nollosi, visco paulo comico; cor....... fruct minor, ovatus, basi rotuniatus, aprice subatremuatis.

Hab. Hungaria, tobonj: Do Botfalu, Kassa (2. Richter).

1939. Crepinia nubilis Gogr mss.

Faculei minuti, brevissimi, inclinato-Silatati, si ramos flor. nulli; fol. latiuscule oborato-suboblonga, basi subatternuata, spice rix acuta, is- 
$-263-$

rincea, intense vividia, subtus albioa, aí costam eglanoulosa; gentes late triingulari-subconvergentes, simplices; pet. longe villose, eglaniaulosi, aculeati; stip. longac, sat angustae, oilute rubentes, Darso hirsutar, auriculis rectes; pes. 1-2, laeres, 4-6 m. longi; tubus ovato-ellipticus; sep. primnulis ammibus foliaceis, inciso- dentatis instructa; styli lanati, exserti, arisco prauls conico; cor...... fruct. amplicescule oratus, fasi subrotuniatus, as apicem abrupte strangulatius.

Hab. Hungarix, Muax- Sest: ad gilla focumix (2. Shichter).

tf Liscas planus.

1940. Crepinia ansmalis Goge Gab. $\mathcal{N}-2565$.

teculai folcato-gilatati, as ramos flor mulli; fol obovato-oblonga, scuta, basi truncata, a costam eglaniulosac, Dentes triangulari-convergentes, inf. biserrati, pet. inermes, inferne glangulosi, stip. maxima, Dorso glaniulosere, auriculis Divergentibus; pet. 1-3, glabri, 14-15 m. longi; tubus svatus; sep. erecta, interaum marginions asperse glaninulosa, pinnulis filiformibus, 3-5-2entato-glaniulosis instructa; - cumine lato, subfoliaces; styli hisuti, gisco plano; cor..........; funct. ovatus, basi subrotunoatus, apice breviter strangulatus.

Hab. Austrix infer. a Kalksburg prope grem (Y. Hiesbaur).

1941. Crepinia crikensis Gogrumss.

teulei valiouli, dilatato-subfalcati, ar ramos flor. nulli; fol-mebiocria, oblongo-acuta, basi attemunta, glouco-viridio, at costam eglaniulosa et subtus paltida, Dentes triangulares, aperti, inf. biserrati; pet. oglanioulosi, inf inermes; stip. sirides, angustae, longrius-culia, dorso pubescentes; auriculis rectis; pei. 1, lueves, 15-16 m.longi; tubus ovatus; sep. pinnulis angustis, sapsius integris instructa, acumine lato; stygli lanati, orisco pino; cor....... puct. late ovatus, basi rotundatus, apice subiopresses. Hab. Eransilvamix, Gik: Ao Mre (2. Richter).

1942. Crepinia megalostigma Gogr \&ab. $N=2568$.

Acculei breves, parvi, falcats - Silatati, ac ramos flor. sat copnisisi, fol. oborato-oblonga, sufattesmata, basi rotundata, as costam eglaniulosa, Gentes simplices, aperto-triangulares; pet. inferne pauls glanoulosi, inf. inermes; stip. virides, auriculis perum Divergentibus; pes. 1-3, glabi, 11-13 m. longr; trebus oratus; sep. juinnulis latis, 1-2-gentatis instuncta, acu- 
$-264-$

mine integro, subfoliaces, styh maximi, lanato-albi, evserti, Disco plano; cor....... fruct. magnus, ovatus, basi rotundatis.

Hab. Scotia, Perthshire; at Peggieden (Drummont-Hay).

1943. Grepinia lutescens Gogr mss.

Acculei brevissimi, minuti, inchinato-Dilatati, às ramos flor. nulli; fol. sat pawa, oblongo- subattemuata, basi contracta, vividi-lutescentia, subs tus canescentia et ad costam eglanioulosa; dentes breses, svato-consargentes, simplices; pet egla nivulosi, onnes subinermes; stip. freves, latiusculac, dorso vilosac, auriculis subrectis; pet.1, glabiri, 1 cent. longi; tubus ovato- ellipticus; seps. pinnulis ammibus foliaceis, 1-2-2entatis instructa, acumine integro; tygli subchmmares, basi lanati, superne glabre;, disco plano; cor.....; fruct. parsus, ovatus, basi subrotuniatus, aprice abmpte strangulatus. Hat. Eranssilvania, Hóromszek: as Diedös (2. Rickter).

1944. Crepinia brachycella Gogr 8ab. N-2569.

Yracilis; aculei tenues, defules, parum fulcati, ar ramos flor. panciores; fol. parra, obovato-obtura, fasi corsata, viridio, ad costam egtaniulose; dentes simplies, ovati, recti; pet. inermes, hine inge glaniaulosi; stip. parrac, angustar, auriculis givergontibus; pet. 1, glabri, breves; tubus ovatus; sep. brevissima, pirmulis foliaceis, 1-2-gentatis isestructa, acumine integro; styli lanati, disco plano; cor......., fruct, parrus, ovatus, basi rotuniatius, apice subattenuatus.

Hab. Eyrol, prope Erente (\%. Gelmi).

1945. Crepinia petasites Ggor \&ab. N-2570. Gogr Herb. nos. N-161. Abenlei debiles, foleato-subvilatati, was ramos flor. sparsi, fol. late obovato-oblonga, attenuata, basi subrotunisata, ad costam eglansulosa; Dentes simplices; pet. eglangulosi, inermes; stip. latinsculae; ped. 1-3, glabri, 8-11 m. longi; tubus freviter ovoidens; sep. pinmulis coprosis, latis, villosis instructa; styli sparse pilosi, disco planiusculo; cor. prallide rosea, fruct. magnus, oratus.

Hab. Rhone, ad tornas et Dencié (M. Gansoger).

1946. Crepinia thyltenii Goge mss.

Atenlei parvi. Debiles, breves, Dilatati, tenuiter falcate, id ramos flor. panciares; fol. sat crassa, sbovato-oblonga, acuta, basi rotunisata, costam eglaresulosa, Dentes simplices, orato-convergentes; pet. eglaniw 
$-265-$

Losi, inermes; stip. latae, virides, subtus pubescentes, averieulis subrectis, ped. 1, glabri, 7-g m. longi; tubus oratus; sep. pirnulis villosis, latis, 2-3-Dentatis instructa, acumine brevi, lats, integro; styli magni, lanadi, Discs plano; cor.......; fuct. ovatus, basi rotundato-truncatus, ad apicem vix attenuatus.

Hab. Luecia, Imailand: - Draf (Hyltán-Cavallius).

1947. Crepinia Peuronü (Ggor 1871, at Qssai p. 32; Goger Ros. Gall. nor. II. p. 28), GJgr Gob. M:2571. C. cinerascens (Cariot Etude ges Pl. 4: id. II, p. 181, non Dumort. nec Halbr); C. aumetorum var a cinerea (Carist loc.

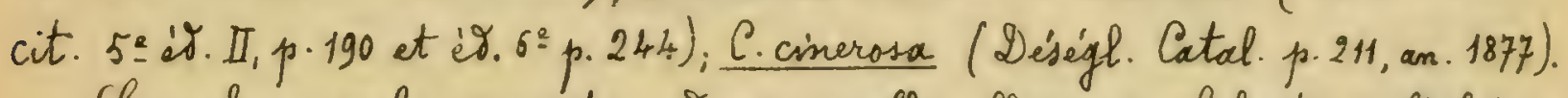
Gracilis, aculei minuti, at ramos flor. flexusos falcato-suboilatati, fol. parvula, ovato-oftusa, unsique cincreo-tomentosa, as costam eglan Dulosa; Dentes simplices; pet. eglanoulosi, parce aculeati, stip. passae, subtus pubescentes, pet. 1-2, glabri, graciles, tubus ovatus, sep. pirmulis parwis, integris, hireutis instructa; styli villosi, disco subplano; cor. parva, rosea, fruct. ovatus.

Hab. Loire, in mantibus ad Chalmayelle (Peyson).

Rosa cineraseens Dumort. Fl. belg p.g3 ad Siegetias (Eomentoseac) pertinet). - Posa cinerascens Walbr. Hist. ras. p. 231 est species ofscura al ommibus ignota.

1948. Crepinia otiosa Gogr mss.

Abculer minuti, brevissini, inchinati, vix dilatati, ab ramos flor.mulli; fol. parva, obovato-oblonga, breviter attenuata, basi contracta, supra pallise viridi-glaucescentix, subtus incana, as costam eglanioulosa; dentes simplices, svato-convergentes, pet. oglansulosi; omnes parce aculeati; stip. angustar, parvar, subtus tomentellar, aurienlis rectis; pes. 1. glabi: 5-7 m. longi, tufus freviter ovoideus; sep. pinnulis frevibus, foliaceis, 1-2 Dentatis instructa; styli lanati, disco plans; cor......; fruct. sat praivus, ovatus, basi subrotimatus, apice comico-strangulatis. Hab. Hungaria, as Budaarsi (2. Richter).

194g. Crepinia Lunata $(G i g r$ Ros.nov. II, p. 2g); Gogr Gab. N=2572.. Gogr Herb. nos. $N: 12$.

Abculai ad ranos glaucos et givaricitos parvi; longuisculi, fol. umple 
$-266-$

oflonga, attenuata, basi rotunsata, oo costam eglanioulssa, terminali vero basi humulata vel coriato-emarginato, Dentes simplices; pet. prarce aculeate, hinc inde subglanidulosi; stip. magnac, ajuce Dilatatae, subtus pubescentes, auriculis divergentibus; pes. 1.2, globri; meviscres; tubss ovatus; sep.pinmulis latis, eglanoulosis instructa; styli hirsuti, aisco plano; cor pallide rosea; fuct parrus, ovatus.

Hab. Rhone, at Montmelas (Mb. Ganooger).

e. Fructus ovoideres vel obovato-oblongus.

+ Foliola basi rotuniata.

\$. Ytugli glabri vel assperse hirtelli.

1950. Greninia astrachamica Gage toat. N-2573.

Focubi falcato-gilatati, ais ramos flor copiosi; fol. ample oblongo-acuta, basi coroato, id costam eglaniubosa; ientes simplices, late ovato-obtusi, convergentes; pet.eglanivulosi, inermes; stip. Dilute rubentes, latissinac, anriculis givergentibus; peov. 1-3, glabri, 5-7 m.longi; tubus ovaideus; sep. pinmulis integris, latis, copiose instructa, acumine lato; stagli glabri, orisco conico; cor eciliata; fruct. maximus, ovoideus, medis turgidus, utrinque attera. Hab. Rossia merio.-orient., fastrakhan:at Darepta (Af. Secker).

1951. Grenimia jasypogon Gog. mss.

Aculei brevissimi, debiles, parim ojilatati, temiter falcati, ad ramos flor. pauciores; fol sat parva, obovata, apice subrotunvata, basi roturnData, pallive virigia, at cosiam eglandulosa; oxntes simplices, triangulariconvorgentes; pet. eglaniulssi, sculsati, stip. latar, breviuscular, virides, subtus toto pubescentes, auriculis aivergentibus; pel. $1-2$, glabsi, $10 \mathrm{~m}$. longi; tabus obovato-shlonias; sep. rubentia, prinnulis ammibus brevibus, integris, latis; styli glabri, gisce proulo conico; con meiscris, ciliata, fruct. ovideus, fasi attenuatus, aprice longe contractus.

Hab. Agloric as. Constantine: wt Rou Chalek (ge Rebuid).

1952. Crepinia concolor Goar tab. N:2574.

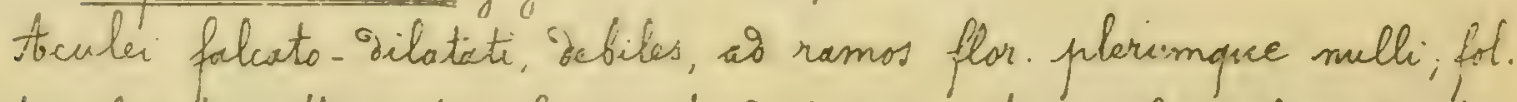
obovata, freviter attemeata, fasi rotinaata, as costam eglaniulosa; gentes simphices; pet eglanaulosi, inermes, stip. mesiocres; ped.1-2, glabri, 8-10 
m. longi; tubus ovoideus, sep............ styh plerumque glabri, Dises sebplano; cor......., fruct. sat parsus, ovoidens, fasi rotunsatus, apice subattemuatus. Hab. Thone, ad bassin (At. Cariot).

1953. Crepinia foliocalyx Gogr mss.

Acculei valivi, foliato-Silatati, ad ramos flor. minores, intersum subgeminati; fol. late obovato-oblonga, acuta, fasi rotuniato-truncata, atrosirentia, subtus pullisiara et ai costam eglanioulosa; dentes simplices, late triangulari-convergantes; pet. eghonioulosi, inf. inermes; stip. longae, latae, Dorso pubescontes, suriculis subrectis; pes. 1-2, glabri, 6-7 m. Congi; tufus sbovato - oblonqus; sep.maxima, pirmulis copiosis, smphissime foliacriss, incis-laciniatis dentatis que instructa; stygli asperse pilosuli, moegni, Jises prulo cancio; cor........ fruct.magnus, bbovato-oblongus, basi gepressus, apice longe attenuato - strangulatus.

Hab. Eranssilvania, Uverhely: Forkaslaka. (2. Michter).

1954. Crewina cidenosontoives Cgogr bab. N:2575.

Bcculei debiles, folcato-Dilatati, ac ramos flor. nulli; fol. obovato-obionga, abrupte acuta, bosi rotunzato, intense virisia, ad costam jaculo glanidulose; dentes sublanceolat-aperte, onnes biserrati, pet-aculexti. sparse glanderlosi; stip. parvar, parum latre, suriculis Divargentibus; pes. 1-2, glabri, 19-21 m. Congi; tubees ovoigeus; sep. magna, pinmulis latis, numerasis, 1-2-Dentatis instructa, acumine longissimo, foliaces, tenviter senticulato; styli inferne paulo pilosuli, gises valde conico; cor.majuscula, sciliata; fuct. svoidens.

Hab. Flgeria, Kabylia in mont. xo Fort Naitional (I. Debeaux).

1955. Crepinia gnaphalodes (Gogr Ros. nor. I, p. 14): Gagr Fab. N22576.Gogr Herb. ros. No89.

Acculei falcato-Dilatati, ad ramos flor.stipulares et geminati; fol. sbovato-acuta, basi rotunaata, unsique canescentia, mollia, as costam non glandulosa; Dentes simplices; pet. inermes, egtansulosi; stip mediocras; per. 1-5, glabri, 12-14 m. longi; tubus avoideus; sep. crass-a, serines decidua, pinnulis latis, eglandulosis instructa; styli subglaberrimi, disco fere plano; cor. pallide rosea, moschata, eciliata; fruct. magnus, ovoigeus, utringue Oefressus.

Haf. Thane, at tornas (M. Gandoger). 
$-268-$

1956. Crepinia verax Gogr mss.

A-culei validi, Jilatati, valde falcati, at ramos flor mulli; fol obovatooblonga, abrupte acuminata, basi rotuniate, viridi-glauca, ad costam eglanDullosa; Ientes simplices, ovato-aperti, pet. eglaniulosi, parce aculeati, stip. sirides, longac, latissimae, subtus toto pubescentes, auriculis apertis; pres. 1-3, glabiri, $8 \mathrm{~m}$.longi; tubus ovoideus; sep. pinnulis smmibus integris et latis instructa, styli assperse hirtelli, Dises comico; car......... fruct. oroidens, basi Depressus, apice attenuatus.

Hab. gear, à Atompus (Jlbert).

1957. Crepinia hallensis Gogr Gab. N:2577. - C. pyenacantha (H. Braun in Kerner Scher. ad exs. an. 1883); C. coriifolic var. (Morba's Ros. hung. 1880).

toculei breves, salibi, folcato-gilatati, ad ramos flor copiosi, fol. late obsvato-acuta, basi cordata, id costam eglandulosa, dentes simplices, oveto-convergents ; pet glanoulosi, aculeatissimi; stip. virives, latissimae., auriculis aivergentibus; pes. 2-3, glabri, 5-6 m. longi; tubus oroidens; sep. pinmelis brevibus, latissimis, 1-2-dentatis instructa, acermine foliaces, in tsgro; styli adsperse pilosuli, disco conice; cor......., fruct.ovoidens, basi agpressus, apice attenuatus.

Hob. ingrot, ai Enaienwali prope Hall (j. Gremblich); Hangaria.

\$. Styli puebascentes.

1958. Cieprimix falcidenta Gogi mss.

toculei vialidi, Dilatato-subfalcati, as ramos flor geminati; fol late svato-elliptiea, oftusa, basi rotundata, lacte viridia, at basin costae acubolato; dentes simplices, profundis, late seato-subconsergentes vel falcati; pet. hirsuti, aculeati, eglongulosi, stip dilute rubentes, latissimas, subtus giabrae; anriculio Divergentibus; pes. 1-2, glabri, 13-15 m. longi; tubus ovoidens; sep. pinmulis hirtellis, omnibus ate foliaceo-incises copiosissime instructa : styli pirsuti, gisco praulo conico, car. magna, rosea, eciliata; fruct. ovoidens, basi altenustus, apice subconitractus.

Hab. Halia orient, as Inenra (2. Caliesi).

1959. Gupinia grisescens Giagr bab. $Y: 2578$.

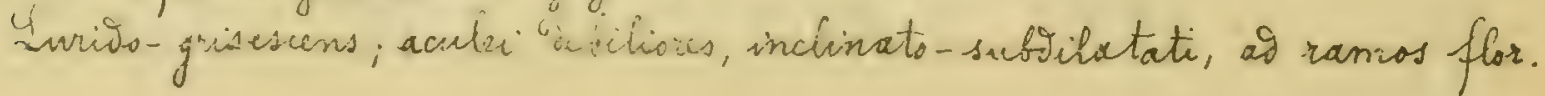


$-269$

panciares; fol midiacria, bovato-attenuata, fasi rotuniacta, supra cinereoglauca, subtus albida, à costam eglanisulasa, bentes simplices, breves, acuti, pet.eglandulosi, inf mermes, stip.longae, subtus pubescentibus, auriculis Divergentibus; per. 1-3, glabri, 13-22 m. longi; tubus obovato-of longus; sep. pinnulis latis, integris, elongatis isstructa, styli villosi; Sises subplano, cor. pallise rosec, fruct. obovatus, apice attemuatres.

Hab. Thone, Dormas (M. Ganooger).

1960. Crepinia pelligera Ggar \&ab. N:2579.

toculei dilatati, tenmiter falcati, ai ramos flor panciores, fol obovatosubacuta, basi rotundata vel subcardata, virisi-glaucescentix, as costam eglanivulosa; gentes simplices, svato-aperti; pet. parce aculeati, hinc inis glonoulosi; stip. pubescentes, suriculis Sivergentibus; feo. 1-3, glabri, $15-18 \mathrm{~m}$. longi; tubus obovato-sblongus; sep. refleza, finnulis integris, foliaceis instructa; styli praulo villosi; gises plano; cor. albo-roser, eciliata; fruct. obosato-sblongus, apice attennatus.

Hab. Gironie, al Bassens (L. Notelay).

1961. Gepinia Dicthyodenoron Giger mss.

foculei minute, debiles, inchinato-Silatati, ai ramos flor-valie arti. culato- Hexussos nulli, for. parva, ohorats-suboblonga, acuta, basi paulation rotuniata, pallidius viridix, subtus canescentia, is costam eglaniulosa; Dentes simplices, late ovato-convergentes; pet eglandulosi; aculeati; stip. angustac, breves, dorso villosae, auriculis rectiusculis; per. 1, glabri, $11-33$ m. longri; tubus sbovato. oblongus; sep............ styli parce villosi, Disco paulo comics; car...... i fruct. parrus, obovato-suboblongus, basi dopressus, apice longe attemuato-strangulatis.

Hab. Eransilranix, Syolnok Doboka: at Cseincfalu (2. Rickter).

1962. Crepinia holophilla Gogr tah. N.2580.

Acculai minuti, recti vel inclinati, as ramos flor pauciara; to oflongssubattenuata, fasi rotumiata, mollissima, viridi-lietescentia .as costam eglansulosa; Dentes simplices, aperti; pet. eglavioulosi, in iresmas;

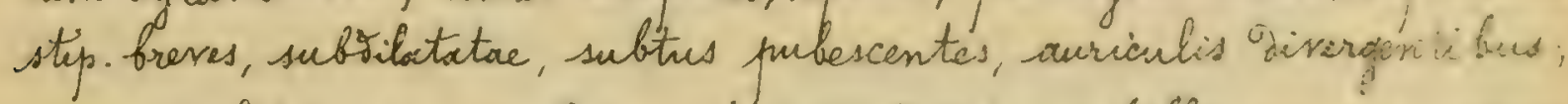
pes. 1-2, glabri; 8-13 m.longi; tubus obovato-suboblon us; s2s. ninatis

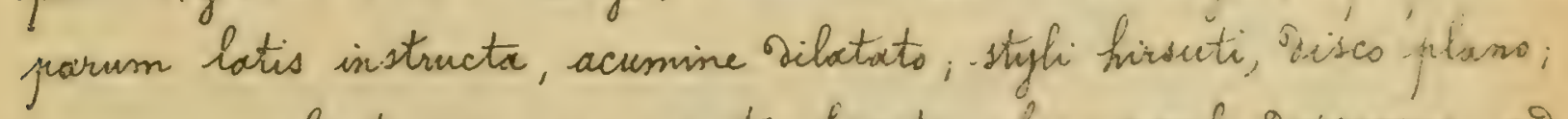

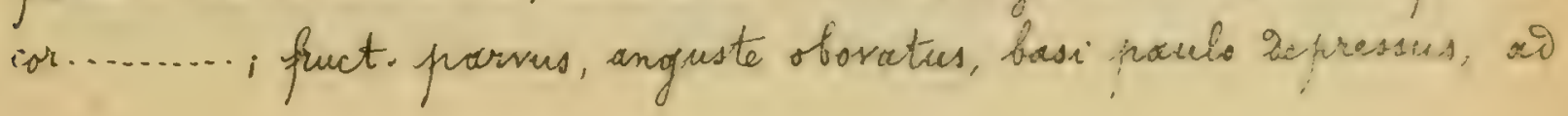


$-270-$.

apiem attemuatus.

Hab. Helvetia, ralais: as Bovernier (Lagger).

1963. Crepinia vistulensis Gigr mss.

teculei breses, Silatati, temiter falcati, aio ramos flot. copiosi, fol. late oblongo-acuta, basi rotuniata, intense viriesia, as costam eglansulosa; Dentes umnes valis biserrati, aperti, latè avato-triangulares; pet. aculeati, glaniulosi; step. latiuscular, virioes, subtus toto probescentes, auriculis Divergentibus; pet. 1-2. glabui, 5-8 m. longi; tubus oborato - oflongus; sep. pionnulis onmibus foliaceis, 2-4-Dentatis instructa, elongatis, villosis; styhi hirsuti, dises plans; cor........, fruct. obovato-sllongus, apice longe strangulatus, basi rotuniatus, centrahi voro gecurrente.

Hab. Bornssia rient, at Grawdenz in gistul. (Lcharlot).

1964. Geminia aralonylom Gogr \&ab. $N=2581$.

Rami elongati, sarmentssi, fraigiles, aculeis valisis, falcato-Dilatatis instructi; fol: ample oblongo-lanceolata et acuminata, basi rotinivata, atrovirentia, subturs albida, adsostam eglanioulosa; Dentes simphices rotunidato-cuspriatic; pat. eglanioulosi, sup. tanterm aculeati; stip. latac, lonqae, subtus pubsecentes, ourriculis vivergentibus; pet. $1-\frac{B}{3}$, glabic, $7-12 \mathrm{~m}$. Congi; tubus sirboblongus, glancus; sep pinmulis integris, latis, interoumve foliaceis instructa; styli pubescentes, aises sat conics, cor.maxima, 55-62 $m$. Tiam. lata, pallise rosea; fruct sbovato-oblongus, apice atternuatus. Hab. Thone, is Fornas (No. Ganaoger).

1965. Creninia lentotricha Coggr mss.

Ttculei parri, cillatato suffaleati, Debiles, ad ramos flor. flexiossos mulli; fol. mesiacria, ollonzo-acuta, basi paulation roturisata, supra intense viridia minute hirsuta, subtus allita, as costam glantulosa; gentes simplices, prolunive ovato - subconvergentes; pet. eglaniulosi; inf. inermes; stip. breves, angustac, gorso pubescentes, auriculis sat Sivergentibus, per.1-2, glabri, 10-12 m. longi; tubus obovato - oblongus; sep. pinnulis latis, 2-9Dentatis instructo, accumine angusto, integro, styli pubesantes, Disco plano; $\operatorname{cor} . . . . . . .$. , fruct. obovato-oblongus, basi repressus, apice attennato-strangulatus, mediceris.

Hab. Eranssilvania, Izolnok Doboka: Dés (2. Richter). 
-271 -

\$§. Ytyli lanati.

$\sim$ Ramiflorales inermes.

1966. Crepinia parata Gogr mss.

teculir sparsi, validi, falcato-gilatati, at ramos flor. mulli; fol.

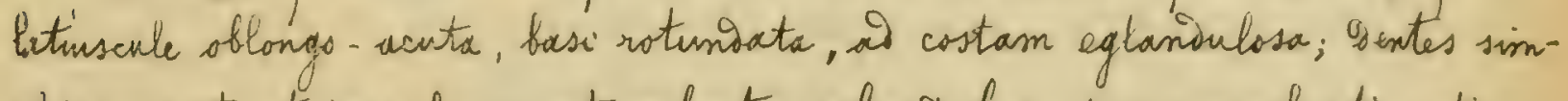
pilics, aperto-triangulares; pet. rubentes, eglansulosi, parce aculeati; stip. purpureae, latissimae, sat breves, aurieulis sirergentibus; ped. 2-4, glabri, $10 \mathrm{~m}$. longi, thelus obovato-oblongus; sep.......... styli lanati, giscs subcanis, car.......; fruct magnus, obovato. oblongus, basi subrotundatus, id apiom atternato-comicus.

Hab. Hungrenia, as Leasyfalu (L. Tichter).

1967. Prepinia reoremicia Cogor Gab. N.2582.

Feculei breves, temiter falcati, io ramos flor. nulli; fol sat late obovato-blonger, acuta, basi rotuniata, al costarn eglarisulasa; Dentes ovati, simplices, aperti, pet inermes, eglaniulosi; stip. sirives, auriculis givergentibus; poi. 1, glabri, $8-9 \mathrm{~m}$. longi; tubus ovoidens; sep. pismulis integris, parvis, angustis instructa; stegh lanati, disco plano; cor........ fruct. ovoidens, utringue opressus.

Hab. Inecia, ad Dref Ioniame, gerentiox (Hybtion-Cavallius).

1968. Grepinia movens Gagr mss.

Acculer panciores, falcato-Dilatati, valisi, ad ramos flor mulli; fol. amplissime oblongo-acuta, basi rotungata, si costarn eglaniulosa; dentes simplices, mucronati, ovato-recti; pet inermes, eglangulosie; stip. sat latae, longiuscular, sirides, auriculis ivergentibus, peo. 1-2, olabsi, 1 cent. Longi, tubus oborato-oblongus; sep. pinnulis amnibus subfoliaceis, paulo Dentatis instructas; stygli lanati, gisco plano; cor........, fruct. majusculus, obovato-oblongus, basi attenuatus, apice longius strangulates.

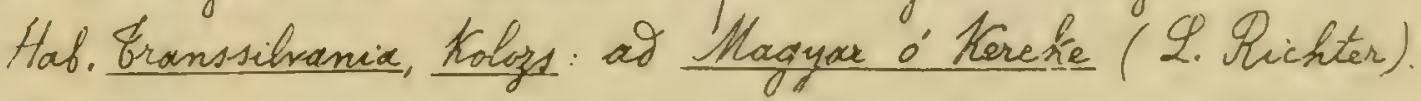

1969. Crepinia feherensis Cligr.

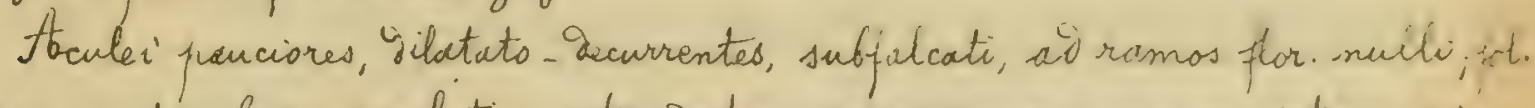
oblongo-acuta, basi paulation rotunoata, crassissima, nervosa, intense virisia, subtus pallioa, ad costam eglaniulssa, Dentes simplices orato-conver- 
$-272-$

gentes; pet. eglanoulosi, plarumque inermes; stip. latae, longae, subtus gorso villosae, auriculis subrectis; ped.1-2. glabri, g- $12 \mathrm{~m}$. Congri; tubus obovato-oblongus, sep. pirnmelis annibus foriaceis, 2-3-sentatis instructo; styli lanati, disco subplano; con....... fruct svoides-comicus, fasci subrotuniatus, apice attenuato-strangulatus.

Hab. Hungaria, Gehier : ad Bieske (2. Richter).

B. acculeslata Gogrmss.- At typo siffert foliolis obovato-oblangis et petiolis fere onmibus aculeolatis.

Hab. arm specie.

1970. Crepinia pachystema (Gogr in Bull. soc. Murith. 2u Galais, 1876, p.34); Gogr tab. N:2583. - C. coniufolive f. frutetorum (Christ Rosen ver 9chiveiz p. 189-191).

teculei rarissimi, Debiles, inchinati, as ramos flor nulki, fol ample obovata, utrimque roturisata, costam eglansulosa; Dentes simplices, lati, aperti; pet. inermes, eglaniuloss; stip. latae, subtus pubescentes, auriculis Sirergentibus; pes. 1-2, glabri, 12-13 m. longi; tubus ovoidens, sep. longissina, crassa, camosa, pinmulis integris, brevibus, foliaceis instructa, pein erecta; styli magni, lanati, Dises plano; con........ fruct magnus, suboroivens, utimgue truneato- strangulatus.

Hab. Helvetia, gelaid at Mayens de Sion (E. O. Holf).

1971. Crepinia crassissima Cojor mss.

toculei panciores, longi, valisi, falcato-Dilatati, ad ramos flor. nulli; fol. late obovato-oblonga, breviter Attenuata, basi notunidata, crassissima, nervose, à cortam eglanioulosa; Pentes simplices, late ovato-convergentes; pet. eglanioulosi, fere onnes inermes; stip. latissinace, subthes pubeccentes, gorso rubae, aurieulis subrectis; per. 1-4, glabri; $12-15 \mathrm{~m}$. longi; tubus oboratoollongus; sep. longa, raflexd, pinmulis anmibus late foliaceis, 2-4- Ientatis instructa, acumine integro; styli lanati, gisco plans; cor.........; fruct. man simus obovato-oblongus, basi depressus (centrali excepto), aprice attemuatosubstrangulatus, medio late turgions.

Hab. Hungria, Tehér: as Briske (L. Richtor).

Frutex crassituitine folislorum, inoumento sublamuginoso purctuque maximo $(12-13 \mathrm{~m}$. Diam. lato) insignis. 
$-273-$

$\sim \sim$ Rami Ploraks aculeat.

X Petioli subinermes.

1972. Crepinia Dumetaides (9uget) in Gogr Gab. N:2584.

foculei ad ramos flor falcato-Dilatati, geminati; fol ovato-acuta, basi rotundata, ad costam eglandulssa; dentes simplices, aperti, pet. hine inve glanialosi, subinermes; stip. subtus pants pubescentes, anriculis sisergentibus; ped. 1-3, glabri, $12 \mathrm{~m}$. longi; tubus ovoidens; sep. pinnulis eglaniaulosis, pubescentibus instructa; styil tanati, exserti, gises plans; cor pallive rosea, fruct. magnus, ovoidens.

Hab. Rhone, at Eassin (Soullu).

1973. Crefinsia tenvicallis Gogr Eab. 1:2585.

Acculei gebiles, Dilatato subfalcati, ad ramos flor copiosi; fol oblongsacuta, basi roturnsata, as costam eglanaulosa; dentes aperto. triangulares, inf. biserrat, pet. subinermes, hine inde ylanoulosi; stipo. madnae, anriculis divergentibus; piò. 1-2, glabri, 7-gm. longi; tubus otovato- oblongus; sep. erecto, decivina, pinnulis integris, angustis isistructa, acumine longissimo iubfoliaces, dentato; styli lanati, disco plano; $\operatorname{cor} . . . . . .$. ; fruct ovisider-comicus, basi rotuniatus, apice valde attenuato-strangulatus.

Hab. Helvetia, Lalais: Lercarin, elt.4000' (L. Favrat).

$X X$ Petiohi omnes inermes.

1974. Crepinia calopetala Gogr 8ab. N-2586.

tcculei breves, Dilatato - subfalcati, as ramos flor geminati; fol. obovatoinlonga, subacuta, basi rotunarta, wo costam eglanaulasa; gentes simpíws, aperto-triangulares; pet eglanoulosi, inermes; stip. rubentes, longse, angustiores, auriculis divergentibus; per. 1-2, glabri, 6-7 m. longi; trebus ovoiders; sep. purpurea, pinnulis latis, 1-2-2intatis instructa, acumine late foliace-Dentato; styli lanati, oisco plano; cor maxima, intense rosea, culiata; fruct. ovoidens, apice attemuatus.'

Hab. Suecia, Hestrogothia: in monte hinnehulle ad Oster ct Yester- have (Y. \&. Latterstest). 
$-274-$

1975. Crepinia malacotricha Gagr mss.

Acculei breves, parum Dilatati, temuiter et valis falcati, as ramos fler. copissi; fol sat late obovato-oblonga, abrupte et parum acuta, basi corbata, intense viridia, mollia, ab costam eglaniulosa; Dentes simplices, ovato-perti, pet inermes, eglaniaulosi; stip. sivides, sat latae, subtus tomentosa, awriculis suboivengentibus; peo. 1-2, glabi; 2-3m.longi; tubus svoidens, valte glaucus; sep. pinnulis tomentosis, omnibus latis, laterahibus 2-3-Dentatis, scumine autem integrs; styli lanati, Dises plano; cor.......; fuct ovideres, basi subrotungatus, apice strangulatus.

Hab. Suecia, Halland: ad Hown (F. t. Gabuielsson).

1976. Crepinia forealis Gogor Gab. No 2587.

Acculei falcato-Dilatati, ramos flor. 2-3-ti; fol. oblongo-obtusiuscula, basi rotuniata, intense sirisia, ad castam eglandulasa; ientes simplices, aperto - triangulares; pet eglasiaulase; inermes, stip. latissimac, anriculis rectis; ped. 2-3, glabri; 15-17 m. longi; tubus ovoideus; sep. erecta, pinmulis integris, angustis instructa, acumine late foliaces, dentats; styli lanati, Disco plano; cor.......... fruct magnus, ovoriens, basi plus minusse aecurrens, aprice breviter strangulatus.

Hab. Inecia, Hestragothia: in monte himnetaulle as Hellekis (y. \&. Letterstegt).

1977. Gepinia solstitialis (Mess. Prim. fl. gahi. I, p. 324; yus Imum. Pood.

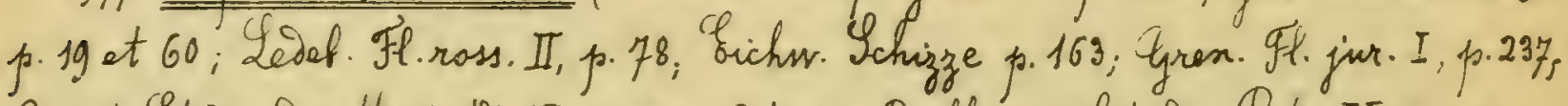

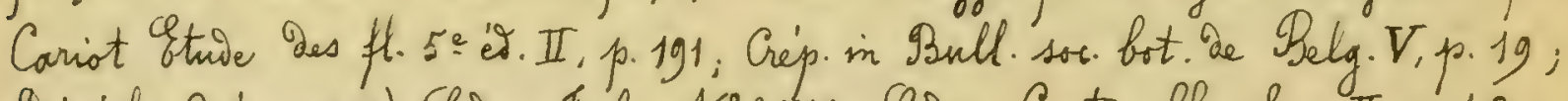
Déségl. Cat. p. 201), Gogr 8ab. Y:2588, Gogr Contr. fl. slar. II, p.42. C. coniufolia var a. (Dumart. Mon ros. belg. p. 62, Déségl. Essai p. 86).

Srutex quarriperaclis; aculei falcato-gilatati, ad ramos flexuosos 1-4mi, stipulares; fol late ovato-acuminata, val elliptica, intersum obtusa, basi rotuniata, soepe biserrata, subtus cinerea; pet. inermes, eglanioulosi; stip. pubescentes, longae, auriculis rectis; ped. 1 3, glabri, 1 cent. longi; twe bus ovsidens, glancus, sep. pirnulis brevibus, ciliatis instructa, acumine elongato, gilatato; Atyli lanati, diseo paulo conico, corrosea, mediocris; fruct. ovoidens.

Hab. Polomix, Racovia et Leopolis, (Besser).

toec est, pro maxima parte, Diagnasis ipsuismet Besseri prims 
$-275-$

erita; sel e specimine wuthentico in herb. OC. a Véséglise locs cita to viso Descriptionem accuratiorem Dex:

1978. Crepinia theratophila (Gogr Essai p. 32, nomen solum, Giggr Ros. nor. I, p.14); Gogr \&ab. N-2589; Gagr Contr. fl. slar.II, p. 43. Gogr Herb. ros. $N=198$.

Atculei falcato-dilatati, ad ramos flor. Densos, purpuress et arcuatos sat copriosi; fol elliptica, breviter acuta, basi corsata, intense viridia, ad costam eglandulosa; Dentes simphices, ovati, pet. inermes, eglandulosi; stip. subtis pubescentes, auriculis divaricatis; per. 2-4, glabri, 7-10 m. longi; tubus oboratus; sep.reflexa, pimmlis Dilatatis, elongatis, eglanaulosis instructa; styli isense villosi, Dises subplans; cor. pallide rosea, moschata; fruct. ovoideus, basi Depressus, aprie attemuatus.

Hab. Ihoone, at tornas (Nh. Ganooger).

Ooor flarum gratus, moschatus non solum in hac specie invenitur sed etiam in onusibus gregis C. corcifolixe, Dumetorum et affinium, saltem accidentalibus, wt ipse jam longs tempore ad virum ego notaxi.

1979. Crepinia phyllostephana Cogr bab. N-2590.

Aculei parri, breves, falcato-dilatati, as namos flor. copriosi; fol. late ovatoelliptica, subsbtusa, basi notungata, intense virisia, al costam eglaninlosa; ventes simplices, ovato-a perti, pet. inermes, eglanoulosi; stip.magnace, anriculis rectis; pes. 1-2, glabri, $12-14 \mathrm{~m}$. longi; tubus ovoidens; sep. erecta, pirnulis ample foliaceis, longis, 1-2-Dentatis instructo, acumine latissimo, inciso; stafli lanati, gises plans; cor........, fruct magnus, ovoidens, basi valde gecurrens, apiee subattemuatus.

Hab. Suecia, Gestrogothia, at Sattra (P. F. Musnguist).

1980. Creninia acanthophora Gogr \&ab. $\mathcal{N}-2591$.

toculei falcato-Dilatati, ad ramos flor. copiosissini; fol sat parra, obovato-acuta, fasi subrotuniata, io costam eglanivulosa; Dentes simplices, aperto-triangulares; pet. inermes, eglaniulosi; stip. mediocres, awriculis givergentibus; pes. 1-2, glabri, 6-7m.longi; tubus ovoideus; sep. hrevia, gecioux, pirmulis integris, angustis instructa; styli lanati, gisco plans; cor........ fuct parrus, ovoidens, basi rotuniatus, aprice subattemeatus.

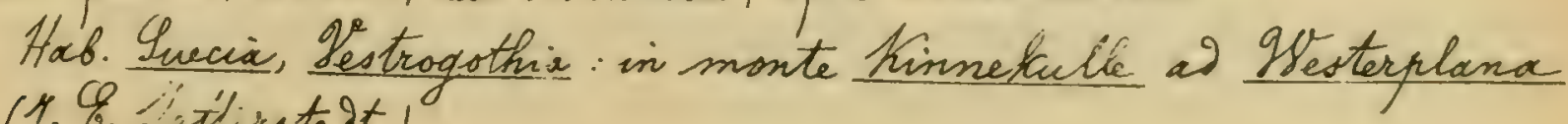
(f. E. Tietirstegt). 
$-276-$

1981. Geninia Nymamiana Giogr Lab. N:2592.

Aceulei Jilatati, temuiter faleati, a ramos flor. copiosi; fol. late cbovato-acutiuscula, basi rotuniata, à costam eglanioulosa; Dentes simplices, ovato-aperti; pet. inermes, eglanoulosi; stip. rubentes, auriculis rectis, ped. 1-2, glabri, 11-19 m. longi; tubus ovoidens; sep. pinnulis integris, latissimis instructa, acumine magno, integro; stygli maximi, lanati, gisco plano icar........ fruct, ovorieus, apice attemiatus.

Hab. Sueciax, Imolanixix: at Barkiryt (Y. N. Ychenty).

XXX Petioli omnes aculeati.

0 . Folisla oborato-oblonga.

1982. Crepinia seleroacantha Gigr \&ab. N-2593.

Acuki turi, valiov, falcato-gilatati, as ramos flor, verticillati; fol. obovato-oblonga, parum acuta, basi rotunioata, ad costarn eglanisulasa; Dentes simplices, aperti, triangulares, pet hine inde glaniulasi, sculeati, stip. latae, longae, auriculis givergentibus; pos 1-3, glabri, 4-6 m. longi; tubus ovoidens; sep. Secirua.......; styh magni, lanati, giseo plano; cot.........; funct maximns, ovoioiens, basi valde athennatus, apice subeontractus.

Hab. Helvetia, galais: as Heremence, grasserleitung (G.0. Ifolf).

1983. Crepinia sacia Gogr mss.

stculei gebiles, subsilatati, temuiter falcati, as ramos flor.copiosi; fol. latiuscule obovato-oblonga, acuta, basi rotuniata, co costam glasioulosa; Dentos simphices, ovato-subconvergentes; pet. basi assperse glanisulosi; aculeati, stip. virides, magnae, aurriculis sat Divergentifus; pes. 1-3, glabri, $12 \mathrm{~m}$. longi; tubus sbovato-oblongus; sep.........., styli lanati, gisco plano; cor.......; fruct. majusculus, obovato - oblongus, basi rotunoatus, apice strangulatus.

Hab. Iranssilvemia, Iorda toranyos: at Iorda Hasadék (L. Richter).

1984. Crepinia cooptata Gogr.

toculei breves, parri, parm falcato-Dilatati, as ramos flor. geminati, fol. sat late oborato-oblonga, breviter acuta, basi rotinioata, ad costasx glaniulosa, gentes simplices, ovato-recti; pet. eglanioulosi; parce aculeati; stip. virives, latac, sat longae, auriculis rectis, pes. 1-2, glabri, 4-5 m. longi; tubus sbovato - oblongus; sep. pirnulis integris, sat angustis ins- 
tructa: styli lanati, Disco plano, car......... finct. mediocris, obovato - suboblongus, basi pauls bepressus, apuice abrupte attenuatus.

Hab. Hungaria bor., Lipto' a Itankova (L. Richter).

o Foliola obovata vel oblonga.

1985. Crepinia cinerascens Gogr 6ob. N=2594.

toculei valivi, falcato-dilatat, as ramos flor. 2-3-ti; fol. ample oblongo-acutiuscula, basi notuniata, ad costam eglaniulosa; Dentes simphices, lanceolato-perti, pet aculeati, basi paulo glanioulosi; stip. latac, auriculis givergentibus; per. 1-9, glabri, 5 -7 m. longi; tubus ovoideus; sep. decioura, pinnulis longis, latis, eglanoulosis instructa, acumine longissimo, foliaces, integro; styli lanati, Siseo plano; car........ fruct.maximus, ovoideus, utrinaue subrotuniatus.

Hab. Helvetia, Talais: 2 Mayens de Sion (L. Forrat).

1986. Crepinia peraueens Gogr mss.

taculei breves, parvi, inclinato-subililatoti, at ramos flor geminati; fol. parva, oblongo-subacuta, basi rotuniacta, costam parce glanoulosa, aentes simplices, triangulari-convergentes; pet. aculeate, hine inde paulo glanoulosi; stip. virides, latae, breves, auriculis suboivergentibus; peo. f, glabri, $7 \mathrm{~m}$. longi; tubus obovato-oblangus; sep.......... styli lanati, Nisco plano; cor........, fruct.majusculus, obovats-oblongus, utrinque attennatus. Hab. Iranssilvania, Gordx toranyos: at Eorda Hasadék (2. . Richter).

1987. Crepinia culmosa Gogr mss.

taculi longuisculi, dilatato-subsecurrentes, tensiter et valie falcati, no ramos flor. geminate; fol mediscria, oblonga, vix attenuata, basi sensim xotionouta, lacte viridia, subtus pallida, as costam eglanioulosa, pet. eglaniaulosi, parce acenleati; stip. longac, sect angustae, subtus pubescentes, vericulis suboirergentibus; pet. 1, glabri, 10-12 m. longi; tubus sbovato- oblonqus; sep. pinmulis onnibus gilatatis plerumque integris instructa; styli lanati, Disco plano; cor......... fruct. amplinscule obovato-oblongus, basi oepressus, apice abrupte attenuato - strangulatus.

Hab. Eranssilvania, Eoria frampos: as Enri Hasadè (2. Richter). 
$-278-$

1988. Ciepinia muricota Gogr 6ab. N-2595.

foculei minutissini, termes, falcato-Dilatati, at ramos flor. copiosi; fol oblongo-acuta, basi rotuniata, as costam eglansulosa; dentes sinphies, lanceolato-aperti; pet eglanioulosi, aculeati; stip magnace, auriculis Divergentibus; peo-1-2, glabri, g-11 m. longi; tubus ovoideus; sep. erecta, gorso glanioulaso-muricata, vix pinnata, pinnulis brevibus, eglasioulosis, latis instructa, weumine foliaceo; sthli lanati, gisco plano; $\operatorname{cor} . . . . .$. , fruct. maximus, ovsideus, basi abrupte sed breviter decurrens, apice strangulatus. Hab. Inecia, Gestrogothia : in monte Kinnehulle (P.F. Lunsoguist).

1989. Crenimia munita Coogr mss.

teculei longi, Dilatati, temuiter falcati, ai ramos flor. sat copiosi; fol. coriacex, ample oblanga, vis acuta, basi rotuniata, inferne as costam 1-2-aculeolata, Ientes simplices, ovato-aperti, pet aculeati, eglanonlos;, stip. vinives, breves, angustae, auriculis suboivergentibus; per. 1-2, glabri, $10 \mathrm{~m}$. Congi; tubus oborato-sblongus; sep. pinnulis latis, ommibus 2-3-gentatis instructa; styli lanati, Disco plano; funct. mesicerris, obovato - oblongus, basi subiecurrens, mivio turgivus, apice strangulatus; cor. ignota.

Hab. Hungaria bor, Haag: as Kováesi (2. Michter).

1990. Crepinia sphaersstigma Gogr \&ab. N-2596.

Acculei valioi, falcato-Dilatati, ao ramos flor. copiosi; fol. late obovato-subacuta, basi coridata, lacte sirisia, costam eglaninlosa; gentes late aperto-triangulares, inf. paulo biserrati, pet. aculeatissimi, eglanDulosi; stip. latissimae, auriculis rectis; ped. 2-3, glabri, 5-7 m. longi; tubus ovoisens; sep. erecta, eglanoulosa, pinmulis latis, longis instructa acumine foliacer, integro; styli maximi, globosi, lanati, gisco plano; cor......., fruct. magrnus, ovoidens, turgions, basi paulo depressus.

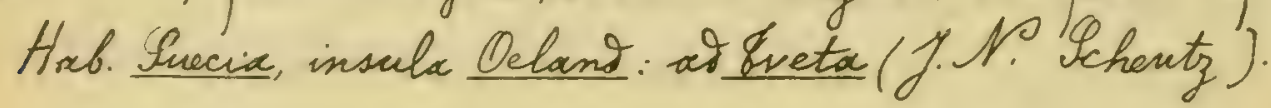

1991. Crepinia Laperrousiana Ciagr Lab. No2597.

foculer minimi, tennissine falcati, at ramos flor. stipulares; fol. parva, obovato-acuta, basi rotuniata, al costam eglaniulosa; gentes simplices, lanceolato-convergentes; pet-eglanivulosi, aculeati; stip. ubbentes, ouriculis Sivergentibus; peo. 1. glabri, 8-g m. longi; tubus ovoidens; sep. pinnulis pancissinns, omnibus integris, angustis instructa; stygh lanati, 
Sisci paulo comico; cor. lacte rosea, eciliata; funct. ovoidens, basi gepressus, of apricem atterneatus.

Hab. Iyrenaer Orient., Copsir; in silva Les tengles (G. Gautier).

1992. Crepinia Borbasiana Gogr mss-C. aumetorum var. leptotricha (Piorbás ex parte).

Atculei robuste, valde folcato-dilatati, ad ramos flor. verticillati; fol ample sbovato-acuta, basi rotunsata, pallisius viritia, subtus fore virescentia, at costam eglanoulosa; Dentes simplices, late triangulari-subconvergentes, pet. eglangulosi, aculeati; stip. maximae, virides, subtus pubescentes, auriculis subsivergentibus; pes. 1-3, glabri; 11-13 m.longi; tubus obovato-oblongus; sep. pinnulis sat angustis, vix dentatis coprose instructa, acumine foliaces, Dentato; stypi lanati, disco jlans; cor.........; fruct. mevioeris, obovato-oblongus, basi subettenuatus, apice contracto-substrangulatus.

Hab. Oranssilvania, Molnok Doboke: as Kaerkes (2. Onichter).

\section{Crepinia subrevoluts Gogrmss.}

Atculei breves, falcato- Dilotati, ad ramos flor-serticillati, fol. mesiscria, Sborset a, vix acuta, basi notumiata, crassa, nervosa, lacte virisia, subtus pailliva, xid basin costae poulo glandulosa, Dentes simplices, subrevoluto-uinirlati, late orato-convergentes; pet. hine inie parcissine glanioulosi; aculeati; stip. breves, sat angustae, viriides, subtus toto villosac, auriculis subsivergentious; per. 1-3, glabri, 1 cont. longi; tubus obovato-suboblongus; sep. villosa, pinnulis integris, latiusenlis instructa, acurnine foliaceo; styli. lanati, exserti, disco subcomico; cor........, fruct. parvus, ovoidens, ettrnique sed aprice proesertion attenuatus, substrongulatus.

Hab. Granssilvania, Solnok Doboke: ad Satorhegy (2. Richter).

t+ Foliola basi attenuata.

§. Petioli imfrior aculeati.

1994. Crepinia Dambialis Gogr Gab. N:2598.

A-culei breves, falcato - Dilatate, ad ramos flor. foliosos panciores; fol. minutissima, oblongo-acutiusenla, basi breviter attenuata, cinerca, ao c.ostam eglanioulosa; ientes parvi, simplices, wato-converyentes, pet. eqlaniselosi, inferiores soli-xculeati, stip. longae, an inulis rectis; ped. 1-2, glabri, 
$-280-$

6-7 m. longi: tutus obovato-oflongus; sep. pirnmlis foliaceis, brevibus, 2-9contatis instructa; acumine foliaces, integro; styli lanati, Disco subplans, cor.magna, pallive rosea, ecihata; fruct. ovsidens

Hab. Hungaria, ins. Damubialis Csepel: al Nillfali (Feichtinger).

1995. Crepinia alpivaga Gogr fab. N-25g9

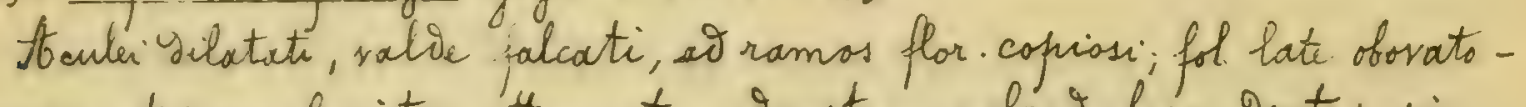
oblonga, utrinque breviter xttenuata, of costam eglandulosa, Dentes simphices, Lanceolato-convergentes; pet. eglanioulosi, inferiores soli aculeati; stp. Silute rubentes, auriculis subrectis, per. 2-3, gtabri, $12-14 \mathrm{~m}$. longi; tivins. ovoidens; Sep. pinnulis anmibus integris, latis, instructa, Diu persistentia; styli. lanati, Lisco plans; cor......., fruct. magnus, ovoidens, basi gepressus, apic.. freviter attemuatus.

Hxb. Basses - tolies, at Bouzohieres (Proal).

§. Setioli superiores aculeati.

1996. Creninia rhactica Gogr bab. N-2600.

Toculei valior, geminati, gilatati, wo ramos flor. rectrusenli vel inclinati; fol. obovata, afsice subotuniata, fasi attenuata, firma, nervosa, id costam eglandulosa; sentes simplices, perti, pet. rubentes, eglanioulosi; sup. soli aculeati; stip. Latoe, rubentes, pubescentes, auriculis givergentibus; ped. 1-2, glabri, 3-7 m. longi; tubus obovatus; sep. pinmulis integris, parm latis instructa; styli lanati, gises subplans; cor. pallive rosea; fruct. ovoidens, utringue roturioitus.

Hab. Helvetia, in Apribus rhaticis (N. Seringe).

1997. Geninia immutata Gagr mss.

tenlei breves, Dilatati, parum falcati, ad ramos flor mulh; fol meiorcria, obovato-subacuta, basi attenuata, intense virivia, as costam egfanDulosa; Dentes simplices, ovato-comvergentes; pet. eglansulosi, superiores soli menleati; stips freres, latae, virides, subtus flabrae, anriculis rectis; pes. 2-4, glabri, $12 \mathrm{~m}$.longi; tubus obovato-oblongus; sep. pisinnlis omnitiow integris, tatis instructa; siyh os perse pilosi; riscs plans; cor........ fruct. ovides-conicus, basi rotuniatus, apice longius aitermatus. Hab. ger, at tompus (Jisert). 
$-281-$

1998. Crepinia cistetorum Gogr mss.

teculei breves, parum Dilatati, valde falcate, ad ramos flor mulki fol. oblongo-acuta, basi sensim attenuata, paltive sirioia, as costam eglanioulosa; Dentes simplices, svato-recti, pet. hine inse glantulosi, superiores ioli aculeati, stip. virides, breves, latae, subtus toto pubescentes, auriculis $g_{i}$ vergentibus; pas. 1-3, glabri, $8 \mathrm{~m}$. longi; tubus ovsideres; sep. pinnulis smmibus integris. lateralibus vero parum latis instructa, acumine foliaces; styli glabri, disco plano; cor. magna, pallixe roser, eciliata; fruct evoisens, basi poulo attenuatus, aprice hais contractus.

Hab. Har, Camarat: in cistotis maritimis (M. Ganoger), ubi. frutex clegans jam sub fine toprikis gat flores.

1999. Crepinia jugularis Gogr \&ab. N-2601.

teculei debiles, parum gilatati, minute et longe falcate; fol obovatooblonga, price fere rotuniata, basi attenuata, costam eglanivilose; dentes simplices, convergentes; pet. inferne aisperse glandulosi, virides, superiores soli et parce aculacti, stip, Songatae, latiusculae, pubescentes, auriculis Divergentibus; pet. 1-3, giabri, $3.9 \mathrm{~m}$. longi; tubus oblongus; sep. erecta, pinnulis angustis, parce gentatis instructa, acumine lato; ityli lanati, Sisco plano; cor......... fruct. magnus, ovoideus, utrinque ralde attenuato-strangulatus.

Hab. Helvetia, galass: Les Favres in valle Champex (De la Toie).

2000. Crepinia culpata Gogr mss.

Atoulei minuti, breves, folcato subsilatato, as ramos flor pactulos stipulares; fol amplinscule oblonga, abrupte acita, basi sensim attenua. $\frac{1}{1 a}$, pallive viridio, subtus canescentia, as costarn oglanialosa; dentes simphices, ovato - recti; pet. eglanoulosi, superiores soli aculeati, stip. latius culae, sat breves, subtus to to pubscentes, auriculis rectiusculis; ped. 1-2, glabri, 10-12 m. longi; tubus suboblongus; sep. pinnulis brevibus, ommibus foliaceis, Dentatis instructa, acurnine inciso, elongato; styli lanati, Diseo plano, cor......., fuct. obovatus, basi gepressus interdumve ao pearne. abupte incrassatus, apice strangulatus.

Hab. Eanssilvamia, Hairomozék: at Bügois (2. Richter). 
$-282=$

§\$\$. Tetioli onnes inermes.

$\sim$. Ityhi glabri, sparse pilosi vel villosi:

2001. Grepinia sarentana Gogr Eab. N:2603.

Acculei breves, falcato-gilatati, at ramos flor mulli; fot ample oblongo-subacuta, basi attenuata, as costasn eglanoulosa, ientes simphies, ovato-convergentes; pet. inermes, eglanoulosi; stip. virides, auriculis divergentibus; per. 1-3, glabii, 12-14 m. longi; ; tubus obovato-oblongus; sep. extro intense rubra, pinnulis annibus foliaceo-laciniatis, longissimis instructa; styli glabri; Disco valte comico; cor. eciliata, fruct magnus, sbovato - oblongus, utrinque paulation sepressus. Hab. Mossia orient., Astrakhan: ar Sarepta (Ao. Mecker).

2002. Grepinia scanicia Gagr bab. N-2604.

Acculei pauciores, breves, falcato-Dilatati, ad ramos flor. Hlexnosos nulli; fol. obovato-senta, basi breviter attennata, lacte viritio, ai costam eglanDulosa, Dintes simplices, lanceolato-aperti; pet. inermes, eglansulosi; - stip. longae, Silatatae, auriculis brevibus; pes. 1-2, glabri; 5-6 m. longi; tubus oforato-oolongus; sep. erecta, pinnulis integris, brevibus, latis instructa.; acumine subfoliaces; styli hirssuti, bises plans; cor........; fruct obovatooblangus, apice valse strangulatus, centrali vers recurrente.

Hab. Suecia, gestrogothia. in monte Kinnehulle (y. G. Tettersteit).

2003. Prepinia blepharophylla Gogr \&at. N-2605.

Eculei sparsi, parum falcato-gilatati, ar ramos flor. Plexuoses nulli; fol. obovato-oblonga, acuminata, basi attenuata, longe pilosa, at costam eglaniulosa; dentes simplices; pet inermes, eglanoulosi, stip. angustae, anriculis sivergentibus; per.1-9, intersum 1-9-glanaulosi; 11-14 m. longi; tubus avoireus; sep. mox geciobra, pirnulis brevibus, villosis instructa; styli aioperse pilosuli, gisco subplano; cor........, fruet ovoirens, basi Depressus, apice abrupte strangulatus.

Hab. Rhone, supra Grancheville (9. Chabert).

2004. Gepinia tephrophylla Gogr Bab. N-2606.

Acculei copiosi, gecurrentes, falcato - Drlatate, o ramos. flor saepe gemi. nati, fol ample elongata, utrinque paulation atternuata, subtus cinerea, 
$-283--$

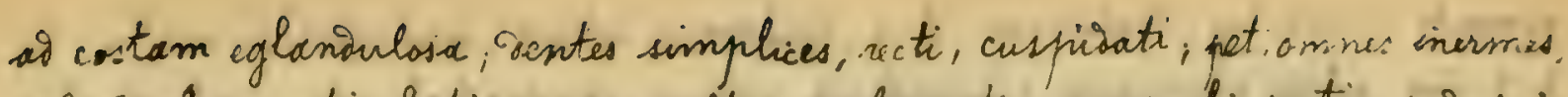
eglansulosi; stip. latissiniae, subtus pubseentis, auriculis rectis; pes. 1-3, glabri, 7-11 m.longi, tabus ovoidens; sep pinmulis brevibus, eglanioulosis, angustis instructa, styli assporse pilosuli, gisco planinsaulo; cor........ , huct. ovoidecus, utrinque paulo depressus.

Hal. Tyrenaei centr., prope Gicre ad viam Heiss (H. Bardère).

$\sim \sim$ Ityli lanati.

X Foliola ofovata vel obovato-solonga.

2005. Crepinia polyacantha Gogr \&ob. N:2607.

Aculei copioss, breves, Dilatati, temuiter folcati, or ramos flor. sparsi; fol. obovato-subacuta, basi breviter attenuata, lacte virisia, ad costam eglansulosa; dentes simplices, ovato-aperti, pet inermes, eglangulosi; stip. latae, Congae, anriculis divergentibus; ped. 1-2, glabri, 6-8 m.longi; tubus or sidens; sep. erecta, gorso sparse glanisulosa, pinnulis integris, parame fatis instructa, acumine foliaces, integro; styli fanati, gises plans; cor......... fruect. obovato. sllongus, utrinque aittenuatus.

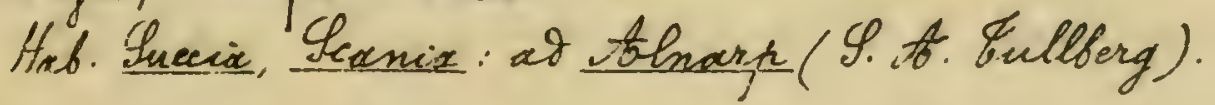

2006. Grepinia colorans Goger inss.

Atcule breves, rari, falcato_Dilatate, ar ramos flot. puerpuress mulli; fol. coriacea, mediocria, obovato-acuta, basi attenuata, al costam eglaninlosa, Dentes simplices, triangulari-convergentes; pet.eglantulosi, inarmes, stip. rubentes, latae, longae, awiculis givorgentibus; per.1-3, glabri; $8 \mathrm{~m}$. longi; tubus obovato-oblongus; sep......... stegli magm; lanati, giseo plano, cor......., fruct. majusculus, obovato-oblongus, basi ritivnoto Dilatatus, puice conics-stranigulatis, centrali vero infine atteruatus.

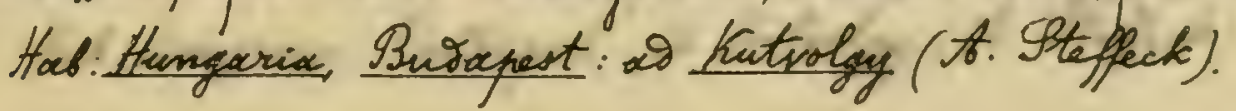

2007. Crepinia lencrphora Gogr Gab. N-2608.

Rubro-canescens; aculei Suri, Dilatato-rectivaculi; à ramos flor. purpureos copiosi; fol. obovato - oflonga, utringue attenuata, abrupte zenta, subtus candida, gentas simplices; pet eglanaulosi, plorumque narmes; stip. inkentes, latissimar ; per.1, glabri, 3-5m.longi; tubis aroideus; sep. brevia, vix pinnate, pirmulis pancioribus, angustis instructa; styli lanate, gises plano; cor......; 
$-284-$

fruct. ovoideus, utringpe gepressers.

Hab. Houte-Sardic, as Nont-Sion (Miget).

.008. Genimia sequax Gigr mas.

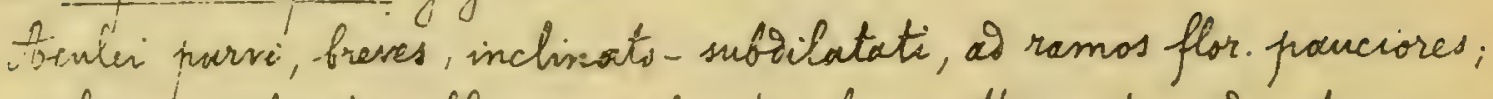
fol amplissime ofovato-oblongi, subacuta; basi attenuati, as costam non

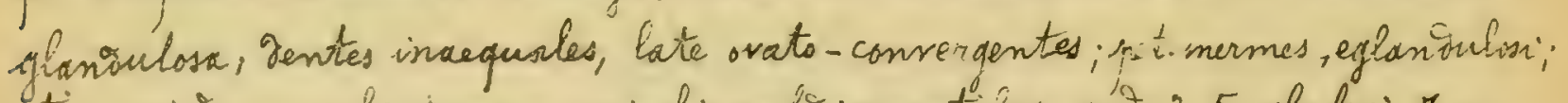
stip. sisitis, amplissimac, auriculis sulivivergentis sov; pe.t. 2-5, glabri, $7 \mathrm{~m}$. longi; tubus ovoiducus; sep. pinmulis ormmigus sat tatis, dentatis instructo, acumine integro, stijli sublanati, oisco plano; cor. eciliata; fruct.ovoiders, basi gepressus, aprice atterinatiss.

Hab. Hungaria, Indapest. ad Driankgyy (2. Pichter).

2009. Qrepinia vellicata Qjogr mss.

Aculei tennes, subdilatato-rectiusculi, ar ramss for intersum verticillati; fol ample obovato - oblonga, subacuta, basi breviter attemeata, as costam eqlanimisa; ientes simplices, late ovato-aparti; pet inarmes, cglandulosi; stip. virides, Lata., tongai, anriculis girsegentibus; ped. 1-2, glabri, 1 cent. - Congis; tubus sovats - oblongus; sep........ styi ianati gises plano, finct. nagnus, obovats - fitangus, basi gepressus, apice attenuats-strangulatus.

Has. Hungaria bor, Lipto' as Lubochene (2. Richter).

2010. Crepinia microphylla Gogr tab. N-260g.

toculei breves, falcato- oilatati, at ramos flor copiosi; fol parra, glace cesientia, obovato - oblonga, acuta, basi attenvata, as costam eglanivelosa; Fentes simplices, aperto-trianqulares; pet. eglanoulosi; inermes; stip. Lonqae, angustar, auriculis givergentibus; pê. 1-2, gabri; 8-10m. Longi; tubus oroisens; sep. vecta, pinmulis sat latis, copiose-gentato-glanialosis instructa, acumine foliaces, integro, styli lanati, giscs plans; cor....... fruct anguste ovoidens, basi rotuniatus, aprice strangulatus.

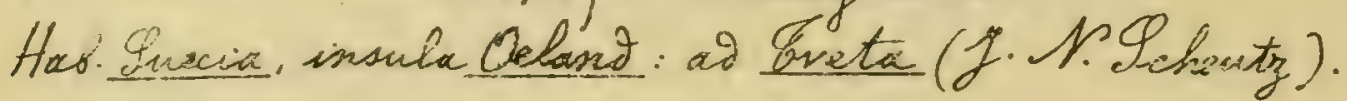

$X X$ Foiviola oblonga:

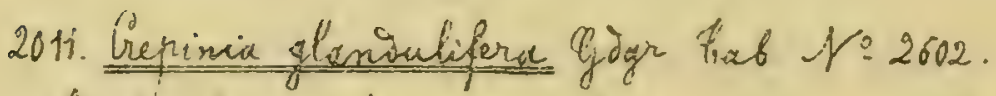

tóculer lingri, tenuiter falcati, as ramos flor. coppiosr; fol. oblanga, 
$-285-$

utrinque attemuats-acuta, $x$ d castam eglaniulos; bentes simplices oratoconvergentes; pet.inermes, eglanoulosi; stip. Latae, rubentes, auriculis Divergentibus; per. 2-4, glabri, 7-g.m.longi; tubus obovato-oblongus; sep. Porso prorsus glanioulosa, pinnulis pancioribus, integris, sublinearibus instructa, ittyli lanati, Diseo plans; cor........ fruct. obovato-oblongus, basi attenuatus, apice strangulatus.

Hab. Inghia, Cheshire: as Mest Kirby. (Jarbori-Lervis).

2012. Crepinia cyanoclada Gigr mss.

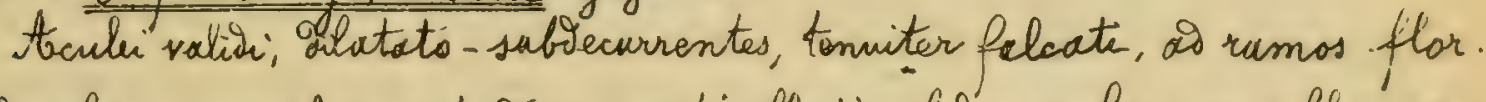
valde glauco-coeruless interousm verticillati; fol amplissime oblonga, utringue paulation attenuata, lacte viridia, subtus grisea et id costam eglanisulasx; Dentes simplices, Late ovato-convergentes; pet inermes, eglanDulosi; stip. maximae, longae, subtus pubescentes, virides, auriculis sub. rectis; ped. 1-3, glabri, 2-4m. longi; tubus avaidens; sep.clongata, pinrulis integris, ommibus foliaceis, pubescentibus instructa, styli magni, lanati; cor...... fruct. svoideus, basi rotingatus, apice semper attenuatotrumeatus, centrali vero vel solitario inferne incrassato-decurrente.

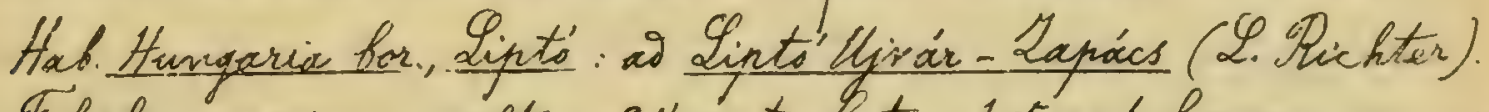

Foliola maxima, saltem $21 / 2$ cent. Lata, $4-5$ cent. longa, eam eximie. pruinos-coerulescentes.

2013. Crepinia Dissidens Gogr Ool. N:2610.

foculei bremes, falcato-gilatati, a ramos flor-geminati; fol sat pouva, viriaia, oblongo-lanceolata, basi attesnuata, id costam eglanivulosa; gentes simplices, aperto-triangulares; pet inermes, eglanoulosi; stip. Dilatatae, awviculis vaise givargentibus; per. 1-2, crassi, glabu; 7-8m. longi; tubus oroidens; sep.erecta, Decidua, pinnulis latis, eglanoulosis instructa, acu mine subfoliacen, integro; Htyli lanati, gisco plano; car.......; fruct magnus, abovati- oblongus, utrinque attenuatus, medis turgious.

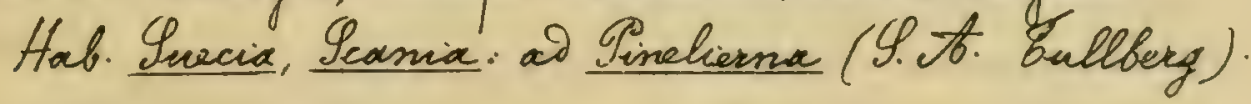

2014. Creminia cerina Gogr Eab. N-2611.

Abculei validi, as ramis flu. falcato- tilatati, fol. ample oblonga, utrinque breviter attenuata, ceriacea, nesvosa, lictescentia, costam non glaniulase; gentes simplices, aperti; pet. virises, eglanioulosi, inermes; 
$-286-$

stip. latae, vinides, subtus pulesccintes, auriculis òvergentibus; ped. 1-3, glabs: 5-10 m.longi; tubus ovoidens; sep. crassa, erecta, die persistentra, finnulis angustis, praucioribus instructa; styli lanati, disco plano; cor..........; fruct. maximus, 17-20 m. giam. latus, avaidens, basi rotuniatus, apuce Sepressus, centraki autem inferne contractus.

Hab. Phone, Craponne (A. Cariot; Boullu).

2015. Crepinia aboensis Gjogr Bab. N:2612.

tcculei gilatati, valve falcati, a namos flor. copriosi; fol ample oblongoacutiuscula, basi attenuata, is costam eglaniulosa; dentes simplices, lancerlato-aperti, pet. inermes, eylan oulosi; stip. virides, auriculis divergentibus; pred. 1-2, glabri, $11-12 \mathrm{~m}$. Congi, tubus obovato - oblongus; sep. pirnnlis parum latis, 1-2-dentatis instructa; acumine integro, styli lanati, giseo plano; cor. ignota; fruct. magnus obovato-oblongus, utringue subrepressus.

Hab. Trossia bor., Tenmix: Hbo, at Uusikaupunki (H. Hollonén).

§§§. Petioli annes aculiati.

2016. Crepinia subuls Gogr Gab. $N=2613$.

Pernmque tota rubens, pure ter folia canescentia; sculei valiti, falcratiDilatati, iramos flor. sat copiosi; for. ampluiscule oblonga, itrinque attenuata, superne virivia, sultus albula, ad costam eglaniulosa; dentes simphices, late, aperti; pat.eglanivulosi; aculeati, unbentes; stip. longue, oilatatae, rubisae, auriculis parum divergentibus; pet. 1-3, glabri, g-13 m.longi; tubus oboratus; sep. pinnulis integris, sat angustis copiose instructa; styli glabri, Biscs convics; cor aiba ant subielute rosea; fruct. bresiter ovoideus, basi subrotuniatus, apice attemeatus.

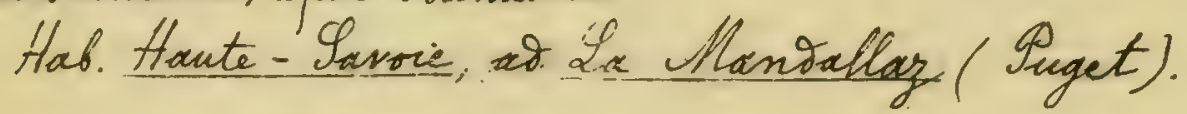

2017. Crepinia olivetorm Gogr tob. No 2614.

Aculei valioi, falcato-Dilatati, ad ramos flor. copiosissism; folmesiocria, oblong-acuta, basi attennata, a costam eglanoulosa; dentes simplices; pet. eglaniulosi, rubentes, aciuleati, stip. Dilute mbentes, auriculis 9 ivergentifus; per. 1-3, glabre; 10-11 m. longi; tubus ovocieus; sep. pinmelis integris, latis, breribus, ut et acumen, instructa; styli hirsuti, gises comico; cor eciliata; fruct. ovoidens, basi'gepressur, aprice attemnatus:"

Hab. Galloprovincia, Noitiqued (to. towtheman). 


\section{Creninia Damanabilis Gogr mss.}

focelei breves, parri, folcato - Dilatati, or ramos flor stipulares; fol, parva, oblonga, utrinque attenuata, pallide viritia, subtus canescentia, as costam eglanoulosa; gentes simplices, profunai, sublanceolato-necti; pet eglandalosi, aculeati; stip. longace, latae, subtus to to villosac, auriculis givorgentibus, pied. 1-2, glabri, 1cent. longi; tubus obovato-oblongus; sep. breviuscula, pinnulis omnibus prebesentibus, subfoliaceis, vix sentatis instructa; styli lanati, dises conic, car....... fruct. obovato- oblongus, utrinque attemeatus, apuce strangul. Hab. Eranssilvania, Solnok Doboka: at Bebes (2. Ricktor).

\section{Crepinia evidens Gogr mos.}

tbculei breves, parri, rectiusculi, parum dilatati; as ramos flor. stipulares; fol. oblonga, utrinque attenuato-acuta, potislulata, intense virisia, subtus grisicentix, oo costam eglanisulsor, Dentes simplices, lanceolato-convergentes; pet. eghaniulose; aculeati; stip. longac, sot latar, subtus tantum gorso villosulas, auriculis Divergentibus; pet. 1-2, glabri, 10-12 m. longi; tubus suboblongus; sep pinmulis glabris, anmibus foliaceis, Pentatis instructa, styli villosi, gisco comico; cor.......; fruct obovato-oblongus, magnus; basi saepius breviter Decurrens, apice abrupte stringulatus.

Hab. Iransilvania, Haromszek: as Bikizäs, Maimis füvö (2. Richter).

2020. Gepinia supracena Gogr \&ab. $\mathcal{N}: 2615$.

Cinereo cana, sculei validi, Silatati, recti vel inclinati, ar ram fos flor. copiosi; fol parra, oborato-oblonga, utringue attemuata, supane pallioe viridi-lutescentia, subtus canescentia, frominule nervosa, ad costam eglansulose, dentes simplices, aperti; pet: aculeati, interoum asperse glanoulosi; stip. parvec, angustac, auriculis givergentibus; ped.1-3, glabri; 10-12m. longi; tribus obovats-oblongus, sep. pinnulis integris, angustis instructa, erecta, style hirsuti, Disco plano, cor....... pruct. parves, fovatus, base subrotunsatus, apice strangul.

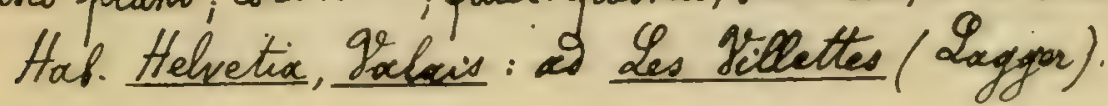

\section{Crepinia temuinernis Gogr mss.}

toculei breses, parri, vir dilatati, tenuiter falcati, ab rams flor. rubros copiosi; fol. crassa, sat late obovato-oblange, acuta, basi breviter attenuata, at costam eglanoulosa, dentes simplices, ivats-convergentes; pet. eglanoulosi, renleati; stip. virides, latae, breviusculae, curciculis Divergentibus; ped. 1-2, 
$-288-$

Habri, 2-3m.longi; tubus ovoiwens; sep......... styli villosi, giseo plano; cor......., fruct. parrus, obovato-oblongus, basi notuniatus, ad apicem atte. mato-conieus.

Hab. Hungaria bor., Iipto': Stankova (L. Michter)

2022. Crepinia intermaria (Kit. apud thanity togat. as fl. hung. in Fora XXXIII 1853, p. 591, non Carrière, nec (rip. nec Desv.). Formanek Rosen Hachges p. 9); Gign Eab. N:2516.

foculi falcato- dilatati, ai ramos floresparsi vel gemunati; fol. ovat.-suó acusminata, basi cuneata, mollia, orentes simplices; pet. eglanielosi; aculeati; stip. subtus pubescentes; ped. 3-4, glabri, tubus oviseus, sap. prinnulis mox irciour, eglanoulosa; style villosi, cor. rosea; fruct ovoiseus.

Hab. Hungaria, ai horenic (Kitaibel): Buiapest (Y. Borbis); Moranis, Hochgesenke. (Formanek).

Calyeis tubus orstus 2ictus, sed pstiver ovoidens.

Rosa intermeivia Carriere in Renue hart, 1868, N:14 est species chi-

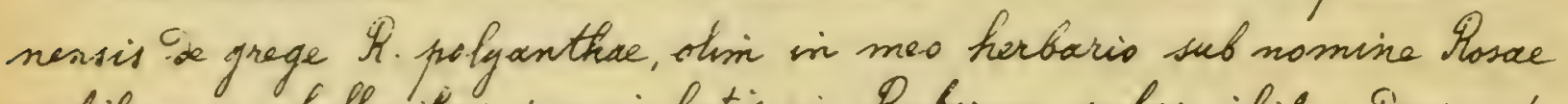
rubiformis, of floribus naniculatis eis Rubarum subsimilibus, iesignata. Rosa intermisia Ciépin m Bull. soc. bot. Maly. DII, p.246, od Sugetias (Gomentosea) pertinet;

Rosa intermedix Dess. Ofs. p. 157, N-6, mihi ignota est.

2023. Crepinia heteroon Gogr mss.

taculei breves, Gilatiti, temuiter falcati, as ramos flar nulli; for ample oblong - acuta, basi attenuata, supra pallive viriai-lutescentia, tandem subglabra, subtus virescentia, nersosa, ais costasin eglaniulosa; Dentes fere immes biserrati, late triangulari-subeonvergentes; pet. aculeat, asperse glaniaulosi, laxe vallosi; stip. longae, sat latac, gorso pubescentes, anriculis rectis; pers. 1-2, glabui, 8-10 m. longi; tubus ovoivens; sep. pinmeles glabris, bresibus, fohiaceis, vativ laciniate-incisis instructa, acumine foliaces, longo; styli magni, inferne sublanati, aprie glabir, bisco subptane; cor. mediocris, rosea, eciliata; frect. svoivens, basi subocurrens, apice stranginlatus.

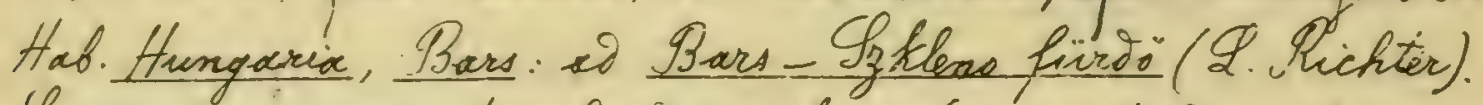

Ipecies, cum sequente, of in iumentom laxius, tandem od fraginam. superiorem fohiolorum fere cranisum, forse melius ad sect- firaceidentern,Iilosac sciliat, pertinet. 
$-289-$

2024. Burvinix inelanophylla Ggar ¿ab. N-2617. Gogr Herb.ros. N-256. toculei falcato-gilatate, as ramos flor. elongatos, flexcrosos copiosi; fol. maxima, 4- $41 / 2$ cent. longa, 25-30 cent. lata, oblongo-rhomboitahia, xcuininata, fasi sittemuata, superne nitive atrovirentia, subtus pallita, as costam eglanoulosa; Gentes simplices, late, aperti; pet acculeati, Dense villosi, eglandulosi; stip. sat angustae, gorso pubescentes, auriculis Divergentibus; per. 1-3, glabri, tennes, 20-27 m. longi; tubus suboblongus, sep. trerria, pinnulis integris, sat latis instructa, styli hirauti, gises plano; cor. albo-rosea; fruct. obovatus, apice prosertion attemuatus.

Hab. Mhone, as tornas (No. Ganooger).

2025. Crepinix tristis Gogr Eab. N=2618. Gogr Herb. ros. Nog1.

Aculei faleato_dilatati, ar ramos flor mox demioatos sat copiosic fol. oblango-acuminata, basi attenuata, pallide virisi-lutescentia, as costam eglaniulosa; Dentes simphices; pet. eglandulosi, aculeati; step. mexiocres; per. 1-3, glabri, 12-15 m. longi; tubus ovoidens; sep. pinnulis angustis, prebes. centibus instructa, acumine lato; styli villosi, Sisco subplans; cor......... fruct. ovoirens, utrinque panlo depressus.

Hab. Hoire, at Charancien (I6. Ganooger).

2026. Crepinia macrosepala Gogr Eab. No2619.

teculei rerticillate, folcato-Dilatati, ad ramos flor.copiosi; fol. anguste oblongs-lanceolata, basi valie attemuata, cinerea, at costam eglaniulosa; sentes simphices, lanceslato-aperti; pet. aculeati, gglanoulosi; stip. magnae, auriculis rectis; per. 1-3, glabri; 10-12 m.longi; tubus opoideus; sep. erecta, maxima, pinnulis integris, latis instructa, acumise longissimo, salse foliaces-dentato; styli lanati, Disco plano; cor....... fruct maximus, or videres, utrinque plus mimis Depressus.

Hab. Suecia, Sestrogothia: in monte Kinnekulle (T. F. Lunoguiat).

2027. Crepinia hypolenca Gogr mss.

Acculei tennes, brerissimi, inclinato-subirilatati, as ramos flor nulli; fol. mediocria, coriacea, oblongo-acuta, basi attenuata, as costam aglanoulosex; Dentes orato-convergentes, inf. pauls biserrati; pet eglanoulosi, subinermes; stip. rubentes, angustae breves, auriculis sat Sirergentibus; po. 1-2, glabri, 6-8 m.longi; tubus obovato-oblongus; sep. pinnulis anmibus latis, 1-2- ben- 
$-290-$

tatis copiose instructo; styli lanati, gesco plano; fruct. magnus, obovatooblongus, hasi noturniatus, aprice longe attemuato-strangulatus, centrali sero basi ebrupte decurrente, cor. ignota.

Hob. Hungarix, Budapest: io kutvoloy (At. Steffeck).

2028. Crepinia Gimorphobasis Giger mss.

Acculei breves, paivi, inclinats - subsilatati, fere teretes, ad ramos flor. articulatos geminati; fol ample oblongo-acuta, fasi atternuata, nerrosa, io costam eglanialosa; Dentes simplices, lanceolato-recti; piet. hime in'se glasiulosi, parce seuleati, stip. virises, longae, latas, anriculis subsivergentibus; pes. saepins 2, glabri, 6-gm.longi; tubus obovato-oblongus; sep. erecta, brevia, pinnulis pancioribus, sat latis, 1-2- rentatis instructa, a. cumine lato, dentato; styli magn; lanati, gives plano; cor......... fruct. sat magrnis, obovato-oblongus, hasi attenuactus, apice strangulatus, centraki vero inferne rostuniato- Dilatato.

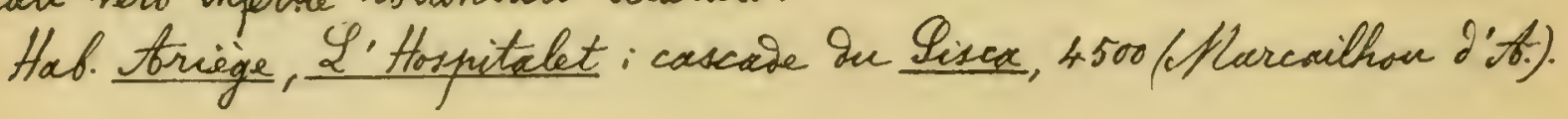

f. Fructus oblongus.

+ Ytyli glabri aut subglaberrimi.

2029. Grepinia eccremaclaia Gogr bab. N-26-20.

toculer minuti, inchinato - subililatati, ad ramos plor. Hlexuosos arcuatos saepe mulli; fol oblongo-acuta, basi attenuato, ad costarn eglanimblosx; gentes simplices, pet. inermes, eglan isulosi; stip. mésiocres, virides; peod.1-2, glabri; 17-22m. longi; tubus oblangus; sep. pinnulis subintegris, pubscentibus, sat latis insiructo; styli glabu; Disco subplano; cor pallide rosea, eciliata; fuct. breviter oblongus, basi ationnatus, aprice abupte strangulatus. Hab. Rhone, at Daroilly et Champagne (T. Chabert).

2030. Crepinia belgraviensis Goger Gab. No2621; Gogr Contr. Al. slav. II, p. 22. focrlei falcato-dilatati, ad nanos flor geminate; fol acute obovata, basi basi roturivata, ai costam eglansuloss; gentes lanceslats-aperti, inf.biserrati; pet. eglandulosi, inermes, stip. rubentes, villosae, anricalis subrectis; per. 1-3. glabri, 11-12 m. longi; tubus oblongus; sep. pinnmlis latis, 2-3-Dentatis inshructe, acumine integro; styhli subglaberrimi, Disco fere plano; cor. eciliata, fruct. ollongus, aprice pracesertion attematus.

Hab. Serbia, ad Meryraie (Yy. Iancic). 
$-291$

2031. Grepinia kabylica Giogr \&ab. N=2622.

trenlei valivi, falcato-gilatati, à rumos flor. copiosissinni, fol. firma, set late obovato-subacuta, basi roturisata, os basin costae paulo aciesulata; Dentes simplices, lanceslato-convergentes; pet. sculeato-glanouloselli, valte muricati, stip. magnae, latar, auriculis girergentibus; per. sacpe ample cssymbosi; laeves, 18 -22 m. longi; tubus oblangus; sep asmplissina, pinsulis integris, pulescentibus, fotiaceis, instructa, acumine longissime, valoe foliaceo-laciniato; styli subglaberrini, gises conics; cor.rosed, eciliata; fruct. slongus, apice proesertion attennatus.

Hab. thgerix, Kabylix: at Fort Mational, alt.3300' (O. Debeawx).

2032. Crepinia Denticalyeina Goger 8ab. N-2623.

Aculei tenues, parum falcato-Dilatati, as ramos flor copiossi; fol. late oblongo-acuta, basi sensim attomuata, ai costam eglansulosa; Dentes simplices, patule lanceslate; pet. eglaniulosi, inferiones soli paulo aculeati; stip. Latas, virites, auriculis subrectis; pes. 1-2, glabri, 20-24 m. langi; tubus sblongus; sep. pinmulis smmibus folisces- laciniatis instructa; stighi subglaberrimi, Diseo csnico; cor........ fruct. oblongus, etringine attenuat. Hab. Gars, as Couvalat prope 2e grizan (D. Ouezhiewicy).

2033. Crepinix stipitata Gogr mos.

Acculei valiai, faleato- Dilatati, a ramos flot geminati, fol ampliuscule suboblonga, set acuminata, basi rotuniata, petiolulata, ar costam non glansouloso, gentes simplices, latissime ovato-convergentes; pet. eglanoulosi, inf. inermes; stip. rubentes, longae, latae, subtus toto pubescentes, auriculis $g_{i}$ vergentibus; peo. 2-4, glabri, 11/2 cont. longi; tubus bblsngus; sep. pinnulis - annibus integris, latis instructs; stylic subghaberrimi, Disco comico; cor magna, exiliata; fruct. oblongus, utrinque attemuatus.

Hob. Har, of tempus (Albert).

2034. Crepinia nigritella Gogr Eab. N-2624.

treilei elongati, subrecti, romos flor. sat copriosi, fol late obovatosblonga, acuta, basi breviter attennata, atrovirentia, costam eglarive. losa; iontes simplices, ovato-recti; pet. eglonioulossi, mesiani soli aculeati; stip. maximore, dilute unbentes, auriculis givergentibus; pe. 2-5. glabri, 12-14 m. longi; tubus oblongus; sep. pir.melis ansibus subintegris, fo- 
$-292-$

Liacis instructo; styli subghaberrimi, Sisco canico; cor..... fruct. oblongues, utrinque abrupte attenuatus.

Hab. Gropol, as Fresilla in Inine (E. Gehni).

tot Ityhi pauls rillosi.

\$. Solich basi rotuniata.

2035. Geninia moesta Gigr Gab. N-2625.

Acenlei pauciores, falcato-Silatati, at ramos flor graciles mulli; fol. obovato-acuta, basi rotuniate, glauca, ad costam eglaniulosa; gentes simplices, svato-convergentes; pet. inarmes, eglanoulosi, stip. virices, surriculis Sivergentibus; pes. 1-2, glabri, 11-13 m. longi; tubus elongatus; sep. pinmulis latis, 1-2-ventatis instructa, acumine lato, integro; styli paulo villosi; gisco comis; cor magna, eciliato; fruct. oblongus, basi subsepressus, apice longe ittenuatus.

Hab. Tustris, Moravia: ao Inain (At. Oboiny).

2036. Crepinia latipinnula Gogr mos.

Acculei breves, parriv, rectivsculi, vix Silatati sal teretes, ad ramos flor. mulli; fol. parra, oblongo-acuta, basi sensim rotundata, supra rividisubglauca, subtus canescentix, ai costarn eglanoulosa; Dentes simplices, ovato-aperti; pet eglaniaulosi, sup. inermes; stip. virides, freves, sat anqustar, subtus toto pubescentes, anriculis dirergentibus; peo. 1. glabri, 11-13 m. tongi; tulus oblongus; sep. pinnulis annibus late foliaceis, incisoDentatis, hresibus, villosis instructa; styli paulo villosi, irise pre plans; cor......... fruct brevitor oblongus, mesiscris, basi abrupte gecurrens, ai apiem attemuato - substrangulatus.

Hab. Gransilvania, Mzomok Doboka: al Déss (L. Pichter).

2037. Crepinia idonea Gogr mss.

Fcculei breves, parri, parum falcato-Dilatati, as ramos flor graciles mulh; fol. late obovato-subacuts, basi notuniseta, ad costam eglaniulosa; Dentes simplices, late orato-aperti; pet. eglanoulosi; vix aculeati; stip. longae, latae, virises, Dorso pubescentes, auricilis divergentibus; peos-1-2, glabri, 2 cent. longi; tubus oblongus; sep. erecta, air porsistentia, pinnulis omnibus integris, latis instructa, styli paulo villosi, Disco plano; cor........ 
$--293-$

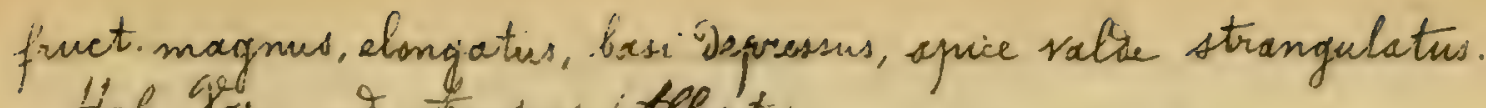
Hab. Gar, at etomnew tobert.

2038. Creymia vealbata Gagr 6x6. 1:2626.

facuici arbiles, faliato-gilatati, as ramss flor copiosi; fol obovato-acuta, bari cariata, al costam eglarioulosa; dentes simplices, aperti; pet eglaniulosi, omnes xculeati; stip. verides, mediocres, pec. 2-5, glabri, 15-18m. longi; tubus anstus, elongatus; sep. pinmulis integris, pubescentibus, latis, elongatis ins-

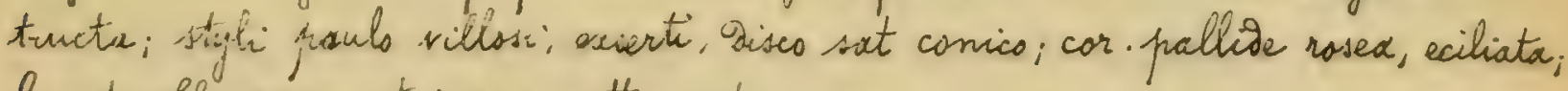
fruct. oblongus, utringue attenuatus.

Hab. Iinne, as Ifortierelas (No. Ganoger).

2039. Cinpinia maura Gogr mss.

toculei validi, felcato-dilatati, so ramos flor. geminati; fol mediscrix, sbosats-oblongx, acuta, basi rotundata, ad costam eglaniulosa; Dentes simplicis, late overto-recti; pat.eglarinitosi, aculeate; stip. longiusenlar, latissimae, ruberites, subtus to to pubescentes, auriculis givergentibus; ped. 2-5, glabri $15 \mathrm{~m}$. Longi; tubus oblongus; sep. pinnulis pinnulis annibus integris,

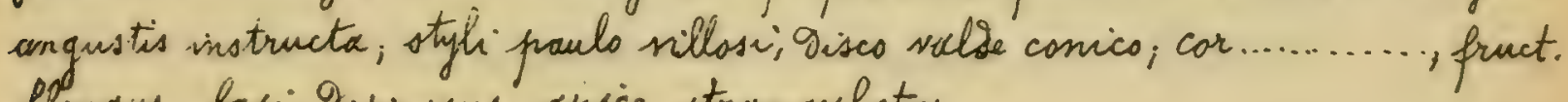
oblingus, basi gepressus, apice strangulatus. Hob. Tlgevia orient., prope Constantine (ge Rebouv).

2040. Crepinia Xatartiana Ggar 8ab. N:262\%-C. Xatartii (Debeaux Mater. ros. Syren., 1878, p. 31 nomen sohum).

Fcullei breves, falcato-Dilatati, as ramos flor. copiosi; fol. oblongo-acuta, basi rotuniata, ai costam eglanoulosa, dentes simplices, ovato-convergentes; pet.eglanimlasi, sup. inermes; stip. virites, axriculis parum divergentibus; hes. 1-2, glabra; 13-14m. longi; tubus oblongus; sep. brevia, pinnulis parum latis, integris, ut et acumine instructa, styhi parce villosi, Discs paulo conico, cor. eciliata; fruct. oflongus, fasi subrotuniatus, apice attenuatus.

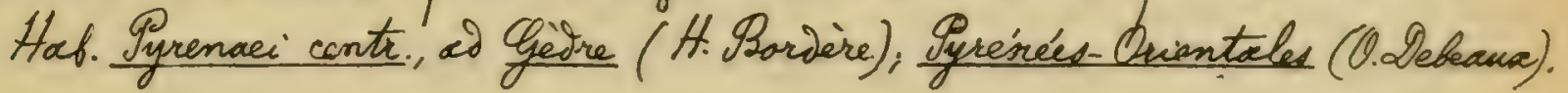

2041. Grepinia Onites Gogr \&ob. N-2628. Gogr Herb ros. $N=42$.

Arm lei caulinares validi, falcato - Dilatati, at ramos flor minores; fol. late obovato-oflonga, vix attenuata, besi rotuniato-cordata, intense siribia, ai costam eglaniaulosa, dentes simplices, lati, aperti; pet. exlanoulosi, sup. 
$-294-$

soli nculesti, stip. Dilatater, pubescentes, aurriculis Sivergentibus; per. 1-3, glabri, 12-20 m. longi; tubus oblongus; sep. pinnulis latiusculis, puerce Dentatis instructa, acumine Dilatato, styli parce villosi, aises comico, cor. pallive rosea; fruct. oblongus, basi Depressus, aprice attemeatus.

Hab. Ahóne, ad trenas (M. Ganooger).

2042. Crepinia convemiens Gogr mss.

Acculei brenes, parri, inclinato - subvilatati, aid remos flor.copiosi; fol. atrovirentia, late obovato- oblonga, vix acuta, basi roturioata, as costam eglas2ulosa; Dentes simphies, breves, svato-recti; pet. eglansulosi, inf. inarmes; stip. virides, freves, latue, auriculis girergentibus; pes. 1-2, glabri, 5 m.longi ; tubis oblongus; sep...........; styli pouclo puebescentes, Disco subplano; cor......., fuct. maximus, oblongus, utringue attemuatus.

Hab. Hungaria bor., Lipto': ag Stankora (L. Shichter).

\$\$. Foliola basi attenuata.

2043. Crepinia subalpina Gour bab. N:2629.

Aculei valivi, falcato-gilatati a ramos flor. panciores; fol. oblongo-obtusa, hasi longe attemuata, aprice Silatata, lacte sirisia, aco costam eglanioulosa; Dentes simplices, trianguleres, aperti, pet eglanioulosi, aculeati, stip.langae, parem latas, auriculis rectis, per.1, glabri, 6-7 m.longi; tubus oblongus; sep. Deciaua, pinnulis latis, lacimatis, eglandulosis instructa, acumine lato, Denticulato, styli paulo pubescentes, Disco comis, cor pallise rosea, eciliata; fruct. oblangus, basi attemuatus.

Hab. Tsere, Is Orande-Chartrense, supre conventum, alt. 3200' (N. Ganooger).

2044. Crepinia Blanoiens Gogrmss.

teculei breves, pawi, parm gilatati, sulfalcati, ai ramos flar. stipulares, pauciores; fol. sat parra, oblongo-acuta, basi attinuata, interse veridia, subtus canescentia, al costam egianiulosa; Dentes profunivi; lanceolato-convergentes; pet. eglanivilosi, sup. inermes, stip. longae, angustac, virites, subtus to to pulescentes, auriculis Sirergentibus; ped. 1, glabri, 6-8 m. longi; trubus oblangus; sep. pirnulis onvibus glabris, parum latis, subintegris instracto; styli paulo villosi; icises comics, cor........, fruct anguste oblongus, basi cepressus, oprice strangulatus. Hab. Eranssivamia, Nolozs: al Selso" Iebes (2. Mrichter). 
$-295-$

2045. Grepinia parvila Gagr Bab. N-2630.

Aculei subrect, gilatati, ad ramos flor. qraciles sparsi, fol prarwa, obovato - obloniga, parum acuta, basi attemeata, glancescentia, as costám eglan-

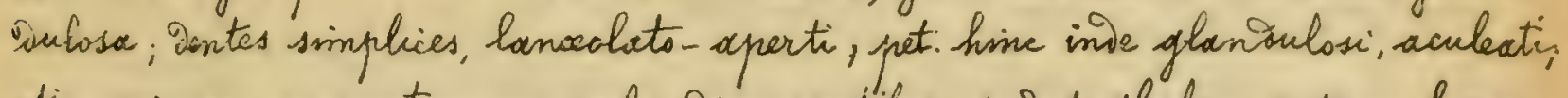
stip. minares, angustac, auriculis givergentibus; pas. 1, glabri, 8-10 m. longi; tubus oblongus; sep. pinmulis latiuscelis, 3-4-dentatis instructa, acmmine angusto; stighi poulo villosi, gisco plano; cor. parra, rosea, eciliata; fruct. blongus, fasi attenuatus.

Hob. Lilesice, gosen: 20 Bsorse (Hülsen).

2046. Creninia politula Ggogr mss.

foculei sat parri, falcato-gilatati, as ramos flor. stipulares, fol.mèviocria, rbovato-oblonga, utringue paulation attenuata, as costam eglansulosa; gentes simplices, triangulari-convergentes; pet. eglandulosi, inf inermes; stip. virities, latar, longiuscular, acoriculis subsirergentibus; per. 1-2, glabri, 1 cent. longi; tubus oblonges, sep........... styli paulo villosi; gisco plass, car ........ fruct. magnus, oblongus, utringue longe attenuatus, meais turgitus.

Hab. Hungaria, Soutapest: as Htenhegy (tt. Steffeck).

2047. Crepinia orgyalis Gogr mss.

Aculei auri, freviuscuh, inclinato- Dilatati, ot ramos flor-geminati, copiasi; fol. lactiusculi sblong-acuminata, basi attenuata, suporne pallive glaves - lutescentia, subtus griseseentia, as costam eglanoulosa, yentes fere omnes beserrate, recti, sublanceolato-triangulares; pot, eglanivulosi, inf. inermes; stip. elangatac, angrestiores, basi rubentes, subtus to to villosace, cusciculis longis, sufrectis; pes. 2-5, glabri, 10-12 m. langi; tribus sblongus; sep. extus rubra, pinnulis anmibus glabris, hinearibus, 2-4-Sentatis instructs, acumine integro; styli paulo villosi, gisco sulplano; cor. magna, rosea, eciliata; fruct oflongess, whinque attenuatus.

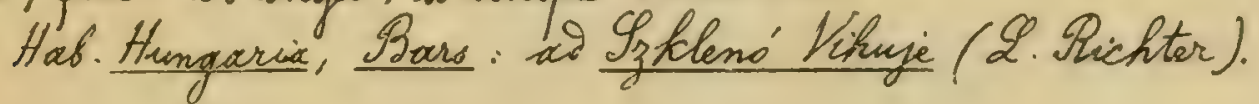

2048. Crepinia prasina Gogr \&ab. N:2631.

touli vix dilatati, recti ral inchiati, al ramos flor. miniti; fol. of longa, utrinque attonuata, af castam eglandulose; Dentes simplices, recti; pet. inermes, onnes eglanisulosi; stip. latee, auricielis givergontikus; per 1-2, glabic, $5 \mathrm{gm}$.longi; tsebus oblongres; sep. pirnulis integris, angustis instructo, stÿti parri, exserti, paulo villosi, gisco subconico; cor......... frecel. 
$-296-$

oblongus, vtrinque attemuatus.

Hab. Haute-Saroie, at Prenthonne (Muget).

2049. Crepinia rhynchantha Giger bab. J/C2632.

Aculei valitissimi, folcato- Dilatati, of ramos flor. copiosissimi, fol. oblongoacuta, basi stternata, os costam eglarimlosa, Dentes simplices, lanceo. Latoaperti, pet. eglanioulosi, inermes; stip. virises, auricilis rectis; per. 2-4, glabri; 12-15 m. longi; tubus elongatus; Asp. pinnulis ommibus latis, laciniatis instructa; styli villosi, giscs subpland, con......., fruct. elongatus, basi gepressus, apice longisisine constrictus.

Hab. Galloprovincia, as Martigues (tt. butheman).

2050. Crepinia obruta Gogr bab. NE2639.

teculei brevissinni, minute et valie falcati, id ramos flor geminati; fol ample blonga, utrinque sensine attenuata, as cestam eglanivelosa; Dentes simplices, aperto - triangulares; pet. eglanisulosi, inermes; stip. virives, auriculis rectis; peo-1-2, glabri, $17-20 \mathrm{~m}$. longi; tubus oblongus; sep. pir nulis lativisculis, 1-2-Pentatis instructo, acumine foliaceo, subventato; styli aisparse pilossi, dises plano; con........, fuct. oblongus, utrinque attemuatus, aprice turgions.

Hab. Iyrenari centr., Do Geire (H. Bordère).

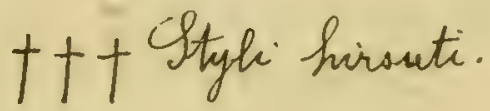

2051. Crepinia oxycarpa Gogr Gab. N:2634.

Acculer bebiles, teretiusculi, inclinate, sat longi; fol obovato-elliptica, utrinque breviter attenuata, so costam eglanivulosa, Dentes simphices, aperti; pet-inermes, eglanoulosi; stip. Dilatatoe, Dorso pulescentes, auriculis Divergentibus; ped. 1-3, glabri, 13-20 m. longi; tubus elongatus; sep.........., styhi hirsuti, Dises subplano, cor........., fruct. elongatus, besi contractus, aprice longissime attemiato. Strangulatus acutusque.

Hab. Ysire, at La Cte-It-Anote' (Moullu).

2052. Gepinia azorica Gogr Eab. N. 2635.

Aculai breves, inclinati, as ramos flos. sat copiosi, fol. oblongo-accuta, basi sensim attenuata, is costam eglanisuloss, Dentes simplices, aperto- triangulares, pet. eglanioulose; aculacti; stip. virives, auriculis girergentibus; ped. 2-4, glabri, 12-14 m. longi, tubes oblongus; sep. pirnmlis foliaceis, 2-3-2en- 
$-297-$

tatis instructa, acumine integro, lato; styli hirsuti, aisco pauls comico; cor. eciliata, fruct. oflongus, utrinque attenuatus.

Hab. Rossia merio, tozoff: as Escherkask (Laupmann).

2053. Crepinia nexa Gogr mss.

toculei breves, minute faleati, subteretes, parum Silatati, as ramos flor. nulli; fol.mediscria, oblongo-subacuta, basi truncata, viridi-lutescentia, subtus canescentia, of costam eglanivulosa; Ientes simplics, triangulares, recti, pet. eglanoulosi, inermes; stip. virides, langar, angustac, aorso pulescentes, auriculis gilatatis, ivergentibus; pes. 1, glabri, 6-8m. longi; tiebus oblongus; sep. hreria, pinnulis puberulis, omnibus latis, 2-3-bentatis copiose instructa; styli hirsuti, Disco comico; cor minor, pallise rosea, eciliata; fruct. oblongus, utrinque attenuatus.

Hab. Hungerix, Bars; od gehme (L. Richter).

2054. Prepinia meganewra Gogr mss.

ticulei tenues, cylinirici, suboilatato-inchinati, as ramos flar sparsi; fol mesiscria, obovato-oblonga, utrinque acuta, subtus nirescentiai, coriacea, prominule nervosa, as costam eglanoulosa, Dentes simplices, profunde triangulari-convergentes, magni; pet aculeati, eglandulosi; stip. virises, langiusculae, sat angustoe, awriculis Divergentibus; pes. 1, glabri, 11/2 cent. longi; tublus oblongus; sep.........., styli villosi, dises phans; cor.........; finct. ample oblmqus, utrinque lange attenuatus, mesio turgidus, apice strangulatus. Hab. Hungaria, Srubapest: a Kuitrolyy (tt. Steffeck).

2055. Crepinia aliena Gagr bab. N-2636.

toculei inchinato-suboilatati, as ramos flor.copiosi; fol elliptica, utrinque paulation rotunsata, nervosa, as costam eglaniulosa, Dentes simphices; pet. eglaniulosi, onnes xculeati; stip. virides; pet. 2-3, glabri, 8-13 m. longi; tubus oblongus; sep........., styli hirsuti, Disco subplano; cor........., fruct. oblongus, utrinque valise attenuatus.

Hab. Thone, at Davilly (9. Chabert).

2056. Crepinia capnotricha Gjoge 4ab. N:2637.

Acculei recti, teretes, as ramos flor.copiosi, fol parva, obovata, aprice subrotumiata, basi fore tiam rotunioata, of costam eglansulosa, Dentes 
$298-$

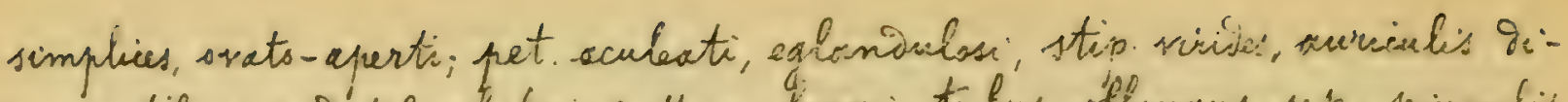

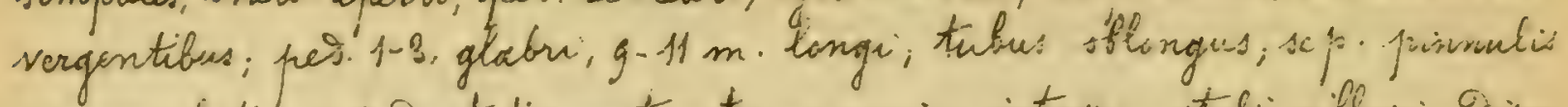
parum latis, 1-2-dentatis instructa, acumine intigro; stigli villosi; Geisec plano, car........, fruct oftingre, utrinque attonnotus.

Hab. Mtalia orient, at Gaenza (2. Coltesi).

205\%. Crepinia leucophulla Gogr inst.

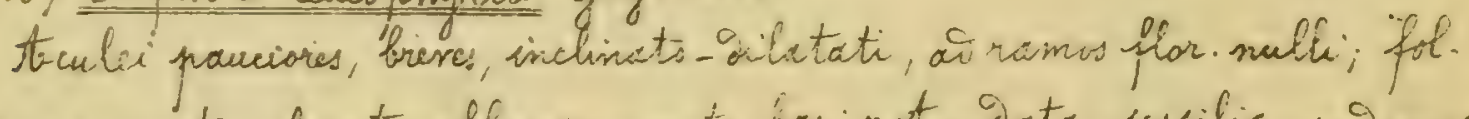

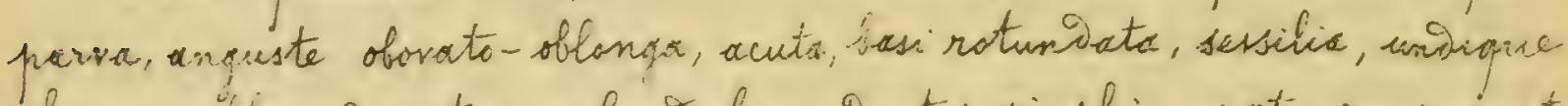

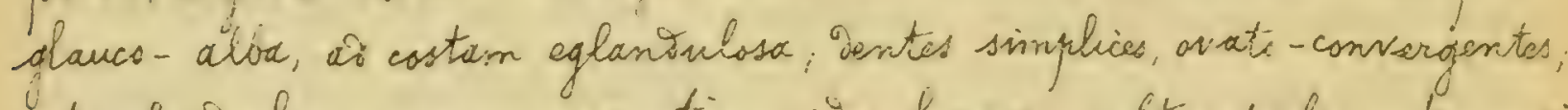

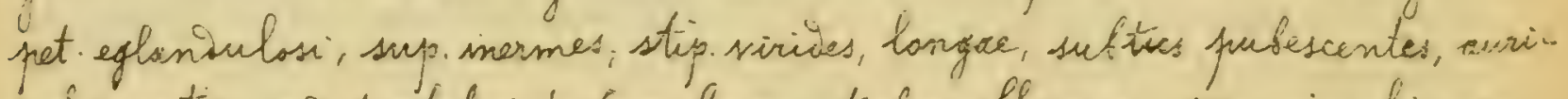
culis rectis; peo. 1, glabri, 4-6 m. longi; tubus oblongus; sep. pinnulis ornsitres integris, pubescentibus, latiusculis instructa; styli hirsuti, isco plans; cor.maxima, eciliata, pallioa, fruct breviter oblongus, bose iepressins, a: apicen attenuatus.

Hab. Holva onint, id Faenzx (I. Calderi).

2058. Crepinia retculata Gogr \&ab. $N-2638$.

Acculei breves, falcato-gilatati, at ramos flor copiosi et geminati; fol. parva, ovato-elliptica, acuto-, basi rotunioata, nerros-reticulata, ai costam eglaniulosa; dentes bimphices, lanceolato-subconvergentes; pet. parce glaniselosi, aculeatissimi; stip. piritas, auriculis givergentibus; pes. 1, glabri, 10-11 m.longi; tubus oblongus; sep. pinnulis annibus latis, 1-2-Dentatis instructa, acumine lats suboentato; styli hirsuti, giseo longissims conico; car. ignota; fruct anguste oblongus, basi longe, apia vers breviter attanuatrs. Hob. Buches-2u- Qhoine, al Martigues (Jo. toutheman).

2059. Crepinia alaucopoia Gogr mss.

tocule: brevissimi, minores, parmm gilatati, tanuiter falcati, ad ramos ffor. geminati, fol parvs, bosato-acuta, basi bresiter attenuata, suporne glavesvirisia, subtus caneseentia, at costam eglanisulosa, gentes simplicas, parvi, ovito-trianqulares, subrecti; pet aculeati, eglarioulosi; stip. latiuscular, breves, rubentes. subtus villosa, arriculis rectis; pes 1, glabri, alauci; 7-g m.longi, tubus sblongus; ser. brevix, pisnshis annibus, integris, axgusti: instructa, acumine lats, styli hinsuti, isise canico, cor. fruct parrus, breviter oblongus, basi gepressus, apice comic-strangulatus. Hab. Iransjilvomi, Izohok Doboka: ao karko (L. Richter). 
$-299-$

2060. Crepinia fusipara Covogr toal. N:2639.

Accelei breves, folcato-gilatati, at ramos flor-mulli, fol parva, ovato. elliptica, sulacuta, basi rotuendatx, à costam eglaniulosa; Dentes simplices, ovato-convergentes; pat eglasioulose; inf. inermes; stip. sividies, auriculis

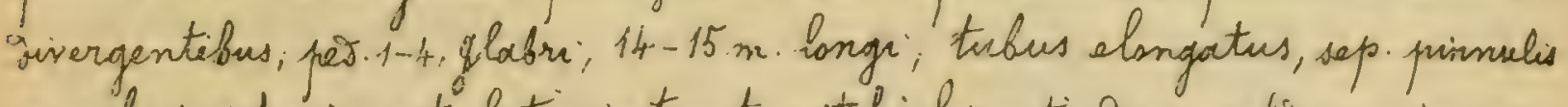

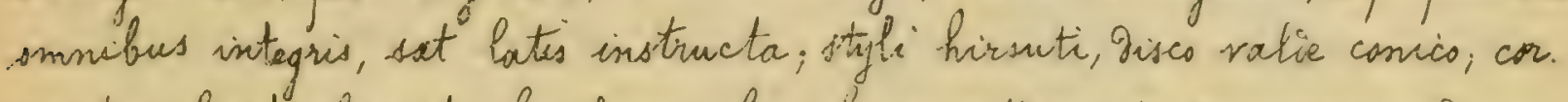
iznota; fruct elongats-fusiformis, basi longe attemuato-Decurrens, id a pioam attenuatus.

Hab. Bouches-2u-Rhone, w gitrolles (to. tutheman).

\$. Foliola maesue rel ommia biar

5. Foliola maeque sel ommia biserrata.

2061. Crepinia Lightfootiana Gogr Gab. N-2540.

Ticula Debiles, falcats-Dilatati, as ramos flor. sparsi; fol. late obovatosubbilonga, acutiuscula, fasi breviter attemiata, ai basin costace glaniulosac; Dentes lancolato-convergentes, onnes fiserrato-glanoulosi; pet. inermes, glanivulosi; stip. latissinae, subtus omnino glaniaulosac, aurriculis sivergentibur; peo. 1-3, glabri, 5-6 m. longi, tubus oblongus; sep. pinnulis latis, 2-3-gentatis instructa, acumine foliaces, integro, stigli lanati, Disco plano; cor........, fruct. breviter oblongus, utrinque aepressus. Hab. Icotia, ffberdeenshire: al Braemar (IShite; Drummond-Hay). Fruticulus stipularum magnarum glandulositate conspicuus.

2062. Prepinia glanialidens Goor mss- C.gothica (Ffinslorr) non Cgogr. Acculei rari, tennes, parri, vix Silatati, tenniter inclinati, as ramos flor.nulli; fol sat late obovato-oblonga, acuta, basi rotunoata, atrovirentia, as costam sparse glanisulosa; ientes bresiter triangulares, patuli, omnibus copriose biserrato-glanioulosi; pet. inermes, inferne puulo glanoulosi; stip. virides, longae, Dorso villoss-glanoulosse, curriculis rectis; peo. 1, glabiri, 5-6 m. longi; tubus oblongus; sep. pinnulis paucioribus, glabris, smnibus parum latis valieque dentato-glanoulosis instructa, styli lanati, Disco plano; cor........, fruct. oflongus, basi subrotundatus, apice strangulatis.

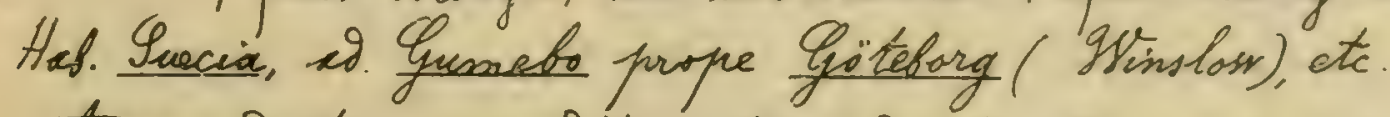
to provecesente cui sccelit, notis indrictis bene recebre videtur. 
$-300-$

2063. Crepinia Bertolomiana Gougr 8ab. N=2641.

toculei falcato- Dilatati, as ramos flor.geminati; fol. late ovato-elliptica, breviter acuta, fasi corvata, at costam eglanoulosa; dentes triangulares, operti, onnos biserrato-glansulosi, pet. inermes, glandulasi; stip. viricies, subtus eqlanaulosae, auriculis rectis; per. 2-4, glabri; $12-14 \mathrm{~m}$.longi; tubus sblongus, sep.pinnulis latis, 3-4-gentatis instructa, acumine angusto, integrs, styli lanati, aisco plano; car eciliata, fruct.oblongus, utrinque atter. Hab Mtalia media, Mavine: is Moteglix (G. Gibelli).

2064. Crepinia stenobasis Kogr \&ab. N:2642.

teculei tenmes, oilatati, temiter falcati, à ramos flor. Iparsi ; fol. oflongoacutiuscula, basi rotuniata, glaucescentix, as costam eglandalosa; sentes lanceslato-aperti, inforisres biserrati; pet. eglanoulosi, inermes; stip.rubentes, anqustare, auriculis givergentibus; per. 1, glabri, 3-4 m.longi; tubus of longus, sep.evecta, pinnulis istegris, latis instructa, acumine longo, parum lato; styli lanati, sises plano; cor...........; fruct. oblongus, basi volle attemato-gecurrens, eppice brevitar strangulatis.

Hab. Luecia. Hestrogothia: in monte Kinnekulle oi Hesterplana (Y. Q. Letterstegt).

2065. Crepinia Yahlenbergiana Gogr bab. N:2643.

foculei debiles, falcato-Dilatati, as ramos flor. sparsi; fol.mediocria, -bovato-acuta, basi paulation attenuata, as costam eglaniulasa; gentes aperto - trianqulares, inf. biserrati; pet. eglaniulosi, inermes; stip. viriedes, duriculis suboirergentibus; pề. 1, glabri, 8-9m. longi; tubus oblongus; sep. pinmulis integris, angustis instructa, dcumine latiisculo; styh lanati, gises plano; cor........... fruct. blongus, basi longe attenuatus, apice subirepressus. Hab. Luecia, Imaland: ad Moheda (Hyltin-Carallius).

2066. Or pinia camptoclada Gggr bab. N-2644.

Atiulei gabiles, inclinato - suboilatati, parum Dilatati, ar ramos flos. flesuoso-arcuatos: geminati, fol. obovato-oblonga, utrinque attemuata, intense siridia, aD costam eglansulosa; Dentes triangulari-subconvergentes, fere omnes biserrati; pet. inermes, hine inde glansulosi; stip. mediocres, parum latae, arriculis sufivergentibus, ped. 1-2, glabri, 7-gm.longi, tubus sblongus, sep. erecta, pinmulis lates, eglandulosis instructa, acumine foliaces, integro; styli 
$-301-$

lanati, Sisce fulans; cor........, fruct. oblongus, basi valle attennatus, as apicem breviter contractus.

Hab. Suecia, Hestrogothia: in monte Kinnekulle (P. . Lunaquist).

§. Foliola ammia simpliciter serrata.

$\sim$ Marmi floriferi inermes.

2067. Crepinia Eauscheri GJogr Eab. N-2645; Gigr Contr. fl. slav. II, p. 42. stculei parri, pauciores, falcati, as ramos flor mulk; fol. sat late ovato-elliptica, freviter attenuata, basi roturioata, is costam eglanisulosa, Dentes Simplices, ovato-convergentes; pet.eglanisulosi, aculeatis; stip.virites, auriculis givergentibus; pes. 1-4, glabir, 10-12 m. longi; tubus oblongus; sep. pinnulis ormibus integris, foliaceis instructa, styli lanati, Gisco conico; cor-magna, eciliata; fruct. oblongus, utrinque valde attenuatus.

Hab. Hungarix, tolbax: as Erg (Y. To. Ganscher), Croatia bor. (Mossi).

2068. Crepinia Bikicsü Gogr mss.

Acculei obbiliores, trervisculi, falcato- Dilatati, as ramos flor. nulli: fol mediacria, sbovato-subacuta, basi truncata, inforiona obtusa val retusa, virivi-glauca, subtus grisescentia, as costam eglanioulosa, gentes simplices, ovato-recti; pect. eglanaulassi, inf. inermes; stip. breves, latae, basi saepuns rubentes, subtus villosae, auriculis sirergentibus; peo. 1 , glabri, 4-6 m. longi; itubus oblangus; sep. pinnulis villasulis, latrusculis, 1-3-gentatis instructa, acumine foliaces, Dentato; styli lanate, girco plano, cor.magna, pallive rosea, eciliata; fruct. oblangus, utringue aepressus.

Hab. Hungaria, as Gelleithegy prope Bruadest (Mikics).

206g. Crepinia oppioana Gogr mss.

Aculei rari, longi, Oelatati, temuiter subfalcate, ad ramos plor nulli; fol mevioeria, oblanga vel obovato-oblange, basi attemeata, aprice annia

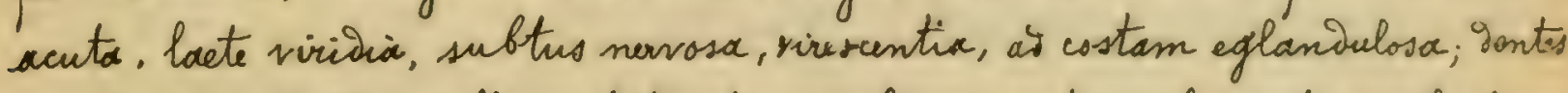
semplices, profande sublanceolato-triangulares, rectirsenli; fect. aculeati, eglanoulosi, stip. virides, latoe, breves, subtus toto pubscentes, awriculis sat divergentibus; ped. 1. glabu;, 14-17 m. longr; tubus oblongua; sep. pin mulis omnibus integerrimis, subfoliaceis, pubescentibus, longe instructa; stygh lanati, disco plans; cor........; fruct. Oblongus, magnus, hasi Depressus, id 
$-302-$

spiem longins aittenuatus.

Hab. Hungaria, ad Disgnify prope Puidapest (J. Steffeck).

2070. Crepinia Anorassyena Gogr mss.

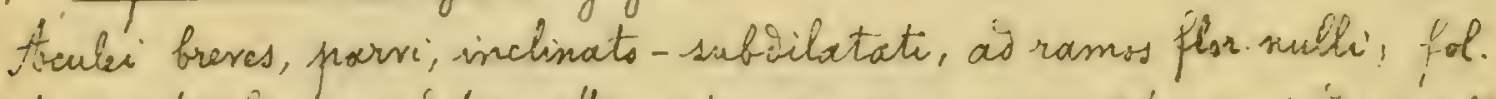

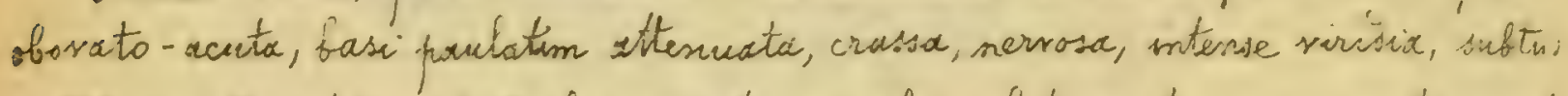

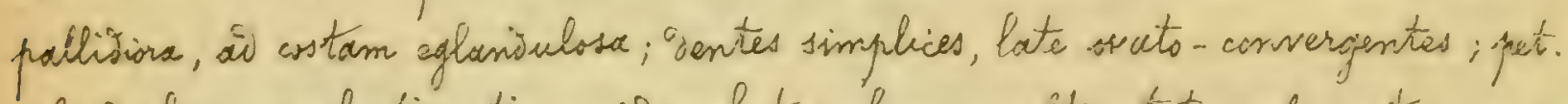

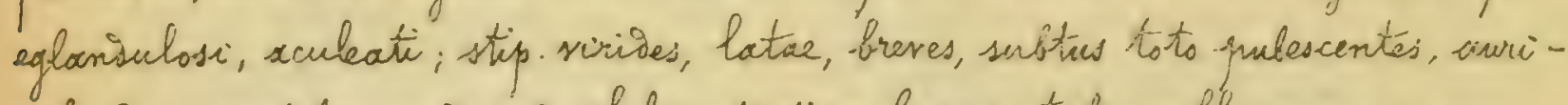
culis Divergentibus; pès. 1-2, glabri, 10-11 m. longi; tubus slongus; sep. ponmulis annibus pubescertibses, integris, sat latis instructio, styli lanati, "iisco plano; cor.......; fruct. magnus, oblongus, basi attenuatus, aprice angreste attenuato-strangulatus, modio turgious.

Hab. Hungaria, ai Gelleithagy prope Murapast (fo. Gteffect).

2071. Grepinia ciritates Gogr mss.

teculei pauciores, longi, Silatati, tenuiter falcati, as ramos flor nulli; fol. late oblongo-acuta, basi rotunivata, as costam eglanioulosa, Sentes simplices, ovato - convergentes; pet. eglaniulasi, aculeati, stip. virides, angustac, Longiusculae, auriculis rectis; ped. 1-2, glabri, $12 \mathrm{~m}$. Congi; tubus oblongus; sep........ styli lanati, disco plano; con ……; fruct. maximus, oblongus, itrinque valie attenuxtus, mevio turgitus.

Hob. Hungarix, ad kutvoloy prope Swapest (It. Iteffect).

2072. Crepinia Gommasinina Gogor bab. N-2846; Gogr Contr. Pl. star.II, p.43. Acculei falcato-silatati, Jebiles, wramos flor. nulli; fol-obovato- oblonga, sucta, basi rotuniata, as costam exlanaulosa; Dentes simplices, ovato-aperti; pet inermes, eglandulosi, stip. Dilute rubentes, auriculis givergentibus; ped.1, glabiv, 12-14 m. longi; tubus Plongus; sep. pirnulis integris, brevibus, latis instructo; styli lanati, sisco conico; cor.........; fruct oblongus, utrinque longe attemiatus.

Hab. Hotria, to Caxifaniaro (J. Freyn).

2073. Crepinia acrocian Go Gopr mss.

Acculei rari, breves, inchinato- osilatati, as ramos flor. nulli, fol.coriacea, sat late suboblonga, utrinque breviter ettenuata, at costam paulo glan aulosa, dentes simplices, ovato-convergentes; pet. hine inde glarioulosi; parce 
$30-2$

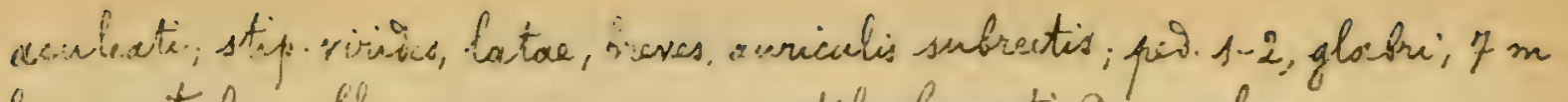
longi; tibess stonqus; sep stili laventia, perec plono; cor........;

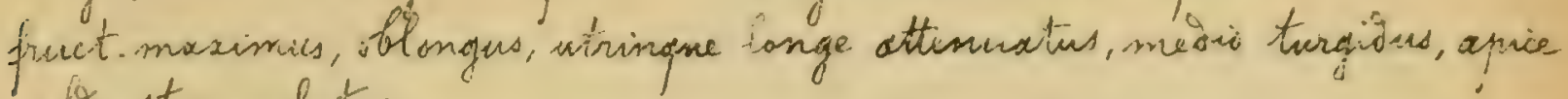
valde strangulatus.

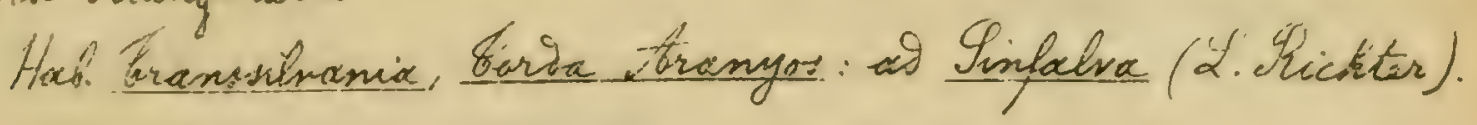

2074. Crecritia major Govar ms.

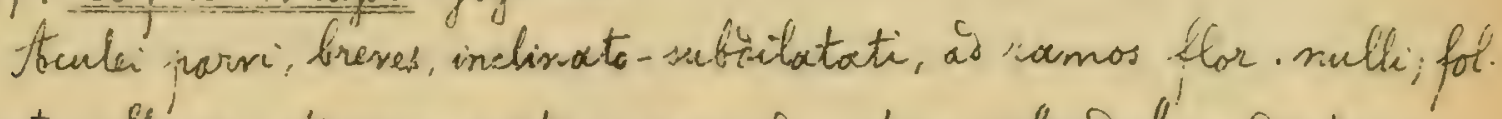

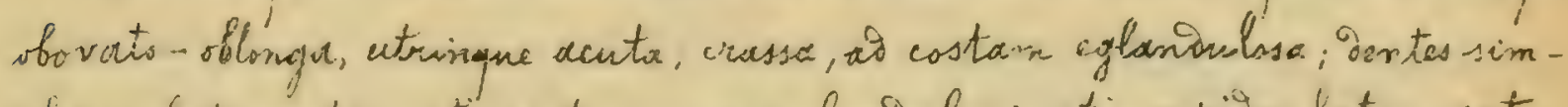

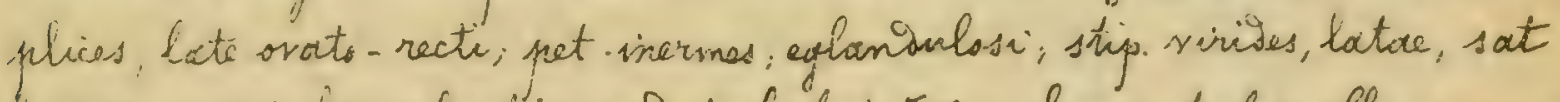
longas, auriculis subrectis; pei. 1, glabri, 5-7 m. longri; tubus oflongus; sep. ignota; styli lanati, gisco plans; cor........., fruct. maximns, glancus, basi attenuatus, in 1/3 sup. Oilatatis, apice vix attenuatus.

Hab. Hungaria bor., Lipto. ad Likanka (L. Michter).

2075. Crepinia impacata Giagr mss.

Aculei rari, breves, Dilatato-inclinati, ad ramos flor nulli; fol. mediocria, oblonga, utringue paulation attenuata, subtus virescentia et a costam eglaniaulosa; Sentes simplices, beriter ovato-convergontes; pet.eglaniulosi, inermes; stip. virises, angustae, breves, auriculis subrectis; ped. 1-9, glabri; 1 cent. Congi; tubus oblongus; sep. Issso glandulosa, pinnulis subintegerrimis, linearibus instructa; styli magni, lansti, Disco conico; cor.......; fruct-magsus, oblangus, utrinque longe attenuatus, turgidus, apice strangulatus. Hab. Eransilsania, Kologs: I Kalota M. Kiraly (2. Richter).

2076. Crepinix abanjensis Ciogr mss.

tocula temes, Tilatati-subfalcati, as ramos flor mulh; fol mediocria. oblonga, utrinque paulation attenuata, supra pallide viriéi-glancescentia, subtus grisea, ad costam eglaniulosa; dentes simplices, lanceolato-recti; pet. eglaniuchsi, onnes inermes; stip. breves, angustar, virides, gorso villosa, ouriculis givargentibus; ped. 1, glabui; 1 cent. longi; tubus oblangus, sep. pinnulis onmibus subtegris, pubescentibus, latiusculis isstructo, styh lanati, Disco comics; cor majuscula, eciliata, pallide rosea; fruct. oblongus, utrinque ottenuatus.

Hab. Hungarix, ffran: a forey Lyanto (2. Richtor). 
$-304-$

$\sim \sim$ Rami florales aculeati.

X Petioli annes aculeati.

2077. Grepinia longifolia Gögr Gab.

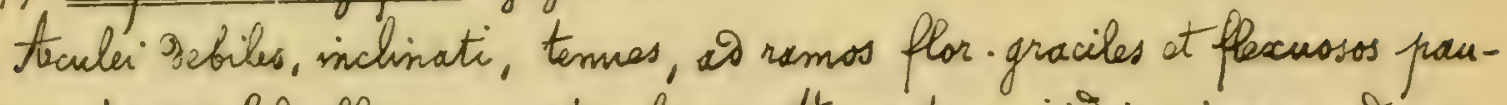
ciores, setacei; foloblongs-acuta, basi attenuata, viridi-cinarea, as costam eglanisuloss; dentes simphies, aperto-triangulares; pet eglaniulosi. tenuiter et parce aculeati; stip. longae, parum latac, auriculis givergentibus, pes. 1, glabri, 7-9 m. longi; tubus longissinus; sep. erecta. pinmulis angustis, eglaniulosis instructa, acumine longo, lato; styli lanati, gises plano; cor........, fruct. elongatus, basi attenuatus, ad apicerm strangulatus. - Tacies Rosace elpinas 2.

Hab. Snecix, Destrogothix: in monte himehulle (T. F. Luniaguist).

2078. Creminia hirastrota Gogr mss.

Tcculei gebiles, brexinsculi, parum Dilatati, minute subfalcati, at ramos flor. Plexassos pacuciones; fot. ample obovato-oblonga, vix acuta, basi subrotuniata, atranrentia, subtus pallida et ao costam eglaniulosa; Dentes simplies, late ovato-aperti, pet. eglaniulosi, onnes prarce aculeati; stip. clangatoe, angustoe, virives, Dorss pubescentes, auriculis subirivergentibus; per. plorumgue 2, glabri, $13-16 \mathrm{~m}$. longi; tubus elangatus; sep. prinnulis latis, glabris, 1-2-Dentatis instructa, acumine hineari, integro; styli lanati, disco plano, cor........., fruct. oblongus, apice semper longe attenuato-rostratus, fasi unus subrotunoatus, alter vers decurrens.

Hab. Eranssibamix, Mrasso': Do Eatrany, Zaizon (2. Richter).

2079. Gepinia obliqua Gogr Gab. N=2648.

Acculei falcato-Dilatati, ai ramos flor yeminate; fol anguste oblongolonceolata, fasi valie attenuata, lacte virioia, ad costam eglaniulosa; gentes simplices, lanceslato-sperti; pet. eglaniulosi, aculeati; stip. longar, Dilatatar, auriculis Sivergentibus; prod. 1-2, glabri, g-11 m. longi; tubus elongatus; sep. Decisua, pinmulis longis, latiusculis, 1-2-Oentatis instructa. acumine parum lato, styli lanati, gises plano, cor.........., fruct. of longus, apice longe attenuatus sicut at at basin.

Hab. Inecia: insula faltica Oelans: inter Eveta et Tarjestaden (Y.N.Seheuts). 
$-305-$

2080. Crepinia chilistricha Gogrmss.

Aculei breves, parri, subsilatato - faleati, ai ramos flor copiosi; fol. meDiscria, obsvato-oblanga, acuta, hasi attenuato-trumcata, coriacea, nervosa, longe piloso-arachnoisea, subtus albida, at basin costac aculeolata; sentes simphies, ovato-convergentes; pet. basi poulo glanioulosi, subinermes; stip. sirives, angustac, breves, auriculis divergentibus; pet. 1-2, glabri, 7-10 min longi; tubus oblongus; sep. pinnulis integris, la tiisculis instructa; styli lanati, Disco plano; con.......; fruct. magnus, elongatus, utringue longe attenuatus, basi subsecurrens, apice valke et anguste strangulatus.

Hab. Iranssilvania, Gorda toranyos: as Sinfalva (L. Richter).

2081. Crepinia Kmeti Goor mss.

toculei minuti, Debiles, suboilatati, temiter falcati, as ramos flor. geminate; fol mediocria, oblanga, subacuta, basi praulation attenuata, pallidius virisi-glowcescentia, subtus grisescentio, ai costam eglanisulosa; Dentes simphices, late triangulares, recti; pet. eglanioulosi, rubentes, parce aculeati, stip. purpureac, breviusculae, latoc, torso pubescentes, auriculis subrectis, per. 1, glabri, 7-g m. longi; tubus oblongus; sep. pirnmlis sat angustis, subintegris, prebescentibus instructa, acumine lato, sty is inferne lanati, aises convic, cor......... fruct. oblongus, fasi proesertion attemuatus. Hab. Hungaria, Hont: $a$ Srencios (A. timet).

XX Petiski annes vel aliguot inermes. - Folisla basi rotunnata.

2082. Crepinix bicallis Gogr Bab. N=2649.

toculei breves, falcato- Silatati, al ramos plor. panuciores; fol late obovatooblonga, abrupte acuminata, basi rotunia ta, laete viritio, ad costam nox glansulosa; Jentes simphices, aparto - triangulares; pat. onnes inermes, eglanSulosi; stip. longue, latiusculae, awniculis subsivergantibus; peo. 2-3, glabri, 7-gm.longi; tubus oblongus; sep. erecto, pinnulis integris, prairum latis breviter instructa, acumine longo, sat lato; styli lanati, Jises plans; cor........., fruct: maximus, oblongus, saepius unsigue salse atternatsstrangulatus.

Hab. Luecix, Gestrogothix: in monte Kinnekulle (9. T. Luniquiat). 
$-305-$

2083. Preminia rorisifolia Gigr inss.

taculei tenmes, recti, sal pauls inchinati, parum bilatati, bieses, at ramos Hor. glaucos sat copriosi; fol mesiscria, obovato-oblongi, vix attenustr, bioi subrotumiata, crassa, nervosa, suparne puallide gliwco-siriscentic, subtus velde glauso-alba, at costam eglansulosa; sentes simplice, orati, recti i pat.

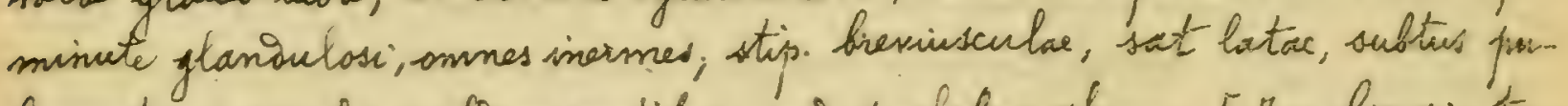
bescentes, auriculis subsivergentibus; pet.1, glabri, ghasei, $5-7 \mathrm{~m}$. lingic; tubus oblongus, rariaus; sep. max eracts, subintegerrima, pionnalis $1-2$, inite-

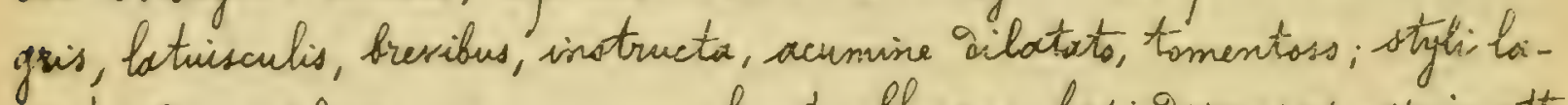
nati, Sisco plans; cor.........; fruct ablongus, basi gepressus, aprie atternuato-strangulatus, glaucescens.

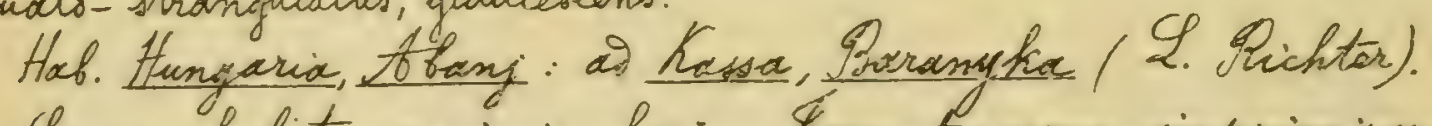

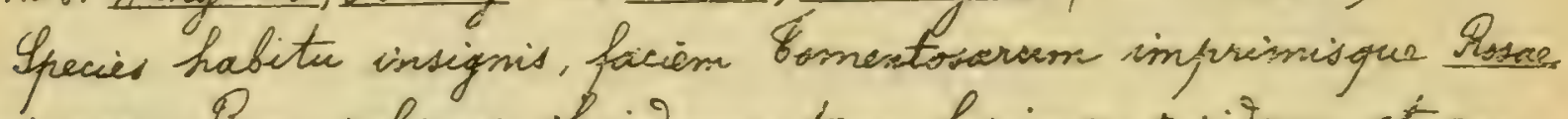

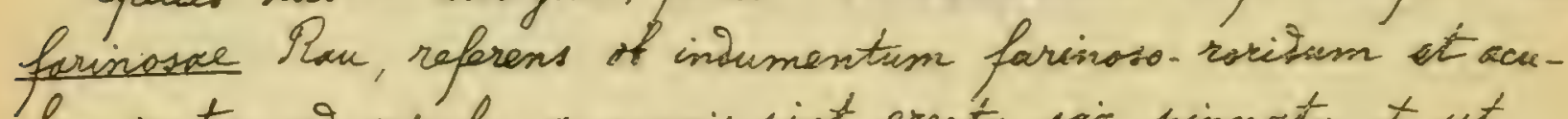
leos rectos; set sefrala, quamris sint erecta, six pinnata at, ut visetur, die persistentic, eqlaniulssa, crossiuscule, eis Sobstivialiom simillima frae se ferunt; stipulase tixm Caninaarum pabesantiver.

2084. Crepinia incrassans Goversos. N.2550.

Acculei breves, folcato-gilatati, w ramos flor. copiosi; fol. sat parra, abovato-acuta, basi rotusiata, virisi-glaucescentia, as cositam eglaniscloss; Dentes simplices, lancealato - apariti; pitt inermes, eglaniulosi; stip. parrace, auriculis Givergentibus; pes. 1-2, glabir; 5-7m-longri; tubus elongatus; sep. pervistentic, pinmulis integris, sat latis instructa; acumine subfoliaces, integro, styli lanati, gises plome, cor.........; fruct. alongatus, basi longe attemuato-gecurrens, apure stranguiatus.

Hab. Iuecia, Imolenoix: as gevie (\%.M. Lchenty).

2085. Prepinia maxima Goar Gab. N-2651.

Aculei breves, silatato - subfalcati, a ramos flor. pauciores; foh oblonga. utrinque rotuniata, as cost sm eglanoulosa, Dentes simplices, triangulares, aperti, pet-inermes, glandandulosi, stip. mediocres, euriculis subsivergentibus, ped. 1-2, glabri, 5.7m. longi; tubus tolongus, sep.erecta, finminclis integris, latis insisucta, acumine integiv, foliaces, longo; styli lanati, Disco plano; c........ fruct. musinuss, oblongus, basi ralde attenuatus, aprice subroturioat. Hob. Grecia, Slekingia: at Gheholm (I) Mp Sheutg). 
$-307-$

2086. Gepinia anceps Gogr toab. N=2652.

Aculei longi, refiles, Dilatati, recti vel inchinati, à ramos flor genninati, fol oblongo-acuminate, basi rotumiata, lacte vividia, as costam eglanSulosa; Dentes sinplices, ovato- rectit; pet inermes, eglanioulosi; stip. longar, lastre, auriculis rectis; per. 1-2, glabri, 5-7 m. Longi; tubus oblongus; sep. Deciovex, Dorso glantulass, fimmulis integris, angustis instructa, ocumne latiusculo, integro; styh lanati, Disco plano, cor........ fruct. oblongus, fasi longe sè sensim attenuatus, apice subrotuniatus.

Hab. Helvetia, Yalais: as Outrerhone, le Mont (2. Favrat).

2087. Gepinia umula Gigr mos.

Accules breses, inchinato - subilatati, as ramos flor. stipulares; fol. me Diacria, oblango-acuta, basi rotuniata, glauca, ad costasn eglarioulosa; Sentes simplices, ovato-recti; pet inermes, eglaniaulosi; stip. virises, latiusculac, breves, auriculis givergentibus; per. 1, glabri, 2-4 m. Congr; tubus oblongus; sep..........; styli lanati, Tisco comiso; cat....... fruct. sat magnus, oblongus, utrinque attenuatus, basi gecurrens.

Hab. Hungaria bou, Liptó: as Rozsahegy Baráthegy (2. Richter).

00 Golisla basi attemuata.

2088. Gepimia cinerex Giger Gab. N=2653.

Acculei falcato-Silatati, ad ramos flor. geminati, fol. cinerea, magna, obovato-oblonga, utringue breviter attemuata, ar costam eglanioulosa, Dentes simphices, aperto - triangulares; pet inermes, parce glanoulosi; stip. longae, Silatatac, auriculis Divergentibus; ped.1-3, glabri, 2-4 m. longi; tubus gracilis, elongatus, sep. pinnulis integris, latis instucta, acumine lato, integro, gir prersistentia; styli lanate, gisco plano; corr rosea, eciliata; fruct. elongatus, basi contractus, apice longissime attemuatus.

Hah. Silesia, ad Lilienthal prope Breslau (Nechtrity).

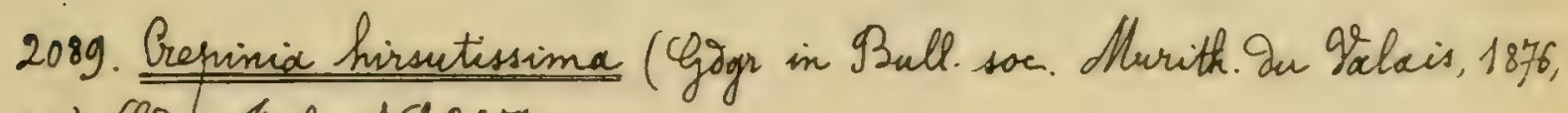
p.34). Gagr Gab. N-2654

taculei vix Silatati, recti val inclinati, aut subaciculares, ad ramos flor. coprosi, fol mollissima, sborata, utinque poulation sttemuata, ai costam zeglanisulose; Dentes simplices, convergentes, pet. inarnes, tennissime glaniou- 
$-308-$

Lsi, stip.magnas, subtus pulescentes, auriculis rectis; per. 1-2, glabri, 4-6 m. longi; tubus oblongus; sep. erecta, pinnulis integris, sat angustis instructa; styli lanati, gisco plano; cor.......... fruet. oblongus, basi valie. attenuatris, aprie strangulatus.

Hab. Hehetia galais: as Bornier (\%.0. Holf).

2090. Orepinia finlanisia Goor Gaf. N-2655.

Aculai elongate, tenniter folcati, so ramos flor.copiosissimn; fol. late oflonga, basi attenuata, inferiora obtusa, sup. vero acutissina; a costam eolaniulosa; Dentes simplices, patule lancealati; pet, inermes, eglanaulosi; stip. virides, suriculis rectis; ped.1. glabri, 8 m. longi; tubus oblongus; sep. pinnulis integris, angustis instructa, scumine lato, integro; styli lanati, sises plano; cor.........; fruct. maximus, olongus, fasi valse attenuatus, apice inflatus visque contractus.

Hab. Mossia bor. Fennia: Abo, at Husikaupunki (H. Hollmén).

2091. Greminia brevipila Gogr orab. $N=2656$.

frculei sat Debiles, falcato-dilatati, wo ramos flor panciores; fol. anguste oblonga, utrinoue paulation attenuate, firma, suparne breviter pilosa, ai costam eglaniulosa; gentes simplices; pet. eglandulosi, inermes; stip. meais rubentes, lator, auriculis givergentibus; per. 1-2, glabri; 5-8 m. longi; tubus oblangus; sep. arecta, firnnulis angustis, parce dentatis instructo; styli magni, lanati, axs plano; cor........ fruct. oblongus, basi subrotumiatus, apice attenuatius:

Hob. Mhone, of Prapone (Moullu).

2092. Crepinia mesophylla Goigr mss.

Abculei mediacres, dilatati, temiter falcati, ad ramos flor geminati; fol.midiacria, oblonga, utringue paulation attenuata, ab costam eglandulosa, gentes simplices, triangulares, aperti, pet-inermes, eglangulosi; stip. sirides, latae, breviusculae, auriculis rectis; peo. 1-2, glabri, 2-4 m. longi; tubus oflongus; sep.crasse, prinmulis inteogris, latis instructa; styli lanati, disco camics; cor....... fruct. ample oblongus, basi abrupte iecurrens, tirgioins, apvice attenuato-stranigulatus.

Hab. Hungaria for, Lifuto' Oo Ozsada (L. Richtor). 
$-309-$

2. Peanculi villosi vel parcissime glaniulosi.

a. Fructus globosus vel subglobosus.

2093. Grenimia tubacycla Gogr Eab. N-2658.

Aculei gure, faleats-gilatati, as ramos flor. dense foliosos copiosi; fol. obsvato-ollonga, utrinque attenucta, strovirentia, mollissima, costam eglaniaulosa; Dentes simplices, aperti, pet. eglandulosi, snnes aculeati; stip. sat latae, pubescentes, iuriculis givergentibus, pes. 1-2, sillasi, 5-11 m.longi; tubus ovaco-rotusiatus; sep. pinnulis integris, sat latis instructa, acumine gilatato; styli hirsutissimi, Dises subplans; cor. rosea; fruct subglobosus, pice breriter strangulatis.

Hab. Houte-Lavaie, Bollevanx (Muget).

2094. Crepinia sarosensis Gigr mss.

toenlei maximi, falcato- Bilatati, as ramos flor glaucos copiosi; fol. late oblonga, utrinque attenuata, intense virisia. subtus grisea th ad costam eglaniulosa; ientes simplias, ovato-lanceolati, recti; pet-eglandulosi, ornnes aculeati, stip. basi urbentes, latissimae, longae, subtus toto pubercentes, auriculis poulb givergentibus, pei. 1-2, parce villosi, 2-4 m. langi; tubus rotundatus; sep. punnulis onmibus foliaceis, Dentratis instructa, acumine magno; styli parce villosi; disco subplans, cor.........; fruct. subglobosus, utrinque fere rotuniatus.

Hab. Hungaria, Laros: as Borkfofirtï Eornyeke (2. Tichter).

2095. Crepinia bractescens (Moods in Frans. linn. soc. XII, p. 216, Imith Engl. fl. II, p. 391; Borrer Brit. Pl. i2. 3:p.242, Dwinort. Mon.ros. belg. p. 10); Gogr Gab. N-265g. - Exs., Cat. soc. bot-of Lono. ad. 1a at 3 a N-351 f. taculer valis; falcato-dilatati, ad ramos flor. saepe geminati; for. late elliptica, subtus canescentiv et ad costam aculeolata; dentes simplices; pet. aculeati, eglanoulosi; stip. angustae. Subtus prubescentes; ped.1-4, slii lacres, alii setulosi, late bracteati, tubus lacris, globosus, scp. pinnulis integris, eglaniulasis instructa; styli lanati; cor. rosex; fruct. jlabosus.

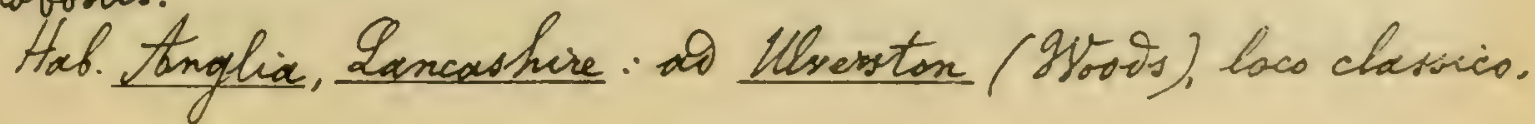


$-310-$

2095. Crepinia juvenilis Gogr mss.

foculer tenses, rectiveculi, vix gilatait, od ramos flor copisisi; fol. latuscule obovato-acuta, abrupte et porum acutix, hasi roturioista, viridiflavescentia, as costam eglanidulose, grentes simplies, lanieshats -aperiti;

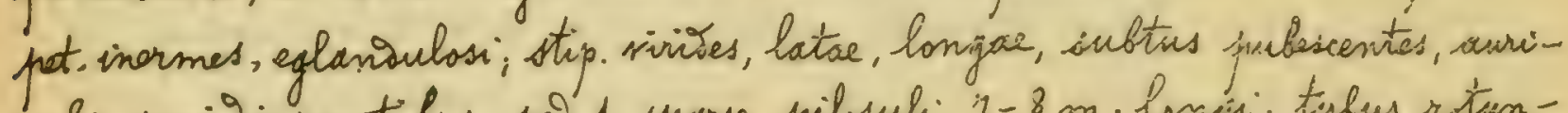
culis semidivergentibus, pea. 1, sparse pilosuli, ${ }^{1}-8$ m. Conjigi; tirbus rotunDatus; sep. pisnulis omnibus integris, foliacesis instincto, acunsine magno; styli maximi, lanati, gises plano; cir. rosea, eciliats; finct. ample sub. globosus, utrinque rotuniatus.

Hab. Hautes -ftres, circa Ie Grave: alt. $4500^{\prime}$ (No. Ganioger).

b. Fructus ovato-rotungates vel ovatus.

2096. Crepinia alpina Gigr Gab. $N=2660$.

Rubens: aculei Debiles, recti vel inclinati, gilatati, ad ramos flor. co. pissi; fol. late obovato-asutiuscula, basi subrotuniata, lacte viridia, at costam eglanduclosa; Pentes simplices, triangulares, acti, pet. eglaniulosi, inermes; stip. breves, latae, auriculis rectis vel conrergentibus; ped-1-2, aliv haves, alii assparse glanialoselli, 6-7 m. longi; tubus ovatus; sep.......; styli hirsuti, giseo plans; cor........., fruct. parrus, ovatus, basi rotem vatus, apric attenuatus.

Hob. Helvetia, in alpibus ganes as Leysin (2. Favrat).

2097. Creminia Havescens Gogr mss.

foculei minuti, recti vel inchinati, vix Silatati, wo ramos flor. stipulares; fol.megiocria, oblongo-acuta, basi paulatim attenuata, superne viri2i-flarescentia, subtus incana et ao costam eglaniulosa, Dentes simphices, breviter triangulares, recti; pet. eglaniulosi, parce aculeati, stip.virides, sat angustae, breviuscular, subtus toto pubescentes, auriculis subDivergentibus; pes.1, pilosi, 6-8 m. longe; tubus ovatus; sep. pinnulis amnibus pubescentibus, integris, latis instructa, acumine longs; stygh villosi, Disco comic; con........, fruct. ovatus, utrinque Depressus.

Hab. Gransilvanix, Heirongsek: Buivos Felso Gorjox (L. Riehter).

2098. Crepinia porphyracarpa Gogr Eab. N:2661. - Gagr Herb.ros. N:116. Acculei mediceres, falcato- diloctati, ad ramos flor. copiosi i fol. late 
$-3 H-$

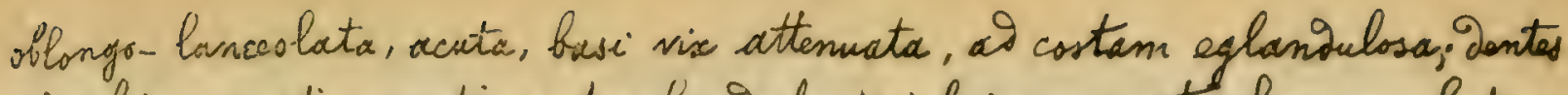
simplies, aperti, remoti, pat. eglaniulosi, inf.inermes; stip.longar, latas,

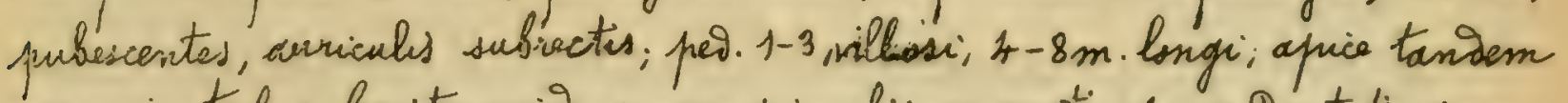
carrssi; tubus breviter svoidens, sep. pimsulis anquistis, parce gentatis instructa; styli parce villosi, gises pauls canico, cor.........; fruct. plesumque ssitus, fasi rotuniatus, apice attenuatus, nitide ruber.

Hab. Irendei centr, Gedre (H. Bordire).

2099. Creninia rubrotioncta Goyr mss.

treulei validuli, falcats-oilatati, acerosi, ai ramos flor-copiosi; fol. smple oblongo-acuta, bosi paulation attenuata, subtus grisea, as costam eglaniulosa; Dentes simplices, triangulari-subanvergantes; pet. eglanZulosi, rubentes, aculeati; stip. prurpurear, breves, anqustae, auriculis rectis; ped. 2-4; sparse pubescentes, $8 \mathrm{~m}$. longi; tubus ellipticus; sep. pinnulis angustis, integris instructa; styli subglaberrimi, aisco plano; fruct. mediscris, ovato-ellipticus, basi subrotundatus, apice contractus.

Hab. Iransilvania, Kolojs: ad Nayar i Kerek (2. Michter).

2100. Crepinia jonides Gagr bab. N-2662.

Aculei falcato-Dilatati, ad ramos flor plerumque nulli; fol. obovatoelliptica, acuta, bai breviter attenwata; Dentes biseriate, aperti, puet eglanjulosi, sapius annes inermes; stip. angustar, gorso pubescentes, acuriculis givergentibus; pred. 1-2, villosi, 8-15 m. longi; tubus oratus; sep. pinnulis integris, latis instructa, styli hirsuti, exserti, gisco paulo conico; cor...... fruct. ovatus, apice valse sthemuato-stranqulatus.

Hab. Savoie, as Salins prope Mouttirs (Muget).

2101. Crepinix Reboutiand (Debeaux) in Gogr Sab. N-2663.

Acculir breves, falcato-dilatate, as ramos flor. saejuis nulli; fol. parwa, corsiacea, sbovato - subobtusa, basi rotuniata, a costam eglandulosa, gentes simplices, ovato-aporti; pet. inermes, eglangulasi; stip virides, freves, curiculis givergentibus; pei. 1-2, villosi, o-8 m. longi; tubus ovatus; sep-pin sulis integris, orevibus, villosis instructa, acumine lato, integro; stipli lanate, esserti, gisco comico; cor - cciliata; fruct. parvus, ovato-rotuniatus, utringue hand ant vix oppressus.

Hab. Syrénees-Arientales, Surg Touan in aprieis, prope Serpiginan (O. Debeaux). 
$-312-$

2102. Preninia phyllochbra (Gogr Mos. nor. I. p.14); Gogr \&ab. N:2664.Gogr Herb.ros. N-372.

Aculei as ramss flor. falcato-Dilatati; fol-ovato-elliptica, acuta, basi coriata, firma, nervosa, molliter villss-lutescentia, so costam eglanoulosa; Gentes simplies; pet. inermes, eglanioulosi; stip. Silatatae, auriculis brevibus; peo. 1-3, berissinn, iasperse pilosuli; tubus ovoideus; sep. pinmulis pulescentibus, subintegris, latis instructa; styli breves, subglabii, sises fere plans; cor subalsa; fruct avatus vel ovato-notundatus. Hob. Thane, at tornas (No. Ganooger).

2103. Crepinix utrigera Gogr mos.

Acculei breves, falcato-gilatati, as ramos flor.geminati; fol. ample oblongo-subacuta, basi prulatim attenuata, subtus grisea, at castam eglanisulosa; Dentes simplices, late triangulari-convergentes; pet. inarmes, eglasioulosi; stip. virides, maximae, auriculis subectis; ped. 1-2, sparse pilasi, 4-5m. longi; tubus ovatus; sep.........., styli lanati, Dises plans; cor........, fruct maximus, turgions, ovatus, it trinque suloepressus. Hab. Hungaria bor. Lipto: as Likenka (2. Aichter).

2104. Crepinia poliochlora Gogr mss.

Acculei falcato dilatati, sat breves, verticillati, as ramos flor. glaveos copriasi; fol ample suboblonga, breviter attenuata, basi subrotunisata, crass, cariaced, superme vinisi-glauca, subtus canescentia et as costam eglanioulosa, Dentes triangulares, recti, inf. subfiserrati; pet. eglanialosi, onnes inermes; stip. sirides, maximae, subtus tomentellae, aurieulis aivergentibus; ped. 1-2, sparse villosi, 7-10 m. longi; tubus ovatus; sep. magna, pinnulis onnibus subintegris, tomontosis, phiaceis instructa, scumine elongato; styli lanati, gises plano, cor........ fuct magnus, ovatus, utrinque rotuniatus, sepalis sape coronatus.

Hab. Gransilwania, Csik: as Eusnád (2. Richter).

2105. Crepinia altera (Gogr Gssai p. 32, Gager Mos. nor. II, p. 29): Gogr Gab. N:2665. - C. silvestris (Ychult. ex Rchb. \$. exc. N:3997 mon alior.). Aculei valivi, falcati-gilatati, sparsi, seo as ramos flor-interoum geminati; fol. oblonga, utrinque acuta, al costame eglanioulosa; gentes simplices; pet glandoulosi, aculeati, stip. pulescentes; ped. basi villosi; tubus oratus; sep. Deciouar pinmelis dentatis, lanceolatis instructa; stigh: hirsuti: 
$-\cdots 3 / 3 \cdots$

cir.minor, carnea, frust. ovatus.

Hob. Permania.

Plures asunt Psac vivestirs, nemke: Mrsa-xiprestris Gaterea

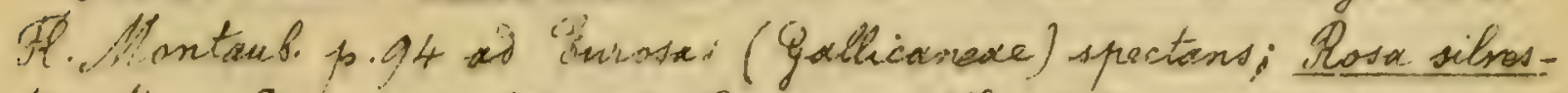

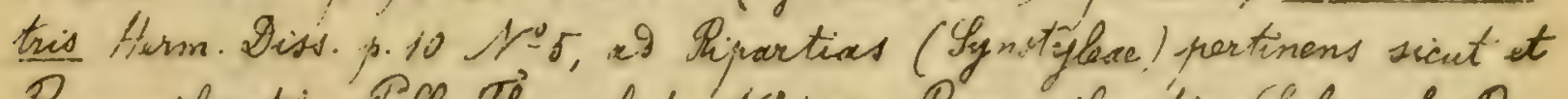

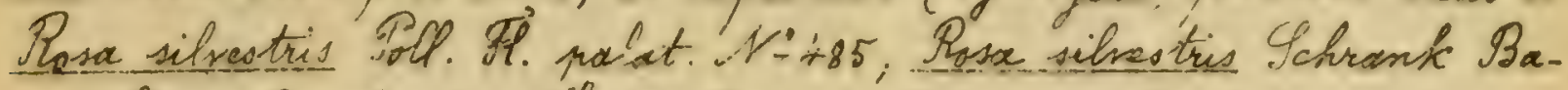

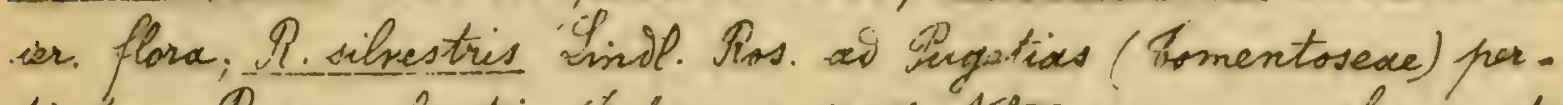

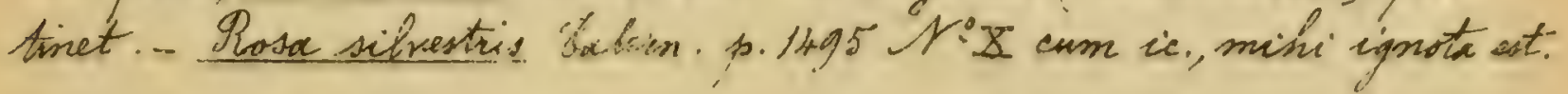

2106. Crepinia verbascina cyatr mss.

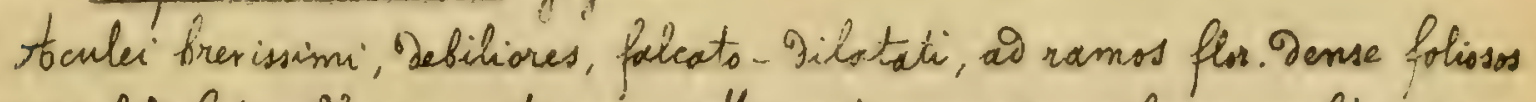

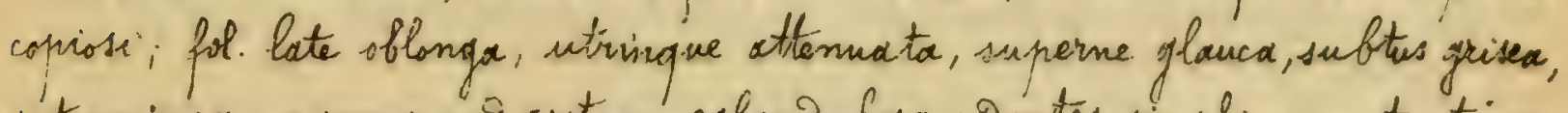
set coriacea, nervosia costam eylanoulosa; bentes simplices, vato-trianFulares, subrecti; pet. rusentes, sucicati, eglansulosi; stip. basi rubentes,

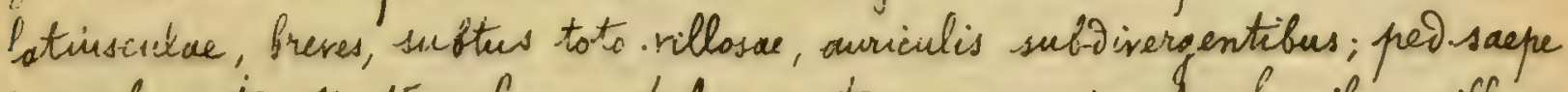
3, pubescentes, 10-15.m. Congi; tufus ovatiss; sep. pinnulis brevibus, villosis, in of oliaceis, 2-3-gentatis instructa, acumine foliaces, elongats; styli lanati, isises praulo conics; cor....... fruct. ovatus, basi rotundortus, apice paulo thenvatus, centrali autem ovoideo, Gasi abrupte gecinriente, aprieque longius contracto.

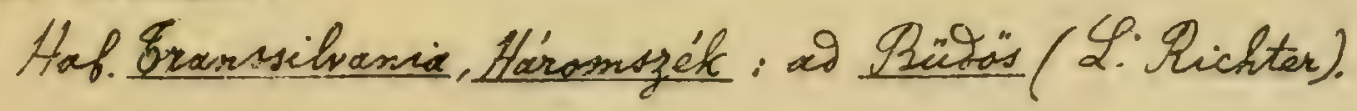

c.- Fructur mideus vel obovato-oblongus.

+ Ityli glabri vel sparse pilosi.

2107. Creninia tomonoda Gogr tab. N:2667.

fculei validi, falcato-Silatate, ad ramos flor.cspiosi; fol oglavescentix, sat late oblongo - acuminata, basi attenuata, as costam eglaniulosa, dentes simphices, lanceslats - aperti, pet. inarmes, eglanoulose; stip. magnae, auriculis subrectis; pet. 3-6, molliter villosi, g-11 m. longi; tubus obovato-oblongus, sep. pimulis latis, numerosis, marginibus copiose glaniulasis instructa, - acumine lato, dentato; styli glabri, giseo paulo canico; cor. magna, pallide rasea, eciliata; fruct. sbovats - oblongus.

Hab. Silesia, gosen: al Staykosvo (R. Hülsen). 
$-314-$

2108. Creprivia haromszekensis Gogr mss.

Accula brevissims, gabiles, parum falcato - Silatati, so ramos flor. nulli; fol.mediscria, sbovato - suboblonga, freviter acuta, basi sat attenvata, nervosa, pallide viridia, subtus albide, at costam eglanivulosa; gentes lanceolato-subconvergentes, inf. paulo biserracti; pet. eglaniculosi, parce aculeati, stip. sivides, sat angustae, freves, gorso sillosae, auriculis rectis; per.1, sparse villosi, 1 cent. longi; thebus suboblongus; sep. pinnuclis glabrescentifus, smmibus foliaceis, ontatis copiose instructa, acumine bate foliaces, inciso; styliswitillosi, gisco

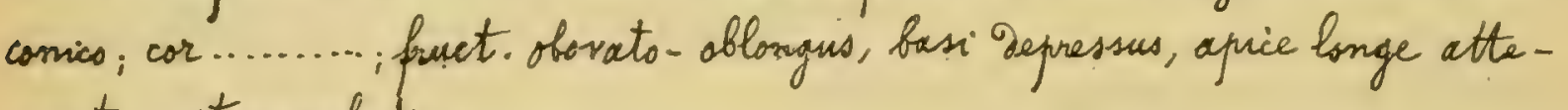
mato - strangulatus.

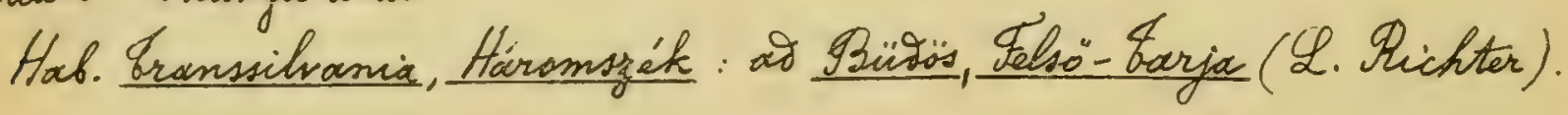

2109. Crepinia monnetierensis (Gouvier) in Gogr bab. N:2668.

Aculei fuleato-dilatate, ro ramos flor.geminati; fol. late oblonga, vix acuta, basi rotunoata, viridi-cinerea, as costam eglanaulosa; gentes simphies, orato-aperti; pet aculeati, eglaniulosi; stip. magnas, latae, avriculis Divergentisus; per. 1-3, villosi, 11-13 m. longi; ; tubus obovato-oblongus; sep. Decisux, pisnulis latis, 1-3-gentatis instructa, acumine subfoliaces, genticulato; styli subghaberrimi, gisco paulo conico; cor..........; fruct. obovato - oblongus, basi phlus minusve Tepressus, aprice atternutus.

Hab. Houte- Saraie: in monte Saleve of Mometier (Mourier).

2110. Crepinia wovarhehensis Goar mss.

Atruli debiles, brevissimi, parm faleato-Dilatati, ad ramos flor. stipulares, folmediscria, oblonga, breviter scuta, basi sensim attemata, viric-glauca, subtus grisea, ad crstam eglaniculosa, ientes simplias, ovato-triangulares, recti, pet. cglanaulosi, parce aculesti; stip. breves, sat latae, virides, gorso

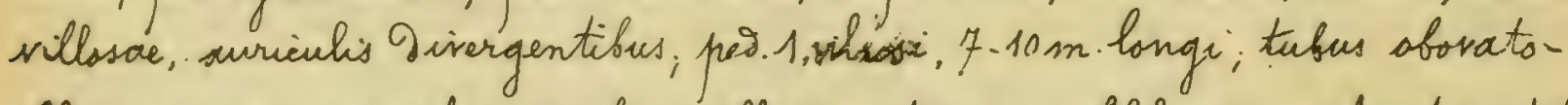
oblongus; sep. primnulis annibus villosis, intrgris, subfolicucis instructa, stigh: Dosperse piloss, gises comis, cor......... fruct. avaideres, basi attemeatus, atrice turgious nee contractus.

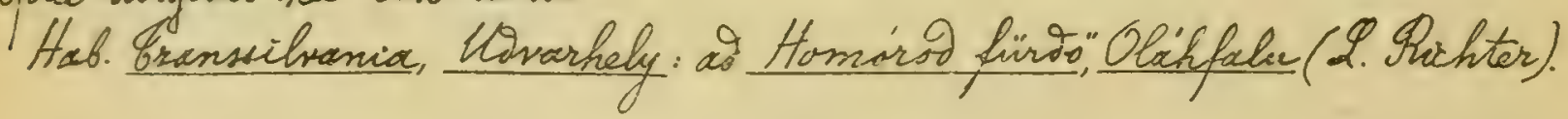

2111. Crepinia auroris Gogr 8.eb. 1'0 2669.

Avenlei faleato-gilatati, ar ramos flor. copriosi; fol late obovato-subattenuati, basifere rotuniata, as costam eglanjoulosa, gentes simplices; pot. 
$-315^{\circ}-$

eghanieloss', alignot parce aculeati, stip. virides, mediseres; hes. 2-4, parce villasi; 13-17 m. longi, tubus oviàeus; sep. pinnulis angustis, sub́glabris instructa; istyli asperse pilosuli, Sisco paulo conico; cor. pallidissime rosea, eciliata; fruct. ovoiciens.

Hab. Phone, as Montmalas: in monte Mt Bonnet (N6. Yjaniogar)

2112. Crepinia lucrata Gigr mss.

Aculer breves, Duri, falcats-Dilatati, at ramos flor. Dense foliosos panciores, fol. oblongs-lanceolata, basi breviter attemuata, coriaced, narvosa, supra viriciglancescentia, subtus pallias et il costam eglanoulosa; dentes lanceolatoconvergentes, fere omnes biserrati; pet. hinc inde glandulosi, aculeati; stip. virives, sat anguste, frever, subtus rillosar, auriculis sat Divergentibus; pro. 1-2, villosi, g-11 m. Congs; tufus suboblongus; sep. pinnulis ansibus longis, glabris, sat latis, inciso-dentatis instructa, acumine foliacer, inciso; styli subglaberrimi, gises convico; cor.......... finct. oborato - oblongus, basi gepressus, ad apiam atteruato - strangulatus.

H.xb. Fransilvania, Hoiromszék: ol Also'-Earja (2. Inichter).

2113. Crepinia constricta Gigr \&ab. N-26\%0.

Acculei gebiles, subilatati, recti sel inclinati, ad ramos flor. Danse folissos sacpe nulli; fol. obovato-elliptica, breviter attennata, basi notunaata, is cstam eglanivulosa; Dentes simplices, convergentes; pet. eglaniolosi, fere omnes inermes; stip. parrace, auriculis givergentibus; ped. 1, villosi, 6-13 m. longi; "ubus oblongus, sep. pinnulis angustis, subientatis copiose instructo, styli parce hirtelli, gisco subplans; cor ......... fruct. obovato-oblongus, basi. rotuniatus, apice breviter attenuatus, sepalis reflexis ise caronatus.

Hab. Helvetia, Halais: ad Sion (Lagger).

t+ tyli villosi.

2114. Crepinia atlantica Gige Gab. N:2666.

toculai falcato-gilatati, as ramos flar. sparsi; fol. ample oborato-oflanga, abrupte acuminata, fasi brevisime contracta, intense sirioic, at costam. paulo glanioulosa; dentes acuti, lanceolato-aperti, inf.valie biserrato-glanqulosi; pet aculexti, glandulosi; stip. longae, auriculis divergentibus; per. saepe corymbosi, prebescentes, 15-17.m.longi; tubus oborato-oblongus; sep. 
-

maxema hinsulis tinuiter marginato-glanivelosis, latis instructa; acamins. fliaces, integro; styli villosi; giseo pare comio; cor eciliata; fruct ovsidens, utrinque attern'stas.

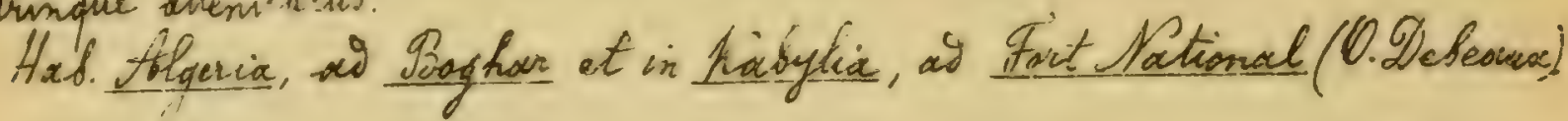

2115. (repuivia prerose Corog mss.

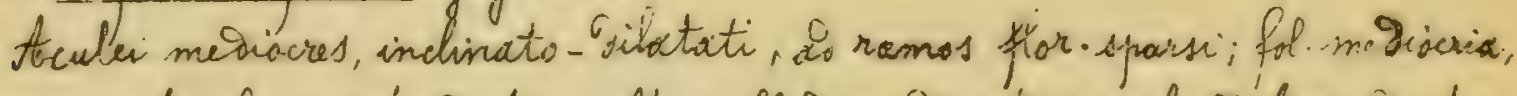

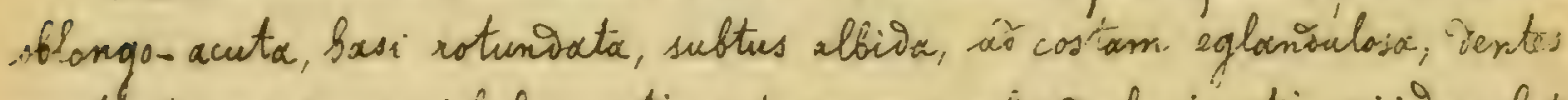

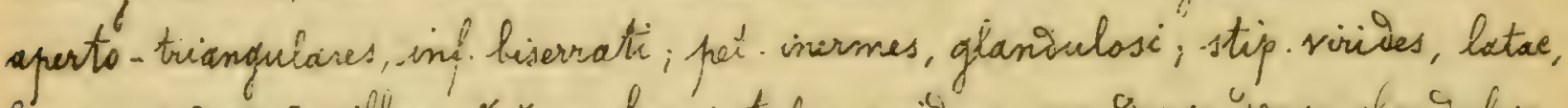

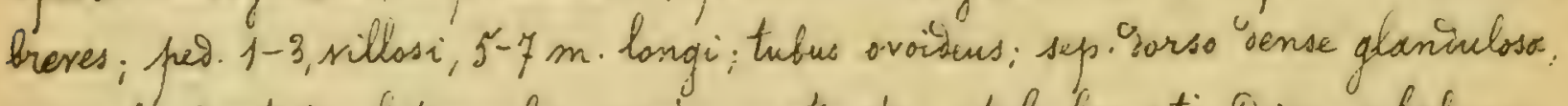
prinnulis dentatis, latiusculis, copiose inotructor; styli tanati, 'dises subpians; cor........ fruct. mediscris, ovoideses, basi gepressus, ipric strangulatus.

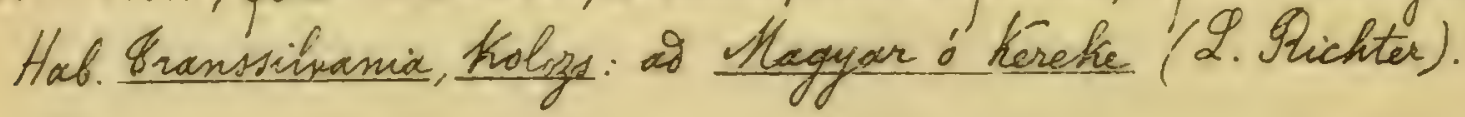

2116. Crepinid glabrior Giger Gab. N:2671.

Aculei falcato-gilatate, a ramos flor. flexuosos geminati; fol. late oblongo-acuta, basi freviter attervata, intense viridia et superne parce villosa, as costam eglaribulosa; Dentes simplices, ovato-aperti; pet. coflaribulosi, inermes, stip. latae, majusculae, arericulis Sivergentibus; peo-1-2, alii loeves, alis aisperse glaniulosi, 6-8 m. hong-; tibus svoideus; sep. reciana,......., styli villosi, gisco plans; cor.......... fruct. ovoidens, basi gepressus, at apicem attenuatus.

Hab. Suciax, Scania: as Kall . Pobbelof (9. T. Luniquist).

2117. Oreprinia oreades Gojo mss.

Acculei rarissimi, freves, inclinato. gilatati, wo ramos flor nulli, fol. laté oforeto-acuta, basi rotundato-coroata, subtus glauco-albida et ad costam eglanioulosa; Dentes simplices, ovato-recti; pet. subinermes, eglanoulosi; stip. Sorso rubentes, sat latas, breves, auriculis divergentibus; ped. 1-2, villosi; $10-12 \mathrm{~m}$. longi'; tubus oborato - sblongus; sep.........; styli villosi; gisco concic; con....... fruct. parrus, oborato-suboblongus, utringpe attenuatus, paulo turgians. Hab. Hungaria for. gSacy: at hovacisi (2. Richter).

2118. Creninia lanuginasa Ggor \&ab. N-2072.

foculei validi, falcati, late gilatati, as ramos flor. nulli; fol. parre, obo-. vato-acuta, basi rotuniata, longe hirsuto-laninginosa, at costam eglaniu- 
Cosic; iontes simplices; patule triangulares; pet aculecti, eglandalosi; Pense et tongissime pilosi; itip. nubentes, awviculis sot sivergentibus, pes. 1, villosi, g-10 pr. Long-; tubui chovats - oblongus; sep. pisinulis omnibus integris,

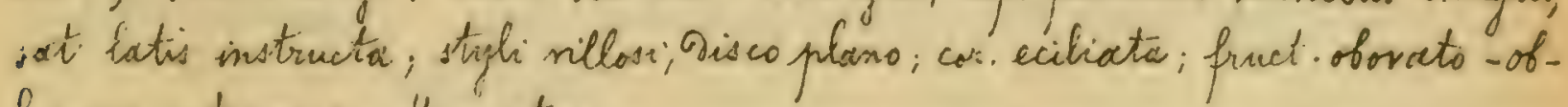
longus, stringuce attemesctis.

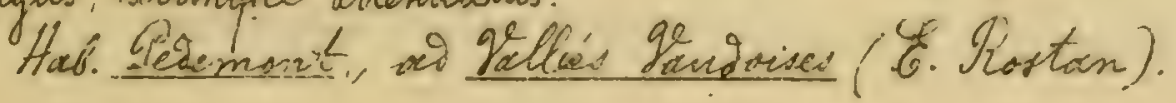

\section{t† Stygli lanati.}

\section{Crepinia macrotricha Gogr. \&ab. N:2673.}

teculei inchinatos silatati, as ramos flor. geminati, rel subnulhi fol. lat oberacto-subacuta, basi roturivatio, mollissine tomentose, ad costam chlanguloss; Jentes simplices, lanceslato-convergentes; pat inermes, eglanoulosi; stip. virides, auriculis Divergentibus; per 1-3, paulo pubescentes, g-10 m. longi; tulus ovoiseus; sep. pisnulis integris, latis, tomentosis instructor; acumine lato, integro; styli lanati, gisce subplano; cor.eciliata; fruct. ovoitens.

Hob. Sasses- Holpes, at La Condamine: alt. H000' (Mroal).

\section{Greninia carpinifolia Gogr mss.}

Aculei breves, folcato-Dilatati, ad ramos flor. glaucos interaum geminati; foi amplissine obovato, abrupte acuta, basi rotuniata, subpetiolulata, lacte viriaia, subtus pallida, ai costam eglanvuloss, Dentes simplices, ovato-triangulares, subconvergentes; pet. eglanuclosi, aculeati; stip. macimae, virides, longissimae, tantum aprice gorsi tomentellae, auriculis subsirergentibus; pel. 2-4, hirtelli, 6-10 m. longi; tubus avoidens; sep. pirnulis annibus glabris, integris, foliaceis longe instructa, acusmine magno; styli lanati, gisco paulo conico; cor-eciliata; fruct. ovoiders, basi gepressus, apice attenuato-substrangulatus, centrali autem inferne gecurrente.

\section{Hab. Oranssilvamia, Uvarhely: at Homoró, Oláhfalu (2. Michter).}

\section{Crepinia poliotricha Gogor mss.}

teculei breves, falcato-gilatati, gecurrentes, ramos flor parce foliosos rari vel mulli; fol amplissime obovato - oblonga, abupte acuta, basi rotunorata, vix petiolulata, atrovirentia, subtus virescentia, xo costam eglanDulosa; Jentes simplices, late svato-recti, pet.sglanoulosi, sup. saepe iner- 
$-318-$

mes; stip. virides, latiuscular, clongatar, gorso pubescentes, auriculis rectis; per. 1-3, villosi; $15 \mathrm{~m}$.longi; tubus suboblongus; sep. pinnulis amnibus foliacio, antatis, vix hirtellis, copiose instructa, acumine saepe ample foliaces, laciniato; styli lasnati, risco subeonico; ca........ funct. obor acto. oblongus, basi depressus, apice attennato - strangulatus.

Hab. Iranssilvania, Kolozs: ad Reketyé prope Kolozvár (2. Richter).

2122. Creminia adenotricha Gogr \&ab. N-2674.

Aculei breves, parri, falcato-dilatati, so ramos flor-verticillati; fol.oblongo-xcuta, basi rotuniata, ob costam eglaniulosa, dentes simplices, aperto-triangulares; pet. eglangulosi, aculeati; stip.majusenlae, auriculis airergentibus; pè. 1-2, villosi et simul parcissime glanoulosi; 7-8 m.longi; tubus ovoiders; sep.erecta, sorso glandulosa, pinnulis integris, foliaceis, acumine maxims, laciniats; styli lanati, dises plano; cor.......; fruct magrus, ovoideus, basi valde et abrupte attenuatus, apice rotuniatus. Hab. Suecix, Eestrogothia: in monte Kinnekulle as Erolmen (y. \&. Letterstest).

2123. Crenisia semipibse Gogr bak. $N=2675$.

toculei caulinares falcato-gilatati, ad ramos flor.minores vel saepe mulli; fol. obovato - oblonga, freviter attenuata, basi notunsata, hair mollia, superne parce villosa; gentes aperti, inf. paulo biserrati, pat. aspperse glangulosi, plerumque annes inermes; stip. pursescentes, auriculis givergentibus; pes. 1-3, villosi, 7-12 m. longi; tubus oblongus; sep. eracta, pinmulis longis, latiusculis, Dentatis instructa; styli lanati, exserti, gisco comic; cor........ fruct sbovato-oblongus, basi plus minusve Depressus, apice longe attenuatus.

Has. Savoie, as Sessons-sur- Dalins prope Moutiers (Muget). Forsan sectionom frace., Pilosece seilicet, pertinet.

2124. Crepinia kinnekullensis Gogr bab. N-2676.

Aculei breves, falcato-Dilatati, ad namos flor. geminati; fol obosata, abrupte at breviter scuta, basi subattenuata, as costam eglanoulosa; Pentes aperti, triangulares, simplices; pet. aculeati, eglaniulosi; stip. breves, sat latace, auriculis aiverger,tibus; prea. 1-2, pouls villssi, 7-8m. longi; tubus orsidens; sep. eglandulosa, pinnulis longiesoulis, sat latis instructa, acumine longs., 
$-319-$

Poliaces, integro, styli lanati, gisco plans; cor..........; fruct. magnus, ovo:teus, basi valie attenuatus, spice breviter strangulatus.

Hef. Suecia, destrogothix: in monte Kimekulle, inter gresterphena ot $\frac{S \text { lomberq }}{0}$ ( $\%$. \%. Letterstegt).

2125. Crepinia bihariensis Gigr mss.

Acculei tenues, frevissimi, subisilatati, temuiter falcati, ag ramos flor. submulli; fol amplissime obovata, breviter attenuata, basi rotuniata, coriacea, nervosa, lacte virioia, subtus pallisula, costam glanoulosa; Dentes ovato-convergentes, fore annes biserrati; pet.glasioulosi; aculeati; stip. langae, latrissinace, subtus villosace, auriculis subrectis; per.saepe ternat, villosi; $7-9 \mathrm{~m}$. longi; tubus sblongus; sep. pirnulis parce villosis, omnibus latis, 2-5-2entato-glaniulosis instructa; styli magni, lanati, Qises subconis; $\operatorname{cor} . . .$. ifruct maximus, sublongus, hosi rotundatus, apice atte"matto-conicus, centrali sutem ivhuc mojore, inierne gecurrenti-incrassato. Hob. Eranssilvania, Bihar: a) Muspok fiil \$i, Nayyvarad (2. Richter).

2126. Crepinia rapta Gogr mss.

Acculei parrs; freves, inchinat o- subs latate, samos flor geminati; fol. iimpile oblong-acuta, fasi poulation attenuata, subtus virescentia, as costam. glangerlosx; Gentes simplices, ovato-recti; pet-gilaniulosi, aculeati; stip. siribes, breves, latae, auriculis subsivergentibus; pes. 1-2, villosi; 8-10 m langi; tubus svoidens; sep.......... stgh lanati, disco prailo conico; cor......... fruct. sat late sviviens, fasi subrotusidatus, aprice freviter sitenuatus.

Hab. Iransilvanix, Solnok Doboka: at Csernefalu (L. Richter).

2127. Crepinia tomophylla Go Gab N 2677.

Acculei minutisimi, recti, fere subulati, as ramos flor. demidatos parceque foliosos sat copiosi; fol. obovata, aprice roturnato-subobtusa, basi abrupte attemuats, unicine mollissime tomentosa, albida; gentes inf. bisernati, pet. anmes inermes, copiose glandulose; stip. latae, subtus tomentrace, inf. insupa glandulosae, auriculis parum givergentibus; ped. 1-2,

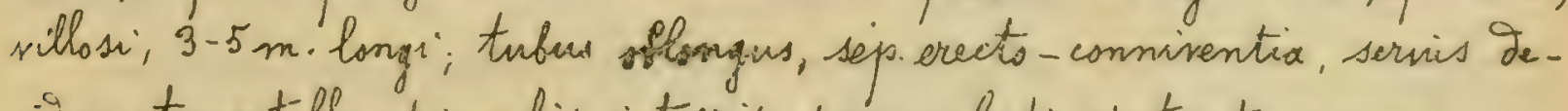
ciisur, tomentella, prinmulis integris, pranm latis instructa, acumine elongato, gilatato; styli maximi, ilib-lanati, gises plano; cor-albo-rosea; pruct uefolongs, hasi attenuatus, aprice contracties. 
$-.320-$

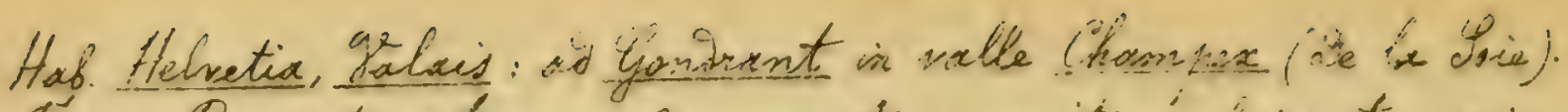

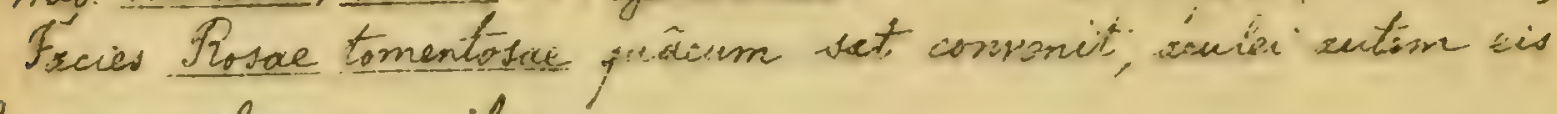
Rosac pomifora similes.

2128. Crepinia jesta Corar mss.

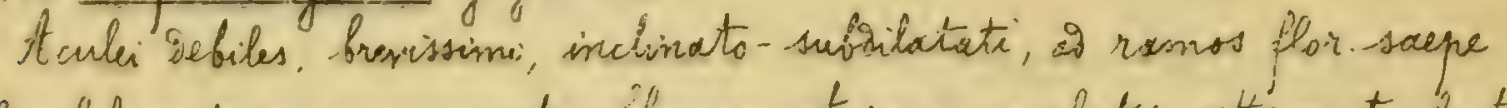

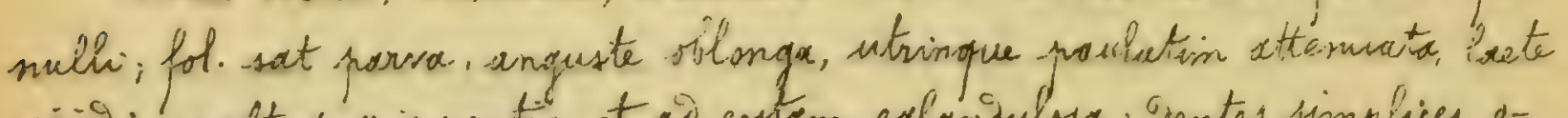
siridia. subtres grisescentia t ai costam eglaridulssa; ventes simplices, o-

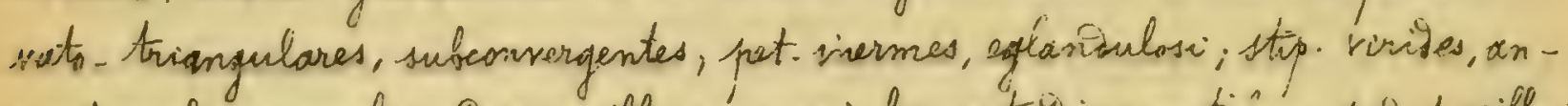

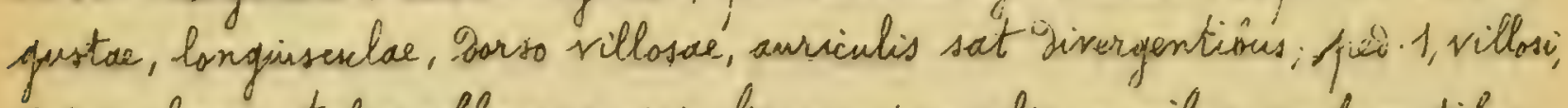
5-8 m.longi; tubus sblongus; sep. hrevia, pinnulis mmibus puseccantibus, late. foliaceis, inciso-gentatis instructa, styli lanati, Disses julans; cor. amsene rosea, meorocris, eciliata; fruct. breviter oblonous, citrin que attiensat.

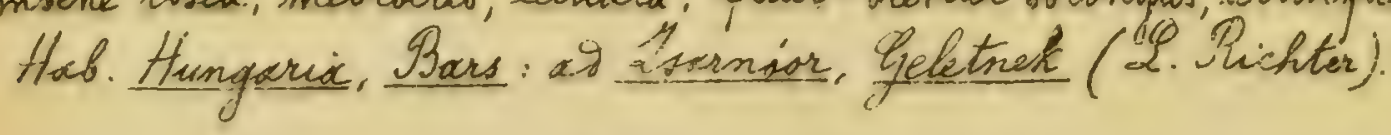

d-Fructus oflongus.

f Styli giabiri, glafrescentes vel paulo hirsuti.

2129. Gepinia punctillata Gogr Gab. N: 2678 .

Acculei breves, gilatati, valde falcati, ol rasnos fler. sat coppiosi; fol parra, svato-elliptica, acutx, basi abrupte cuneata, unorique asporo-punetato, costam paulo glarisulosa; rentes simphices, sublanceslato-consersentes; pet aculeati, eglanoulosi; stip. rubentes, auriculis rectis; peos 1-3, villosi, 7-8m. longi; tubus elongatus; sep. pinnulis onsibus linearibus instructa, styhi poulo hirtelli, Disco valse comics; cor......... fruct oblon gus, basi subsepressus, apice longe attenuato-conicus. Hab Bouches-gu-Mhone, as Mognac (f. Autheman).

2130. Crepimia onerato Groge mss.

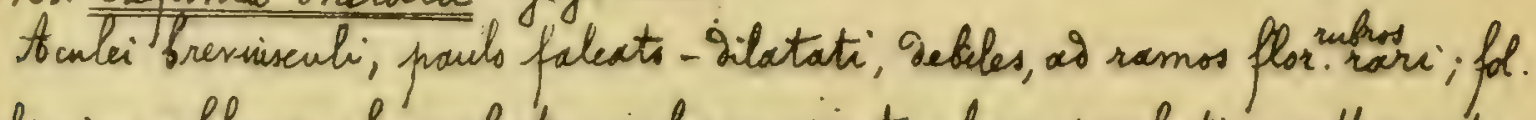
amplissime oblong-lanceolata, subacuminata, basi paulation atternata, lnete virioi-sublancescentix; subtis canescentia, or costam eglanoulosa; Dentes simphies, breves, triangulares, racti, pet. eglanoulosi, eubentes, culeati; stip.elongatre, latae, rubentes, Iorso villosae, awriculis rectis; peo. 2. 4, villosi, 7-10 m. longi; tubus oblongus; sep......... styli assparse pilosuli, giscs subplano; cor........ fruct. sat magnus, oblongus, utringue attenuatus. Hab. Hungaria, ot Nas (L. Richter, At. Steffeck). 
$321 \ldots$

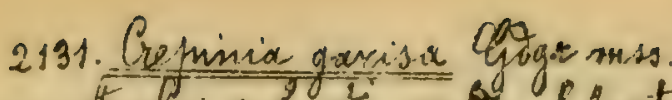

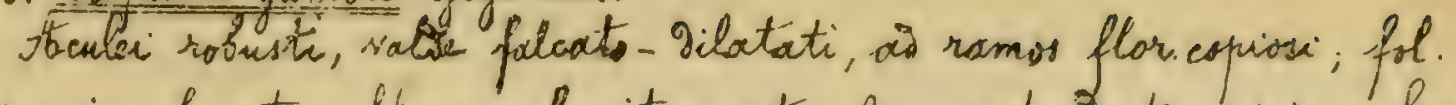
mesiocria, obovato - oftanga, freviter acuta, fosi rotuniacta, cariacua, lacte. viridia, subtus virescentia st as astam eglanidulosa i entes simplices, sati, triangulares; pet. aglanoulasi, inermes; stip. basi rubentes, sat longar, latra, yorso pubescentes, euriculis oivergentibus; pres. 1-2, villosi, 8-10 m. longi; inbus sblongus; sep. pirmulis villosulis, anmibus latis, 2-4-dentatis paras instructa, acumine subloliaces, integrs; styli glabrescentes, gisco corriais; cor........ fruct oblongus, basi attenucatis, apice longissime contruct. strangulatus.

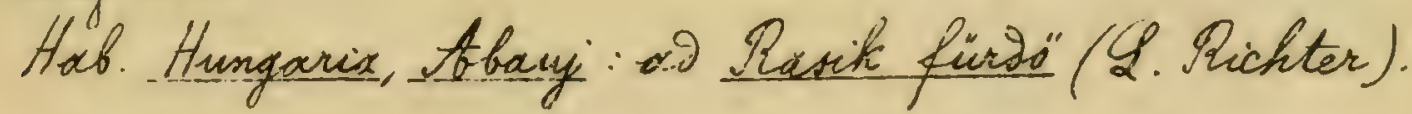

2132. Crepinia opposita Ggar mss.

Acculei minores, Greves, falcato-Dilatate, a ramos flor. nuilli; fol. amplissime oblongo-auta, fasi attenuata, superne intense siridia, subtus canescentia et à costam eglanisulosa, Dentes simplices, propunde ovatotriangulares, subrecti; pet, inermes, eglanivulosi; stip. lattae, longae, virises, subtus toto pubescentes, awriculis givergentibus; pes. 1-3, villosi, 12-15 m. longi; tuburs oblongus; spp.elongata, pinnulis glabrescentibus, annibus foliaceis, 2-3-ientatis instructa, stgli glabrescentes, disco plano; cor majuscula, rosea, eciliata; fruct oblongus, fasi attemuatus, apie-longe contractus. Hab. Hungaria, Bars: ad Geletrek, Harnois (2. Richteir).

2133. Crepinia stictochlanys Gogr dab. N-267a.

toculei breves, falcato-dilabati, or ramos flor. sparsi; fol obovato-alliptica, apice subrotuniacta, fasi rotuniata, costam eglomauloss; Dentes svato-aperti, inf. biverrati; pet. basi asoperse glansulosi, inermes; stip. Dilute rubentes, auriculis givergentibus; pres.1-2, viliosi, 23-25 m. longi; tubus slanguj; sep. pinnulis latis, copiose sentato-glandulas is instructa, acumine ralde foliaceo- Dentrato; styli glabri; Sises comico; cor. esiliate; fruct. oblongus, basi subrotundatus, apice longe attinuatis.

Hab. Sedemont, ad It Geant (o. Astan).

2134. Crepinix locato Gogr mss.

Aculei rari, breves, parum faleato-gilatati, o ranw flor-mulli; fol. parra, anguste oblongo-acuta, basi altemwatho, apeses iscrivic-sublauces- 
$-322-$

centio, subtus grisec, nersose et ai costam eglenaulssa, Jentes simplices, triangulares, recti; pot inermes, eglanialosi; stip. viriotes, angustae, sat longae, subtus fubescentes, auriculis subivivergentions; peo 1, sparse rillosi, 4-6m. lange; thbus oblangus; sep. pirnmlis giubris, angustioribus, 1-2-iven. tatis instructa, acumine Catrisseulo, integro, styli glabri: aisco conico; cor. magna, rosea, eciliats, fruct. oflongus, utrinque longa attennativs.

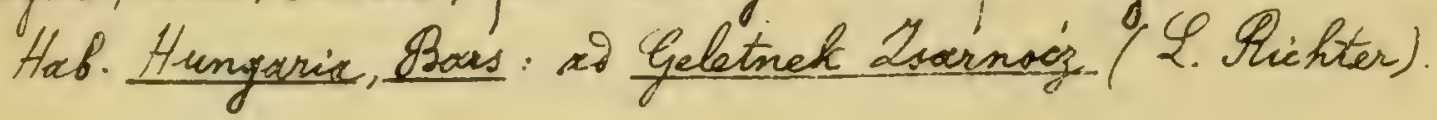

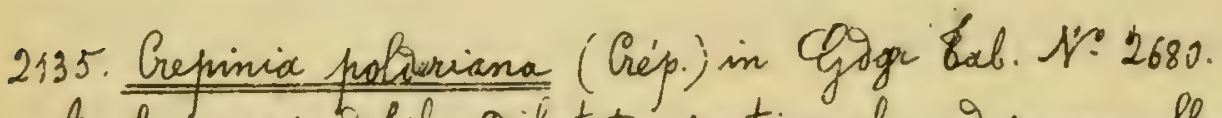

foculei rari, Jzbiles, gibetato-rectiusculi, ad ramos flor mulli; fol. late obovata, breviter senta, basi abrupte truncata, a basin costac praesertion minute glaniulosa; Jentes lati, patiuli, fore smnes biserrati; pet laxe pubescentes, aculeati, copiose glanaulosi; stip. glabrescentes, "Sorso, paulo glansulosa, auriculis givergentibus; pes. 2-3, villosi, 5-11 m. longi; tiebus oblongus; sep. pinnulis clongatis, foliaceis, Dentatis instructs, acumine amplo: styli glabri, Siseo sublans; cor. fore alba; fruct. Oblangus, Gan gejressus, ai apicem valia attemuatus.

Hab. Melogium occit., as Lan Hoek prope Dixmuide (F. Créfin).

2136. Crepinia Hulsemii Gagr Lab. N-2681.

toculei breves, falcato- Bilctate, as ramos for graciles mulli; fol prarva, oblang-lanceolata, basi sttemuata, pallide viridia, at costam eglanaulase; gentes simplices, lancesalato-recth; pet eglanoulosi, parce aculeati; stip. parvace, angustac, auriculis divergentibus; peis-1, paulo hirtelh; g-10 m. longi graciles; tubus sblongies; "ap. pinnulis latis, frevibus, 2-3-9rentatis instructa, acumine parms lato, integru, styli glabri, Disco poulo

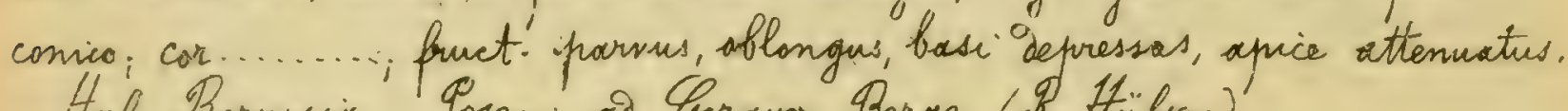
Hab. Borussia, gosen: ad Gorayor Merge (R. Hiilsen).

if Stylimislosi.

2127. Creminia accurata Gogr mss.

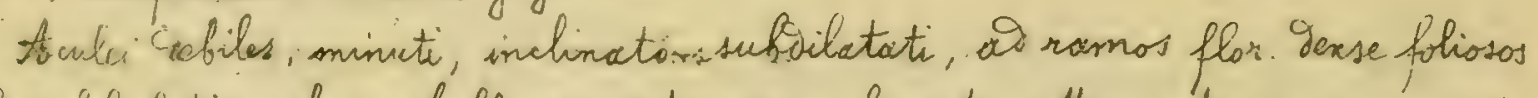
sull: for hatiuscule suboblonga, utringue breviter attenurita, crassa, coris -

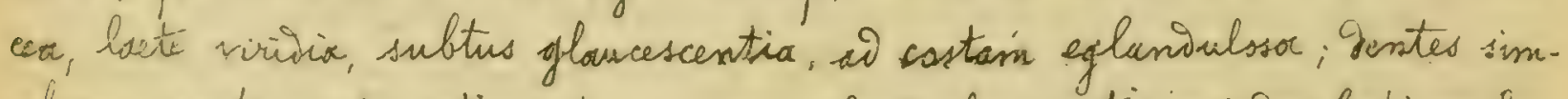

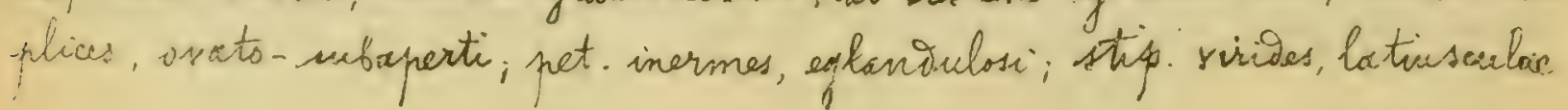


$-323-$

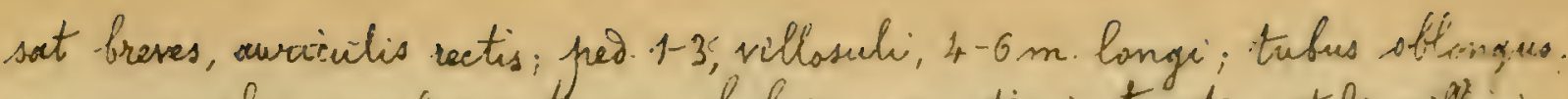
sep. purnulis ansibus integris, plabris, angustis instructa; stigi velsisi, Dises plano; cor......... fruct, sot snagmes, sflongus, base Topressies, sinses, sttemeato-conicus, strangulatus, turgious.

Hab. Hungaria, at Buvakesjieriso (2. Richter).

2138. Crepinia folliomin Goyr toab. N-2682.

terelei falcato-ailatati, so ramos flor gominati; fol. late vato-elliptica, acuta, basi rotuniata, costasm eglandulosa; gentes simplices, ovato apati, pet. aculeati, hasi paulo glanimulosi; stip. sivides, auriculis valte Pivergontibus; ped. 2-4, villori, 12-14 m. langi; tubus oblongus; sep. pinnulis latis, 4-5Dentatis instructa, acusmine subfolvacer, Pentato; styli villosi, Disco conico, cor.eciliata; fruct slongus, itringue atternucitus. Hal. Gedemont occis, at It Ieconi (E. Rastan).

2139. Grenmia Michaelis Cgoge mss.

toculei mediseres, gilatati, tenuiter falcati, as ramos flor. foliosos copissi, fol obovato slonga, abupte acent, basi truncata, superne atrovisentia, et spers-punctulata, subtus prallita, at costam eglansulasa, Sentes simplices, profunde sublanceolato-convergentes, pat, inf a asperse glanoulaselli, parce aculeati, stip. breses, angustaes viribes, auriculis sivergentibus, gorss villosae; peo.1, villosi, 7-10 m. longi; Aubus oblongus; sep. pinnulis villosis, sat latis, 4-5-gentato-glandulosis instructa, acumine integro; sityli villasi, Disco paulo cirvico; cor .........; pruct. majusculus, oblongus, Sasi gepress. apice longissime ttemuato- strangulatus.

Hab. Iransiliania, Izeben: ao Michelsberg, Kis-Dingnod (2. Richtor).

2140. Crepinia evagata Gogr mss.

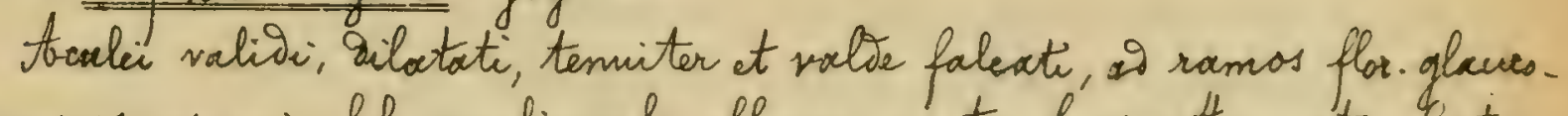
purpuress coprosi; fol ampliuscule sblongo-acuta, basi stemuata, irete virisia, subtus glauca ct os costam eglaniulosa; dentes simplices, trianguelariconvergentes; pat-eglanisulosi, aculeati; stip. sirises, elongatae, sat angrestae, subtus pubescentes, aurviculis Divergentibus; pes. 2-3. sparse scllosi, 12-15 m. longi; tubus clangatus; sep. primulis glabis, latis, 2-3-dentatis instructa, acumine subfoliaces; sty ti flarce villosi; gisco valde conico; cor. ignota; fruct magnus, oblongus, basi de pressus, apice longe atterniato- 
$-324-$

Strangulatus, centrali autam majore, basi decurrente tirgiaulo. Has. Iransilvanix. Nolojs: a Canthegy prope Kologsvar (2. Richter).

2141. Crepinix Pentocalyx Goor Gab. M-2683.

stenki longinsenli, falcate - Dilstati, as ramos flor. foliosos pauiciores; fol. oborato-oftusiuscula, basi breviter attenserta, glauca, as costam eglonivulosa, Jentes simplices, ovato-aperti; pet. inermes, eglanivilasi; stip. oilatata, auriculis rectis; pes.1-3, sillosi, g-11 m. longi; tubus gracilis, oblongis, sep. Decianx, elongata, pinnulis foliaceis, copiose dentato-glanianclose instructa, acumine folinces-oenticulato; styli villosi, dises comico; cor. pollive rosea, eciliata; fruct. anguste shiongus, basi abrupte at parum recurrens; apice valse strangulatus. Hal. Mtalia arient., at Sonze (2. Collesi).

2142. Grepinia barsensis Ggigr mss.

Stcrlei breves, sebiliores, silatati, minute falcati, somos flor. plerumque mulli; fol oblongo-subacuta, basi freviter attemuata, intense viridia, subtur grisea at as costam eglaniaulosa; Qentes simplices, sublanceolato - convergentas; pet.colantulosi, inf. inames; stip. sirides, longoe, latar, subtus pubescentes, auviculis sat givergentifus; ped. 1-2, villosi, 5-7 m. longe; tubus oblongres; sep. pin mulis hirtellis, brevibus, amnibus latis, 1-2-ientatis instructs; stagl villasi; giseo paulo conics; cor-meoriscris, subalba, eciliata: fruct. oblangus, utringre. attenuatus.

Hab. Hungaria, Sars: as Geletrak, Zsarnocy (2. Michter).

2143. Crepinia cylinarocarpa Gagr mss.

toculei Debiles, temiter inchinati, parum Dilatati, id ramos flor. graciles nulli; fol. suboblonga, brevita acuminats, basi attenveata, ad castam eglanaulasa; Dentes simplices, aperto-triangulares; pet-inermes, eglaniulosi; stips. angustissimas, virites, auriculis parmm givergentibus, prod. 1, basi pubeseentes, 11-13 m. longi; tubis elongatus; sep. pirmulis lostis, 1-2-dentatis instructa, acumine integrs; stygh villosi, disco páulo cosnics, cor....... frict. clongatus, basi attenuatus, apicis longe et valse attenuato-strangulatus.

Hab. Exprol ai Gabbiols propre Erente (\%. Gelmi).

2144. Crepinia offermata Gogr mss.

Aculei freves, faleato-gilatati, a ramos fior nulli; fol. late ofo- 
$-325-$

vato.oblongar, apice subattemuata, basi poulation rotumiata, crasso, finm nersosa, intense riridia, subtus pallidula et à costam eglandulose, ientes simplices, late avato-convergentes, pat.eglandulasi, vif incomes; stip.latisimae, longatac, sirides, subtus as yorsum pubescentes, auricilis vivergontibus; per. 2-6, pilosuli, 11-14 im. longi; tubus oblangus; sep........., sighti villosi, gisco poulo corrico; cor.......; fruct. oblongus, base Genressus, is opiriem atérivito-cosicus.

Hab. Hungaria, Bubapest: a kis Lvobhegy (2. Olichter).

t t ' Ptyli lanati.

\$. Petioli annes aculeate vel tantum inferiores inermes.

2145: Genimia Letterstestii Gigr bat. N-2686.

Acula debiles, vix dilatati, recti vel inclinate, ad ramos flor.verticillati, fol parva, anguste oblong-acuta, basi breviter attemuata, cinerea, at costam eglanivulosx; gentes simplices, triangulares, aperti; pet. eglanaulosi, aculeati: stip angustac, sat parvore, auriculis givergentibus; pos. 1-2, villosi, 7-8 m. langi; tribus oblongus; sep. erecta, pirnulis anaustis, eglanaulosis, instructa, acumine parum lato, integro; styli lanati, disco plano; cor........ fruct. oblongus, utrinque valde attemuato-strangulatus, metio turgious.

Hob. Suecia, Gestrogothise: in monte Kinnekulle ag Gesterplanx ( $\%$. E. Ketterstest).

2146. Geninia clausiopolitana Gogr mss.

tbculci tenves, elongati, parum Silatati, tenuiter subfalcati, as ramos Por.copiosissime; fol-minuta, oblongs-acuta, borsi attemuata, supra palliceviridia, subtus polliva, at costam eglanisulosa, dentes simplices, lanceolatorecti, pet. eglanoulosi, inf. inermes; stip. brevinsculoce, sat angustac, virides, porso villosac, auri-ulis subsivergentibus; pet. 1, villosi: 7-10 m. longe; tubus oblongus; sep. brevia, pinmelis glabrescentibus, latiusculis, ommibus copriose Dentato-glanoulosis instructa; styli albo-lanati, Bisco plano; cor. fruct. magnus, oblangus, basi atternatus, apice abrupte strangulatus.

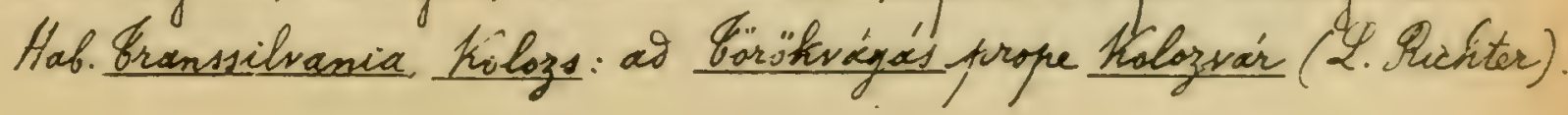

2117. Crepinia scaphusiensis (Gremli) in Gogr \&ab. Y 1687.

Abculei breves, falcato-Dilatati, at ramos plot. geninati; fol. oblongo- 
$-326-$

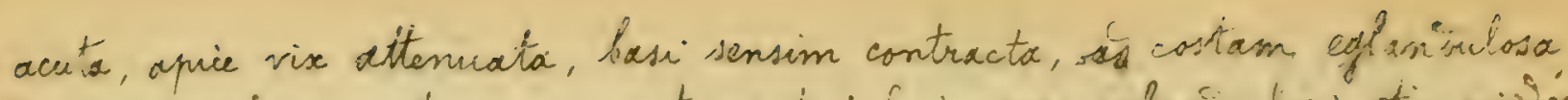

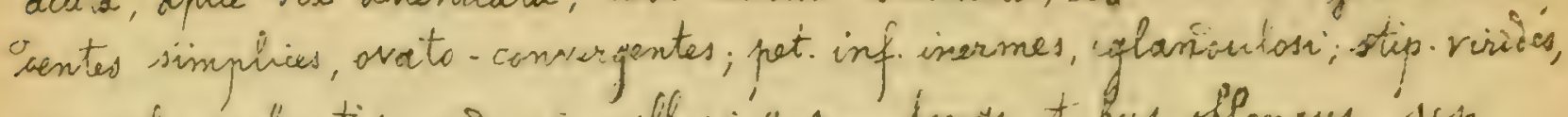
surriculis subrectis; pes. 1-i, villosi; $8-9 \mathrm{~m}$. Conge, tivous ollongres; - sep. pinnulis integris, brevissinis, latis instructa, acumini angusto, stifh lanati gisco phano; cor. cciliata, fruct. breviter oblangus, fasi subrotuniatus, if apicem stierivatus.

Hab. Helvetia, Ichafforese: a' Haengenthalt (K. Lehmann).

§E. Tetish anines inarmes.

2148. Orepinia suecica Gogr Gab. N:2685.

Acentei sebiles, Bilatato - subfaleati, at ramos flor. copiosi; fol. oblongoacuta, basi breviter attemuata, costam eglasimilosex; gentes simphies, aperts. triangulares; pet. inermes, eglavaulosi; stip. mesiocres, auriculis givergentibus; pier-1-2, sparse pilasi, 9-11 m. longi, tubus oblongus; sep. pinnulis longis, integris, latiusculis instructa, acumine integro, lato, styli lanati, oisco plans; cor. rosea, sciliato; fruct oblongus, apuice attemuatus.

Hab. Suecia, Gestrogothia: in monte Kinnehulle al Raback ( I. Q. Thettersteot).

2149. Crepinia megapoox Gogr mss.

Eeulei temiter, inclinati, parum oilatati, ad ramos flor melli; f. ample. obovato-suboblonga, utrinque attemuato, midis gilatata, subtus virescentica, at costam eglanoulosa, Dentes simplices, triangulari-subconsergéntes; pet. eglanioulosi, inermes; stip. viriies, latoe, breves, surviculis sat givergentibus; peid 1-3, villosi, 11/2 cent. longr; tubus oblongus; sep........., stygli lanati, siseo conico; cor. fruct maximus, elongatus, basi: gecurrens, apice attemuato - strangulatus.

Hab. Oransilvania, Szolnok Doboka: ad Cornefalu (2. Richter):

2150. Crepinia amplissima Goger mss.

Trulei valivissinn; gilatati, valio falcati, as ramos flor. pauciores; fol. amplissime sbovato - oblonga, acuta, basi rotuniacta, supe ne lacte viridia, Subtres glanco-alliax, ai costam eglanaulase, ientes aperti, ample ovate, omnes biserrati ses eglansulissi; pet inermes, eglanoulosi; stip. latae, sirives, subtus tomentosae, auriculis semidivergentifus; per.1-3, villosi, 
$-327-$

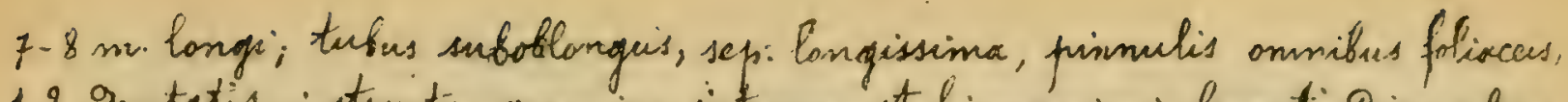
1-3. Ientatis instrueta, acumine integro; styli maximi, lanati, gisco plano; cor. intense rosea, eciliata: fruct. set magnus, subohlongus, basi attenuatus, aprice longe attemunto-strangulatus.

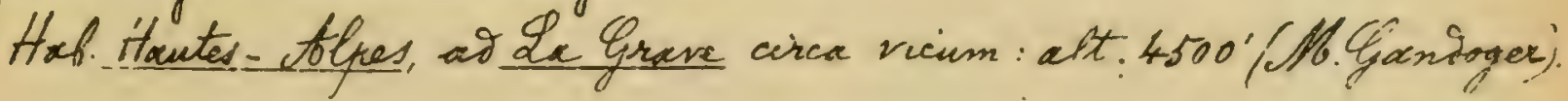

2151. Grepinia alpestricola Gogr mss.

Aoculir maxinui, valie faleato-ditatati, as ramoj flor phus minore

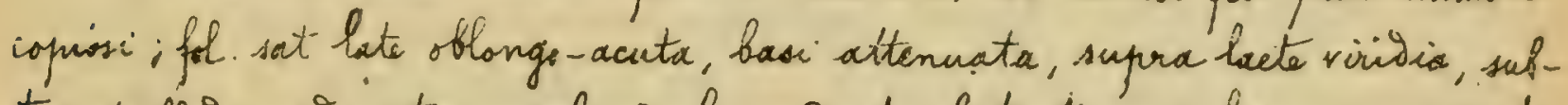
tus palliba, wi costam eglaniulosa, rentes late triangulari-convergentes, inacquales, fere annes fiserrati, nae glanoulasi, pet. inermes, eglansouloic; stip. viribes, latae, longae, suftus tomentoswe, auriculis subrectis, pes.1.: proulo villssi, 8 m. longi; tubus oblongus; sep. pinnulis paucionibus, perom Cotis, 1-3-gentatis, acumine integro, fotiaceo; styli maximi, lanati, onssco plano; esr. intense rosea, eciliata; fruct. oflongus, bosi attenuastur, apice. longe strangulatus.

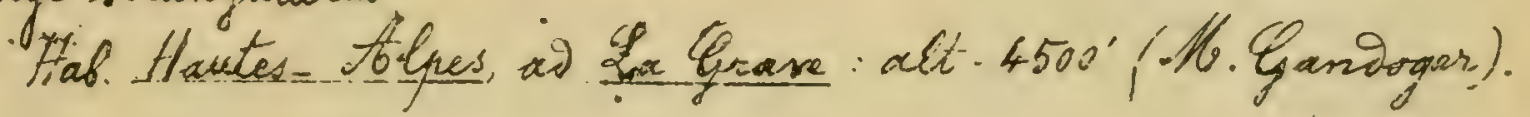

2157 Creninia firelia Gogr mus.

Acciha mediocres, inchinato-Silotati, a remos flor. nulli; fol parva, -blang-lancislata, basi acuta, subtus sufverescentia, a castam eglanisilosa; Pentes simphees, lanceslato-subaperti, pet. eglarsielesi, inermes, stip. sirisies, angustac, breves, auriculis subrectis; pei. 1, villosi, $1 / 1 / 2$ cent. longi; tubus elongatus, sep. Gorso glanidulosa, pinmulis latis, inciso-gantatis instructa; styh lanati, give comis; cor........ fruct ample clongatus, basi attennato-subiecurrens, in 1/3 sup-tiurgians, apicé vix attinsuates.

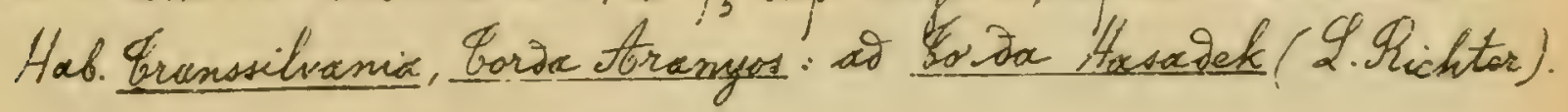

2153. Crepinia jacterns Gogr ms:

toculei vicivieli, gilatati, temiter faleati, ar ramos flor. stipulares; foh medioeria, obleng-acuta, bain attunuata, subtus albio-glanco, ic costam eglaniulase; dentes simplices, triangulari-convergentes; pot. eglingalosi, inermes: stip. virides, latec, brovusculne, curcichlis givergentisus, ped. 2-3, villosi, 8-10 m. longi; tibus oflongus; sep pinnulis omvibus integris, binearibus instructa; styli lanati, gises paulo conico; fruct. sat magnus, oblongus, basi gepressus, aprie longe attonuato-stianqulatis, centrali au- 
$-328-$

tem basi valts attemuatus.

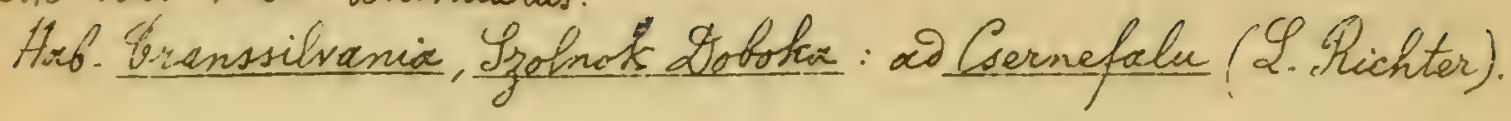

2154. Crepinia inensifolia Goar mss.

Acolei meviacres, falicato-oilatate, ad ramos flar. geminati, fol parva, oflonga, aprice subattermata, in $1 / 3$ sup. Dilatata et inde vique as bais contraceta, glauco-virioia, subtus canescentia, costam eglaniulosa; gentes simplices, profunsi, lancealato - recie ; pet eglanioulosi, inermes; stip. basi rubentes, sat latae, elongatae, subtus puborular, auriculis givergentibus; peo. 1. viélosuli, 4-5 m. longi; tubus oblongus; seps pinnulis pubescentibis, elongatis, integris, latis instructa; styli tanate, disco subplano; cor......... fruet, maximus, ohlongus, basi longe attenuatiss; in 1/3 sup. turgidus et 'trangulatus.

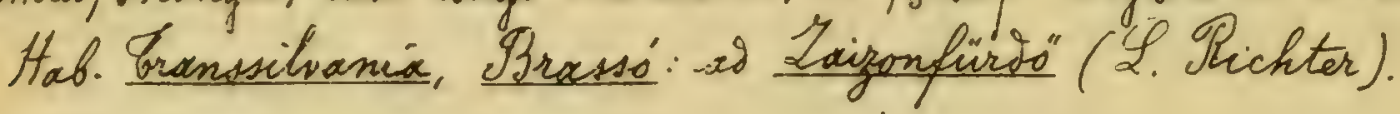

2155. Crepinia rostrilera Gogr mss.

teculei Conquasculi, parum gilatati, temiter subfalcati, à ramos flor. articulatos nulli, fol. ampliusecile oblong-lanceslata, basi attenuato-acuta, inténse viridia, subtus eglandulosa, Jentes simplices, oratotriangulares, subconvergentes; pet. inermes, hinc inde adsperse glaniaulosi; stip. latae, breves, virides, subtus villosae, auriculis rectiusculis; ped.1,2, hirtelli, 11/2 cent. longi; tubus sblongus; sep. elangata, pinnulis villosulis, integris, subfoliaceis, longe et copiose instructa; styli lanati, gisco plans; cor........ fruct. elongatus, mxior, basi gecurrens, apice longissime at-

Hab. Eranssitranix, Brasso': ad Laizonfïide" (2. Michter).

2156. Crepinia brossensis Gogir inss.

Acculi Gebiles, parum dilateti, tenuiter subfolcati, ramssflor. rigidos denseque foliosos instructa; fol parve, obovato oblonga, basi pouelation rotuniacta, apice ireviter acuta, inf. vero obtusa, pallide viridia, subtus grisea, xo costam eqlanoulosa; Dentes simplices, sublanceolato-recti; pet. inermes, eglaniulosi; stip.virites, "stae, sat longae, subtus pubsescentes, auriculis girergentibus; peo. 1, villosi, 7-9m. longi; tubus oblangus; sep. brevio, pinnuli smmibus angustis, 1-2-Dentatis, pubescontibus instructa, acumine integro; styli lanati, disco subplano; cor.........; fruct mojor; sblongus, thasi gepressass, aprice freviter strangulatus. Hab Iransilvania, Mrasso": 2o Laizonfiirgo" (2. Richtor). 
$-32 y-$

2157. Greninia termiflora Gogr mss.

taculei breves, late falcato- Dilatati, wo ramos flor. Densos pauciores vel nulli; fol. late sblong-acuta, basi paulation attenuata, ar castam eglanoulosa, lucide viridix, subtus virescentia; gentes simplices, profuniti, lancerlato-subconvergentes; pet. inermes, eglandulosi; stip. virides., elongatoe, latai, gorso pubescentes; ped. plerumgue 3, villosi, $7-10 \mathrm{~m}$. longi; tubus oblangus; sep. longiuscula, pinnulis annibus parce villasis, foliaceis, incisis, 3-5-Dentato-glanivulasis instructo, tyli sublanati, gises sat conico, cor......... fruct. ampliuscule sed breviter oblangus, basi subrotuniatus, apice abripte strangulatus.

Hab. Hungaria, tobayj: a hassa, Mankingaralo' (L. Richter).

II. AOdenophorace Gogr Essai p.32; Gogr Eab. rhor. p. 193. - Caminear collinas et tomentellar Crep. Drim. I, p.18.

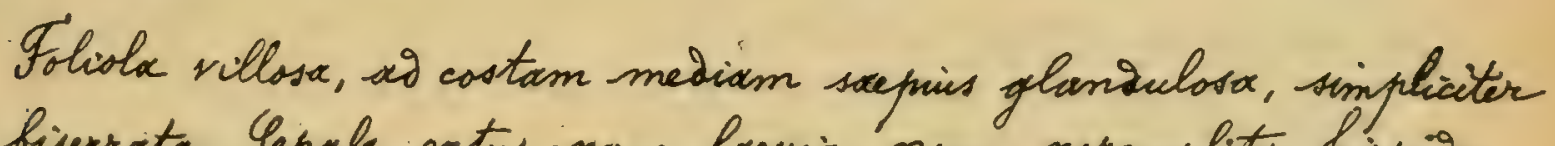
vel biserrata. Sepala extus nanc laevia, nune normaliter hispiva vel rubiginosa. Megmonli (saltem in quibusdarn invivisuis) semper vil. losi; hispiti vel villaso-glandulosi. Corolla rosea tunc ampla petalisque cordatis gonata, aut albita vel achrolenca, parra, ergo petalis coriato-cuneatis. Sructus glaber glandulasusve. Scular robust, conformes, rarissime in setas prodeuntes. - Sectio sat abnormis spreciesque inter se subissimiles continens, 'et forsan, ut nupa Qiai; ulteruis aliter ordi nansa; sel charactores firmiores hucusoque non novi.

Foliolo simphiciter serrata; pied. han normaliter hispidi; sepo. Darso alia loeria; alia glaniulosa

1. Recedentes.

Foliola simpliciter serrata, pei. normaliter hispisi vel tomentosi; sep.

A Darso glaniulosa necne

2. Collinare.

Foliola inaeque serrata

3. Mixtar.

Foliola anmia biserrata

4. Eomentellac.

1.- Recedentes Giag Gab. hos. p. 19k.

Foliolis unsigue pubesentibus rel supra rarius glabris, impli- 
citer serratis; peounculis numquam normaliter hisfisis; sepalis nune lavibus, nune gorso phus mismsse glandulosis; petalis selbis vel carneis, mediscribus, coroatis, odorem maschatam iroinarie avidentius resolentibus.- Iprecis subsectionis hujus eas Golstiticlium fore omnino referunt.

Foliola univique subtomentosa; sep. saepines glandulosa; cor. moschata (a). Crep solstitialem Bess. acceinnt)

1 Foliola supra subglabra vel glabra; sep. Dorso sacpines eglanioulosa; cor. haid ent vix moschata (grex C. Deseghisei Mor.) 4

Fruct. ovoidess vel obovato-slongus

$2\{$ Eruct. globosus vel rotuniatuo

Fruct avato - rotur atus, ovatus vel oblongus

Petioli inferiores inermes

o Petioli onnes aculeati

Petioli annes inermes

4 Petiolis inermes

Ipecies $N=2158-2159$.

Inecies N-2160-2166.

Ipecies $N: 2167-2109$.

Irecies N $N 2172-2172$.

Ipecies N-2173-2177.

Satioli aculeati

Ipecies N:2197-2204

Ityki villosi vel lanati

5 Styli glabri

Styli paulo hirsute

Foliola basi rotuniata

6 Foliola basi attenuatic

Fruct. ovoideus

$7\{$ Fruct. oblongus

Species N-2178-2182.

Ipries $\mathcal{N}^{6}-2183-2185$.

Qrecies N:2194-2196.

Ipecies N.2186-2187.

Ipecies $N^{2} 2188-2193$.

1. Foliola unsique subtomentosa, sep. sacpius Dorso glavinulose;

cor.moschata (a) C. solotiticlem Sess accedunt).

a. Fructurs globosus vel rotunsatus.

2158. Crevinix lewcographa (Gigr Ros.nor. I. p. 14): Gogr Gab. N:2688; Gogr Contr. fi. Slav. II, p. 43. - Gage Herb. nos. N.145.

Faculei faleato - Dilatati, ai ramos arcuatos plus misusve copriosi, fol. obovata, breviter attenuata, basi subrotensata, utrinque malliter hirsuta, os costam eglaniulasa; Dentes simplices, subconvergentes; pet inermes, aglanoulasi. stip. lntar. viculis Sivergentibus; pes. 2-3, elii glabri, alii porce glandulo.., 
$-331 \div$

$11.13 \mathrm{~m}$. longi; tubus oratus, glaber; sep. Sorso fere ommix eglanialosa, pinnulis integris, pubescentibus, sat angustis instructa, styli subglabui, giseo fere plano; car-pallive rosea, eciliata; fruct.magnus, subrotuniatus.

4ib. If cone, as Montmelas (No. Ganooger).

2159. Crepinia aceuthothamnos Gogr qab. N-2689.

Aculei defiles, parmm Dilatati, tenniter folcati, wo remos flor. copiosissimi; foll unique pubescentia, intense viribia, oblongo-acutuscula, basi attenuata, coriacea, ad costam pauls glanoulosa, oerutes simphies, apato. triangulares; pet. glanivulasi, inf. inermes; stip. magnae, latae, aursiculis rectis,

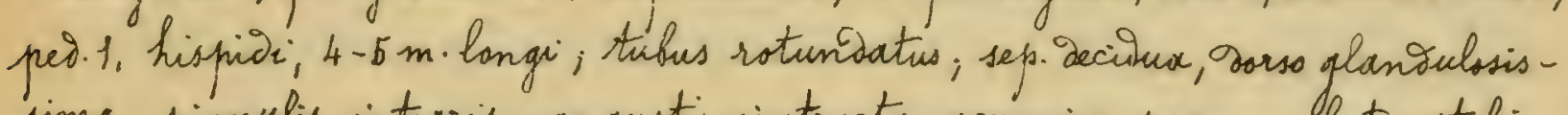
sima, finnulis integris, angustis instructa, acumine parmm lato, styli lanati, discs plano; cor......... fruct magnus, sphoericus.

Hab. Sweix. Scanix: at Frillestad (J.N. Schents).

6. Fuctus ovato-rotuniatus, ovatus rel oblongus.

2160. Gepinia transcaucasica Gogr Eab. N:2690.

Henlei......... is ramos For.milli, fot. obvecto-rotiesa, basi subrotumData, undique minutissime hirtella et glauca, ad costam eglanidulosa, dentes simplices, valie profundi, lancealato-sufconvergentes; pet. inermes, tomentosi, eglanaulosi, stip. glancae, subtus paulo pubescentes, marginibus copiose glaninuloso-serrulatae, aurieulis subrectis; pes. 2-3, paulo villosi, 10-12 m. longi, tubus ovato-ellipticus, in $1 / 2$ sup. hispitus; sep. integra, dorso glonaulosa, acumine lato, ontato-glandulaso; styli lanati, aiseo plano; cas. magna, eciliata; fruct. ovatus, apuce subattemeatus.

Hab. Cancasus, as Kutais (G. Raste).

Species singularis faciem Rosa alfince quadammoto refarens; - servaturac eis Frogariace similes.

2161. Crepuinia vepreticola Gogr mss.

Accula freves, parum Dilatati, temiter at valde faleati, as ramos flor. sparsi; fol sot late obovato - sblanga, subacuta, basi rotuniata, ungoigue villosa, supra glauca, wo costam eglanoulosa, gentes simplices, swato aperti, pet. inermes, eglanaulosi, tamentosi, stip. viriess, magnace, auriculis aivergentibus; per.1, asperse glanisulosi, 13-15.m. longi; tubus ovato- 
$-332-$

ollipticus, glaber; sep. frevia, Dorso paulo glanoulosa, finnulis ammibus angustis, parce dentatis instructa; styli lanati, disco flano; cor.........; fruct. ovato- ellipticus, basi notuniartus, apice vix attenuatus.

Hab. Suecia, Hallani: as tos Haster (\%. To. Gabrilsson).

2162. Crepinia stephanomorpha Gogr \&ab. N:26g1.

teculei Defiles, filcato- Dilatati, id ramos flor pauciores, fol. ollongoacuta, lasi subrotundata, lacte virivia, unoique pubescentix, do costam non glaniulosa; rentes simplices, aperto-triangulares; pet. eglanoulosi, pubescentes, parce aculeati; stip. breves, latoe, auriculis subsivergentebus; per. 1, parce glani ulosi, 8-g m. longi; tubus ovatus, laevis, sep.erecta, gorso glanoulose, pinnis annibus foliaces- Dentatis instructa, acumine magno, in tegro; styli lanati, Sisce plans; cor......... funct. magnus, ovotus, basi prosertion paulo Tepressus.

Hab. Iuecia, Gestrogothia: in monte Kinnekulle a grinosater (Y. G. Letterstegt).

2163: Creninia flabellata Gigr Gab. M. 2692.

foculei dilatato-subfalcate, is ramos rubros et givaricatos geminati; fol. parva obovato-oblonga, acuta, basi breviter attenuata, univie villosa, al costam eglaniouloso, Dentes simplices, lanceolato-aperti; pet.eglaxsulosi, pukscentes, aculeati, stip. majusculac, auriculis inf. patulis; pel. 1. paree glanioulosi, 8-9m. longi; tubus ovatus, larpis; sep. erecta, gorso glaniulosa, pinmulis integris, langis, parum latis instructi, acumine integro, foliaces; styli lanati, discs flano; cor.........., fruct. ovatorotundatus, utringue rotunaxtus.

Hab. Inecia, gestrinsthix: in monte Kinnehulle, as Mjornsater (P. F. Lungavint].

2164. Crepinia cerino-albe Gogr bab. N-2693.

Gortuosa, humilis; aculei parum falcato-ailatati, as ramos flor. pauciares; fol. parra, obovato-oblonga, utrinque paulation attenuata, villosa, as costam eglaniulosa; Dentes simplices, ovati; pet. eglaniulosi; tomentosi, fere onnes inermes; stip. mediocres, villosar; pes. 1-2, ahipuot sparse glaninlosi, 9-10 m. longi; tubus oblongus, lavis; sep. gorso plerumque eglanoulosa, pinnulis brevibus, ingustis, pubtescentibus iristructa; styli villosi, 
$-333$

sisco subplans; cor. albo- rosea, sciliata; fruct. parves, oblongus, angustus, utrinque lange attenuatus, apuce strangulatus.

Hob Mhone, ad tornas (N. Ganooger).

2165. Bepinia cylingriea Gage 8ab. N:2694.

Acculei gefiles, falcato-Dilatati, ad ramos flor. flexuosos et elangactos geminati val ternatic; fol. oblongo-acuta, basi rotuniata, unsique villosa, intense sirisia, ad costam eglanioulosa; Dentes simplices, Triangulares, aperti; pet. eglanioulosi, inermes, villosi, stip. medioeres, subtus glabrac, auriculis givergentibus; ped.1-2, sat glomaulasi; 17-19m. longi; tubus hasnis, elongatus, sep. Deciaur, aress glandulosa, pinmulis integris, latiusenlis instructa it et acumine; styli lanati, Disco plans; cor...........; fruct. elongatus, cylinoricus, magnus, utrinque attemuatus.

Hab. Snecia, Scania: Sinelierna (\$. A. Eallberg).

2166. Crepinia societatis Gogr mss.

Foculei Preves, parri, falcato- Dilatati, od ramos flor. geminati; fol. sat late oflanga, breviter acuta, basi sensim rotunioata, crassa, subtris prisea, uniaque villosa, supra pallise viribia, at costam eglanioulosa, identes simplices, ovato-recte; pet. hine inde glanioulosi, inermes, tomentosi; stip. sirises, latar, longore, subtus pubescentes, auriculis rectis; pos.1-3, mone glabri, nuc pauls glanieulosi, 10-13 m. longi; tubus oblongus, lacevis, sep. Jorso laevia, pinnulis onnibus latis, 2-4-Dentatis coprose instructo; styli lanati, giscs proulo conico, con.........; fruct. sat late sel brexiter oblongus, basi gapressus apice abrupte strangulatus. Hab. toriege: in valle La Lauge, at Montinija (havcaithou J'Ayma).

c. Fructus ovoidens val obovato-oblangus. + Tetioli inferiores inermes.

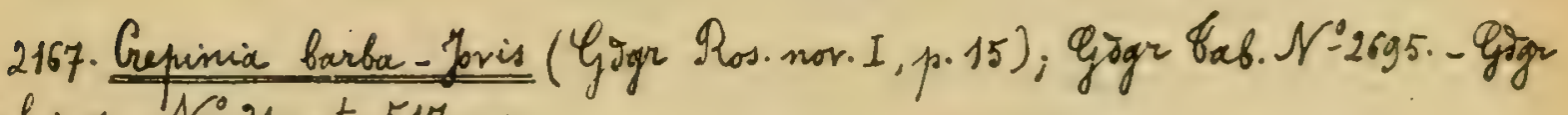
Herb.ros. N:34 at 517 var.

Acculei falcato-Dilatate, al ramos flor.copiosi, fol. obovato-oblanga, acuta, basi sensim attenuatr, intense virisia, utrinque villosa, vel subtamentosa, a costem eglandoulosa, Dentes simplices, aperti, pet. eglanaulosi; tomentosi, sup. aculeati; stip. subtus pubescentes, auriculis givergentibus; pes. 2-3, aliqust 
glaniulosi, g-14 m. longi; tubus ovoideus, laevis; sep. gorso sacpe annia glanoulosa, pisnulis angustis, pubescentibus instructa; styli villosi, Dises subplans; cor. subalba, eciliata, fruct ampliuscule sed breviter svoidens, utrinoue gepressus.

Hab. Mhöne, as Montmelas (M. Ganioger).

\section{Crepisia palumbetis Goge inss.}

tbeulei gebiles, subfolcati, tenues, parum Silatati, as ramos flor geminati; fol. obovato-oblonga, acuta, basi attenvesto, lacte viritia, undipue villosa, as costam eglandaulosa, Dentes simplices, ovato-aperti, pet. eglanaulosi, intinermes, tomentosi, stip.magnae, virives, auriculis vivergentibus; pes. 1, paululum glanaulosi, g-10 m. longi; tubus ovoideus, glaber; sep. Sorso assperse glanSalosi, pinnulis latis; 2-3-ientatis instructa, acumine foliaces, integro; styli lanati, gises plano; cor. magna, eciliata; fruct ovoideus, utrinque attenuaties. Hab. Suecia, ad Halmstad (\%. A. Gabrielsson).

2169. Crepinia niveseens Gogr Eab. N:2696.

Aculei inclinato-subirlatati, as ramos flor. pauciores; fol. sat parwa, elliptico-contata, breviter acuta, unisique villoss-canescentix, as costam non glanaulosa, Dentes simphies, pet.eglanaulosi, villosi, inf inermes, stip. metiocres; ped. 2-3, fere onnes eglaniulosi, 10-15 m. longi; tubus ovidieus, laevis, sep. Oorso plerumque glangulosa, pinnulis brevibus, latiusculis, gentatis instructa; stijli magni, villosi, gisco plans; cor. magna, rosea, eciliata; fruct. magnus, ovoideus, basi subiapressus, apice abrupte strangulatus Hab. Mhome, at Mont-Cingre supra Lyon (M. Chabert).

\section{t† Petioli ammes aculeati.}

2170. Crepinia echinopoda Gogr Qab. No2697.

Acculei validi, falcato - Silatati, as ramos flor.copiosi; fol. obovata, utrinque rotuniata, glaucescéntia, ungique villosa, at costam glandulosa, rentes simplices, ovato-convergentes, pet. toto glanoulosi, villosi, aculeati; stip.magnac, gorso glanioulosae, auriculis subsivergentibus; pes. 1-2, hispitoaculeati, 4-5 m. longi; tubus subovötens, basi glanioulosus; sep. erecta, Dorso glanoulosia, pinmulis latirsculis, 3-5-gentato-glanimiosis instructa; styli lanati, gisco plans; cor........; fruct. subaroideus, utringue gepressus. Hab. Suevia, ins: Ocland: inter \&reta et Thorshinda (Y. N. Ichenty). 
$-335-$

2i11. Rupinia pulchra tiggr tok. N=2698.

Juculei debiles, falcato- Dilatati, ai ramos flor. copiosi; fol. oblonga,

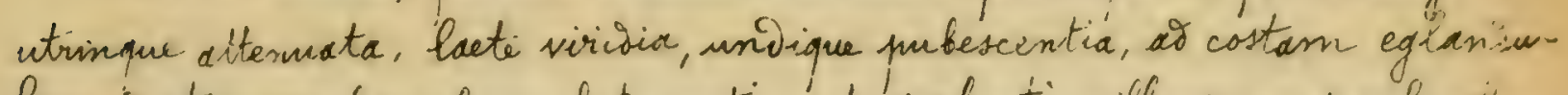
losa, Yentes simplices, lo rceolato..recti; pet doculeati, villosi, sup pauls gianDulosi; stip. breves, sat lator, mbentes, auriculis divergentibus; pés-1, glansieeloxi, 4-5m.longi; tubus ovoideus, laevis; sep. rutra, erscta, corso glonitulosa, prinnulis integris, latis instructa, acumine villoso, folixces, integro; stygh timati, gisco plano; cor. rosea, eciliata; fruct. ovoivens, utrincine a ttemeat.

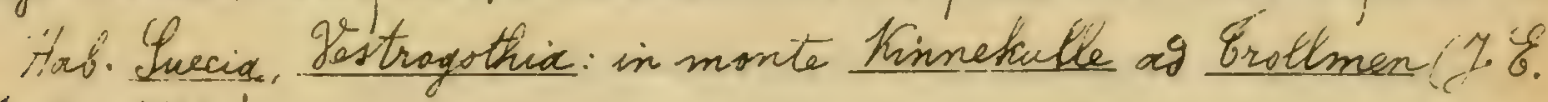
hetterstegt).

2172. Gepinix aryadea Gogr bab. N-269g.

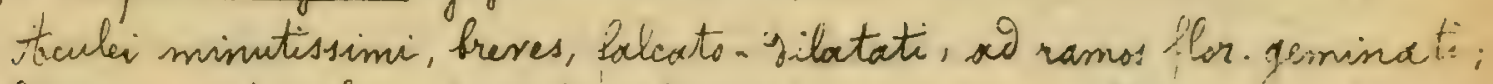
floblong-acuta, basi breviter attenuacto, univique villosa, costam-non Canialosor; gentes simphies, lanceolats-aperti, pet eglanisulosi, pubescentes, prarce aculeati, stip. majusculac, arricilis inf. valse is ivergentibus; ped.1, pare glaniulosi, 8-g m. longi; tuebus ovosideus, lowis; spp. Erecta, rubra, gorso glaniulosa, pinnulis integris, anqustis instructa; scumine lato, integro; stali magni, lanati, disco plans; car......... fruct. ovoideus, basi abupte plus minusve decurrens, apice breviter strangulatus.

Hab. Luecix, Hestragothix: in mante Himakulle as Hesterplana (J. E. Letterstest).

t t Setioli onmes inermes.

2173. Oreprinia conyzoides (Gggr 920s. nov. II, p. 29); Ggar 8ab. N-2700.Gogr Herb. ros. $N=233$.

Fcculei falcato-Dilatati, as ramos flor-geminati; fol: latiuscule obovata, paulatim attenuata, basi breviter rotuniata, inoique molliter serice-tamentosa, id costam eglaniulosa; dentes simplices, subeonvergentes; pet. eglandulosi; inermes, tomentosi; stip. virides, subtus villosar; pes: 3-4, alii hispioi, alii lacres, $10-15 \mathrm{~m}$. longi; tubus oforato sblongus, glaber, sep. purpurea, dorso plerumque glaniulosa, ódecioua, pinmulis pubescentibus, integris late instructa; styli villosi, oiscs sat conico; cor.rosea, eciliata; fruct. obovato-oblongus, basidepressus, of apriem abrupte angusteque constrictus.

Hab. Rhone, a tornas (M. Gandoger). 
2172. Crepinia signata Gogr mss.

toculei breves, parri, paum Dilatati, tenuiter falcati, as ramos flor-geminati, fol. sat late obovato-acuta, basi cordata, glaucescentix, unalique villasa, ad costam eglandulasa, Dentes simplices, aperti, ovatotriangulares, pet. tomentosi; inermes, eglanaulosi; stip. virides, longae, auriculis Divergentibus; ped. 1, glabri, 5-8 m. longi; tubus ovoidens, glaucus, loevis; sep. Darso glanioulosa, pinnulis amnibus foliaceis, 2-4Tentato-glaniulosis instructa; styli lanati, gisco plano; cor. rosea, eciliata ifruct. Ovoidens, utrinque attenuatus.

Hab. Suecix, Hallant: at Itravalla (\%. A. Gabrielsson).

2175. Crevinia goniogonta Gogr Bab. N-2701.

toculir falcato-gilatati, as ramos flor. tennes plerumque nulli; fol. obovato - oblonga, attenuata, basi coriata, undique molliter sericestomentosa, canescentia, at costam eglanioulosa, Dentes simphice, lati, aperto-triangulares; pet. eglanoulosi; tomentosi, inermes; stip. villosae, auriculis sat givergentibus; pes. 1-3, fere annes glandulosi, 10-14 m. longi; tubus oblongus, laevis; sep. Dorso plerungue glantulosa, pinnulis brevibus, sat latis, villosis instructa; styli breves, villosi, Disco subplano; cor-pallide nosea; fruct. obovato-oblongus, utringue attenuatus. Hab. Rhóne, is Trancheville et Daroilly (IP Chabert).

2176. Crepinia filamentosa Giog \&ab. N:2702.

toculei breves, minuti, parusn falcats-Dilatati, at ramos flor. Subinermes, fol. parva, oblongo-lanceolata, basi breviter attenuata, pallide virivia, unsique pilis eslongatis, filamentosis copiose obiveta, ad castam eglaniulosa; Dentes simplices, lanceolato-aperti; pot. eglandulosi, inermes, villosi; stip. virides, auriculis subrectis; pes. 1-3, fere omnes glanoulosi; 13-14 m. longi; tubus obovato - oblongus, glaber; sep. virisia, Darso fere onnia eglanaulosa, brevia, pinnulis integris parum latis instructa; styli villosi, gises sat comico; con. rosea? eciliata; fruct. obovato-oflongus, basi gepressus, aprice valde attenuatus.

Hab. Rhone, io Chaponost (9. Chabert).

2177. Crepinia intertesta Gigr Gab. N-2703.

Aculei breves, falcato- Dilatati, as ramos flor. gense foliosos, intertastos 
$-337-$

nulli, fol oblango-acutiuscula, basi breviter atternuata, glaucescentic, univique villosa, ad costam eglaniulosa; Jentes simplices, aperto-triangulares, pat. eglanialosi, inermes, villosi; stip. longar, angustiores, auriculis givesgentibus, pes. 1, glandulosi, 8-g m. longi, tubus orvideus, basi paulo hispisus; sep.erecta, Dorso glanoulosa, pinnulis integris, angustis instructa, acumine sat lato, styli lanate, gises plano; cor........, fruct. ovoidens, basi appressus, apice strangulatus.

Hab. Sluecia, insula Oeland: Eveta (Y. M. Schentz).

2. Toliola supra glabra vel subglabra; sep. Dorso sacpius eglanoulosa; corolla haui aut vix moschata (grea (. Deseghisei Mor).

a. Petioli inermis.

+ Styli glabri.

2178. Crepinia gossypina Gogr toab. N'2704.

Asculei falcato-gilatati, as ramos flor. mulli vel 1-2; fol. sat parva, obovata, utrinque attemuata, supra glabra, subtres villoso-canescentia, ad costam eglandulosa; dentes simplices, pet eglanoulosi, inermes, villosi; stios. sirides; pes-1-2, glan'sulosi et sirmul aosperse pilosuli, 12-16 m. longi; tubus sblongus; sep. brevia, pinnulis hirsutis, angustis instructa; styli glabri, Qises conico, cor. rosea, eciliata; fruct. oblongus, sitrinque attenuatus. Hab. Thone, as toinas (Ho. Ganooger).

2179. Crepinia leiostigma Gogr 8 ab. N:2705.

Acenlei grefiles, parum dilatati, ai ramos flor. plus minusve copiosi; fol. elliptica, utrinque sensim rotumbata, lacte virivia, supra glabra, subtus toto villosa et as costam eglanoulosa; Dentes simplices, recti; pet. inermes, egtaniulosi, villosi; stip. virides; pes. 2-3, sasperse glaraulosi, 5-g m. longi;

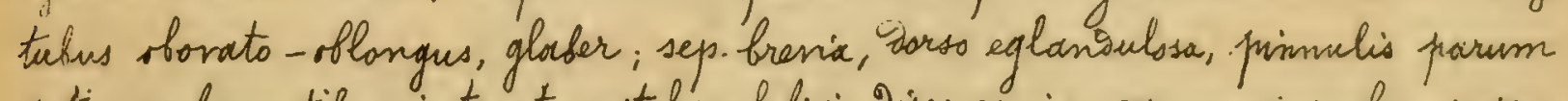
ratis, pubescentibus instructa; styli glebri, dises conics; cor majuscula, rosea, eciliata, fruct parrus, ovoidens, utrinque paulo gepressus.

Hab. Thane; at Darvilly et Francheville (9. Chabert).

2180. Crepinia geficiens Gogr Gab. N-2706. Ggogr Herb. ros. N-3:16. Aculei of ramss flor. falcato. Dilatati; fol. obovata, ettrinque attenuata, lacte viribia, subtus villasa et ad costam paulo glandeclosea, Jentes sim- 
$-338-$

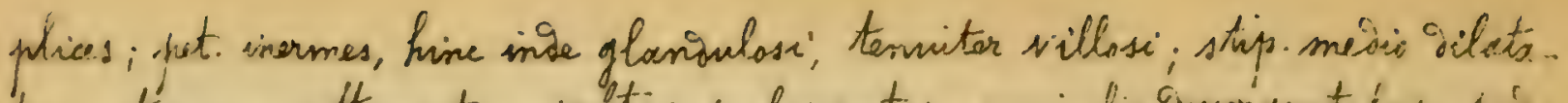
toe, utrinque attenuatar, subtis pubescentes, auriculis suvergentióus: péo.

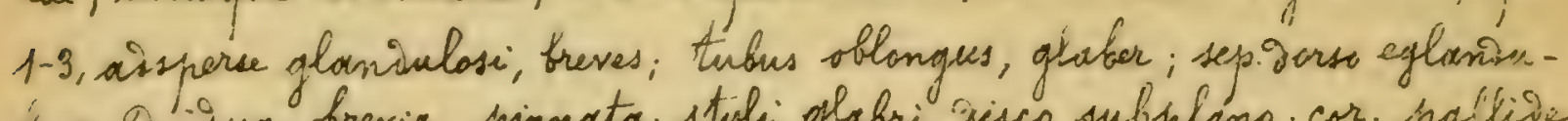
cosa, oecisua, frevia, pinnata; stigh ghabri, sisco subplano; cor. pallivio rosed fruct. orvidens, fase rotunioates, apic attenuatus.

Hab. Thone, at trnas (Iti. Ganioger).

2181. Crepinia errabuniax Gigr \&ab. N-2707.

tbculei gebiles, parum folcato-gilatati, ai ramos flor. subnulli; fil. oblongr, utrinque attenuata, sutitus toto villasa, as costam sglanisulosa, sentes simplices; pat hinc inse glansulosi, inermes, villosi; stip subtus vix pubscentes, aunculis rectis; ped. 1-3, villso-glanaulosi, meviscres; tubus oborato- oblongus, laevis, sep. Dorso sglangulasa, pinmulis longis, hatiusculis instructo; styli; subelaberrimi, dises sat conico; cor. albx; fruct. obovaitus. Hab. Rhone, ad tormas (M. Ganianyer).

2182. Crepinia variabilis Gogr Gab. N-2708. - Gogr Herb.ros. N-504. Aculei breves, minuti, vix falcato-gilatati, as namos flor. nulli; fol. oborcta, firma, intense siribia, subtus toto villosa, frominule nervosa; Sentes simplices; pet. inermes, eglandulosi, villosi; stip. angustac, subtus villosa, auriculis acutis, subrectis, per.2-3, sat glanivulosi ses glabri, mediocres; tubus ovats notuniatus, laevis, sep. Sin persistentia, porso eglanDulosa, pinnulis brevibus, Oentato-glandulosis copiose instructa; styli breves, subglaberrismi, Diseo plano; cor.rosea; fruct magnus, globosus, apice pauls contractus.

Hab. Thone, as Chaponost (P. Chabert).

$$
\text { t+ Iityli proulo hirsuti. }
$$

2183. Crepinia pachybasis Gogr Gab. N-270g. - Gogr Herb.ros. N-532 var. Aculei falcato- dilatati, as ramos flor. dixaricatos gebiles; fol oborata, utrinque paulation rotunidata, subtus toto villosa; Bentes simplices; pet villosi, inermes, eglanoulosi; stip. virides; ped. 3-4, glangulssi, villosissimi; 14-16 m. longi; tubus oblongus, laevis; sep. Sorso eglaniaulosa, pinnulis brevibus, angustis instructa, styli poulo hirsuti, disco conico; cor. pallide rosea, nox ciliata; fruct.magnus, oblongus, basi longissime attemiato-racurrens, 
$-339-$

apice breriter attemiatus, in $1 / 3$ sup. tius aisus.

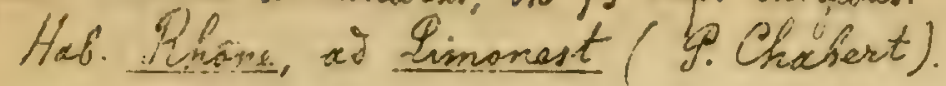

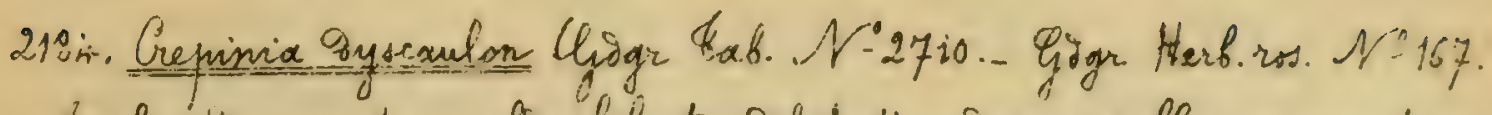
Toculci "iecurrentes, valide faleato-Dilatati, ais ramos flor sarmentosoflesuasss, aresertis moxque denvidectos pauciores; fol. late oblongs-rhombizalia, iringque parum attenuata, subtus toto villosa, Dentes simphices; pet. eglanivi Losi, incermss, villasi; stip. mediscres; ped. 1, tantum villosi, 14-18 m. longe; tabis oblongus, Laevis; sep. garso eglanioulosa, elongata, pinnulis integris, pubescentibus, folixceis instructa; stugli pauls hirsuti, sises sat conico; cor........... fruct magrus, oblangus, basi abrupte attennatus, Dein bonge Decurrens, appice vix. Dapressus.

Hab. Hoire, al St-Anire'-le-Gay (M. Ganirger).

2185. Crepinix podosticta Goor Tab. No2711. Gogr Herb ros. N: 361.

Acculer volisi, falcato dii atati, ad ramos flor.copioss; fol anguste oblonga, utrinque paulation attermata, nervosx, subtus -tots villosa; ientes simplices, filcatis, pat. egleniaulosi: inermes, longe pilosi; stip. elongatre,

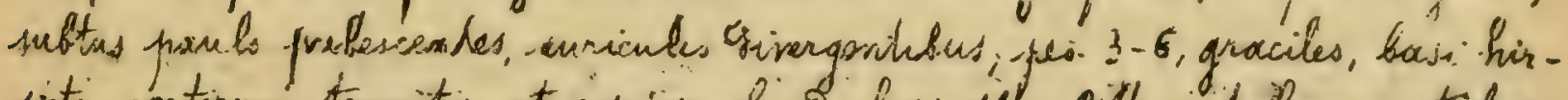
sist, coeterum temuiter et copinse glanivilosi, $1 / / 2-2 \pi / 2$ cent. longi; tubus oblongus, laevis; sep. Darso églansulosa, pinmulis elongatis, foliaces-lacimiatis instructa; styli poulo hirsuti, disses comics, cor. pallive rosea; fruct. anguste oblongus, hasi longe securrens, spice valde atternatus.

Hab. Thoone, à tornas (N. Ganooger).

tft Ytyli villosi val lanati.

\$. Foliola basi rotuniata.

,, Iructus avoidens.

2186: Crepinia vexata Gogr \&ab. N-2712.

Bculei valive; falcato-silatati, ai ramos flor sot copiosi; fol. elliptica, utrinque poulation rotiendata: subtus laxe villosa; rentes simplices; pet. eglanaulosi, inarmes, villosi; stip. medioeres, pers. 1-3, villoso-glanoulosi, 13-18 m. longi; tubus avoìens, glaber; sep. Darso eglaniaulose, pirnulis angustis, glabrescentibus instructa; styli villose; Dises paulo conico; cor.......... fruct. ovoidens, utrinque breviter attenceatus.

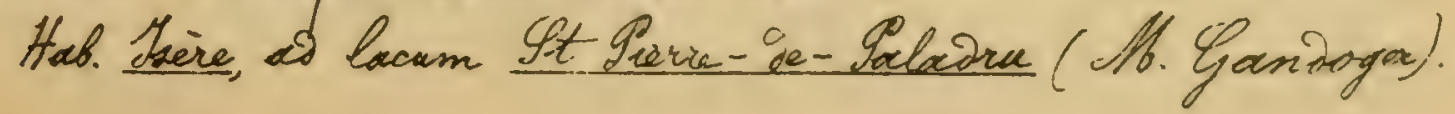


$-340 \cdots$

2187. Crepinia polychlora Giogr mos.

Bculei breves, parum gilatati, valde faleati, od ramos flor. stipulares; fol. latiuscule sbovato - suboblonga, abrupte acuta, basi rotuniata, supra gla fra, lucida pallideque viricia, subtus to to villosa et ad costam eglaniulosa; Dentes simplices, ovato - recti, pet-tamentosi, inermes, eqlanidulosi; stip. virio is, - Cossgae, latar, gorso pubescentes, suriculis sat givergentibus; pes.1-3, villosi et paulo glaniulosi, 10-12 m. longi; tubus obovato - oblongus, laevis; sep. Iorso eglandulosa, pirnulis annibus integris, latis instructa; styli hirsenti, Sisco plans; cor. sat parva, albo-carsea, basi subcarnea; fruct.obovatooblangus, basi paulo rotuniatus, aprice six attenuaties.

Hab. Laône-et-Loire, it St Laurent-g'sondenay (N. Ganooger).

$\sim \sim$ Fructus oflongus.

2188. Creninia stictanoda (Gjar Mos nov. II, p. 30); Gogr Fab. N-2713. - Gogre Herb.ros. N-85.

tculi debiles, subfalcati, as rumos flor. flexuosos sat copiosi; fol ovatoelliptica, breviter atiemuata, basi rotungata, utrinque villosa; oentes simplices, aperti; pet. inermes, villosi, eglaniolosi; stip. latue, subtus pubescentes, auriculis givergentibus; ped.3-4, villosi at glanoulasi; 12-20 m. longi; tubus sblongus. Liveris, sep. Dorso eglandulose, pirmulis integris, sat latis instructa; style villosi, Disco subconico, car.magna, pallise rasea; fruct. oblongus, basi rotundatus, aprice rotungatus. Hal. Rhone, at tornat (M. Gandoger).

2189. Crevinia amphibola Gogr mss.

Acculai breves, parum gilatati, valie falcati, at namos flor. stipulares; fol.mevisiria, obovato-oblonga, breviter acuta, basi sensim rotungata, supra glabra, lacte ac hucide viridia, sultus to to villosa, al costam non

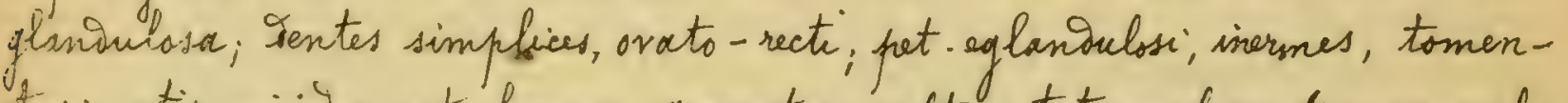
Aosi, stip. virides, sat longare, angustar, subtus to to pubescentes, auriculis semidivergentious; pes.1-2, villosi, sparse glandulosi, $10 \mathrm{~m}$. longi; tubus oblongres, lavis; sep. Darso eglandulosa, pinnulis omnibus integris, subfoliaceis instructa; styli lanate, gises paulo conics, cor. albo-carnea, eciliata; fruct.magnes, oblongus, utrinque attenuatius. Hab. Ioane-et. Loire, at Ecuises (N. Gangoger). 
$-341-$

2190. Crepinia 2icthyơes Gojor mss.

Heculei rarissimi, Sebiles, parum Silatati, temuiter falcati, as ramos flor. nulli, rami valde tortuoso-articulati, fol latiuscule obovato-oblonga, fresiter acuta, basi roteiniata, supra glabra, luciba lacteque virioisa, subtus toto sillosa, ai costam eglanialosa; ientes simplices, aperti, arato-lanceolati; pet. eglanisulosi, inermes, tomentosi, stip. beeriusculace, parum longac, virives, Sorsto pubescentes, auriculis subrectis; pes. 1-2, vis hirsuti, sat glanivulosi, $12 \mathrm{~m} \cdot$ longi; tubus oblongus, laevis, sep. Dorso eglanioulosa, pinnulis omnibus integris, lates instructar, styli sublanati, Disco plano; cor. subalba, eciliata; fruct misiacris, oblongus, utringue sat attemuatus.

Hub. Iaone-et-Loire, as It Laurent-g'tonienay. (M. Ganooger).

2191. Crepinia enqustispina Gogr Gab. N:2714.

toculei tenues, recti, parum silatati, at ramos flor geminati ifol. obovato-oblonga, acuta, basi rotuniata, pallise viridia, supra glabra, subtus to to villosa et as costasn eglanaulosa; gentes simplices, oratoaperti; pet. eglanioulosi, inermes, villosi; stip. rubentes, angustae, subtus Glabrae, auriculis givergentibus; ped 1, parce glanoulosi, mediseres; tubus elongatus, laevis, sep. erecto, gorso eglanoulosa, pinnulis integris, parum latis instructa, acumine foliaces, integro; style lanati, gisco plano; cor. ignota; fruct. anguste elongatus, utringue longe attenuatus.

Hab. Surcia, Halland: ad Halmstad (J. NP. Ichents).

Exdem est forsan ac C. Rallondica (Pchenty see. Gigr Essai p. 26), sed notae Distinctivar minus congreusent.

2192. Crepinia abnormis Gogr mss.

Acculei Deliles, Dilatati, temuiter falcati, as ramos flor. geminati, fol. obovato-oblonga, parum acuta, basi subroturioata, laete viricia, supra gilabra, subtus toto sed parce villose, at costam eglaniulose, Sentes rect, simplices, triangulares ; pet. eglanoulosi, inermes, tomentosi; stip. Dilute rubentes, parvae, auriculis givergentifus; ped-1, sat hispisi, 10-19 m. longi; trubus suboblongus, lavis; sep. Porso glanoulosa, pirnulis ommibus integris, angustis instructa; acumine lato; styli lanati, sises plano; cor...... puct. suboblangies, bosi longe attenuatus, apice breviter strangielatus. Hab. Inecia, Halland: as geaberg (J. Io. Gabrielsson). 
$-31.2-$

2193. Geminia trichadeniopoda Giar mss.

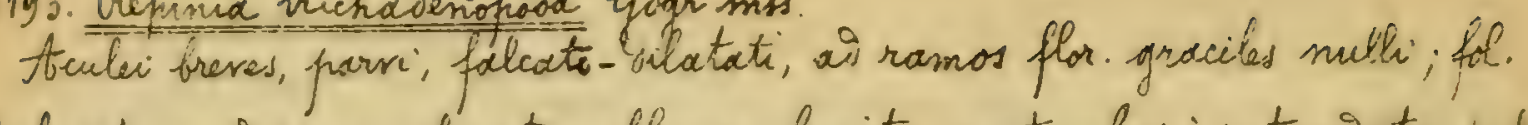
subplicata, mediacria, obovato-oblonga, frevitor acuta, basi rotasnaxta, pal live virisia et suparne glabra, subtus niescentix et toto sei parce villosa, at costam eglanisulosa; Dentes simplices, ovato-recti; pet. eglanioulisi, iner-

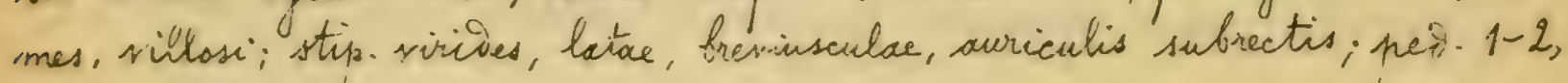
copiose villoso-glarioulosi, 15-17.m. Longi; tubus sblongus, laevis'; sej. Forso eglanoulosa, pinnulis latiusculis, 1-2-asentates parce instructa; styli villosi, Desco plano; cor albo-carnea, eciliata; fruct mesiocris, breviter oflongus, basi aepressus, apice parem attenuatus.

Hab. Laine-et-Laire, of It Laurent-9'Anienay (H. Ganooger).

§. Foliolx basi attenuata.

2194. Ceninia goniophylla Gyogr Gab. N:2715.

ticulei minuti, parum falcato-Dilatati, as ramos flor. pranciones, fol. obovata, utrinque abrupte truncata, subtus tots villosa, as costam eglanaulosa, superne glabra; Dentes simpliaes, lanceolati; pet. eglanisulose; inormes, villosi; step.meviseres; ped. 1-3, sparse pilosi, 13-18 m. longi; tubus stlongus, laeris, sep. elongata, gorso eglandulosa, prinnelis integris, puebescentibus, saepe folidces-laciniatis instructer; styli villose; gises conico; cor. rosea; fruct. oblongus, utrinque valde attemuatus.

Hab. Rhòne, at Darilly, Ecully, Francherille, 2a Gour (9. Chabat).

2195. Grepinia Sholeyromiana Giogr Gab. F:2716. G Gogr Herb. ros. Ni223 at 223 bis var.

Acculei elongati, gecurrentes, falcati, ai ramos flor. rigivos sat copiosi; fol. anguste oblonga, apice fere rotuniata, basi attenuata et in $1 / 3$ infer. integra, subtus toto villosa; gentes simplices, mucronati; pet. eglanoulosi, iner mes, villosi; stip. Oorso publeventes; per. 2-4, hirsuti et sparse glansulosi; longinsculi; tubus clongatus, laevis; sep. gorso eglandulosa, pisnulis longis, latis instructo; styli sublanati, Tises conico; con-pallide rosea; fruct. anguste oblongus, basi valbe attenuatus. Hab. Rhone, od tornas (Nh. Ganooger). 
$-343-$

2196. Crepinix - pilifers goar \&ab. j:2717.

bculai ad ramsi flor. faleato-silatati; fol. ample oblong-lances. lata, basi attenuata, wistus toto villose et a costam eglanioulosa; sentes simplices, lati, remati; pet.eglanisulosi, inermes, villosi; stip. apice dilatatae, subtus pubesentes, auriculis ivirgentibus; pes. 2-4, villose; sparse géanoulosi, graciles; tubus oblongus, lavis, sep. gorso eglansulosa, pinnulis longis, latis instructr; styli hirsuti, giscs conics; cor-rosea; fruct. oblongus, $\mu$ tringue attemuatus.

Hab. Thone, at Lx bour-De-Calvagry (P. Chabert).

b. Petiok aculeati.

2197. Crepinia cryptostylis (Govgr Ros. nov. II, p. 30); Gogr \&ab. N=2718. Goger Herb.ros. $N^{-} 335$.

Acculei falcato-gilatati, wamos flor. panciores, fol. parva, ellipticoacuta, basi rotunacta, superne glabra, subtus to to villosa, al costam eglardulosa; Dentes simplices; pet.eglaniulasi, villosi; sup. aculeati; stip. mearicres; pes. 2-5, hispisi; sparse pilosi, 11-13 m. longi; tubus obovatus, laevis; sep. brenia, gorso eglanaulose; pinnulis angustis, ylabris inotructs; styli panciores, brevissimi aut rix exserte, pilasuli, Qiscs conico; cor subalba, non ciliata; fruct. parvus, ovoitens, basi rotunisatus, apice attenuatus. Hab. Phone, as Jornas (N. Ganioger).

2198. Crepinia stupenia (Crép. in Poiss. Th orient. II, p. 684); Gogr Gab. N-2719. - C.macrocarpa (93oiss. Loco cit. non Mérat), C. meaalocarpa (Désegh. in Mém, acad. Itongers, XXVIT, 1873, p. 117, extr. p. 21) non Gogr..

Acula panciones, graciles, minuti, decurrentes, falcati; fol ample ovatoelliptica, utrinque glaberrima vel tantum as basin costae mesiac villosula, Dentes acuti, pet. glabri, aculeati; stip. Dilatatac, glabriusculae, auriculis ovato-lanceolatis, acuminatis, pes. saepe 3, breves, alii laeves, alii parcissime setulosi; tubus subrotungatus, glaber; sep. erecta, geciaux, gorso vix glanaulosa, pinnata, tomentella; styli villosi; cor....... fuct. ma. gnus, subglobosus, depressus, loveris.

Hab. Itsia thinor, in valle Dimil Sonti Lazici: $6000^{\prime}$ (Baclansa). If Dorisier prope. R. albam 2. collocata, af ex tamen muttum giffert et Sorsan ad Prepinias Lewphyllas reTucenda. 
$-344-$

2199. Crepinia adulterina Gogr \&ab. N:2720.

fcculei elangati, tennissimi, haus dilatati, recti val inclinati, as ramos flexussos intersum setacei aut tineares; fol. obovata, utrinque rotuniata, is costam mediam glandulosam tantum villosa; Dentes aperti, simplices, pet amnes aculeati, paulo glaniaulosi, tenuiter et laxe villosi; stip. longae, angustor, intense rubentes, auriculis subrectis; ped.1, aprice tantum glaniaulosi, 10-13 m. langi; tubus obovotus, glaber; sep. brevia, oorso eglanivulosa, purpiurea, pinnulis angustis, deciduis, glabrescentibus, 1-3gentatis instructa; styli villosi, sat exserti, osiscs paulo conico; cor. Loate rosea, eciliata; fruct, parwus, ovoidens, utrinque orapressus, sape abortivus. Hab. Phone, at Francheville (P Chabert).

Plantre spectabilis, aculesum folislarumgire forma Rosam pisilsphyllam Rau referens; an hijus hybrida cum guadam trichophylla?

2200. Crepinia cicura Gogr mss.

Abculei breves, parum silatati, valde falcati, as ramos flor.copiosi; fol. lativiscule obovata, freviter acuta, fasi notundata, supra glabra, lucida, lacteque viridia, subtus toto villosa, al costam eglangulosa, gentes simplices, ovato-recte; pet. eglandulosi, tamentosi, parce aculeati; stip. virides, latissimac, longae, Dorso pubescentes, auriculis Divergentibus; ped-1-3, villosi; paulo glanaulosi, $15 \mathrm{~m}$.longi; tubus obovato sblongus, laevis; sep. gorso eglanaulosa, pinnulis amnibus integris, suffoliaceis instructa; styli sublanati, giseo plans; cor. subalba, eciliata; fruct. sbovato-sblongus, basi fore rotuniatus, apice sat attemuato-substrangulatus:

Hab. Laone-et-Laire, à It Luvent-g'fondenay (M. Gandoger).

2201. Crepinia gocteborgensis Gogr mss.

Acculei tenuiter folcati, parum silatati, as ramos flor. sat copiosi, fol. oblsngo-acuta, basi caroata, suparne glabra et virioia, subtus minute hirsuta, as costam eglandulasa; dentes simplices, svato-aperti; pet-eglanDulasi, tamentosi, inf.inermes; stip.latae, virides, auriculis avivergontibus; pei. 1, laves, 5-7 m.longi; tubus ovoisens, astperse setulosus; sep. Dorso glandulose, pinnulis onnibus integris, latis instructa; styli lanati, sisco plano; cor......... fruct. ovoidens, basi gepressus, aprice non attenuatus. Hab. Inecic meris., at Goeiteborg (J. A. Gabrielson). 


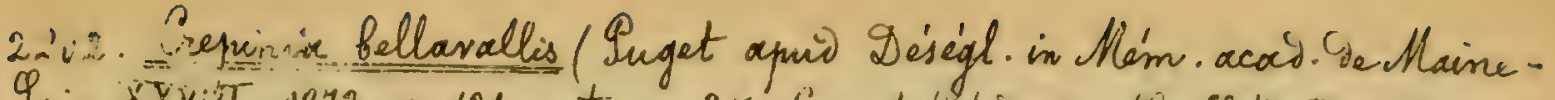

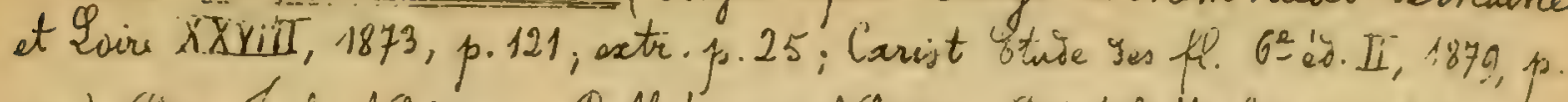

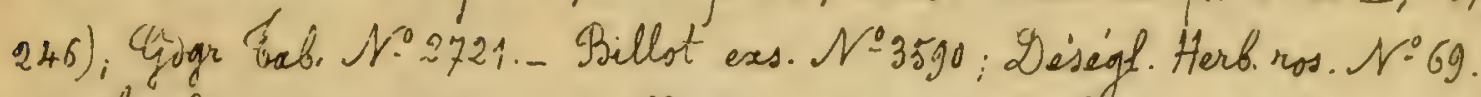

Hacilei validi, wo ramos flor. Densos falcato-gilatate; fol ovato-acuta, firmo, fictiohulecta, nervosa, subtus tsto villosa; dentes simphices, mucronati; pet. Nillosi, sculeati, eglanaulosi, stip. subtus paulo pubescentes; per. 1-3, sparse glanioulasi, 3-5 m. longi; tubus oblongus, laevis; sep. Sii persistentia.

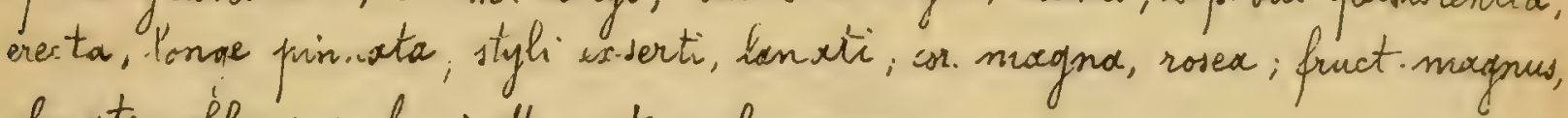
obovato-oblangus, basi attenuatus, glancescens.

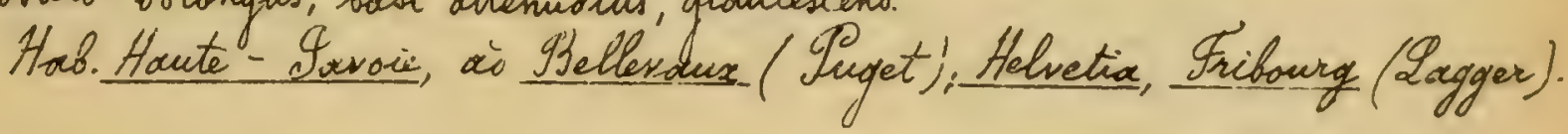

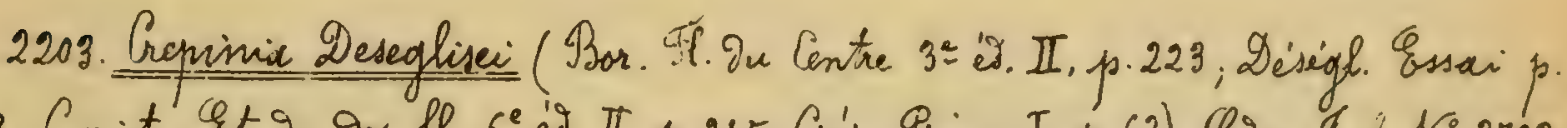

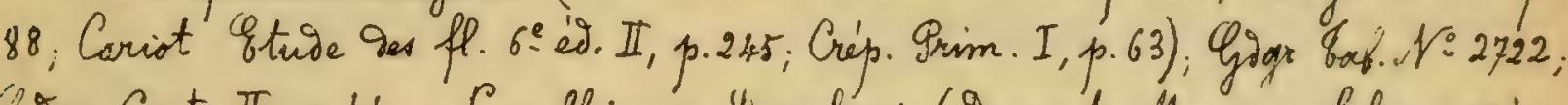
Gogr Contr. II, p. 44. - C. collina Y. Deseghisei (Dumart. Mon. ros. belg. p. 58); C.aumetorum f. Deseglisei (Christ Mosen ger Mchw. p. 187). - Gogr Herb. ros. W-507. teculei salisi, fielcats- Lilatate; fol ovato-acuta, supra subolabra palliveque virioia, subtus toto villosa, so costam eglaniulosa; sentes simphices; pet. parce aculeati, eglaniaulssi, tamentosi; stip. pubescentes; pes. 1-4, nilloso-glancoutosi; thibus ovoisens, glaler, sep. ovrso eglanduloso, Gecicua, pinnulis pubescentibus, sat angustes instructa; stigh hirsute, Diseo plano; cor. pallide rosea, eciliata, fruct. parwus, ovioiens.

Hab. Gallia meivia, etc.

2204. Crepinia Cessacui Gigr Essai p.32, Gogr \&ab. N.2723. - C.corymbifera? (q. Cessac Cat.plant. ye la Crense in Grull. sac. Ges sciences natur. et archeiol. ge $l_{\alpha}$ Crense III, p. 311, nan Morck.h.).

Acculei validi, falcato - vilatati, as ramos flor robustos coprissi; fol ovatoreuta, nitive saturateque sividia, supra glabriuscula, subtus villosa, "sentes simphices; pet eglaniulosi, villosi, porce aculeati; stip. subtus imsesicentes, arriculis Divergentibus; pes. corymbosi, sosperse gloniulosi; tubus ovioiseus, loevis vel basi setulosus; sep. reflexa, Decisua, pirmulis foliaceis instructa; styli hirsuti, dises subcomics; cor. pallise rosea vel subalba, majusculor; fruct aroidens.

Hat Preuse, Grand-Moury (G. ge Cessac). 


\section{2.- Collinale Giép. Tim. I, p. 18 part, Gogr Gab. p. 195.}

Foliolis pubescentifres, sourius maopis, simpliciter serratis; pedunculis normáliter glemoulosis, hisinisis vich tomentosis; sep. Sorso gitanduiosis necne; petalis amplis, coriatis, florumave amoene roseis, hacie sut vix olentitus.

1 Yepala Barso glandiclasa vel hispisa

1 Sepala dorso eglaniulosa

Gructus oblangus

$2\{$ Fruct. glabosus sel ovatus

Iruct. ovirieus val obovato- oblongus

Styli glabri

3 Sogli hirsuti

$\Phi_{\text {pecies }} f^{2205-2214}$.

$\varphi_{\text {mecies }} \mathcal{N}-2215-2222$

Inecies $N: 2223-2228$.

Inecies N-2229-2231.

Sparies N'2232-2236.

1. Sepala gorso eglanioulosa.

2205. Crepinia iseriensis (Iuget) in Ggogr God. F:2724.

toulei valigi, tantam inclinati, oo ramos flor pauciores, fol ellipticoacuta, basi attemuata, supra glatra, subtus ai costam villosa, inf. vera ai nerwos hirsuta; bentes simphices, acuti, pet. villosi, aculeati, sparse glanaulosi; stip. elongatar, aprice gorsi pulescentes, curriculis burvitus, rectio; ped.1, inspivi; tirsus oroisens, havis vel basi glanoulosus; sep. brevia, gecisua, pinnulis poulo ientato-glanoulosis instructa, Gorso laevia; styli plerumque glabri; cor-palliae rosea; fruct. ovoidens.

Hab. Ysere, in silva Gortes prope Grenoble (Mouthe).

2206. Crepinia collina (Jacq. Tl. austr. II, p.58, tab. 194; Gsillo. Ip. pl. II, p. 1078; Spreng. Syat.II, p.553; DC. FP. frane. IV, p. 441, Gren. at Goor. FP. De Fr. I.p. 558; Gand. Fl. hoh. III, p. 350, Math. Fl. betg. I. p. 175; All. FP. ped.II, p. 140; Pert. Fl. ital. V, p. 200, Koch Pyn.ed. 2a p. 251; Host Fl anstr. II, p.22;

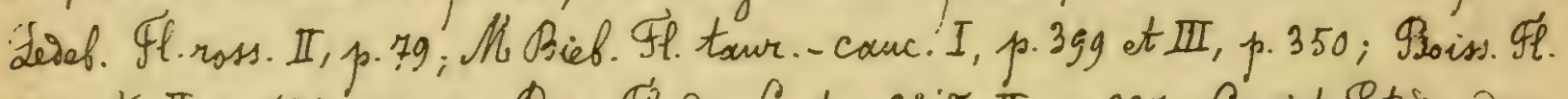

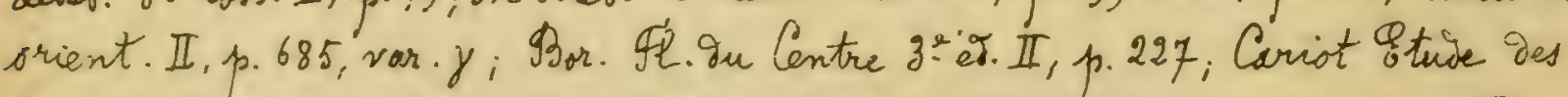

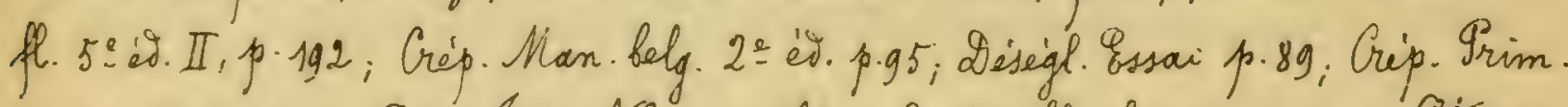
I. sp. 53 et 196); Goor Gas. N:2725; Gogr Contr. fl. shar. II. p. 44.- Gturm

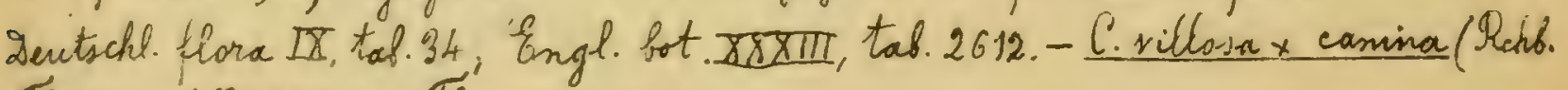
Ff. exe. $N^{\circ}=3996 ;$ of. Tf. sex. p. 345). 
$-347-$

A cuivi Lalcato - dilatati. prarai if subgeminati; fol. ovata val ovatorotuniata, supra viridia ac subylabra, subtus toto villosa et as castam

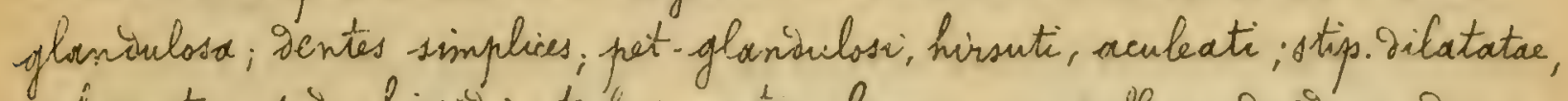
pubescentes, pet. hispitiv; tubus ovakrs, laevis; sep. reflexa, decioud, gorss eglosioulosa, pinmulis gentato-glanisulosis isistructa; styli villosis; cor. laete rosea, fruct magnus, ovatus.

Hah. Austria. ot ahbi sec. aucióves var, sed alteras species sistunt. Sex varietates e Massix merid.-vecis. in sur Enum. Gorol. At Holk.

p.63 enumerat Besser, quoe onmibus ignotas esse videntur, si tamen exceparis var. S. Rosam solotitialem Bers. constituens.

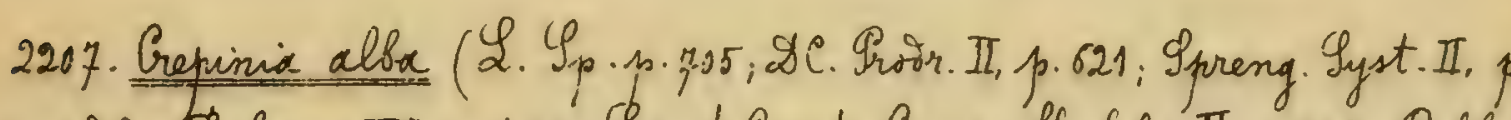

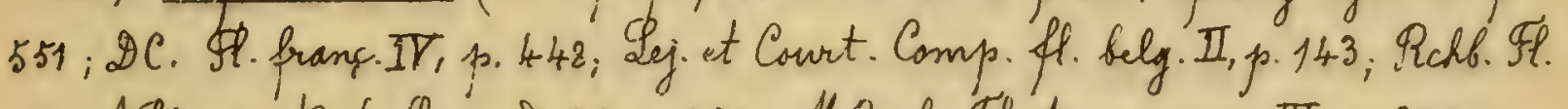

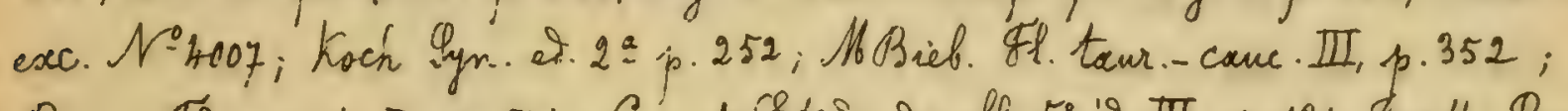
Bois. Il orient. II, p. 584; Cariot Ottuid ges fl. 5: id. III, p.131; Gratt. Ros. mon. II, p. 41; Por. Tl. au Cenire 3ะe's. II, p. 227; Crep. Trim. I, pp. 63 et 197; Christ Rosen ger Ychw. p.207; Deseije. Cat.p.228); Gogr Goeb. N:2726. - C. procera ( Takis. Hort. Ablart. 35g). - Mcan., Te. Lan. VII, tab. 1215; Hayne torch. II, tarb. 31: Grimp. Hob. tiah.g6; Maur. Duham. VII, tab.16; Lawn. Mos. tah. 37.Hoppe exs. (1793) $N^{\circ} 73$; Gogr Herb. ros. $N^{\circ} 387$ et 404 var.

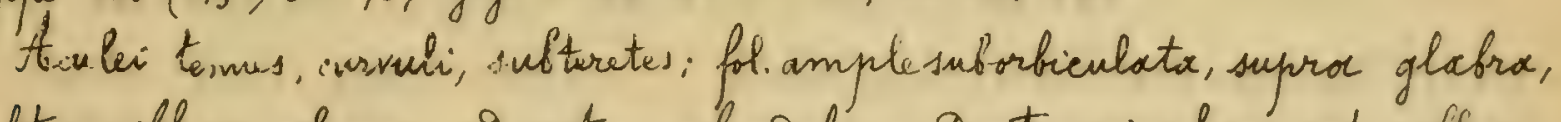
subtus villasa, glouca, ai costam glaniulosa; sentes simplices; pet. villosi, pauls glaniulasi, aculeati, stip. latar, subtus pubeseentes; pea 1-2, longi, glanJubsi; tubus ovatus, havis val glaniulasus; sep. gecitux, pinnulis gentatsglanonlosis instructa; styli hirsuti; cor magno, alfa; fruct. ovatus. Hab. Cancasus. - In buropa vulgo culto st ince subspontarea.

2208. Crepinid trichairea (Mip. in Désegl. Cat.p. 217); Giger \&al. N:2727. foculai gebiles, falcati, ad ramos flar. plerumque nulli; fol. orato-obtusa, superme assperse pilosa, subtus toto villose, oentes simplices; pat. hirsuti, saepe inermes; stip. ad apioem parce pubescentes; peo. 1-3, paulo villoso-glanduLosi, 夜bus oltongus; sep. Secidua, longa, pinmulis publescentibus instructor, styli hirsuti; cor. alba; fruct. oblongus. Hat. Savore, ad Salins firope. Nlantiers! (Paget). 
$-318-$

2209. Pepinia phalacrochtamys Goor tab. N:2728.

Foculei caulisnares valte follati, gecurrentes, as ramos flor. Debiles vel submulli; fol.oborato-elliptica, attenuata, basi subrotuniata, superne glabra, subtus toto villosa, às costam eglangulosa; Dentes simphices, sub. convergentes; pat. aglaniulosi, tomentelki, inermes; stip. Dorso pubescentes, duriculis givergentibus; pes. 1-3, basi pilosi et simul to to glanoulosi, $19-25 \mathrm{~m}$. longi; tubus oblongus, laevis; sep. Gorss eglanioulose, pinnulis subintegris, lates copriose instructa, acusmine fotiaces; styli lanati, gisco comic; cor.alba vel gilute rosea; fruct. oblongus, utringue sed apice proesertion attenuatus. Hab. Haute-Saroie, as Sonnecy (Puget).

2210. Grepinia encophylla (Gogr Essai p.32, Gogr Ros. nor. II. p. 30); Yijar Gab. $\sqrt{ } \cdot 272 g$. - C. collina (Cariot \&tude ges ft. 5: ig. II, p. 192, non Jacq.). - Gogr Herb-ros. N-251 et 516.

- toculer subverticillati, falcato- "oilatati, as ramos flor-copiosi; fol. ample ovata, utrinque rotuniata, supra pilosula saturategue viriaia, sub. tus toto villosa, ar basin costare glanidulosd, vientes simplices; pet. villosoGlaivioulosi, aculeati; stip. silatatac, auriculis givergentibus; ped. corymbosi, ganse hispidi, $17-20 \mathrm{~m}$. congi; tubus ovatus, glaber vel basi infima. setulosus; sep. decisua, Gorso eglangulosa, pinmulis foliaceis, pauls dentatis instructa; styli maximi, lanati, Disco plans; cor. magna, rosea; fruct. obovatus, utrinque depressus, soridide ruber.

Hab. Thone, ad Craponne, Francherille, Chabonnières, ete.

2211. Crepinia hypoglauca Gogr mss.

Aculei irlatati, parum falicati, as ramos flor. glaucos saepe nulli, fol. meiviseria, subblonga, aprice parum attenuata, basi cuneata, supra glabra, glaucessentia, subtus à nervos villosa, toto valic albico-glauca, costa mèdia eglanoulosa; dentes simplices, lanceslato-convergentes; pot. tenviter glangulosi, tomentosi, acculeati; stip. sat longae, porum latai, sarso tamentellae, glauco-rubentes, auriculis sat sivergentibus, per. 1, sparse glandulosi, 5-7 m. langi; tubus svoïens, laesis; sep.reflesa, gorss Laevia, pirmulis foliaceis, integris, qlabreseentibus instructa; sigli sublanati, disco sat conico; cor......; fruct. avoidens, hasi valde attenuatus, apice turgisus, haud aut vix contractus. "ab. Hungaria, Saris: ad Csercmyés (W. Lichter).

Fries fore Rrue cinnomamear, sed sepala pinnata, decitua et stiquelar cum Lracteis Giversee. 
$-349-$

2212. Prepinia hemixiena Goger mss.

t-culei sat brens, falcato-yilatati, as ramos Plor. Densos sacpe gemimatic; fol sat late suboblonga, breviter acuto, bossi attemuata, superne gla brescentia et lacte viridia, subtus toto vellosa ac virescentia, io costam non glaniculosa; pentes simplices, lanceolato-aperti; pet. hine inde glesioulosi, to mentosi, inermes, stip. virises, longae, anoustac, Dorso hirsutac, auriculis subrectis; pers. 1. basi laeres, a mice hispioi, 10-12 m. longi; tubus sublongus, basi planiviosus; sip.reflexa, gorso eglaniulosa, pinnulis onnibus latiusulis,

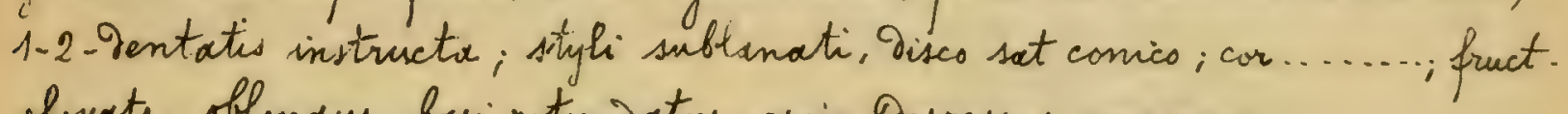
obovats - oblongus, basi rotuniatus, apice depressus, magnus.

Hab. Hungaria, Sars's: at Hoszúzétfalu, 'L. Richter).

2213. Cupinia Ychergiana (Moiss. Fl. arient. II, p. 686); Gogr 8ab. N.2730.-

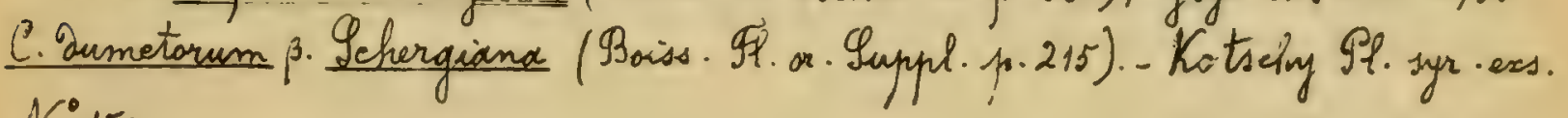
$N^{\circ}-150$.

Asculei valioi, freves, falcati, saepe geminati, fol minuta, oflonga, acuta, utringue rillosa, prominule nerwosa; gentes simplices, arguti; pet. hirsuti, aculeati; stip. subsilatatar, villosac, pes. 3-4, brevissimi, sense tomentosi; tubus oblangus, tomentellus; sep. Oeflexa, pinnulis pancioribus, silatatis, hirsutis instructa; styli ignoti; cor parvo, alba; fruct. oblongus, utrinque attenuatus.

Hab. Iyria, in monte Ichergi as Leborm tontilibani (Oh. Kotschy).

2214. Crepinia ssetica Googr mss. - Rosa ......... Grép. Prim. VI, p.22. Brotherus Plant. canc. (Rosae) N:35.

Acenler rectiusculi, parum compressi, sat longi, ad ramos flor. copiosi; fol. ample oblongo-acuta, basi rotuinata, crassa, gein supra glabra, subtes toto ses parce villosa, à costam eglan'sulos , Dentes simplices, ovato-lanceolati, recti; pet. eglanidulosi, hirsuti, sup. inermes; stip. maximae, silute rubentes, auriculis rectiss; ped. 3-7, parce glanoulosi, $17-19 \mathrm{~m}$. longi; thbus oblonqus, laeris; sep. vorso eglanimulosa, pinnulis pubeseentifes, parum latis, 1-3-bentatis instructa, acumine integro; styli lanati, Disco comics; cor......; fruct magnus, oblongo-pyriformis, basi attenuaters, apuice longriscule strangulatis, in $1 / 3$ sup. Aurgious.

Hab. Coucasur, Ossetic: prope fluvium forton (Brotharus). Species molesta, ut jam sixit $d$. Frepin. Acculei at inflorexcentia Rosae tomentosac Im., sed evidenter in Canineis collocanax. 
2. Sepala yerso ylanioulosa sel hispiaia.

a. Fructus globosus vel avatis.

2215. Crepinid conasifera. (Gimb. in 93ull. soc. bot. Ge. Grance XI, 1864; jus esetr.p.22; Grep. Trim. I, p. 62). Giogr Goub. N:2731.

Atculei is ramos foliosos, falcato-gilatati, fol. elliptica, firma, unirigue villosa, in 1/3 inf. istegra; Dentes simplices; pet. Tomentosi; inermes; stip. maxsimor, pubescentes, uuriculis brevibus, ostusis; pes. setulosi; tufus globosus, caesius, saltem basi hispianus; sup. Oarso glandulosa, reflexa, pirmata; styli hirsuti, cor rosex; fruct.globosus.

Hab. Tyrensei, inter Sénasque et Sarle' vo viam (Bimbal-Lagreve).

2216. Crepinia Lionjü (Dèigk. Cat. p.222), Gogr Eah. N22732.

toculei cinarphi, nunc falcato- Silatati, nune setacer; fol ovato-rota, isatia, atrovirentia, supra glabra, subtus tantum ad costam villosa; oentes simplices, aperti, profunbi; pet. tomentosi, aculeati, glanioulosi; stip.mediseres; pel. 1-5, hispisuli; tious rotinioatus; sep. Oorss glañoulose, pinnata, -acumine lato; sightivillosi; cor. rosea; fruct.ovatus.

Hob. Laire-Infer., at St Fibadien (Lloyo).

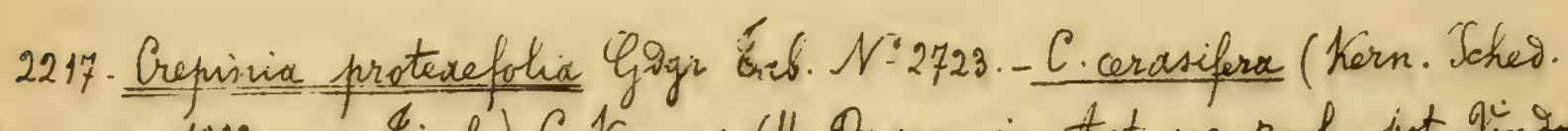
io exs.an. 1883, non Gimb), C. Ferneri (H. Praun in toct soc. Zool-bot. Jinioob. an. 1885).

toculai breves, forleato-jilatati, is ramos flor. pauciores; fol amplissime obovato-rhomboivalia, utrinque paulation truncata, intense sirivia, supra glabra, subtus toto villosa; sentes simplices, profunisi, paulo convergentes; pet eglaniulosi, inermes, hirsuti, stip. virices, tatae; ped.1-3, glanoulosi, 4-7 m.longi; tubus svoisens, laevis; sep. Dorso sparse glandulosa, pinmulis integris, subfoliaceis instructa, styli breves, glabri, Disco plano; cor. eciliata; fruct. overtus.

Hab. Auy-ch-Dome, as Sary (N. 2ubosclard), toustria infer.

2218. Cuppivia tentaculata Gige Gab. N:2734.

Acculei longi, inclinato - ielatati, as ramos flor. "oprosissimi; forl anguste oblango-lanciolata, fasi rotuniata, supra glabra lacteque viridia, sustis titc hirs-cta, as csitam eglanoulosa; dentes simplices, lanceolato-aperti, 
$-351-$

pet. eglowimlosi, pubescentis, aculeati, stip.magnae, latae, ghaprescentis, anvieulis givergentibus; pret.1. glanaulosi, 7.9 m. longi; tubus ovatus, basi hispitas, sep crecta, dorso eglandulosa, pinnulis integris, latis instructa., acumine foliace, integro, styli magrn; tanati, gisw fulano; cor....... fruct. maximus, vatus, utrinoine rotuniatus.

Hab. Danix, Teelang: Holleboek (9: T:Luniquist).

2119. Crepinia Gantzü (Schultes Ofs. bot.p.g4; if. Tl austr. II, p.29): Ggyr Gab. N:2735. Rosa ....... Crantz Stirp. sustr. II. p.87; C. Loysacana (Oborny apuid H. Braun in tict. sac cool. - bot. Ginoob. an 1885), C.numidica (Gren. apuis Dèsegh in Journ. of 990

toculei panciores, sparsi, falcato - dilatati, at ramos flor saspe nulli; fol.oblongo-acuminata, intense viricia, pilosula, os costam mesiam aculeolata; sentes simplices, ciliati, pet. sillasi, aculeati; stip. subtus pubescentes; per. hispini:; tubus ovatus, lavis; sep. gorso glansulosa, parce pinnata, styli villosi; cor amoene rosea; fruct ovatus.

Hob. toustria; Morevia; Galicia. - tgeria, ad Constantine (Coste).

2220. Brepinix torta (Muget) in Gogr Eab. N:2736.

Rami freves, tortussi, dense foliosi, florales inermes; aculei breves, foulcato-ailatati; fol. obovato-elliptica, breviter attenuata, basi rotunioata uniaique villosa, ai costam eqlansulosa, identes inf. biserrati, pet eqlanivulosi, inermes, tamentosi; stip. angustae, subtus pubescentes, auriculis givergentibus, peid, tenuiter et parce glanioulosi, 8-12 m. longi; tubus brevites obovatus, sparse setulosus; sep. brevissinna, gecidua, gorso glaniulosa, pinnata, acumine Cilatats; stijli villosissimi, Disco subplano; cor. parra, pallide rosea; fruct. parses, svotus.

Hab. Savoie, prope Moütiers (Siget).

2221. Gerinia transsilvamica (Mchur Enum pl. Eranss. p. 202 ; Halpers Ann. bot. VII, p. 879; H. Irraun in Kern. Iched. is exs. IV, 18-21); Gogr Gab. $\mathcal{N}=2737$.

Aculei pancieres, falcato- Dilotati, valivi, do ramos flor purpureos subnulli, fol. Elliptici, inf minora, subrotunida, lucida, subtus as nervos pilosula, ientes subfiserrati, convergentes; pet aculeati, sparse pilosi; stip. glanisulasa, auriculis ovato-acuminatis; per. Sense hispidi; tubus ovatus, 
- setulesus; sep. pinnata, 'deciona, gorss glanidulosa; styli ignote; cor. purpureorosea, bionata; fruct. ovatus, inipions.

Hob. Eranssibvanix, is kronstixt, te.; Mosnia; thengaria, Moloasia.

2222. Crepinia divonum ( Scheatz Ykano. Posa, p. 28; Chep. Trim. I, p. 125); Goar bab. N:2738.

touli falcato-Silatati, ai ramos flor. sparsi; fol. alliptica-ovata vel obo. veta, unvique se's praesertion subtus villosa; oentes simplices; pet. hirsuti; aculeati; stip.subtus prebescentes; pess. hispios; tubus suboviraeus, setulosres;

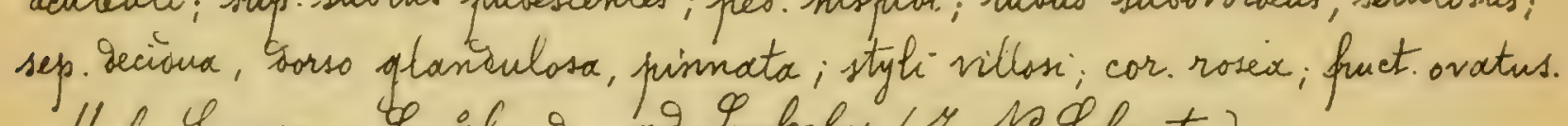

Hib. Inecia: Imokand, al Lyckeby (\%. No. Icheuty).

\section{Fructus ovideus val obovato-oblongus.}

2223. Greninia Gautiori Gogr Eab. N-2739. - C. rupestris (Eausch mss, non (ianty). C. Gauschiand (H. Mraun in toct. sac. zool-bot. Gingob, an. 1885).

foculei debiles, as ramos flor. parum dilatati, subrecti, fol. obovato-oblonga, subacula, basi attenuato - cuneato, sat parra, crassa, nervosa, superne pilosa, subtus fere tomentosa, as costam eglandoulosa; ventes simplices, aperti, triangulares, pet. eglanoulosi, tomentosi, inf. inermes; stip. angustae, subtus pubescentes, acuriculis subrectis; ped. 1, hispidi, 10-11 m. longi; tubus ovoideus, glavcus, basi infina setulosus, sep. brevissiona, villosa, Sorso sparse glanouLase, pinnuhi brevissimis, folixceis, parce dentatis isstructa, acumine foliaces, Dentato; styli hirsuti, siscs subplans; cor........; fruct ovoidens, bxui subrotumsatus, upice palo attenuatus.

Hes. Abrizige in valle Boxtoirul prope Mlijiones (G. Gautior).

\section{Crepinia liocarna Gagr Cab. N:2740}

Lape tota rubers, aculei gebiles, gilatati, teretes, recti nel inclinati, a ramos flor. Iense foliosos geminati aut verticillati, fol.oblonga, utrinque paulation attenuata, superme teniiter pilosa, subtus to to villosa, pallide sirisia; cientes simplices, lanceolato-recti; pet. hine inde glanidulosi, villosi, inf. inermes; stip. Tarso pubascentes, auriculis sivergentibus; peo sappe 2-4, hispicis, 11/2-21/2 cent. longi, rpice tantum hispici; tufus obovatus, glaber; sep. Sorss sparse itandulasa, seciour, anguste pinnatia, acumine lictiusculo, dongato; ityli villosissimi, disco rubro, subplano; cor. rosea; fruct obovatus, 
$\therefore 353-$

laveris, apice setterniatus.

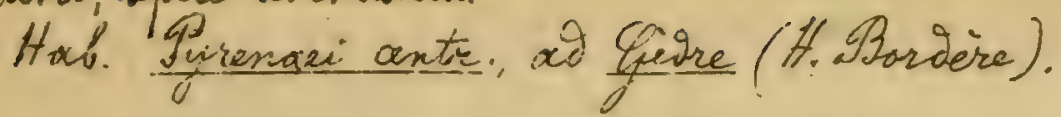

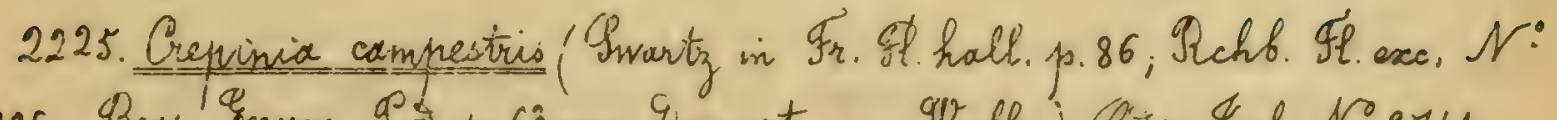

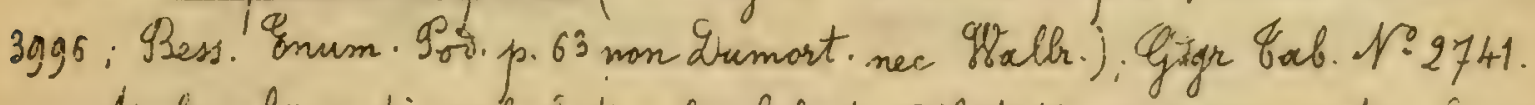
timbic ahi recti, subsalati, alii falcato-gilatati, compressi, cortex fuscus,

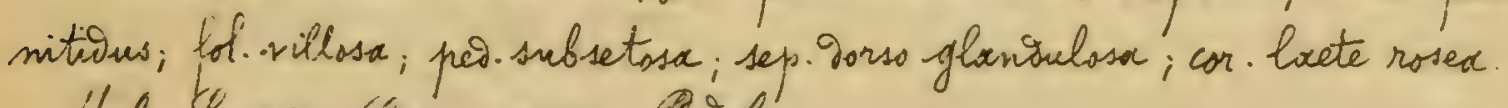
Hab. Suacix; Germana; Togolia.

Irecies thinem maxime ofscura vixape nota. Sequentia adit Rchb. laco it.: habitu Mosare collince, sed Tensiuscula, corolla facte rosea.

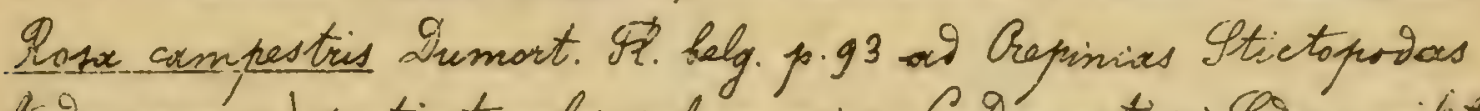
(itrieguvenses) pertinct, ubi sut nomine (D) Dumotiori Goge militat. Eisa campestris Hallr. Ros. Kist. p. Ho wo Cottetias / Mimpinallifoheoe) shactare videtur.

2225. Crenimia Echiane Gogr Eab. N:2742.

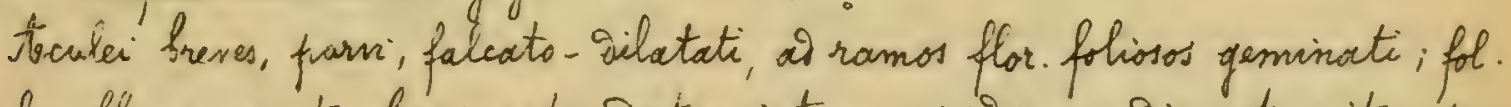
ample sblong-acuta, basi rotunvata, intense viriaia, unirique tenuiter pubescentïr, so costam eglanioulosa; Dentes simphices, triangulares, aperti; pet. glanoulosi, villosi, sup. soli paulo aculeati, stip. longace, parum latai, glabrescentes, aurriculis Sivergentibus; ped. 1, hispidi, $11-12 \mathrm{~m}$. longi; tíbus ov videus, hispitus; sep. erecta, oorso glanaulosissima, pinmulis integris, latiusculis instructa, acumine integro, foliaces; styli lanate, gisce plans; cor ........ fruct. magnus, breviter ovoidens, basi abrupte depressis, hispious.

Hab. Dania, at Igalt Mark (Tahrtmann).

2227. Crepinia implicata (Suget) in Gogro 8.ab. N-2743.

Areulei oebiles, falcati, as ramos flor. laxos stitipulares et geminati, fol. oborato-suboblonga, utrinque ses apice praesertion attesnuato, unioique viliosa, as costam eglarioulosam albiar; oentes simplices, lati, rotunioato-cuspioati; pet inferne poulo glanoulosi, tomentelli, plerumque inermes; stip. subitu, pubescentes, auriculis rectis; pet. 1-4, glanaulositemuitor, 9-16 m. longi; thitus or oideus, minute hispistulus; sep. brevia, Decidua, Forss glanoulasa, primulis sat latis. instructa, staghi breves, hirsuti, aisco comico; cor prallise essea: fruct. orvideus, apice ralte attenuatus.

Hab. Savoie, oo Bois Champion prope Noütiers (Muget). 
$-35 \%$

2228. Crepinia platypenthos Giagr \$ad. N-2744.

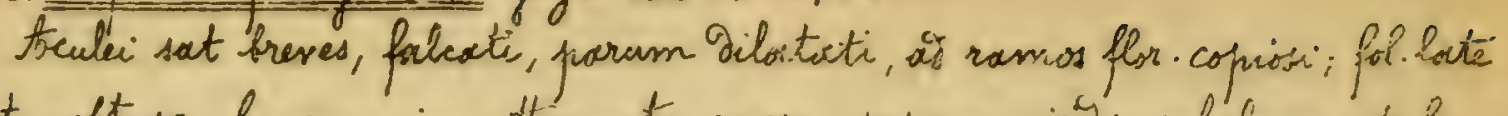

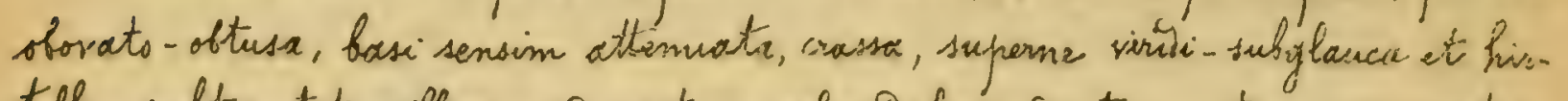
tella, subtus to to villosa, at costam aglamoalosa; ientes ovato-convergentiss, simplices; pet. eglaniulesi, inermes, tomentosi; stitip. gilute rulentes, latissirnue, auriculis parum givergentibus; pes. 2-3, hispioi, 4-6 m. longi; tubus ovoideus,

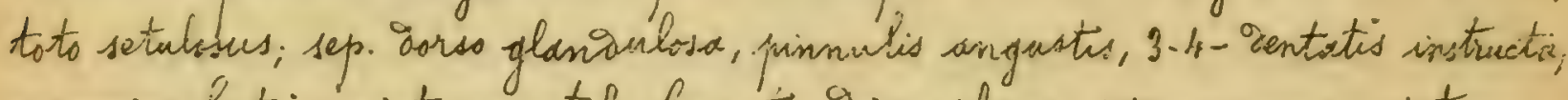
acumine lactione, integro; styli lanatr, giseo plano; cor-maxima, intense rosea, eciliata; fruct. wovidens, utringue attanisatiss.

Hoch. Basses. Alpes, ad cLa Conamine (Mraal).

c. Iructus eflongues.

† Ptagli glabri.

2229. Crepinia wenstema Gogr Gob. N-2745.

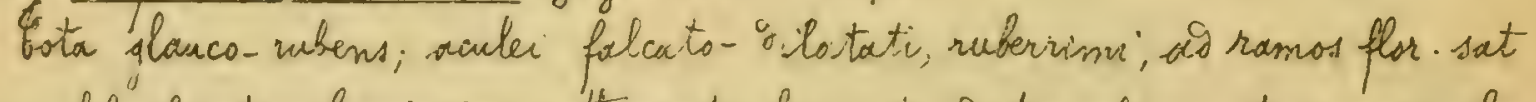

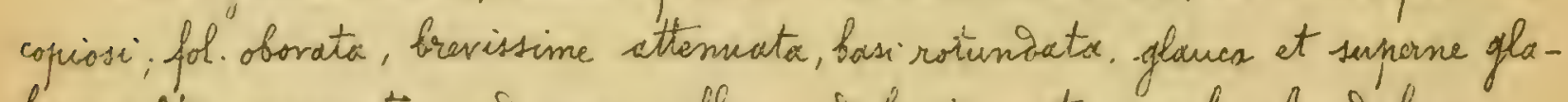

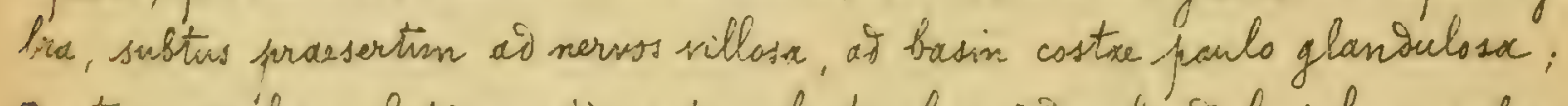
Dentes simplices, lati, aperti, pet rubentes, hime inde glanioulosi, laxe puses-

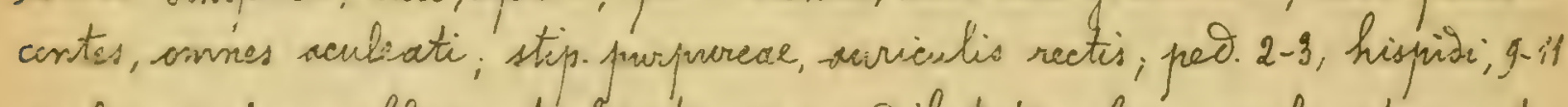

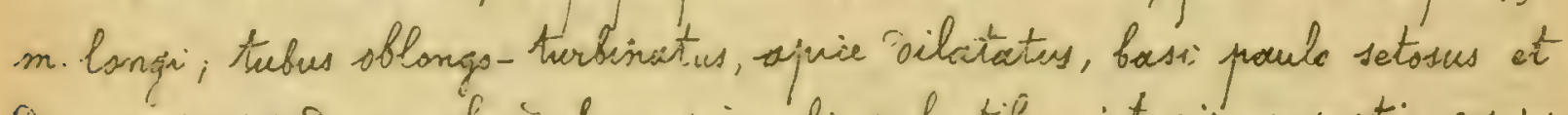
Decivisens; sep. Dorso glaniouloses, pinnulis rubertibus, integris, angustis coprose

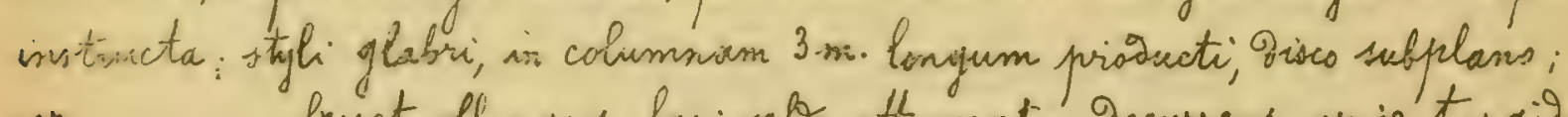

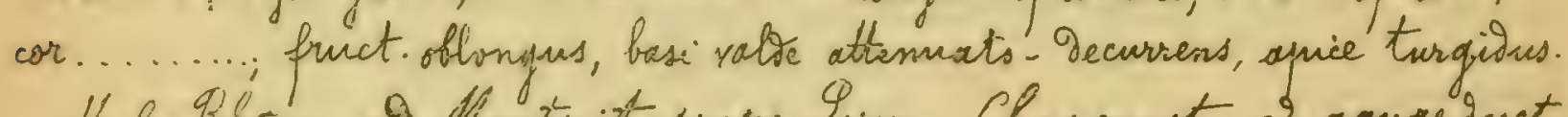

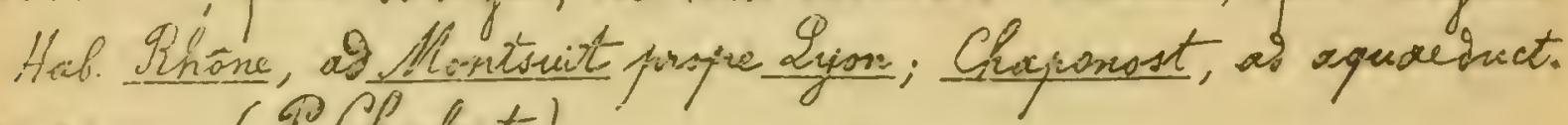
somanum (T. Chabert).

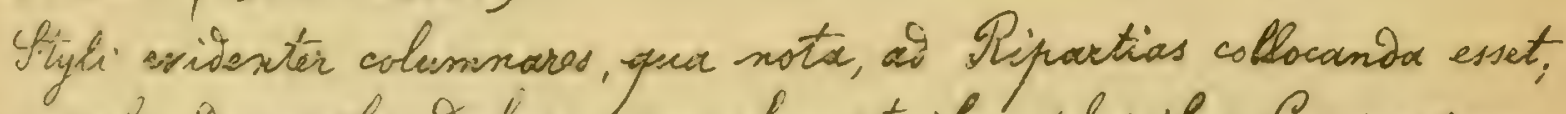
sed sepala gorso glongulosa cum characteribus phuribus Canineorum,

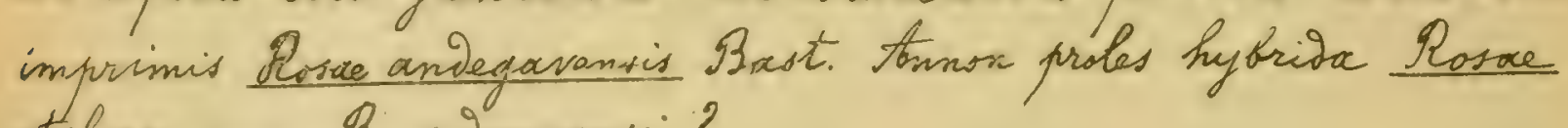
stylosas cum M. diniegavense:?

2230. Premina comia Gogr Gab. M:2746.

Acculei falcato-gilatati, ad ramos flor.genninati, fol. firma, oborato-obTisiuscula, basi rotuniacta, unsique pubescentia, atrovirentia, subtus palhiviora, ad costam eglanivulosa; Dentes simplices, profunis: late aperto-triangulares; pet. lase pubessentes, glanisulosi, aculeati; stip. rubentes, subtus 
$-355-$

glabue, latae, suriculis rectis; por. 1-9, glandulosi; $13-15 \mathrm{~m}$. longr; tubus

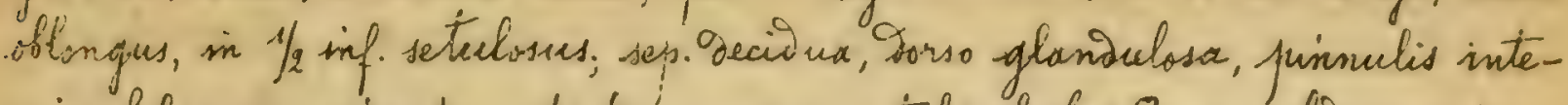
Fris, foliaceis irstructa, ut at scumine; ityli flabri, oise valie conico; cor sinagna, eciliata, fruct parvess, oblongus, apice athenuatus.

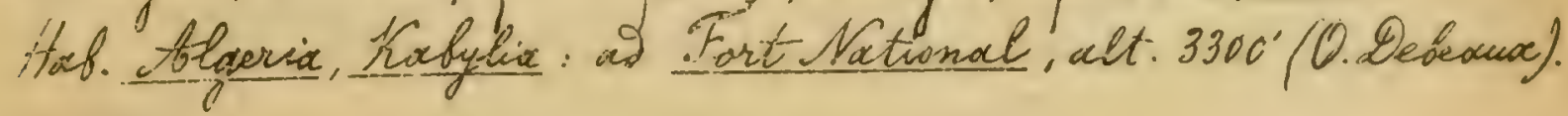

2231. Crepinix mauritanica Gogr mss.

Toculic breves, faicato-vilatat, ai ra nos flor mulli vel rarissimi; fot. wmple oboruito-oblmag, vix acuta, fasi rotindata, intense viridia, jupra tomicter subtus vor prorsus villosa, canescentix, as estam eglaniulosa: ientes simplices, late orato-recti, pet hine inde glanoulasi, tamentose, vior

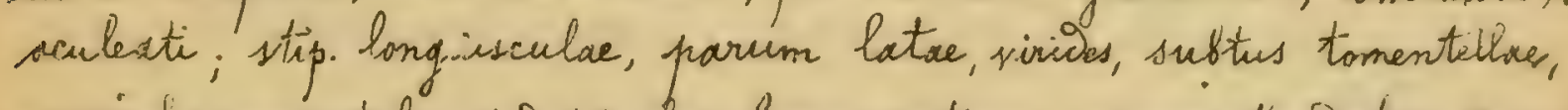
invicielis semipatulis; peo. 1-3, basi Caevs, cacterum sparse glaniulosi, 2 cent. longi; tubres flongu, laevis; sep. oorso paululum glangulosa, pinnulis integris, parem latis instrencta, acumine foliaies, Dentato; styli glabri, Dises

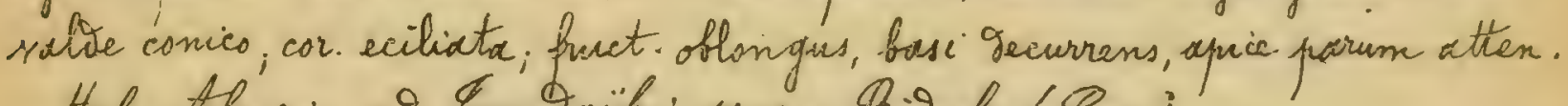
Hab. Algeria, al Eamaigh prope Bidah (Quay).

Tt Pigh hisonte.

2232. Crepinia filisinina Gover Gab. 1r:271\%.

Acular recti, subulati, temes, as ramos flor rari; fol gtaucescentia.

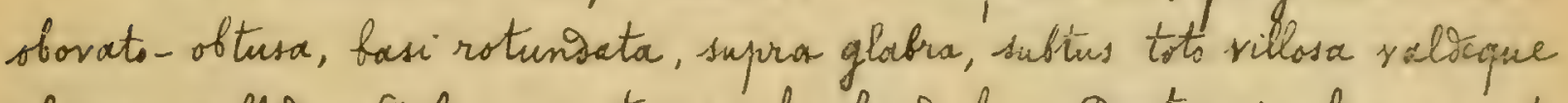
glaucs-pallita, as basin costoe paulo glaniulosiz; Dentes simplices, aperitotriangulares; pet. villasi, gloniulosi, sculeati; stip. "irides, auricilis rectis; per. 1, hispiai, 20-22 m. longi; tubus elangatus, toto sed parce setosus; scp. Sorso prorsus hispida, pinnulis integri, hinearibus instructa, acumine lato, integro; styli lanati, Quisco plans; cor. eciliata; fruct. elongatus, utringue longe attenuatus.

Hab. Hispania bor., Navarra: at Caparsoio (Y. Muiz-Casovidlla).

2233. Crepinia pisica Gogr mss.

Toculei rarissimi, clongati, recti, cylininici, as ramos flor. mulli; fol anplissime obovato- subacuti, basi rotunsata, supros glabrx lateque virisia, subtus to to villosa et albita, acntes simplices, late triangulares, apertic; pret. tomentosi subinermes, inforne aspuerse glanoulosi, stip. virides, langissimac, 
$-356-$

sprice Dilatato, gonso tantum pubescentes, aurieulis subrectis; ped. 1-2, hisfidi, 3 cent. longi; tubus oblongus, sparse setosus; sep. Sorso sense glanionlosa, pinnulis annibus salie gentato-glansulosis, acumine longo, foliaces; sityli magni, lanati, gises plano; cor......... fruct. oblongus, wirinque thenuatus. Hab. Thône, is Denicé (M. Ganioger); affinis R xitac.

2234. Crepinia illudens Gogrmss.

taculei longissimi, sat gilatati, inclinati, at ramos flor. copiosi, fol. ample obovata, apuce subrotuniata, basi rotungata, lacte virisia, superne glabra, subtus glancescentia et to to villosa, ad costam eglasioulosa, Sentes simplices, late ovato-recti; pet. hine inide glaniulosi, tomentosi, aculeatic; stip. virises, longissimac; latos, gorso aculeolatac, auriculis rectis; ped. 1-3,-sparse setosi, 21/2 cent. longi; tubus sbiongus, toto assperse hispians; sep. 90 rso prorsus glaniulosa, pinnulis innibus integris, valie dentats-glanidulosis instrueta, acumine integro; styli lanati, gises plano; cor. alba, eciliata; fruct. glancus, oblongus, basi Fepressus, apice breviter strangulatus.

Hab. Hispania media, as Ablarracin (9. Lapater).

Species, ut et antecevens, as grequm C.albae (2.) pertinens, ab ex tamen sepalis gorso glanoulosis frimo intuitu Differt.

2235. Crepinia acanthsearpa Gogr Gab. N-2748.

Aculei breves, dilatato-subfalcati, ad ramos flor. geminati; fol. oblongoacuminata, basi valse attemuato-cuneata, intense siricia, supra pulis sparsis iein evaniois obsita, subtus io nervos villosa et ris costam eglaniulosx; Jentes simplices, lanceolato-convergentes, pet, eglandulosi, inermes, longe pilosi; stip. virides, subtus smnins pubescentes, auriculis givergentibus; pel-1, aculeati, 5-7 m.longi; tubus oblongus, to to aculeolatus; sep. Dorso gense glansulosa, pinnulis hinearibus parce instructo, acumine parum latox, integro, longissino; styli lanati, gisco subplans; cor. eciliata; fruct. oblongus, utrinque attenuatus, aculeolatus.

Hob. Sedemant, as galleés gaudoises (E. Tostan).

2236. Crepinia rapa Gogr mss. - Rosa......... Crép. Prim.VI, p. 31. Protherus Slant. cauc. (Mosac) $N=12$ bis.

toculei tennes, subcylinarici, inclinati vel rectrisculi, ad ramos flor. copiosi; fol. late oborato-acuta, basi rotuniata, supra glabra, subtus as nervos 
$-357--$

parce villosa, ad costam eglaniaulosa; Ientes simplices, lancealato-convergentes, pet eglanoulosi, hirsuti, inf inermes; stip. latissimae, medis rubentes, auriculis ivergentibus; ped 2-3, toto hispisi, $15 \mathrm{~m}$. longi; tubus elongatus, glauens, to to glandulosus, sep. Forso prorsus glaniouloso-muricata, pinmulis glabis, latis, 2-3-gentatis instructa, acumine integro; stghli magni, basi pruilo pubescentes, disco sat comico, cor magna, albo-carnea, eciliata; fuct. probabilius oblangus, à apicem longe strangulatus.

Hab. Cancasus, Ossetia: inter Moki et Jedisi; as fontes Cluvii Didi Siachna (Brotherus).

Certe Collinas, nec Domentosas (Singetia) ut suspicatus est ch. Crepin, howe spectat.

2236 bis. Crepinia superba Gagr mss. Rosa.

$\therefore$ Bip. Prim. II p. 30. - Mrotherus Mlant. couce. (Mosac) N: 17 bis.

Ramosissina, Dense folioss; aculei panciores, longi, subteretes, tennes, inclinati, as ramos flor. mulli; fol late obovato - subacuta, basi sensim roAunvata, lacte vinivia, superne paulo hirtella, subtus toto et breviter villosa, costam eglanioulosa; dentes simphies, triangulari-subconvergentes; pet.eglanioulosi, inermes, tomentosi, stip. virives, latac, subtus pubescentes, auriculis deirergentibus; ped. 2-3, toto hispiiai, 16-17 m. longi; tubus ollongus, toto hispians, sep. Dorso prorsus glangulosa, pinnulis pubescentibus, latis, 1-3-gentatis instructa, acumine integro, longo; styli parce villosi, Disco comico, cor. magna, alba, eciliata, fruct probabilius sblongus, at apicem longe attemuatus.

Hab. Caucasus, Carthahinia: It Itrasneaksop prope flureium hiura (Brotherus).

Inecies sane Difficilis atoue classificatione molestissima. Aculei At inflorescentia ut in Rosa tomentosa; sed petala, habitus Dense foliosus, serraturace foliolorum atoue foliola ipso cum Canineis benequairant: planta igitur illa hoc in genere aptime militare protest.

3. Noixtae Gige Gak. p. 198.

Foliolis pubescentibus, magnitudine mediscritus, infimis fiserratis, superioribus vero simpliciter serratis; pequnculis normaliter glanoulo- 
$-358-$

sis vel hispidis; sepalis gorso ghandulasis wel non; petalis sat piorvis, albis vel palkivis.

1 Sepala gorso eglanioulosia

Fructus ghobossis rel oxatus

$2\{$ Fructus sroitens vel oblongus Inuctus rotunisatus vel ovatus $3\left\{\begin{array}{l}\text { Fructus ovoisens } \\ \text { Fructus oblongus }\end{array}\right.$

2

3

Shecien N N $2237-3212$

Iflocie $1-2243-2245$.

Precies $N 2246-2250$.

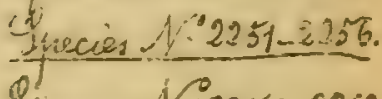

Ipecies $\sqrt{22257-2262}$.

1. Yepala dorso eglansulosa.

a. Fuctus globosies vel ovatus.

2237. Crepinia lasionhylla Gogr bab. N-2750.

Acculei minutissinu, recti vel inchinati, xo ramos flor pranciores; fol. sbovata, apice serbrotunoata, basi vis attemuater, unsipue pubescentia,

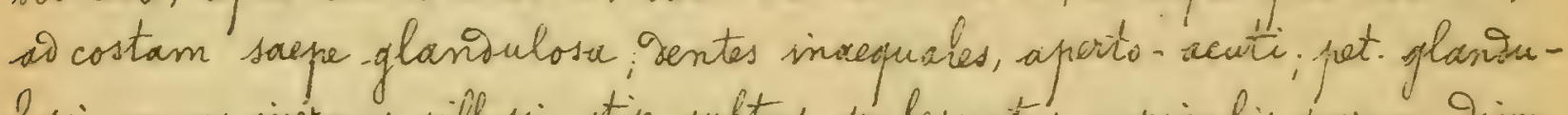
Losi, onmes inermes, villosi; stip. subtus pubescentes, curciculis parum Divorjentibus; jes. 1, alii laeves, alii spoorse glanioulosi, 4-10 m. Congi; tubus rotunvatus, laer is; sep.erecta, sat elongatia, primmilis pracioribus, copiose

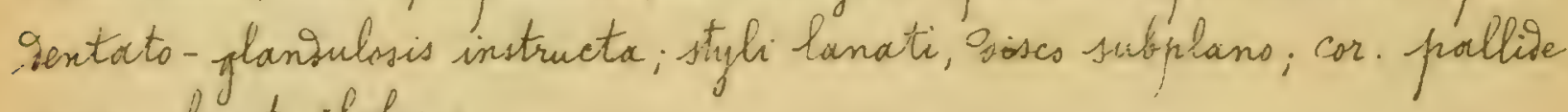
rosea; fruct. ylobosus.

Hab. Savore, to Salins prope Hoütiers (Suget).

2238. Crepinia obtusifohia (Desw. Yowrn. bot. II. p.317, Gren. ct Goor. Fh De

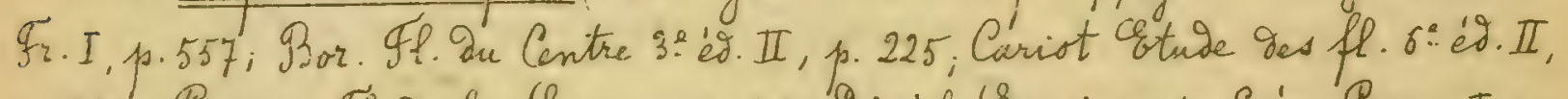
p. 243; Pauquy FP. Do la Yorme p. 118; Diseigl. Gssai p. 81; Grép. Prim. I, p. 57; Christ Rosen ger Gehw. p. 186); Gjogr Gab. N:2751; Gogr Contr. fl. slav. II. p. 44. - C.canina var obtusifolia (2C. Tisor. II, p. 613). - C. puberula (Desv. in herb. DC.), C. Drachiata (Déségh. in Him. acad. ge Maine-et-Loire XXVIII, 1873, p. 103; extr. p.7. - Millot exs. N:1654, G. Ychulty Herb norm. N:473; xisigh. Herb.ros. $N=21$.

teculei falcato-gilatati; fol orbiculatx vel ovoto-obtusx, froesertion inferiora, nervosa, unisioue villosa; Ventes subbiserrati; pet. Tomentose; aculexti, stip. pubescentes, denticulatac; ped. olie laeves, alii parcissime glan- 
$-359-$

Tuloss; tubus subrotuniatus, Laevis, sep. brevia, gorss cglanialosa, pinmulid gentatis instructa; stighi hirsuti; car. parva, alba; fruct. globosus. Hiat Gollia media, cte.

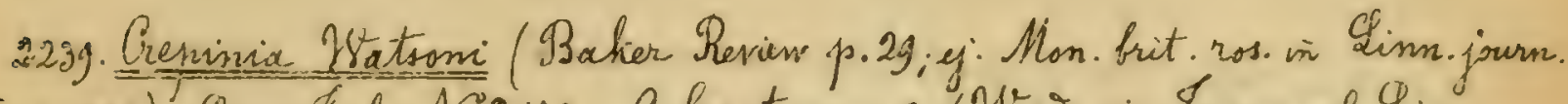

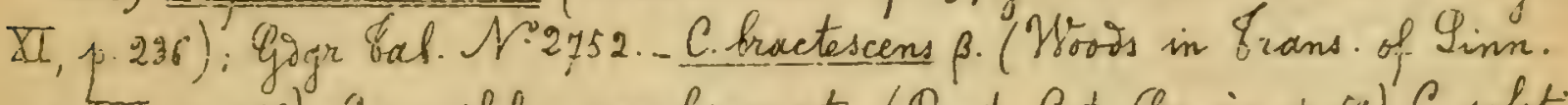

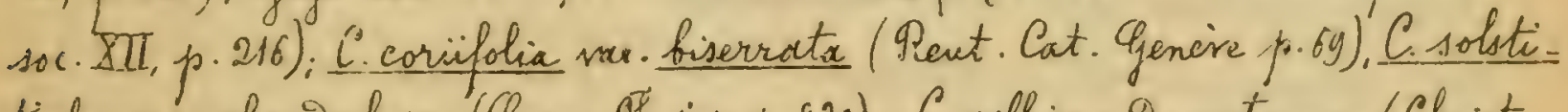

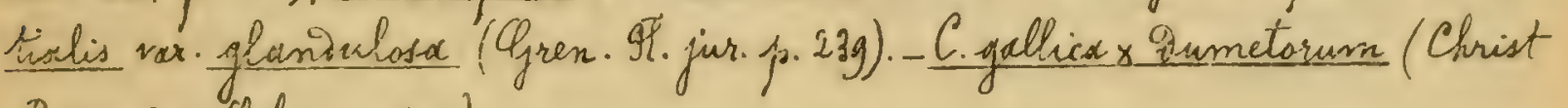
Roien ger Ychw. p. 202).

Tbichei Silatati, rectiusculi; fol. obovata, superne glabresantia, subtus toto villosa, is costam glaniulosa; dentes inf. biserrati; pet. glandulosi, aculeati, hissti, stip. angusta, prebscentes, marginibus glañoulosar, pros. hispiti; tubus oveto-iotunivatus, Cacvis; sep. Dorso saguins laevia, pinnulis foliacsis instructa; styli hirsuti; cor. rosen; fruct. subglobosus.

Hab. Anglim: Hurassus.

2240. Crepinia fallaciosa (Déségl. Cat p.222), Gogr bab. N-2753.

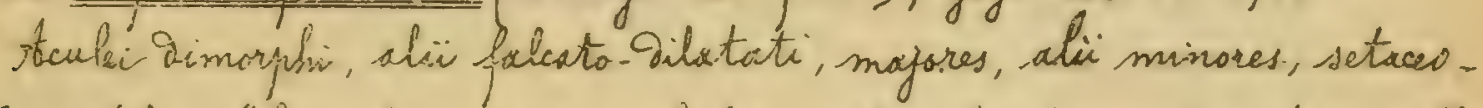
sublanouliferi; fol ovata, superne glabia, subtris tantum al costam villoser, gentes inf. biserrati; pet glanaulosi, glabri; stip. laves; pet saepius glan aulosi, trebus svatus; styli pauds villosi (omn, sec. Dése'glise).

Hab. Gallix oceid, prope tongers (At. Boreau).

2241. Grepinia nova (Gogr Essai p. 33; Gyogr Ros. II, p.31), Gogr \&ab. N-2754.. Rosa......... Désegl. Gssai p.go, in nota.

Áculei condensati, falcati vel rectiusculi; fol coriocea, late elliptrica, superme glabra saturateque viridia, subtus al nervos villasa; ientes inf. biserrati; pet. sparse pilosuli, hine inde glangulosi, aculexti; stip. subtus probescentes, auriculis oirergentibus; pes. hispidi; tubus ovatus, lacris; sep.

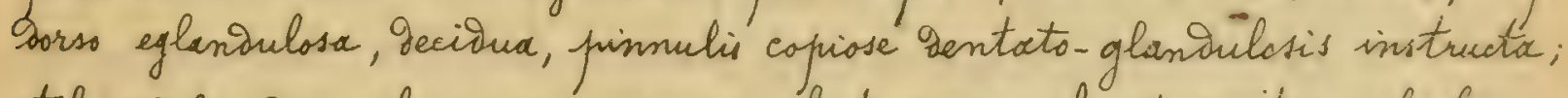
styli glabri; disco plano; cor.magna, lacte rosea; fruct oritus, glaber.

Hab. Gallia vacio, as tongers, et in Gallia austro-arient

2242. Prepinia obtusifolio $\times$ tomentella (Pugct). M Gogr Gubb. NP2755.Formanek Rosen Hochges.p. 10.

Aculei falcato-gilatati, as ramos flor. pauciores; fol ample obovata, 
$-360-$

aprie subrotuniata, baxi cuneata, superne assperse pilosule, subtus toto villosa, is costam piaulo glaniulos-a, ientes lati, aperti, rotinaati, fere smnes biserrati, pet. hirsuti, sparse glandulosi; inf. inermes, stip. elongatar; ped. 1-3, rillosi, 5-11 m. longi; tubus breviter ovoideus, lavis, sep. Isrso non glanoulosa, pinnulis latis, gentatis copiose instructa; styli hirsuti; Disco subplano; cor alba; fruct. parvus, ovatus.

Hab. Phône, ad Charbonmières (Moullu); Moravia (Formanek). os speciminibus quace suppeditaverunt, flanta haece minime misida est iteogue nomen inconoruum esse visetur.

b. Fructus woidens vel oblongus.

2243. Crepinia eriogyna (Gogr Gssai p. 31; Gogr Ros. II. p. 31); Gogr Gab. N-274g:- C. Frieslanieriana (Cariot ottide ges fl. 5: ì. II. p.192; Hort.Lug. an. 1867. 73, non Mess.). - Gagr Herb. ros. $1=122$.

foculei elongati, falcato-gilatati, aro ramos Plor copiosissinn; fol. magna, obovato-crisata, apice vix atterwata, superne mose glabra, subtus toto villosa, as costam flanoulosa; Tentes inf. biserrati; pet. villosi, aculeati, copiose glaniulosi; stip. Dorso glanisulosae, auriculis ovivergentibus; pees. corymbosi, hispidi, tubus oboratus, lavis; sep. Jeciaua, gorso aglaniulosa, pinnulis foliaceis, copiose gentato-glandulosis instructa; styli lanati, gisco conico; cor. lacte rosea, basi flavescens; fruct parrus, ovsidens, basi rotundatus, $x$ apicem attemiatus, coriaceus.

Hab. Gallia merid, circa Lyon of Francheville, Dardilly, te.

2244. Crepinia calochlora Gogr Eab. N-2756.

Aculei vix gilatate, decurrentes, tenniter falcati, as ramos flor. nulli, fol. obovata, apice rotundata, basi sensim attemuata, petiolulata, lacte viridia, suprex glabra, subtus proesertim ad nervos villosa, glarce et ad costam glanInlos a; dentes orato-convergentes, inf. biserrati; pet glandulosi, pubescentes, parce sculeati; stip. subtus glancae, glabrac, arriculis sivergentibus; pes. 1 . tenviter et parce glaniulosi, 16-18 m. longi; tubus oblongus, laevis; sep. Ocrss eglanoulosa, pinnulis hinearibus, integris, pauciaribus instructa, acumine lato, integro; styli lanati, giseo flano; cor eciliata, fruct of longus, glaucus, utrinque attenuatus.

Hab. Helvetia, firope Lwich alt. 1800' (K. Lehmann). 
$-361-$

2245. Crepinie gasucalyx Gogr \&́ab. N=2757.

oceutei treves, falcats. subicilatate, a ramos flor. temues; fol obovata, coniscea, stritia ungrque glabra, as csstam eglangulosa, supra intense ac Eucive virentia, gentes extra-inf. biserrati; pet aculeati, tomiter tomentelk; sitip. awriculis parum Iovergentibus, pes 1-2, glanoulosi, 6-8 m. longi; tubus elongatus, baxi infina hisfindies; sep. replexa, secidus, pinnulis brevibus, angas tis, 2-4-Gentato-glandulasis instructa, stighi breves, hirsuti, Bisco parvo, paulo cornis, cor. rosea; fruct. magnus, elongatus (3 cent), utionque valie atternuatus, glancescens.

Hab. Phone, at Charbonnieres (Soullu).

2. Pepala dorso ylanoulasa.

a. Fuctus roturivatus vel opatus.

2246. Crepinia areana (Désésl. Cat. p. 216), Gigr Gab. N'2758.

Toculei falcati, ai ramos flor submuli; fol. oreato-acuta, suprob breviter prissa, subtus toto villssa, gentes inf biserrati; pet. inermes, tomentosi; stip. angustar, auriculis acutis, rectis, pet. 1-3, parce glangulosi; tubus parwus, globosus; sep. Gorso glandulaso, purnulis latis, Sentatis instructa, styli lanati, sis-co plani; cor parra, pallive rosea; fruct purrus, rotunsatus.

Hab. Delphinatus; Sabouina; Heh., Makxis.

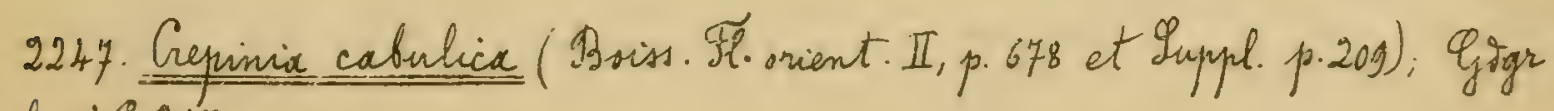
bab. N:2759.

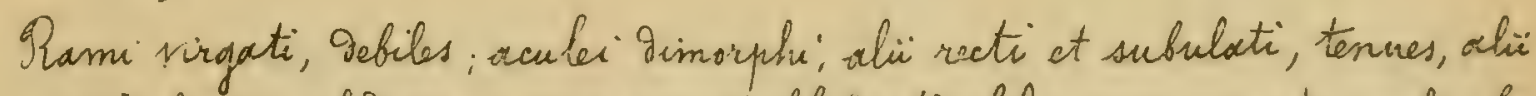
outem stipulares validiores, compresso-subfalcati, fol parva, ovato rel oforato-rotundata, obtusissima, anpra sparse, subtus autom dense ac longe villosa; Dentes magni, inf. Siserrati, pet. villosi, aculeati, parce glanioulosi, stip. basi angustar, superme gilatatore, auriculis lanceolatis; pet. corynbosi, flaniulssi; tubus oroidens, sparse ylanouloses, sep. reflexa, geciaura, Torso

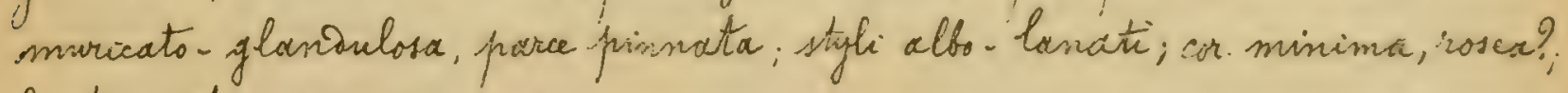
Pruct. oratus, pios vis major.

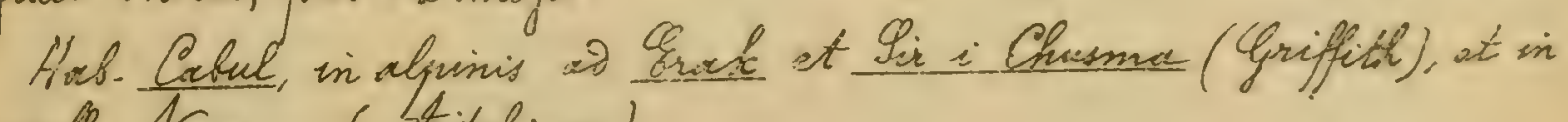
vale Lwram (Btitchison). 
$-362-$

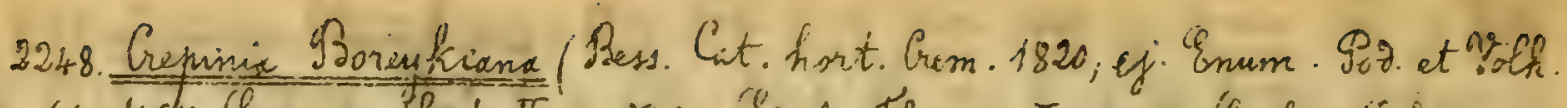

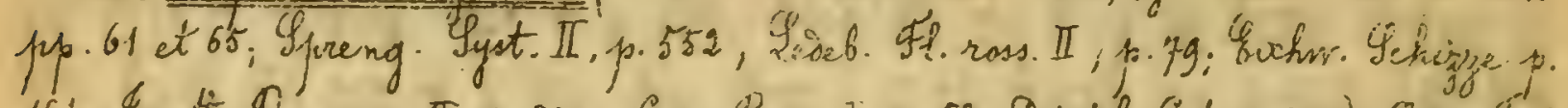

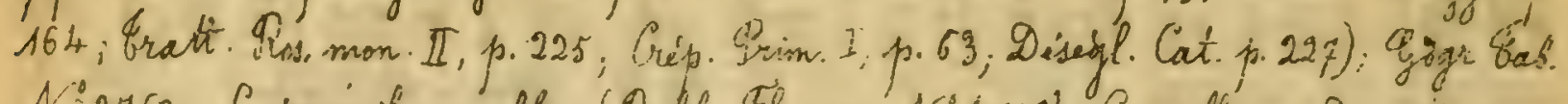

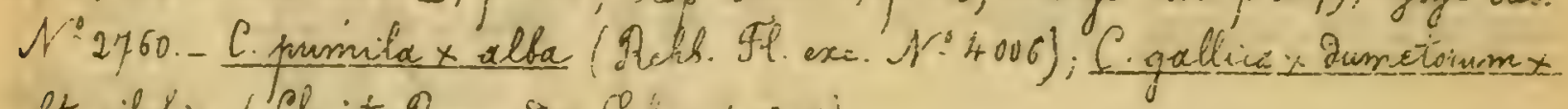
obtasiftix (Christ Rosen var Terur.p. 205).

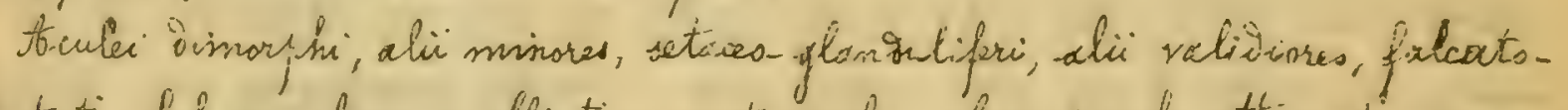

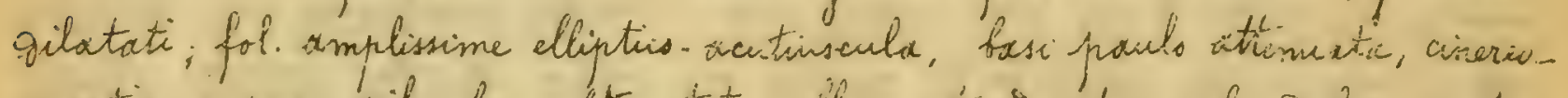

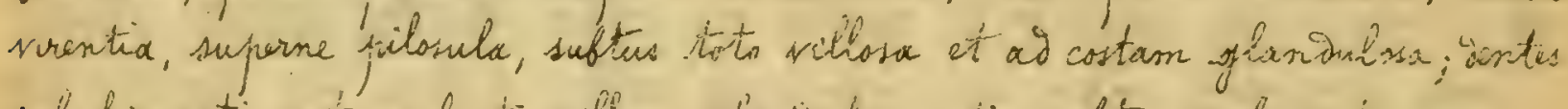
inf. biserrati, pet aculeati, villoso-glavisulosi, stip. subtus pubescentes, -1ersu-

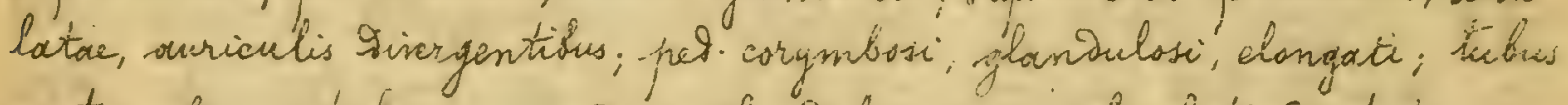
oratus, fasi setulosus; sep. iorio hanoulas, juinnulis latis, gentatis instructa, acumine elongato, dentats; ty param villosi, Risco subphano, cor.magna, rosea, fruct:oratus, mitidus.

Hab. Rossia merid. sec, in Favolia, etc.

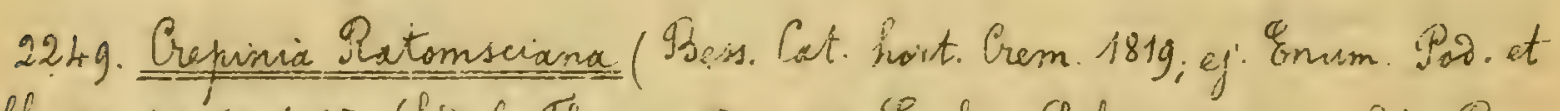

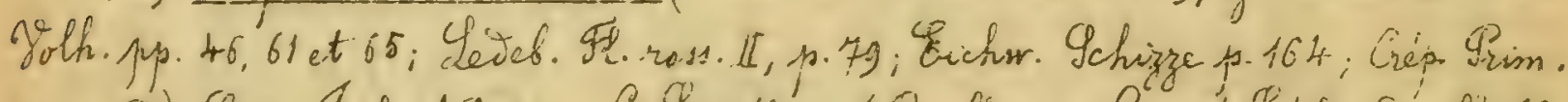

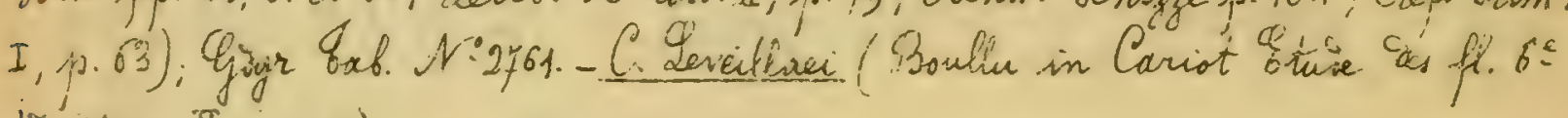
iv., $1879, \mathbb{I}, 2.242$ ).

Sccolei Timorphii, ahii majors, falcato-gilatati, alii minores rel setaceoslanouliperi; fol miaioaria, ovata, basi paulo attemuata, viridix, superme ylabra, subtus villose, gentes parri, inf biserrati; pet.villose; aculeati; stip. subtus pubeseentes; per. hispioi; itubus ovatus, basi setulosus; sep. reflexa, derioux, Darso glaninclose, angustivis pinnata; styli villosi; cor. pallide rosea; fruct. ovatus.

Hab. Rossia occio, in Podolia (Messer); Galla merid, circa Lyon (Soullu).

2250. Crepinia hissivacalyx Giar sinss.

Aberlei conformes, silatati, salde fibleati, as ramos flor verticillati; fol. late obovato-oblonga, acuta, basi sensim rotunidata, medis gilatacta, intense sinidia, supra glabra, subtus ad nervos villosa, et ab costam eglaniulasa; ientes triangulari-convergentes, inf. biserrati; pet tomentosi, aculeati, pauls glandulosi; - stip. virives, longare, subtus paulo pubescentes, auriculis oivergentitus; pes. 2-3, hispiiti, 5-6 m. longi; tubus ovato-elliptiens, tots aculaolatus; sepcisso prorsus glanisuloser, pinnulis onnibus foliaceis, parce Dentatis instructa, 
$-363-$.

acumine integro: styli lanati, disce subplans; cor. cosea, sciliata, fruct ovatoollipticus, uthinque rotuniatus, centrole autim hasi decurrente.

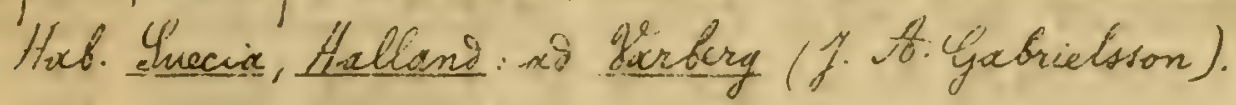

6. Fructus overiocus.

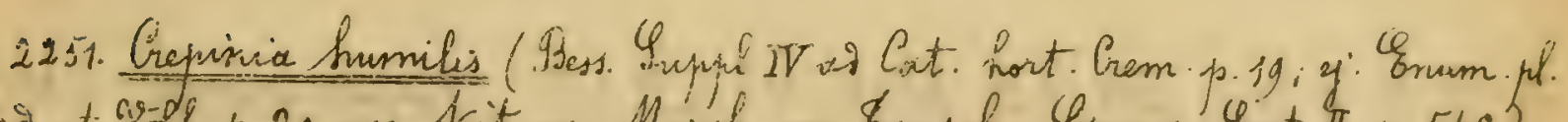

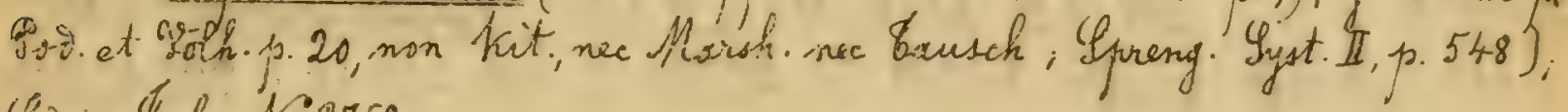
Gagr tab. 19762.

Aculer Timorphi, alii masoses, falcato- Dilatati, panciores, alii minores et setacer; fol.elliptica, sustus villosa; Dentes inf. biserrati, pet. aculeati, villosi, glaniulosi; stip. subtus pubscenties; peg. hispiti; thobus ellipticus, glaber;

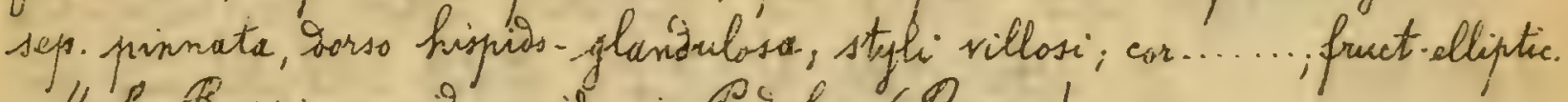

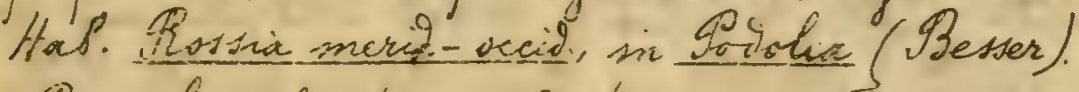

Rosa humilis Kit. apus trienity in Fora XX811, (1869), p. 590 idem sit ai panta Besseri, mini ignotim est. Hab. in Hungario, ai montom Nors in prope Korenic.

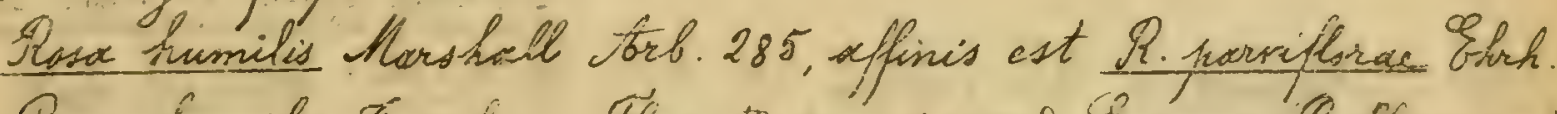

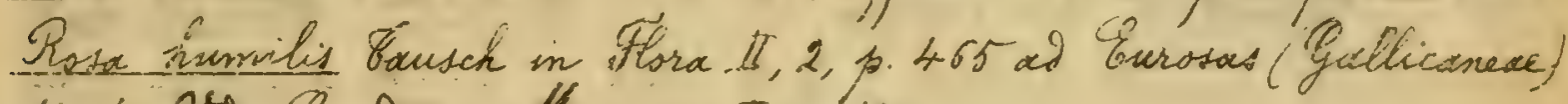
pertinet, Wide Ganooger Kon. ros. I.p. Hoo.

2252. Creninia Obornyi Gogr Gab. N:2763. - C. Mnenkiana (gallies x obtusifolia) (Bdaenity Herb.europ. an. 1378).

Acculei imorphi, alii breves, inchinato - Dilatati, as ramss flor sparsi, alii setacei, tenus; fol amplissime elliptico-sbtusa, basi subtrumeata, superne tenniter hirtella ac lacte viridia, subtus pallioa totoque villosa, nervosa et as csstam glaniulosa; Dentes late ovato-aperti, fere onnes biserrati; pet villosi, aculeati, glanidulosissimi; stip. latissima, virides, subtus pubescentes, valdo marginato-glanivelosac, arriculis rectis; pet.1-2, aculeatissimi, 12-15 m. longi; tubus oroidens, toto hispious; sep. gorso gense glanidulosa, pirmulis latis, 1-3-gentatis instructa, acumine imple foliaces sacpeque laciniacto; styli lanati, gisso sat conico; cor magna, rosea, eciliata; fruct. ovideus, utrinque attematus.

Hab. Austria, Moravix: at Anaim (A. Oborny). 
$-364-$

2253. Crepninia Blekingensis Gigr N-2764.

tbculei parvi, breves, conformes, falcats-gilatati, at ramos flor. sparsi; fol. parsa, coriacea, obovato-subacutax, basi truncato-attemuata, suprá glabra, viritia, subtus ai nervos villosa et as costam eglanaulosa, Dentes subaperti, triangulares, inf. biserrati; pet. eglanioulosi, villosi, aculeati; stip. virides, subtus hubescentes, auriculis sirergentibus; pes. 1-2, glandulasi, 8-g m. longi: tubus ovoidens, in $1 / 3$ inf. glanoulosus; sep. Dorso parce glandulosex, pinnulis parum latis, 1-3-gentatis instructa; acumine lato, integro; styli hirsuti, giseo plans; cor......... fruct. ovoiveus, utrinque abrupte attenuatus.

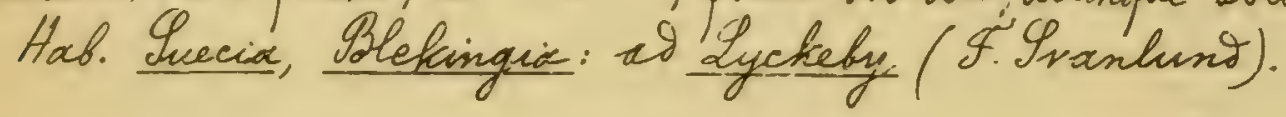

2254. Crepinia multiglandulosa Googr mss.

teculei conformes, parvi, sat longi, sinchinati, parum gilatati, as ranos flor mulli; fol sat parva, obsiato-subacuta, basi fere rotuniata, inf. obtusa, subtus ylabra ac pallide viridia, subtus valis nervosa, albisa, toto hirsuta, as costam eglaniulosa, Dentes profunde triangulares, aperti, inf biserrati; pet inermes, tomentosi, parmm glansulosi; stip. brevissimae, angustar, virivis, subries toto pubescentes, auriculis subrectis; ped. 1-3, to to hispisi, 12 m. longi; tirbus ovoibens, Laexis; sep. Dorso gense setoso, pinnulis valde gentatoglaniulosis instructa, acumine foliaces, laciniats; styli glabri, disco plano; coi. parva, eciliata, fruct. ovvisess, basi subroturnatus, apice sensim attenuat. Has. Hispanix orient, Daiencio: at Barranco ge Cabrera prope Gegorbe (C. Pour).

2255. Crepinia sideralis Gigr mss.

tcuchi conformes, sot brevas, folcato-Silatati, at ramos flor. Sparsi; fol amplissime obovats -bblonga, subacuta, basi attemuata, coriacea, supra glabra intenseque viritia, subtus toto villosa, virescontia, sat nersosa et is costam eglanisulosa; Dentes late tirangulari-convergentes, inf. biserrate, pet. villosi, aculacti, parce olanoulosi, stip. virites, longac, latwe, tantum apice Sersi pubescentes, auriculis latis, subivergentibus, pes. 1-3, dense hispiidi, 6-g.m. longi, tufus ovoidcis, in $1 / 3$ inf setosus; sep. Dorso glanidulosa, latiuscule pimato-dentata; ityli lanate, Disco plano; cor............ fruct sat late ovoideus, turgians, utringiue attenuartus.

Hab. Hungaria, ag. Bubakersierso (to. Steffeck). 
$-365-$

2256. Gepinia incerta (Déségl. Cat.p. 215), Gogr \&ab. N-2765.

toulei falcato-Silatati; fol ovato-alliptica, supra glabra, nervosa; Destes profunivi, inf biserracti; pet. villosi, inf inermes, stip. angustae, glabrae, auriculs givergentibus; peod 1-4, parce glanoulosi; sep. Sorso glandulass, pinnulis onnibus latis instructa; styli hirsuti; cor........; fruct. oroideus. Hoob. Anghia, in oyerkshire (Y. G. Maker).

c. Fructus oblongus.

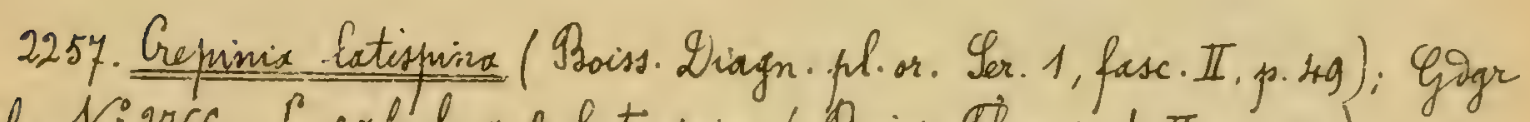

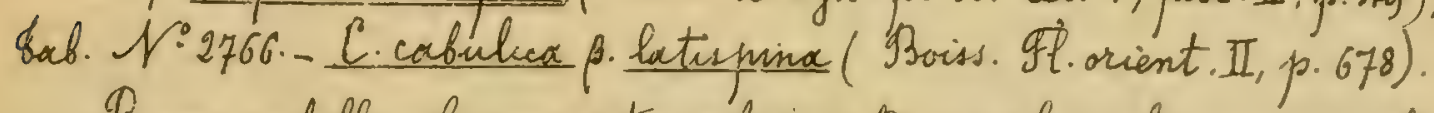

Tarni rubello-glacescentes; alir suboimarphi, alii majores, latissime Dilatato-falcato, aliu minores, pauciores, subsilatati vixque incurvi; fol. parra, ovato - subrotusiata, approsimato, witrinque tomentella; gentes iviangulares, inf biserrati; pet. Tomentosi, aculeati, stip. anguste, pilosuibe,

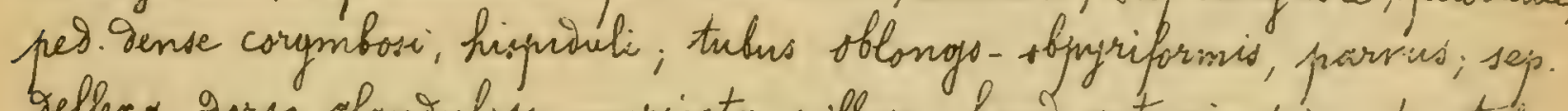

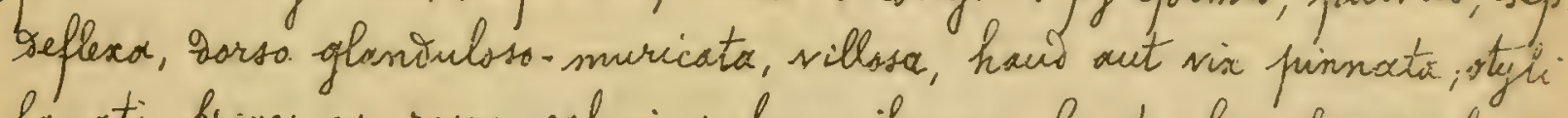
lanati, breves; cor. rosea, calyci subaequilonga; fruct. obpyriformis, basi subrotuniatus, apice attematio-strangulatus, setulosus. Hob. Cabul, in alpinis prope Kaloo (Griffith).

2258. Quepinia seelanicia Gogr mos.

Atculer sat breves, falcato-Dilatati, aid ramos flor. copissi; fol sat late elliptico-subacuta, basi rotuniata, supera glabra, subtus as nerros villosa, ao costam eglanialosor, Dientes late aperto - triangulares, inf pailo biserrati, pet. tomentosi, parce aculeati, hinc inse glaniulosi; stip. mugnace, virides, gorso pubescentes, auriculis givergentibus; per. 1-2, hisfiati, 6-8 m. longi; tubus oblsngus, sparse aculeatis; sej. gorso aculeslate, pirnulis onnibis integerrimis, latis instructor; styli lanati, gisco subplesis; cor........ f fred: sblongus, utringue attenuatus, apice longie contractus.

Hob. Lamia, Leeland: Wo Hellebelk (7. to. Gobrieloson).

2259. Prepinim prassiva Gogr mss.

toculer brevisism, parum falcato. Silatati, as rumos for parciores; fol. parwa, obovato-subainta, basi paulo attemuata, supro glabra', locteque sinisia, subtus-cervose, tots villose et ad costam parcissine ylaniaulosex; 
ientes valie profunis; lanceslato - recii, fere annes fiserrati, fret. tomantosi, sculeati, sat glaninlosi; stip. Grercissimae, sat lutae, vivides, sorst villosae, ouriculis rectis; ped. 1-2, toto hippidi, 10 m. Eorgi; tubus sblangus, laevis. sep. Forso glaniulosa, pinnulis onnibus sat andestis, Sentatis instructer. styli villosi, gises plano; cor........., fruct. anguste oblongus, istrinque longe attenuatus.

Hab. Hispania arient, Yelencix: as Yegorbe (C. Sau).

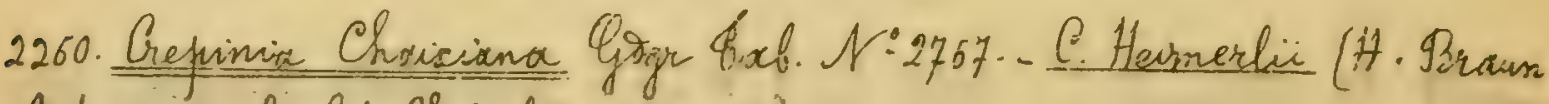
in tect. soe zool. bot. Vingol. ans. 1885).

tberlei dilatati, parum falcati, is ramos flor. sat copisse; fot obrats-acuta, basi attenuata, viridi-subglinca, siuperne ylabra, subins prasiertion as nervos villisa et as costam eglarisulos; sentes lanceslato-convergentes, fere ommes biserrati; pet. villosi, sparse glanisulssi, inf.inermes; -2 ip. viricides, subtus pubescentes, auriculis aivergentilus, ped. 1-2, 1 parse of tonsulosi, 7 -gm longi; tubus oblongus, to to glandulosus; sep. sorso glansulosa, pinnulis hinearibus, 3-4 Dentatis instructa, acumine filiformi, integro; sty li hisutis-

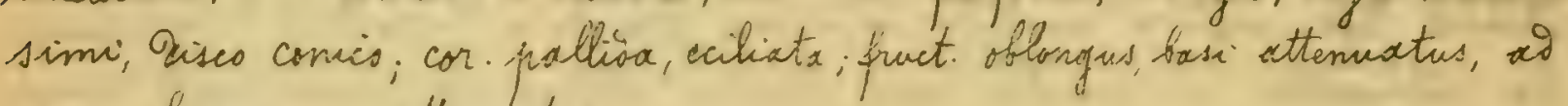
apriem longissine attemiatus.

Hab. Gasses - thes, at La Consamine (Proal); - Hungaria (H. Droun).

2261. Creininia stictosepala (Gogr Essai p. 33 nomen solum; Gogr 路os nor. II. p.32). Gage Bab. M.2768.

Aculi panciores, falcato silatati, as ramos flor. sappe nulli; fol. oboratooblonga, uininque sensim attenuata, supra sparse pilosa, subtus toto villosa et as costam eglaniulosa; Dentes aperti, inf biserrati, pet. tenuiter qlaniulosi, inermes, villosi; stip. pubescentes, auriculis rectiusculis; peo. 1-3, Gispivic, 10-15 m. longi; tubus ollongus, tots et minute glanaulosus; sep seciour, elangata, gorss glaniulosx, pinnulis parce gentato-glanivulosis intructa; styli lanati, Sisco subconico; cor........ fruct. Blongus, basi gepressus, apice solde attenuatus.

Hab. Iavore, ad Salins prope Moñties in colle Maraivis (Muget).

2261. Crepinia niaiulula (Lagger et Puget) in Goger Qab. N:2769.

Aculei Debiles, recti sel inclinati, \& ramos flor. panciores, fol oberato, utringue sensim attenunta, supra hirtella ac intense virioia. subtus glau- 


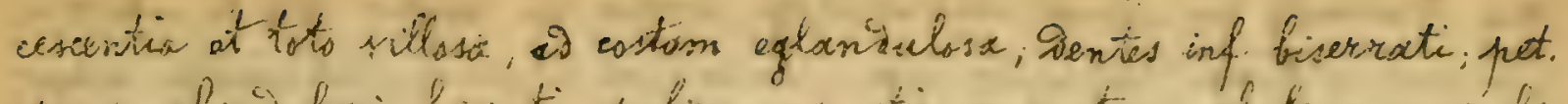

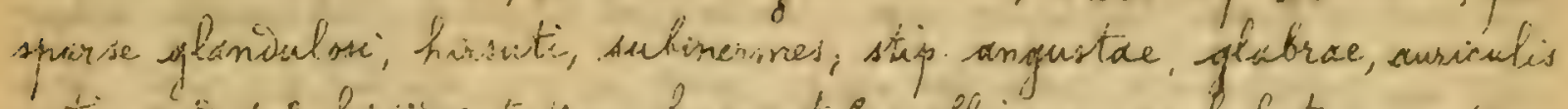

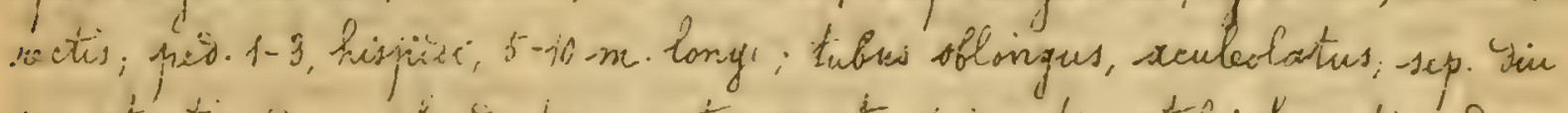

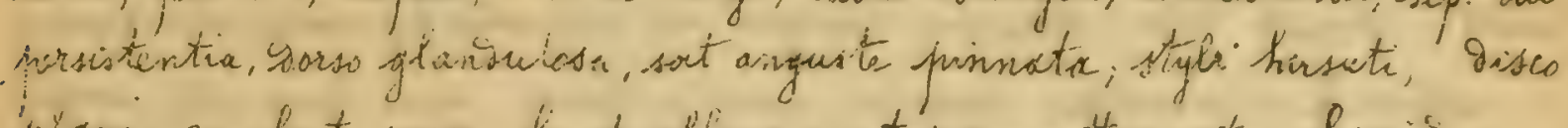

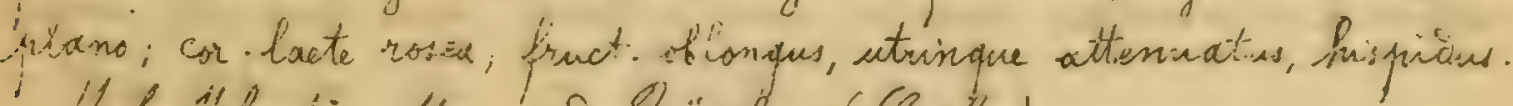

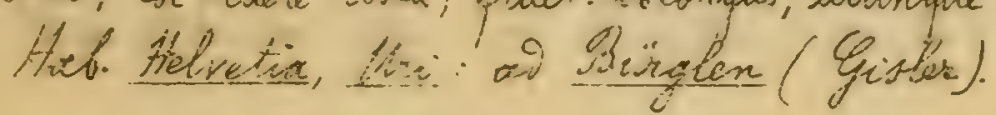

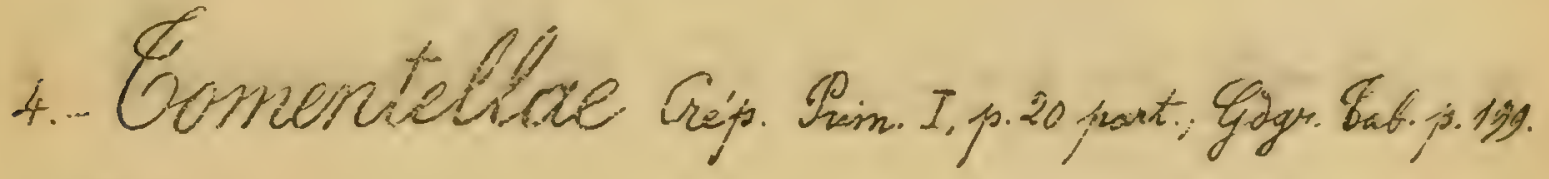

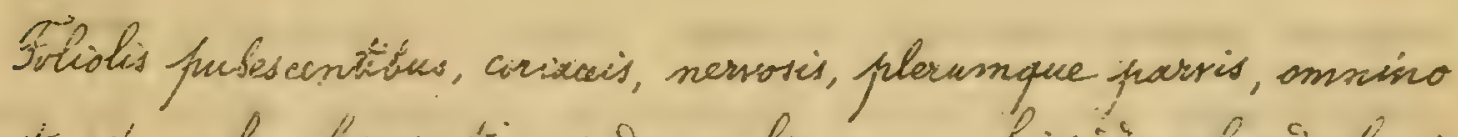

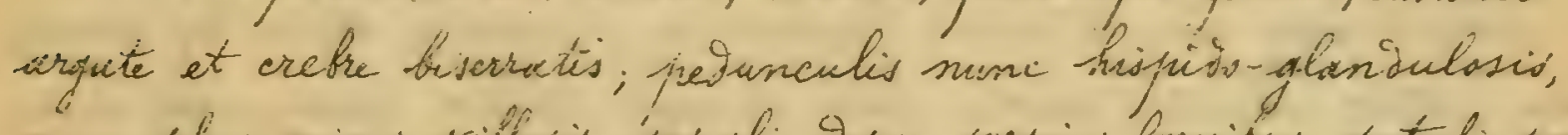

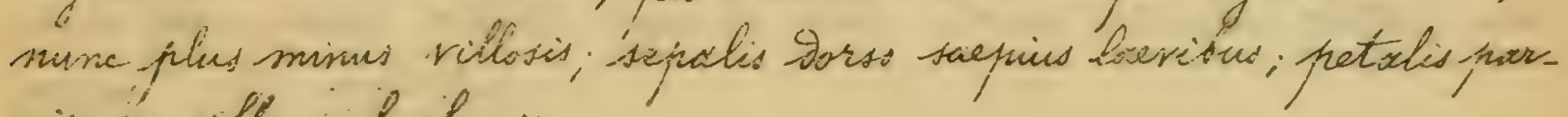
ris, atb ochromeis.

Pedunculi normaliter et gense glandulosi

1 Sosunculi glabri, villosi, vel 1-4-glaniulosi (grex C. trmentellac Lem.) iruectus alobosus val rotunisarios

$\int$ Gruetirs ovatus

$2\{$ Fruetus avoideus val obovato-sblongus

Fruct. sflongus

Qpesies N-2263-2265.

Shevies N.2256-2261)

Specus $12270-2276$.

Yrecies N.2277-2282. Foliola ovato-rotunsata vel ovata

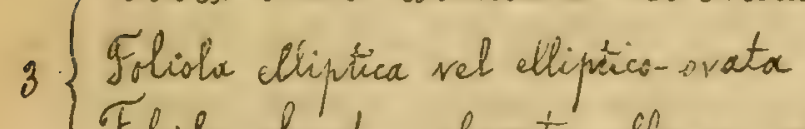

Foliola oborata, obovato-oblanga vel oblanga

Sruct globosus vel ovato-rotunsatus

4 Fruct ovatus

Fruct. oroidens

Fruct. ovoitens vel obovato-oblasgus

5 Fruet. globosus, rotuniatu, ovato-rotuniatus vel ovatus Setioli anmes aculeati

6 Tetioli inf. inermes

Irvetus ovoiseus, vel obovato-oblongus

Fructus rotundatus vel globosus

7 Fructus ovato-rotuniatus, ovatus vel ellipiticus

Fructus oblongus

Grecies N:2283-2287.

Inecies N-2288-2290.

Lpecies N:2291-2297.

Yrecies N-2298-2303.

grecies N-2304-2307.

Ipries 1:2308-2312.

Inecies $\mathcal{N} 2313-2316$. 9precies N:2317-2322. Grecies N-2337-2342. 
$-368 \ldots$

Thili glabs: vel paulo villosi

8 SOEthli hirsuti vel' lanati

Qjecies N:2323-2329.

Inecies N:2330-2336.

1. Peouncali normaliter et iense glantulosi:

a. Fructus globosus vel rotumiactus.

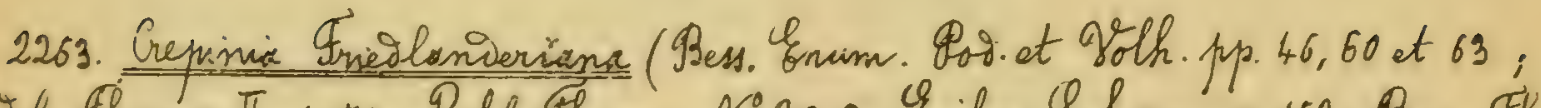

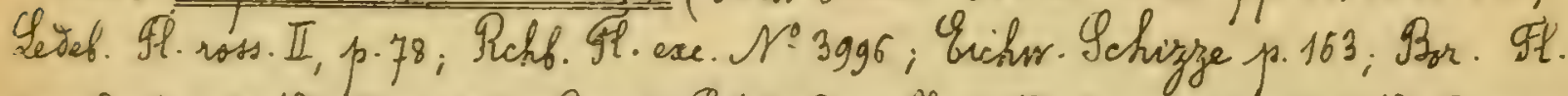

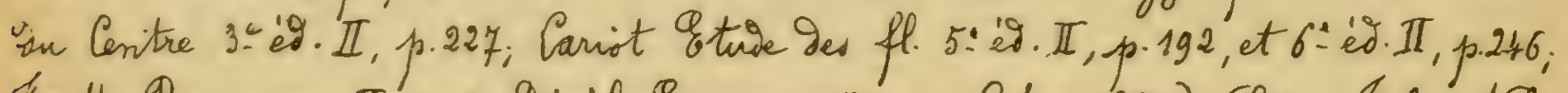

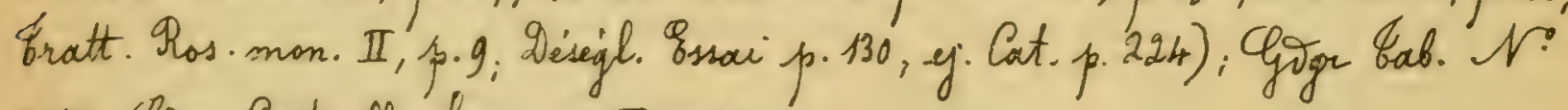
2770; CGogr Conte. Pl. Slar. mer. II, p. 4.5.

toculei conformes, faleato-Silatati, fol orato-subrotuniata, firma, superne glabrescentia satwrateque virisia, subtus toto villosa et ad costam glanioulosa: Sentes argute biserrati, pet glaniouloso-pubescentes, aculeati; stip. subtus villosae, sorso at marginibus ghenimlosar; per. hispivis; tubus subrotimoatus,

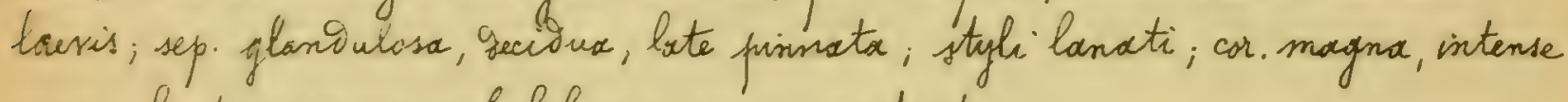
rosea; fruct magnus, subglobosus, apice censtrictus.

Has. Rossia merid-accio, Dacia, etc.

2251. Crepinia arguta (Muss- Pusshk. in M6. Breb. Fl. Taur-canc. III, p. 348; Ipreng. Gyst. II, p. 549; Crep. Prim. I, p. 194), Gogr Bah. N: 2771.

toculei corformes, subfalcati, parum Dilatati, fere geminati; fol. ovato - elliptica, ineviscria, supra glabia vel glabriuscula, subtus à nerros villosa; gentes biserrati, pet- seuleati, pare glanoulosi, inf villasi, sup. glabrescontes, stip.

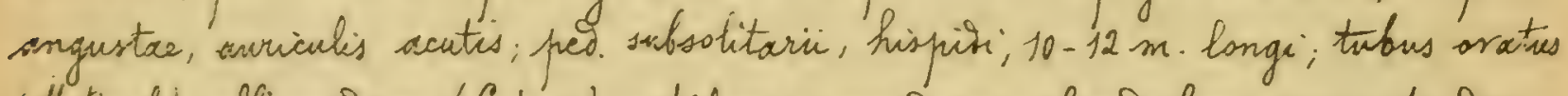

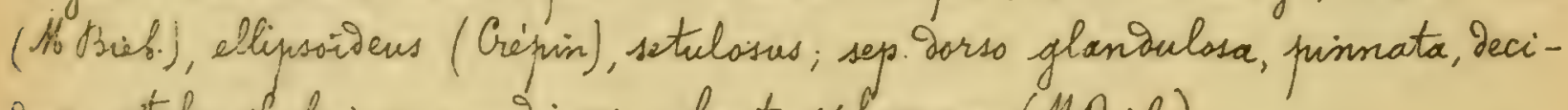

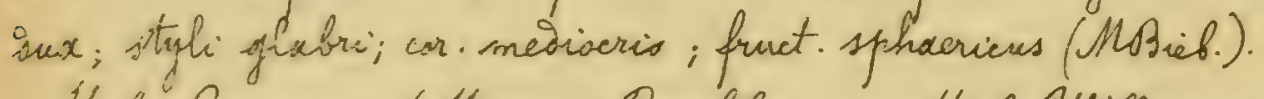

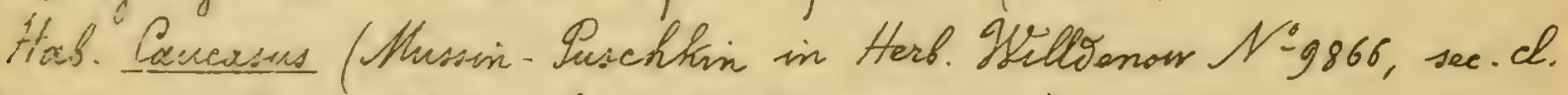
Secfion copjes Tescriptione, laco cit, abjutus sum).

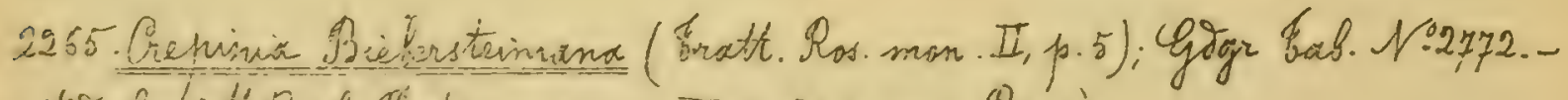

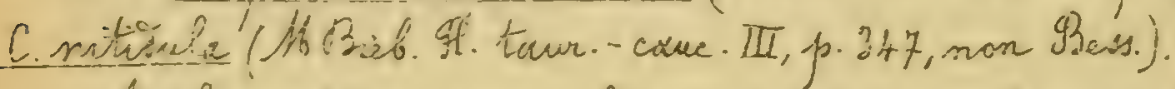

Acculei valias, spersi, faliato-gilatati, conformes; fol mesioeria, elliptico. sonta, acuta, laste virioix, sitsinque glabiniscula, sentes argute biserrate,

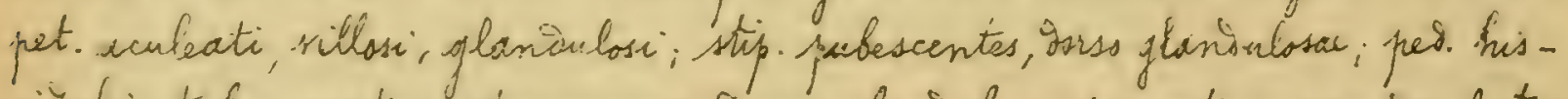

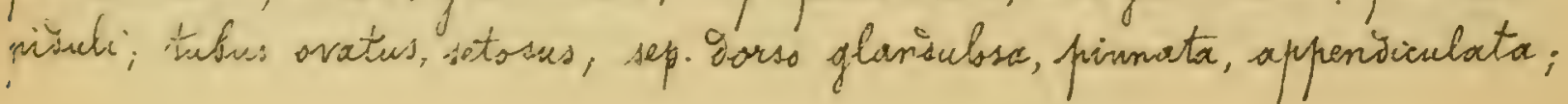


$-369-$

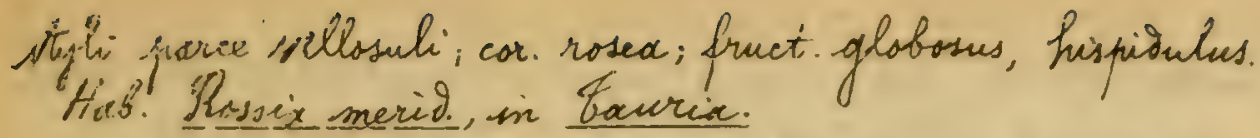

7." Disegitise Cat.p.253 at Chaviniam leveantham/Lois, sect. GlanSulosece situct.) pessime relata.

6. Fructus ovatus.

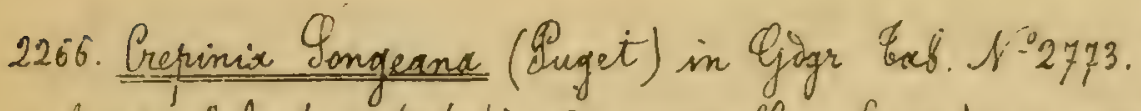

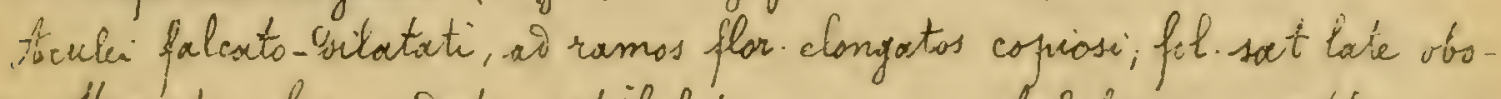

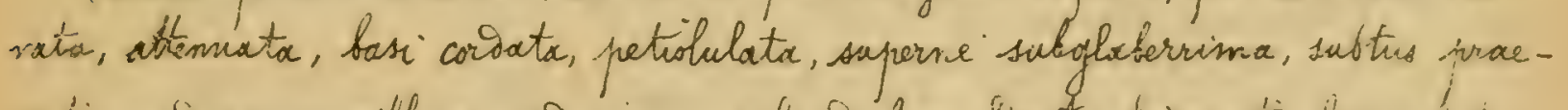

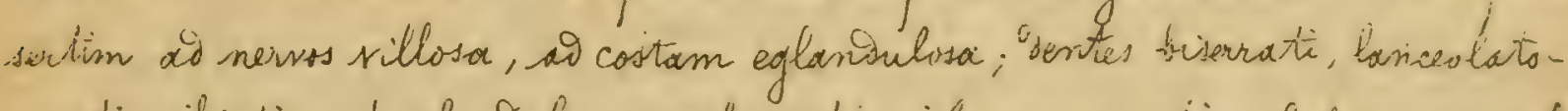
presti, ciliate; pet glaninulosi; putescentes, infinermes; stip. glaborxe, auriculis

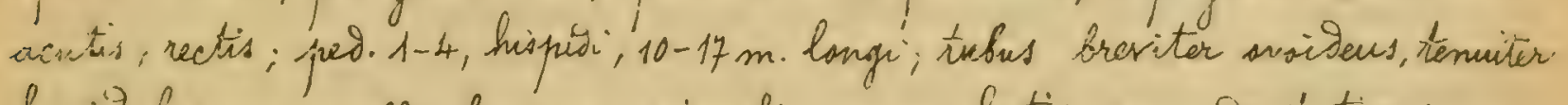
hispitulus; sep. corolla bieviora, pinnulis parum latis, parce crentatis instructa; styh hirsute, gisco conico; con. rosea; fruct. plorumpue oratus.s., Has. Gavori, at Jacob prope Chambéry (Mongeon).

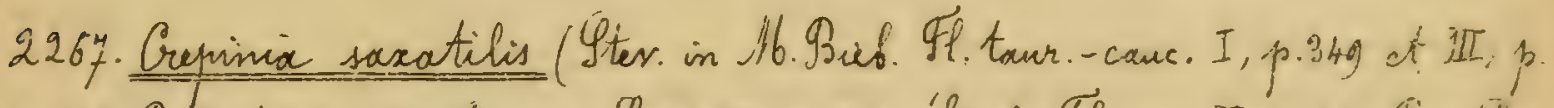

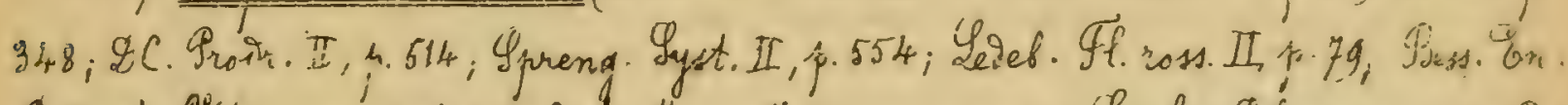

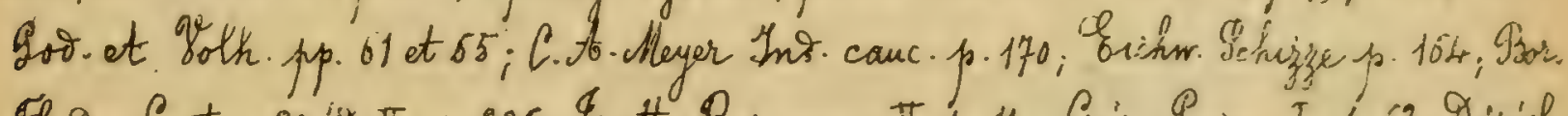

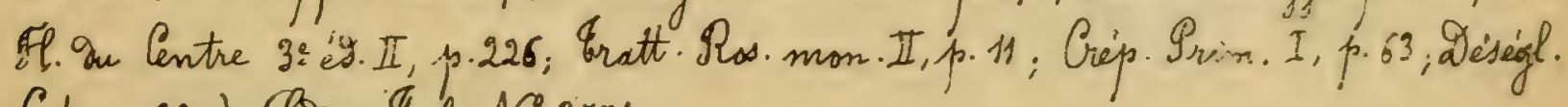
Cat. p. 225); Googr \&ab. N.2774.

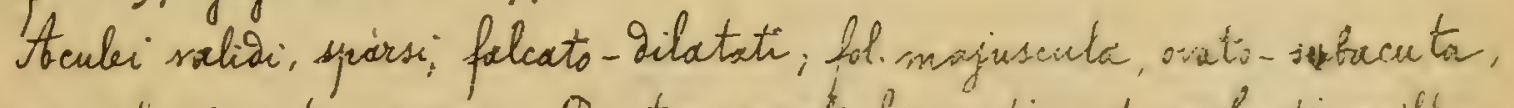
utringue $g^{\prime}$ inscula, nervosa, Pentes argute hierratis; pet aculeati, villosi,

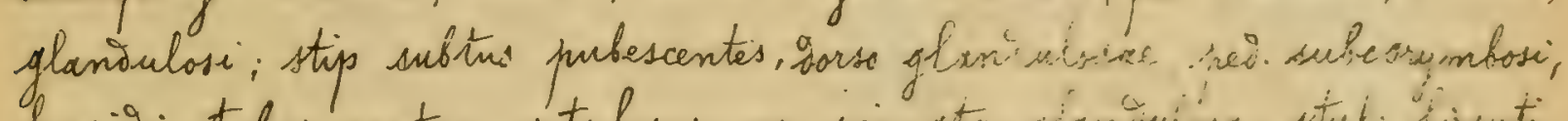

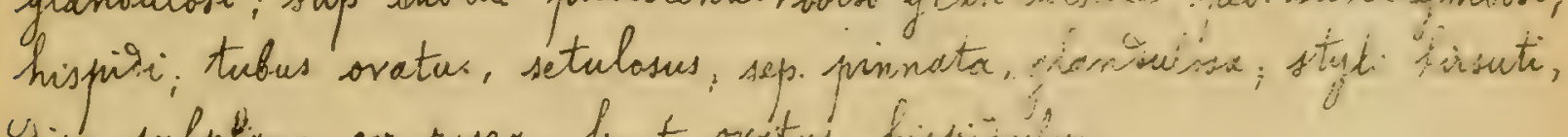

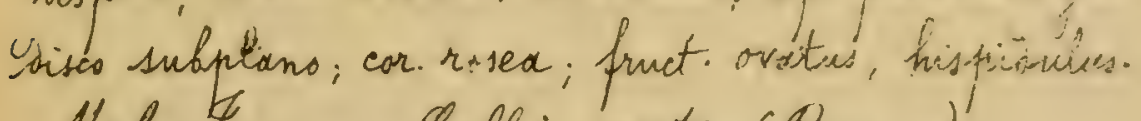

Hab. Eaurix; Gallix centr. (Moreau).'

Deveriftis Soraci cum illa Htevenui optime congruit, num autom. eximen sit planta, gubium.

2268. Crepinia macrantha (Desportes Fi. De la Sarthe p. 77 ; Gren. et Goor.

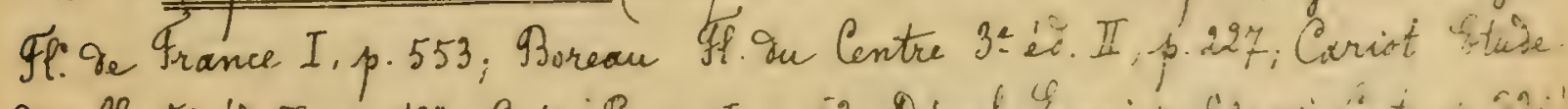

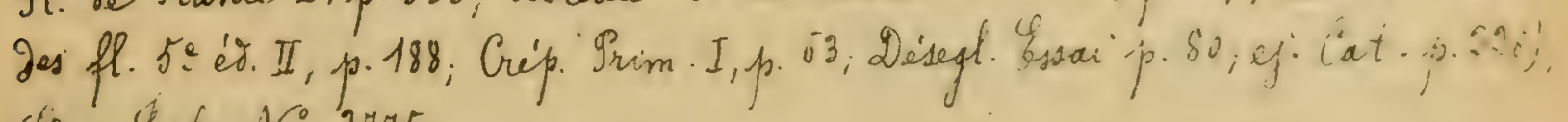
Gogr \&ab. $\mathcal{H}: 2775$

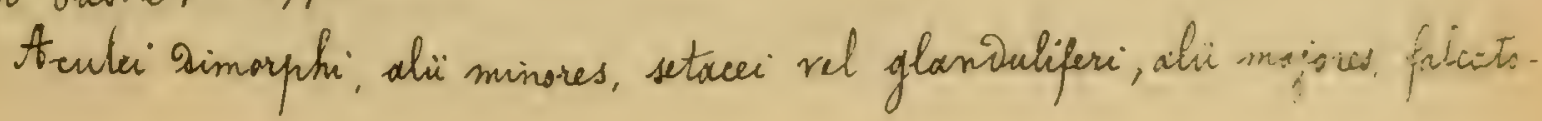


$-370-$

Dilatati; fol ovato-acutiuscula, basi subatternuata, nitita, subtus tantum ai costam planiulosam villosa, sentes crebre biserrati; pert. glaniulosi; puhescentes, rculeati; stip. Dorso glaniulosae, auriculis acutis; pes. 2-4, hispiati; itubus ovatus, laeris, sep. Decisua, pinnata, Dorso glanivubsa: styli hirsutit: cor. magna, intense nosea; fruct magnus, oratus.

Hab. Gallia centr., ad Garthe, Mthone, etc.

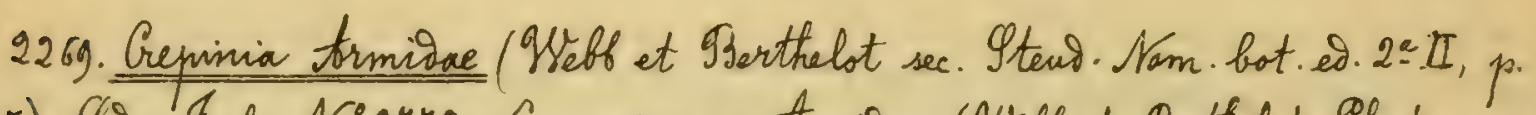
465): Gagr bab. N:2776. C.camina y. Armidar (Mebb et Berthelot Thyt. canar. II, p. 16)... Mourgean SR canat. N-352.

Acculei conformes, falcato - Dilatati, aid ramos flor. nulli; fol. late ovatorotumiata, coriacea, unioigue villosa; dentes profunovi, argutissine biserrati; pet. Tamentossi, aculeati, stip. subtus molliter pubescentes; pret. hispicic; trbus svatis, hispious, sep. Secioura, pinnata; iglan Sulosa, styli ignoti, cor. rosea; fruct. ovatis, magnus, pulposus.

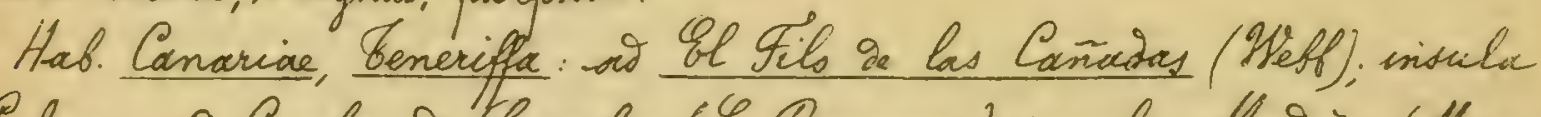
Salma, al Cumbre de Garahia (G. Banrgeau); insula Madere (Noxson).

c. Fructus ovoideres vel obovato-oblongus.

2270. Grenimia obodonskiensis (Gogr ossai p. 33); Giogr Exb. N-2777- - C.

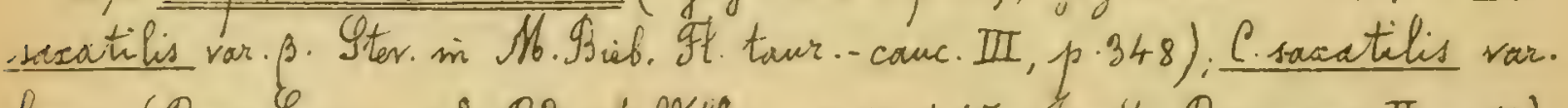

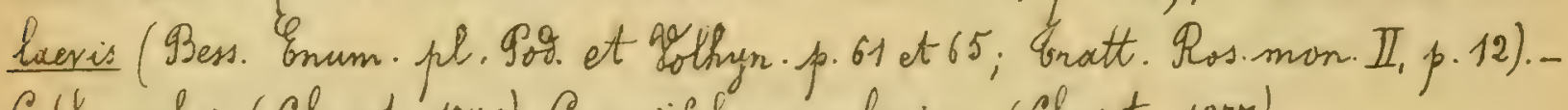
CEereschii (Christ, 1877). C corifolia $x$ alpina (Christ, 1877).

Acculei grimorphi, ahï majoires, falcato-oilatati, alii tennes, stipulares ac setacer; fol. elliptica vel ovato-elliptica, acuminata, basi atternuata, superne glabra, subtus glaucescentia et ad nervos villosia; Dentes argute biserrati; pet. aculeati, villosi; stip. Dentato-glaniulosac, auriculis lanceolatis, ped. subhisfigi, tubus oborato - oblongus, laevis, sep. Dorso glaniulosa, pinnulis inciso-sen tatis instructa, styli hirsutic, cor. sat parva, rosea, calyce brevior, frect. sbovato-oblongus, laeris.

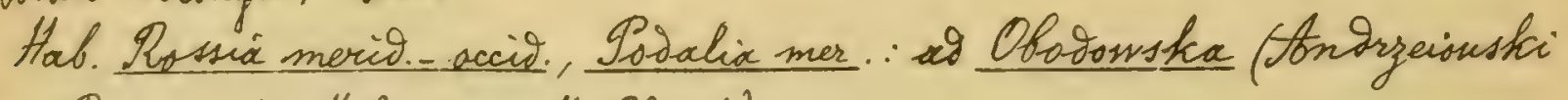
in Pess. l.e.)i Helvetia (H. Christ).

2271. Gepuinix Kabothi (tonsarge) in Gogr Gab. N=2778.

Acculei Dimorphi, recti, tennes, ar ramos flor. setaceo-glanguliferi, fol. 
ovorato-acutiuscula, basi rotuniata, intense virisia, supra tenuiter villosa. subtus ad nervos hirsuta st io costam glansulosa, ientes fiserrati, aperto. triangulares; pet. Nillosi, aculeati, minutissime glasidulosi; stip rubentes, "vorso glansulcsae, auriculis givergentibus; pet. 1, hispisix; $11-13 \mathrm{~m}$. Long; tubus ofovato-oblongus, sparse setosus; sep. Oorso glanduloser, puinnulis angustis, valde jentato-glandulosis instructa, acumine integrs, parum lato; styli villosi, sisco plano; cor-rosea, valise ciliata; fruct, obovato-oblongus, fasi rotinioxhist, aprie longe attenuatus.

\section{Hab. Silesix, at Mansern prope Breshue (Ansonge! Kaboth!).}

2272. Crepinia Nrausiu Goor Gab. N-2779. C.cominix yalbica. (Krause!).

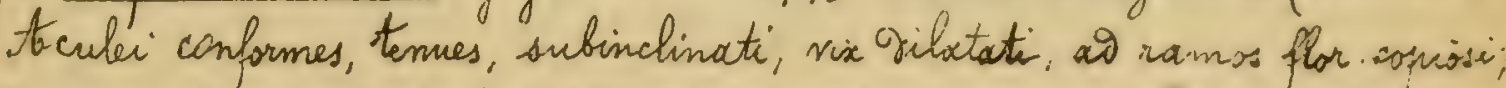
fil. oblongo-acuta, fasi subrotinivata, glancescentia, superne glabra, subtu's ad nerres villosa, et a costam glanoulosx; Dentes biserrati, aperto-triangulares; pet glangulssissinn; aculeate, villosi; stip. virides, gorso glangulosae, awriculis subivergentikus; ped. 1-2, hispidi; 5-7 m. longi; tubus ovoideus, laevis, sep. garso eglan Dulosa. pinmulis latis, 4-5-9entatis instructor, acusnine integro, longo; styti villosi, gisco plano; cor. rosea, inferne copiose ciliata; fruct. ovoidens, utingue astemuatus.

Hab. Iilevia, wo Carlowitz prope Breshou (Kranse; Ansorge).

\section{Crepinia rufioula Gogr Gab. N-2780}

Acculei breves, parri, silatati, parum faleati, wo ramos flor sat copiosi, foll late suboblongo-acuta, basi abrupte attenuata, undigue as. presse hirsuta, as costam eglanguloso; Dentes biserrati, lanceolato-aperti; pet. temiter glaniulosi, pubescentes, aculeati, stip. rubentes, eglangulosi, auriculis subrectis; peg-1, parce hispisi, 11-14 m.longi; tubus svoideus, lacris, sep. mufescentia, gorso glandulosa, pinnulis angustis, 3-5-gentato-glandahisis instructa, acumine foliaces, gentato; styli hirsuti, aiseo plano; cor. rosea, inferne ciliata; funct. Ovoidens, glancescens, utringue attenuatus.

Hab. Seotia, Gerthshere: ad Dunkeld (Drummont-Hay; Istrite).

2774. Crepinia reperta Gogr mss.

Ficulei Debiles, nic Dilatati, teretes, inchinati, as ramos flor. graciles rare fol sat para, suboblongo-acuta, basi atternuata, coriacea, superne glabra ae glaucescentia, subtus albioa, tantum ad costorm mediam paulo glanou- 
$-3,72-$

Cosan villosz, nervulosa; Gentes fiserrati. lanceslato-recti; put. tomentosi,

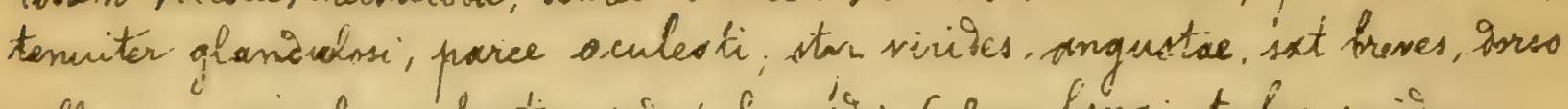

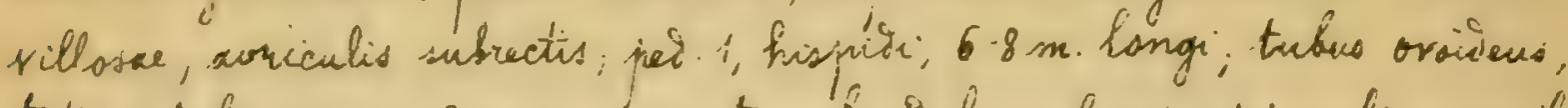

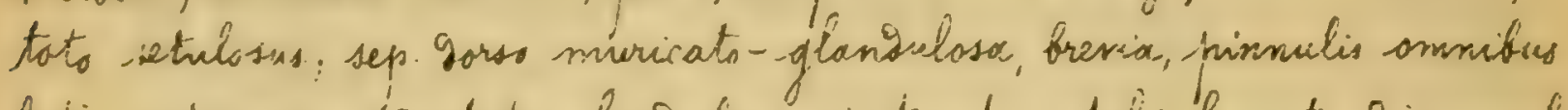

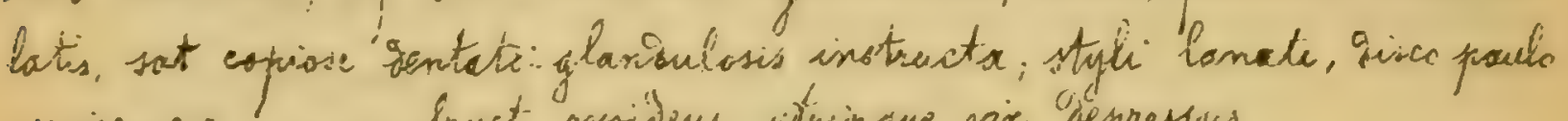

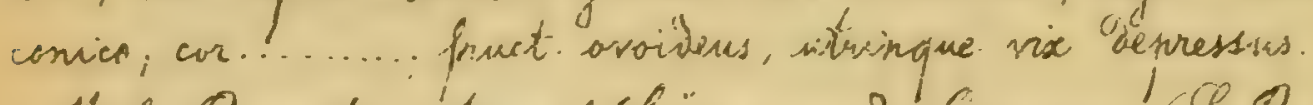

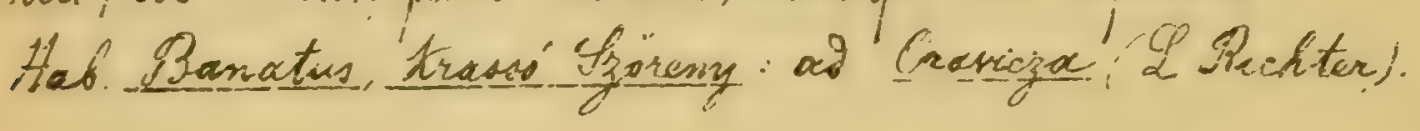

2275. Crepinia noijenensis Giagr mss.

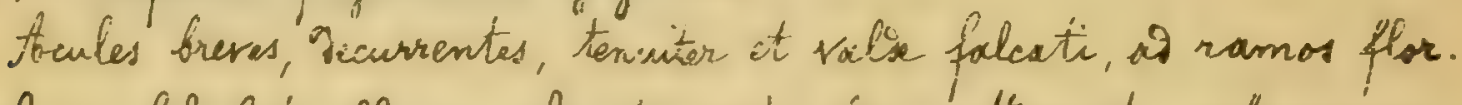
stipualares; fol late oblenga, abrupte acuta, basi attemuata, subcoriacera,

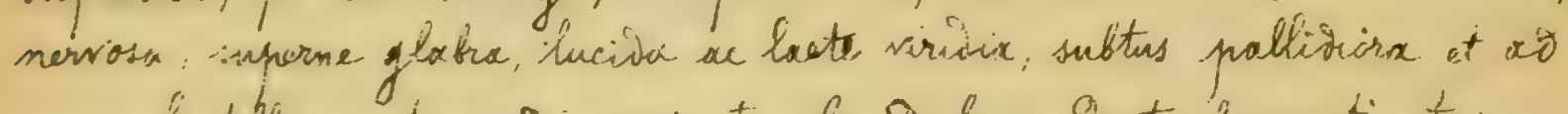

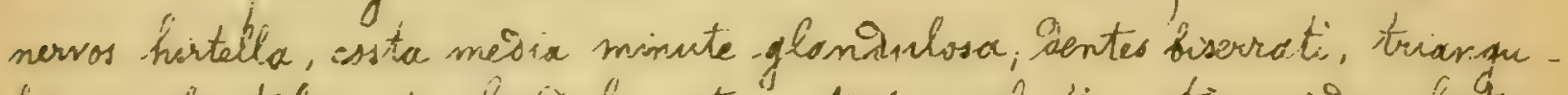

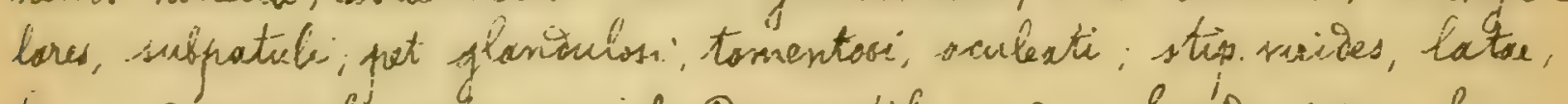

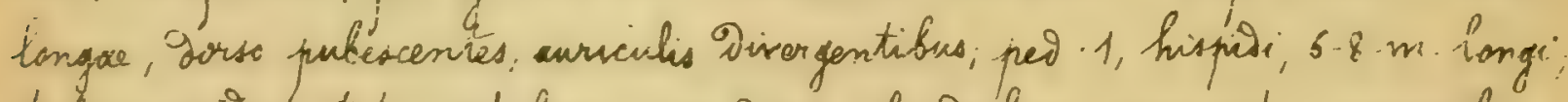
tubus ovo-dens, tots setulosus; sep gorso glantuloso-muricata, pismeclis armibus hates sat copiose Dentacto-glanioulosis instructa, sty'ti lunati, inses plano; cor......... fruct. ovöiens, basi abrupte gecurrens, apuic tergianlus ree- atternutiti.

Hab. Sunatú, Krasso' Söreny: as Sojena, Marilla (2. Richter).

2276. Gepinia champosticta Gyogi Gab. No2781.

toculei coublenares valisi, falcato-gilatati, copiosi, ad ramos flor minores, subrecti. fo. thovato-sblonga, utrinque paulation attemuata, sapins supra glabia, subtus is nerros villosa, et as costam paulo glanivulosa, gentes suerrati, breves, recti; pet-glonsulosi, hirsuti, aculeati; stip. latac, Gorso glanisulosar, auriculis patulis; ped 1-3, vix glanialosi, 5-15 m longi; tubus vbovato-oblongus, lavis, sep. gorso ipso eglanialoss, pimulis cosiosissime sentatis sel sermulato-glanoulosis abunse instructa; styli hirsuti, gisco sat corvic; cor rosea, fruct parwus, sbovatus, utrinque Depressus.

Hab. Hante-Savoie, is It Martion (Suget).

d. Fructus sblongus.

2277 Crepinia murcuca Giagr bab. N:2782.

Rubens, rami elongati, flexussi, arcuati, aculer foliato-Dilatati, ad 
$-393 . . .-$

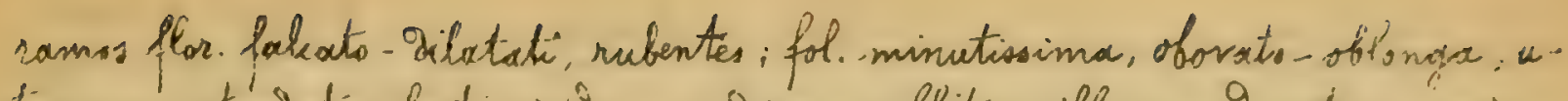

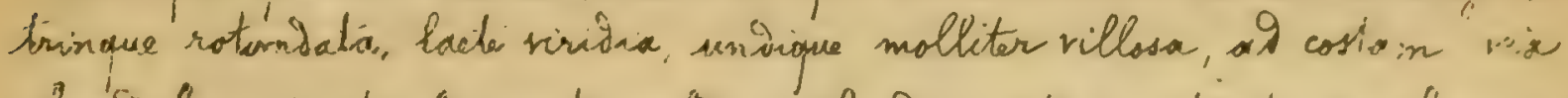

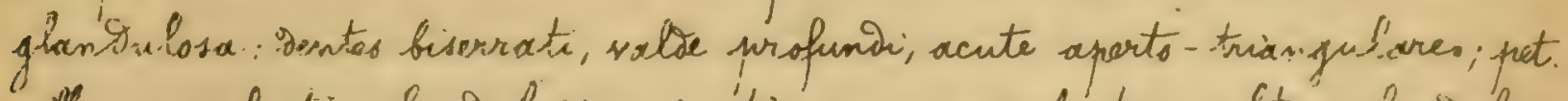

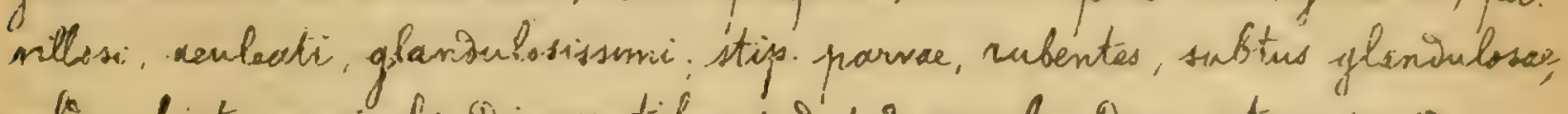

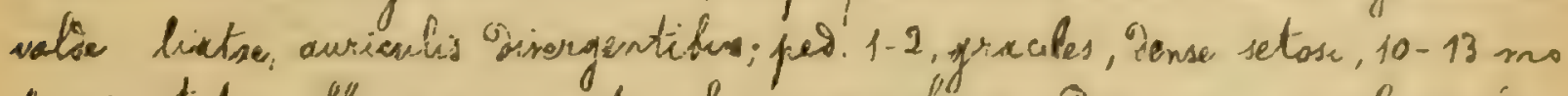

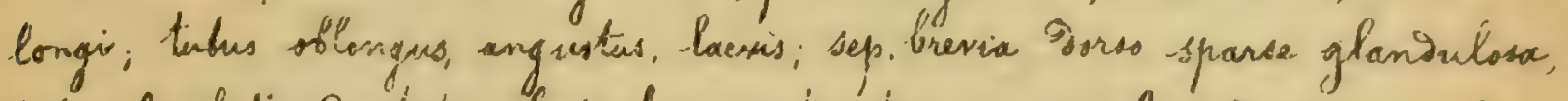
prinmalis latis dontait gfanialosis instructa, acumine lato, gentats, consllam

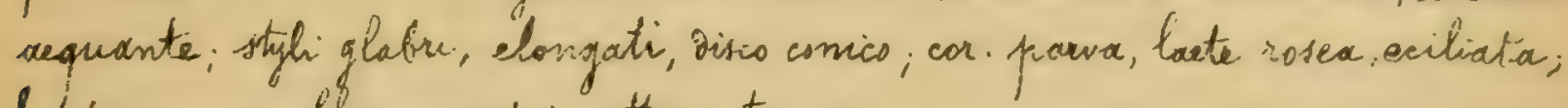

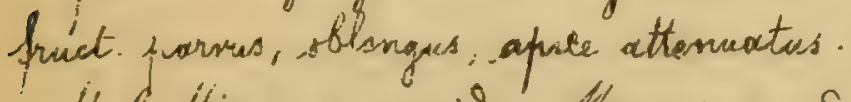

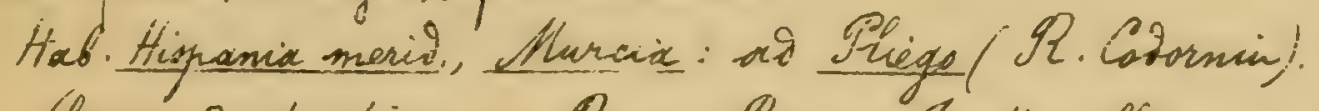

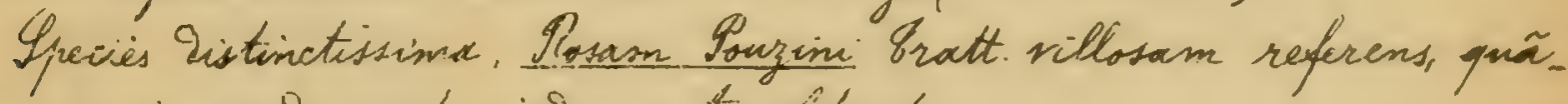
cum confingen 9 asset, inoumento of tainte.

2278. Crepinix Decalwans G.9gr mus.

Acule longi, tenaitir falrati, parum gilotati, as rarnos flor. nulli, fot. parva, onorate-subacuta, basi rotunsata, superne glabea ac virix-ylaucescentia, subtus to to villosa et so costam glangiclosa; Dentes bierrati, profunde. tanceslati-aperti, pat rubentés, glanoulasi, villosi, parce xculeati; stip.

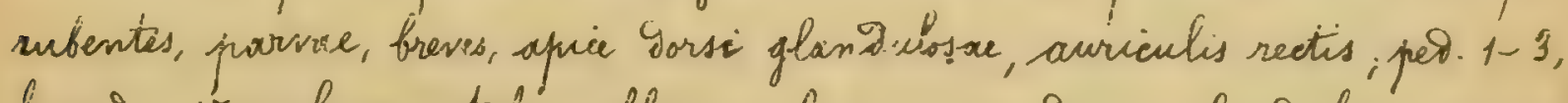
hispigi, $15 \mathrm{~m}$. longi; tubus oblonqus, heenis; sep. Dorso eglanoulosa, pionulis ammibus foliace- Dentatis instructa, styli glabri, Disco subplons; cor. rosex, mesiscris, eciliata; fruct oblangus, basi gepressus, apice longius attenuaters. Hab. Hispania orient, gelencriv: av Legorbe (C. Sau).

Affinis procedentis, ut at sequens, faciem etiam Itosoce onyini Iratt. reperens, sei foliola villosó.

2279. Crepinia eminens Gogr mss.

treculei longi, inclinati, frarum Dilatati, as ramos por stipulares; fol. sat pawa, elliptica, etrinque subrotuniata, supra hirta ac intense viridia, subtus toto villoso-grisescentia, al costam eglandulosa; Dentes biserrati, ovato-aperti; pet glandulosi, tomentosi, aculeati, ililute rubentes; stip.breres, latae, subtus pubescentes et gorso glangulosar, auriculis rectis; ped. 2-5, tots hispisi, 12 m. longi; tubus sflangus, lovis; sep. Torso eglandulosa, rubra, pinnulis ammibes latis, gentato-glaniulosis isstructa, acumine foliaces; Atyli hirsuti, gises paulo conics; cor pallide rosea, sciliata, mediocrir; fract. oblongus, utrinque attonuatus.

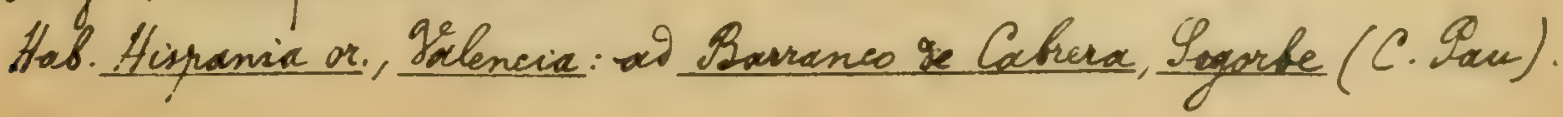


$-374-$

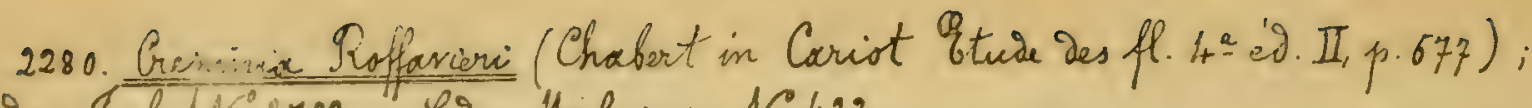
Gogr vab. N.2783. - Gogr Herb. ros. N. 423.

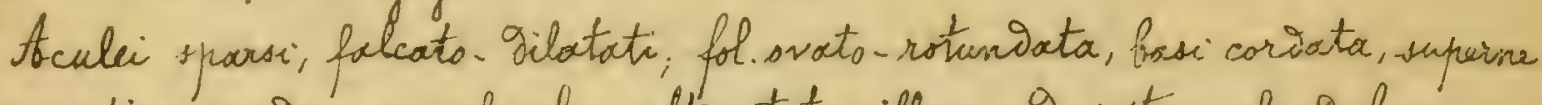
atrovirentia ac appresse pilosula, subtas toto sillosa, ob costam glanisulasa. gentes argute biserrato, pet. glandulosi, sillosi, aculecuti, stip. subtus pubescentes, auriculis rectis, lancealatis; pes. hispidi; tubus oblongus, loveris; sep. Fecidua, pinmulis dentats-glandulasis instructa; styli villasi, gisco plano, cor-majuscula, laete rosea; fruct oblongus, laevis.

Hab. Mhone, at Franckenille, Pont d' thai (P. Chabert).

2281. Chapinia orawiogensis Gogr mss.

teculei breves, patri, falcato-Dilatate, ai ramos flor.copiosissini; fol sat late oblongo-lanceslata, basi breviter wttenuato, supra glabra ac virioi-glaucescentia, subtus fuellidula et ai costam meviam glandulosam villosa, Dentes biserrati, triangulares, subpatuli; pet. glansulosi, aculeati, tomentelli; stip. sirides, breves, latiusculac, Gorso hirtellae, aurieulis Divergentibus; pro.1-2, hispidi, 7-9m.longi; tubus oblongus, toto sparse glandulosus; sep. elongata, gorso glanaulosa, pinnulis annibus sat folixceis, 4-5-gentatis instructa. acumine longissims; styli lanati, Diseo conico; cor......... fruct. anguste oblangus, basi attenuatus, apice longe contracto-strangulatus. Hab. Manatus, Krass' Yoreny: Ot Orawioga (2. Michter).

2282. Crepinia Wienzickiana Goger mse.

Toculei Debiles, therissinni, parum falcato-gilatati, as ramos fla. plus minusve espiosi; fol. late suboflonga, tcuta, basi breviter attenuata, supra glabra ac virivi-glancescentia, subtes pallida, nervulosa, ai nervos villosula et at costam poulo glanivulosox; Dentes biserrati, frofunde sublanceslato-recti; pot-glansulosi, vculeati, tomentosi; stip. virises, longrisculac, sat latar, oorso tomentellae, auriculis givergentibus; fret. 1, hispidi, g-11 m. Longi: tubus sblongus, toto glanoulosus, sep. elongata, gorso glandoulosa, prisnulis annibus sat foliaceis, Dentatis instructa, acumine longs; styh. villaxi, Sisco canico; cor........., fruct maximus, oblangus, besi longjus attesmatus, in $1 / 3$ sup. targialuhs ac vix contractus.

Ha\&. Manatur, Krasso' Göreny: ad Marilla (2. Michter).

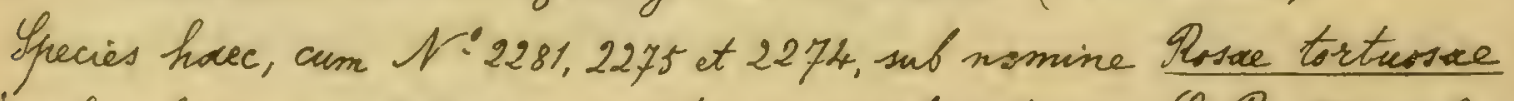
Hriengh. ab amico inaepssiosimo atopue munificentissins 2. Richter plevies accepi; sed Rosa tortuosa verx at R. onidegavensem pertinat, ciujus 
$-375-$

movem synosymon esse videtur. Chactuor illow igitur species a Mosa andegerens folishis subius plus minusve quebscentibus frimo intritu

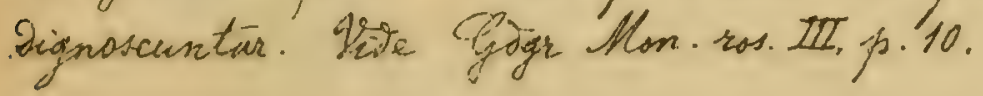

2. Sedunculi glabri, villosi vel 1-4-glandulosi.

a. Folicla ovato-rotuniata vel ovata.

+ Fructus globsus vel ovato-rotuniatus.

2283. Gepinia betusiuscula Goge Gab. N:2784.

toculei elongati, Dilatati, valis folcati, as ramos fior pauciores; fol. ovata, vitringue rotundata, sed apice soepe obtusa, supane subolaberrima, subtus to to villose, w costam eglanioulosa; gentes viserrati, aperti; pet. ghansulosi; aculeati, hirsutic; stip. auriculis givergentibus; ped. 1-3, adsperse pilosuli, g-15 m. longi; tubus oratus, sep. goroo laeria, pirnutis glabis, sat angustis, 4-6-dentatis instructa; styli pubescentes, gises pauls conico; cor-ex albo-rosea, eciliata, fruct. prarrus, ovato-rotuniatus, apuie vix attenuatus.

Hab. Rhone, at Eassin et Charbonnieres (9. Chabert). Ifecies, ut et omines seopuentes, al gregem C. Tomentellae (Lem.) portinet, nisi contrarium indicatum.

2284. Crepinia litterata Gogr Bab. N-2785. - Googr Herb. nos. N-204.

Acculai validi, late falcato-gilatati, aid ramos flor. tortuosos nulli, fol ovato-attemuata, basi roteniacta, coriacea, superne glabrata, subtus toto villosa, sape punctulata et al costam paulo glansulosa; gentes biserrati, accetic; pet, parce glanisulosi, hirsuti, soepius inermes; stip. subtus paulo pubescentes, awriculis latis, Divergentibus; ped.1-3, oisperse pilasuli, 1 cent. longi; tubus ovatus; sep. Dorso Laevia, pinnulis latis, rentatis copiose instructa; styli hirsueti, Disco subplano; cor albo-carnea; fruct.parrus, globosus, aprice attemeatus, striato-lineatus, fracecox. Hab. Thane, at Montmelas (M. Gandoger).

2285. Crepinia Pchneideri (Cottet et Puget) in Googr Gab. N:2786. Aculei parum falcato-gilatati, at ramis flor. rubellos pauciores; fol. sata, utringue rotumiata aut apice vis attemuata, corixcea, glavea, nervosa, supira glabra, subtus tanturn as navos pauls villosa; gentes 
-- $376 \cdots$

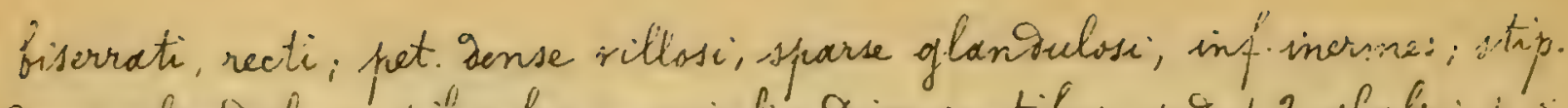

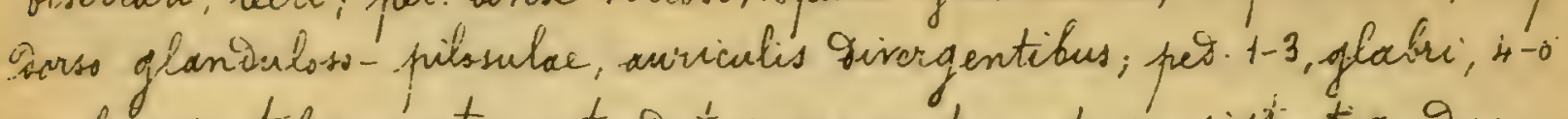
m. longi; tubus ovato-rotuniates; sep.erecta, sat pursistentia, gorss

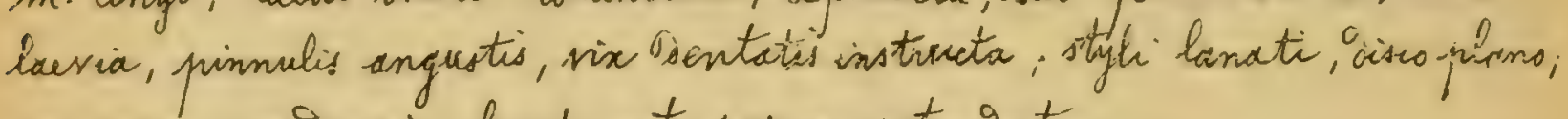
cor rosea, mevioeris, fruct. sat parwus, rotungatus.

Hab. Helvetia, Fribourg: at Hontheren (Cottet).

Hue forsen abnomis, unie melius in sect. Empubescentes Trmiglabiace collocanod foret.

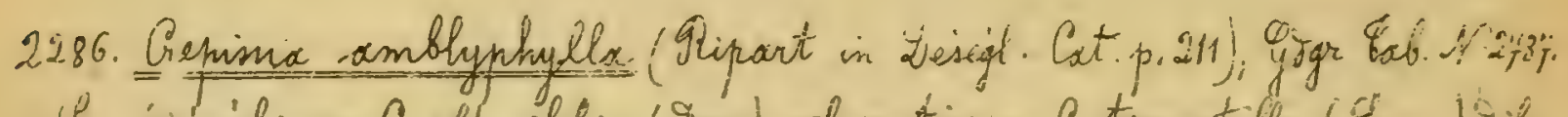

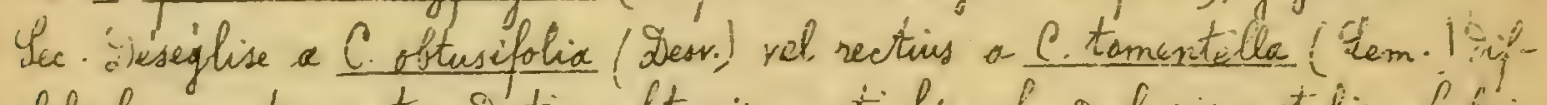

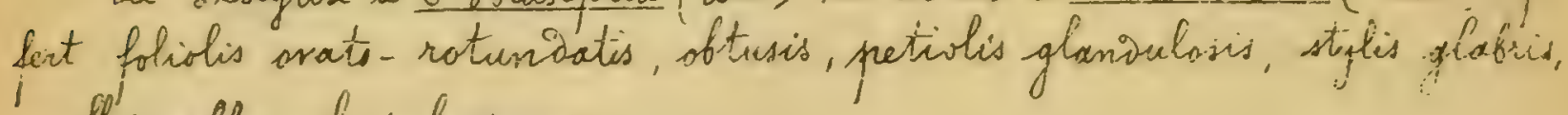
corolla alba vel subcarnea.

Hab. Gallia; Holvetia; toustia.

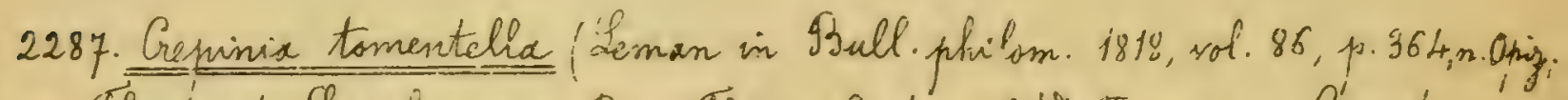

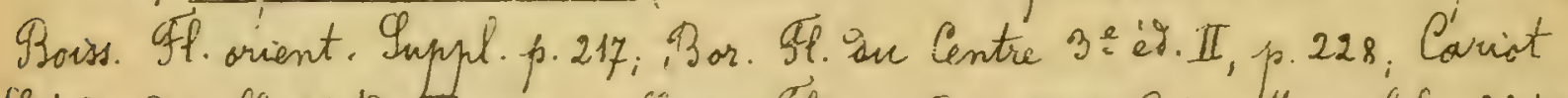

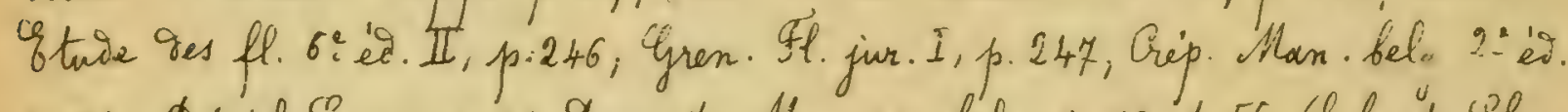
p.94; Désigl. Coss xi p. 71 ; Dhmort. Mon. ros. belg. p. 12 et 56; Schenty Thant.

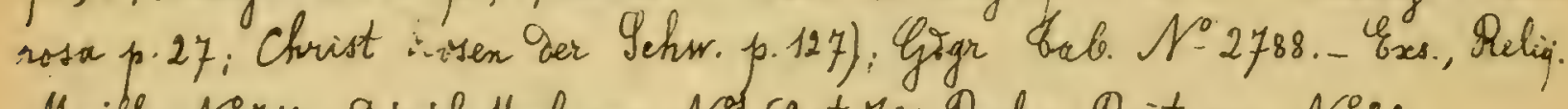
Maill. No714; Déseigl. Herb. ros. No 69 et 70; Gaker Grit. ros. No2g.

Acculei valie falcato. Silatati, plus minus copiosi; fol ovato-rotusniata, coriacea, nervosa, supra glabra, luciote atrovirentia, subtus to to villosa, et as costam glanaulosa; gentes triangulares, argute biserrati; pet. sparse glanaulosi; villosi; aculeati; stip. meviceres, subtus pubescentes; peos. pilosuli; tubus ovatus, glaber; sep. Dorso eglanoulosa, pirmulis sentatis, latis copiose instructa, decioura; styli lanati, aisco subplano; cor. piallida, vel ailiox reciliata; fruct, piarrus, rotuniatus.

Hab. Buropa plur., etiam Groecia et Caucasus (sec. Boissier). Existit quaedam Mosa tomentella. Opis, sed mishi frorsus ignota.

$+f$ Fruetus ovatus.

2288. Prepinia jemiata Gogr Eab. N-2789 - Gigr Herb. nos. N-519. Aculei faleato- vilatati, ad ramos flor mox denudatos stipulares; fol. narra, orato-acuta, base rotimdata, supra glabra intenseque vinisia, 
$-377-$

sustus toto villosa et à costam alanioulosa; ientes fiserrate; pet. sparse glaniulosi, hirsutic, aculeati; stip. Silatatio, auriculis oirergentibus; pes. 1-9, villosi, breves, tubus ovato-ellipticus; sep. brevia, prinmulis latis, Sentatis instructa; styli hirsuti, gesco sat comico; car. parra, albo-rosea; fruit. parrus, ovatus, basi rotuniatus, appice breviter attemuatus. Hab. Mhone, at Denice' (N). Ganooger).

2289. Creminia aractmoidea Gogr Gab. N-2790. - Gagre Herb. ros. N-87. fbculei valide, falcato- dilatati, so ramos flor. prouciores; fol ovata, basi notiuniata, apice fere etiam rotunisata, superne hirtello, subtus tots pilis longis, araneosis copiose instructa, Dentes biserrati; pot aculeati, villesi, glangulosi; stip. anoriculis givergentibus; ped. 3-5, paulo hirtelli; tubus ovato-ellipticus; sep. pinmulis latis, villosis, 4-6-gentairo-glanaulosis instructa, acumine foliaceo, laciniata, styli villose, exsert, gisco rubro, comic; cor achrolenca, eciliata; funct. ovatus, utrinque breviter depressus. Hab. Thoñe, at fornas (M. Ganooger).

2290. Crepinia nervosa (Crép) in Gggr Gab. N-2791.

Acculei valisi, falcato- Dilatati, fol. sat late ovato-subattenuata, basi rotumiata, crassa, coriacea, superne glabra, subtus al nerros prominulos villosa, it costam paulo glaniulosa ; tentes simplices, acuti; pet parce gianiulosi, hirsute, aculeati; stip. subvilatatar, auriculis orato. triangu-

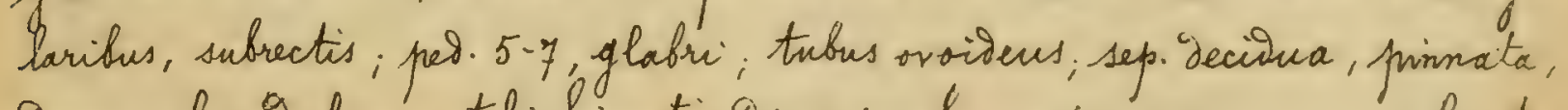

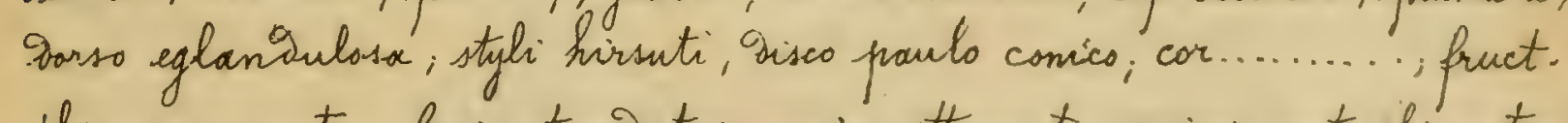
plerumque oratus, besi rotumiatus, apice attemuato-conicus, centrahi autem orrides.

Hab. toude, al Conques (Eimbal-Lagrave).

t.t Gructus ovoidens.

2291. Crepinia sunantha Gogr Eub. J':2796.

Aculei falcato - Silatati, as ramos flor. coprisis; fol ovato-obtesa.

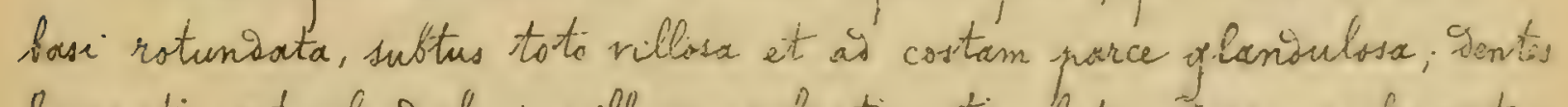

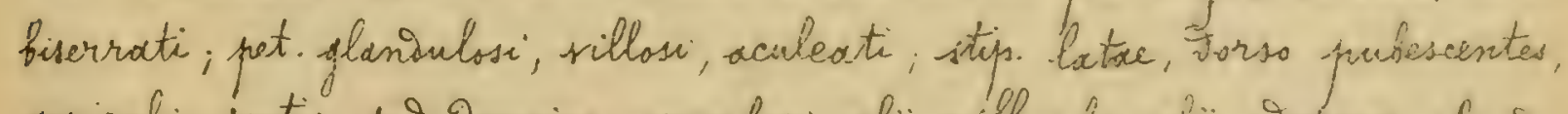

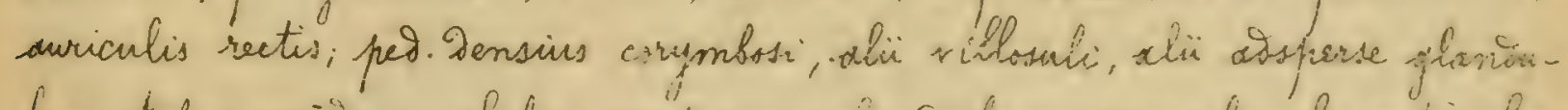

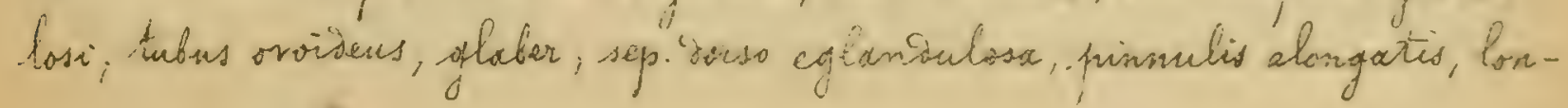


$-378-$

gis, incisis, eopiose instructa; stagli breves, hirsuti, gisco conico; cor.alborosea; fruct. ovoideus.

Hab. Thane, at Eassin (At. Cariot).

2292. Cuppinic gespicita Gogr \&ab. N:2792.

A pracecidente Tiffert ramis at ctian caulib toto inarmibus, petiolis plorumque inarmibus, floribusque paucioribus.

Hab. Rhone, at Eassin (fo. Cariot).

2293. Crepinia holotenca Gige toab. N:2793.

Acculei tenues, Longi; as ramos flor graciles param gilatati sed valie falcati, foll parva, svato-rotuniata, utringue rotunisata, rel aprice saepe oftusa, superne hirtella, subtus albiva totoque longe pilasa; rentes acute biserrati; pet glanoulosi, villosi, inf. inermes; stip. breves, Dorso hirsutac; pio-1-2, subglaberrimi; tubus oroidens; sep. brevia, pinnulis glabris, angustis, 4-6-9entato-glangulosis instructa; styli minores, parce villosi, gises subplano; cor. subalba, eciliacta; fruct. avoiseus, utringue atten. Hab. IRhone, ai fornas (N. Ganioger).

2294. Crepinia Jacobi (Gggr in 92ull. soc. Murith. 1876, p.35); Ggogr Grab. No 2794. - C. Tomentella (Jacob in Soc. hehr. exs. an. 1874, non Lem.).

toculei Silatati, temuiter falcati, as ramos flor. pauciores, geminati; fol. latissime ovato-subacuto, basi rotunsata, petiolulata, atrovirentia, subtus toto villosa; Bentes biserrati, late aparto-triangulares; pet. glanioulosi, inf. inermes, toto hirsuti, stip. subtur pubescentes, orso glansulosac, auriculis givergentibus; pet.1-2, villosi interaumque hispioiuli, 14-17 m. longi; tubus ovoisens, laesis; sep. pinmulis latis, copisse gentatis instructa, stylivillssi, Sisco plano; cor. alba, eciliata, fruct sroitens, parrus.

Hab. Hehretia accid, at Comonoreche prope Neichatele (Jacob).

2295. Crepinia ochrolencsites Gogr bab. N-2795- Gogr Herb. nos. N-157. tbculei falcato-bilatati, as ramos flor. sat copiosi; fol firma, ovatosubattemuata, basi notundata, subtus toto villosa, prominule nervosa, al costam glanamlosa, Dentes biserrati; pet-glanamlosi, aculeati, villosi; stip. subtus pubescentes, auriculis givergentifus; peo. 2-5, sparse pilos;, brevissimi; tubus obovato-oblongus, sep. Longinscula, pinnulis foliaceis, dentatis, 
$-379-$

copiose instructa, styli freves, hirsuti, lisco proulo comic, cor. parwa, ocholenca, eciliata; fruct. parrus, ovoidens.

Hab. Rhone, at Itlix (M. Ganorger).

2296. Crepinia brachyphylla Gogr Gab. N:2797. Gogr Herb.ros. N:515. Heculei elongati, as ramos flor. abbreriatos falcato-Dilatati, fol minuta, ovato-subacuta, interivens sbtura, basi attenuata, suparne sparse pilssa, subtus toto villosa, ai costam parce glanoulosa, ventes biserrati; pect. hirsuti, glanoulosi, sculeati; stip. subtus pubescentes, aurriculis parum divergentibus; pet.1-2, hirtelli, sat breves; tubus ovoisens; sep. brevia, pirnulis latis, gentatis instructa; thgli hirsuitissimi, Disco plano; cor. ex albo-roses; fruct. parvus, breviter ovoideus.

Hab. Mhone, al Gleizé (No. Gandoger).

2297. Crepinia Lucandiana (2érégl. et Gillot in Bull. sac. bot. Feelg. XIX, 1830, p. 36), Gojr Zab. N:2798.

Acenlei faleato-Dilatati, ad ramos flor. panciores; fol. orats-elliptica, obtusa, supra glabra ac virioia, subtus as sevvos villosa, Dentes fiserrat,; pet. sellosi, gionioniosi, taculeain, sop. Sonqua, angustiae, auriculis ovivergentious;

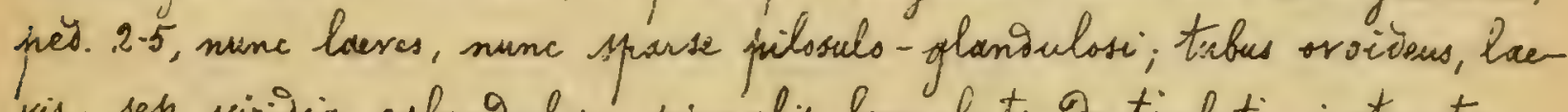
vis; sep. virisia, eglanisulisa, pirmalis lanceolito-Denticulatis instuncta; stigli hirsuti, Pisco plano; cor paillise rosed; grict. orsisens.

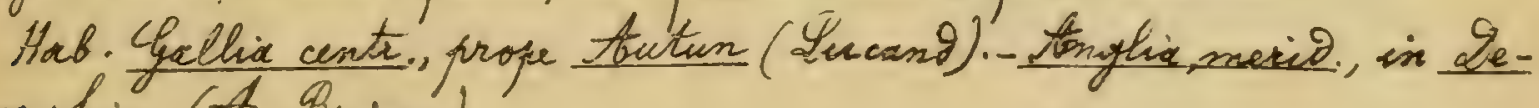
sonshire (fo. Mriags).

b. Toliola elliptica vel avato-elliptica.

T Fructus globosess, rotuniatus, ovato-rotuniatus vel ovatus.

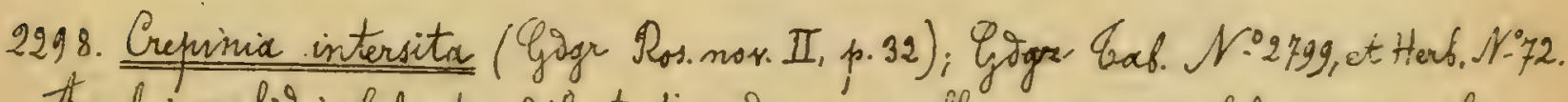

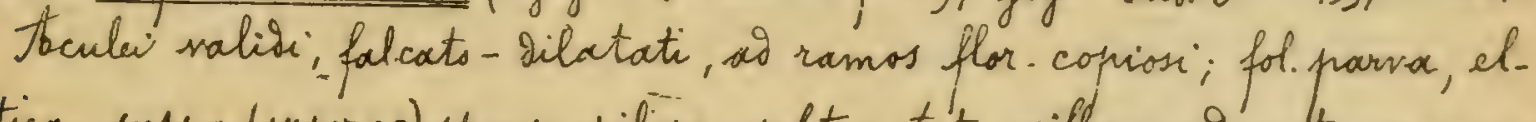
liptica, supra (superne) sparse pilissa, subtus toto villosa, is costam parce Giansulosa, newosx. Dentes wingute biserrati, pet. hine inse glansulosi,

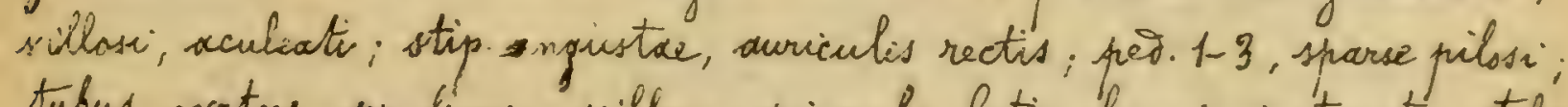
tubus ovatus, sep. firevia, villose, pianulis latis, longis instructa, styli Aseves parce hirouti, panciores, sisco plano; cor-albo-subrosea, ecitiata, fruct. parres rotosingatus.

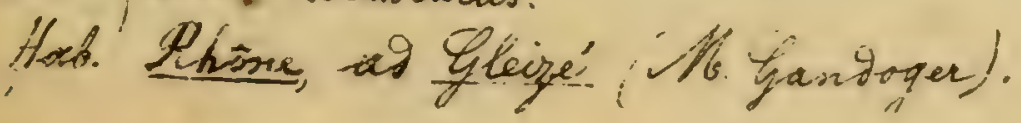


$-380-$

2299. Cepinia stilbocarpa Gigr \&ab. N-2800. C. Tomentella (Hort: lugo. an. 1870-72, non 2em. . Gogr Herb ros. $1=235$.

toculei caubinares valivissimi, falcato- Dilatati, ad ramos flor. "rebiliores et geminati, fol. elliptica, breviter acuta, basi rotunisata, supraasperse pilosa, subtus toto villosa; ientes biserrati; pet glanivulosi, aceuleati, villosi; stip. mésiocres; ped. 3-5, villosi; ' tubus ovoideus; sep. pinmulis glabris, angustis, 1-4. Tentatis instructa; styli subglaberrimi, siseo conico; cor........, fruct, parrus, ovatus, basi rotuniatus, apice poulo Depresssi, 1 cent. Fiam. Latus.

Hab. Atin, as Neron (Hoit. Lugaun.).

2300. Greninia giscors Gogr Eab. N-2801.

Acculei falcato-Silatati, a ramss flor. Deminatos nulli; fol. firma, late elliptica, supra atsperse pilasulx, subtus toto villosa et as costam eglaniulosa; rentes argute biserrati; pet. hinc inde glanoulosi, villosi, parce aculeati, stip. subtus pubescentes, auriculis brevibus; ped. 2-4, sillosi; tubus ovatus, glamiescens; sep. brevia, pinnulis latis, dentatis instructa, stygh hirsuti, Gises plans; cor.albo-rosea, fruct-sat magnus, ovato-rotuniatus, glaucescens.

Hab. Ihòne, Eassin (to. Cariot).

2301. Prepinia Hilobbandtii (Neitenweber in Limnaea XIII, p. 17; Opiz NaTur. p. 450); Giogr bab. N-2802. C. collina f. Kutbergensis (Keller in Formanek Rosen Hochges. p. 7 ).

Acculer sparsi, falcato- Dilatati; fol. elliptica, norvosa, superne g-labriuscula, -jubters toto villosa; aentes argute fisserati; pect inermes, tomentosi; parce glanoulosi; stip. subtus pubescentes; pes. glabri; trubus ovatues, lacris; sep. pinnatta, eglanidulose, gecitua; styli villosi; cor.......... fruct-ovatus. Hat. Mohemia, at Prague (Heitenweler, Oriz), Stustrix, in Noravia (Formanek).

2302. Crepinia Guepini (Wess. Ft. ge l' toryou p. 325; Désegt. Cat.p. 212);

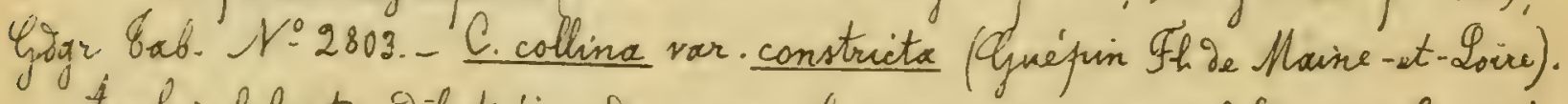
Iculei falcato Silatati, as ramos plus minus copiose; fol. ample ovatoelliptica, as newos villosa, experne glabro, Dentes biserrati; pet. parem imbescentes; per. glabii; tubus ovatus, laeris; sep. Deciana, styli hirsuti.

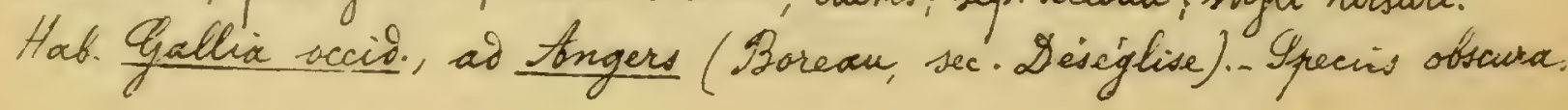


- $33 i-$

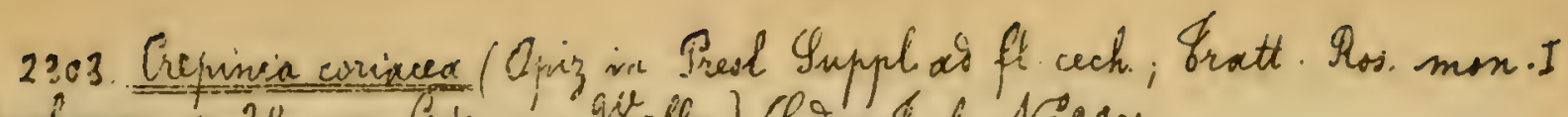

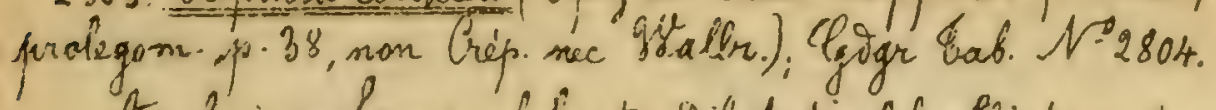

- teuler conformes, falcato- Dillatati fol elliptica, firms, coriacea, superne glabra, subtices to nerros vilisia, et at costom glanaulosa; aentes biserrati;

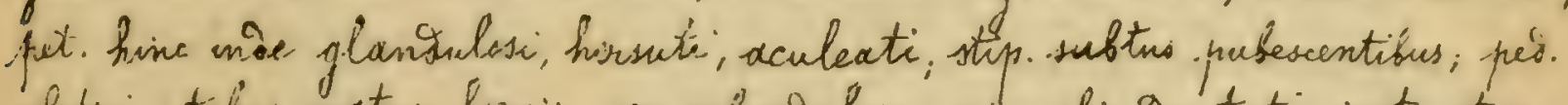

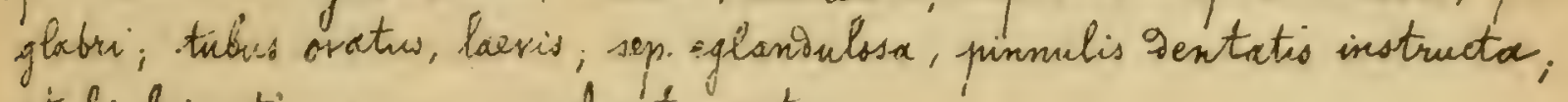

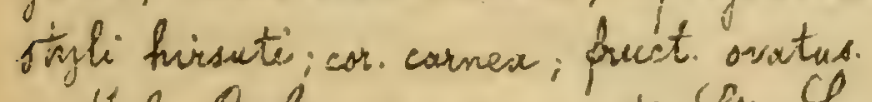

Hâ. Mohemix, in monte Sti Lourentii.

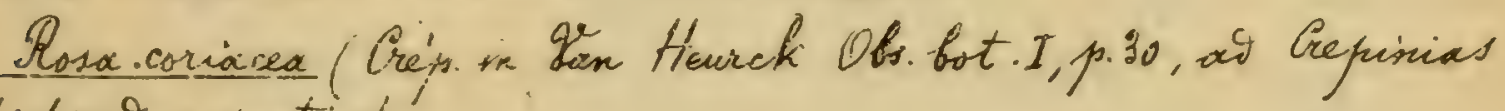
Stictoposas pertinet.

Rosa coriacea Mrallr. Mos. Hist. p. 223, penities ignote, non ast specis propuic gicta.

+ Fructus avoidens vel obovato-oblangus.

§. Petioli unnes aculeate.

2304. Grepinia phagnalocies Gogr Bah. N-2805.- Gogr Herb. ros. Ni2g.

Acculei falcato-bilatati, so ramos flor. flexussos sat copiosi, fol. parro, elliptica, utringue sensirn et parum attemeata, elbida, suferne aipresse pitosula, subtu's toto villosa et as costam saepe rubellam glanioulosa; Dentes biserrati; pet. glandulosi, aculeati, villosi; stip. angustre; pet. sacpe 1, glabri', 15-18 m. longi; tulus ovoineus, sep. rubescentia, pinmulis latis, ciliatis, 4-6-Dentatis instructa, acumine laciniato; styli alabri, isisco subplano; cor, parros, albiira; fruct ovoidens.

Hab. Iry-2e-2ime, at Rionsat (N6. Diuboselard).

2305. Crepinia nematellk Goor \&ab. N-2806.

Rubens; aculei follato - Silatati, as ramos flor. pauciores, for poorro, elliptica; parum attemuata, basi abrupte truncata, firma, superne aboperse

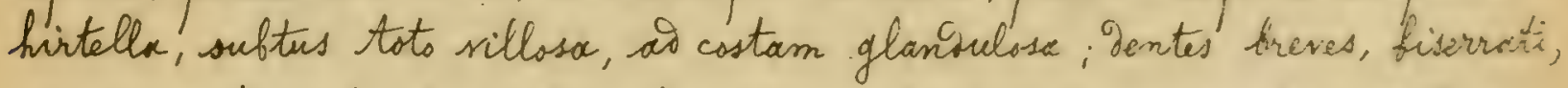
parvi, recti, pet glanoulose; hirsuti, aculeati, stip. angustace, sublitus

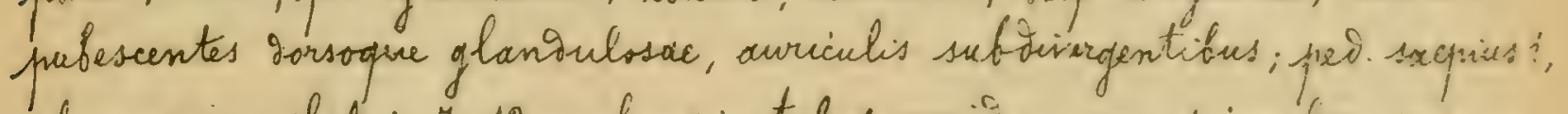
plerumque glabir, 7-12 m.longi; tubus ovoineres; sep. fuinmelis parsum

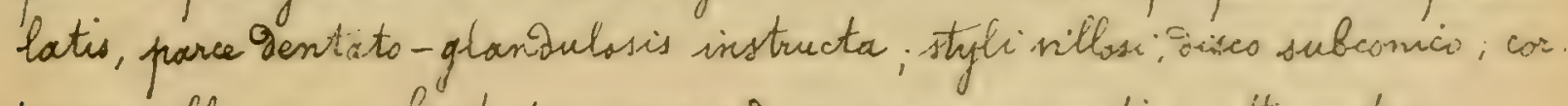
harva, albo-rosea; fruct. frorsus, oroidens, apice procestion a tismatus. Hab. Haute- Farsie, al Mingy (Muget ${ }^{2}$ ). 
$-382-$

2306. Brepinia Benthamiana Gogr \&ub. N:2807.

teculer falcato-Dilatati, as ramos flor gensinate, fol. elliptico-acuta, basi subrotunaata, meinocria, superme glabra ac intense viribia, subtus to to villosa et at costam paree glanioulosa, sentes biserrati, aperto-triangulares; pet.glanoulosi, villose; aculeati; stip. sirises, auriculis divergentibus; ped. 1-2, parce palosuli, 11-13 m. longi; tubus sbovato-oblongus; sep. Dorss non glangulasx, pirmulis brevibus, foliaceis, 2-4-Dentatis instructa, acumine integro, angusto; styli parce villosi, gisco plans; cor.......; fruct. obovato-oblongus, basi rotunaatus, aquice longe et valve attemuatus.

Hab. Anglia bor., prope York (G. Hebster).

2307. Crepisia minutifolia Ggar Eab. N-2808.

Acculer silatati, tenuiter falcate, ramos flor.copiosissimi; fol parra, srato-elliptica, acuta, basi rotundata, rividi-glaucescentia, unsique hirsuta, ad costam parce glanaulosa; ientes biserrati, aperts - triangulares; pot. villosi; glaniulosi, aculeati, stip. rubentes, subtus pulescentes, auriculis givergentibus; peo.1-2, paulo villosi, 6-8 m. longi; tubus osoinew; sep. pinnulis latis, 2-3bentatis instructa, acumine integro, styli parce hictelli, gisco plano; cor.......... fruct. freviter ovoidens, utrinque sensim oepressus.

Hab. Anglia, Surrey: ad Barnes (Groves).

§. Setioli inferiores inermes.

2308. Grepinia bioclada Gagr \&ab. N-280g.

Acculei pauciores, sparsi; falcato-vilatati, as ramos flor. nulli; fol. ovatoelliptica, vix attervato, basi rotunsata, superne adpresse hirtella, subturs toto villosa, at costam glaniulosa; pet. glaniulosi, sillosi, inf. inermes; sentes biserrati, aperti; stup.parvar, angustae, subtus villos-glangulosac, auriculis givergentibus; peo. 1-2, parce pilosi, 10-14 m. longi; tubus oblongus; sep. brevia, pinnulis latis, gentato-glanisulosis instructa, styli parum villosi, gisco sat conis; cor. rosea; fruct. oborato-oblonges, basi subrotunDotus, apuice valoe attenuatus.

Hab. Haute- Savsie, al St Martin frope tonnecy (Muget).

2309. Repinia mitoacantha Gogr 4 ab , $r-2810$.

Abculei subteretes, parum falcesto-délatati, al ramos flor. copriosi; fol. 
$-383-$

Ulliptica, apice fere rotunaata, basi rotuniata, ubtus prasentim toto villosa, al costam parce glonioul, sentes biserrati, lati, patuli; pet. glanDulosi, hisuti, inf inarmes; stip. breves, sat latae, acuriculis givergentitur; ped. 1-3, parce villoss: 12-16 m. longi; tubus oblongus; sep. pinnulis parum latis, gentato-glanoulosis instructa; styli hirsuti, Dises sat comico; cor-rosed, fruct. ovoidens, quice attemuatus.

Hab. Haute-Savou, at torgonnex (Suget).

2310. Crepinia bellula Gogr bab. $N=2811$.

toculei breves, rectiusculi, gilatati, ramos flor. copiosissimi; fol.ovatoelliptica, subacuta, basi coriata, unique sed subtus proesertion villosa; is entes biserrati, aperto-triangulares; pet. glaniulosi, villosi, inf.inermes; stip. oorso pubescentes, surricalis aivergentibus; ped. 3-5, pilosi, 9-11 m. longi, tubus obovatooblongus; sep. pinnulis latis, copiose marginato-glandulosis instructa; styli villosuli, Gisco subplans; cor. alba, inferne subciliata; fruct. ovoisens. Hab. Gironde, at Bursac (2. Matelay).

2311. Crepinia Tagiana (Crép. in Fritge et Mlse Karpat. Reise, 1870, in nota; Goyr ofsai p. 33). Gogr \&ab. N-2812.

Abculei falcato- Dilatati; fol amplissime elliptica, acuta, basi abrupte truncata, superne nitida, glabra, subtus as nervos parce villosa; gentes biserrati; pet. villosi, eglandulosi; inf inermes; stip. clongatar, latissaclac, auriculis patulis; per. 3-6, patule pilosi, 3-5 m. longi; tubus oblongus; sep. pinnata, eglandulosa, styli hirsuti; cor.........; fruct sat magnus, obosato-oblongus, intertum ovatus (sec. Crép.).

Hab. Hungaria bor, in valle glaxg at Hradek (Mtse).

2312. Crefinia pulvinaris Gogr \&ab. Y-2813.

Acculei rubelli, falcato-gilatati, wo ramos flor-geminati; fol parva, elliptica, utrinque abrupte et farum truncata, undique villosissima, subtus albita et fere floccoss; dentes acuti, onnes simplices; pet. eglanoulosi; tamentosi; inf.inermes; stip. subtus pubescentes; per.2-4, sparse pilosuli, 4-7 m.longi; tubus suboblangus; sep. pinnulis latis, sat villosis, gentatolacimiatis, saepe foliaceis instructa; stigh pronce villosi, gises subplans; cor. eciliata, fruct. oroidens, basi notuniatus, aprice attenuatus. Hob. Rhone, as Mrrinais (P. Chabert). 
$-384 \ldots$

Ipecies illa, of foliola simphiciter serrata, arogulo Eamentethaum alienam esse videtier meliesque in sect. Solstitiatium colicaniam; sed fropter ahis notas hue tamen aiscribendam censes.

c. Foliola oboveta, sbovats-oblonga vel oblonga. + Fructus rotunidatu: vel giobosus.

2313. Crepima celerata (Baker Teview p.31, ut var), Gogr Gab. M:2814.. C. cannina var. (Baker Mrit.mon ros. in Limn journ. XI, p.235). - D. catilaumica (Costa).

Tbcules conformes, falcato-Dilatati, fol oflonga, superse glabra pallideque sirivia, subtus as nervos villosa; Dentes biserati, pet villosi, glansulosi, aculeati; stip. Subtus pubescentes; ped glabre; tubus suboglobosus, laevis; sep. erecta, Dir parsistentia, pinnulis pectinato- ontatis copiose isestructa, styli rillosi; cor; rosea; fruct. subglobosus.

Hab. Anglix, Northumberlant: Itolywel (Maker). Hispania bor. (Sosta).

2314. Orepinia Robulata Gige Gab. N-2815.

tocule robusti, valde folcato- Dilatoti, as ramos flor panciores; fol. late obovato-oblanga, freviter attemuata, basi abrupte et subtruncata, sub-

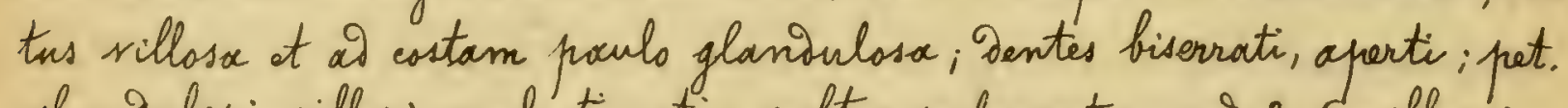
glanoulosi, villosi, aculeati, stip. subtus pubescentes; pes. 3-6, villosi, 10-14m. longi; tubus oroideus, sep pininulis latis, paulo pubescentibus, volve rentato-glanoulosis copiose instructa; styli pubescentes, majusculi, sisco subplans; cor. ex albo rosea; fruct. rotundatus, $1 \frac{1}{2}$ cent. latus. Hab. Ithone, at Ecully (P.Chabert).

2315. Prepimix villistula Goor Eab. N:2816.

- H-culei falcato - ilatati, is namos flor copiosi; fol late oborato-ob. longa, freiter weuta, bisi rotunivata, atrovirentia ac superne fere glabra, subtu: albida titopese villosi, al costam glangulosa, Dentes fiserrati, apertotriangulares, isct. foniulase; aculeati, tomentosi; stip. Dorss glanisulosac,

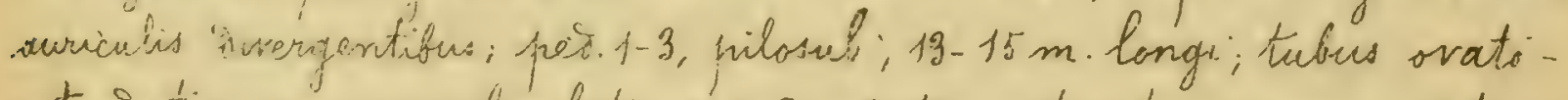
roturiatius; sep promelis batis, 3-4-gentatis instructa, acumine integre,

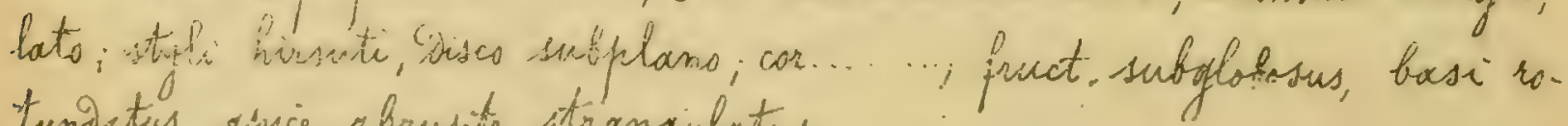
tundrtias, apice abrupice strangialatws.

How 
2316 Creprisia sphoenoyna Gogr oxb N:2817.

Foculer fuciato. Dilatati, freves, as ramos flor. sat copiosi; fol. late of ovato-oblonga, acuta, fasi rotuniata, laete viridix, ungique villosa, at costam vix glandulosa; gentes vix biserrati, aperto-triangulares, pet. hinc inde glandulosi, pubescentes, aculeati, stip. sirides, subtus villosae, auriculis subrectis; ped. 2-4, pilosi; 7 -g m. longi; tubus ovatus; sep. pinnulis parum latis, 2-3-gentatis instructa, acumine angusto, integrs; styli magni, albolanati, Dises plano; cor......... fruct. subglobosus, basi rotunvatus, aprice vix gepressus.

Hob. Lilesia, prope Greslau (Knebel).

$$
\text { T+ Fructus ovato-roterviatus, svatus vel allipticus. }
$$

\section{Crepinia mucionata Gogr \&ab. $\mathcal{N}^{\circ} 2818$}

Aculei breves, parvi; Dilatato-subfalcati, at ramos flor graciles rari, fol. ample oforato - obtusiuscula, basi rotungata, undigue villosa, 2 costam non glanivulosa, Dentes biserrati, profunde lanceolato-aperti; pet eglandulasi, inermes, villosi, stip. longae, anojustace, auriculis Sivergentibus; per. 1-2, alii laeves, alii pachlulum glansulosi, 7-8 m. longi; tubus ovatus, laeris; sep. Forso eglandulose, pinnulis pancioribus, integris, angustis instructa, acumine integro, subilitatato; styli lanati, Sisco plano; cor..........; fruct. ovatus, basi rotundatus, apice strangulatus.

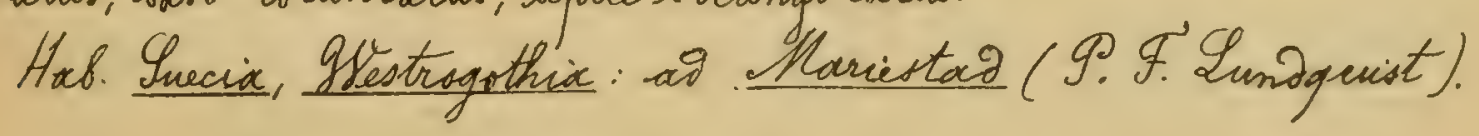

2318. Crepinia psendo-ochrolencoides Gggr \&.ef. N-2819._Gogr Herb.ros. N-22. toculer valivissimi, falcati, late gilatati, as ramos flor minores, copiosi; fol ample oborato-oblonga, attenuata, basi rotunivata, subtus jraesertim villose, at costam parce glanisulosa. ientes crebre biserrati; pet. aculeati, glandulosi, villosi; stip. subtus pubescentes, auriculis givergentibus; pis. 2-5, pilosi, 10-15 m. longr; tubus or-ideus; sep. pinnulis latis, gentatis, longis copiose instructa; styh hirsuti, gises subconico; cor.alba, sat parra ; fruct. ovatus.

Hab. Rhome, ad tomas (N6. Ganooger).

2319. Crepinia caucasica (Tall. Th. ross. I, pars 2a p. 62, M6. Mrib. Tl. taur-

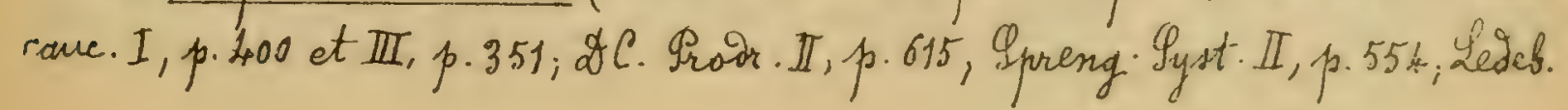


$-386-$

TP. ross. II, 6.78; Georg. Meischr. y. Muss. R. III, 4, p. 1027; Rchb. Fl exc. N-4005; Gichw. Cisp.-canc., 24,30, Henning in Mém. soe. nat. Mose. VI, p.72,88;

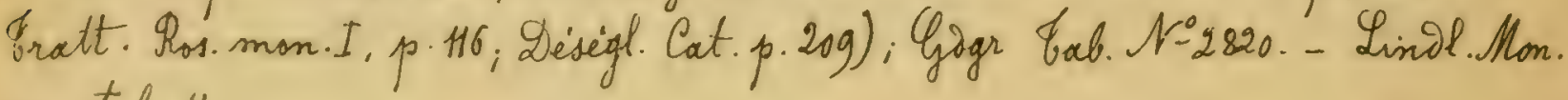
ros. Tab. 11 .

Th.cuin conformes, falcato-Silatati; fol late oborato-cuspicata, glabrescentia; ventes biserrati, convergentes, cuspibati, pet. villosi, aculeati, stip. subtus ipubsesentes; pei. subternati, glabri; tubus oratus, laevis, sep. pirnulislatis, copiose dentato-glanivulosis, longis instructa; styli hirsuti; cor. pallide. roses; fruct ovatus.

Hob. Cancarus. - Thecies a caciteris bamentellis lange jistat.

2320. Crepinia cosmocarna Goger Gab. N-2821.

Aculir falcato-dilatati, wo ramis flor copiosi; fol. late oborata, sensim whesmato, bisi cordata, subtus prasertion villsa, ai costam fuaclo glanidulasa; ientes biserrati, aperti, pet. aculeati, hine inve glanivulosi, villosi; stip. subtres pribescentes; peo. 6-10, sparse pilosuli, 13-20 m.longi;

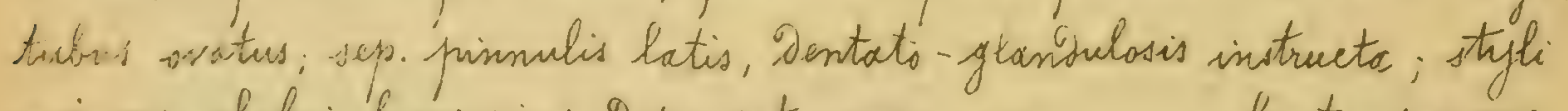
minares, olafri, brevissimi, Deseo sat canico; cor.........; fruct - prarrus, ivato-roturidatus, nitious.

t'iob. Thone, as Moy trope Fontaines (M. Chabert).

2321. Crepinia Fraseri Gogr \&ab. N.2822.

It culei Salcato- dilatati, as ramos flor. copiosi; fol. latissime oboratooblonga, acuta, basi sensim attemuata, intense virivia, unjigue villosa, at costam glanidulosa; dentes biserrati, patule triangulares; pet. eglanisulosi, villosi, aculeate; stip. Dorso eglendulosac, avriculis Divergentibus; pe. 2-5, sisperse pilosuli, 12-14m. longi; tubus ovato-ellipticus; sep. Dorso eglandulosa, pinmulis latis, 3-5-'dentatis instructa, acrimine dentato; styli parce hirsuti, dises plano; cor......... fruct. ovato-ellipticus, basi rotundatus, anice abrupte strangulatus.

Hab. Anghax, Harwickshire: as Hatton (f. Fraser).

2322: Grepini indecora Gige tab. $N=2823$.

toculei veres, rilatiti, ralie falcati, ad ramos flor nulli; fol sborato- of iorer, acu'ta, basi attemats, uninque temiter villosa, as costam glanoulosa: ientes biserrati, triangulari-convergentes, pet. glanioulosi, acceleati, villosi: 
$-387-$

stip. virides, latae, eglañulosae, awriculis divergentibus, hes. 1-3, ärperse piCosuli, 10-12 m. longi; tubus ovatus; sep..........; styli villosi, sisco plans; cor........ fruct. purves, ovats-rotunidatus, comiens, basi rotunisatus, aprice atten. Hab. Anglia, Cheohire: at Brombarough (1. Horbort-Leswis).

T†T Fructus avoideus vel obovato-oblongus.

\$. Ytyli glabir vel paulo villosi:

2323. Crepinid breviuscula Gogr Bab. N'2824. C. Tomentella f Hasclbergeri - Keller in Leimbach 9oot. Monats. 1884).

Thechli fakcato - vilatati, res ramos flor. geminati, fol parva, obovato-oblonga, acuta, basi rotuniata, intense virisio, unsique villosa, ad costam ylanisulosa, Sontes bisersati, aperto-triangulares, pet. glanioulasi, sillosi, aculeati; stip. parvere, anqustae, auriculis givergentibus; neo. 1-3, graciles, pilosuli, 14.-16 m. langi, tubus sbovato-oblongus; sep. pisnata, marginibus glanionlosa; styli minures, glabri; Sisco sat comico; cor..........; fruct. parrus, oborats - oblongirs, bresi roturioatus, comicus, aprice lorge attenuatis.

Hab. Hahia orient. at Fanza (2. Caisesi).

2324. Bremina stenocoma Gygr 8ab. N-2825.

triulei ailatati, parnm falcati, vel inchinati, as ramos flor pancióres sel mulli; fol. late oblanga, sat acuta, basi roturiatei, intense virioir, swireme glabra, subtus to to nillosa, as costam ghindulosa; dentes biserrati, lanceslatoaperti; pot. glangulosissimi, subinermes, parce villosi; stip. Dorso glanioulosae, auriculis givargentibus; ped.1-9, subglabri; 6-8m.longi; tubus ovoisens; sp. pinnulis hinearibus, 1-2-jentatis instructa, acumine integro; sijli glabri, Jisco tonge canics; cor......... fruct svicieus, parkus, conicus, basi rotuñoatus, aprice longe stranguelatus.

Hob. tonglix, ad Gork (-y. gebster).

9325. Gepinia inducta yjar bab. N:2826.

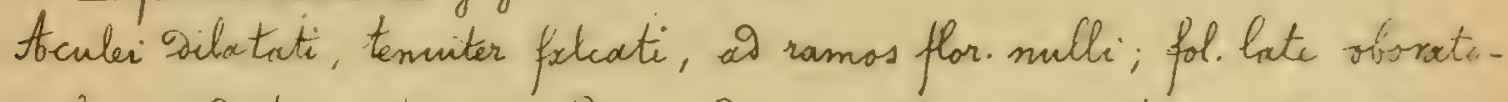
acuta, fasi cordata, intense, vietia, undique villosa, ai costam givisectoser.

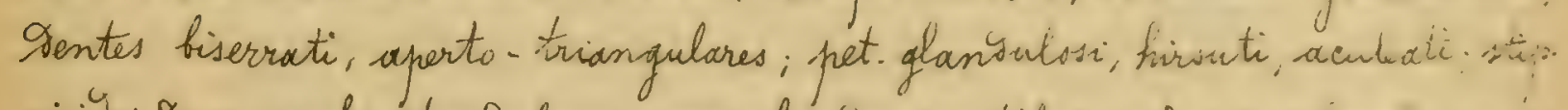
sirides, gorso paulo glansulosac, auriculis divergentisus; pes. 1-3, pilasuli, 10-12

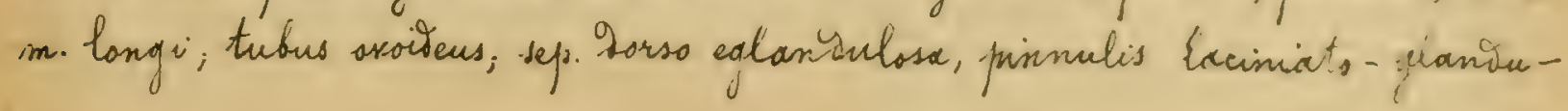


$-388-$

losis, ample foliaceis, cum acumine instructa; styli paulo villosi; Pisco comico, car..........; fruet ovaideus, basi subrotuniatus, apice strangulato-atten. Hab. Anglix, prope York (G. Héster).

2326. Crepinia fragilia Gogr mss.

tbculer validi, falcato-gilatati, as ramos flor nulli; fol medicaria, obovato-acuta, basi rotuniata, supra lucire atroverentia ac sparse pilosula, subtur to to villosa et so csstam glansulasa; Dentes biserrati, aperto - trisngulares; pet parce glanoulosi, rillosi, inf inermes, stip. virides, garso sillosar, mesiacres, auriculis rectis; peot. 1-2, villosi; 10-12 m. longi; tirbus obovato-oblangus, basi pubescens, sep. Forso laeria, pinnulis latis, 4-5Dentatis coprosius instructa, acumine lato, dentato; styli sparse pilosi, sises comico; car. albo-subcarnea, eciliata; fruct. parvus, oforatus, utrinque paulo attenuatus.

Hab. Saine - t Loire, at Ht Miand (No. Goondoger).

2327. Qupinia Harbordü Giogr Gab. N=2827.

Aculei valivi, falcato-Dilatati, as ramos flor geminati; fol late obovato. oblonga, breviter acuminata, basi subattemuata, pallise virisica, utrinque temuiter villosa, à costam eglanioulose, Dentes biserrati, aperto-triangulares; pet. glanoulosi, aculeate, villosi; stip. rubentes, gorso eglandulasac, auriculis sivergentibus; per. 2-4, pilosuli; 11-12 m. longi; tubus ovorideus; sep. pinnulis parrm latis, 2-3-gentatis instructa, acumine angusto, integro; styli villosuli;

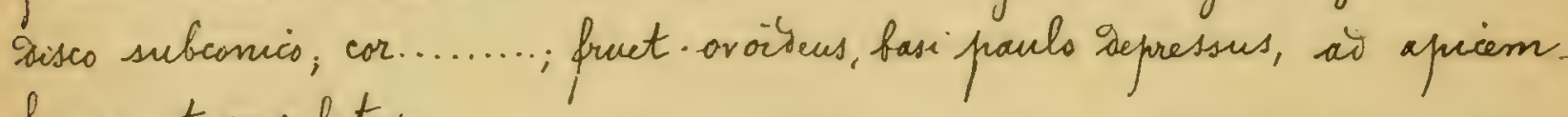
longe strangulatus.

Hab. Anglia, Cheshire: at Iromborough (J. Harbort Lewris, Fishor).

2328. Crepinia avellanaefolia Gogr Gab. N-2828. - Gagr Herb ros. To-g4. itculer breves, gilatato-subfalcati, at ramos flor. plerumgue nulli; fol. steritia amplissima ( 3 cent.), floralia vers oborato oblongo, utringue bievis sime attenuata, villos costam parce glanoulosa, gentes biserrati, lanceolati. longi; pet six glanoulosi; villosi, inf inermes; stip. subtus fubescentes; peo. 2-4, pilasuli et simul asprerse glanoulosi, 8-10 m. longi, tubus late oroicens, laevis; sep. primulis ventatis, pubescentibus, subfoliaceis instructa, stagli aisperse pilosi, Sisco subplans; cor pallive rosed; fruct breviter ovoidens.

Hab. Suy-2e-Dáme, at Iavy (H. Dubosclarg). 
$-389-$

232g. Crepinia praeruntorum Gagr \&ab. Ni2829.

Aculi lalcato-vilatati, as romos flor. milki; fol. obovato-acuta, fasi breinter attemuato - truncata, subtus prosestim villosa; ${ }^{\circ}$ dentes biserrati, apertotriangulares; pet. glaniulosi, villosi, inf inermes; stip. pubescentes, auriculis subsivergentisus; per. 1-2, hirtelli, 4-6m. longi; tubus obovato-allipticus; sap. heria, pinnulis foliaceis, 4-6-gentato-glaniulosis instructa; styli villosuli, Sisco plans; cor. albar; fruct. parvers, breviter ovoidens. Hab. Love, as Lauvain : alt 250"' (N. Ganioger).

§5. Gtyli hirsuti vel lanati.

2330. Crepinia megacantha Gogr Gat. N-2830.

to culei valisi, aut maximi, falcati, late gilatato-Decurrentes, ai ramos flor. sat copiosi; fol. ample obovato-acuta, basi rotunisata, subtus praesertion villosa, is costam paree glandulosa, ientes biserrati, remoti, ovato-aperti; pet. glanoulasi, hirsuti, aculeati, stip. sat latae, garso glanouloso-pubescentes, auriculis divergentibus; ped saepe 3-10, subhirtelli, 12-18 m. longi; tubus oblongus; sep. pinnulis ontatis, parmm latis instructa; styli hirsuti, aisco comico, cor. alba, fruct. woidens, basi rotunoatus, apice attenustus. Hab. Rhone, at ternos (At. Granoger).

231. Brepinix barbiseta Gogr Eab. N-2831.

Aculei falcato gilatati, as ramos flor. plus minus copiosi fol parve, obovato - attenuata, basi rotungata, subtus praesertion villose; gentes biserrati, magni; pet hisic inde glandulosi, villosi, parce aculeati; stip. subtus pubescentes; pot. 2-3, villosissimi; 10-15 m.longi; tubus obovato-oblongus; sep. pinmulis latis, brevibus, gentatis instructa; styh hirsuti, Disco subptans; cor......... fruct. oroisens, basi rotundatus, apuice conico-aitanuatus, interoum strangulatus, $11 / 2$ cent. giam. latus.

Hab. Irhone, ad Charbonmieres ( T. Chabert).

2332. Crepinia Loretiana Gogr Eab V:2832.

Aculei silatato-subfalcati, as ramos flor. sparsi; fol. sext tate oboratoobtusuiscula, basi notundata, ungique villosa, as costam ioflaniulosa; isntes simplices, aperto-trianpulares; pet glandulasi, inermss, tamentosi; stip. For so eglansulosar, rebentes, wiviculis sivergentifus; per. 1u3, villosissini, g-10 sn. 
$-390-$

Wngi; tutus ovideus; sep. Gorso eglandulosa, pinnulis angustis, 2-3-oentatis instructo, asumine integro; styli sublanate, jisco plano; cor. ecitiata; fruct. ovoidens, fresi rotuniatus, apice longe attermatus.

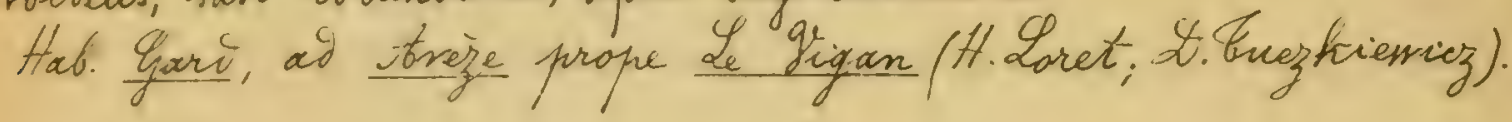

2333. Gepinia reluctans Giogr mss.

Aculir iefiles, subiliatati, inclinati rut vetiresculi, xo ramos flor. stiputares; fol parve, oftong-renta rel abrupte acuminata, basi subattenusta, - superne glabia, glavecscentia, subtus ad nerros (excepta media eglanioulosa) Nise hirtilla, albisa; sentes biserrati, sperto-tringulares; pat. inferne paculo glanoulosi, trmentosi, aculeati, stip. Gasi rubentes, brevinsulac, sat tatiae, -vorso glabrae, auriculis subrectis; pet. 1-2, aprice glañoulosi; $5-7 \mathrm{~m}$. Eongi; tubus ove üeus, saepe prorsus setulosus; sep. oorso glanidulosa, pinnulis sat

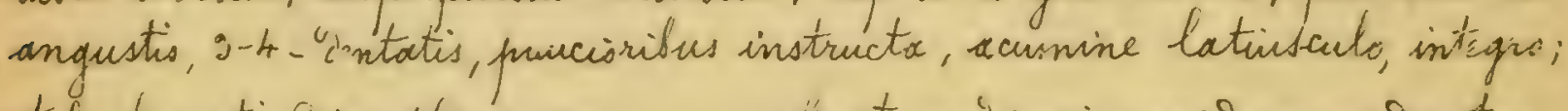

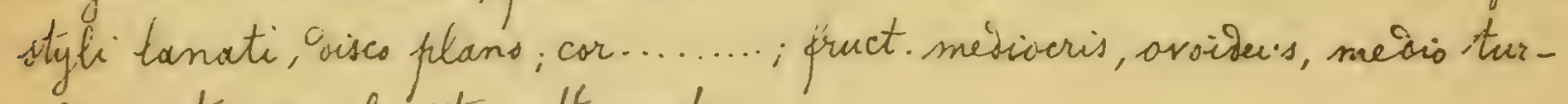
giaus, utrinque breviter attenuatus.

Hol. Banatus, Mrasso' Isoireny: of Traiseisa. (2. Thichter). Ifrecies, foliolis decalvatis, painculis fere nomaliter glandalosis ut et sefrala, ob affioibus mox Distinguenda.

2334. Crepinia oxybasis Ggar tob. N:2833.

steula varisiom, ciebiles, falcato-silatati, ad ramos sup. et flor.nulli;

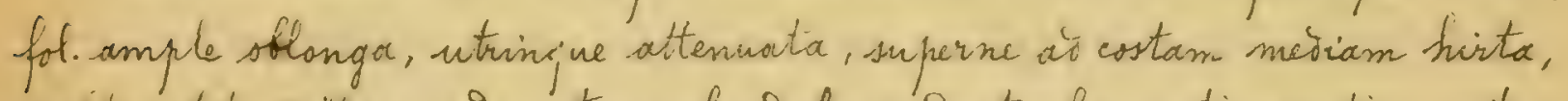
subius toto viltasa, w2 cistam ylaniulssa, ientes biserrati, aperti; pet. glaninlosi, frilosi, subinermes; stip. inguestac, Iirso purbescentes, duriculis rectis; ped.1-3, glabri, g-10̂m. langi; tubus ovovato-oblongus; sep. pesimulis latis, dentatis ses eglaniulosis copiose instructa; stapli breves', hirsuti, aisco conico; cor. palkide rosex; frect. sat magnus, ovoiveus, basi, rotuniortus, afuce altemiatis.

Hab. Here, ad Charvinx. (Moullu).

Cum prowsesente et sequentibus haee a vara C. Tomintella (Lem.) multum dejfort.

2335. Crepinia ciliaris (Wilson sec. Maker. Gint. ros, in Linn. Journ. XI, p. aig); Goar Sab. N-2834. - C.arvatiea (Mahser Review p.33, non Buget). C. canina var.

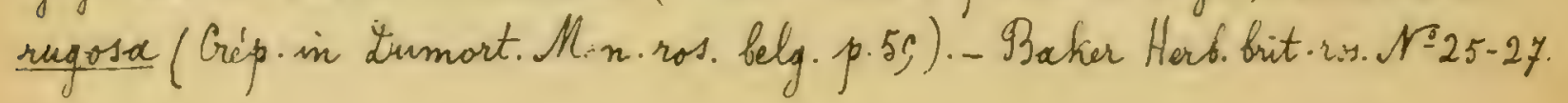


THeulei conforms, faliato vilatati; fof. obovato - Silongor, subtus villosa t $a$ J costam ytansulosa, Dentes valde biserrati; pet. glanjoulosi, tomentosi, aculeati; stip. subtus pubescentes at garso glaniulosac; peic laeves; tubus ellipticus, glater; sep. torso ylan'sulosa, secioner, pinnata; styli villosi, Nisco plano; cor. rosea; fruct ellipticus, vel ovatus.

Hab. Anghir bor., Yorkshire, tc. (Yarington Mnse sec. Foorrer 9rit. fl. ei. 3a, , is: 241).

2336. Crepinia exsurgens Giagr Gab. $\mathcal{N}: 2835$.

foculei panciores, falcato-dilatati, as ramos flor rari; fol ample ofovato-oblonga, acuta, basi attanuata, juperne intenss virisia ac glabrescensia, subtess toto villosa, is costara glanáulosa, dentes fiserrati, lanceolato apierti; pet. glandsulosi, aculeati, parce villosi; stip. sat maynae, latae, auriculis Givergentibus; peiv-it, piloss, 7-10 m. longi; iubus shovato-oblangus;

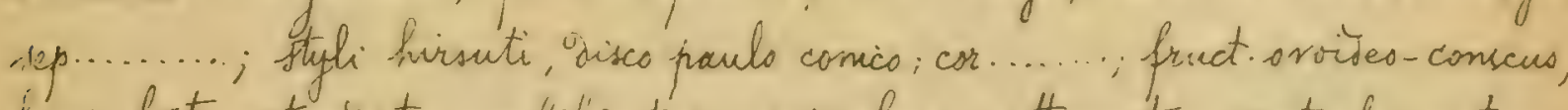
trasi late notungato-umbilicatus, apice longe attemuatus, centrah autem inferne contracto.

Hab. Honorre, ad Cassel (Label).

$$
\text { t†t Fructus oblongus. }
$$

2337. Crepinia comosa Gogr toab. N:2836.

Arulei falcato- Silietati, at ramos flor stipulares; fol. sat parva, obovactoacutiuscula basi truncata, supra glabra et glauca, subtus ao nervos villoseat as costam parce alaniulosa; Dentes tenues, crebre biserrati, lanceolatoconvergentes; pet. villosi, aculeati, glanivulosi; stip. rubentes, andus tsec, gorso glaniulosar, auriculis subsivergentibus; neod.2-3, graciles, ahi laces, alii parcissime glanaulosi, $10-11 \mathrm{~m}$. longi; inbus gracilis, oftongus, basi saepe temuissime setulosus; sep. elongata, gorso eglondulosa, pinnulis lon. gis, angustis, valie bentato-glandulosis copiose instucto, acumine fohiaces, ylariulosissino; styli villosuli; gises paulo conico; cor........ fruct. iolonqus, aprice attemuatus

Hab. Mtalix orient: ad Taensa (2. Caldesi).

Pisunculis nonnumauam ivevibus, tubo calycis hisi soepe tenuiter glanisuloso, sepalisque valde pectinato-glanoulosis, hace a seguentivis ontime giversa. 
$-392-$

2338. Crepinia palnata Cyagr mss.

toculer maximi, falcato-Silatati, at ramos flor. glaveas panciores: fol méviscria, suboblangon acuta, basi poulation attenuata, superne glabra ac viribi-glaucescentia, subtus albila, à nervos parce villosa, costa mesia vero hirsutior ac eglanisulose; o ventes biserrati, subpatulo - triangulares; pet. parce aculeati, tomaentosi; hinc inise glansulosi; stip. sirivaes, magnae, longae, yorss villoso-subgh anioulosar, auriculis rectis; prés-1, vix glaniulose, 4-6 m. longi; tubus oblongus, prululum glantulosus; sep. reflexa, brevinscula, Dorso glanioulosa, pirnulis sat angustis, copiose dentato-glanaulosis instructa, acumine latiusculo, parum gentato; sty hi lanati, gisces paulo conics, cor.......... fruct. oblangus, majusculus, utionque attemuatus, apice strangulatus.

Hab. Sanatus, hrasso' Ysoreny: OD Narilla (2. Brichter).

2339. Repinia squarrosula Gige Gab. A :2837. - Gogr Herb. nos. N:49. Aculei nobusti, Silatati, Lalcati, as ramos flor robustos Geminati; fol obovato-attenuata, basi subrotuniata, subtus praesertim villosa, ac costam parce glanisulosa; Dentes biserrati, breves, aperti; pot. hirsuti, glanoulosi, aculeati; stip. sat angustac, varso pubescentes sed fere eglananlosae, auriculis sivergentibus, peo. 2-3, pilosi, 7-12 m.longi; tubus oblangus; sep. Dorso eglaniabsa, pinnulis latiusculis, gentato-glanisulosis instructa, styli exserti, sublanati, gises comis, cor pallige rosea, fruct. oblangus, basi rotuniatus, apice volthe attemiatus.

Hab. Rhone, as tomas (No. Gandoger).

2340. Crepinix zelotes Goor mss. - Magnier FP. selecta exs. N:260.

Toculi breves, gilatati, parum falcati, at ramos flor geminati, fol. oborata, sensim attemuata, basi subrotuniata, unoique villosa, as costam eglaniulosix; dentes biserrati, apesto - triangulares; pet vix glanoulasi;, tomentosi, aculeatr; stip mésiocres, sirieses, subtus villosae, auriculis subtivergentibus; peo. 1-2, glabri, g-10 m. longi; tubus oblongus, laevis: sep. Dorss eglaniwiosa, pinmulis parum latis, sat ientato-glandulosis instructa, acumine integro; styli subglxberrimi, Sises fere plano; cor.alba,

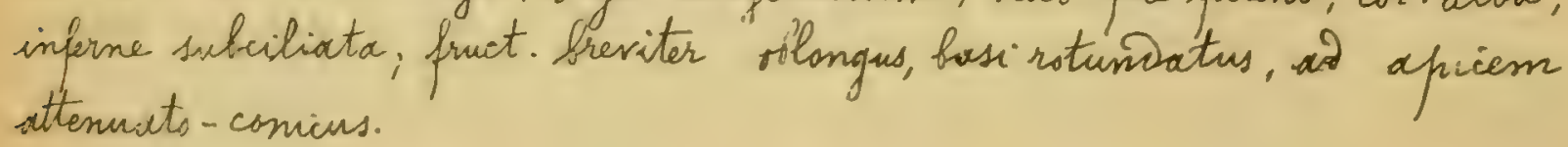

Hab. Thòne, as Lassin (S: sullu). 
$-393-$

2341. Crepinia andeniaca Gagr mss.

taculei misiocres, sat falcato-gilatati, as ramos flor panciores; fol. mesiseria, obovato subattemuata, basi fere rotuniata, superne laste virisia et sparse pilosula, subtus to to sec laxe villosa, às costam paulo glanioulosa, Dentes biserrati, aperto - triangulares; pet. hine inde glangulosi, villosi, parce aculeati; stip.virides, mediscres, sorso hirsutae, auriculis sivergentibus; ped.1-4, villosi; 1 cent. longi; tubus stilsngus, losvis; sep. Dorss cglangulss-a, pinnulis latiusculis, pubescentibus, 2-5. Dentato-ylansulosis instructa, acumine lato, saepius integro; styli sparse pilosi; Yiscus subconicus, cor-parra, albo- subcarnea, eciliicta; fruct. obtongus, utringue attenuatus.

Hab. Laone-et-Loire: ad It Laurent-9'tonanay (N. Ganooger).

2342. Crepinia amabilis Gjoge Gab. N.2838.

toculer valioi, falcato- dilatati, to ramos flor geminati; fol parra, obovectooblonga, acuta, basi rotuniata, pallise viridix, unsique villose, as costam glanidulosa; Dentes biserrati, late triangulares, subpatulis; pet. glañuliosi, piLosi, aculeati, stip. rabentes, latac, longae, auriculis Divergentibus; pes. 2-4, sillosi, $14-15 \mathrm{~m}$. longi; tubus oblongus; sep. Iorso eglanioulosa, pinmilis for hiaceis, margine glaniulosis, acunine suffoliaces, Dantato; styli parce villosi, cisco plano; cor pallige rosex; fruct. oblongus, basi pracsertion attenuates. Hof. Htalia orient: at Fonzax (2. (ailosi).

Pog. 308, post Grepiniam brevipilam adiv:

2091 fis. Creninia proxima Gogr bab. N-2657.

Aculer freves, dilatali, tenviter falcati, as ramos flor. geminati; fol. Slongo-acuta, basi freviter attenuata, as costam eglandulosa, ientes simphices, lanceolato-recti; pet. inermes, glandulosi; stip. londae, paum latoe, - muriculis sivergentibus; frè.1-2, glabri, 6-9m. tongi; tubus oblongus; sep. iccitux, pinnulis intégris, parum latis sinstructa, acumine sat anginsto, styli har a ti, sisco plans; cor. pallise rosex eciliata; fruct oslongus, basi longissime decurrens, apice valty jtranzulatus.

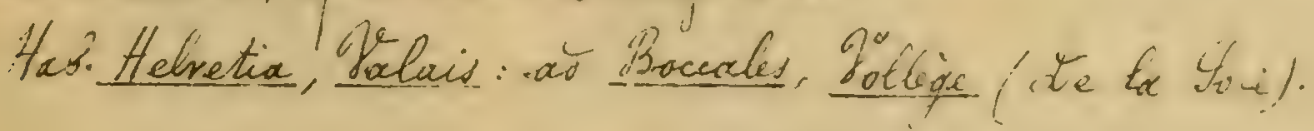




\section{Index specierum.}

M.93. Whi nomen auctoris deest, prone: Gogr. - Numeri primani votumen secundam sol tertium hujus operis, secundarii varo boum speciei in his duolus roluminibus indicant.

Gepinia abayjensis, 3,303 abhorems, 3, 44

abjecta, 2,304

abnormis, 3,341

aboensis, 3, 286

abolita, 2,312

abrogata, 2,311

abripta, 3,14

absens, 2, 33\%

abstenta (Désége), , ,7

abungans, 2, 383

accanthina (2resisgl.), 2,222

acanthocarpa, 3,356

acanthoclaio, 3, 188

acanthophylla, 3,232

acanthophora, 3,275

reanthothamnos, 3,391

rccetens (Mip.),3,107

accipitrina (Eakeaux),

3,218

accurata, 3,322

accusativa, 2,341

acerina, 2,186́

focharii (Millb), 3, 75

rcicarpa, 2,276

Acicularis , 2,376
Repuinia aciculifera, 3,68 Brepinia sedita (Léségl), 3,12 aciculifotia, 2, 266 acioula, 3,231 xciostenta, 2, 437 aciphylla (Rau),2,282 aciphalloides (Brip.). 2,200

acmosonta, 2,231

acmophylla, 2,296

acora, 3,95

acridentato, 2,367

acrocarpa, 3, 202

acrocomata, 3, 154

octinochlarnys, 2,467

actirioclaiva, 2, 379

actinophlaea, 3, 30

actinooroma 2,453

aculeata, 2, 410

acuta (Grep.), 3, 12

acuta (Fisch), 3, 12

acuta, 2,279

acutella, 2,279

acutipitia, 3,101

acutispina, 2,442

acutrisisula , 2,119,

adauntia, 3, 208 abenochlamida, 2,264 idenogontsides, 3,267 adenovontes, 3, 49 adenophora, 2,394 aisenopoidx, 3, 120 adenssepala, 2,326 Wenostephana, 3, 128 adenstricha, 3,318 ajjectiva, 3,188 asmissa (Gép),2,331 aimixta, 3, 53 adornata 2,237. adriatica, 3, 148 riocensionis, 3, 18 aosscita (Lésigl.), 2,330 atulterina, 3, 344 acouensis, 2, 305 aequalis, 3,119 affabilis, 3,g6 affinis (Rau), 2,398 affinita (Treget), 3, 121 africana, 3, 17 Affzeliana (Fries), 2, 4no oggerixaga, 2,187 aienostema, 3,354 
irepinia aggregata, 2,450 Crepinia ambigens, 3,21 aginensis (Mip.),2,183

agpina, 3,92

aggrivaga, 3, 227

agraria (.tip.), 3, 31

-ogrestina (Crép.), 3, 31

agricolx, 3, 340

slanthoides, 2,338

alba $(2), 3,$.

albanorum, 2,446

Febarracini, 2,384

albereana (2) beaux),

$$
2,20
$$

folberti, 2,256

albidulat, 3,245

aldistigma, 3,52

albistyla, 2, 300

relforyitra, $3, g^{3}$

elbo-luteseens (Mip.),

2,229

sbula , 3, 286

abiena, 3,297

alligata, 3,168

tollionü, 3, 323

alnicolx, 3, 19

alpestricola , 3, 327

olpestris, 2,438

alpicola, 2,165.

alpina, 3,30

alpuina $x$ canina

(Réb), 3,242

Apiraga, 3, 280

altera, 3,3/2

foltobellii, 2,372

amabilis, 3, 393

Aomansii (Dis. ,2,183 ambigua, 3, 53

mulify $x$ cosn tix $2,4 \%$

anblyotis, 3,46

amblyphylla (Ilip.),

3,376

timbrosianx, 2, 214

amsenx,2,2g1

amoeneriens, 2,291

amoenicorona, 3,154

amoemiflora, 2,461

emonifolia, 2,4:7

amoenipetala , 3,152

cmphifola, 3, 340

mphriearpa, 3, 198

amphiloga, 2,215

anifhirhyncha, 3.176

amphiovena, 3,184

amphora, 2, 413

amphoricarpa, 3, 116

ampla, 3,240

smplioens, 3,135

amplifolia, 2,178

amplior, 3,10t

amplissima, 3,326

ampinscula , 2,191

amygoalinx, 3, 118

anacantha, 3,200

incucanpserss ,3,235

inalogx (Des.) , 2,212

inceps, 3, 307

ancoritand, 3,191

incy tacantha, 3,211

andalusica, 2,167

andegarens is (Dast. $, 3,6$ amvenipes, 3.247
Crepinid anviemaca, 3, 393

andeniacinsi:, 995

toncersaniana, 3,259

tonarassyana, 3,302

andoropogon, 3.239

ancrosacea, 3,206

anglica, 3,145

anglorum ,3,129

angoris, 3,125

angulosa, 2,392

angustifolia, 3,222

congustior, 2, 24g

angustituba, 3,41

angustippina, 3, 341

angustula, 2,204

anhelens, 2,479

anisotonta, 2,139

anisophloea, 2,417

Arrnonianc ( 5 uget),

annuens, 3, 10

anarmalis, 3,253

anteferrens, 2, 447

anthophora, 3, 121

antigua, 2,188

anticaria, 3,75

anseia, 2, 22

apalasyton, 3,270

apensuina, 2, 203

- Mertivens, 3,263

apertionum, 2,291

aprieacutax, 2,243

apriculata, 2.389

apostigma, 2, 438

appeniculata, 3,230

appenariclose, 2, 178 
Crepinia apposita, 3,45 Crepinia suturica, 2, 195 Crepinia Bavarica, 2,258

appropinopuata, 2,251

epproximata, 3, 198

aptata, 2, 33g

toquacesetiae, 3,98

aquilina, 2,409

araborum, $3,18 \mathrm{~g}$

aractunoidea, 3, 377

arougonensis, 2,222

aranyensis, 2,319

irbutifolid, 2,232

arcana (Disegl.), 3,361

Arcturus, 3, 210

areuata, 3,143

arelatensis, 3, 12

arenosta, 3,3

-argutiserrecta, 2, 258

arquta (Muss) ), 3,368

armata (Iter.) 2,300

armativerra, 2,241

armatissima Déségl.

$$
2,425
$$

Armitac (Juebs),

armoricana, 2,361

arnossensis, 3, 126

arrigens, 2,407

arvatica (Jakter) 3,3,30

aselchixived, 2,411

aspers. 2, 405

isperulasa, 3,28

-spernata (Dis.), 3,57

aspratilis (Grep.),3,57

assentiens, 2, 385

asseverans, 2,417

istrachanica, 3,266

astreata, 2, 254 atlantica, 3, 315

atrovirens, 2,235

attenuata (Mip.), 2,359

attenuatifolia, 3,175

augescens, 2,464

auctior, 3, 248

Aumieri (Cariot), 2.25

aurasiaca, 2,285

aurigerano, 2,418

aurita, 2,249

auroris, 3, 314

australis, 3,205

austriaca, 3, 114

touthemani, 3,106

avellana, 2,426

avellanxefolia, 3,388

axensis, 3, 210

azonica, 3,296

babensis, 3, 102

batica, 3,18

Bakeri, 2,271

baltica, 2.461

banflyens is, 2,307

Manksiand, 2,447

barba - Jovis, 3, 333

barbara, 3,58

barbulata, 2, 447

barbiseta, 3, 389

bareinanensis, 3,237

barsensis, 3,324

basaltici, 3, 109

fasilacens is, 3,24g

batarica, 2, 276

Scumiaritamana.

2,335 gravouxii, 3,5

bayomensis, 3,220

Belgiea, 2, 412

belgrabiensis, 3,290

bella, 2,305

bellarallis (Guget), 3,345

bellissima, 2, 463

bellojisensit, 2,194

bellula, 3, 385

belonioimorptha, 3, 194

benearnensis, 2,415

Benthamiana, 3,382

Berheri, 2.279

barnensis, 2, 376

Bertolamiana, 3,300

fiacuta, 2,201

biattenuato, 2,170

bicollis, 3,305

bicontracta, 2.441

Siebersteiniana (bratt.).

3,368

gienerti, 2,203

biformis, 3,246

bihariensis, 3,319

Bickiesii, 3, 301

bilingris, 2, 217

Gillotiana, 2,347

birostrata, 3,304

biserrata (Neiat), 2,453

biturigensis, 3.5

Givondeara, 3,25

blandiens, $3,-94$

blekingems is, 3.36\%

blephrielk 2,284

blepharapetaix, 2.380 
Crepuniar Blepharophyilla, 3,282 hepnsia brevepubens, 3,133 Orepiana calorhyncha, 2,223

Blepharoposa, 3,140

Bluffiana, 2, 460

Tolyttii, ? 223

bohernica, 2,309

Boitrayana, 2,357

bambaxe, 3,99

bambugella, 2, 416

bombyaina, 2, 192

Parbasiana, 3, 279

Gorideri, 3,165

borealis, 3,274

Boregchiana (Bess.), 3,362

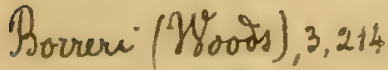

borussica, 3,67

fosmiacex, 3,224

botruoclada, 3, 168

brachiata (Dér), 3,358

frachyacantha, 3,191

brachycalyx , 2,227

brachyearpa, 3,172

brachycella, 3,264

brachyeacca, 3,26

brachyoion, 3,179

brachypood (2sis. R.), 2, too brachyphylla ,3,379 brachystema, 3,22 Practhystaglis, 3; 196 fracteosa (Gép) , 3,75 fractescens ( $98000-$ ),

biassensis, 3, 328

Braumi, 2, 214

bregillinsis, 2,430

brevearsnata. 3,155 brevicalyz, 2,322

brevicallis, 2,259

brevifolia , 3, 176

brevior, 2, 451

brevipila, 3,308

brevispina, 2, 426

brevituba, 3, 138

brevinscula, 3,387

Tringssii, 3,211

britannica, 2.291

Broteriona, 3,55

Protheri, 2, 190

buecina, 3,90

Buidapestina, 2,425

buidensis, 2,329

buridigalensis, 2, 179

burgoram, 3, 139

buxeigena, 2,199

cabulica (93oiss) ), 3,361

cacens, 2, 453

coesarangustana, 9,203

cassia $(4 \mathrm{~m}), 3,$.

calamaria, 3,21

calcarea, 2,231

Calsesï, 2,184

calestamiorum ,2,407

callicanthos, 2, 402

callichroa, 3,304

calliphylla(Tip),2,4ho

callosa, 2,222

calochlamys, 2,222

calochlora, 3,360

caloosonta, 2,224

calopetala, 3,273

calophytion, 2,375 colostephouna, 2, 468

calosphoera, 3, 182

caloxys, 3,212

calyeifera, 3. 42

calyeina (M. Buib) , 2,273

calyeschlamys, 3, 134

calyesgloba, 2,400

calypitocaly $x, 2,401$

calva, 2, 173

calvescens, 3, 160

camerina, 2,172

campestris (2umont.), 3,68

compestris $\left(y_{W}\right), 3,353$

campicola, 3,254

campiratga, 2,372

camporum, 2, 373

camputoclain., 3, 300

camnitodes, 2,178

comptomorpha, 3, 132

campylophylla, 2,20\%

canescens (Maker), 3,220

comina (L). 2,215

canina $x$ gallica (Krause), 3,374

Camina $\times$ Tamentosa

(Nitschke), 3,258

cantabrica, 2, 164

contalica, 3,137

capnotricha, 3,297

caprifica, 2,221

capromiana, 2,453

cardiophora, 3,163

Carestiae, 2:227

Cariomii (2isifi. et

$$
\text { Tisillot;, 3, } 182
$$


Crepinia Carioti (Chabert), Grepinia Cessacii, 3,345 Crepinia clavata (Crép), 2,283

\section{2,346}

carneiflora, 2,250

carmcipetala, 3,226

carpattrica, 2,475

carpathorum, 3,125

carpetanx, 2, 420

carpinifolix, 3, 317

corpinoides, 3,130

carpocyclar, 2,189

Carrace, 3,30

Carriezii, 3,104

Carthaliniar, 3, 9

Lasterneanx, 2, 273

castanuicola, 3,61

custanearfolix, 3,17

castellanx, 3,7

cetalaumica (Costa), 3,384

catulaunica, 2,182

causasice (MB.) 3,385

caisata, 3,59

caulifolix, 2,420

cebennensis, 2,365

celerata (Baker) ,3,384

celtiberica, 3,177

censix, 3,221

crasifera (Kern.), 3, 350

crasifera (Gimb) $, 3,350$

crasiformis, 2,89

cerasoides, 3, 217

cratophora, 2, 196 ceratophylla, 3,60 Creosiana, 2,465

cering, 3,285

cerino-alba, 3,332

cervina, 2, 456
Chaberti (Dis.), 3,81

Chabertioives, 3,48

Chaisiana, 3,966

chamaenespilus,2,456

Chapusie (Gren),2,242

Characias, 3,7

charybiaca, 2,274

Chaubardiana, 3,56

chersonensis, 3,147

chilistricha, 3,305

chlamyoódiena, 2,266

chlamiorocilyx, 3,356

chlarmyoosticta, 3,372

intoromelanos, 3, 186

chlorophana, 2, 4.15

cieura, 3,344

citiouris (9lilison), 9,390

cincinnata, 3,166

asierxteens (Cariot),

3,265

cinerascent, 3, 277

cincrea, 3, 367

cineressula, 3,157

cinerose (thes) , 3, 265

annamomicolor, 3,29

citata, 2, 433

cistatorm, 3,281

ciris, 3, 28

ciritatis, 3,302

clasobotrys, 2, 351

clavocampta, 3,31

cladoleia (Mip),2.35y

clasophora, 2,265

claram -tana, 2,444

claugisjotitana, 3,325 clarigera, 3, 115 claroides, 3,177

clinacantha, 2, 458

clinella, 2,292

chinophlaea, 2,203

chirorum (Schenty) 3,352

chpeolaria, 3, 37

cootunata, 2,184

carretans, 2, 330

Cosormii, 3,57

coerulescens, 2,279

cognata, 3, 148

collaris, 2, 168

Collieri, 3, 186

collina (Joeeg), 3, 346

collincola, 2,265

collocato, 2, 222

colorans, 3,283

colorata, 2, 194

columnifera (Fries),

3,204

comis, 2,430

comitata, 2, $32 \mathrm{~g}$

commiserata, 3,234

comosa, 3, 391

compracta. 3,155

Companyoü (Deleaux)

Companyonis, 2,24,2

compressispina, 2,2g8

concolot, 3,266

conidensata (Muget), 2,192

condigns, 2, 420

conperta, 2,428

comfertiflora, 2, 426

comfortifolix, 2,298 
Crepinia confertior, 2,193 Crepinia coniceas (Crépin) Crepinia crinits, 2, 399 confivens, 2, 4th confinis, 3, 231 conflictata, 3, 41 confusa, 2,367 conglofata, 3,240 congruent, 2,238 comica, 3,354 conifer $x, 3,89$ comimbricensis, 2, 351 conoacantha, 2, 463 conolasis, 2,337 conocarpa, 2,175 consitea, 2.421 conomorpha, 2,365 conophora, 2,381 consobrina, 2,2g3 consociatio, 2,463 consolation's, 2.208 consolicata, 2,404 constricta, 3,315 continens, 3,176 contorta, 309 converniens, 3.294 convergend, 3, 157 conversor 3,157 converula, 3.181 conyzoices, 3,335 cooptata, 3,276 corbariensis (Debeaux).

$\operatorname{Cos} \alpha, 2,213$

cordati, 3,go corbifalia, 3,19 corvigera, 2,244. coridubensis, 2,392

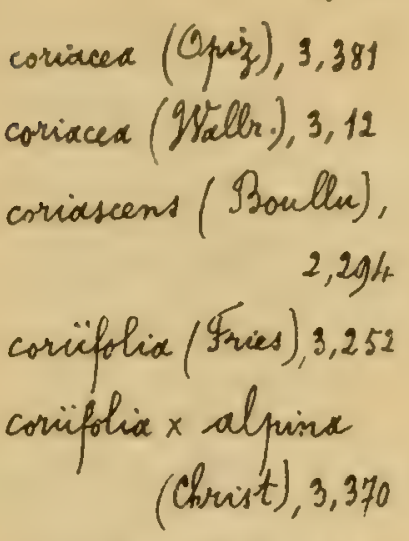
cormubiensis, 2,163 coranata, 3,254 Corvaliana, 3, 136 corylicola, 2, 173 coryloides, 2,247 corymbifera (Borkh), 3,241

corymbulos $x, 2,302$ coennorarifa, 2, 250 cosmophylla, 3,86 Costace, 3, 131 Crantzii (Ichultes),

crassifolia (Wallm), 3,253

orassidens, 2.217 crassinervia, 3,103 crassior, 2,170 crassifues, 3, 71 crassissina, 3,272 crassinescula, 2, 186 crataginaria, 2,204 cremoclada, 2,165 cretica, 3,232 Giacanthe 12,278 cribrose, 3,256 crimisepala, 2,370 crispetula, 3,114 cristulata, 3,253 croatica, 2,292 civentata, 2,267 cryptostylis, 3,343 csepaliensis, 2,256 csucsensis, 2,301 culmosa, 3,277 culprata, 3,281 cultellata, 9,39 cuneata (Chastaingt), 3,44

cuncata, 3,66 cunvibatis, 3, 122 cuncifolix, 3,183 cunciformis, 2,465 ansinetrala, 2, 169 Cupariaina, 3,124 curticola ( Suget),2,384 curtifolia, 2,434 curtipes, 2, 448 Curtisian a, 2, 395 curtispina, 3,233 curridens, 2,208 curvispina, 2,257 curvila (Gimb), 3,253 cyanocalyre, 3, 134 cyanoclada, 3, 285 cyanofioda, 3,260 eyclobasis , 2,214 cyclocalyx, 3, 35 cyclocarpa, 2, 189 cyelosonta, 3,240 cycloidex, 2, 35 g cyclomarpha, 3,14 
Crepinia ugcisphylla, 2,421 Crepuicia denticalycina, 3,291 Crepinia gimitiens, 3, 178.

cylinirocantha, 2,359

cylintive, 3,333

cyliniarocarpra, 3,324

cylinionorhyncha,2,358

cymigera, 2,208

orikensis, 3,263

galmatica, 3,246

damnabilis, 3,287

Lanica, 3,148

Lambialis, 3,279

Dasycalyx, 3,361

Sasycarpa, 3, 216

gasystyla, 2, 195

Sasyphora, 3, 183

Dasyphylla, 2,202

Dasypogon, 3,266

Davisiana, 3,117

iebeauxii, 3.60

decalvans, 3,373

secalvata (Crép.), 3,89

decepta, 2, 42g

secisux, 3, 146

iecipriens, 2, 186

secumbens, 3,83

Decurrens, 3,136

oiecurtata, 3,166

Dejotata, 2,220

Oseficiens, 3,397

delectabelis, 2, 176

Deleta, 2, 312

Peliciosa, 3,22

Delileand, 2,378

Gohminensis, 2,258

iensifolix, 2, 405

Densiuscula; 2,209
Dentifera, 3, 225

Sentisepala, 3, 167

gentosa,2,265

genuidata, 3,376

Depressa, 3,253

Derbesiana, 3, 122

Deseglisei (Mor.),3,345

Desiserabilis, 2,219

Desolata, 2,321

gespricata, 3,378

Deswanxii, 2,223

Setracta, 2, 217

getruncata, 2,407

Dencalion, 3, 56

Seriata, 2, 199

Devota, 2,348

giachylon, 3,61

dichrofhylla , 2, 450

gieranoclaba, 2, 317

Dicranoibes, 2,409

Lichthii, 3, 158

xicthyocliera, 2,177

gicttyodeniron, 3,26g

bicthyoies, 3, 3401

Dicthyophora, 3, 201

gidoensis (Isaiss) , 3, 27

disymocarpa, 2, 385

dilumovonta , 3, 121

dicymopoda, 3, 70

giaynorimyncha 2,167

ciaymoxis Goop et

sebeasse, 3,214

Differins, 2,198

Gifficilis, 3. 244

giluivex Mos. Os. ), 2,196 giminuta, 2,218

Dimarphacantha, 3,82

Dimoriphofisis, 3, 2go

Dimorphacarfio, 3, 241

Dimorphocycla, 3,27

Sinariea, 2, 478

Dismesis (Grex) , 3,58

Dioxcys , 2, 315

Diphtheritis 2,266

discolor, 2,370

Piscons, 3, 380

giscostyla, 2,370

Discursira, 2, 171

Disparilis (Encand),

2,285 et $3,3 \mathrm{~g}$

Pissidens, 3,285

jissimilis, 2, $38 \mathrm{~g}$

sistentissima, 2,324

Tisaricata, 2,26́

giversidens, 3, 142

Dinerspina, 3, 82

gobokensis, 2,324

Dodonacana, 3,50

dolabrifolia 3,29

Dolata, 2, 403

oolenia, 3.97

golosa (Gooet), 3, 36

Lommartini, 2, 406

Dambensis, 2,350

Dimiand, 2,29?

Dormiensis, 2, 169,

Urepanoton, 3.46

sryagea, 3, 335

vinumophila, 2,293

Jubic irast.) 3,8 
Crepinia Dnboselardii, 2, 320 Crepinia eivocenda, 2, 360

Dufftii, 2,274

sumalis (Bechot), 2, L.27

isunatioides (Muget),

$$
2,475
$$

Dumetoides (Muget),

$$
3,273
$$

Dum toroides (Guip.),

$$
3,116
$$

goumctirum ("6huill).

$$
3,258
$$

Dumortisi, 3,68

Sumosa, 2, 220

gurd, 2,200

Iurriaena, 3,6\%

gurifolia, 3,63

Gawriformis, 2,202

Durispina, 3,246

Inriuscula, 2, 202

iysphoroidex, 3, 10

Syscoulon, 3,339

Iysclados, 2, 405

aysphorica, 2, 270

Aysthamnos, 2,318

eboracensis, 2,289

eceremoclada, 3,290

eccremophlaex, 2, 440

Gcheaniviana, 2,234

Echidne; 3,353

echonopota, 3,334

eximburgensis, 2, 166

esita (Diseigl.), 3, 6

ésita, 2, 387

exiticaulis, 2,287

editicola, 2, 390

Sitorum, 3,20 ensecta, 9,283

évecta, 3,24

Eunensis, 2, 392

esuresuns, 3,3

effecto, 2, 358

efferta, 3,9 \%

efficax, 3230

ifficas, 2,422

eflansulose, 3, 219

clegars, 3: 233

eleventula, 2,2:89,

clesata, 3, 1 \%

Chisabinopolensis, 3,115

elisantha, 2,240

exisocalyse, 2,479

elisophylla, 3,200

elisothora , 3, 212

elliptica, 3,135

elongata, 2,338

emaicinata , 2, 4.49

emiliensis, 3,229

eminens, 3.373

empita, 2,340

enormis, 2,337

epimetium 3,222

epipactis, 2, 451

erecta, 3, 132.

erectisefiala, 3.74

erectiuscula, 2,286

eremacharis, 3,39

ricctorum, 2,262

eriogyna, 3,360

eriostyla (Disegl. et Thip. $, 2,468$

errabunsa, 3,338
Crepinia erratied, 3,168

erronea, 2,205

erythreantha (Mor). 3,219

erythrella (Mip.), 3,21

erythrocarpa (Mip2),2,275

ery tir opeta. I, 3, 178

scurialensis, 3, 63

ctrusca, 3,170

encharis, 2,417

enisithyon, 2, 288

suncerte, 3.34

eños co, 2,355

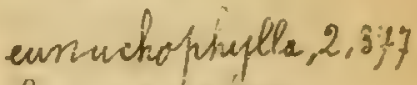

\&unajale, 2, 156

cusynphyella, 2:361

evagatix, 3,323

evecta, 3,261

evidens, 2,287

exalbata, 3, 293

exarmata (Gép), 2,188

excerrans, 3, 225

exhausta, 2,327

exilis (Crép. Wirtg.), 2,231

exoptata, 2, 23y

expallixi, 3,31

expansa, 2,396

explanata, 3.9

exsertistyla, 2,237

exsul, 2,381

exsurgend, 3, 391

extranea, 3,9

fabactolia, 2, 44.

fagifolia, 2,287

falcatula, 2,255

ackidenta, 3,268

falcifera, 3,35 
Crepinix falcinella, 2,386 Crepinia firso, 2,452

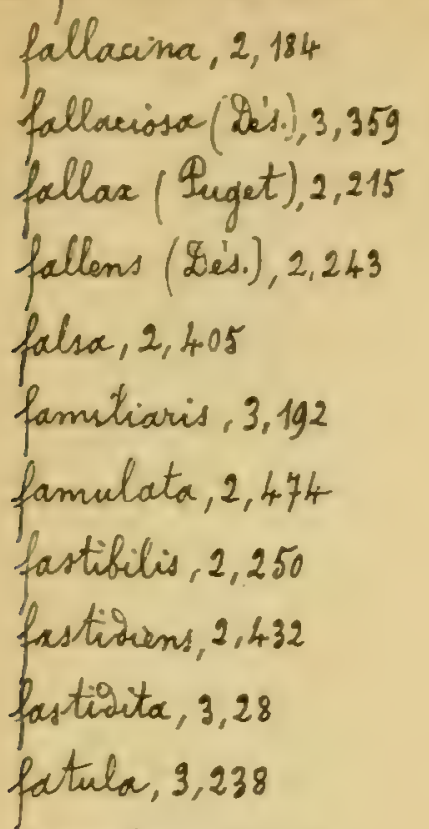

fistidens, 2, 217

fissifotix, 2, 192

Crepinix fuburgensis, 3, $14 \mathrm{k}$

Friedanderiana (Sess),

fissisfuna (MSierzb.).

3,368

Fresii (Lagger et Lug.),

2,427

3.219

flatellata, 3,332

thabilis, 2, 327

flagrans, 2,336

flavescens, 3,310

flavioula,2,335

Harispina, 3,146

flexibilis (Dérégl), 2, 162

Hexibilis, 2.299

farentina, 2,219

Farrati, 2, 174

feherensis, 3,271

Lelix, 3, 101

Sennica, 2,194

ferens 2,24g

Fernaniezii, 3,49

feros (Chabert), 2,282

fertilis, 2, $46 \mathrm{~g}$

Lestinata, 3,184

tosting

ficaides, 3,107

ficta, 2,314

fictilis, 2,466

fiselia, 3, 327

filamentosa, 3,336

filiformis (Ozanon), 2,182

flexicaulis ( $h_{c}^{\prime}$ p. $), 2,363$

flexilis, 2,29g

Hexiramea, 3, 173

foinarum, 3,112

foetos $\alpha, 3,13$

foliocalyze, 3,267

foliolosa, 2,260

folingera, 2,190

Fontancsï, 2,210

foresiaca, 2,211

forgesti, 3, 144

formicata, 3, 179

Forsteri (Im.), 3,169

froniose (9tas.) , 3, 100

fruteticola, 2,233

frutetorum (Soss.),

3,213

fruticetorum, 2, 466

funesta, 3,145

furcellaria, 2,183

furtiva, 2. 335

fusifera, 3, 299

fusiformis (Chabert),

3,115

Gabuelssonii, 1,302

gallica $\times$ biserrato (Christ), 3,84

gallica $x$ canina

$$
\text { (Reut.), 3,83 }
$$

gallica $\times$ gumetorum

(Christ), 3.359

gallica $x$ oumetorum $x$

obtusifolia (Christ),

3,362

fortis, 2.379

forticita, 3,172

Silisfind, 3, 355

filopes, 3, 133

fint.

finitiona, 2,186

finlanicica, 3,308

firma (Suget), 3,66

firmifolia, 3,42

firmiot, 2, 34.

firmula (Gicet) , 2,165

fracta, 2,317

fragilior, 3, 388

fragilis, 2,374

Fraseri, 3,386

fraternx, 9,478

frasinoides, 2,462

Sreynï (Gommasiri),

3,150

gallica $\times$ lutetiana

(Christ), 3,83

gallicax obtusifolia

(Oborny; Baenitz),

3,363

gallinaria, 2,418

galloccica, 3, 106

galloprovincialis, 2, 422

Ganteri; 2,459

Ganoogeri ( $\left.9_{\text {uget }}\right), 2,171$ 
Grepinia Garumnac, 2,253 Grepinia glancescens (20sv.), Grepinia grañissina, 2, 295

gastroivea, 2,300

goundens, 3, 20

Gacisimianx,3,197

gauciosa, 2, 213

Goution: 3,352

gavista, 3, 321

Gelmii, 2, 415 generalis (Chastaingt),

gencrensis, $3,2 \%$

genistacola, 2,320

Gensiarie (Huet), 3,87

geophila, 2, 476

georgiea, 3,199

geracantha, 2,329

geraphyll a, 3, 168

Gerardiana, 3, 107

germana, 3,55

geruniensis, 2,389

jibfosa, 2,298

Wibellie, 3,97

jlabella, 2, if

glaberrima (Iumort),

2,346

glabrata (kit),2,171

glabrescens, 3, 122

ylabrior, 3,316

jlabristyla, 3,5

alungulidens, 3, 299

danonlifera, 3, 284

glanoulinaeva, 2,200

glanisulisepala, 2,252

glanisulosa, 3,87

glandulosella ,3,135

glan

glanca (Ychott), 3,68

glanceseens ( 92 ess.),2,466
2,223

glancifolia (0piz), 2,434

glancina (Mip), 2,452

glauciramea, 3,235

olancocarpa, 3,8

glanco proda, 3, 298

glancorubra, 2,435

glaucovirens, 3,54

globatoc (D\&'s), 3,107

globosa, 2,289

globularis (Eranchet),

2,246

globulosa, 3, 257

gloniosa, 2,461

gnaphalodes, 2,267

gnara, 3,56

Gorionianx, 3,98

goetchorgensis, 3,344

gonisearpa, 2, 180

goniogonto, 3,336

gomiophylla, 3,31+2

gossypina, 3, 397

gothica, 2,295

gothica (Ninsl.),3,299

gracilenta, 3, 195

gracilicanlis, 2,437

graciltuba, 2,230

gracilior, 2,249

graciliramea, 2,296

gracilis, 2,188

gracilispina, 3, 11

granatensis, 2, 34.7

grandicoronia, 3,179

grankidentata, 3,54

jrandiflor $x, 2,309$ grasiticx, 2, 183

frato, 2,432

gravisensa, 2,271

gravis, 3, 186

gregarix, 2,425

Gremblichii, 2,457

yrisescers, 3, 208

grossularioides, 2,287

Guxdalajara, 3, 2.06

Guadarramae, 2,236

Emepini (Dlesv.), 2, 380

Guilloti, 2, 216

Giviraiana, 3,62

Gussoneana, 2,374

gymnophlaed, 3,17

gysmnostigma, 3,209

aynoleix , 2, 161

Haberiana (Muget),9,66

haemantha, 3, 110

haematoses, 3,57

Hailstomi (Macker),3,80

hallanisica, 2,300

hallensis, 3,268

hamata, 3, 134

Hanausetiana (Keller),

2,442

hanonensis, 3,236

hansverana, 2,991

Hansyiana, 3, 119

Harborsii, 3,388

haromizekensis, 3, 314

has achetensis, 2,338

Haussknechtii, 2, 426

Haussmanmiana, 2,382

hebechlamess, 2,33: 
Crepina helagyna, 3,14 nefescens, 2,430

hebetata, 2,283

Heanigare (93lacki),3,15i

Hermerlie (H. Iroum),

3,366

helvetica, 3.217

hemiádenc, 3, 2irg

hemicycla, 2, 288

hemiglaucos, 3, 137

hemitricha (Mip.).

2,376

Henrici, 2,35\%

heteracantha, 2,464

Herbichiana (Blacki),

heterocarpa 12,191

hoteromorpha, 3,187

heterooon, 3,288

heterostigma, 3,20k

hibernorwm, 2,459

Hilsebranstii (Wein

$$
\text { tenweber), 3,380 }
$$

Hirsuta (Des.). 3,30

hirsutissima, 3, 307

hirta, 2, 4r52

hirtella (Rip.), 3,2

hirtistyla, 2,450

hirtipes, 3,248

hispanica (Boiss. et Dent.), 3,75

hispancia (Mill) 13,256 hispicula (Rip.),3,9g hispiciscalyx , 3, 362

histricoso (Gép.), 3,66

hololenex, 3, 378

holopetala, 2,401
Crepinia nolophaer, 2,370

holophylla, 3,269

holonoida, 3,249

Holubyi: 3,237

Hookeriana, 2, 476

horridula (Dis.), 2,282

Hubsoniana, 3,38

Hulsenii, 3, 322

humcampita, 2,434

toumilis (3ess.), 3,363

Huncariale, 2,333

hungarica, 2, 368

ityetemi, 3,264

hymenodes, 2,210

humyadentis, 2,323

hypochionaex, 3,239

hyprehlora, 2,281

hypocyela, 2, 445

hypoglanca, 3,348

hypoleuca, 3,289

hypotricha, 3,209

iberensis, 3, 146

iberorum, 9, 152

iimaiophyylla, 2,284

icogloss , 3, 192

itanensis, 3,7

idonea, 3, 292

ignobilis, 2,306

Yesefonsii, 2,396

ilioatorum, 3, 181

illecebrosa, 3, 139

iflecta, 2,454

illibata, 2, 310

itligata, 3,120

illinella, 2,408

illuivens, 3,356
Crefimia Dthstrata, 2,279

initata (Dis.), 3, 180

immutata, 3, 280

impracatix, 3,303

impaciosa, 2,333

implexa (Gren $), 3,133$

implicata (Tuget), 3, 35s

impolita, 2,248

imponens, 2, 454

improbata, 2, 318

improrita $a, 3,208$

incequalis, 3, 123

incerqueserrata, 3,94

incequidens, 2,427

inanis, 2,218

incerta (Dis.), 3,365

incita, 3,19

incisifolia, 3,100

incompta, 2,266

inconcessa, 2,234

inconsiderata (Deiseigl.),

3,75

inconspicua (Déségl.),

3,52

incrassans, 3, 306

incurva , 2,252

insecarx, 3, 386

indifferems, 2,406

insomita, 2,213

insucta, 3,387

inermifolia, 3, 328

inermis, 2,302

inescato, 2.418

infatuata, 3, 113

infelix, 2,460

infibulata, 2,427. 
Gepimia inflata, 3,151

infracordata; 3,207

infraglauca, 3,160

infundifuliformis, 3,246

ingenue, 2,402

snnocua (Mip.), 2,475

insidiosa (Rip), 3, 83

insignis (Sip) 2,462

insolata, 3,71

insularis, 2,393

insulsa, 2,162

intacta, 2, 173

integrata, 3,233

intenta, 3,237

intermedia (Kit.), 3,288

interobseura, 2.336

intersita, 9,379

intervallata, 2, 245

intertexta, 3,336

intervenciens (Déségl.),

3.18

intricata, 2, 206

involens, 3, 158

involucrata, 2, 264

iosophylla, 3, 260

Irpex, 3,26

ischnoclata, 3,99

ischnoteniron, 3,33

iserientis (Muget), 3,346

isotes, 2,392

istriaca, 2,306

italica, 2,239

iterata, 2, 238

Jacsbi, 3,378

Jacouimiana, 2,230

factans, 3,327

Crepinia jactata (2és) , 3,125 Crepinia tresiramea, 2, 174

Janus; 3.260

jomides, 3, 311

juidicabilis, 3, 163

jugularis, 3, 281

juncta (Luget), 3,138

jumperorum, 2,193

justa, 3, 120

jurenilis, 3,310

Kabathi (tonsorge),

3,370

Kabylica, 3,291

Keckii, 2,375

kerekenvis, 2,351

Kerneri (H. Iramn),

3,350

Kenneriana , 2,414

Kinnetiullensis, 3, 318

Kitaibeliana, 2,180

Kmeti., 3, 305

kolozsensis, 2,320

Kosinsciana (Bess.).

3,37

Kovatsian $\alpha, 2,377$

Krausii, 3,371

Krockeri (Eratt.), 3,252

kullabergensis, 3,88

labrellata, 3, 186

laburnifolix, 2.368

lacertosa, 3,103

lacessens, 2,433

Lacoizquatae, 2,388

lactaglancescens, 2,381

lacterubens, 2,44t

lacterciens, 2, 303

lavipes, 3, 5 k lacris, $2.26 \mathrm{~g}$

laesissima, 3.22

laeriuscula, 2, 214

lagenaria, 3,229

lagenoides, 3, 232

laguncula, 2,362

Lamatti, 2, 236

lampocseca, 3,245

lampophinglla, 3, 238

lancarfolia, 2, 314

lancaeformis, 3,79

Lanceslata (Opiz), 2,493

lancifolia, 2,297

Langeana, 3, 159

Lannesiana, 2.207

lanuginosa, 3.316

Lapeyrousiana, 3,278

lapidicola, 2, 271

Laristamica, 3, 12

lasiophylla, 3,358

latefrans, 2, 209

latebrosa (Dés.) , 3,81

latesentata, 2,2/8

latens, 3,5

latepartita, 1,333

latifasis, $2: 351$

latiflora, 3,91

Catifolix, 3, 166

latipetala, 3,128

latipinmuls, 3,292

latisepala, 2, 460

latiopiona (Boiss), 3,365

latistipula , 2, 423

lauta, 2,328

lase , 2, 4,20 
Crepinia laxapilosa, 3,143

laxifolia, 2, 371

laxipula, 3, 179

laxistyla, 3,70

lascula, 3,96

Legallii, 3, 69

bainen

ledionensis, 2,349

legitima, 2,235

leiocarpa, 3,352

hiscklamys, 2, 272

lioclado, 3,382

leiophlaca, 2, 444

levisepala, 2,362

leiostigma, 3, 337

leiostyla (Trip) ,2,348

Lejeumi (Orép.), 2,424

Lemaitrei (Mip.),3,32

lemoricentis, 2,923

lenis, 9, 156

lenta, 2,254

bentula, 3,51

Lesntomyx , 3,227

lespohinsis (Mlacki),

\section{3,215}

Penida, 3,45

lepivillora, 2,353

lepicota, 3, 152

leporina, 3,76

leptocalyge, 9,324

lentocar ha, 2,363

lentoclacia, 2, 383

lertorias, 3, 16

leutiphloex, 3, 141

leptostephand, 3,248

leptosticta, 2,398

leptoticicha, 3,270
Gepinia Lerefrii, 2, 163,

Lereschii (Christ),3,390

leveantha, 3,218

lencantha (2ois), 3,231

lencellac, 3,218

leucoacantha (Deleaux),

leucochlora, 3,212

leucographa, 3,330

lenconoma, 3,116

lencophora, 3, 283

lencophylla, 3,298

lenesstigma, 3,192

levcostyla, 3,109

lencotricha, 3,161

Leveillaei (\$2oullu),

3,362

Leyboltiona, 3,141

Levi, 2,176

liberalis, 2,315

libertina, 3, 112

Lightfortiano, 3,299

limitanea (Gép.), 3,81

limata, 2,325

liptensis, 3, 325

litigans, 2,165

litigiosa (Grep.), 3,3

litterata, 3,375

livonica, 3, 116

Lloygü (2⿰冫广). , 3, 350

lobulata, 3,384

localis, 3 ,

locata, 3, 321

locsensis, 3,97

Loeflingiand, 2,382

Lojuconor, 2,228

lom, $x, 3,144$
Crepinia Conchitis, 3,236

lonisinensis, 3, 161

longa, 3,11

longecoronata, 2,328

langeasiscos $\alpha, 2,261$

longifolix, 3, 304

langiox, 2, 197

longiramea, 2,375

longiseapa, 2,278

lomgispina, 3, 183

longissina, 2, 442

longistyla, 3,219

longituba, 2,306

longuiscula, 3, 248

Qoretiana, 3, 389

Loscosiana, 2,2g2

Qucanisiana (Déségl. et Gillot), 3.379

lucoum, 2, 996

lucratia, 3, 315

heromiensis, 2,360

lidens, 3, 185

ludibundax (Gren.), 3,30

Lusovici, 3,79

hugaunensis (bimeroy),

lunata, 3,265

Lunioquistrii, 3.242

Lupheana, 2,366

lisitamica, 2, 355

lustrata, 2,381

lutescens, 3,264

lutetiana (2em.),2,183

lupulina, 2,258

luxembirgiana (Grép.)

2.446 
irepinia lnxuriosa, 3,126

Maasï, 3, 196

macranthax (2lesportes),
3,369

macroacantha (Rip.),

$$
2,368
$$

macrocalyze, 3,200

macrocarpa (ho piss.),

3,343

macrocarpa (Merat),

$$
2,468
$$

macrochlamys, 2,287 inacrotisens, 2, 419 macroton, 3,33

macromegas, 3,44 macropmylla, 3, 114 macrasepala, 3,289 macrosphaer a, 2, 169 macrastigma, 3, 220 macrastylis, 3, 152 macrotricha, 3,317 maculisparsa, 2,206 matriensis, 2,308 Masomiac, 2, 259 magna, 3,8 Nigmeri; 2, 160 magnifica, 2, 411 magnoliafolia, 3,96 magyarica, 2,414 major, 3,303 majuscula, 3,46 malacotricha, 3,274 malmungariensis

$$
\text { (Lej), 2, 400 }
$$

mamss, 2,270

manca, 2,233
Crepinia manceps, 2,340 Crepinia megatricha, 2,308

Manionir (2)és.),2,435 melancholica, 2,22g

manens, 3,163

marcescens, 3,30

Marcailhowi, 3,157

marensis, 2.337

Marchantiana,2,428 marillana (9oorfias),

Martini, 2,366

melanochloros, 2,223

melanoidea, 2,280

melanophylla, 3,289 melastoma, 2,216

mentacea (9uget), 2,316 merioionalis, 2,253

mersa, 2,470

mesocarpa, 3.122

mesoclada, 2,455

mesocycla, 2,445

mesamorpha, 3,221

mesoneurot, 2,165

mesophylla, 3,308

mespeota, 2, 193

mesostema, 3.26

mestanensis, 2,272

Micheliane, 3.142

Michaielis, 3,323

micradena; 3,76

microdeantha, 2,369

micrabasis, 3,227

mierocalyse, 2.358

microcappa, 2,247

micrachada, 2,248

mediterranea, 2,391

megacantha, 3,389

megalocarpa (2vis), 3,343 megalocar por, 3,163 meganeura, 3,297 megabphylla, 2,209 mega poosa, 3.326 megalosepala ,2,383 megalostigma, 3,263 megalostyla, 3.15 megastyla, 3,171 miciacocess, 3,138

micromegas, 2,247

micrapappa, 2,263

microphylla, 3,284 mierarhyncha, 3,35 micostyla, 2,165 microtuba, 2, 475

micrsphicara, 3,109 militans, 3,196 smiroifea, 2,278 
Crepinia millefoliata, 2,350 Crepinia mucronata, 3,385 Ciefinia nemausensis, 3, 94

minor, 2,290

minuscula, 2,281

minuta, 3,136

minutifolia, 3, 382

minutiramea, 1,358

minutispina, 2, 185

minutula, 3, 173

miraniox, 3, 153

miscens, 3, 154

misella, 3,199

misera, 3, 13g

mitigata, 2,297

mition, 2,445

mitiramed, 3, 156

mitis, 2, 270

mitodcantha, 3,382

mitoclada, 2, 235

molesta, 2,247

mobica, 2,175

moerens, 3, 100

moesta, 3, 292

Momnetierensis (Mouvier), 3,314

montorna, 3,167

monticola (Mapin),

montivaga (Dése'st.),

Montsuiana (Chabert),

3.114

Morocer (Crép.), 3,216

moravica, 3,78

Marelie, 3,11

mortaniensis, $2.36 \mathrm{~g}$

onosqueruclensis, 2,278

Motelayi, 2, 314

movens, 3,271 mueromulata (Deségl),
2,165

muletra, 2,332

multicers, 2, 322

multiglandalosa, 3,361 multimervis, 2, 429

multispina (Puget), 2,318

multivaga, 2,412

munita, 3,278

murcica, 3,972

muricata, 3,278

muscaria, 3,63

muscescens, 3,98

mutata (Rip.), 3,80

mutinensis, 3, 140

myacantho, 3, 178

empriocarpa, 3, 25

myiriodon, 3,87

myzriophylla, 2,270

myriosticts, 2, 397

myriotricha, 2, 431

myrophylla, 3,4

myrtillsides, 2,282

myrtaides, 2,273

moerulifolia, 2,205

Sacias 2,354

marbonensis, 2,423

nava, 2, 4,77

mavarrensis, 3,95

nebrodensis, 2,274

Neibreichianx, 3, 145

nematella, 3, 381

nematochroa, 3,35

nematostipula, 3,29 nemtcharis, 3,238

nemophila (Mìs.), 2, 16?

norrifolia, 2,307

nervigerd, 3,198

merrosa (Crép), 5,377

nervose (guget), 2,356

newrulosa, 2, 406

nesciens, 2,182

neuroides, 2,462

newrophyllix, 3,254

nevacensis, 3,77

nexa, 3,297

nesibitis, 2,387

nitulans, 2,319

nigreseens, 2,280

migritella, 3, 291

Misus, 3, 194

sitens (Desr.) 2,175

nitioula (P2ess.), 3,65

nitisula (16. \$3).,3,368

mivescens, 3,334

normalis, 2, 292

Notarisiana, 3,170

notata, 3,216

nova, 3, 359

novella, 3,89

nubilis, 3,262

miva (Hooss),2,242

nuivicaulis, 3, 113

milifes, 3,24

inimidica (Gren.),3,351

mummularioides, 3, 105

Nymamiana, 3, 276

obattenuacta, 2,181

obeocidata, 2,330 
Crepinia uhmoides, 3,71 ullulans, 2,389 ultiona, 3,51 umbilicata, 2, 248 umbraticola, 3,22 umbrigend, 2, 206 umbrina (Muget), 3,118 unibros $\alpha, 2,409$ uncata, 3,92 uncinella (\$2ess), 3, 124 uncinellocides (Guget), 3,187

unainula, 2,234

inicolor, 3,47 urbana, 2,274 urbica (2em.), 3. 199 urbicivioes (Grep.), 3, 150 usmula, 3.807 utingera, 3, 312 Utefke, 2, 360 vacans, 2,397 vaccinisides, 2,232 rarrendice, 3, 271 Nafia, 2,390 raga, 2,393. Nagensis, 9,175 Gagiana (Crép). 3,383 raginata, 2, 451 valachica, 2,167 valtefoliosa, 2,179 volde puiferis , 2, 428 valentiva, 3, 64 salisa, 2, 372 vallesioca, 3,176 vallicola, 2, 198 vena, 2,238
Crepinia Yapullomii, 3,244 rariabilis, 3,338

varians, 2.477

rascomica, 3,130

rascula, 3,95

veudensis, 3,174

Layresac, 2,243

vellicata, 3,284

vensibilos, 2,312

remins, 2, 309

renix, 9, 149

senosa $\left[I_{w_{0}}\right], 2,407$

Neprecularum, 3,248

vepraticola, 3,391

verax, 3,268

verbascina, 3,313

vabascoises, 3,262

verecunde, 2,24

Nor.w: 3,24

Gerbloti (Grép.), 3,78

veromansuorum, 3,118

versuto, 2,262

verticillacontha (Misat), 3,65

verticillaris, 3,259

vestita, 2,332

vetula: , 2,301

vesata, $3,33 \mathrm{~g}$

vexionensis, 2,296

mialis (2ie. Rip.), 2,247

vinum, 2.199

ricana, 3, 7n

vicermalis, 2,458

vicinse, 2,377

vilis, 2,3/3

Sillarsiana, 2,244
Crepuinia villigera, 2,431 rillimacere, 3,92 rillipes, 2, 474 villistyla, 3, 384 villosa $\times$ canina ( $(2 \mathrm{chb})$, 3,346

rillosinscula (Mip.). 1,376

rimminalis, 2, 181

rinacea (IBaker), 3,73

rimidex, 2, 395

singolonensis, 3, 181

vingicans, 3,13

rinealis (Mip.), 3,23

vinosa, 2,257

violascens (Mugst), 3, 158

rivens, 2,278

vireticola, 3,194

viretorem, 2,272

ningata (Gromli), 3,68

sirginea (Mip), 2,382

rengrsax, 3,242

siridicata (\$uget),

viribiglanea, 2,393

rividis, 2,32i

rise, 2,335

visentox 2,320

Gisiamiana, 2, 185 ristalensis, 3,270

vitivaga, 2,371

virifica, 2, 480

voless, 2,480

volmyrice, 2, 339

volite, 2, 390

volutans, 2,306 


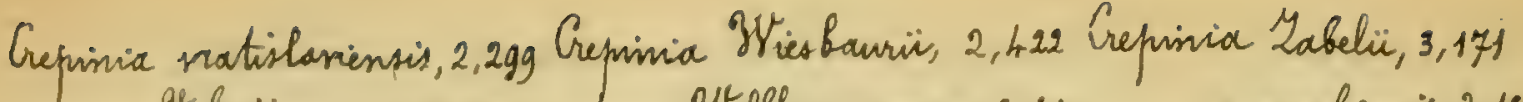

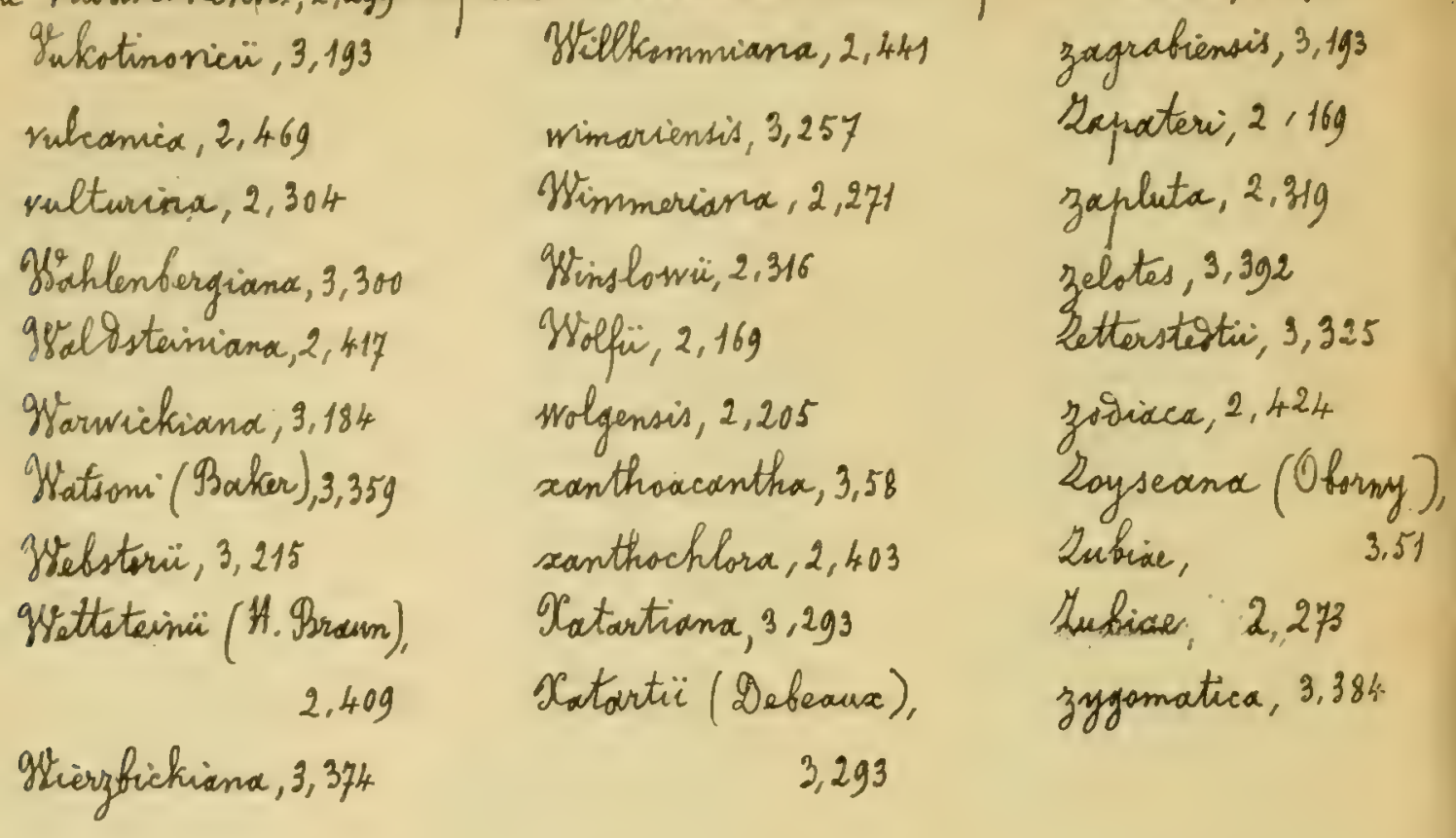

Yrdex revum tomi III.

Adenophorae Gogr, 329

Caninear Collinae Gep., 3146

"Hispiase Crép., 1

- Pubuscentes Criep. 85

" Gomentellar Cripo, 367

Decalvatac Gogr, 85 Euansegavenses Gyogr, 1 Eupubescentes Googr, 85 Naricidac Gogr, 16 Mixtae Gigr, 357
Osmophyllae, lygar, 49

Silasae Googr, 201

Gilosiuscular Gogr, 127

Recidentes Gogr, $32 g$

Senvi-glabrae Gogr, 108

Golstitiales Giogr, 250

Stictopotac Gigr, 1

Enchophyllae Googe, 84

Urbicas Goge, 164

Finis tomi tertic. 




QL 145. R6 G29 vit 3

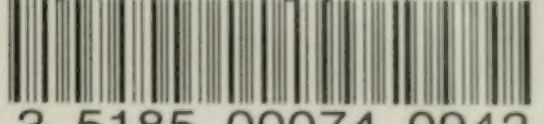

35185000740942 
